\title{
Formal Synthesis of
}

\section{Salinosporamide A}

Isabel Villanueva Margalef

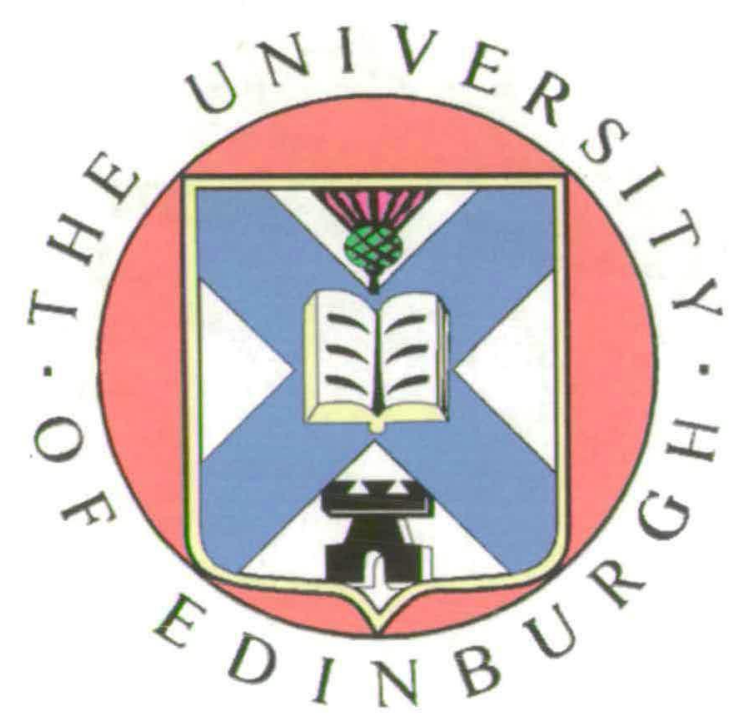

A thesis submitted for the degree of Doctor of Philosophy

The University of Edinburgh

February 2008 


\section{Canción tonta}

Mamá.

Yo quiero ser de plata.

Hijo,

tendrás mucho frío.

Mamá.

Yo quiero ser de agua.

Hijo,

tendrás mucho frío.

Mamá.

Bórdame en tu almohada.

¡Eso sí!

¡Ahora mismo!

Federico Garcia Lorca 


\section{Declaration}

I hereby declare that, except where specific reference is made to other sources, the work contained in this thesis is the original work of my own research since the registration of the $\mathrm{PhD}$ degree in September 2004, and any collaboration has been clearly indicated. This thesis has been composed by myself and has not been submitted, in whole or part, for any other degree, diploma or other qualification.

Isabel Villanueva Margalef 


\section{Acknowledgements}

First of all, I would like to thank Hon for giving me the opportunity of coming to Edinburgh to do my PhD with him. It's been an interesting experience and I think that, after all, I've learnt a lot. If only I could start the $\mathrm{PhD}$ now... Well, not sure about that... Also thank you for giving me this project, which was, in a slightly masochistic kind of way, what I wanted.

I don't think I can say thank you to everyone the way I would like to do it so, against my will, I'll try my best to be brief.

A big thank you to ALL past and present members of the Lam group. I truly believe that the good atmosphere we have in the lab has helped me a lot in numerous occasions to keep on going and not to give up. Thanks to "the boys", Pekka and Gordon; well done, boys. Thanks to Euan and Oscar, for all the encouraging chats. Thank you to Ralph, Myriam, Claire, Mairi and Yi, for many different reasons and for being great to work with. Thank you to Katherine and Leszek, people I've shared this project with. Double thanks to my proof readers: Ralph, Claire, Mairi and Euan. And good luck to everyone! I'm sure somehow we'll keep in touch.

Huge hug to all the very important people with whom I've luckily shared my time in Edinburgh: Miky, Laurent, Emiliano, Mike, Giai, Agoustinos, Marc, Alessandro, Manu, Fred, Alessandro, Javi, Javi, Luis, Iria, Silvia, Irene, Daniella, Pilar, Maria, Cecile, Aileen, Amber, Eric, Carla, Hannah and more poeple than I'll be forgetting right now; it's been great to meet you all, thanks for all those great times and days or nights out. Thanks also to Alex, for the good chats in and out of the lab. Finally, a special kiss to three excellent girls: Ana, Jone and Tijana. Thanks also to all my friends back at home. Girls, I love you, you know me far too well, and I can't imagine my life without you.

My friends are great and my family too. Special big hug and kiss to my aunty Pilar. For all the support, all the phonecalls and for always being there. Te quiero, tía. And to my brother, because he is a great man and I am lucky to have him as my brother. Thanks also to all my crazy Margalef family: aunts and uncles (Ricardo, Ana 
y Mónica), cousins (Ricky, Pepe, Ana, Diego, Lucía y Paula) and very especially to my querida abue. And of course I won't forget my English "family", the Woods. Love you all.

Another very special person I need to say thanks to is Scott Michael Rafferty. Not sure if you wanted to see your name written here but I think you should and that's why I've written it in full. Thanks for being there, for the love and for the understanding, for making me happy, for making me challenge myself and for making me see things in a different way. With all my love, thank you.

Finally, I want to dedicate this work to my parents. Because I am what they made me and because I owe it all to them. To my mum, for being the great woman and mum she was. And to my dad, for everything he has done and for all the support. Thank you.

And now in Spanish:

Finalmente, quiero dedicar este trabajo a mis padres. Porque soy lo que ellos me han hecho y porque se lo debo todo a ellos. A mi madre, por ser esa increible mujer y madre que fue. Y a mi padre, por ser un padrazo y por todo lo que ha hecho. Papá, GRACIAS por todo el apoyo, y por todo lo que siempre has hecho y haces por nosotros. Cada día lo valoro más. Sólo deseo que ahora escuches un poco a tu cuerpo y seas capaz de relajarte y disfrutar. Que tú también te lo mereces. Te quiero. 


\section{Abstract}

Salinosporamide A (1) is a secondary metabolite that was isolated in 2003 by Fenical and co-workers from marine actinomycete bacteria of Salinospora strain CNB-392. Salinosporamide A is a highly potent and specific inhibitor of the $20 \mathrm{~S}$ proteasome. The proteasome, a multicatalytic proteolytic complex that regulates protein degradation within the cells, is receiving considerable attention as a target for treating cancer. Salinosporamide A is currently undergoing clinical trials as potential drug for cancer treatment. It contains a densely functionalised fused $\gamma$-lactam- $\beta$-lactone bicyclic ring structure, with five contiguous stereocentres. Due to the impressive biological activity, as well as its complex chemical structure, this compound has become an important target for the synthetic community.

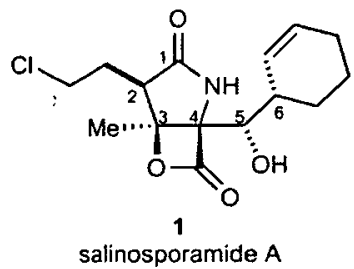

This thesis describes the investigations undertaken towards the synthesis of 1 . After studies on several different approaches, a concise formal synthesis was achieved. This route employed a sequential Ni-catalysed reductive aldol cyclisation-lactonisation reaction in the construction of the $\gamma$-lactam core of 1 , which contained the required stereochemistry. This approach represents an attractive method to install the $\mathrm{C} 2$ side-chain with simultaneous protection of the $\mathrm{C} 5$ oxygen and saves several steps over alternative routes. The product of the cyclisation-lactonisation sequence (489) was readily transformed into an intermediate in the synthesis of 1 by Corey and co-workers (26), which had been converted into salinosporamide $\mathrm{A}$ in four steps.

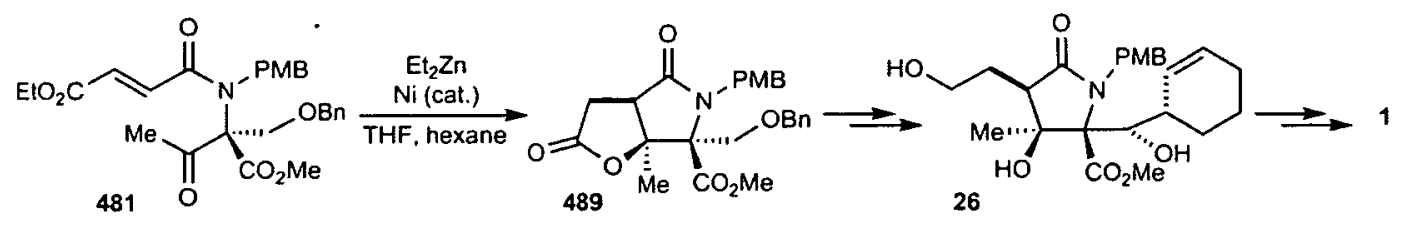




\section{List of Abbreviations}

$\alpha$

${ }^{\circ} \mathrm{C}$

Ac

acac

AIBN

$\mathrm{Aq}$

$\mathrm{Ar}$

Atm

ATP

$\mathrm{BBN}$

BINAP

MeO-BIPHEP

$\mathrm{Bn}$

Boc

$\mathrm{BOPCl}$

BOX

BroP

$\mathrm{Bu}$

$\mathrm{Bz}$

Calcd

CAN

Cat

CDI

CloP

COD

$\mathrm{Cp}$

CSA

cy

d

DBAD observed optical rotation

degrees celsius

acetyl

acetyl acetonate

2,2'-azobis(isobutyronitrile)

aqueous

aromatic group

atmosphere(s)

adenosine 5'-triphosphate

9-borabicyclo[3.3.1]nonane

2,2'-bis(diphenylphosphino)-1,1'-binaphthyl

(6,6'-dimethoxybiphenyl-2,2'-diyl)bis(diphenylphosphine))

benzyl

tert-butoxycarbonyl

$N, N$ '-bis(2-oxo-3-oxazolidinyl)-phosphinic chloride

2,2'-isopropylidenebis-2-oxazoline

bromotris(dimethylamino)phosphonium hexafluorophosphate

butyl

benzyl

Calculated

Cerium(IV) ammonium nitrate

catalytic; catalyst

1,1'-carbonyldiimidazole

chlorotri(dimethylamino)phosphonium hexafluorophosphate

cyclooctadiene

cyclopentadiene

camphor sulfonic acid

cyclohexyl

doublet; day(s)

dibenzylazodicarboxylate 
DCC

dd

DEPT

DIBAL-H

DIC

DMAP

DME

DMF

DMIPS

DMPU

DMS

DMSO

Dpm

DPPB

DPPE

DPPF

dr

$(S, S)$-Et-DuPhos

EDC

ee

EI

equiv

ES

Et

FAB

FDA

$\mathrm{g}$

$\mathrm{GI}_{50}$

GSH

$\mathrm{h}$

HBTU

HIV

HMPA
$N, N$ 'dicyclohexylcarbodiimide

doublet of doublets

distortionless enhancement by polarization transfer

diisobutylaluminium hydride

$N, N^{\prime}$-diisopropylcarbodiimide

4-dimethylaminopyridine

dimethylether

dimethylformamide

dimethylisopropylsilyl

1,3-dimethyl hexahydro-2-pyrimidinone

dimethyl sulfide

dimethylsulfoxide

dipivaloylmethane

1,2-bis(diphenyl-phosphino)butane

1,2-bis(diphenylphosphino)ethane

1,1'-bis(diphenylphosphino)ferrocene

diastereomeric ratio

(+)-1,2-bis((2S,5S)-2,5-diethylphospholano)benzene

1-[3-(dimethylamino)propyl]-3-ethylcarbodiimide hydrochloride enantiomeric excess

electron ionization

equivalents

electrospray ionisation

ethyl

fast atom bombardment

Food and Drug Administration

gram(s)

concentration to achieve 50\% growth inhibition

glutathione

hour(s)

$O$-(benzotriazol-1-yl)-1,1,3,3-tetramethyluronium

hexafluorophosphate

human immunodeficiency virus

hexametylphosphoramide 


\begin{tabular}{|c|c|}
\hline $\mathrm{HOBt}$ & 1-hydroxybenzotriazole \\
\hline HRMS & high resolution mass spectrometry \\
\hline $\mathrm{Hz}$ & Hertz \\
\hline $\mathrm{IC}_{50}$ & half maximal (50\%) inhibitory concentration \\
\hline${ }^{\mathrm{i}} \mathrm{Pr}$ & iso-propyl \\
\hline IR & infra red \\
\hline $\mathrm{I} \kappa \mathrm{B}$ & inhibitor of nuclear factor $\mathrm{kB}$. \\
\hline$J$ & coupling constant \\
\hline KHMDS & potassium bis-(trimethylsilyl)amide \\
\hline $\mathrm{L}$ & litre(s) \\
\hline LAB & lithium triamidoborohydride \\
\hline LDA & lithium diisopropylamide \\
\hline LHMDS & lithium bis-(trimethylsilyl)amide \\
\hline LRMS & low resolution mass spectrometry \\
\hline LTMP & lithium 2,2,6,6-tetramethylpiperidine \\
\hline M & Molar; metal \\
\hline m.p. & melting point \\
\hline $\mathrm{Me}$ & methyl \\
\hline $\mathrm{MeCN}$ & acetonitrile \\
\hline Me-DuPhos & 1,2-Bis(2,5-dimethylphospholano)benzene \\
\hline $\mathrm{mg}$ & milligram \\
\hline $\min$ & Minute(s) \\
\hline $\mathrm{mL}$ & millilitre \\
\hline $\mathrm{mmol}$ & millimole \\
\hline mol & mole(s) \\
\hline $\mathrm{MsCl}$ & methanesulfonyl chloride \\
\hline NaHMDS & sodium bis-(trimethylsilyl)amide \\
\hline NBS & $N$-bromosuccinimide \\
\hline $\mathrm{NCI}$ & National cancer institute \\
\hline$N F-\kappa B$ & nuclear factor $\mathrm{kB}$ \\
\hline NIS & $N$-iodosuccinimide \\
\hline NMM & $N$-methylmorpholine \\
\hline NMO & $N$-methylmorpholine- $N$-oxide \\
\hline
\end{tabular}




$\begin{array}{ll}\text { NMR } & \text { nuclear magnetic resonance } \\ \text { NOESY } & \text { nuclear overhauser enhancement spectroscopy } \\ \text { PCC } & \text { pyridinium chlorochromate } \\ \text { PDC } & \text { pyridinium dichromate } \\ \text { Ph } & \text { phenyl } \\ \text { PMB } & p \text {-methoxybenzyl } \\ \text { PMHS } & \text { polymethylhydrosiloxane } \\ \text { PMP } & p \text {-methoxyphenyl } \\ \text { ppm } & \text { parts per million } \\ \text { PPY } & \text { 4-pyrrolidinopyridine } \\ \text { pr } & \text { propyl } \\ p \text {-TsOH } & p \text {-toluenesulfonic acid } \\ \text { pybox } & 2,6 \text {-bis(oxazolinyl)pyridine } \\ \text { PyBrop } & \text { bromotri(pyrrolidino)phosphonium hexafluorophosphate } \\ \text { q } & \text { quartet } \\ \text { rac-BINAP } & \text { racemic 2,2'-bis(diphenylphosphino)-1,1'-binaphthyl } \\ \text { rt } & \text { room temperature } \\ \text { SAR } & \text { structure-activity relationship } \\ \text { SEGPHOS } & \text { (S)-(4,4-bi-1,3-benzodioxole)-5,5-diylbis(diphenylphosphine) } \\ \text { t } & \text { triplet } \\ \text { TBDPS } & \text { tert-Butyldiphenylsilyl } \\ \text { TBS } & \text { tert-Butyldimethylsilyl } \\ \text { TBTU } & \text { tetrapropylammotriazol-1-yl-1,1,3,3-tetramethyluronium } \\ t \text {-Bu } & \text { tetrafluoroborate } \\ \text { TfOH } & \text { tert-butyl } \\ \text { THF } & \text { trifluoromethanesulfonic acid (triflic acid) } \\ \text { THP } & \text { tetrahydrofuran } \\ \text { Thr } & \text { tetrahydropyrane } \\ \text { TLC } & \text { threonine } \\ \text { TMDS } & \text { thin layer chromatography } \\ \text { TMEDA } & 1,1,3,3 \text {-tetramethylhydrosiloxane } \\ \text { TMS } & \text { tetramethylethylenediamine } \\ \text { TPAP } & \end{array}$




\section{Contents}

Declaration i

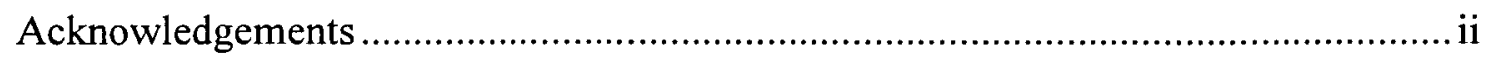

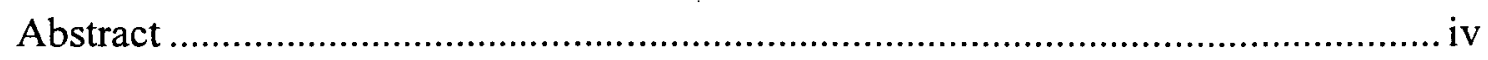

List of Abbreviations.....................................................................................

1. Introduction ...................................................................................... 1

1.1. Ubiquitin-mediated proteolysis and proteasome inhibitors ....................... 2

1.1.1. The discovery of ubiquitin-mediated proteolysis............................ 2

1.1.2. Biological functions of the ubiquitin mediated proteolysis ............... 4

1.1.3. Proteasome inhibitors and mode of action ..................................... 5

1.1.3.1. Lactacystin, omuralide and salinoporamide A. Mode of action and Structure-Activity Relationships (SAR) studies................................. 7

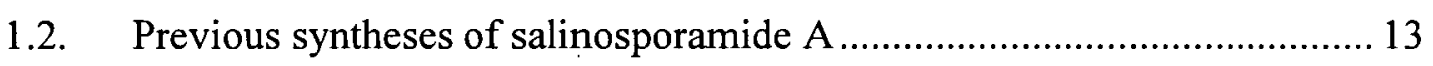

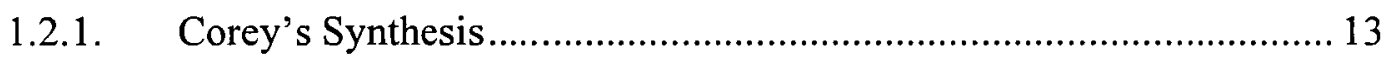

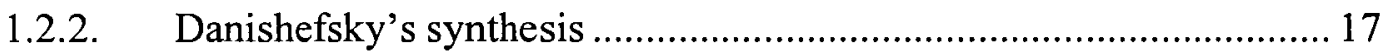

1.2.3. Pattenden Racemic Synthesis.................................................... 20

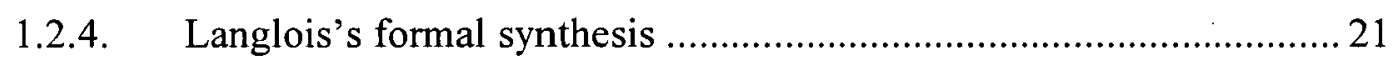

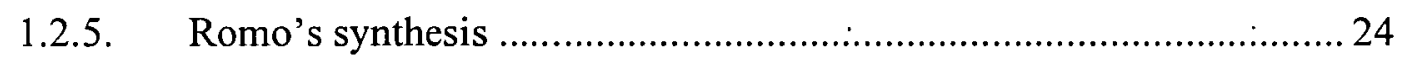

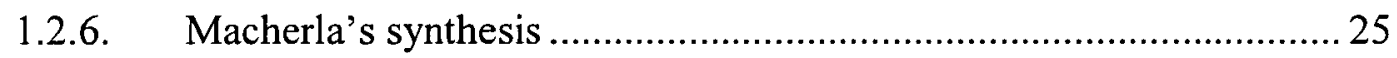

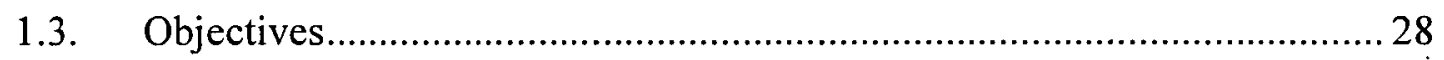

2. Racemic approach based on amidomalonate aldol cyclisations ..................... 30

2.1. Retrosynthetic analysis................................................................ 30

2.2. Results and discussion ............................................................ 31

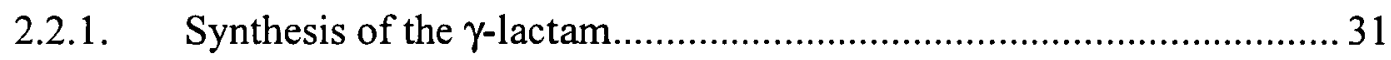

2.2.2. Regioselective reduction of the ester and cyclohexenyl insertion ..... 35

2.2.3. Introduction of the $\mathrm{C} 2$ substituent............................................. 40

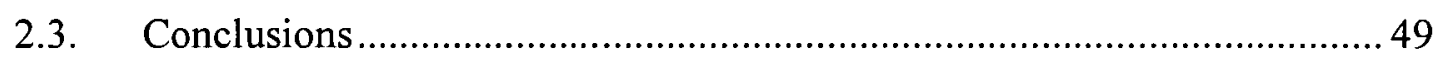

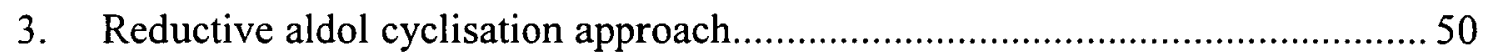

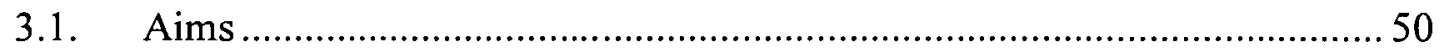

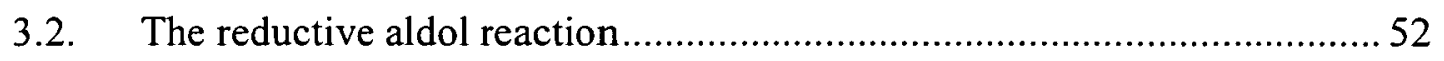

3.3. Development of the reductive aldol cyclisation in the Lam group ............ 68 
3.3.1. Copper-catalysed reductive aldol cyclisation...................................... 68

3.3.2. Cobalt-catalysed reductive aldol cyclisation..................................... 73

3.3.3. Nickel-catalysed reductive aldol cyclisation...................................... 76

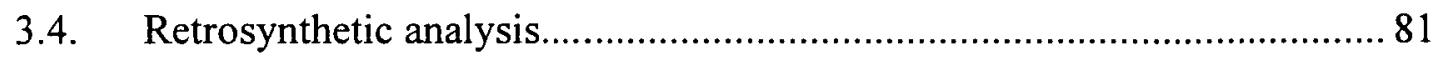

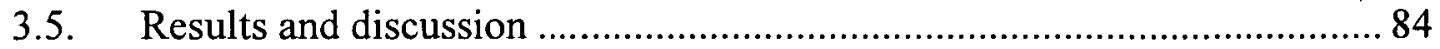

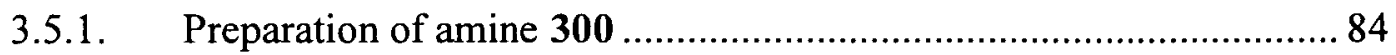

3.5.2. Studies on the amide coupling and reductive aldol cyclisation ......... 90

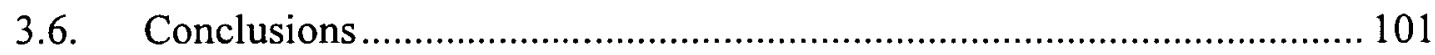

4. Reformatsky reaction approach in the synthesis of salinoporamide A ............ 102

4.1. Retrosynthetic analysis..................................................................... 102

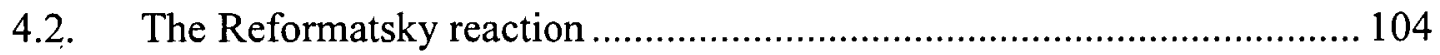

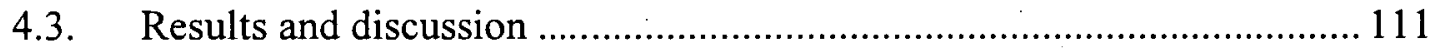

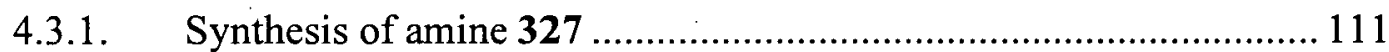

4.3.2. Study of the Reformatsky cyclisation reaction ................................. 113

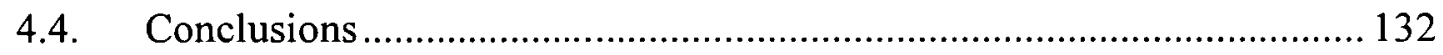

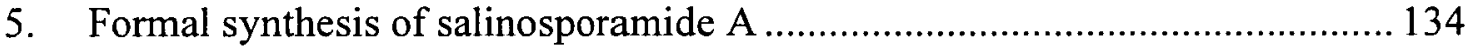

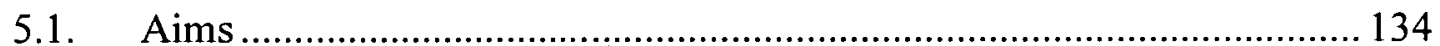

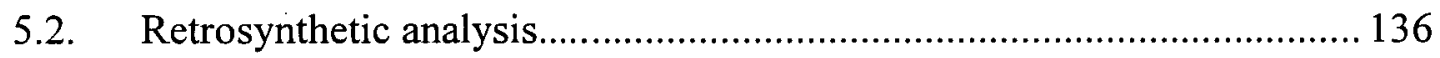

5.3. Preliminary studies on the reductive aldol cyclisation reaction................ 136

5.4. Completion of the formal synthesis ..................................................... 141

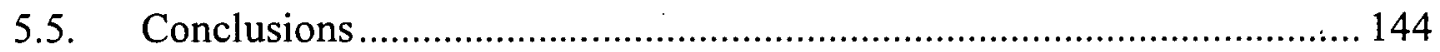

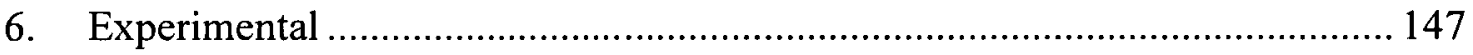

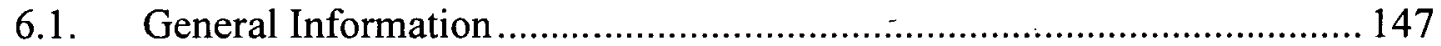

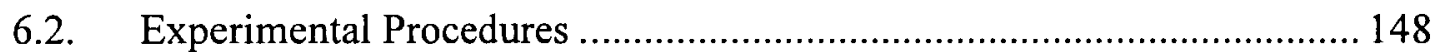

6.2.1. Racemic approaches based on amidomalonate aldol cyclisations ... 148

6.2.2. Reductive aldol cyclisation approach.............................................. 168

6.2.3. Reformatsky reaction approach.................................................... 184

6.2.4. Formal synthesis .......................................................................... 203

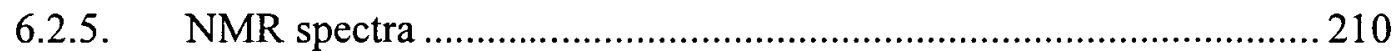

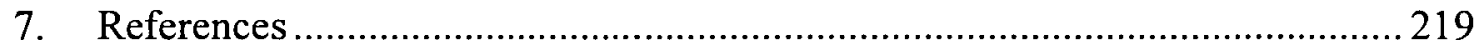




\section{Introduction}

Salinosporamide A (1) is a secondary metabolite that was isolated in 2003 by Fenical and co-workers from the marine actinomycete bacteria of Salinospora strain CNB-392 (Figure 1.1). ${ }^{1}$ Salinosporamide A is structurally related to omuralide (2), a transformation product of the soil microbial metabolite (+)-lactacystin (3) isolated by Omura et al. in 1991. ${ }^{2}$ Omuralide was the first specific $20 \mathrm{~S}$ proteasome inhibitor identified. The proteasome is a multicatalytic proteolytic complex that regulates protein degradation within cells and is receiving considerable attention as a target for treating cancer. Omuralide (2) has an $\mathrm{IC}_{50}$ value of $49 \mathrm{nM}$ when tested against purified $20 \mathrm{~S}$ proteasome, but salinosporamide A (1) was found to be approximately 35 times more potent than omuralide, with an $\mathrm{IC}_{50}$ value of $1.3 \mathrm{nM}$ in the same assay. Salinosporamide $A$ also exhibited high in vitro cytotoxicity with a mean $\mathrm{GI}_{50}$ value of less than $10 \mathrm{nM}$ when tested against the NCI's 60 cell-line panel. 20S Proteasome inhibitors are of interest for the treatment of several types of cancer and the drug Velcade $($ (bortezomib) (4) has been approved by the FDA for the treatment of multiple melanoma. ${ }^{3}$ Salinosporamide A (NPI-0052) is currently in phase I human clinical trials for the treatment of multiple myeloma and other cancers. ${ }^{4}$
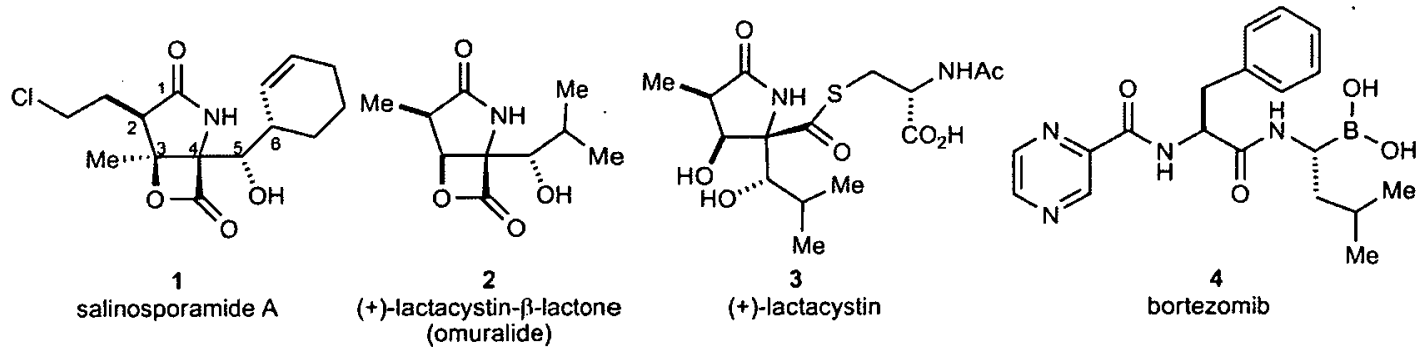

Figure 1.1 Salinosporamide A, omuralide, lactacystin and bortezomib

The synthesis of proteasome inhibitors such as omuralide and salinosporamide A is therefore of great pharmaceutical value, since it would allow greater quantities to be produced which would permit more extensive testing, as well as the synthesis of analogues. The aim of this project was to investigate synthetic approaches to salinosporamide $\mathrm{A}$, the most interesting $20 \mathrm{~S}$ proteasome inhibitor recently isolated. 


\subsection{Ubiquitin-mediated proteolysis and proteasome inhibitors}

\subsubsection{The discovery of ubiquitin-mediated proteolysis}

While there had been great interest and much research on understanding how the cell controls the synthesis of certain proteins, control of the degradation of proteins had for a long time not received so much attention. The first system known for protein degradation in eukaryotic cells was the lysosomal apparatus, a membrane-enclosed vacuole containing multiple acid proteases and other hydrolases. It was long believed to be the only site for protein breakdown but, from studies initiated in the fifties, it was established that the bulk of cytosolic proteins are hydrolysed by a distinct pathway which is highly specific and requires ATP and the $26 \mathrm{~S}$ proteasome. Knowledge in this area developed rather slowly but in the early nineties the studies were greatly simplified thanks to the availability of proteasome inhibitors. ${ }^{5}$ Avram Hersko, Aaron Ciechanover and Ernie Rose were awarded the Nobel Prize in chemistry in 2004 for their studies of this system. ${ }^{6}$

Ubiquitination is the process, present both in the nucleus and the cytosol, whereby proteins are marked for degradation by covalent linkage to the protein ubiquitin. This process requires the initial ATP-dependent activation of ubiquitin's terminal carboxy group by the ubiquitin-activating enzyme, E1 (Figure 1.2). The activated ubiquitin is then transferred to the ubiquitin carrier proteins, E2s. There is some selectivity in this step but the great selectivity of this process comes from the ubiquitin ligases, or E3s, which reorganise one or a set of protein substrates and catalyse the transfer of the activated ubiquitin from the E2 to the substrate, forming processively a chain of ubiquitin molecules. A chain composed of four or more ubiquitin molecules leads to the degradation of the protein in the proteosome. ${ }^{3}$ 


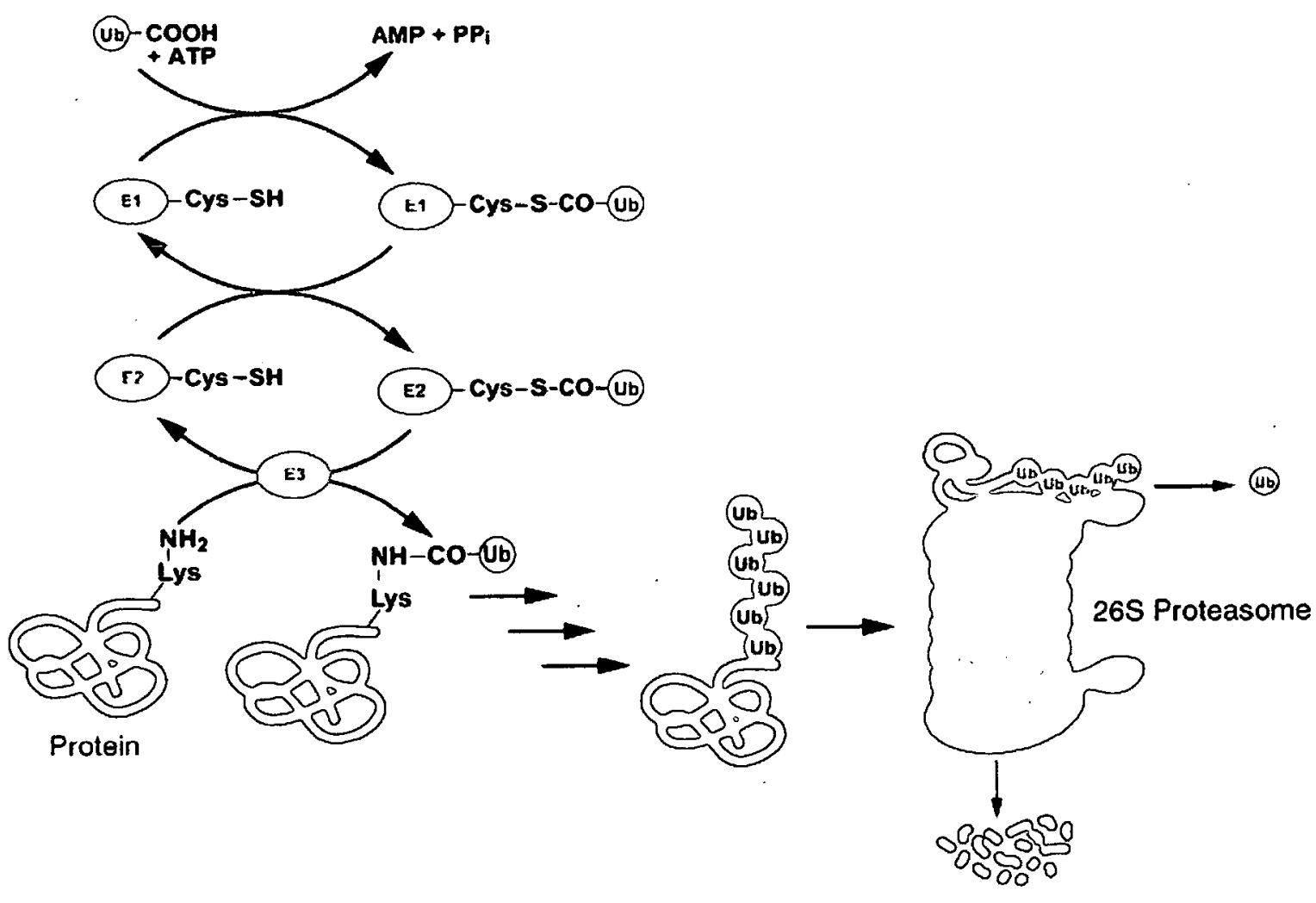

Figure 1.2 Mechanism of the E1/E2/E3-catalysed attachment of ubiquitin to proteins $^{7}$

The 26S proteasome is an ATP-dependent proteolytic complex, formed by two $19 \mathrm{~S}(890 \mathrm{kDa})$ "cap" complexes, which provide the specificity and regulation, and the central $20 \mathrm{~S}$ proteosome $(720 \mathrm{kDa})$, where proteins are degraded. The $19 \mathrm{~S}$ complexes contain binding sites for ubiquitinated proteins, enzymes that depolymerise the ubiquitin chain, and six different ATPases that unfold the substrate and make the entry to the $20 \mathrm{~S}$ complex easier. The barrel-shaped $20 \mathrm{~S}$ proteasome is formed from four stacked rings that enclose a central chamber where proteolysis occurs. This proteasome uses the hydroxyl group of a terminal threonine residue as the catalytic nucleophile that attacks and breaks peptide bonds. A protein, once taken up by the proteasome, is completely degraded into small peptides of 3-20 residues that are further hydrolysed into amino acids by other peptidases. The isolation of the active sites within the $20 \mathrm{~S}$ complex away from cytosolic proteins and the restriction of substrate entry must have evolved to avoid non-specific degradation of cell proteins. $^{8}$ 


\subsubsection{Biological functions of the ubiquitin mediated proteolysis}

The availability of proteasomal inhibitors with increasing specificity and potency has helped greatly in understanding the role of the proteasomal-ubiquitin protein degradation in many biological processes. ${ }^{5}$ Since the late 1980 s a number of physiologically important substrates involved in this proteolysis pathway have been identified. Only as an illustration, a few examples will be briefly described.

I $\mathrm{B}$ was the first substrate of the ubiquitin-proteasome pathway identified with the help of proteasome inhibitors. ${ }^{9}$ It is an inhibitor of transcription factor NF- $k B$, which activates the expression of many genes encoding inflammatory mediators. The signal for the initiation of the inflammatory response is the rapid destruction of the inhibitory protein IKB. Consequently, proteasome inhibitors, by ștabilising I $\mathrm{B}$, maintain NF- $\kappa B$ in the inhibited state and prevent production of the inflammatory proteins. $^{3}$

The proteasome is also involved in degradation of numerous proteins regulating the cell cycle. Inhibition of the proteasomal activity was reported to stop the cell cycle at different stages. Several studies demonstrated that cancer cells are usually more sensitive to proteasome inhibitors than normal cells. The use of proteasome inhibitors also demonstrated that the proteasomal-ubiquitin pathway has a decisive effect on cell death and survival: several substrates of this proteolytic pathway are short-lived proteins which are critical for cell progression and transcriptional regulation, and they can therefore become the key players in the control of the cell death program. ${ }^{10}$

Proteasome inhibitors have been associated with suppression of angiogenesis. ${ }^{11}$ Cancerous tissues stimulate angiogenesis and that leads to increased tumour formation and possible metastases. Many of the factors involved in angiogenesis are regulated by the proteasome.

The ability of proteasome inhibitors to inhibit cell growth and selectively induce apoptosis in cancer cells, together with the ability to inhibit angiogenesis, renders these agents attractive candidates as anticancer drugs. The development of proteasome inhibitors in cancer treatment is therefore one of the fastest growing fields in modern biomedical science. ${ }^{8}$ 


\subsubsection{Proteasome inhibitors and mode of action}

More than $90 \%$ of cell protein degradation occurs through the ubiquitin-proteasomal pathway. The proteasome is the main component of this mechanism and the regulation of its activity is very important for an enormous number of essential biological processes. For that reason, compounds that inhibit or modulate proteasomal activity have great biological importance. Proteasome inhibitors can be used as tools to investigate regulation of the ubiquitin-proteasomal system, and also as leads for possible drug development. They are generally classified based on the pharmacophore with preference for the proteasome's active site, the hydroxyl group of an $N$-terminal threonine. They are also classified based on the specificity and the reversibility of binding. ${ }^{12}$

Peptide aldehydes (Scheme 1.1) were the first proteasome inhibitors to be developed and are still the most widely used inhibitors. Aldehyde inhibitors enter the cell rapidly and their effect is reversible. They have fast dissociation rates, are rapidly oxidised into inactive acids by cells and are easily transported out of the cell. Consequently, the effects of these inhibitors can be rapidly reversed by removal of the inhibitor.

Peptides with a vinyl sulfone are another class of proteosomal inhibitor. These bind to proteasomes irreversibly but are less reactive than aldehydes. Vinyl sulfones act as conjugate addition acceptors for the hydroxyl group of the proteasome's catalytic threonine, forming a covalent bond.

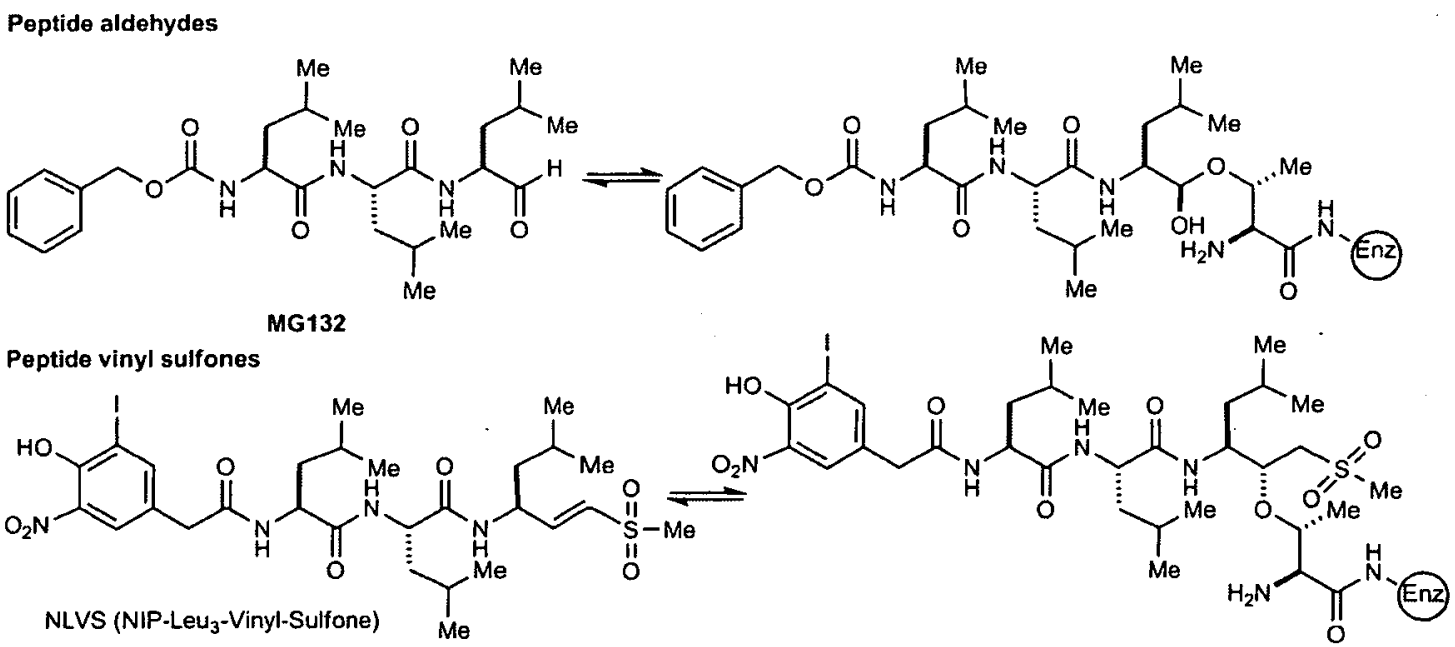

Scheme 1.1 Peptide aldehydes and peptide vinyl sulfones proteasome inhibitors 
Peptide boronates (Scheme 1.2) are much more potent inhibitors than aldehydes and vinyl sulfones. Although boronates are considered reversible inhibitors, the inhibition is practically irreversible over the duration of several hours. Their high inhibition efficiency, high selectivity (they do not inhibit other cellular proteases) and low dissociation rates render these chemical compounds the focus of intense research. The boronate derivative bortezomib (4) has already passed through clinical trials and represents a new drug against multiple myelanoma. ${ }^{4}$

Apart from synthetic peptide inhibitors, there are a variety of natural compounds, like peptide epoxyketones and $\beta$-lactones. Epoxyketones act by an interesting and unique mechanism. They react with both the hydroxyl and amino groups of the catalytic $N$-terminal threonine of the proteasome. Due to their unique mechanism, they are the most selective inhibitors of the proteasome known.

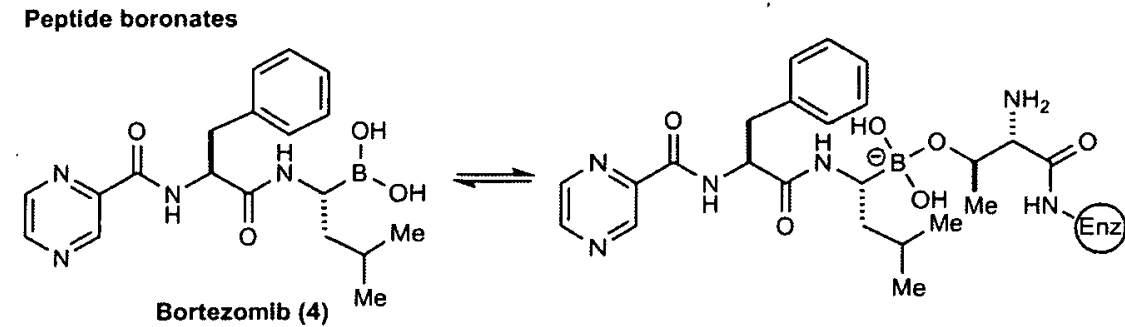

Peptide epoxyketones

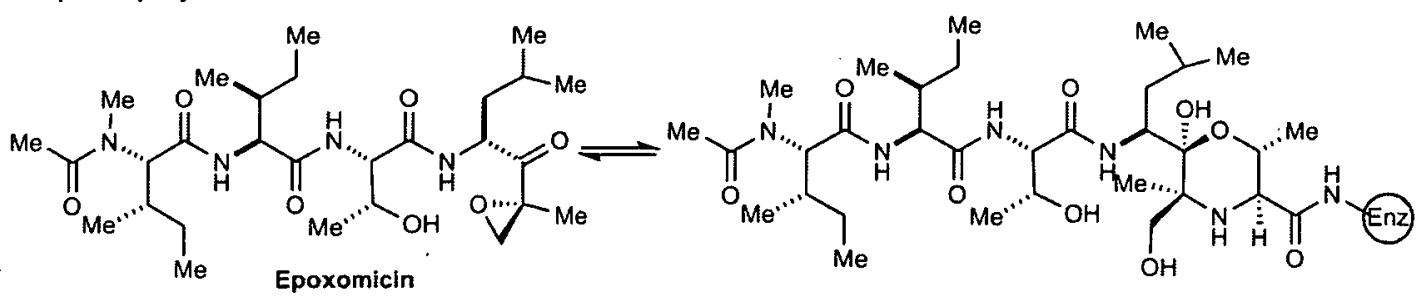

Scheme 1.2 Peptide boronates and peptide epoxyketones proteasome inhibitors

Lactacystin, omuralide and salinosporamide $A$ are $\beta$-lactone inhibitors and their mode of action and reactivity will be explained in more detail in the next section. 


\subsubsection{Lactacystin, omuralide and salinoporamide A. Mode of action} and Structure-Activity Relationships (SAR) studies

In 1991, Omura's group isolated and characterised (+)-lactacystin (3), a secondary metabolite derived from a Streptomyces bacterial strain. ${ }^{2}$ It was initially considered a neurotrophic agent but has since found application in models of arthritis, ischemia, and asthma. By further interpretation of the biological activity, Fenteany discovered that its molecular target was the $20 \mathrm{~S}$ proteasome. ${ }^{13}$ Experiments done by Fenteany and co-workers as well as the crystallographic work of Huber ${ }^{14}$ clearly defined the mode of inhibition of $(+)$-lactacystin and explained its high potency and selectivity.

(+)-Lactacystin was shown to be in fact a pro-drug for the active inhibitor omuralide (2), which is the only species to enter the cell. ${ }^{15}(+)$-Lactacystin spontaneously eliminates $N$-acetylcysteine in a reversible manner to produce the $\beta$ lactone. Once inside the cell, omuralide either: (i) is hydrolysed by water to give the carboxylic acid 5; (ii) reacts with glutathione to form the glutathione derivative (lactathione) 6 which acts as a reservoir of the drug; or (iii) binds to the $20 \mathrm{~S}$ proteasome to form ester 7 , as illustrated in Scheme $1.3 .^{16}$

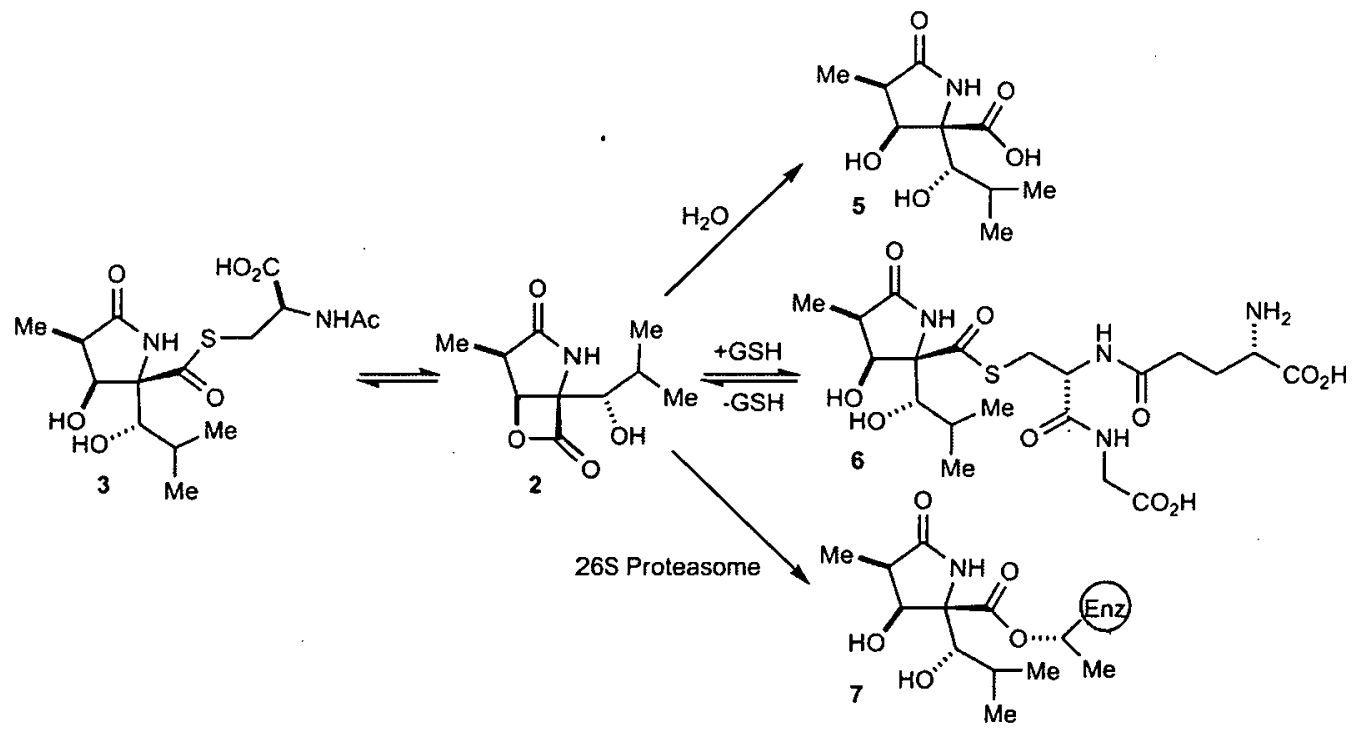

Scheme 1.3 Formation of omuralide (2) from (+)-lactacystin 3 and its subsequent reaction inside the cell with water, glutathione (GSH) and the $20 \mathrm{~S}$ proteasome 
The electrophilic centre of the inhibitor is the $\beta$-lactone. Omuralide binds to the $20 \mathrm{~S}$ proteasome through a covalent bond in the form of an ester between the $\mathrm{C} 4$ carbonyl and the hydroxyl group of an $N$-terminal threonine residue in the active site. Although the inhibitor is covalently bound the resultant ester is subject to hydrolysis and the inhibition is therefore reversible, with enzymatic activity restored in a matter of hours. ${ }^{8}$

The synthesis of $(+)$-lactacystin has received widespread attention since its isolation in 1991. The first synthesis of 2 was reported by Corey and co-workers in 1992. ${ }^{17}$ Many refinements to the synthesis have been reported by the Corey group, allowing $(+)$-lactacystin to be synthesised via omuralide and allowing the synthesis of $\mathrm{C} 2$ and $\mathrm{C} 5$ analogues. ${ }^{16}$ It was found that replacing the $\mathrm{C} 2$ methyl group with larger alkyl substituents increased the potency of proteasome inhibition relative to omuralide but that alteration of the isopropyl group at the $\mathrm{C} 5$ position resulted in a loss of activity. ${ }^{18}$ The $\beta$-lactone has been shown to be essential for biological activity through synthesis of the ' $3 R$ diastereoisomer' $(8)$ of $(+)$-lactacystin $(3)$ which is unable to form the $\beta$-lactone but shares all of the other structural features. ${ }^{19}$ This compound was found to be inactive which is consistent with the mechanism of inhibition.
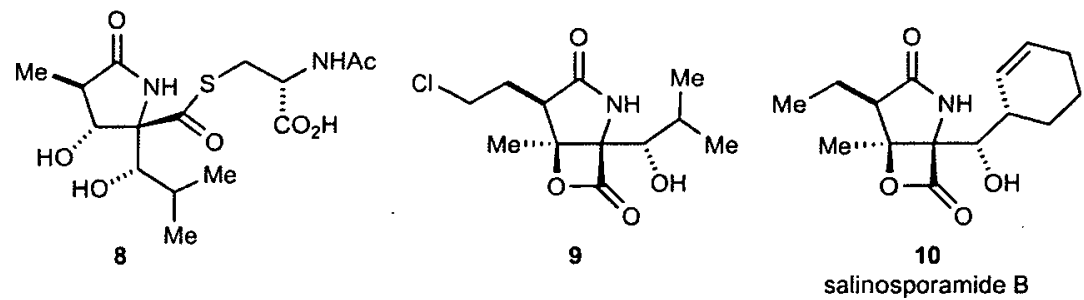

Figure 1.3 Lactacystin, omuralide and salinosporamide $A$ analogues

Salinosporamide A not only represents a more attractive synthetic target than omuralide due to its increased biological activity, but it is also a more challenging one. It is even more densely functionalised than omuralide, containing five contiguous stereogenic centres. Salinosporamide A shares the key $\gamma$-lactam- $\beta$-lactone pharmacophore with omuralide but differs in the C2, C3 and C5 substituents. It contains a cloroethyl group at $\mathrm{C} 2$, it is methylated at $\mathrm{C} 3$ creating a second quaternary 
stereogenic centre and carries a cyclohexenyl group at C5 in place of the isopropyl group of omuralide. It is this unique substitution that, collectively, makes salinosporamide A approximately 35 times more potent that omuralide in proteasome inhibition.

Given the structural similarities between omuralide and salinosporamide A, a similar binding mode with the proteasome could be expected, namely attack of the Thrl-OH of the proteasome to the $\beta$-lactone carbonyl, opening the ring and forming an ester. This was confirmed by a crystal structure analysis of salinosporamide A in complex with the $20 \mathrm{~S}$ proteasome, by Potts and co-workers (Figure 1.4). ${ }^{20}$

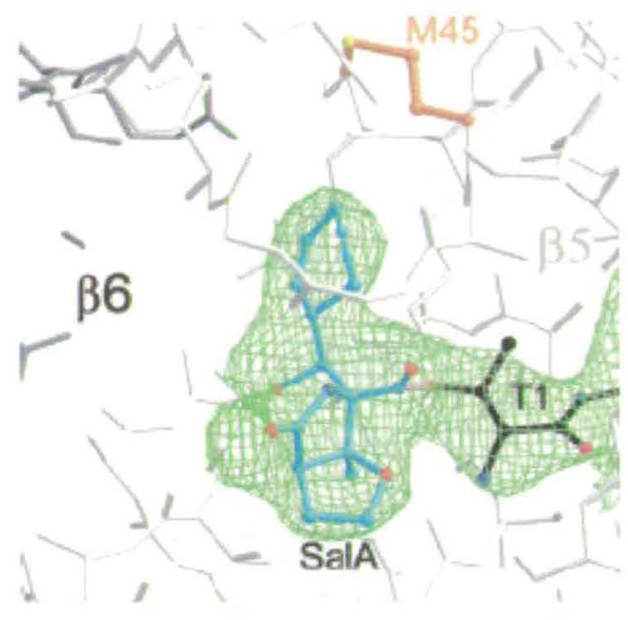

Figure 1.4 Crystal structure analysis of salinosporamide $A(1)$ in complex with the 20 s proteasome 20

The $\beta$-lactone is therefore recognised as the key pharmacophore, but the enhanced potency of salinosporamide A must be due to certain substituents of the bicyclic ring. Therefore, full structure-activity relationship (SAR) studies of salinosporamide A were undertaken. ${ }^{21}$ Table 1.1 outlines some biological data of in vivo (cytotoxicity against multiple myeloma cell line RPMI 8226) and in vitro proteasome inhibition. This SAR study together with the analysis of the crystal structure of salinosporamide A (1) and of the des-chloro analogue (10, Figure 1.3) in complex with the proteasome, provided explanations for its enhanced potency. ${ }^{20}$ This des-chloro analogue $\mathbf{1 0}$ (salinosporamide B) was isolated, in smaller quantities, from the same microbial strain. 
The fact that the cyclohexenyl group of 1 does not result in a loss of activity (Table 1.1) contradicts the SAR data obtained for omuralide (2) by Corey and coworkers and suggests that salinosporamide A may interact with the proteasome in a different manner to omuralide. The C5 isopropyl analogue of salinosporamide A (9, Figure 1.3, vide supra) has been synthesised by the Corey group ${ }^{22}$ and was found to be 2.5 times more potent than 2 but less active than 1 against the purified proteasome. ${ }^{23}$ Various $\mathrm{C} 5$ analogues were synthesised by modifying the cyclohexene substituent but all were found to be less potent than the parent compound (Table 1.1, entries 3-5). The hydrogen bonds formed between $\mathrm{C} 5-\mathrm{OH}$ and the proteasome also have an important role in causing inhibition. Epimerisation of this centre or oxidation to the ketone results in analogues without proteosomal inhibition (entry 6).

It was observed that the C3 methyl group points toward a small pocket and interacts weakly with the protein. Both a hydrogen atom and a methyl group fit into this pocket, but an analogue containing an ethyl group at this position does not inhibit the proteasome (entry 7). The ethyl group is too big and causes steric interactions that do not allow binding.

\begin{tabular}{|c|c|c|c|}
\hline & \multirow{2}{*}{$\begin{array}{c}\text { Modification from } \\
\text { salinosporamide } A(1) \\
\left(\mathrm{R}^{2}=\mathrm{CH}_{2} \mathrm{CH}_{2} \mathrm{Cl}, \mathrm{R}^{3}=\mathrm{Me}, \mathrm{R}^{1}=\right. \\
\text { cyclohexenyl) }\end{array}$} & \multicolumn{2}{|c|}{$\begin{array}{c}\text { Biological activities } \\
\text { (IC50 } n M)\end{array}$} \\
\hline & & $\begin{array}{l}\text { Cytotoxicity } \\
\text { RPMI 8226 } \\
\text { (MM) }\end{array}$ & $\begin{array}{c}\text { Proteasome } \\
\text { Inhibition }\end{array}$ \\
\hline 1 & None (1) & $8.2 \pm 2.0$ & $21 \pm 3$ \\
\hline 2 & Omuralide (2) & $3300 \pm 1600$ & $540 \pm 60$ \\
\hline 3 & cyclohexyl & $52 \pm 13$ & $250 \pm 30$ \\
\hline 4 & & $32 \pm 3$ & $92 \pm 6$ \\
\hline 5 & & $320 \pm 5$ & $770 \pm 50$ \\
\hline 6 & $e p i-\mathrm{C}-5(\mathrm{OH})$ & $>20000$ & $>20000$ \\
\hline 7 & $\mathrm{R}^{3}=\mathrm{CH}_{2} \mathrm{CH}_{3}$ & $1900 \pm 400$ & $3300 \pm 200$ \\
\hline 8 & $\mathrm{R}^{2}=\mathrm{CH}_{2} \mathrm{CH}_{2} \mathrm{Br}$ & $7.4 \pm 1.6$ & $14 \pm 2$ \\
\hline 9 & $\mathrm{R}^{2}=\mathrm{CH}_{2} \mathrm{CH}_{2} \mathrm{I}$ & $6.8 \pm 0.8$ & $13 \pm 3$ \\
\hline 10 & $\mathrm{R}^{2}=e p i-\mathrm{CH}_{2} \mathrm{CH}_{2} \mathrm{Cl}$ & $1900 \pm 300$ & $2500 \pm 500$ \\
\hline 11 & $\mathrm{R}^{2}=\mathrm{CH}_{2} \mathrm{CH}_{3}$ & $6100 \pm 3300$ & $640 \pm 60$ \\
\hline
\end{tabular}

Table 1.1 Biological activities of salinosporamide $A$, omuralide and analogues 
The hydrolysis of the $\beta$-lactone was also evaluated. In aqueous solutions salinosporamide $\mathrm{A}$ hydrolyses to the acid $\mathbf{1 1}$ which rapidly eliminates $\mathrm{HCl}$ to give the bicyclic product 12, as illustrated in Scheme 1.4.

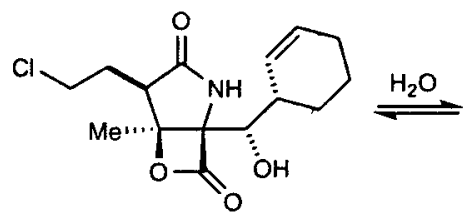

1

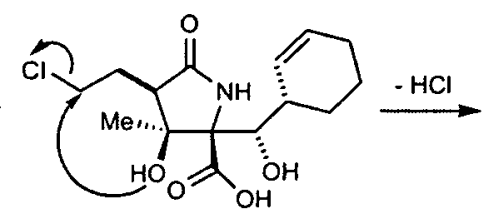

11

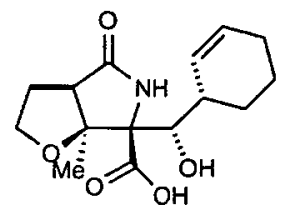

12

Scheme 1.4: Formation of the bicyclic tetrahydrofuran derivative 12 following displacement of the C2-chloro substituent

A potential problem with salinosporamide $\mathrm{A}$ as a pharmaceutical agent is therefore its instability in aqueous solution at $\mathrm{pH} 7\left(\mathrm{t}_{1 / 2}=5-10 \mathrm{~min}\right)$. In an attempt to get round this problem, Corey and co-workers have synthesised $\beta$-lactam 13 which is stable at $\mathrm{pH} 7$ for at least 24 hours (Figure 1.5). ${ }^{24}$ This is proposed to compensate for the decreased potency of proteasome inhibition exhibited for this compound.

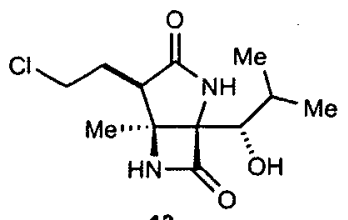

13

Figure $1.5 \beta$-Lactam 13

Salinosporamide A (1) contains a chloroethyl group at the $\mathrm{C} 2$ position; the des-chloro analogue of salinosporamide A, salinosporamide B (Figure 1.3), is found to be 500-fold less cytotoxic than salinosporamide A against the human colon HCT-116 cancer cell line. ${ }^{25}$. Substitution of chlorine with bromine or iodine (Table 1.1, entries 8 and 9) gave analogues of similar potency. The C2 epimer (entry 10) also resulted in a marked decreased potency.

The chloride substituent is proposed to be responsible for the increase in potency by ensuring that the covalent attachment to the proteasome is irreversible. It is suggested a two-step binding mechanism, in which $\beta$-lactone ring opening (14) is followed by the facile chloride displacement from the side chain by the free hydroxyl 
at $\mathrm{C} 3$, forming a bicyclic tetrahydrofuran derivative (15) (Scheme 1.5). For omuralide (2), the formation of the ester bond with the proteasome is reversible because the hydroxyl at C3 is free and can attack the carbonyl again, but this is not possible for salinosporamide A.

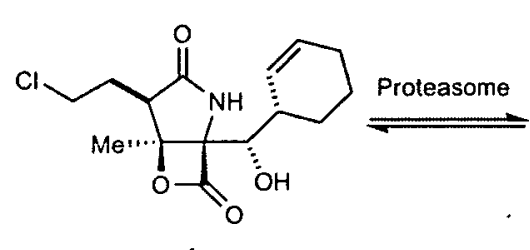

1

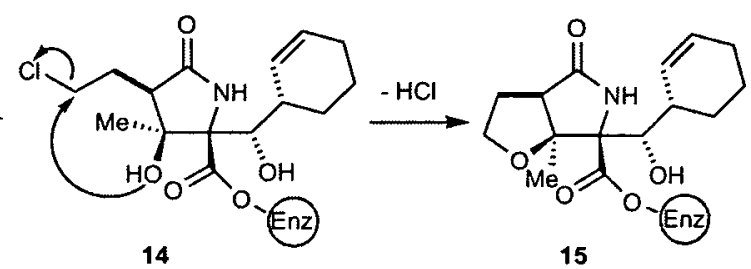

14

15

Scheme 1.5 Salinosporamide A binds irreversibly

Cleavage of the acyl ester by a nucleophilic water molecule is challenged by the spatial arrangement of $\mathrm{C} 3-\mathrm{O}$ in the tetrahydrofuran derivative. Proteasome activity is only slowly recovered after 7 days in mice treated with $\mathbf{1}$, which could be attributed to red blood cell turnover. ${ }^{4}$ 


\subsection{Previous syntheses of salinosporamide $\mathbf{A}$}

The discovery of proteasome inhibitors has facilitated a greater understanding of the mechanism of the proteasome and an increased awareness of its biological importance. They have shown the proteasome as a viable target for drug development.

Because of the potent biological activity, as well as its complex structure, salinosporamide A (1) has generated considerable interest in the synthetic community. Corey completed the first total synthesis in $2004^{26}$. Since then, other total syntheses (Danishefsky, ${ }^{27}$ Macherla, $^{28}$ Pattenden $^{29}$ (racemic) and $\operatorname{Romo}^{30}$ (racemic)) and a formal synthesis (Langlois) ${ }^{31,32}$ have been reported. The remainder of this section will review the synthetic strategies towards the natural product.

\subsubsection{Corey's Synthesis}

The first total synthesis of salinosporamide A was achieved by Corey and co-workers in $2004,{ }^{26}$ following on from extensive work by the group on the synthesis of (+)-lactacystin, omuralide and related analogues. ${ }^{17,33,34}$ This synthesis is outlined in Scheme 1.6

$(S)$-Threonine methyl ester was treated with 4-methoxybenzoyl chloride to give the $\mathrm{N}$-acylated product 16 which cyclised to an ozaxoline in the presence of $p$-TsOH. Stereoselective alkylation of the oxazoline with benzyloxymethyl chloride afforded a new oxazoline 17, with the required stereochemistry at the tetrasubstituted $\mathrm{C} 4$ carbon. Selective reductive ring-opening of 17 using $\mathrm{NaBH}_{3} \mathrm{CN} / \mathrm{HOAc}$ gave the $N$-PMB-protected amino alcohol 18. This intermediate was acylated in a one-flask sequence: temporary protection of the secondary alcohol with a trimethylsilyl group, $N$-acylation with acryloyl chloride, followed by desilylation, to yield the $N$-acrylyl$N$-PMB derivative 19 in $96 \%$ yield. Oxidation of 19 with Dess-Martin periodinane ${ }^{35}$ produced keto-acrylamide $\mathbf{2 0}$.

The key formation of the $\gamma$-lactam was successfully achieved via a Baylis-Hillman cyclisation, operation that installs the stereocentre at C3. Using quinuclidine as the catalyst in DME at $0^{\circ} \mathrm{C}$ for 7 days, 20 cyclised to give the desired 
diastereomer of the $\gamma$-lactam with high diastereoselectivity (9:1). Silylation of the cyclised products allowed separation of the two diastereomers and desired 21 was isolated in $95 \%$ yield. Subsequent tributyltin-mediated radical-chain cyclisation of $\mathbf{2 1}$ gave the syn-fused $\gamma$-lactam 22, setting up the desired stereochemical relationship at $\mathrm{C} 2$ and $\mathrm{C} 3$. Cleavage of the benzyl ether and oxidation of the resulting primary alcohol afforded aldehyde 23.

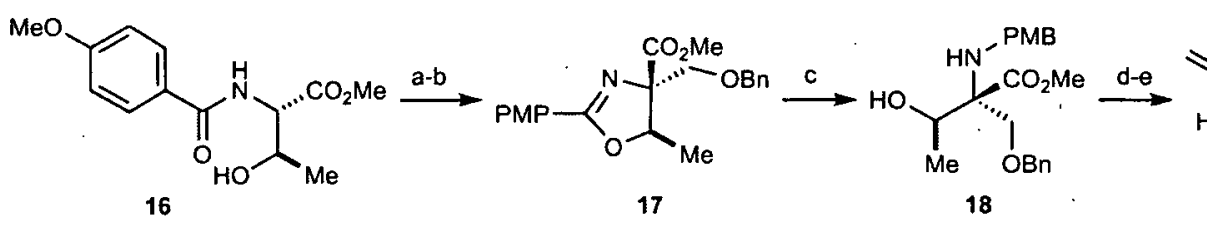

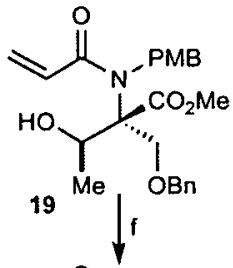

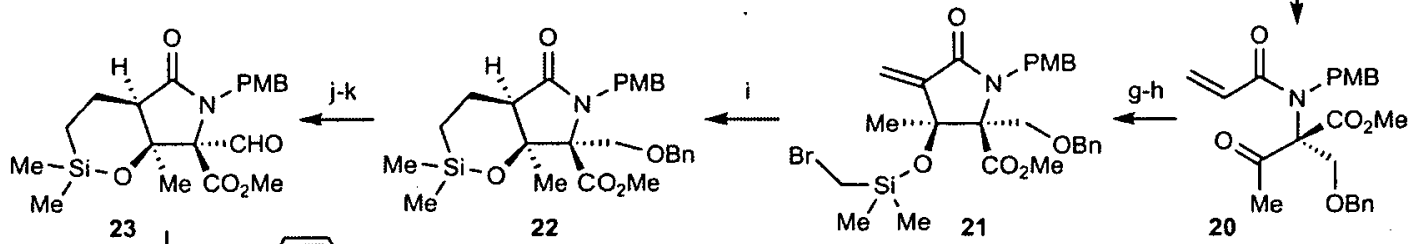<smiles>[14CH3][14C]([Te])[14CH]1C=CCCC1</smiles>

22

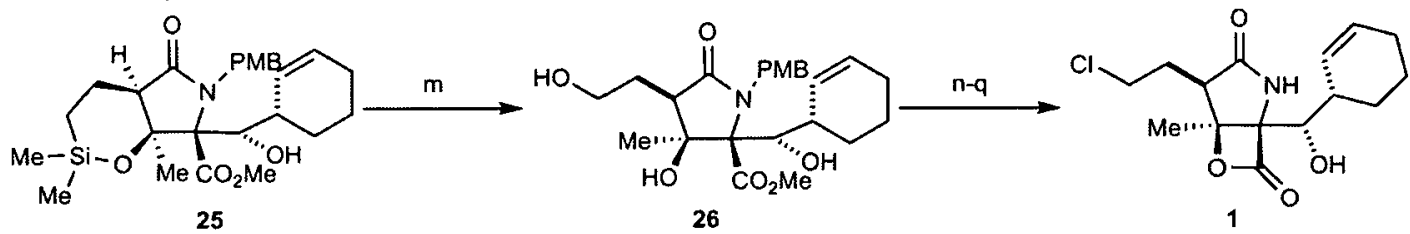

Reagents and conditions: (a) $p$ - TsOH, toluene, reflux, $12 \mathrm{~h}, 80 \%$; (b) LDA, THF-HMPA, $-78^{\circ} \mathrm{C}$ then $\mathrm{ClCH}_{2} \mathrm{OBn}, 4 \mathrm{~h}, 69 \%$; (c) $\mathrm{NaCNBH}_{3}, \mathrm{AcOH}, 40^{\circ} \mathrm{C}, 12 \mathrm{~h}, 90$; (d) TMSCl, Et ${ }_{2} \mathrm{O}, 23{ }^{\circ} \mathrm{C}, 12 \mathrm{~h}$; (e) Acryloyl Chloride, ${ }^{\mathrm{P}} \mathrm{Pr}_{2} \mathrm{NEt}, \mathrm{CH}_{2} \mathrm{Cl}_{2}, 1 \mathrm{~h}, 0^{\circ} \mathrm{C}$ then $\mathrm{H}^{+}, \mathrm{Et}_{2} \mathrm{O}, 23{ }^{\circ} \mathrm{C}, 1 \mathrm{~h}, 96 \%$; (f) Dess-Martin periodinane, $23{ }^{\circ} \mathrm{C}, 1 \mathrm{~h}, 96 \%$; (g) Quinuclidine, DME, $0^{\circ} \mathrm{C}, 7 \mathrm{~d}, 90 \%$; (h) $\mathrm{BrCH}_{2} \mathrm{Si}\left(\mathrm{CH}_{3}\right)_{2} \mathrm{Cl}, \mathrm{Et}_{3} \mathrm{~N}$, DMAP, $\mathrm{CH}_{2} \mathrm{Cl}_{2}, 0^{\circ} \mathrm{C}$, $30 \mathrm{~min}, 95 \%$; (i) $\mathrm{Bu}_{3} \mathrm{SnH}, \mathrm{AIBN}$, Benzene, reflux, $8 \mathrm{~h}, 89 \%$; (j) Pd-C, EtOH, $\mathrm{H}_{2}$ (1 atm), $18 \mathrm{~h}, 95 \%$;. (k) Dess-Martin periodinane, $23^{\circ} \mathrm{C}, 1 \mathrm{~h}, 95 \%$; (l) 24, THF, $-78{ }^{\circ} \mathrm{C}, 5$ h, 88\%; (m) KF, $\mathrm{KHCO}_{3}, \mathrm{H}_{2} \mathrm{O}_{2}$, THF-MeOH (1:1), $23^{\circ} \mathrm{C}, 18 \mathrm{~h}, 92 \%$; (n) CAN, MeCN$\mathrm{H}_{2} \mathrm{O}$; (3:1), $0{ }^{\circ} \mathrm{C}, 1 \mathrm{~h}, 83 \%$; (o) $3 \mathrm{~N} \mathrm{LiOH-THF} \mathrm{(3:1),} 5^{\circ} \mathrm{C}, 4 \mathrm{~d}$; (p) $\mathrm{BOPCl}$, pyridine, $\mathrm{CH}_{2} \mathrm{Cl}_{2}, 23^{\circ} \mathrm{C}, 1 \mathrm{~h}$; (q) $\mathrm{Ph}_{3} \mathrm{PCl}_{2}, \mathrm{MeCN}$, pyridine, $23{ }^{\circ} \mathrm{C}, 1 \mathrm{~h}, 65 \%$ (3 steps).

\section{Scheme 1.6: First total synthesis of 1}

The next step was the installation of the 2-cyclohexenyl group via an organometallic addition of 2-cyclohexenylzinc chloride ${ }^{36}(24)$ into aldehyde 23 . This reaction proceeded with excellent diastereoselectivity (20:1), setting up the desired stereochemical relationship at C5 and C6 (Scheme 1.6). 
Tamao-Fleming oxidation ${ }^{37}$ of 25 afforded triol 26. This intermediate was successfully converted into 1 in four final steps: cleavage of the PMB group using CAN (83\% yield), hydrolysis of the methyl ester employing $\mathrm{LiOH}, \beta$-lactone formation with $\mathrm{BOPCl}$, and finally chlorination of the primary alcohol with triphenylphosphine dichloride (65\% yield over three steps).

The Baylis-Hilman cyclisation step was revised by Corey and co-workers in 2005 to give complete diastereoselectivity $(\mathrm{dr}>99: 1) .{ }^{22}, 23$ In this new methodology, keto-acrylamide 20 was reacted with the Kulinkovich ${ }^{38}$ reagent 27 , formed from reaction of four equivalents of $\mathrm{Ti}^{\mathrm{i}}(\mathrm{PrO})_{4}$ with seven equivalents of cyclopentylmagnesium chloride (Scheme 1.7). The reaction mixture was treated then with iodine to form the $\alpha$-iodomethyl- $\gamma$-lactam 28. Finally, the addition of triethylamine afforded the $\gamma$-lactam 29 in $83 \%$ overall yield.

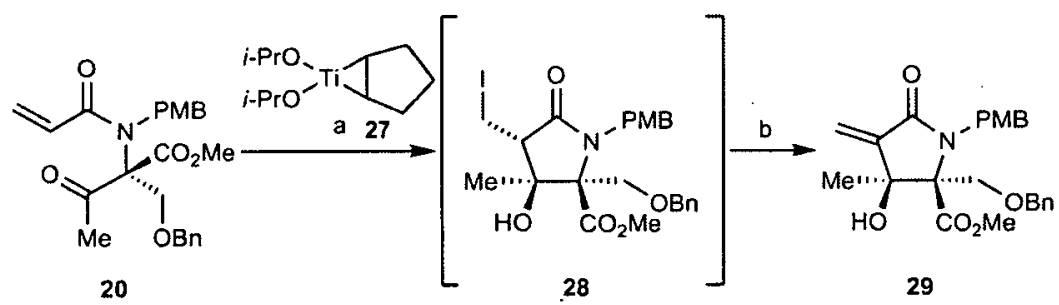

Reagents and conditions: (a) $\mathrm{Ti}(\mathrm{O} i-\mathrm{Pr})_{4}(4 \mathrm{eq}), \mathrm{c}^{-} \mathrm{C}_{5} \mathrm{H}_{9} \mathrm{MgCl}(7 \mathrm{eq}), t-\mathrm{BuOMe},-40^{\circ} \mathrm{C}$, $30 \mathrm{~min}, \mathrm{I}_{2}(5 \mathrm{eq}),-40{ }^{\circ} \mathrm{C}, 2 \mathrm{~h}$ then $0{ }^{\circ} \mathrm{C}, 2 \mathrm{~h}$.; (b) $\mathrm{NEt}_{3}, \mathrm{CH}_{2} \mathrm{Cl}_{2}, 30 \mathrm{~min}, 83 \%$.

Scheme 1.7 Improved $\gamma$ lactam formation

This new methodology was also employed in the synthesis of 3-methyl-2-epi-omuralide 32. ${ }^{39}$ Keto acrylamide 30 was added to the Kulinchovich reagent in a similar procedure but the cyclised titanium-containing intermediate formed was then quenched with $1 \mathrm{~N} \mathrm{HCl}$, an operation that afforded $\gamma$-lactam 31 in $95 \%$ yield and as a single diastereomer (Scheme 1.8). 


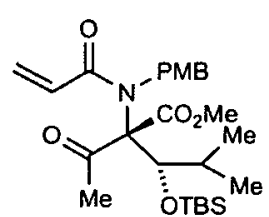

30

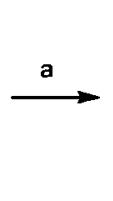

(20)

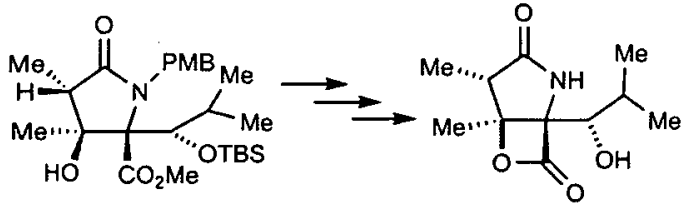

32

Reagents and conditions: (a) $\mathrm{Ti}(\mathrm{O} i \text { - } \mathrm{Pr})_{4}$ (4 eq), $\mathrm{c}-\mathrm{C}_{5} \mathrm{H}_{9} \mathrm{MgCl}$ ( $\left.7 \mathrm{eq}\right), t-\mathrm{BuOMe},-40^{\circ} \mathrm{C}$ to $-20^{\circ} \mathrm{C}$, then $1 \mathrm{~N}$ aq $\mathrm{HCl}, 95 \%$.

\section{Scheme 1.8 Synthesis of 3-methyl-2-epi-omuralide 32}

It was surprising that $\gamma$-lactam 31 was obtained with a anti relationship between the methyl substituent at $\mathrm{C} 2$ and the hydroxyl group at $\mathrm{C} 3$. The Ti-mediated cyclisations of $\gamma, \varepsilon$-unsaturated ketones ${ }^{40}$ have been proposed to occur through a bicyclic organotitanium intermediate such as $\mathbf{3 3}$ (Scheme 1.9). However, the anti arrangement in $\gamma$-lactam 31 of substituents at $\mathrm{C} 2$ and $\mathrm{C} 3$ suggested that the cyclisation occurs via a monocyclic intermediate such as $\mathbf{3 4}$. The formation of $\mathbf{3 4}$ could be explained via a two-step mechanism involving transfer of $(\mathrm{RO})_{2} \mathrm{Ti}$ from the Kulinchovich reagent 27 to the acrylamide $\alpha, \beta$-double bond of $\mathbf{3 0}$, followed by radical addition ${ }^{41}$ of that intermediate to the methyl ketone, which is chelated through $\mathrm{MgCl}$ with the methyl ester carbonyl.

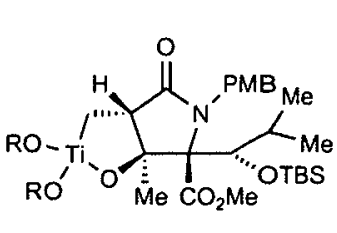

33

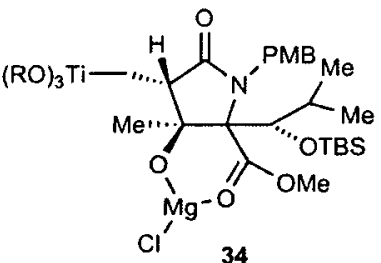

34

Scheme 1.9 Bicyclic organotitanium intermediate vs radical mechanism

Novel methodology for the methyl ester cleavage, $\beta$-lactone formation and the chlorination procedure was also reported. ${ }^{22,} 23$ A novel sequence of three reactions was developed and applied in the transformation of dihydroxylactam 35 to the salinosporamide-omuralide hybrid 9 (Scheme 1.10). The cleavage of the methyl ester was a challenging step due to the strong steric hindrance of the $\mathrm{CO}_{2} \mathrm{Me}$ carbonyl and the tendency of $\mathbf{3 5}$ to undergo retroaldol cleavage and other undesired reactions. A new reagent was successfully developed, $\left(\mathrm{Me}_{2} \mathrm{TeAlMe}\right)_{2}$, readily generated by 
heating tellurium powder and $\mathrm{AlMe}_{3}$ in toluene for $6 \mathrm{~h}$ and cooling the product to room temperature. ${ }^{42}$ This reagent selectively attacks the methyl group of the methyl ester, instead of the carbonyl, and it can operate under mild conditions in terms of temperature and basicity.

Without further purification, the resulting carboxylic acid was reacted with $\mathrm{Ph}_{3} \mathrm{PCl}_{2}$ in dry 1:1 $\mathrm{MeCN}$-pyridine to give directly 36 in $89 \%$ yield. This efficient operation combined the side chain chlorination with a novel method of $\beta$-lactonisation in a single step. Finally, desilylation afforded the salinosporamideomuralide hybrid 9.

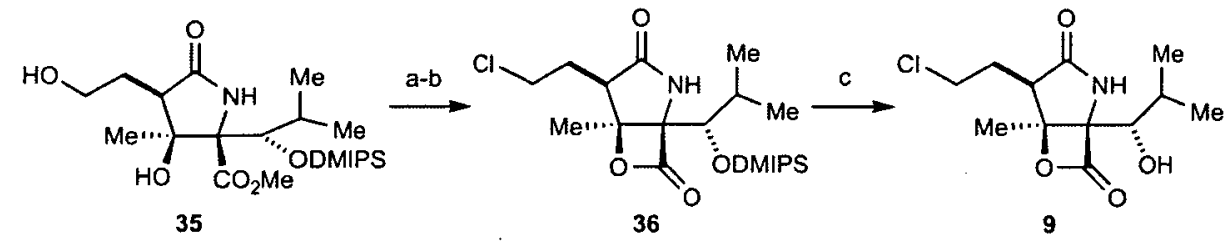

Reagents and conditions: (a) $[\mathrm{MeTeAlMe}]_{2}$ Toluene, $23{ }^{\circ} \mathrm{C}, 12 \mathrm{~h}$; (b) $\mathrm{PPh}_{3} \mathrm{Cl}_{2}$, $\mathrm{CH}_{2} \mathrm{Cl}_{2}$, pyridine, $12 \mathrm{~h}, 89 \%$; (c) $\mathrm{NEt}_{3} \cdot 3 \mathrm{HF}, \mathrm{THF}, 23^{\circ} \mathrm{C}, 4 \mathrm{~h}, 92 \%$.

Scheme 1.10 Completion of the synthesis of salinosporamide-omuralide hybrid 9 via a novel sequence of three reactions

Corey's total synthesis of salinosporamide A via a Baylis-Hillman cyclisation to construct $\gamma$-lactam 29 was achieved in $8.7 \%$ overall yield over eighteen steps. This was improved with a less time consuming, very efficient and completely diastereoselective cyclisation reaction employing the Kulinkovich reagent 27, methodology that allows the synthesis of salinosporamide A in eightteen steps and an improved $9.1 \%$ overall yield.

\subsubsection{Danishefsky's synthesis}

The second total synthesis of 1 was published by Danishefsky in $2005 .{ }^{27}$ This synthetic route afforded salinosporamide A from 37 in twenty-eight steps and in $1.8 \%$ overall yield . It started from the pyroglutamate derivative 37 derived from $L$ glutamic acid (Scheme 1.11). ${ }^{43}$ The strong facial bias of this bicyclo[2.2.0] compound 37 allowed the creation of the stereocentre at $\mathrm{C} 3$ through treatment with divinyl cuprate, attacking from the $\alpha$-face (addition completely diastereoselective). 
This was followed by alkylation at $\mathrm{C} 2$ with $\beta$-benzyloxy iodoethane, with attack now from the $\beta$-face, to give lactam 38 as a $14: 1$ mixture of diastereoisomers.

Acylation at $\mathrm{C} 4$ would be achieved via conversion of the vinyl group into a carbonate ester acylating agent. Ozonolysis of $\mathbf{3 8}$ and reductive treatment with sodium borohydride produced alcohol 39. Carbonate formation and aminal cleavage using TfOH afforded alcohol $\mathbf{4 0}$, which was subjected to Jones oxidation to the carboxylic acid, tert-butyl ester formation and imidate formation with Meerwein's reagent $\left(\mathrm{Et}_{3} \mathrm{OBF}_{4}\right)$ to produce $\mathbf{4 1}$.
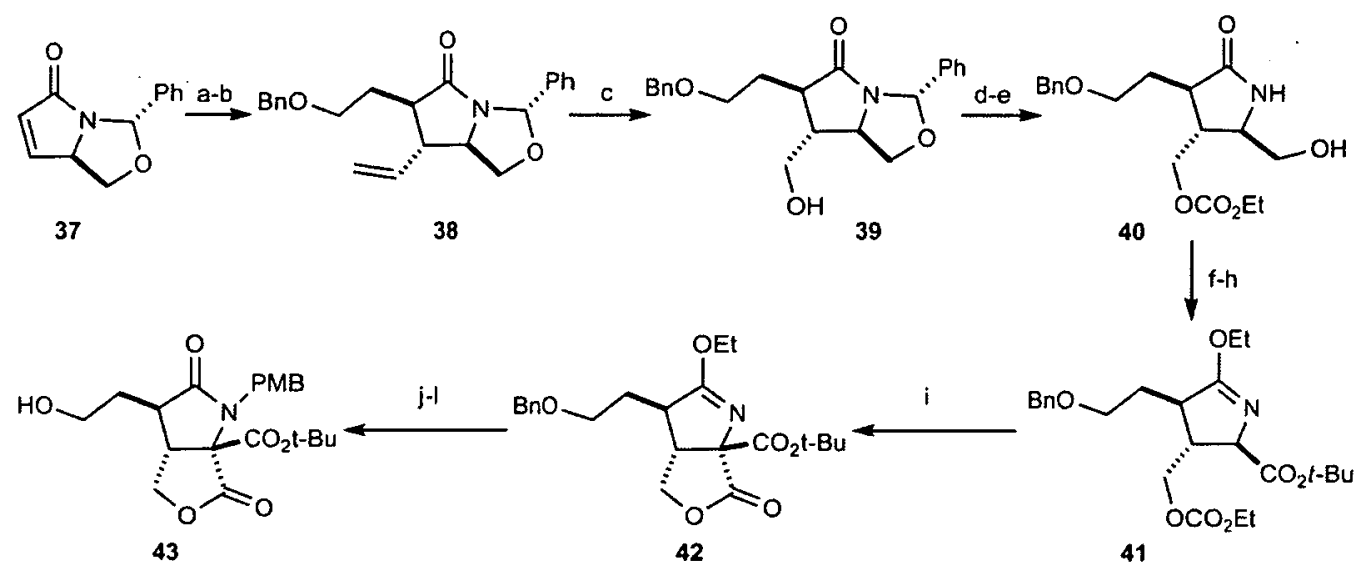

Reagents and conditions: (a) vinylmagnesium bromide, TMSCl, $\mathrm{CuI},-78^{\circ} \mathrm{C}, 75 \%$; (b) [(2-iodoethoxy)methyl]benzene, LDA, THF, rt, 77\%, dr $=14: 1$; (c) $\mathrm{O}_{3}, \mathrm{CH}_{2} \mathrm{Cl}_{2}-\mathrm{MeOH}$ (3:1), $-78{ }^{\circ} \mathrm{C}$ then $\mathrm{NaBH}_{4}, 0{ }^{\circ} \mathrm{C}\left(86 \%\right.$ ); (d) $\mathrm{ClCO}_{2} \mathrm{Et}$, pyridine, rt, 96\%; (e) $\mathrm{TfOH}$, THF- $\mathrm{H}_{2} \mathrm{O}$ (9:1), rt, 100\%; (f) Jones reagent, acetone, rt; (g) $\mathrm{Me}_{2} \mathrm{NCH}(\mathrm{O} t-\mathrm{Bu})_{2}$, toluene, reflux, $72 \%$ over 2 steps; (h) $\mathrm{Et}_{3} \mathrm{OBF}_{4}, \mathrm{~K}_{2} \mathrm{CO}_{3}, \mathrm{CH}_{2} \mathrm{Cl}_{2}, \mathrm{rt}, 88 \%$; (i) LHMDS, THF, $-20{ }^{\circ} \mathrm{C}, 82 \%$; (j) $1 \mathrm{M} \mathrm{HCl}$ aq, THF, $0{ }^{\circ} \mathrm{C}, 90 \%$; (k) PMBCl, NaH, DMF, rt, 61\%; (l) $\mathrm{Pd}(\mathrm{OH})_{2}-\mathrm{C}, \mathrm{H}_{2}$, EtOH, $\mathrm{rt}, 100 \%$.

\section{Scheme 1.11 Danishefsky's synthesis (I)}

With the lactam functionality thus masked, internal acylation of $\mathrm{C} 4$ with the pendant carbonate occurred in good yield using LHMDS, forming intermediate $\mathbf{4 2}$. In this step, the stereogenic centre at $\mathrm{C} 4$ was constructed. The lactam was unmasked under acidic conditions, the amide was then protected with $\mathrm{PMBCl}$ and the benzyl ether was cleaved to give lactam $\mathbf{4 3}$.

Nucleophilic lactone ring-opening of $\mathbf{4 3}$ with a phenylselenium anion generated from $(\mathrm{PhSe})_{2}$ and $\mathrm{NaBH}_{4}$ followed by benzyl ester formation of the resulting carboxylic acid afforded 44 (Scheme 1.12). The subsequent selenide 
oxidation/elimination sequence afforded a mixture of the expected alcohol $45(72 \%)$, along with aldehyde $46(22 \%)$. Upon purification, the unoxidised material was converted into aldehyde 46 via a Dess-Martin periodinane ${ }^{35}$ oxidation ( $89 \%$ in three steps from 44).

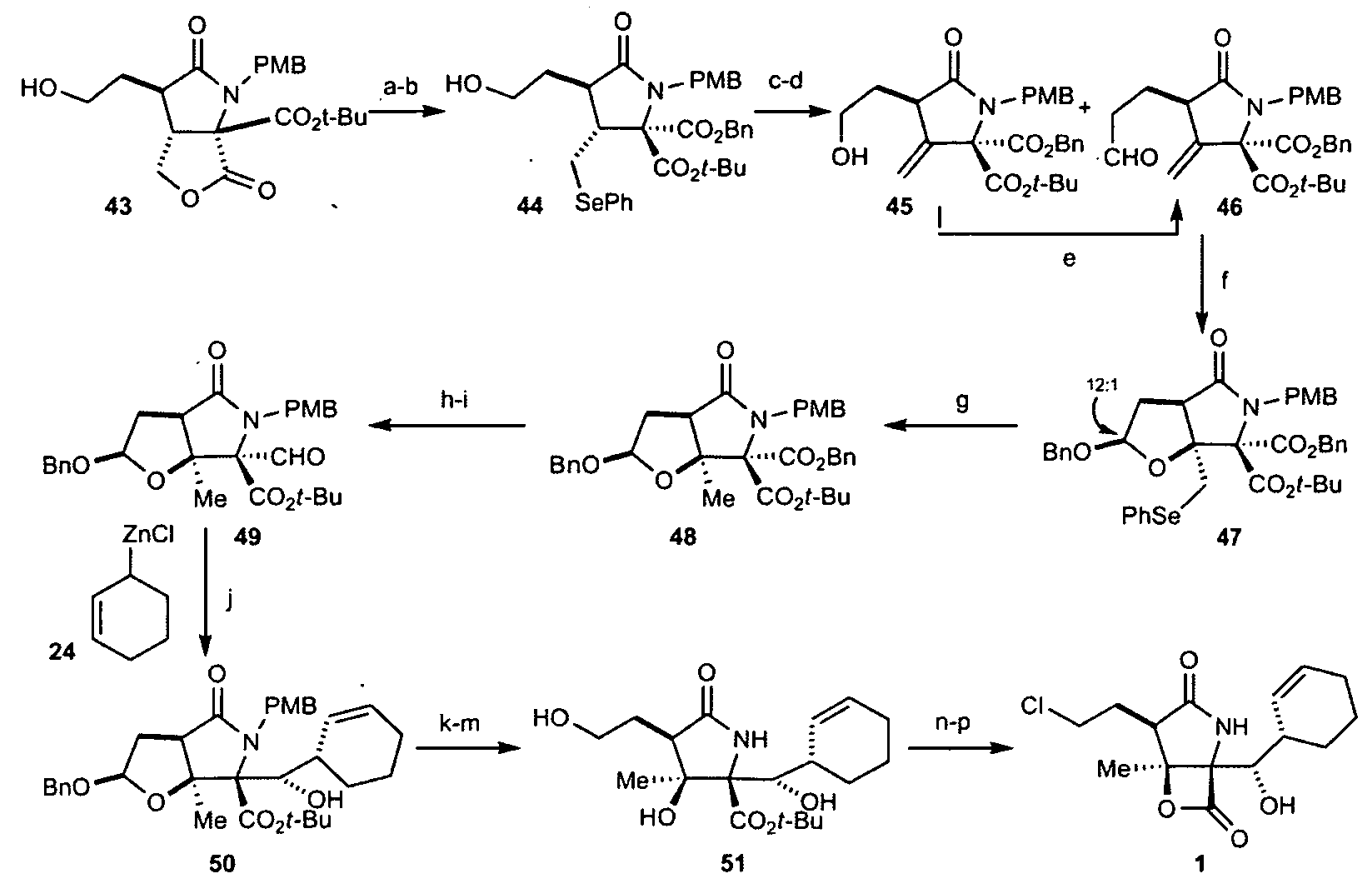

Reagents and conditions: (a) $\mathrm{PhSeSePh}, \mathrm{NaBH}_{4}, \mathrm{EtOH}, 60{ }^{\circ} \mathrm{C}$; (b) $\mathrm{BnBr}, \mathrm{K}_{2} \mathrm{CO}_{3}$, DMF, rt, $72 \%$ over 2 steps; (c) $30 \% \mathrm{H}_{2} \mathrm{O}_{2}$ aq, THF, rt; (d) toluene, $100{ }^{\circ} \mathrm{C}, 72 \% 45+$ $22 \% 46$ over 2 steps; (e) Dess-Martin periodinane, $\mathrm{CH}_{2} \mathrm{Cl}_{2}, \mathrm{rt}, 92 \%$ (89\% in three steps from 44); (f) $\mathrm{PhSeBr}, \mathrm{AgBF}_{4}, \mathrm{BnOH}, \mathrm{CH}_{2} \mathrm{Cl}_{2},-20$ to $0{ }^{\circ} \mathrm{C}, 74 \%$ as an anomeric mixture (12:1); (g) AIBN, $n \mathrm{Bu}_{3} \mathrm{SnH}$, toluene, $100{ }^{\circ} \mathrm{C}, 98 \%$; (h) $\mathrm{NaBH}_{4}$, THF-EtOH (3:1), rt, 85\%; (i) Dess-Martin periodinane, $\mathrm{CH}_{2} \mathrm{Cl}_{2}, \mathrm{rt}, 95 \%$; (j) cyclohexenyl zinc chloride 24, THF, $-78{ }^{\circ} \mathrm{C}$; (k) $\mathrm{CAN}, \mathrm{CH}_{2} \mathrm{Cl}_{2}-\mathrm{H}_{2} \mathrm{O}, 0^{\circ} \mathrm{C}, 90 \%$; (l) $\mathrm{Na}$, liq $\mathrm{NH}_{3},-78^{\circ} \mathrm{C}$; (m) $\mathrm{NaBH}_{4}$, THF- $\mathrm{H}_{2} \mathrm{O}$ (2:1), rt, 97\% over two steps; (n) $\mathrm{BCl}_{3}, \mathrm{CH}_{2} \mathrm{Cl}_{2}, 0{ }^{\circ} \mathrm{C}$; (o) $\mathrm{BOPCl}, \mathrm{NEt}_{3}$, $\mathrm{CH}_{2} \mathrm{Cl}_{2}, \mathrm{rt}$; (p) $\mathrm{PPh}_{3} \mathrm{Cl}_{2}$, pyridine, $\mathrm{CH}_{2} \mathrm{Cl}_{2}, \mathrm{rt}, 51 \%$ over 3 steps.

\section{Scheme 1.12 Danishefsky's synthesis (II)}

Treatment of 46 with phenylselenium bromide and $\mathrm{AgBF}_{4}$ in the presence of benzyl alcohol gave acetal $\mathbf{4 7}$, setting up the desired stereochemical relationship at $\mathrm{C} 2$ and $\mathrm{C} 3$. Subsequent radical deselenenylation of 47 yielded 48 , which contains the desired C3 methyl substituent. Selective reduction of the benzyl ester of $\mathbf{4 8}$ to the alcohol followed by a Dess Martin oxidation ${ }^{35}$ afforded aldehyde 49. Stereoselective introduction of the cyclohexenyl group was conducted using Corey's procedure ${ }^{26}$ : 
treatment of the PMB protected $\gamma$-lactam 49 with the cyclohexenyl zinc reagent 24, produced 50 with an excellent yield and diastereoselectivity. It was reported that the use of this methodology on the corresponding imidate aldehyde derived from $\mathbf{4 2}$ (Scheme 1.11) instead of on $\mathbf{4 9}$ resulted in poor diastereoselectivity (78\% yield, 4:3, configuration not determined). This showed that the PMB group played a critical role in diastereoselection in this novel addition.

Removal of the PMB group, followed by reductive opening of the benzyl glycoside, gave rise to triol 51. Lewis acidic cleavage of the tert-butyl ester was effected through treatment with $\mathrm{BCl}_{3}$, and the crude trihydroxy acid was then subjected to lactonisation-chlorination to provide salinosporamide A (1).

\subsubsection{Pattenden Racemic Synthesis}

Pattenden and co-workers reported in 2006 a racemic synthesis of salinosporamide A, outlined in Scheme $1.13 .{ }^{29}$ In this route, $( \pm)-1$ was obtained from 52 in fourteen steps and $11.5 \%$ overall yield.

Protection of known ketone $\mathbf{5 2}^{\mathbf{4 4}}$ as an acetal was followed by ester hydrolysis and coupling of the resulting acid with dimethyl 2-aminomalonate to afford amide 53. This intermediate was transformed into amide $\gamma$-lactam ( \pm -54 via a diastereoselective acid catalysed intramolecular cyclisation. When a solution of 53 in acetic acid/water (4:1) was heated at $65^{\circ} \mathrm{C}$ for 4 days, deprotection of the dioxolane and in situ cyclisation occurred, leading to a single diastereomer of ( \pm )-54.

The tertiary alcohol of $( \pm)-54$ was then TMS-protected and the lactam nitrogen was protected with a PMB group, forming ( \pm )-55. The ester anti to the protected alcohol was then selectively reduced to the aldehyde using lithium superhydride. The cyclohexenyl side chain was inserted into $( \pm)-56$ following Corey's procedure, ${ }^{26}$ obtaining the addition product $( \pm)-57$ with excellent diastereoselectivity. 


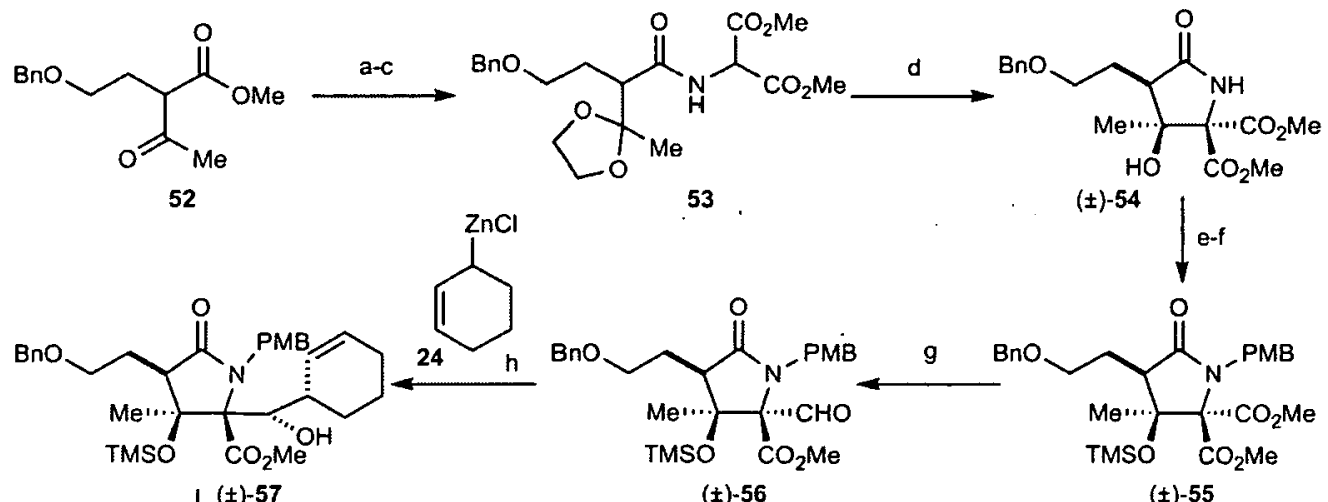

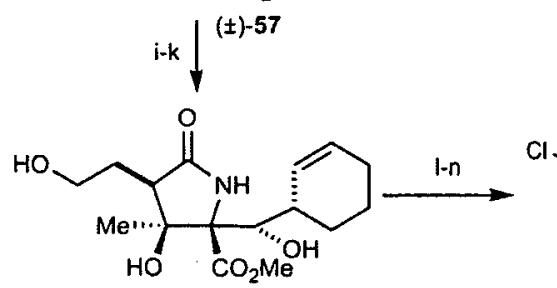

(士)-58

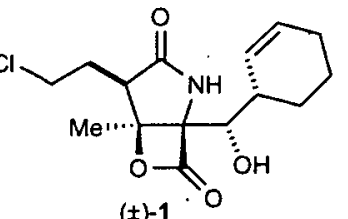

$( \pm)-1$

Reagents and conditions: (a) ethylene glycol, $p$ - TsOH, benzene, $110^{\circ} \mathrm{C}, 14 \mathrm{~h}$; (b) $2 \mathrm{M}$ $\mathrm{NaOH}, \mathrm{EtOH}, 70^{\circ} \mathrm{C}, 3 \mathrm{~h}$; (c) dimethyl aminomalonate $\mathrm{HCl}, \mathrm{HOBt}, \mathrm{EDC} \cdot \mathrm{HCl}, \mathrm{CH}_{2} \mathrm{Cl}_{2}$, NMM, $0{ }^{\circ} \mathrm{C}$ to rt (82\% over 3 steps); (d) $4: 1 \mathrm{AcOH}-\mathrm{H}_{2} \mathrm{O}, 65^{\circ} \mathrm{C}, 4 \mathrm{~d}(71 \%)$; (e) excess TMSOTf, 2,6-lutidine, $\mathrm{CH}_{2} \mathrm{Cl}_{2},-78{ }^{\circ} \mathrm{C}$ to $0{ }^{\circ} \mathrm{C}$, then $1 \mathrm{M} \mathrm{HCl}(91 \%$ ); (f) $\mathrm{PMB}-\mathrm{Br}, \mathrm{NaH}$, DMF, $0{ }^{\circ} \mathrm{C}$ to $\mathrm{rt}, 14 \mathrm{~h}(82 \%)$; (g) Super-hydride (1.0 M in THF), $\mathrm{CH}_{2} \mathrm{Cl}_{2},-78{ }^{\circ} \mathrm{C}, 3 \mathrm{~h}$ (78\%); (h) 2-cyclohexenylzinc chloride 24 , THF, $-78{ }^{\circ} \mathrm{C}$ (87\%); (i) $\mathrm{BCl}_{3} \cdot \mathrm{DMS}, \mathrm{CH}_{2} \mathrm{Cl}_{2}$, $24 \mathrm{~h}, 0^{\circ} \mathrm{C}$ to $\mathrm{rt}$; (j) $48 \% \mathrm{HF}$ in $\mathrm{H}_{2} \mathrm{O}-\mathrm{MeCN}$ (1:9), rt, $22 \mathrm{~h}$; (k) CAN, MeCN, $\mathrm{H}_{2} \mathrm{O}$ (3:1), 0 ${ }^{\circ} \mathrm{C}, 1 \mathrm{~h}$ (87\% over 3 steps); (l) [MeTeAlMe $]_{2}$, toluene, $\mathrm{rt}, 24 \mathrm{~h}$; (m) BOP-Cl, $\mathrm{CH}_{2} \mathrm{Cl}_{2}$, pyridine, $\mathrm{rt}, 3 \mathrm{~h}$; (n) $\mathrm{PPh}_{3} \mathrm{Cl}_{2}, \mathrm{MeCN}$, pyridine, $\mathrm{rt}, 4 \mathrm{~h}$ ( $45 \%$ over 3 steps).

\section{Scheme 1.13 Pattenden's synthesis}

Cleavage of the benzyl ether and trimethylsilyl ether, followed by deprotection of the PMB group on the lactam nitrogen afforded Corey intermediate $( \pm)-58,{ }^{26}$ which was then converted into $( \pm)$-salinosporamide A following reported methods. ${ }^{22,23,42}$

\subsubsection{Langlois's formal synthesis}

In 2006, Langlois and co-workers reported a formal synthesis of Salinosporamide A. ${ }^{31,32}$ As it has been shown, Corey's synthesis involved the highly functionalised $\alpha$-methylene-lactam 29 as a key intermediate (Figure 1.6). Langlois first published a route to $( \pm)-59$, a closely related scaffold which contains all the required functionality to be transformed into racemic salinosporamide $A$. Their synthetic strategy was based on a regio- and stereoselective $N$-methylnitrone cycloaddition. 


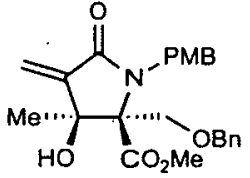

29

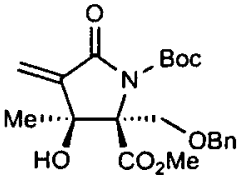

(t) -59

Figure 1.6 $\alpha$-Methylene-lactam key intermediates 29 and $( \pm)-59$

Selective alkylation of methyl pyroglutamate 60 with chloromethylbenzylether was successfully achieved using LHMDS (Scheme 1.14). $\mathrm{N}$-Boc protection afforded intermediate 61. This protection improved the reactivity of the lactam carbonyl, allowing efficient classical introduction of the double bond to give 62. A stereoselective methylcuprate addition was then performed (product obtained as a single diastereomer), followed by reintroduction of the double bond, yielding 63. A regio- and stereoselective 1,3-dipolar cycloaddition of $N$-methylnitrone with 63 was achieved affording ( \pm )-64 in 57\% yield. In this step the C3 hydroxyl group and the precursor for the $\mathrm{C} 2$ exo-methylene group are introduced. The cleavage of the isoxaline $\mathrm{N}-\mathrm{O}$ bond was performed chemoselectively by hydrogenolysis using Pearlman's catalyst, without affecting the $O$-benzyl group. Finally, methylation with an excess of iodomethane and treatment with triethylamine afforded the target intermediate $( \pm)-59$.

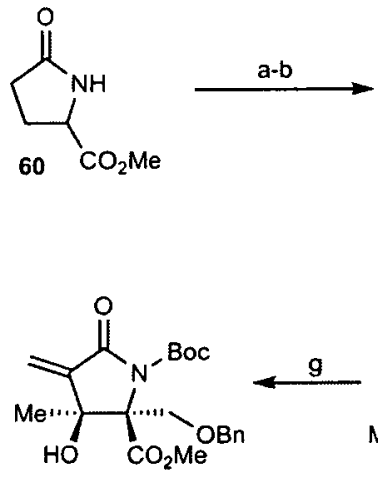

$( \pm)-59$
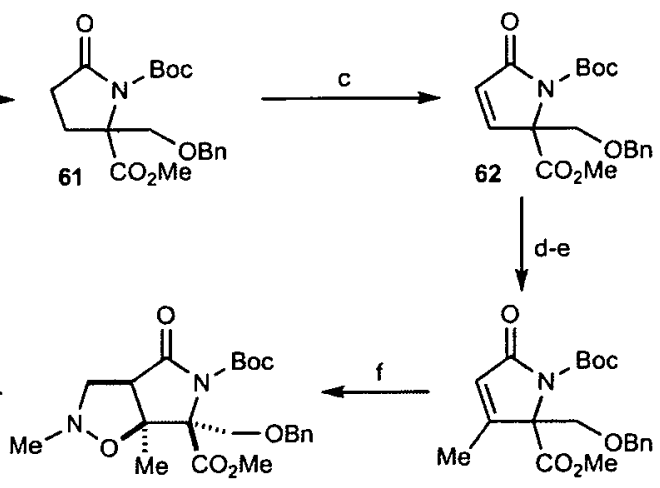

$( \pm)-64$

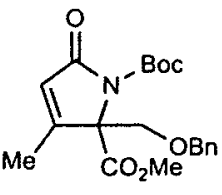

63

Reagents and conditions: (a) LHMDS, THF, $\mathrm{BnOCH}_{2} \mathrm{Cl}$ (53\%); (b) (Boc) ${ }_{2} \mathrm{O}$, DMAP, $\mathrm{MeCN}$ (100\%); (c) (i) LHMDS, THF, $\mathrm{PhSeCl}$; (ii) $\mathrm{H}_{2} \mathrm{O}_{2}, \mathrm{CH}_{2} \mathrm{Cl}_{2}$, pyridine (89\%); (d) $\mathrm{Me}_{2} \mathrm{CuLi}$, THF, TMSCl (83\%); (e) (i) LHMDS, THF, $\mathrm{PhSeCl}$; (ii) $\mathrm{H}_{2} \mathrm{O}_{2}, \mathrm{CH}_{2} \mathrm{Cl}_{2}$, pyridine (75\%); (f) $N$-methylnitrone, toluene, reflux (57\%); (g) (i) $\mathrm{H}_{2}, \mathrm{Pd}(\mathrm{OH})_{2}$, EtOAc-MeOH (72\%); (ii) MeI, THF, $\mathrm{Et}_{3} \mathrm{~N}$ (65\%).

Scheme 1.14 Racemic formal synthesis 
A second publication by Langlois reported an asymmetric route to 1 . To obtain enantiopure 29 and 59 (Figure 1.6), (S)-methyl 2-hydroxymethylpyroglutamate 67 was chosen as the chiral precursor (Scheme 1.15). This compound has been prepared before from bicyclic nitrile $65^{45}$ Selective $O$-benzylation of 67 was successfully achieved using 2-benzyloxy-1-methylpyridinium triflate (68), leading to 69 in $75 \%$ yield. From intermediate 69, the synthesis of enantiopure 59 (Figure 1.6) is achievable, because the subsequent steps of the racemic synthesis (Scheme 1.14) cannot produce racemisation.

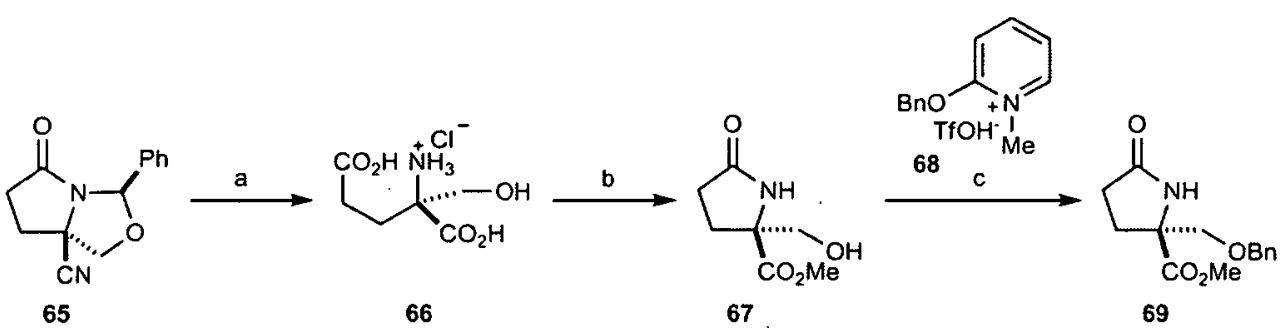

Reagents and conditions: (a) $6 \mathrm{~N} \mathrm{HCl}$, reflux (98\%); (b) $\mathrm{CH}_{2} \mathrm{~N}_{2}, \mathrm{Et}_{2} \mathrm{O}(67 \%)$; (c) 68 , $\mathrm{MgO}, \mathrm{PhCF}_{3}$, reflux (75\%).

\section{Scheme 1.15 Langlois' formal synthesis}

This chemistry was also employed for the synthesis of Corey's intermediate 29. Starting from PMB-protected 69, the double bond introduction (Scheme 1.14) was achieved in a very low yield. Therefore 69 was transformed into the $N$-Boc derivative 59, which was followed by removal of the Boc protecting group and PMB protection, to afford pyrrolinone 70 (Scheme 1.16).

The $N$-methylnitrone cycloaddition with $\mathbf{7 0}$ occurred with the expected regioselectivity, but slightly lower stereoselectivity (ratio 4:1), giving the desired diastereomer 72 in $54 \%$ yield (14\% of 71). After hydrogenolysis (73), $N$-methylation using iodomethane and elimination in a biphasic mixture of aqueous $\mathrm{Na}_{2} \mathrm{CO}_{3}$, and $\mathrm{CH}_{2} \mathrm{Cl}_{2}$ afforded the target $\alpha$-methylenelactam 29. This route represents a formal synthesis of the natural product salinosporamide $A$ from 67 in eighteen steps and $4 \%$ overall yield. 


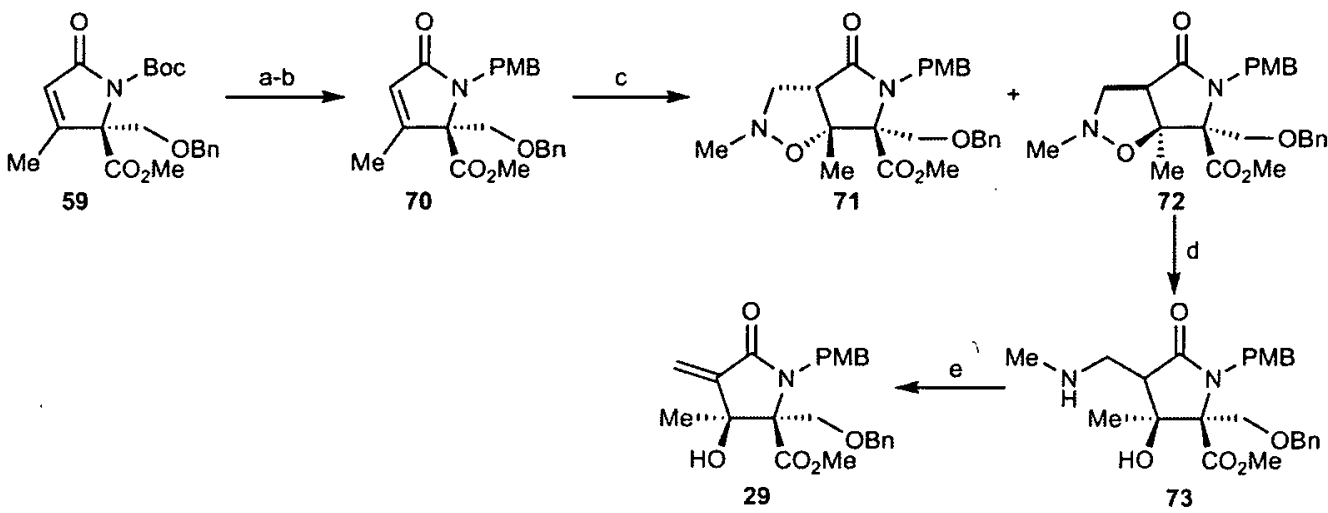

Reagents and conditions: (a) $\mathrm{CF}_{3} \mathrm{CO}_{2} \mathrm{H}, \mathrm{CH}_{2} \mathrm{Cl}_{2}$ (100\%); (b) $\mathrm{CsCO}_{3}, \mathrm{PMBBr}$, DMF (57\%); (c) $\mathrm{N}$-methylnitrone, toluene, reflux (14\% for 71, 54\% for 72); (d) $\mathrm{H}_{2}, \mathrm{Pd}(\mathrm{OH})_{2}$, EtOAc-MeOH (64\%); (e) (i) MeI, $\mathrm{MeOH}$; (ii) $\mathrm{Na}_{2} \mathrm{CO}_{3}, \mathrm{CH}_{2} \mathrm{Cl}_{2}$ (90\%).

Scheme 1.16: Langlois' formal synthesis

\subsubsection{Romo's synthesis}

A racemic synthesis of salinosporamide $A$ was published by Romo and co-workers in March 2007..$^{30}$ In this publication, they reported new methodology to form the bicyclic core of the natural product, via an intramolecular bis-cyclisation process which forms the $\mathrm{C}-\mathrm{C}$ and the $\mathrm{C}-\mathrm{O}$ bonds simultaneously (Scheme 1.17). This bis-cyclisation process permitted the synthesis of the $( \pm)$-bicyclic- $\beta$-lactone with the required relative configuration. Potential enolisation and subsequent racemisation at C2 was not problematic since, due to the conformationally controlled acidity of $\beta$ ketoamides owing to $\mathrm{A}^{1,3}$ strain, ${ }^{46}$ retention of this stereocentre occurred. This route allowed the synthesis of $( \pm)$-salinosporamide $A$ in nine steps and $1.2 \%$ overall yield.

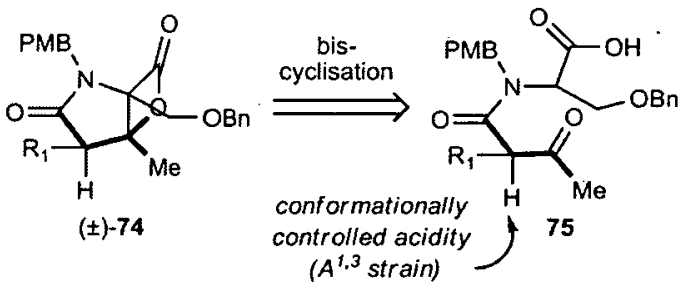

Scheme 1.17: Racemic formal synthesis

In this synthesis, $N$-PMB serine allyl ester 77 was obtained in two steps from benzyl protected serine $\mathbf{7 6}$ and then coupled with heteroketene dimer $\mathbf{7 8}$, providing . 
79, which afforded ketoacid 80 after Pd-mediated ester deprotection (Scheme 1.18). Bis-cyclisation of 80 gave bicyclic- $\beta$-lactones $( \pm)-82$ and $( \pm)-83$ in $25-35 \%$ yield, in favour of the relative configuration of salinosporamide $A$ (dr 2-3:1). This was a much lower yield than the ones obtained in bis-cyclisation reactions with C4-unsubstituted substrates, most likely due to increased steric crowding.

Deprotection of the benzyl ether allowed enrichment of the major diastereomer $( \pm)-84$ to 6-10:1 after purification. A modified Moffat oxidation ${ }^{47}$ using EDCI and dichloroacetic acid, followed by addition of 2-cyclohexenyl zinc chloride gave two diastereomeric alcohols (dr 3.5:1) in 33\% yield. Finally, PMB deprotection of $( \pm)$-85 permitted isolation of diastereomerically pure $( \pm)$-salinosporamide A.
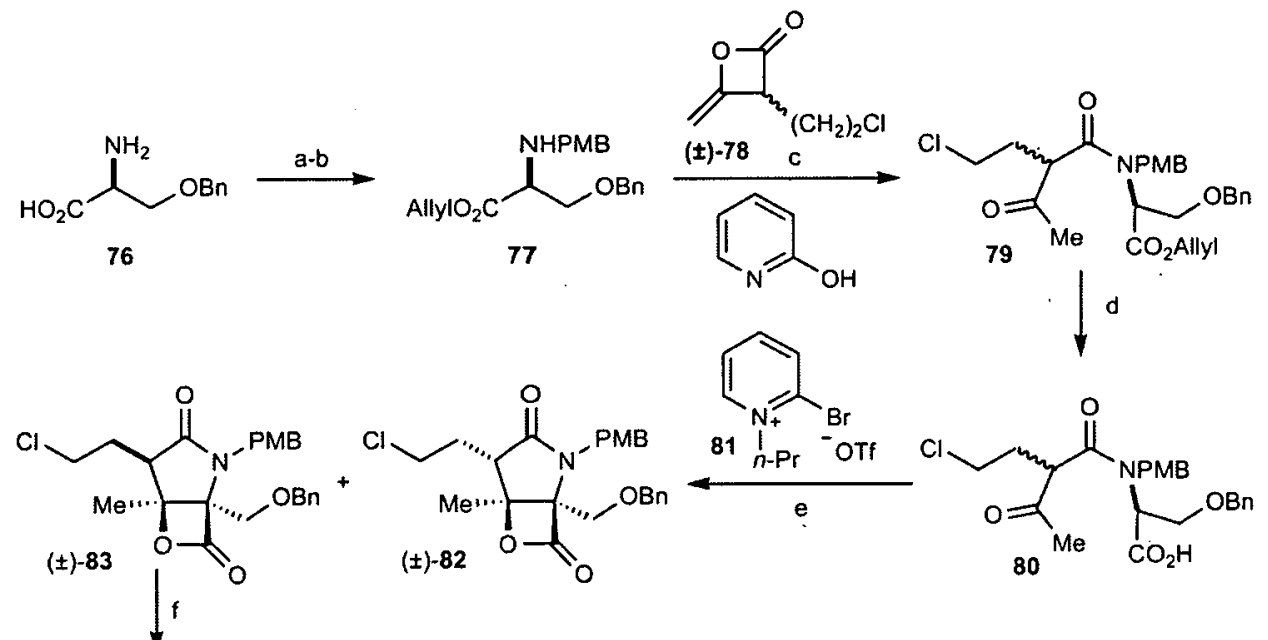

$+84$<smiles>CC1C(=O)N([Pb])[C@@]2(CO)C(=O)O[C@@]12C</smiles><smiles>CCN1C(=O)[C@H](CCCl)[C@@]2(C)OC(=O)[C@]12[C@H](O)[C@H]1C=CCC(I)C1</smiles><smiles>C[C@]1([Ga])N[C@@](O)([C@H]2C=CCCC2)C(=O)O1</smiles>

Reagents and conditions: (a) p-anisaldehyde, $\mathrm{MeOH}, \mathrm{NaBH}_{4}$; (b) $p$ - $\mathrm{TsOH}$, allyl alcohol (74\%, 2 steps); (c) THF, $60{ }^{\circ} \mathrm{C}, 36 \mathrm{~h}(80 \%)$; (d) $\mathrm{Pd}\left(\mathrm{PPh}_{3}\right)_{4}$, morpholine (75\%); (e) DIPEA, PPY, $\mathrm{CH}_{2} \mathrm{Cl}_{2},-10{ }^{\circ} \mathrm{C}, 6 \mathrm{~h}\left(25-35 \%\right.$, dr 2-3:1); (f) $\mathrm{H}_{2}, \mathrm{Pd} / \mathrm{C}, \mathrm{THF}, 25{ }^{\circ} \mathrm{C}$ (98\%); (g) EDCI, DMSO, $\mathrm{Cl}_{2} \mathrm{CHCO}_{2} \mathrm{H}$; (h) 2-cyclohexenylzinc chloride 24, THF, $-78{ }^{\circ} \mathrm{C}$ (33\%, 2 steps, dr 3.5:1); (i) $\mathrm{CAN}, \mathrm{MeCN} / \mathrm{H}_{2} \mathrm{O}$ (49\%, major diast.).

\section{Scheme 1.18 Racemic formal synthesis}

\subsubsection{Macherla's synthesis}

Another enantioselective synthesis of salinosporamide $\mathrm{A}$ was reported by Macherla and co-workers in 2007. ${ }^{28}$ This synthesis starts with a $\mathrm{MsCl}$-mediated amide coupling of $\mathbf{8 6}$ and 87 to give protected $\beta$-ketoamide 88 (Scheme 1.19). 
Oxazolidine 87 serves both as a group to induce chirality in the aldol cyclisation performed in a later step and as a protecting group. Deprotection of the ketone generated $\beta$-ketoamide 89 as a mixture of epimers at the $\alpha$-carbon containing the allyl group.

The next step generated key intermediate 90, via a novel enantioselective intramolecular aldol cyclisation that uses the self-regeneration of stereocentres principle $^{48}$ to generate simultaneously chiral stereocentres $C 2$ and $C 3$. $\beta$-Ketoamide 89 was treated with $t$-BuOK to give rise to 90 in $64 \%$ yield and $70 \%$ d.e., along with minor diastereomers and dehydration product. The chirality of $\mathrm{C} 4$ stereocentre was maintained in the reaction.

The highly functionalised intermediate 90 was then transformed into 91 (and its diastereomer, 5:1 ratio) via oxidative cleavage of the allyl group with $\mathrm{OsO}_{4} / \mathrm{NMO}$ followed by treatment with $\mathrm{NaIO}_{4}$ and then treatment with $\mathrm{BnBr}$ in the presence of $t$-BuOK. The methyl ester of 91 was reduced to the alcohol and reoxidised to the aldehyde 92 using a TPAP/NMO oxidation ${ }^{49}$. The use of Corey's methodology ${ }^{26}$ to install the cyclohexene ring on 92 was not successful in this system and Brown's allylboration chemistry ${ }^{50}$ had to be used instead. Aldehyde 92 was reacted with $B$-2-cyclohexen-1-yl-9-BBN (93) to give the expected syn addition product 94, having the desired stereochemistry at $\mathrm{C} 6$, but the required stereocentre at $\mathrm{C} 5$ would have to be regenerated later.

The benzyl acetal in 94 was opened and transformed into the benzoyl protected alcohol 96. This transformation aimed to obtain a protecting group that would withstand the strong acidic conditions of the aminal deprotection (1,3-propanedithiol, $\mathrm{HCl}$, TfOH), because if the benzyl alcohol was deprotected, it could not be differentiated later in the synthesis from the other primary alcohol ( $\mathrm{C} 15$ in 96). Oxidation of the $\mathrm{C} 15$ alcohol was only possible after protection of the C5 hydroxyl group. It was found out that TMS protection of C5 hydroxyl followed by a 2-step oxidation afforded the corresponding carboxylic acid 97.

Deprotection of the benzoyl group of 97 was followed by $\beta$-lactone formation with $\mathrm{BOPCl}$ and chlorination of the primary alcohol with $\mathrm{Ph}_{3} \mathrm{PCl}_{2}$ afforded 98 , the C5 epimer of 1. The C5 hydroxyl group was oxidised to the ketone using Dess- 
Martin periodinane ${ }^{35}$, which was stereoselectively reduced by a ketoreductase enzyme to afford the desired (-)-1.

The synthesis of salinosporamide $A$ was achieved in twenty-four steps and $0.2 \%$ overall yield. The key formation of 90 was accomplished via an impressive enantioselective intramolecular aldol cyclisation, but protecting group manipulations and the regeneration of the $\mathrm{C} 5$ stereocentre added extra steps and lowered the yield of this synthetic route.

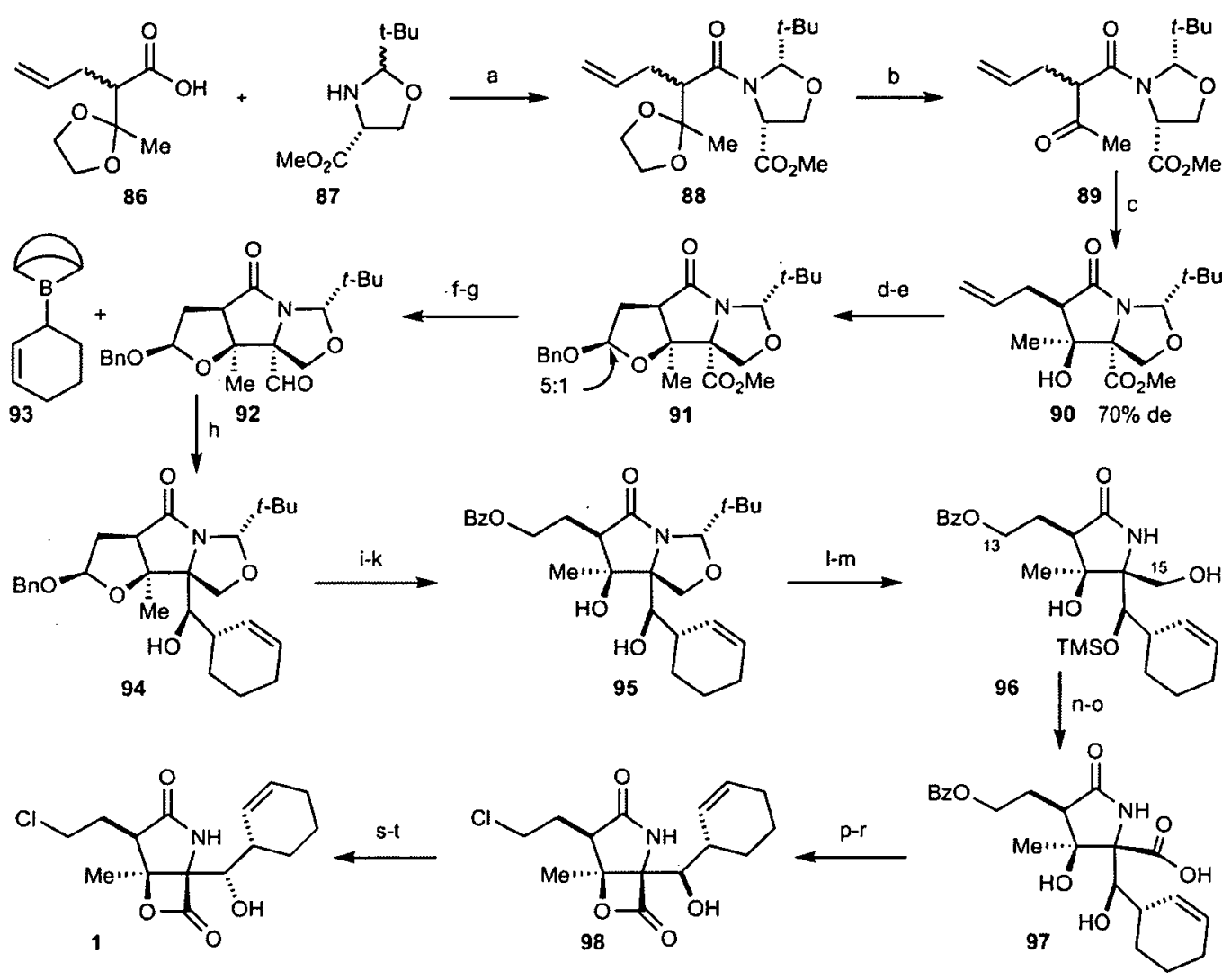

Reagents and conditions: (a) $\mathrm{MsCl}, \mathrm{Et}_{3} \mathrm{~N}, \mathrm{CH}_{2} \mathrm{Cl}_{2}, 0{ }^{\circ} \mathrm{C}$ to $\mathrm{rt}, \mathrm{o} / \mathrm{n}(66 \%$ ); (b) $\mathrm{CeCl}_{3} .7 \mathrm{H}_{2} \mathrm{O}, \mathrm{NaI}, \mathrm{MeCN}, 60^{\circ} \mathrm{C}, 4 \mathrm{~h}(84 \%)$; (c) $t$-BuOK, THF, rt, $15 \min (64 \%, 70 \%$ de); (d) $\mathrm{OsO}_{4}$, NMO, $\mathrm{H}_{2} \mathrm{O} / \mathrm{THF}, 3 \mathrm{~h}$ then $\mathrm{NaIO}_{4}, 2 \mathrm{~h},(99 \%)$; (e) $\mathrm{BnBr}, t$-BuOK, THF, 6 h, 76\%; (f) $\mathrm{LiBH}_{4}$, THF, 24 h (94\%); (g) TPAP (cat.), NMO, $\mathrm{CH}_{2} \mathrm{Cl}_{2}, 18$ h (79\%); (h) THF, $-78^{\circ} \mathrm{C}$ to $\mathrm{rt}, 11.5 \mathrm{~h}\left(80 \%\right.$ ); (i) $\mathrm{HCl}$, THF, $60^{\circ} \mathrm{C}, 10 \mathrm{~h}$; (j) $\mathrm{NaBH}_{4}, \mathrm{THF} / \mathrm{H}_{2} \mathrm{O}, 30$ min (81\%, 2 steps); (k) BzCl, $\mathrm{Et}_{3} \mathrm{~N}, \mathrm{CH}_{2} \mathrm{Cl}_{2}, 10 \mathrm{~h}$ (87\%); (l) 1,3-propanedithiol, $\mathrm{HCl}$, $\mathrm{CF}_{3} \mathrm{CH}_{2} \mathrm{OH}, 60^{\circ} \mathrm{C}, 4 \mathrm{~h}(94 \%)$; (m) TMSCl, $\mathrm{Et}_{3} \mathrm{~N}, \mathrm{CH}_{2} \mathrm{Cl}_{2}, 12 \mathrm{~h}(53 \%)$; (n) Dess-Martin periodinane, $\mathrm{CH}_{2} \mathrm{Cl}_{2}, 2 \mathrm{~h}$; (o) $\mathrm{NaH}_{2} \mathrm{PO}_{4}, \mathrm{NaClO}_{2}, 2$-methyl-2-butene, $t$ - $\mathrm{BuOH} / \mathrm{H}_{2} \mathrm{O}, 0$ ${ }^{\circ} \mathrm{C}$, 1.5 h (63\%, 2 steps); (p) $\mathrm{K}_{2} \mathrm{CO}_{3}, \mathrm{MeOH}, 15 \mathrm{~h}$; (q) BOPCl, pyridine, MeCN, $2 \mathrm{~h}$ (60\%, 2 steps); (r) $\mathrm{Ph}_{3} \mathrm{PCl}_{2}$, pyridine, MeCN, 18 h (57\%); (s) Dess-Martin periodinane, $\mathrm{CH}_{2} \mathrm{Cl}_{2}, 2 \mathrm{~h}(60 \%)$; (t) KRED-EXP-BlY, NAD+, GDH-103, glucose, $\mathrm{pH} 6.9,37-39^{\circ} \mathrm{C}$, $40 \min (56 \%)$.

Scheme 1.19 Macherla's total synthesis 


\subsection{Objectives}

The outstanding biological activity of salinosporamide A together with the challenge of synthesising the densely functionalised and compact structure prompted us to investigate a synthetic route to this natural product. The ultimate aim of this project was to develop a synthesis of salinosporamide A which is efficient, stereocontrolled and amenable to the preparation of significant quantities of the natural product.

Our objective was to integrate the development of new catalytic reaction methodologies into the synthesis of 1 . In parallel studies conducted by other $\mathrm{PhD}$ students within our group, the development of copper-, cobalt-, and nickel-catalysed reductive aldol cyclisations resulting in the production of $\beta$-hydroxylactones and $\beta$ hydroxylactams was being undertaken (Scheme 1.20). The successful application of a suitable variant of these methodologies within a complex setting, such as in a total synthesis of $\mathbf{1}$, would serve to demonstrate the utility of this chemistry.

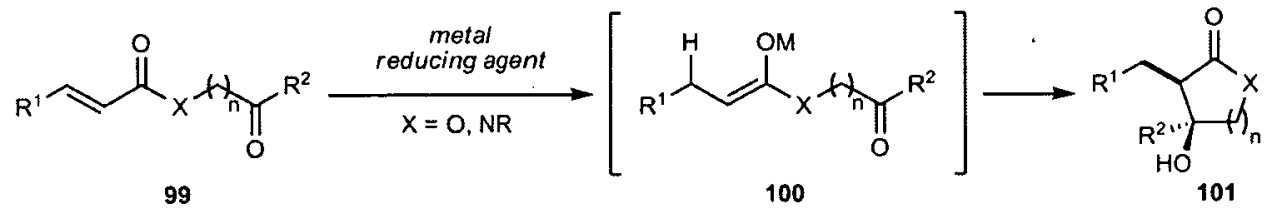

Scheme 1.20 Reductive aldol cyclisation methodology

To examine the feasibility of the use of this approach in our system, an amide represented by 102 would be first prepared (Scheme 1.21). With this substrate, the conditions for the reductive aldol cyclisation would be investigated, aiming to obtain $\gamma$-lactam 103 with good yields and diastereoselectivities. We envisaged this advanced intermediate could be converted into salinosporamide $A(1)$ in a few straightforward operations.

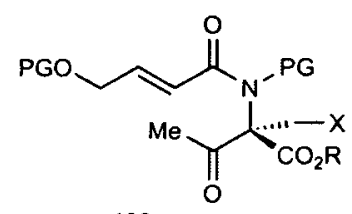

102

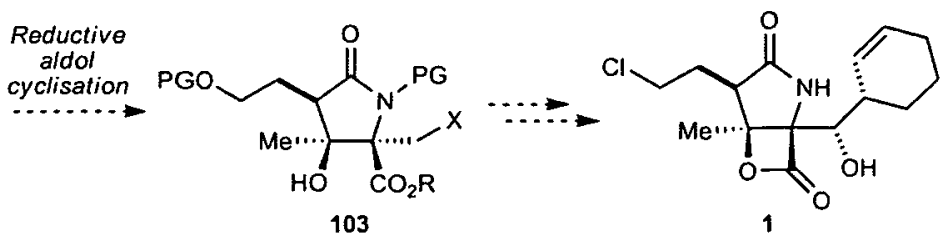

103

Scheme 1.21 Reductive aldol cyclisation approach 
However, at the inception of our studies these methodologies were not available. Therefore, we would initially focus our efforts on a racemic synthetic approach that looked to be concise, allowing rapid assembly of much of the functionality of 1 (Scheme 1.22). The first target of this route was the synthesis of a $\gamma$-lactam such as 104. Three key transformations would be then performed on this substrate: selective reduction to the aldehyde of the diester functionality, insertion of the cyclohexenyl side chain and alkylation at $\mathrm{C} 2$. If these three operations were successful, a few final transformations would afford $( \pm)-1$.

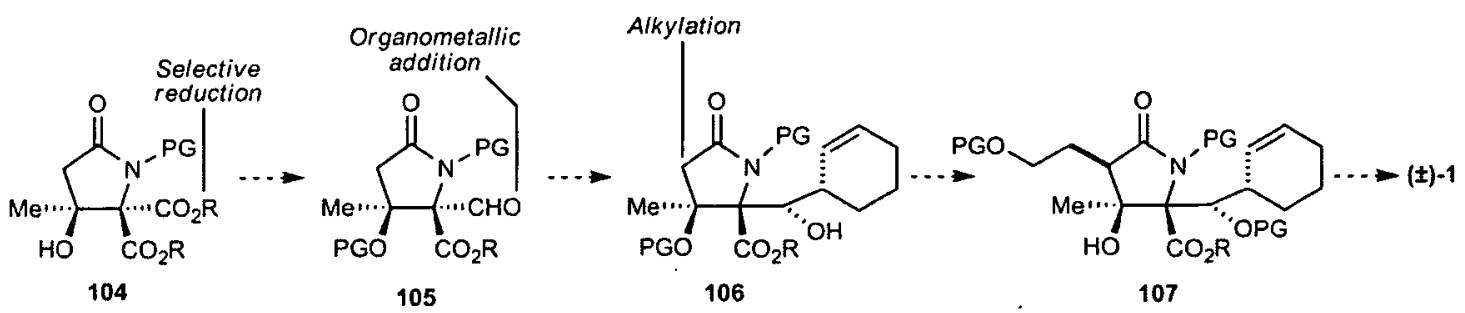

Scheme 1.22 Initial investigations to $( \pm)-1$

If this route was successful, we hoped to investigate the development of an asymmetric variant. With this approach we would also learn about the chemical behaviour of these $\gamma$-lactam intermediates and this knowledge could also be applied eventually into the route developed with the reductive aldol cyclisation approach. 


\section{Racemic approach based on amidomalonate aldol cyclisations}

When this project began, the methodology studies on the catalysed reductive aldol cyclisation were simultaneously initiated. Until that methodology was developed, efforts were focused on another synthetic approach, the development of which is described herein.

\subsection{Retrosynthetic analysis}

Our first approach to salinosporamide A is outlined in Scheme 2.1. Disconnection of the $\beta$-lactone of 1 , transformation of the chlorine atom on the ethyl side chain and deprotection of the lactam nitrogen atom lead back to intermediate 107. It was envisioned that the hydroxyethyl side chain of 107 could be inserted, after protecting group manipulations, via an enolate alkylation on 106. Introduction of the side chain at $\mathrm{C} 2$ at a late stage in the synthesis would facilitate the preparation of derivatives of 1 .

Disconnection of the cyclohexenyl side chain of 106 reveals aldehyde 105 and cyclohexenyl zinc reagent $\mathbf{2 4}$, methodology that had been successfully developed by Corey and co-workers in their total synthesis of $1 .{ }^{26}$ This reaction constructs stereoselectively the two contiguous stereogenic centres at C5 and at C6. It was anticipated that aldehyde $\mathbf{1 0 5}$ could be prepared by selective mono-reduction of the diester functionality of 104. Our initial efforts targeted the construction of a $\gamma$-lactam such as $\mathbf{1 0 4}$. 

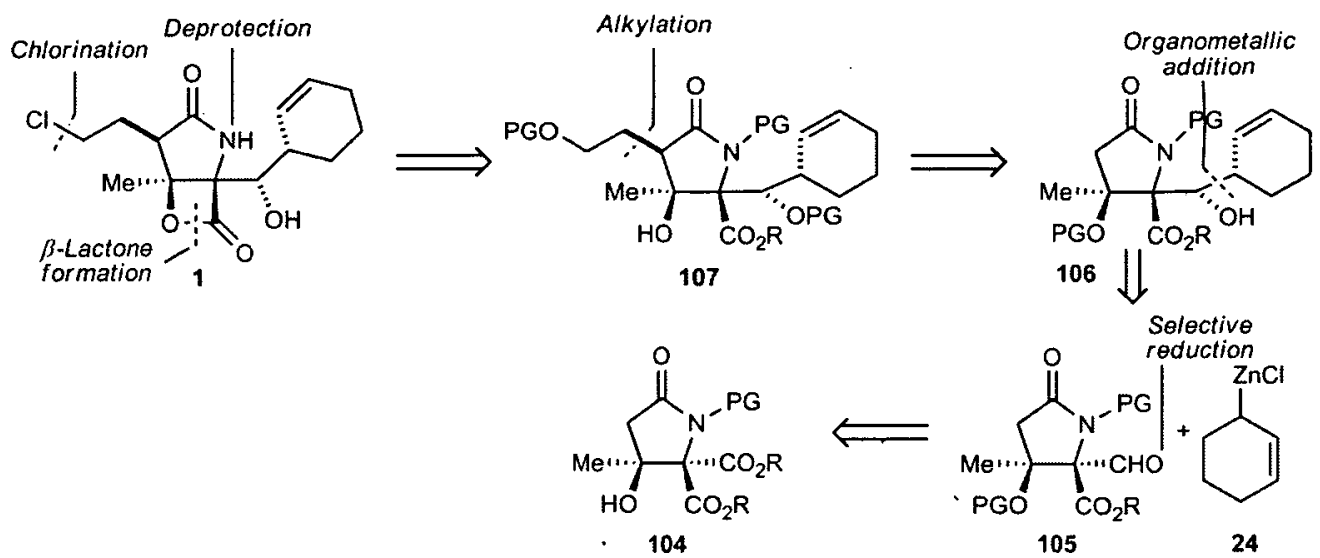

Scheme 2.1 First retrosynthetic analysis of salinosporamide $A$ (1)

\subsection{Results and discussion}

\subsubsection{Synthesis of the $\gamma$-lactam}

Inspection of the literature revealed that a $\gamma$-lactam of general structure 104 had been prepared previously. 51 Pyrrolidinone 110 was obtained via an acid catalysed reaction of $N$-PMP diethyl aminomalonate 109 with diketene 108 (Scheme 2.2). $\gamma$-Lactam 110 was then transformed into $\beta$-lactam 111, which was proposed as a suitable intermediate in the synthesis of $\beta$-lactam antibiotics such as thienamycin (113)..$^{52}$

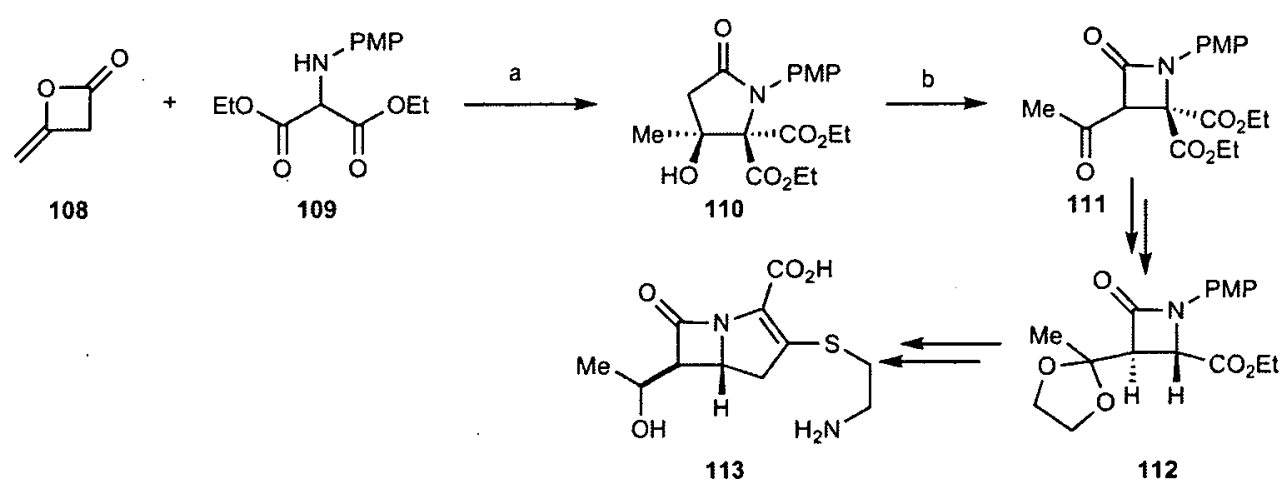

Reagents and conditions: (a) HOAc, reflux, 72\%; (b) $\mathrm{I}_{2}$, EtONa, $62 \%$.

Scheme 2.2 Reported example of the synthesis of 110 using diketene

Diketene (108) is a reactive and versatile compound which is best known as a reagent for the preparation of acetoacetic acid derivatives (Scheme 2.3). It is a strained molecule which is readily ring-opened and therefore it frequently appears to 
react as acetylketene (114). ${ }^{53}$ It is known that aliphatic amines are rapidly acetoacetylated by diketene, without the need for a catalyst. Therefore, in this example, reaction of amine 109 with diketene presumably forms acetoacetamide $\mathbf{1 1 5}$, which provides $\gamma$-lactam 110 via an acid catalysed aldol cyclisation.

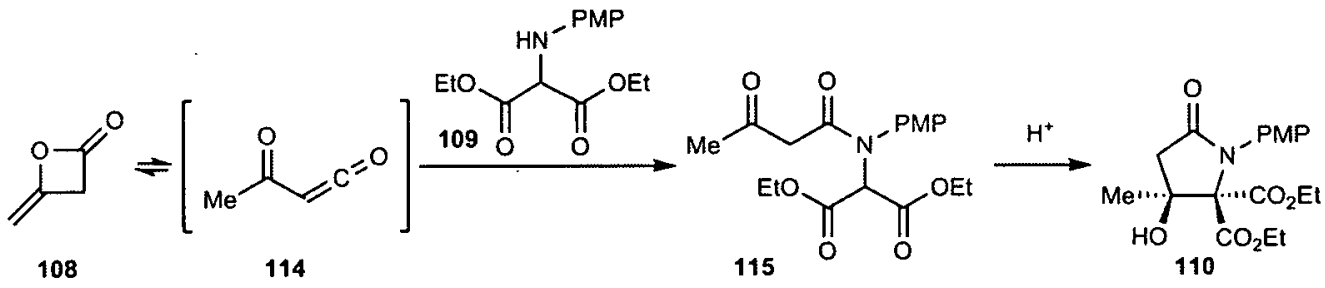

Scheme 2.3 Synthesis of 110

It was therefore decided to exploit this procedure in the preparation of the $\gamma$-lactam, which would be the starting point of our synthetic route. For this purpose, three aminomalonate derivatives were first prepared. The synthesis of $N$-PMP diethyl ester malonate 109 was successfully achieved reacting $p$-anisidine (117) and diethyl bromomalonate (116) in refluxing $\mathrm{Et}_{2} \mathrm{O}$. Following the reaction, a simple recrystallisation afforded amine $\mathbf{1 0 9}$ in $85 \%$ yield (Scheme 2.4 ).

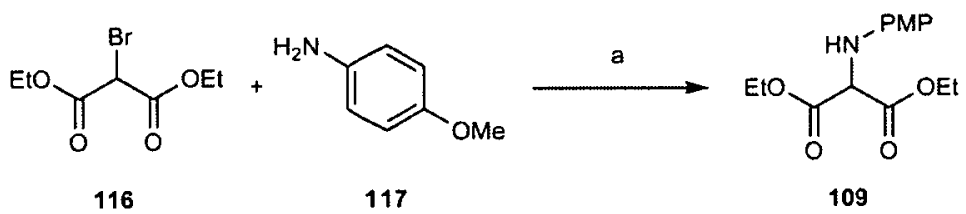

Reagents and conditions: (a) $\mathrm{Et}_{2} \mathrm{O}, 2 \mathrm{~h}$ heating under reflux, $14 \mathrm{~h} \mathrm{rt}, 85 \%$.

Scheme 2.4 Synthesis of amine 109

$N$-PMB-protected aminomalonates 119 and 121 were also prepared (Scheme 2.5). The use of a PMB protecting group on the nitrogen was desirable, because although similar conditions are employed for the deprotection of both PMP and PMB groups (CAN reagent), the PMB group is much more readily cleaved than the PMP group. ${ }^{54}$ In addition to this, Corey employed a $N$-PMB protecting group in the organometallic addition into aldehyde 23 (Scheme 1.6) to introduce the cyclohexenyl side chain. ${ }^{26}$ 
Diethyl bromomalonate (116) and p-methoxybenzyl amine (118) were reacted in the presence of $\mathrm{Et}_{3} \mathrm{~N}$ to yield amine $119(52 \%)$, although with a lower yield than that obtained for the N-PMP equivalent. Similarly, 118 was reacted with dimethyl 2-bromomalonate (120), which afforded 121 in a 35-40\% yield.

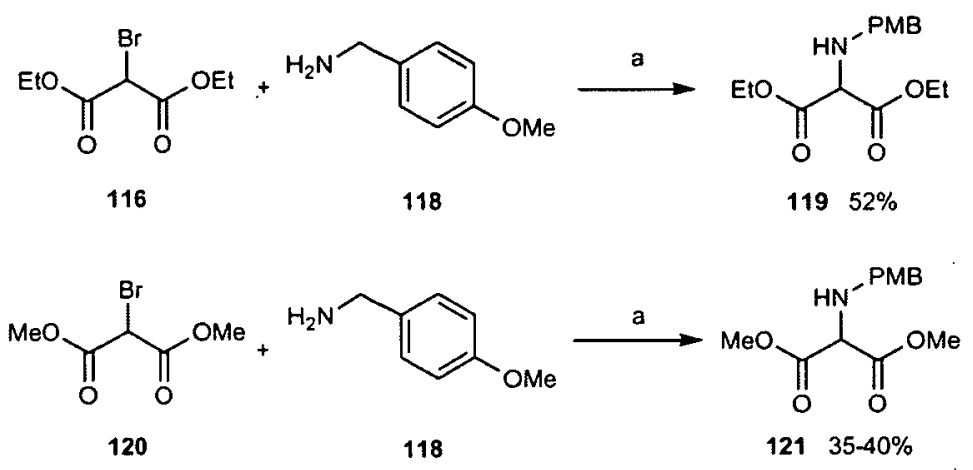

Reagents and conditions: (a) $\mathrm{Et}_{3} \mathrm{~N}(1 \mathrm{eq}), \mathrm{MeCN}, 0^{\circ} \mathrm{C}$ to $\mathrm{rt}, 14 \mathrm{~h}$.

Scheme 2.5 Synthesis of N-PMB protected amines 119 and 121

We proceeded then to study the formation of the $\gamma$-lactam. Two procedures were investigated. Initially, we conducted the reported procedure, which involved reaction of diketene with the aminomalonate. ${ }^{51}$ Due to the fact that diketene is now difficult to access from commercial sources; we decided to modify slightly the original procedure, by generating diketene in situ. Therefore, acetyl chloride was treated with $\mathrm{Et}_{3} \mathrm{~N}$, followed by addition of amine 121, to successfully afford $\gamma$-lactam 123 . Scheme 2.6 outlines the mechanism of this reaction. One equivalent of $\mathrm{Et}_{3} \mathrm{~N}$ reacts with acetyl chloride (122), eliminating $\mathrm{HCl}$ and forming ketene (124). A substoichiometric quantity of base then reacts with some of this ketene to form an ammonium enolate (125), which attacks ketene present in the media to give diketene 108. Then, in the presence of DMAP as a catalyst, amine 121 reacts with diketene, forming the uncyclised amide 127, which spontaneously cyclises to form $\gamma$-lactam 123 in good yield. 


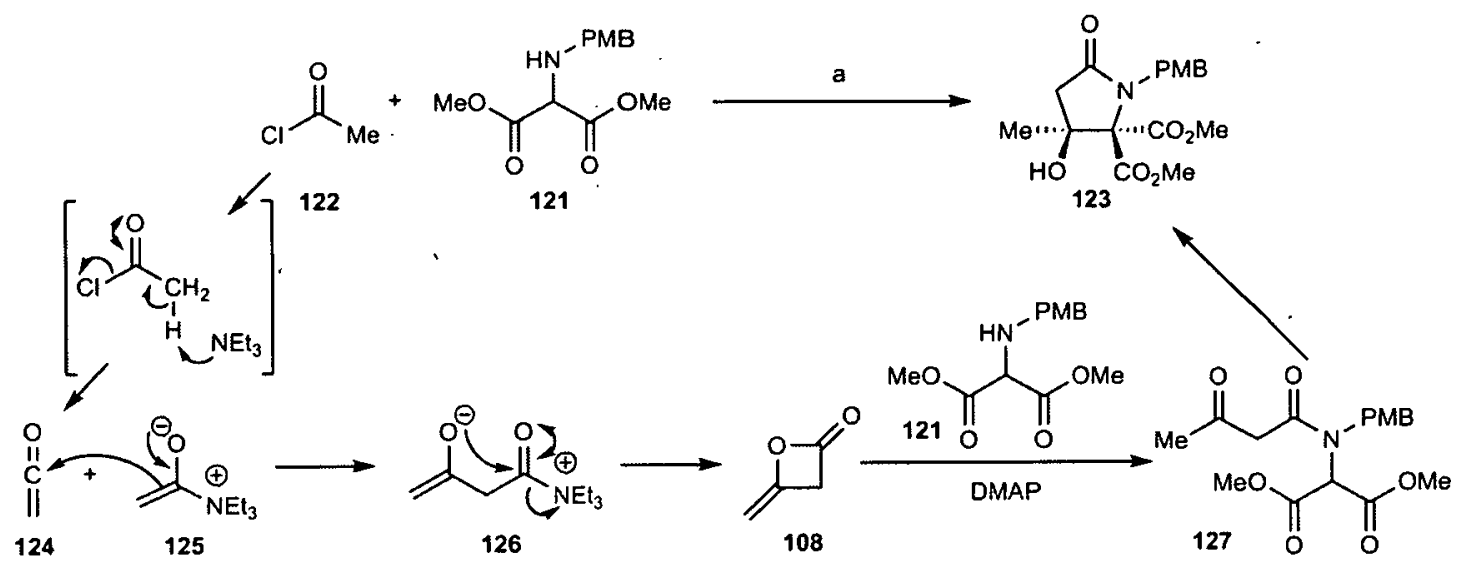

Reagents and conditions: (a) $\mathrm{Et}_{3} \mathrm{~N}$ (1.1 eq), DMAP (0.2 eq), $\mathrm{CH}_{2} \mathrm{Cl}_{2}$, $0{ }^{\circ} \mathrm{C}$ to rt, $14 \mathrm{~h}, 70-80 \%$.

Scheme 2.6 Mechanism of the formation of $\gamma$ lactam 123

The second procedure investigated involved the reaction of an aminomalonate with dioxinone 129. In 1952, Carroll and Bader described the acid catalysed reaction of diketene and acetone forming the dioxinone (Scheme 2.7). Subsequently, they reported that 129, when pyrolysed, decomposes into acetylketene (114) and acetone, via a retro-Diels-Alder fragmentation. ${ }^{55}$ Clemens reported in 1985 the pyrolysis of 129 in the presence of nucleophiles as a general purpose acetoacetylation procedure. ${ }^{56}$ Dioxinone 129 is a commercially available liquid, which is easily handled and stored, and the acetylation reactions are rapid and provide only volatile products.

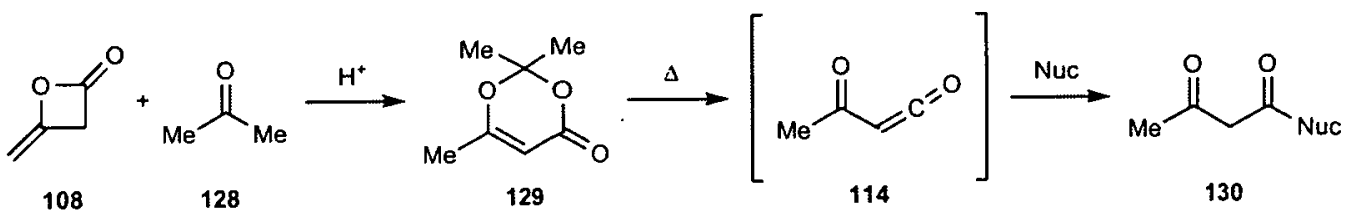

Scheme 2.7 Synthesis and use of dioxinone 129

We were pleased to find that $\gamma$-lactams 110 and 131 were readily prepared by mixing dioxinone 129 and the corresponding amine (109 or 119) in refluxing toluene (Scheme 2.8). Under the reflux conditions, the amine reacts with the acetylketene (114) formed in the reaction, to give an acetoacetamide which spontaneously affords the desired $\gamma$-lactam via an aldol cyclisation. 


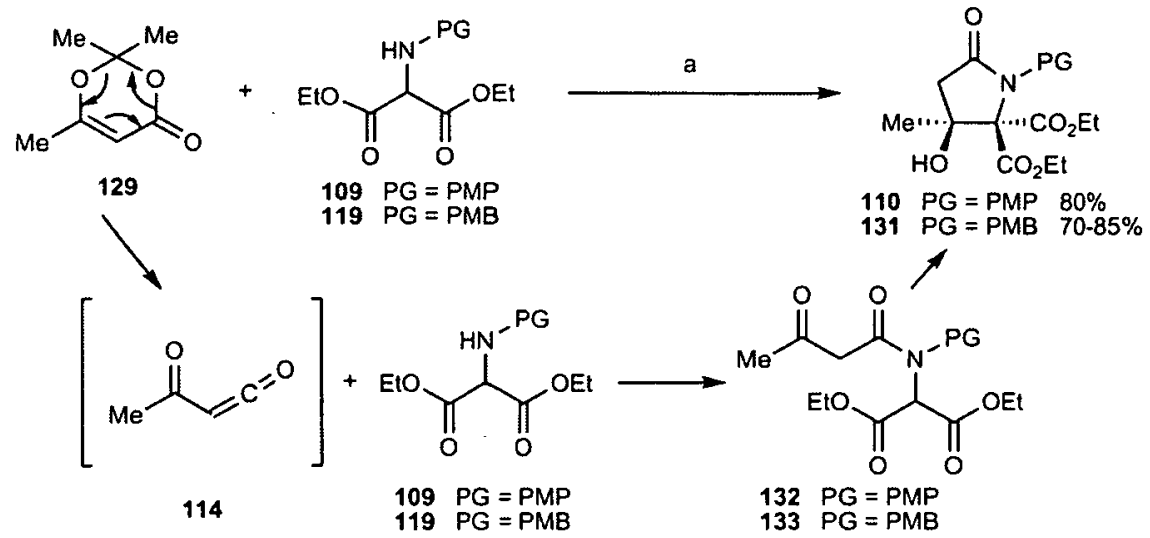

Reagents and conditions: (a) Toluene, $110^{\circ} \mathrm{C}, 14 \mathrm{~h}$.

Scheme 2.8 Synthesis of $\gamma$ lactams 110 and 131 using dioxinone 129

This procedure was very easily performed and afforded the desired cyclised products in good yields. Due to this efficiency and practicality, it became our preferred method for the synthesis of the $\gamma$-lactam. It was decided to use amines $\gamma$-lactams 110 and 131 in parallel studies in the development of the synthesis, since amines 109 and 119 were obtained in higher yields than amine 121. Moreover, the use of these two $\gamma$-lactams would keep consistency in the ester moiety, and only the nitrogen protecting group would vary. It was assumed that having an ethyl ester instead of a methyl ester as the intermediates in Corey's synthesis would not have an important effect in the synthetic route.

\subsubsection{Regioselective reduction of the ester and cyclohexenyl insertion}

With the $\gamma$-lactam in hand, the next step was the selective reduction of the ester anti to C3 hydroxyl to the aldehyde (Scheme 2.9). By having the aldehyde anti to the tertiary alcohol (135), the side chain at $\mathrm{C} 5$ would be correctly inserted at this position. It was anticipated that protection of $\mathrm{C} 3$ hydroxyl with a suitably bulky group (134) would be sufficient to direct reduction of the ester anti to it. The ester syn to the alcohol (136) is necessary in order to form the $\beta$-lactone of salinosporamide A. 


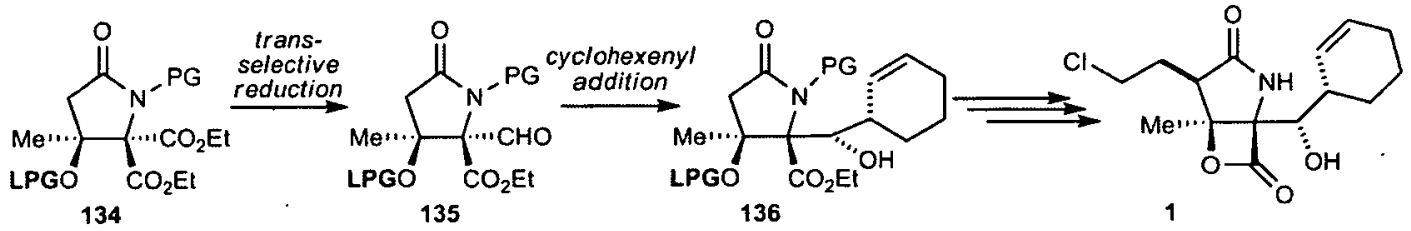

Scheme 2.9 Selective reduction of the diester functionality

Several protection attempts using trimethylsilyl triflate or tert-butyldimethylsilyl triflate were unsuccessful, with none or very little desired product obtained. Finally, it was found that $\gamma$-lactams $\mathbf{1 3 0}$ and $\mathbf{1 3 1}$ were successfully protected with TMSCl to give the protected silyl ethers $\mathbf{1 3 7}$ and 138, as shown in Scheme 2.10.
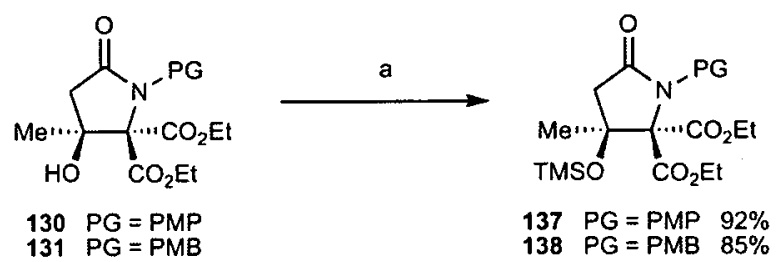

Reagents and conditions: (a) TMSCl (1.5 eq), DMAP ( $0.2 \mathrm{eq}), \mathrm{Et}_{3} \mathrm{~N}(3 \mathrm{eq})$, $\mathrm{CH}_{2} \mathrm{Cl}_{2}, 0^{\circ} \mathrm{C}$ to rt, $14 \mathrm{~h}$.

Scheme 2.10 TMS protection of C3 hydroxyl

Initial investigations on the selective mono-reduction to the aldehyde, and subsequent addition of the side chain, were done employing the PMP-derivative. A selective reduction of diester $\mathbf{1 3 7}$ to yield an $\alpha$-formyl ester was achieved using a method described by Burton. ${ }^{57}$ In 1993, the Burton group reported the transformation of 139 to predominantly trans-1,4-dialdehyde 140 via a stereoselective lowtemperature reduction with an excess of DIBAL-H (Scheme 2.11).

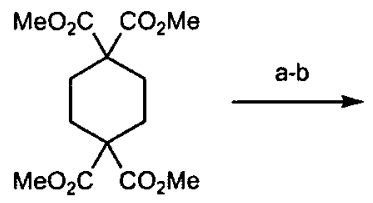

139

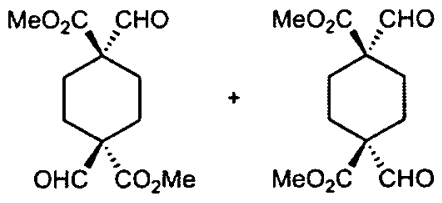

$140 \quad(90: 10) 63 \% \quad .141$

Reagents and conditions: (a) DIBAL-H (4 eq), $\mathrm{CH}_{2} \mathrm{Cl}_{2},-78^{\circ} \mathrm{C}, 3 \mathrm{~h}$; (b) $\mathrm{HCl}(\mathrm{aq}),-65^{\circ} \mathrm{C}, 63 \%$.

Scheme 2.11 Burton's stereoselective reduction using DIBAL-H 
After some investigation, the optimal conditions for the reduction of 137 were found to be a slow addition of 3.5 equivalents of DIBAL-H to a solution of the ester in $\mathrm{CH}_{2} \mathrm{Cl}_{2}$ at $-78^{\circ} \mathrm{C}$. This reaction did in fact provide a mono-aldehyde product with high selectivity, and minimal traces of over-reduced or doubly reduced products were detected in the unpurified mixture. Initially, we assumed that reduction had occurred anti- to the bulky trimethylsilyloxy group to provide the desired aldehyde. In fact, reduction had occurred in the undesired sense to provide aldehyde 144 (Scheme 2.13, vide infra).

This outcome was discovered with the next transformation, the insertion of the cyclohexenyl side chain, which was done according to Corey's procedure. ${ }^{26}$ 2-Cyclohexenyl tributyltin was first prepared, via a 1,4-palladium catalysed addition of tributyltin hydride into 1,3-cyclohexadiene (Scheme 2.12). Initially, this reaction was carried out following the reported method, ${ }^{36,58}$ which utilises $\mathrm{Pd}\left(\mathrm{PPh}_{3}\right)_{4}$ in THF. However, it was discovered that the reaction also proceeds using only $0.5 \mathrm{~mol} \%$ of the less expensive $\mathrm{Pd}_{2}(\mathrm{dba})_{3}$ and $4 \mathrm{~mol} \%$ of $\mathrm{PPh}_{3}$. Toluene was observed to be a better solvent than THF. After stirring overnight, the catalyst was removed by filtration of the reaction mixture through a silica plug and distillation of the crude product under high vacuum gave allylstannane 143 (85\% purity) ${ }^{i}$.

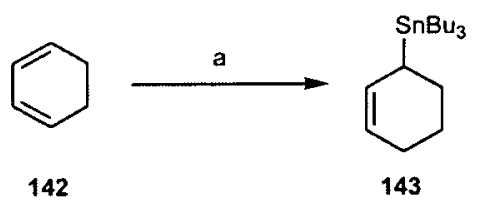

Reagents and conditions: (a) $\mathrm{Bu}_{3} \mathrm{SnH}(1 \mathrm{eq}), \mathrm{Pd}_{2}(\mathrm{dba})_{3}(0.5 \mathrm{~mol} \%), \mathrm{PPh}_{3}$ (4 mol\%), toluene, $\mathrm{rt}, 14 \mathrm{~h}, 52 \%$.

Scheme 2.12 Synthesis of 2-cyclohexenyl tributyltin 143

Subsequently, 2-cyclohexenyl tributyltin 143 was transmetallated at $-78{ }^{\circ} \mathrm{C}$, first with $n$-BuLi and then with zinc chloride, forming a solution of 2-cyclohexenyl zinc chloride 24. A solution of aldehyde 144 in THF at $-78^{\circ} \mathrm{C}$ was transferred into a solution of the zinc chloride reagent, which provided homoallylic alcohol 145 in $83 \%$ yield (Scheme 2.13).

\footnotetext{
${ }^{i}$ The purity of 143 was assessed by ${ }^{1} \mathrm{H}$ NMR spectroscopy, calculating the amount of $\mathrm{Bu}_{3} \mathrm{SnH}$ remaining in the sample by integration of the expected $15 \mathrm{H}$ from $3 \times \mathrm{CH}_{2} \mathrm{CH}_{2} \mathrm{CH}_{2} \mathrm{CH}_{3}$.
} 


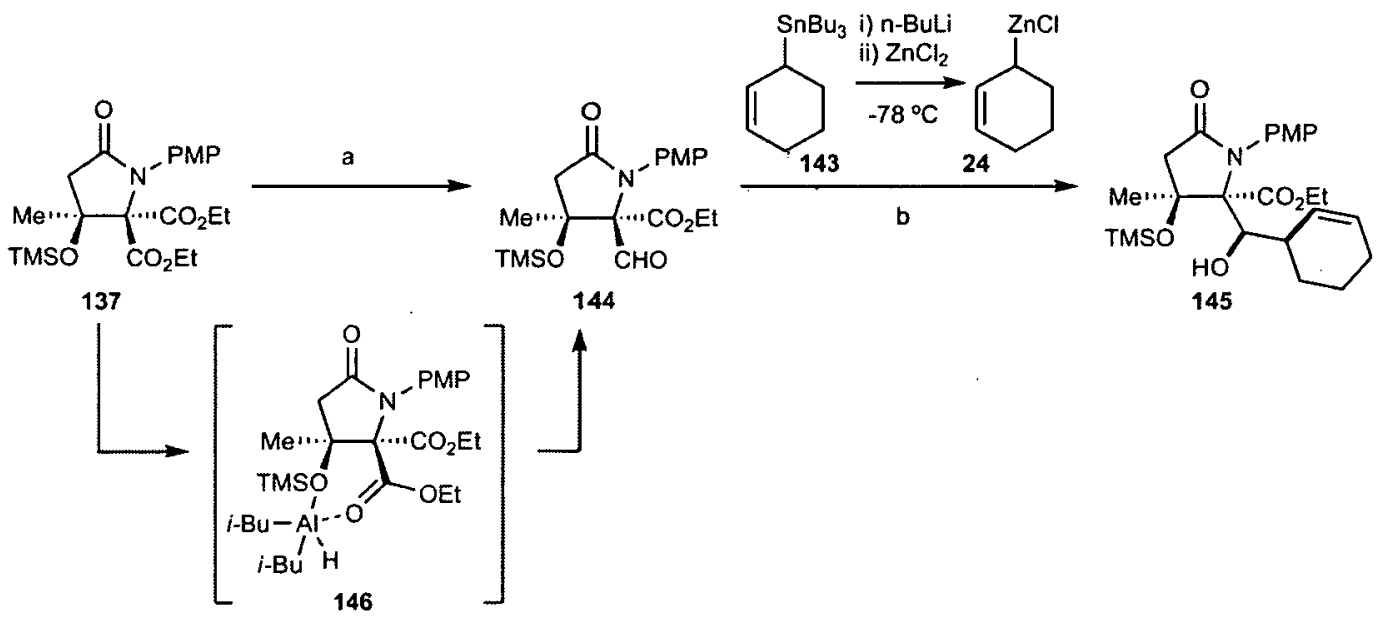

Reagents and conditions: (a) DIBAL-H (3.5 eq), $\mathrm{CH}_{2} \mathrm{Cl}_{2},-78{ }^{\circ} \mathrm{C}, 1.5 \mathrm{~h}$, $69 \%$; (b) 2-cyclohexenyl zinc chloride 24 (2.5 eq), THF, $-78^{\circ} \mathrm{C}, 4 \mathrm{~h}, 83 \%$.

Scheme 2.13 Synthesis of undesired addition product 145

Alcohol 145 was isolated as a crystalline solid and it was possible to establish its connectivity and relative stereochemistry using X-ray crystallography (Figure 2.1), which unfortunately, revealed unambiguously that reduction of $\mathbf{1 3 7}$ had occurred in the undesired sense. Presumably, prior coordination of DIBAL-H to the oxygen atom of the TMS ether was responsible for the outcome of these reactions (chelate 146, Scheme 2.13). Although not expected, similar effects have been observed by Evans and co-workers in carbonyl addition reactions of silyloxyaldehydes using aluminium Lewis acids. ${ }^{59}$

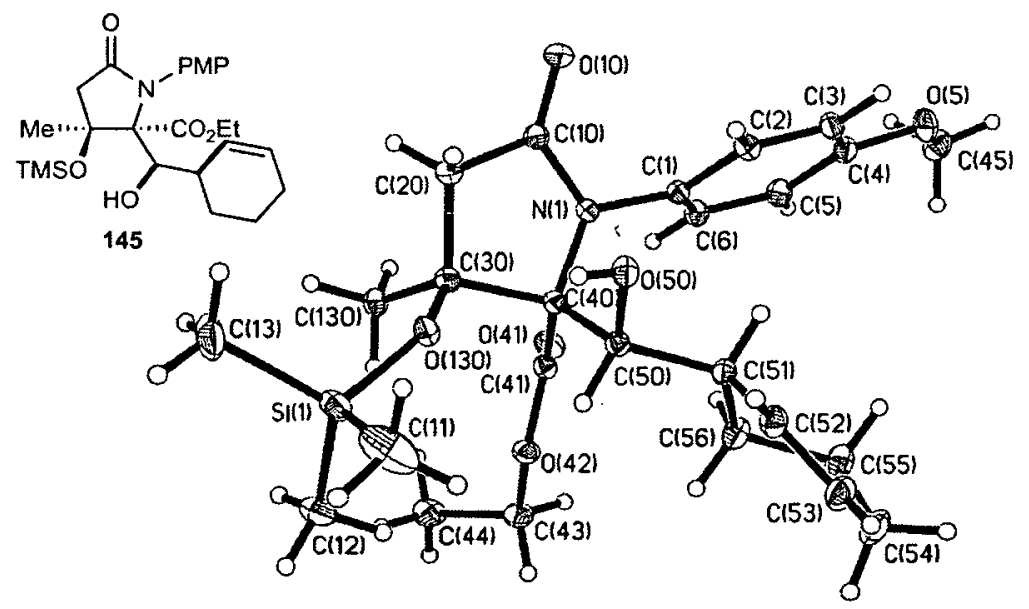

Figure 2.1 X-ray crystal structure of 145 
Therefore, a reducing agent without this coordinating ability was required, in the hope that the steric hindrance would this time be the determining factor for the selectivity of the reaction. A range of alternative procedures for selective monoreduction of 137 were evaluated and it was discovered that $\mathrm{NaBH}_{4} / \mathrm{LiCl}$ in $\mathrm{THF} / \mathrm{EtOH}^{60}$ was efficient in this regard to provide alcohol 147 in $90 \%$ yield (Scheme 2.14). Re-oxidation of 147 with either Dess-Martin periodinane ${ }^{35}$ or under Swern conditions ${ }^{61}$ provided aldehyde 148, which showed slight differences in its ${ }^{1} \mathrm{H}$ NMR spectra to aldehyde 144. The introduction of the 2-cyclohexenyl chain was carried out following the same procedure as with undesired aldehyde 144, to provide desired homoallylic alcohol 149 in $85 \%$ yield (Scheme 2.14).

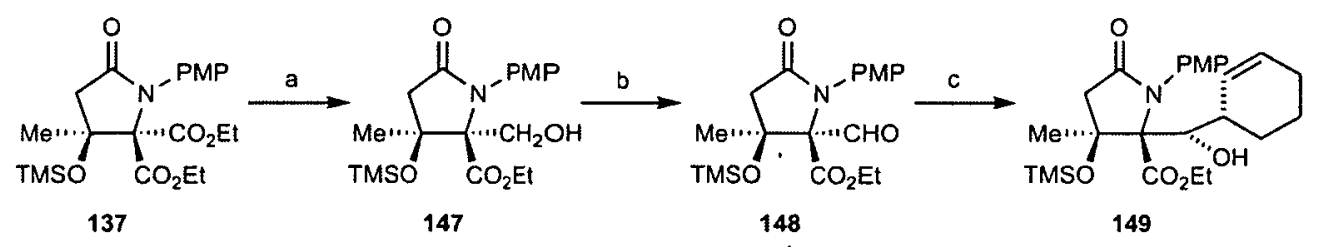

Reagents and conditions: (a) $\mathrm{NaBH}_{4}(5.5 \mathrm{eq}), \mathrm{LiCl}(5.5 \mathrm{eq}), \mathrm{THF}, \mathrm{EtOH}$, $0{ }^{\circ} \mathrm{C}$ to rt, $14 \mathrm{~h}, 90 \%$; (b) $(\mathrm{COCl})_{2}(2.1 \mathrm{eq})$, DMSO (4.2 eq), $\mathrm{Et}_{3} \mathrm{~N}$ (4.2 eq), $\mathrm{CH}_{2} \mathrm{Cl}_{2},-78^{\circ} \mathrm{C}, 3 \mathrm{~h}, 90 \%$; (c) 2-cyclohexenyl zinc chloride 24 (2.5 eq), THF, $-78^{\circ} \mathrm{C}, 4 \mathrm{~h}, 84 \%$.

Scheme 2.14 Synthesis of desired addition product 149

The X-ray crystal structure of alcohol 149 was obtained, which further confirmed that reduction of $\mathbf{1 3 7}$ had occurred in the desired sense, in addition to showing that 149 possessed the desired relative stereochemistry (Figure 2.2).

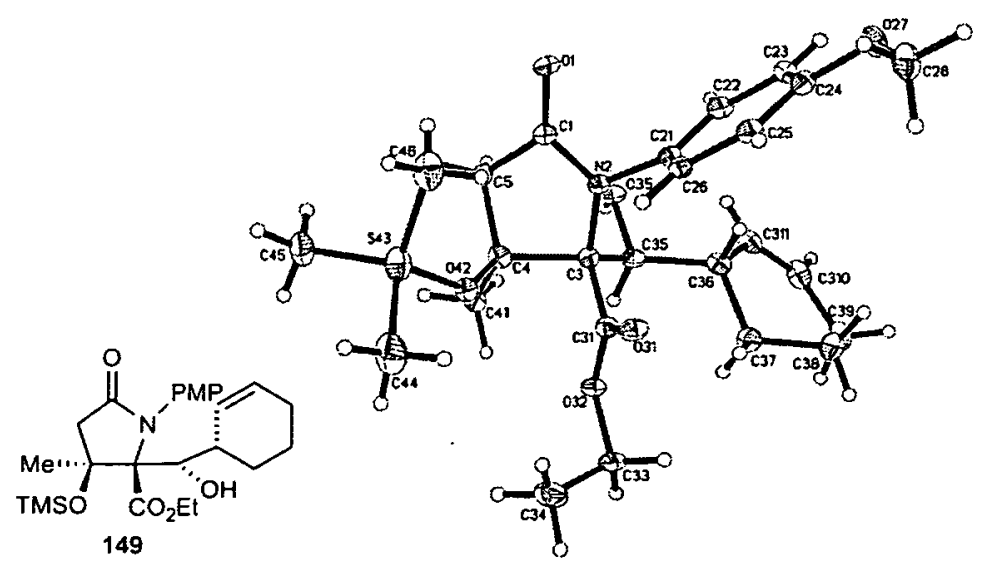

Figure 2.2 X-ray crystal structure of 149 
Once the optimal conditions for the mono-reduction and subsequent insertion of the cyclohexenyl group were found, these were applied to the PMB-derivative 138. Selective reduction of $\mathbf{1 3 8}$ to the alcohol was successfully achieved (Scheme 2.15), but in slightly lower yields (68\%) than those for the PMP $\gamma$-lactam. This was due to formation of the diol, product of reduction of both esters, before starting diester 138 was consumed. The cyclohexenyl side chain was inserted following the same procedure employed for 148 with good yield and high diastereoselectivity. An X-ray crystal structure of 151 was not obtained, but by analogy with the PMP-derivatives, it was assumed that alcohol $\mathbf{1 5 1}$ had the desired relative stereochemistry.

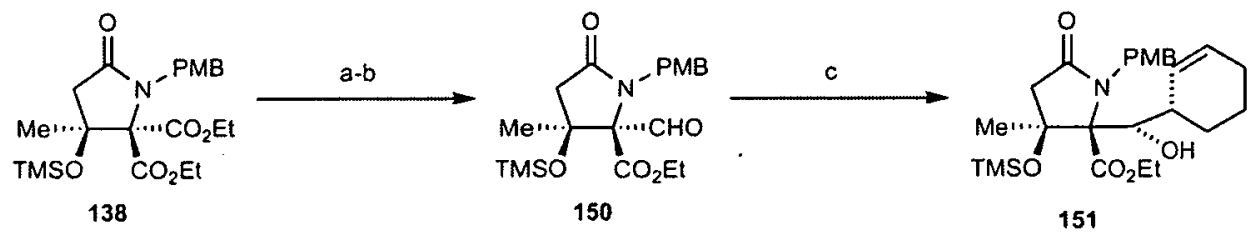

Reagents and conditions: (a) $\mathrm{NaBH}_{4}$ (2 eq), $\mathrm{LiCl}(2 \mathrm{eq}), \mathrm{THF}, \mathrm{EtOH}$, $0^{\circ} \mathrm{C}$ to rt, $14 \mathrm{~h}, 68 \%$; (b) $(\mathrm{COCl})_{2}(2.1 \mathrm{eq})$, DMSO (4.2 eq), $\mathrm{Et}_{3} \mathrm{~N}$ (4.2 eq), $\mathrm{CH}_{2} \mathrm{Cl}_{2},-78{ }^{\circ} \mathrm{C}, 4 \mathrm{~h}, 78 \%$; (c) 2-cyclohexenyl zinc chloride 24 (2.5 eq), THF, $-78^{\circ} \mathrm{C}, 4 \mathrm{~h}, 71 \%$.

\section{Scheme 2.15 Synthesis of PMB protected alcohol 151}

\subsubsection{Introduction of the $\mathrm{C} 2$ substituent}

Focus was turned then to the installation of the side chain at $\mathrm{C} 2$ via enolate alkylation. The best available precedent for this transformation was found in the total synthesis of omuralide (2) by the Donohoe group. ${ }^{62}$ In their route, $\beta$-hydroxylactam 152 was alkylated with MeI using LDA as base to provide 153 in $63 \%$ yield, along with the undesired diastereomer 154 in $10 \%$ yield (Scheme 2.16 ).

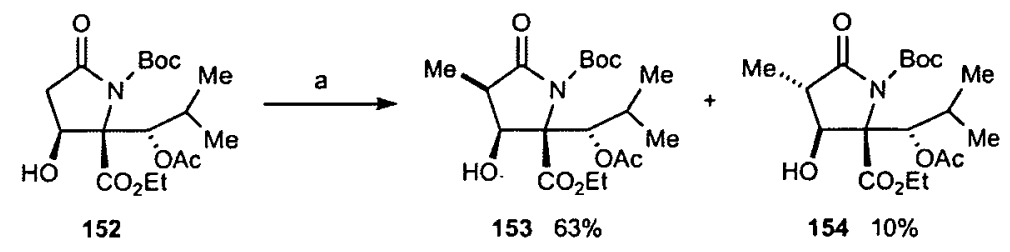

Reagents and conditions: (a) LDA (2.4 eq), HMPA (15 eq), MeI (9.3 eq), THF, $-78^{\circ} \mathrm{C}$.

Scheme 2.16 Donohoe's alkylation at C2 in the synthesis of omuralide 
$\gamma$-Lactam 152 had the C5 hydroxyl group acetate protected but a free C3 hydroxyl group. Surprisingly, no alkylation of the acetate ester was observed. However, we believed that the free C3 hydroxyl group was required to avoid undesired elimination to form the corresponding $\alpha, \beta$-unsaturated lactam. The tertiary alcohol would be deprotonated under the basic conditions of the reaction and therefore a minimum of two equivalents of base was needed for the formation of the enolate.

In light of this precedent, we desired access to a $\beta$-hydroxylactam 157 (Scheme 2.17), in which the C5 secondary alcohol is protected and the C3 tertiary alcohol unprotected. Protection of the secondary alcohol of 149 was first attempted. Unfortunately, this hydroxyl group proved to be extremely unreactive, presumably due to steric crowding, and many attempts to install a suitable protecting group (such as acetate, benzyl, pivaloate, TBS etc) were fruitless. It was therefore decided to deprotect the C3 hydroxyl group and attempt a selective protection of the secondary over the tertiary alcohol. Diol 156 was easily obtained from 149 using CSA in EtOH. However, all efforts to protect selectively the secondary alcohol were unsuccessful and forcing conditions resulted in reprotection of the tertiary alcohol.

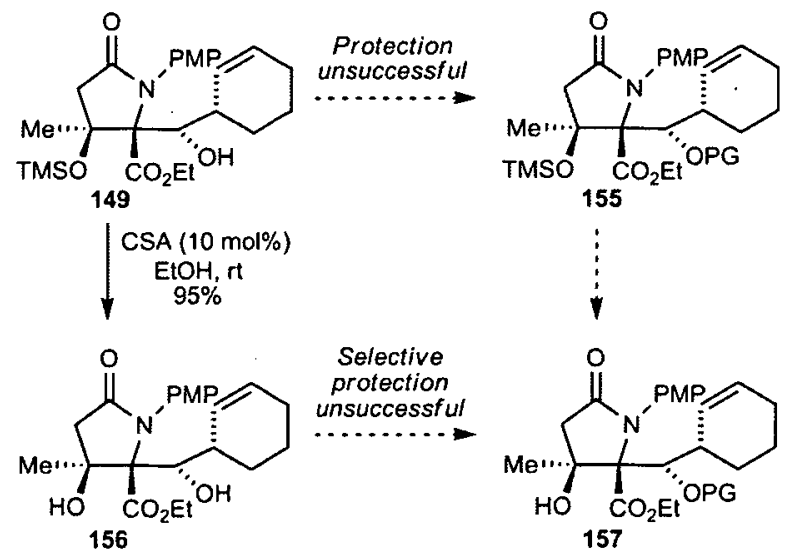

Scheme 2.17 Unsuccessful attempts to $\beta$-hydroxylactam 157

The protection of the secondary alcohol of the PMB derivative 151 also proved to be very challenging. Protection with a benzyl, pivaloate, TBS or TES group under several conditions were investigated but all attempts met with failure. However, 
protection as the acetate ester was successful, and subsequent deprotection of the TMS ether provided $\beta$-hydroxylactam 159 (Scheme 2.18).

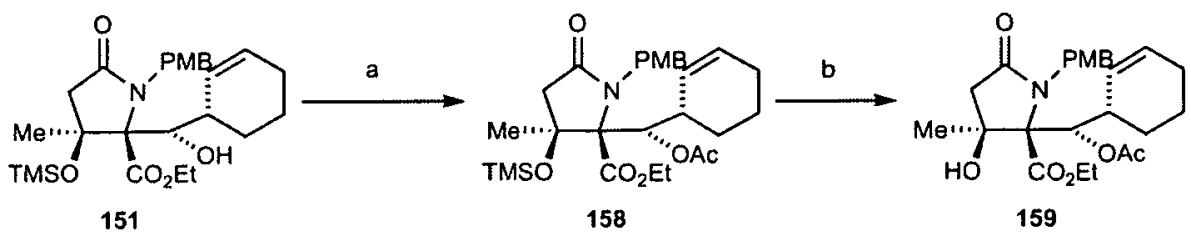

Reagents and conditions: (a) $\mathrm{Ac}_{2} \mathrm{O}$ ( $\left.3 \mathrm{eq}\right), \mathrm{Et}_{3} \mathrm{~N}$ ( 5 eq), DMAP (0.8 eq), $\mathrm{CH}_{2} \mathrm{Cl}_{2}, 0{ }^{\circ} \mathrm{C}$ to rt, $14 \mathrm{~h}, 90 \%$. (b) $\mathrm{CSA}$ (2 eq), $\mathrm{EtOH}, \mathrm{rt}, 0.5 \mathrm{~h}, 91 \%$.

Scheme 2.18 Successful transformations to alkylation substrate 159

$\gamma$-Lactam 159 was ready for alkylation. Although it displays differences to the compound that Donohoe and co-workers had successfully alkylated in their synthesis of omuralide (152), ${ }^{62}$ most notably in a denser, more sterically crowded substitution pattern and a different nitrogen protecting group, we felt that there might be enough similarities to provide a good chance of success.

Donohoe's procedure involved the addition of methyl iodide (9.3 equivalents) to a solution of LDA ( 2.4 equivalents) at $-78^{\circ} \mathrm{C}$, followed by the addition of a solution of alcohol 152 and HMPA (15 equivalents) in THF (Scheme 2.16). Additives such as HMPA, DMPU or TMEDA are often used as a cosolvent in reactions with organolithium reagents because they coordinate to the lithium cations, breaking up oligomers of lithium species and leaving a more reactive monomeric carbanion available for the reaction. ${ }^{63}$ Because HMPA is highly toxic (it is a reported carcinogen), the use of DMPU or TMEDA is preferred. The influence of $\mathrm{LiCl}$ on the structure and reactivity of lithium enolates has also been reported. ${ }^{64}$

When we initiated the investigation of the alkylation reaction we decided to initially employ the very reactive $\mathrm{MeI}$ as alkylating agent, in order to test the reaction. Failure with this electrophile was likely to mean failure with a more complex alkylating agent required to give the desired chloroethyl side-chain. Numerous conditions were attempted: various multiple equivalents of LDA, NaHMDS or KHMDS as base, with or without HMPA or DMPU, with or without $\mathrm{LiCl}$ additive; but no evidence for the desired product $\mathbf{1 6 0}$ was obtained (Scheme 
2.19). In some cases, only unreacted starting material was recovered, while in others, complex mixtures of products were obtained.

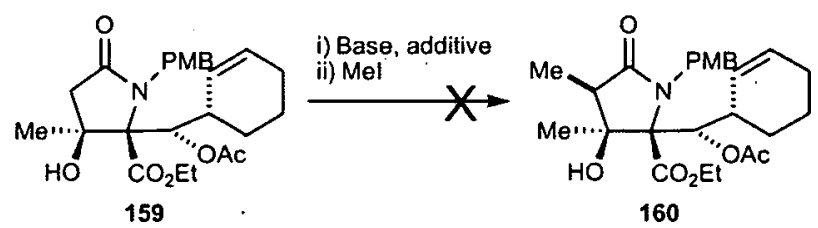

Scheme 2.19 Alkylation reaction trials with substrate 159

\section{Model Studies}

Analysis of the NMR spectra of the alkylation reactions performed on $\mathbf{1 5 9}$ proved to be complicated; furthermore, valuable 2-cyclohexenyl tributyltin 143 was being consumed during the study. Therefore, we decided to prepare a structurally less complex substrate, aiming for a more experimentally convenient preparation and also in order to facilitate the analysis of the NMR spectra. A thorough study of the alkylation reaction would then be undertaken on these derivatives. After finding the optimal conditions for the alkylation reaction, these would be applied to 159 , which would allow the synthesis of our natural product.

In one of the synthetic routes to omuralide (2) developed by Corey's group, the reaction of aldehyde 161 with isopropylmagnesium bromide 162 afforded alcohol 163 stereospecifically and in very good yields (Scheme 2.20). ${ }^{33}$ In this transformation, the reaction of isopropenylmagnesium bromide with TMSCl at $-40^{\circ} \mathrm{C}$ is slower than with the aldehyde; attack of the Grignard reagent forms an alkoxide, which is rapidly silylated, avoiding a retroaldol cleavage. The isopropenyl group was then reduced to give the isopropyl side chain required for the synthesis of 2.

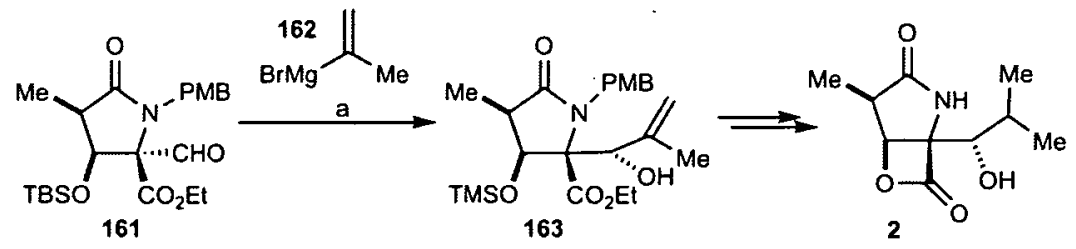

Reagents and conditions: (a) $162, \mathrm{TMSCl}, \mathrm{THF},-40^{\circ} \mathrm{C}, 0.5 \mathrm{~h}, 97 \%$.

Scheme 2.20 Corey's insertion of the isopropenyl group in the synthesis of omuralide 
Consequently, for our model system we decided to insert an isopropyl group at C5 instead of the cyclohexenyl group, since this was an efficient and simple reaction, performed with a commercially available reagent. Moreover, if the route was successful, we would be preparing an omuralide-salinosporamide hybrid, compound that had also been recently synthesised by Corey. ${ }^{23}$ Isopropenyl magnesium bromide was reacted with aldehyde 150 , in the presence of $\mathrm{TMSCl}$ to obtain alcohol 164 in good yield (Scheme 2.21). C5 of alcohol 164 was expected to have the desired stereochemistry, by analogy with Corey's reaction.

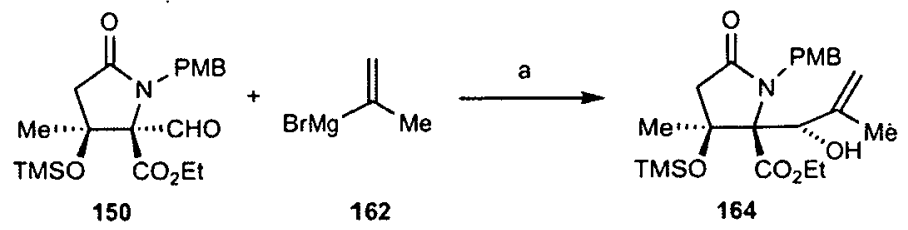

Reagents and conditions: (a) TMSCl (5 eq), 162 (3 eq), THF, $-78^{\circ} \mathrm{C}, 3 \mathrm{~h}, 76 \%$.

Scheme 2.21 Introduction of the isopropenyl side chain

As previously described, before attempting the alkylating reaction, alcohol 164 needed to be transformed into a $\beta$-hydroxylactam with a free $\mathrm{C} 3$ hydroxyl group and the C5 hydroxyl group protected. Acetate protection of the secondary alcohol of 164 was readily achieved using acetic anhydride and pyridine as base (Scheme 2.22). It should be noted that, protection attempts using other protecting groups, such as benzyl or pivaloate, were fruitless. Deprotection of the TMS group of 165 in the presence of CSA was completed overnight, affording isopropenyl derivative 166, which was then ready for alkylation.

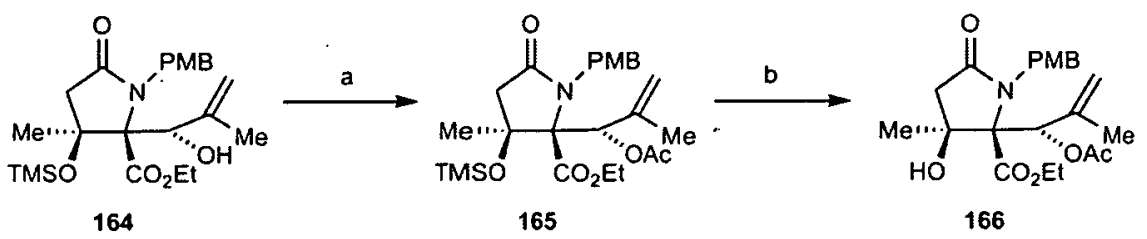

Reagents and conditions: (a) $\mathrm{Ac}_{2} \mathrm{O}$ ( 6 eq), pyridine ( $\left.3.5 \mathrm{eq}\right)$, DMAP $(0.2$ eq), $\mathrm{CH}_{2} \mathrm{Cl}_{2}, 0{ }^{\circ} \mathrm{C}$ to rt, $14 \mathrm{~h}, 92 \%$; (b) CSA (4 eq), EtOH, rt, $14 \mathrm{~h}, 83 \%$.

Scheme 2.22 Obtention of $\beta$-hydroxylactam 166 
Hydrogenation of 166 using $\mathrm{Pd} / \mathrm{C}$ afforded the new alcohol 167 containing the isopropyl group, albeit in low yield (Scheme 2.23).
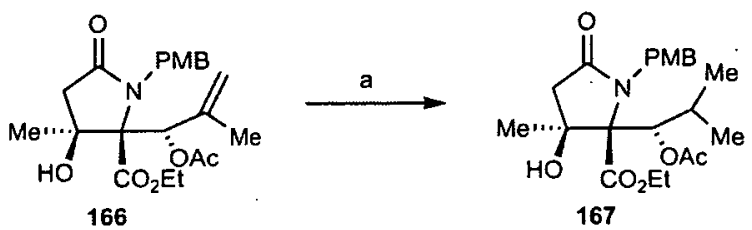

Reagents and conditions: (a) $\mathrm{H}_{2}, \mathrm{Pd} / \mathrm{C}(0.7 \mathrm{eq}), \mathrm{EtOH}, \mathrm{rt}, 18 \mathrm{~h}, 50 \%$.

Scheme 2.23 Hydrogenation of 166 reveals the isopropyl side chain

The investigation of the reaction was initiated employing alcohol 167 and again utilising the very reactive $\mathrm{MeI}$ as the alkylating agent. Numerous conditions were attempted. Firstly, Donohoe's conditions ${ }^{62}$ were repeated, procedure that involved the addition of a solution of the alcohol to a solution of LDA and MeI; however, none of the desired methylated product was observed. This reaction was repeated without HMPA or using DMPU instead of HMPA. The use of other bases, such as KHMDS and LTMP, was also examined. An attempt was made altering the order of addition, therefore adding a solution of alcohol 167 into a solution of LDA, to form the enolate, followed by addition of MeI. The reaction was also performed using allyl iodide instead of methyl iodide. However, all efforts to obtain the alkylated product were unsuccessful (Scheme 2.24).

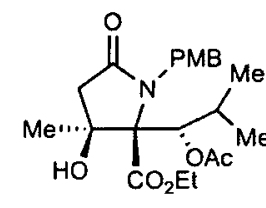

167

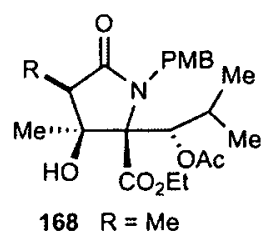

$169 \mathrm{R}=\mathrm{Allyl}$

\section{Scheme 2.24 Alkylation reaction trials with substrate 167}

Several variables had been studied for this reaction but, in general, the procedure involved the addition of a base (typically LDA) to a solution of 167 , the reaction mixture was then stirred at $-78{ }^{\circ} \mathrm{C}$ for $30-60 \mathrm{~min}$, followed by the addition of MeI. In none of the conditions attempted desired alkylated product 168 was formed; however, a methylated product was obtained as a major product (50-75\%). Careful 
analysis of these reactions revealed the formation of a methylated compound, which displayed spectroscopic data consistent with the structure represented by enamide 172 (Scheme 2.25). We assumed that 172 was formed as a result of alkoxide $\mathbf{1 7 0}$ undergoing elimination of the acetate to provide ring-opened acetoacetamide 171, which is then methylated under the reaction conditions. The ${ }^{1} \mathrm{H}$ NMR spectrum showed a doublet at high chemical shift $(6.7 \mathrm{ppm})$ and there was no signal for the methyl of the acetate group. This doublet was assigned to the proton on the double bond formed in the elimination of the acetate group $\left(\mathrm{H}_{A}\right)$. When the ${ }^{13} \mathrm{C} N M R$ spectrum was examined, we observed a new quaternary carbon at $205.3 \mathrm{ppm}$, which was assigned to the newly formed methyl ketone. The ${ }^{1} \mathrm{H}$ NMR spectrum of this substrate showed in fact that $\mathbf{1 7 2}$ was a mixture of two compounds, due to the formation of either the double bond $Z / E$ isomers or to a mixture of two amide rotamers.

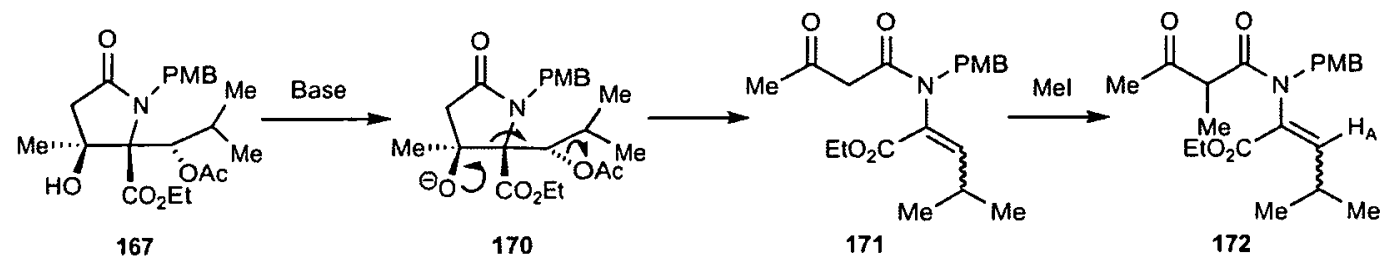

Scheme 2.25 Fragmentation of 167: acetate elimination and ring opening

When repeating the reaction in the absence of methyl iodide, the non-alkylated amide 171 was obtained (also as a mixture of two isomers or rotamers), thus demonstrating that the alkylation at $\mathrm{C} 2$ occurred in a second step, after the elimination and ring opening. The reaction was also performed using allyl iodide instead of methyl iodide and it afforded the analogous allylated amide.

The C2 alkylation of the isopropenyl derivative 166 was also investigated. The same combinations of conditions (different bases, addition of HMPA or DMPU, order of addition) were explored, however, all attempts failed to give the desired product (173). In this occasion, a methylated product, with spectroscopic data consistent with acetoamide 174 was isolated (Scheme 2.26). This product is a result 
of a similar elimination and ring-opening process, followed by alkylation, to the one detailed for the formation of 172 (Scheme 2.25).

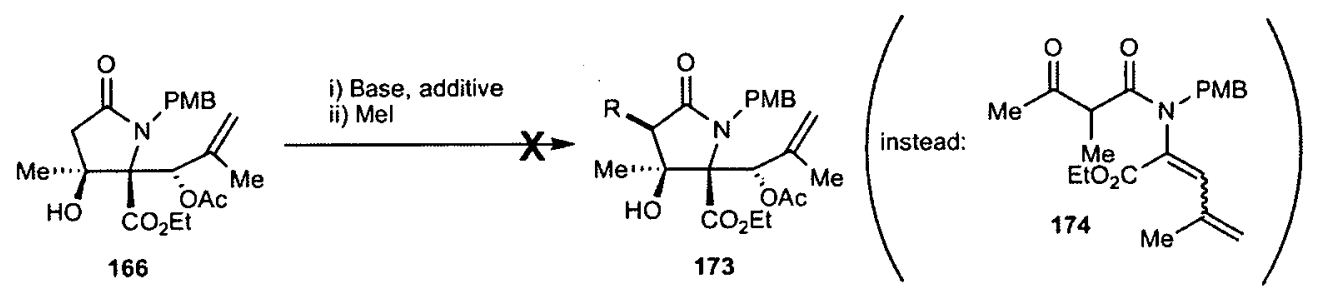

Scheme 2.26 Failure in the preparation of 173 and formation of 174

Employing the model substrates, we had identified the fragmentation process that was occurring under the reaction conditions. Despite this discouraging result, it was necessary to verify whether a similar ring-opened product was obtained when performing the reaction on $\gamma$-lactam 159, the substrate with all the required functionalities for the synthesis of $( \pm)-1$.

The alkylation reactions attempted on $\mathbf{1 5 9}$ produced a slightly more complex mixture of products than 167 and $\mathbf{1 6 6}$. However, now that we were able to recognise the pattern of the fragmentation product in the ${ }^{1} \mathrm{H}$ NMR spectrum, it was confirmed that the same ring-opening process was clearly taking place and compound $\mathbf{1 7 5}$ was being formed (Scheme 2.27).
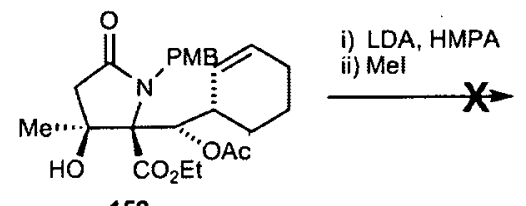

159

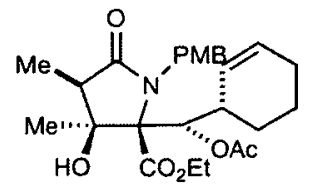

160
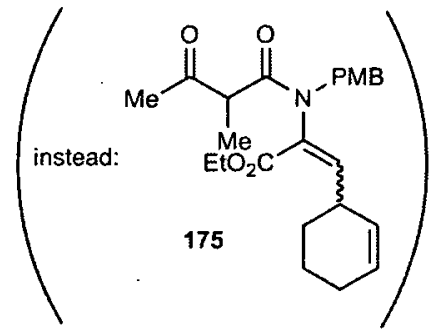

Scheme 2.27 Final attempt of the alkylation on 159

Although for $\mathrm{C} 2$ alkylation we had opted to target substrates containing a free alcohol at $\mathrm{C} 3$ to avoid hydroxide elimination, clearly this feature was resulting in the undesired ring-opening/acetate elimination described above. Therefore for comparative purposes, we attempted the alkylation of $\mathbf{1 5 8}$, which has the $\mathrm{C} 3$ alcohol TMS protected (Scheme 2.28). Unreacted starting material was recovered when only 
1.2 equivalents of base were employed. However, when using an excess of LDA (2.2 eq) in HMPA/THF for enolisation, followed by addition of MeI (9 equivalents), 177 was obtained in a rapid reaction, which is the product of methylation of the acetate ester. There was no evidence of methylation at $\mathrm{C} 2$, or elimination of the trimethylsilyloxy substituent.
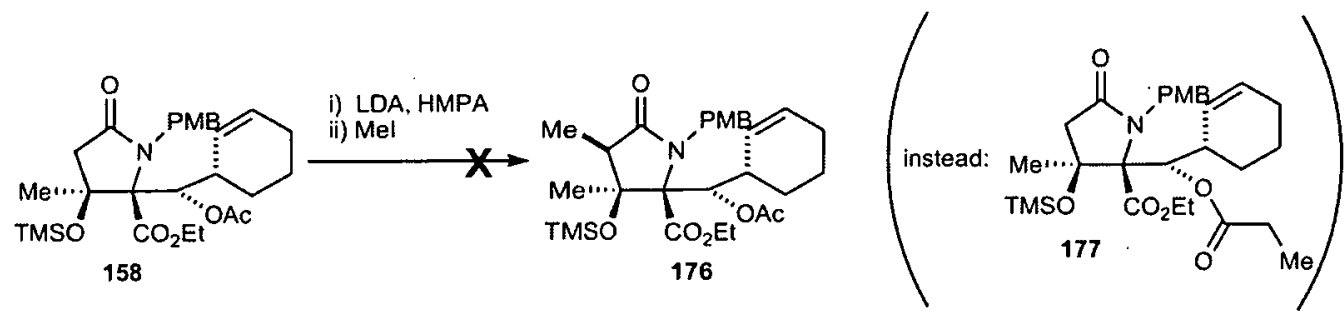

Scheme 2.28 Acetate methylation of 158

The reaction was also performed on $\mathbf{1 6 5}$, and acetate methylation occurred again. Therefore, protection of the secondary alcohol at C5 with a non-enolisable protecting group was necessary. However, as previously described all efforts to protect the C5 hydroxyl group of 151 and also of 164 with any other group than acetate were unsuccessful.

The alkylation reaction was also performed with $N$-PMP diol 156 and $\gamma$-lactam 138 (Scheme 2.29), but mostly starting material was recovered in both cases. Comparing our substrates with Donohoe's (152, Scheme 2.29), ${ }^{62}$ we wondered why the enolate formation and alkylation was unfavoured in our system. Although the presence of the C3 methyl made a more dense structure, we believed that the main difference with our system was the protecting group on the amide nitrogen. A possible explanation for the failure of these reactions was the lower reactivity of the $N$-PMP- or $N$-PMB-protected amides compared to the $N$-Boc substrate, due to electronic factors.
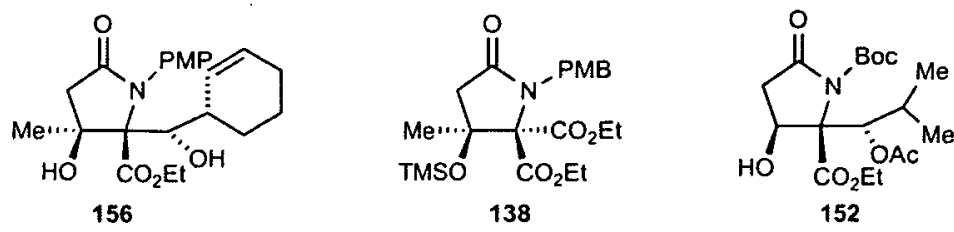

Scheme 2.29 Comparison of our substrates with Donohoe's 
The $N$-Boc $\gamma$-lactam derivative could potentially be prepared, but Danishefsky reported $^{27}$ that the success of Corey's procedure ${ }^{26}$ for the insertion of the cyclohexenyl side chain is very dependent on the protecting group of the amide. Therefore, a Boc group could be not beneficial for that step. The PMB group could have also been replaced by a Boc group only prior to the alkylation reaction, but this would mean adding extra steps to the synthesis.

\subsection{Conclusions}

We employed an easy and efficient way to prepare $\gamma$-lactams of general structure 104. A selective mono-reduction of the ester to the aldehyde, followed by insertion of the cyclohexenyl side chain into the aldehyde were successfully performed.

The next key step in the synthetic sequence was the introduction of the side chain at C2. Unfortunately, all efforts to induce the formation of the enolate and subsequent reaction with the alkylating agent were fruitless, and the desired alkylated product was not obtained. When the reaction was performed on $\beta$-hydroxy $\gamma$-lactam substrates protected at $\mathrm{C} 5$, a fragmentation process ocurred. Throughout the study, we had no evidence for the formation of the amide enolate. It was speculated that changing the protecting group on the nitrogen could make the enolate more reactive.

The investigation of the racemic synthetic route proved more challenging than originally expected. Therefore, although we had managed to prepare compounds with much of the functionality required for salinosporamide A (1), the unsuccessful C2 alkylation studies prompted us to consider alternative strategies. Although we were not able to complete the route, with this approach we gained valuable insight into the reactivity and chemical behaviour of the $\gamma$-lactam substrates. This knowledge was fundamental for the development of the rest of the project. Most importantly, we were able to readily apply Corey's method for the insertion of the cyclohexenyl chain in the final approach, whereby we achieved the formal synthesis. 


\section{Reductive aldol cyclisation approach}

A series of metal-catalysed reductive aldol cyclisation reactions for the formation of $\beta$-hydroxylactones and $\beta$-hydroxylactams in a highly diastereoselective fashion had been developed in our laboratories. ${ }^{65-67}$ The studies carried out on the application of this methodology for the introduction of the $\gamma$-lactam moiety in salinosporamide $A$ will be described in this chapter.

\subsection{Aims}

Disconnection of the $\beta$-lactone and the cyclohexenyl group of $\mathbf{1}$, along with appropriate functional group manipulations/interconversions lead back to $\gamma$-lactam 103. We envisaged that $\gamma$-lactam 103 could be synthesised from an amide enolate such as 178, via an intramolecular aldol reaction with the methyl ketone moiety (Scheme 3.1). A crucial advantage over the previous approach is that the reductive aldol cyclisation is expected to afford the $\gamma$-lactam intermediate 103 having the side chain at $\mathrm{C} 2$ in place.
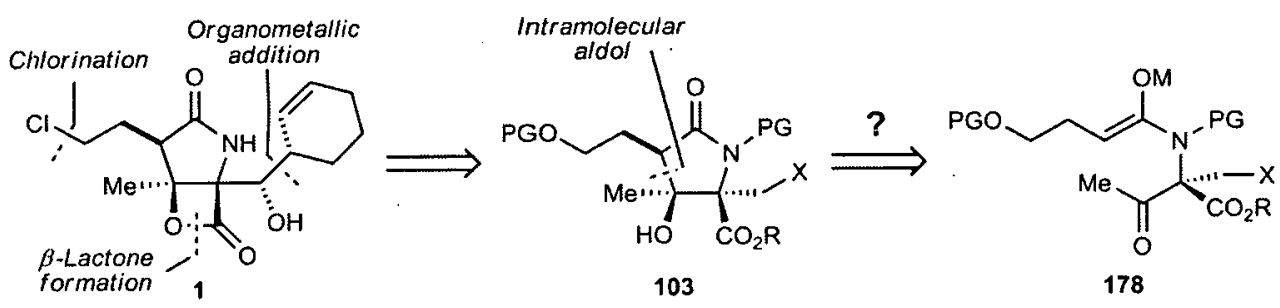

Scheme 3.1 Formation of the $\gamma$ lactam via an intramolecular aldol reaction

In order to obtain $\beta$-hydroxylactam 103 in an intramolecular aldol fashion, the main challenge was to regioselectively form the amide enolate over the ketone enolate. Control of the regioselectivity of the enolisation was essential, because under traditional aldol reaction conditions ketone enolate 180 would be the first to form, due to a higher acidity of the protons and also to a lower steric hindrance. (Scheme 3.2). 


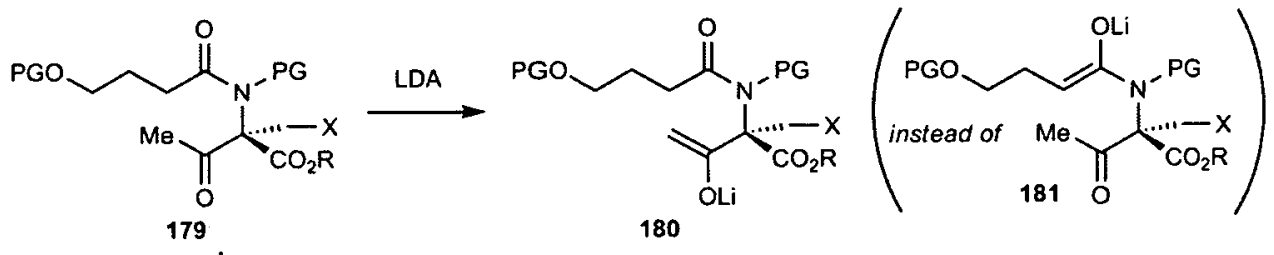

Scheme 3.2 Favoured formation of ketone enolate 180 under classical aldol conditions

We reasoned that the best approach to overcome this selectivity problem would be a reductive aldol protocol, which utilises an $\alpha, \beta$-unsaturated amide as a latent enolate. Reductive enolate formation of $\alpha, \beta$-unsaturated amide 102, followed by aldol reaction with the methyl ketone, would provide access to the target $\gamma$-lactam 103, as represented in Scheme 3.3.

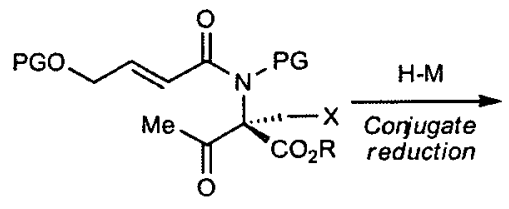

102

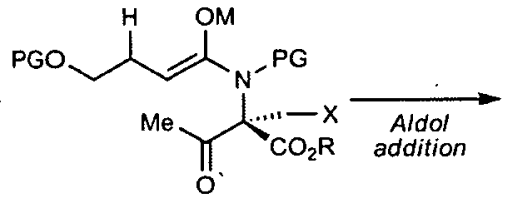

178

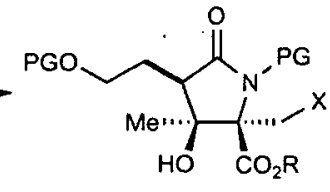

103

Scheme 3.3 Reductive aldol approach for the preparation of $\gamma$ lactam 103

Important advances have been made in the field of the reductive aldol chemistry in recent years. This technique has become an important tool in organic synthesis and the application of this methodology in synthesis of natural products has already been possible, as it will be described in the next section. Although the intramolecular aldol reaction of enones with ketones or aldehydes for the formation of carbocyclic rings had been widely described in the literature, when this project was started there were no examples of reductive aldol cyclisations of $\alpha, \beta$-unsaturated amides to afford lactams. With this observation, our group initiated a programme for the development of d-block catalysed reductive aldol cyclisation of $\alpha, \beta$-unsaturated amides and, although not relevant to this project of $\alpha, \beta$-unsaturated esters, to yield heterocyclic products. The aim was to subsequently apply this methodology into the synthesis of the $\beta$-hydroxy- $\gamma$-lactam moiety of salinosporamide $A$. 


\subsection{The reductive aldol reaction}

\section{The aldol reaction}

The aldol reaction is an extraordinarily important carbon-carbon bond-forming tool in organic synthesis, and is considered the most important method for the construction of $\beta$-hydroxy carbonyl compounds. ${ }^{68}$ The addition of an enolisable carbonyl to a ketone or, more typically, to an aldehyde (due to its higher electrophilicity) affords a $\beta$-hydroxy carbonyl compound, forming a new $\mathrm{C}-\mathrm{C}$ bond and setting two new stereogenic centres in a single transformation (Scheme 3.4).

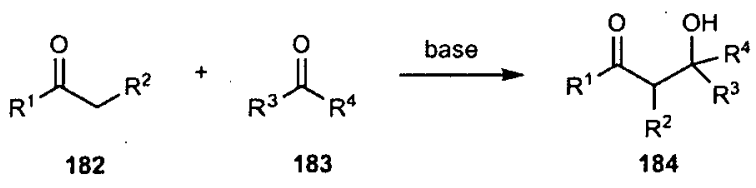

Scheme 3.4 The aldol reaction

The classic aldol reaction is a highly atom efficient process but the chemo- and regioselectivity in the formation of enolates from unsymmetrical precursors has represented a big challenge. The development of the asymmetric aldol reaction has been one of the most important topics in modern catalysis and the control of its stereoselectivity has now reached impressive levels. In natural product synthesis, control of the absolute stereochemistry when creating new stereocentres is fundamental and that is the reason why the asymmetric aldol reaction has been extensively used for the construction of complex molecules over the last three decades. ${ }^{69}$

In the early days of asymmetric synthesis, studies were limited to stepwise aldol approaches that utilised preformed enolates. ${ }^{70}$ Two methods were extensively developed: the use of stoichiometric amounts of chirality in the form of auxiliaries or ligands (asymmetric modification of the enolate or of the electrophile) and the catalytic approach using chiral Lewis acids.

Crimmins $^{71}$, Evans ${ }^{72}$, Oppolzer ${ }^{73}$, Massamune ${ }^{74}$, Paterson $^{75}$ and Denmark ${ }^{76}$ groups have been the main contributors to the methodology employing chiral auxiliaries or highly efficient catalysts to generate aldol products with high enantioselectivity. 
In order to avoid the use of stoichiometric amounts of chiral promoters, the catalytic version of the enantioselective aldol reaction was investigated. This methodology required a reaction catalyst to initiate and mediate the reaction. Therefore, the catalyst represented the vehicle for influencing reaction stereoselection. This reaction design needed the identification of a suitable latent enolate that would not react with the electrophile without the presence of the activating agent. Mukaiyama ${ }^{77}$ first recognised enolsilanes as the requisite latent enolate equivalents to which the addition of a chiral metal-based Lewis acid would promote the asymmetric catalysed aldol reaction (Scheme 3.5). Since the discovery of the Mukaiyama aldol reaction, there has been a great progress in the development of the asymmetric catalytic aldol reaction, becoming thus a very reliable methodology. ${ }^{78}$

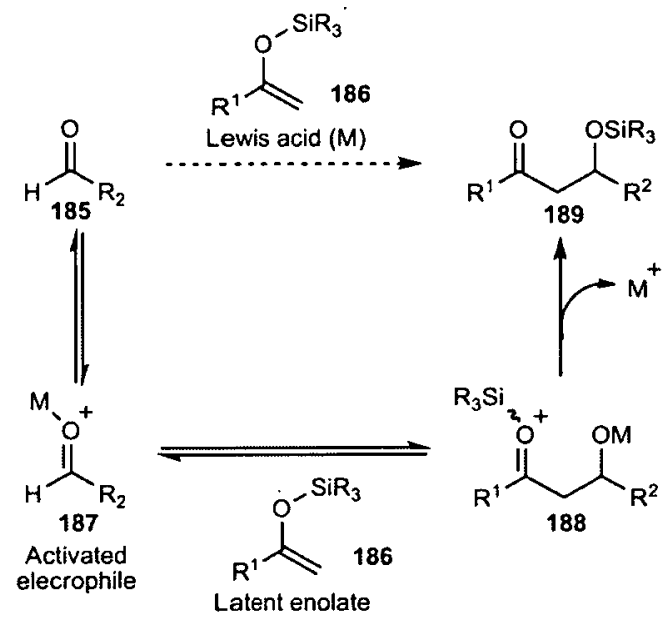

Scheme 3.5 Lewis acid catalysed aldol reaction

Although achieving high levels of diastereo- and enantioselectivity, these strategies always require the transformation of one of the reagents prior to the aldol addition. Ideally, catalytic methodologies should employ unmodified substrates. The direct catalytic asymmetric aldol reaction has been extensively developed in recent years, but these methodologies are still limited to the use of symmetric ketones, ketones possessing a single set of acidic hydrogens, or ketones which incorporate hydroxyl directing groups. ${ }^{79}$ 


\section{The reductive aldol reaction}

The use of enones represents an alternative way of achieving a selective enolate formation. Stork established that tandem enone reduction-enolate alkylation is an effective method to control the regiochemistry in enolate-mediated $\mathrm{C}-\mathrm{C}$ bond formations (Scheme 3.6). ${ }^{80}$

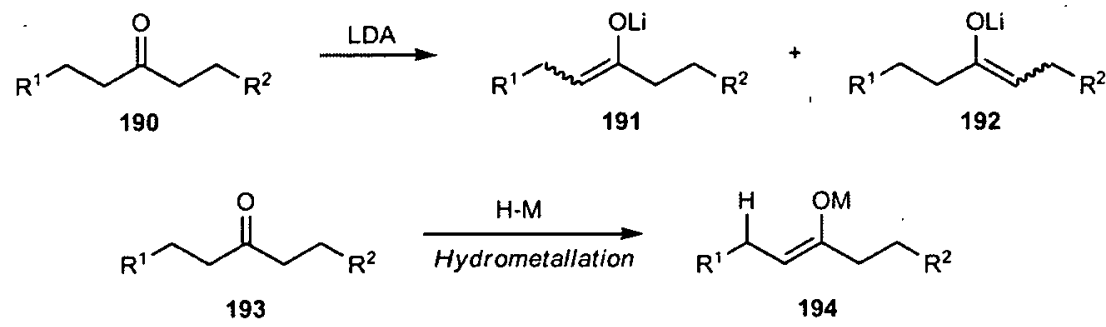

Scheme 3.6 Control of the enolisation regioselectivity using enones as latent enolates equivalents

The hydrometallation of $\alpha, \beta$-unsaturated carbonyl compounds has proven to be a mild and versatile method for enolate formation, being amenable to catalysis. ${ }^{81}$ The reductive aldol reaction involves the direct metal-catalysed reductive addition of $\alpha, \beta$-unsaturated carbonyl compounds with aldehydes or ketones (Scheme 3.7).

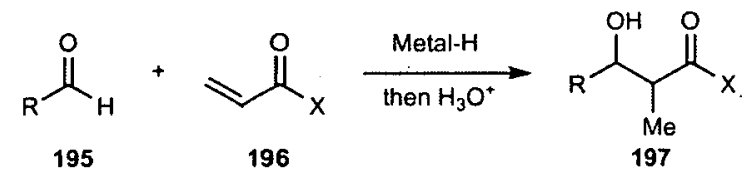

Scheme 3.7 Transition metal-catalysed reductive aldol reaction

- The first example of a reductive aldol coupling was reported by Revis and Hilty in $1987 .{ }^{82}$ They demonstrated that the reaction of $\alpha, \beta$-unsaturated esters with ketones or aldehydes in the presence of substoichiometric amounts of $\mathrm{RhCl}_{3} \cdot 3 \mathrm{H}_{2} \mathrm{O}(0.09$ mol\%) and trimethylsilane ( $1.3 \mathrm{~mol} \%)$ represents a general and mild method to form $\beta$-siloxy esters (Scheme 3.8). The coupling proceeded well for several types of ketones and also for acetaldehyde. As nucleophiles, only esters were employed, since methyl vinyl ketone did not couple to acetone, and silyl enol ether (200) was formed instead. 


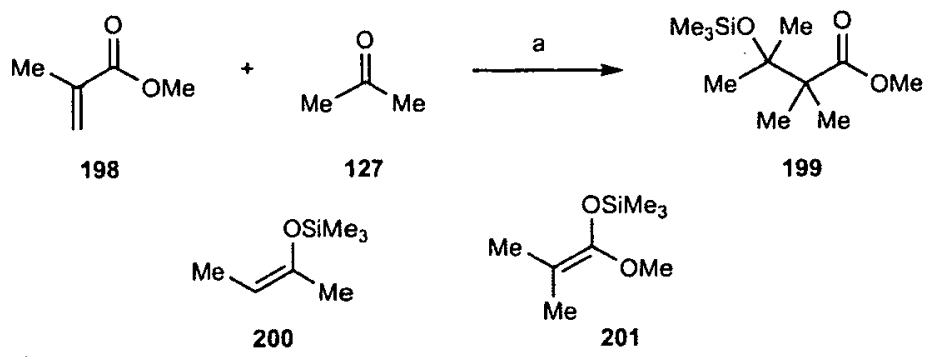

Reagents and conditions: (a) cat $\mathrm{RhCl}_{3} \cdot 3 \mathrm{H}_{2} \mathrm{O}, \mathrm{Me}_{3} \mathrm{SiH}, \mathrm{rt}, 95 \%$.

Scheme 3.8 First example of a catalytic reductive aldol reaction

Silyl ketene acetal (201) was prepared independently and reacted with acetone in the presence of a substoichiometric quantity of $\mathrm{RhCl}_{3} \cdot 3 \mathrm{H}_{2} \mathrm{O}$, but only traces of the aldol product were formed. This eliminated the possibility of the Rh species acting as a Lewis acid in the reaction, thus supporting the conjugate reduction followed by aldol reaction mechanism.

Since the Revis and Hilty report, there have been numerous advances in the reductive aldol reaction, employing a broad variety of transition metal catalysts and reaction conditions. The reductive aldol reaction has already been fully reviewed in the literature; ${ }^{81,83,84}$ the rest of this section will therefore focus on developments in the field that are directly related to this project. Two aspects will be covered: methodologies that have been successfully applied towards the synthesis of natural products and the methodologies reported for the formation of carbocyclic rings, which constitute the precedents for the methodology studies carried out in our group.

In 1990, Matsuda made an important contribution in the rhodium-catalysed reductive aldol reaction, reporting the first coupling of $\alpha, \beta$-unsaturated ketones with aldehydes, to form $\beta$-siloxy ketone aldols, using $\mathrm{Rh}_{4}(\mathrm{CO})_{12}$ as catalyst. ${ }^{85}$ Nevertheless, Morken has been one of the main contributors to the Rh-catalysed reductive aldol reaction. As little was known about the mechanism, his aim was to find the most efficient catalyst system. A high-throughput evaluation of 192 independent catalytic systems was performed. ${ }^{86}$ The reaction chosen for the screening was the reaction of methyl acrylate with benzyldehyde. He examined four complexes, $\mathrm{Co}(\mathrm{acac})_{2} \cdot 2 \mathrm{H}_{2} \mathrm{O},[\mathrm{Pd}(\text { allyl }) \mathrm{Cl}]_{2},[\operatorname{Ir}(\mathrm{COD}) \mathrm{Cl}]_{2}$ and $[\mathrm{Rh}(\mathrm{COD}) \mathrm{Cl}]_{2}$, six 
hydride sources and seven chiral ligands. The combination of $[\mathrm{Rh}(\mathrm{COD}) \mathrm{Cl}]_{2}$, $\mathrm{Cl}_{2} \mathrm{MeSiH}$ and Me-DuPhos (204) was found to be the most successful one (Scheme 3.9), providing diastereoselectivities of up to 23:1 (syn:anti) and relatively high yields (15-82\%).

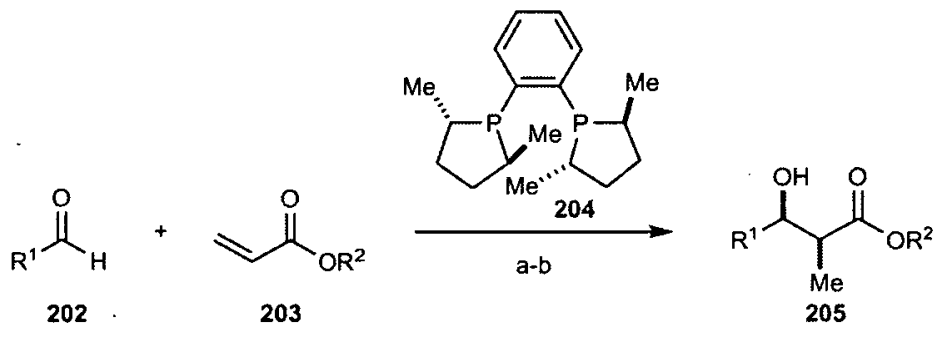

Reagents and conditions: (a) $5.5 \mathrm{~mol} \%$ Me-DuPhos, $2.5 \mathrm{~mol} \%$ $[(\mathrm{COD}) \mathrm{RhCl}]_{2}, \mathrm{Cl}_{2} \mathrm{MeSiH} ;$ (b) $\mathrm{H}_{3} \mathrm{O}^{+}$.

Scheme 3.9 Morken's catalytic stereoselective reductive aldol reaction

Morken also reported the first example of a catalytic asymmetric reductive aldol reaction. ${ }^{87}$ Coupling of methyl acrylate with benzaldehyde in the presence of $\mathrm{Rh}(\mathrm{BINAP})$ as catalyst and $\mathrm{Et}_{2} \mathrm{MeSiH}$ as reductant, afforded a syn selective (1.7:1, $37 \%$ yield) reaction in which the syn aldol product was obtained in $91 \%$ ee and the anti in $88 \%$ ee. It was discovered that the yields were much more favourable using phenyl acrylate (207) (Scheme 3.10).

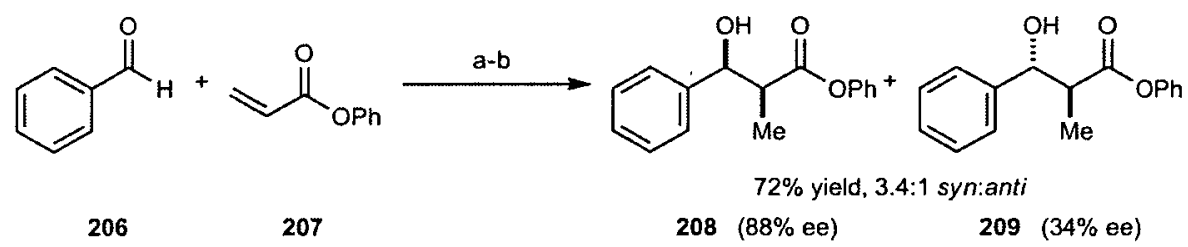

Reagents and conditions: (a) $2.5 \mathrm{~mol} \%[(\mathrm{COD}) \mathrm{RhCl}]_{2}, 6.5 \mathrm{~mol} \%$ $R$-BINAP, $\mathrm{Et}_{2} \mathrm{MeSiH}$; (b) $\mathrm{H}_{3} \mathrm{O}^{+}$.

\section{Scheme 3.10 First Rh-catalysed asymmetric reductive aldol reaction}

With the advances made in the Rh-catalysed reductive aldol reaction its application in total synthesis was now possible. Morken applied this methodology in the synthesis of the C10-C24 ketone fragment of the inostamycin family of compounds (Figure 3.1). ${ }^{88}$ These compounds are known to possess anti-HIV and anticancer activity. 


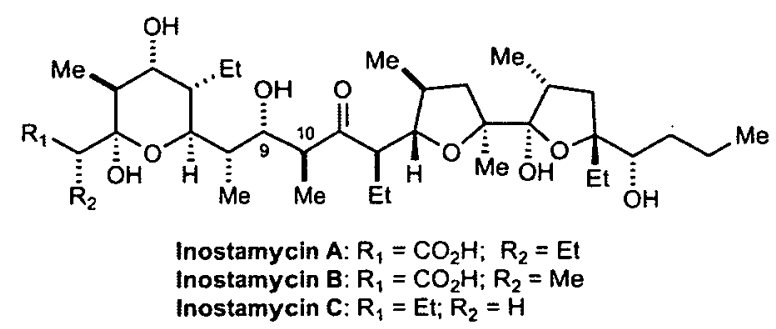

Figure 3.1 Compounds of the Inostamycin family

The Rh-catalysed asymmetric reductive aldol reaction between propionaldehyde (210) and phenyl crotonate (211) afforded the corresponding $\beta$-hydroxy ester 212 in $76 \%$ yield and with 4.3:1 syn diastereoselectivity and $88 \%$ enantiomeric excess (Scheme 3.11). This intermediate was then used for the synthesis of the C10-C24 ketone fragment 213.

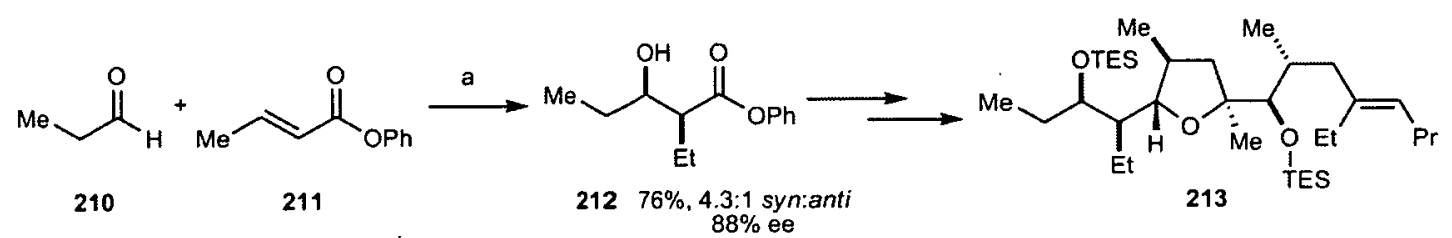

Reagents and conditions: (a) $2.5 \mathrm{~mol} \%[\mathrm{Rh}(\mathrm{COD}) \mathrm{Cl}]_{2}, 5.5 \mathrm{~mol} \%$ $S$-BINAP, $\mathrm{Et}_{2} \mathrm{MeSiH}(1.2 \mathrm{eq}), \mathrm{Cl}\left(\mathrm{CH}_{2}\right) \mathrm{Cl}$, $\mathrm{rt}$; then $\mathrm{H}_{3} \mathrm{O}^{+}, 76 \%$.

Scheme 3.11 Synthesis of the C10-C24 ketone fragment of the inostamycin family

In 2001, Morken and co-workers reported that $\operatorname{Ir}(\mathrm{I})$ salts, when complexed to an appropriate 2,6-bis(oxazolinyl)pyridine (pybox) ligand (Figure 3.2), are able to catalyse reductive aldol reactions to give the products in good yields, good syn-diastereoselectivity and good to excellent enantioselectivities. ${ }^{89}$
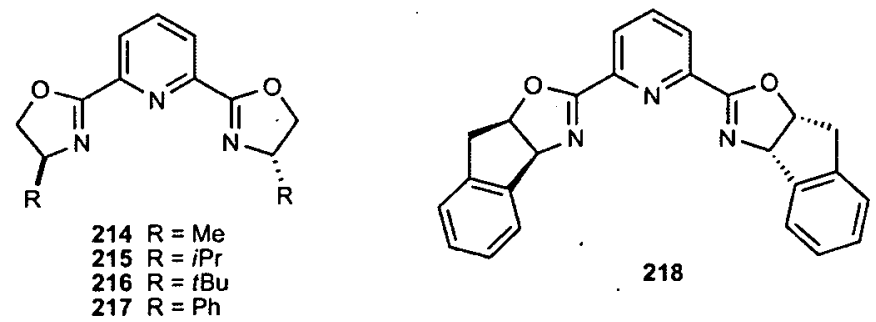

Figure 3.2 Pybox ligands employed in the Ir-catalysed asymmetric reaction 
An initial survey of the impact of ligand architecture revealed that the nature of the pybox ligand substitution has a significant impact on stereoselectivity. These investigations identified the aminoindanol-derived indane pybox 218 as the best ligand with regards to both enantio- and diastereoselectivity.

Therefore, ligand $\mathbf{2 1 8}$ was successfully used in the iridium-based catalytic reductive aldol reaction of various aldehydes. This catalyst system showed remarkable enantioselectivity with benzaldehyde and $\alpha$-alkoxy aldehydes, however poor reaction with simple aldehydes. It was also observed that oxygenation adjacent to the aldehydes enhances reactivity relative to non-oxygenated aliphatic substrates (Table 3.1).

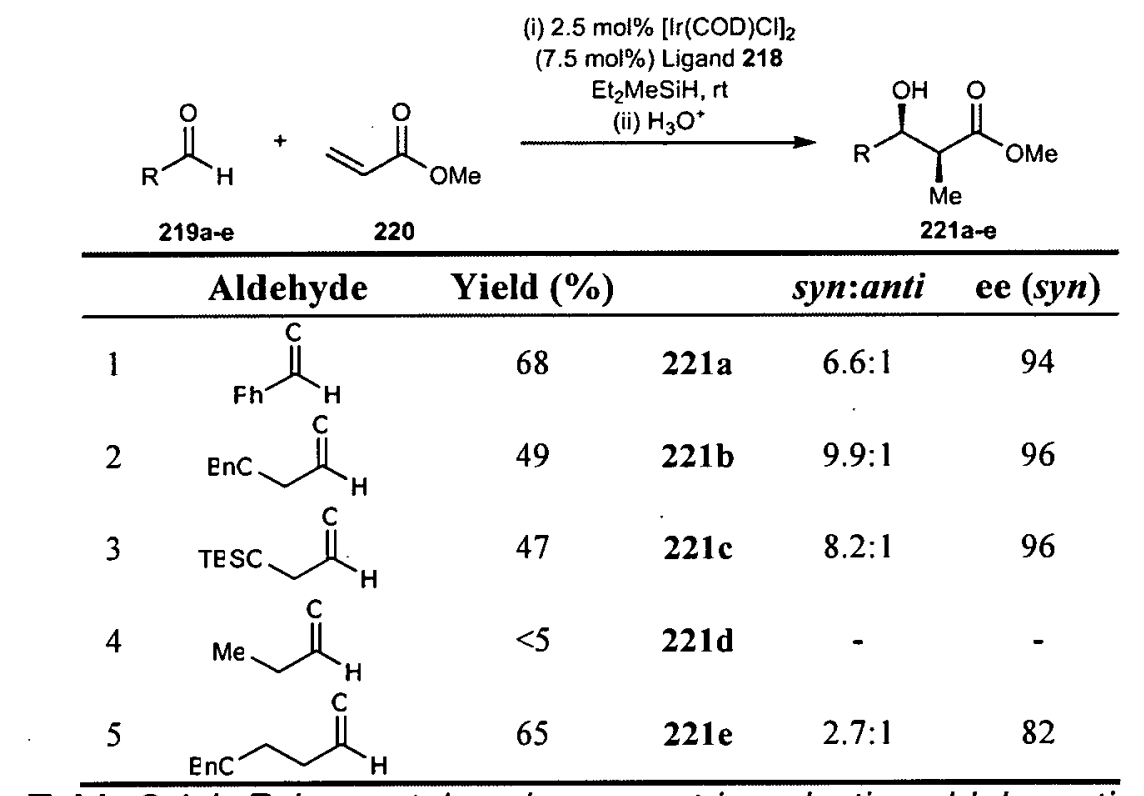

Table 3.1 /r-Pybox catalysed asymmetric reductive aldol reaction

Morken applied this methodology in the total synthesis of borrelidin (222), a potential anti-cancer agent. ${ }^{90}$ The structure of borrelidin is characterised by an 18-membered macrolactone carrying a cyclopentane carboxylic acid and a unique conjugated cyanodiene (Figure 3.3). 


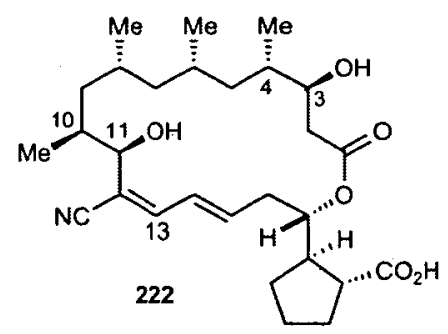

Figure 3.3 Borrelidin

Reductive aldol methodology was used to establish the stereogenic centres at $\mathrm{C} 3$, $\mathrm{C} 4, \mathrm{C} 10$ and $\mathrm{C} 11$. Both steps in which this methodology was used went in good yield, with high diastereoselectivity and good to excellent enantioselectivity (Scheme 3.12).

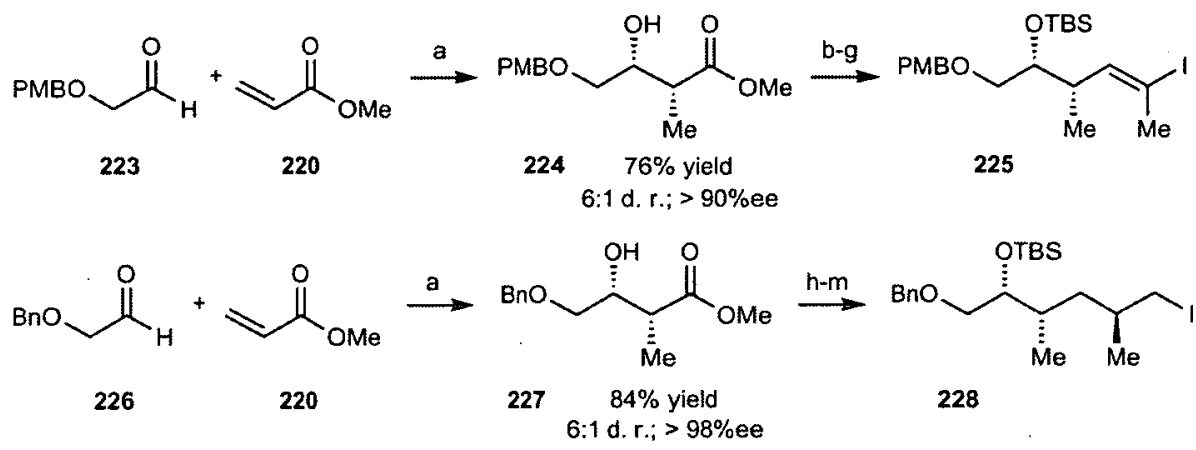

Reagents and conditions: (a) $2.5 \mathrm{~mol} \%[\operatorname{Ir}(\mathrm{COD}) \mathrm{Cl}]_{2}$, Ligand $218,7.5 \mathrm{~mol} \%$, $\mathrm{Et}_{2} \mathrm{MeSiH}$, rt; (b) TBSOTf, 2,6-lutidine, 90\%; (c) DIBAL-H, 79\%; (d) Dess-Martin periodinane, 92\%; (e) $\mathrm{CBr}_{4}, \mathrm{PPh}_{3}, 94 \%$; (f) $n$-BuLi, MeI, 97\%; (g) (i) $\mathrm{Cp}_{2} \mathrm{ZrHCl}$, (ii) $\mathrm{I}_{2}, 89 \%$; (h) TBSOTf, 2,6-lutidine, 99\%; (i) DIBAL-H, 94\%; (j) $\mathrm{PPh}_{3}, \mathrm{I}_{2}, 96 \%$; (k) pseudoephedrine propionamide, LDA, 93\%; (l) LAB, 93\%; (m) $\mathrm{PPh}_{3}, \mathrm{I}_{2}, 95 \%$.

Scheme 3.12 Application of the Ir-Pybox catalysed asymmetric reductive aldol reaction in the synthesis of borrelidin

Fragments 225 and 228 were coupled in a modified Negishi coupling. Alkyl iodide 228 was converted to the dialkyl zinc and reacted with vinyl iodide $\mathbf{2 2 5}$ yielding intermediate 229, which ultimately allowed the synthesis of borrelidin (Scheme 3.13). 


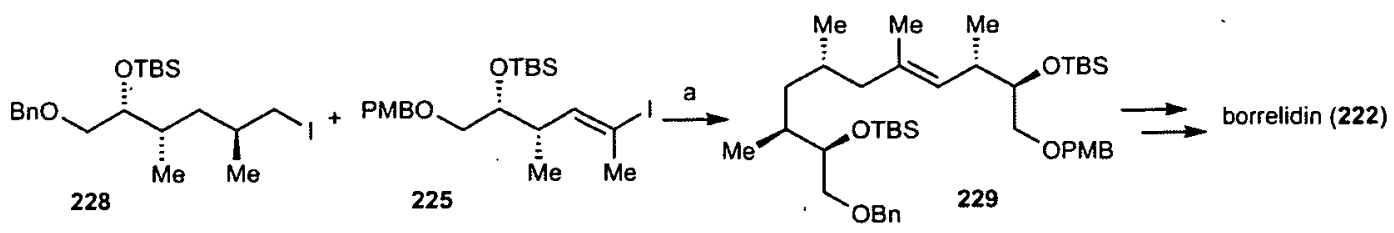

Reagents and conditions: (a) (i) $t-\mathrm{BuLi}, \mathrm{ZnCl}_{2}$, (ii) $\mathrm{Pd}\left(\mathrm{PPh}_{3}\right)_{4}, 225$, (58\%).

\section{Scheme 3.13 Synthesis of borrelidin 222}

Motherwell reported in 2004 a Rh-catalysed intramolecular reductive aldol reaction of aldehyde-enoate derivatives. ${ }^{91}$ 6-Oxo-enoates were cyclised with $\mathrm{RhCl}\left(\mathrm{PPh}_{3}\right)_{3}$ or $\mathrm{RhH}\left(\mathrm{PPh}_{3}\right)_{4}$ as the $\mathrm{Rh}$ source $(1 \mathrm{~mol} \%)$ and $\mathrm{Et}_{3} \mathrm{SiH}(2.1 \mathrm{eq})$ as the hydride source, in toluene. Aldol products were obtained with syn-selectivity when using $\mathrm{RhCl}\left(\mathrm{PPh}_{3}\right)_{3}$, but $\mathrm{RhH}\left(\mathrm{PPh}_{3}\right)_{4}$ showed higher catalytic activity and high anti-selectivity, except for the cyclisation of $\mathbf{2 3 2}$ (Table 3.2).

\begin{tabular}{|c|c|c|c|c|c|}
\hline & Substrate & Product & Catalyst & Yield (\%) & syn/anti \\
\hline 1 & 230 & 231 & $\mathrm{RhCl}\left(\mathrm{PPh}_{3}\right)_{3}$ & 81 & $3: 1$ \\
\hline 2 & & & $\mathrm{RhH}\left(\mathrm{PPh}_{3}\right)_{4}$ & 81 & 1:11 \\
\hline 3 & 232 & 233 & $\mathrm{RhCl}\left(\mathrm{PPh}_{3}\right)_{3}$ & 56 & $1: 1$ \\
\hline 4 & & & $\mathrm{RhH}\left(\mathrm{PPh}_{3}\right)_{4}$ & 62 & $6.4: 1$ \\
\hline 5 & 234 & & $\mathrm{RhCl}\left(\mathrm{PPh}_{3}\right)_{3}$ & 93 & $2.2: 1$ \\
\hline 6 & $\mathrm{cos}^{2}$ & he & $\mathrm{RhH}\left(\mathrm{PPh}_{3}\right)_{4}$ & 61 & $1: 11$ \\
\hline
\end{tabular}

Table 3.2 Rh-catalysed reductive cyclisation of 6-oxo-enoates derivatives

They also applied this methodology in the synthesis of the highly potent anti-HIV carbocyclic nucleosides (-)-carbovir (239) and (-)-abacavir (240). ${ }^{92}$ The Rh-catalysed reductive aldol reaction allowed access to key intermediate 237 from cis-cyclopentane 236 (Scheme 3.14). Protected triol 237 was converted into protected tetraol 238, which is a key intermediate in the synthesis of the carbocyclic nucleosides. 


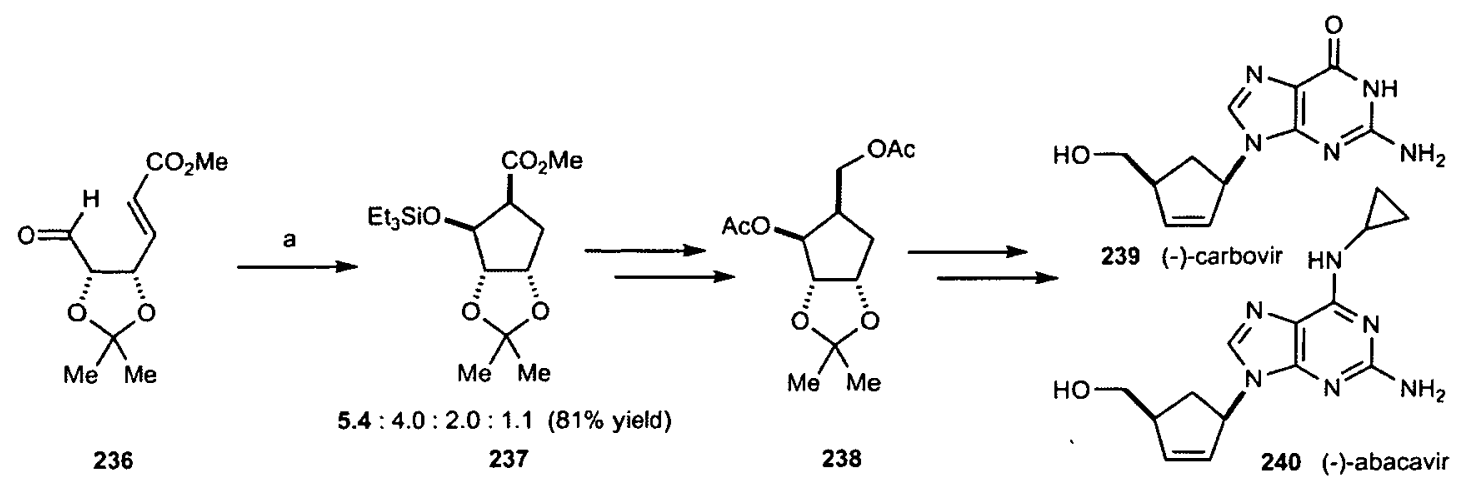

Reagents and conditions: (a) $1 \mathrm{~mol} \% \mathrm{RhH}\left(\mathrm{PPh}_{3}\right)_{4}, \mathrm{Et} \mathrm{SiH}_{3}(2.1 \mathrm{eq})$, toluene, $50^{\circ} \mathrm{C}$.

Scheme 3.14 Use of the Rh-catalysed reductive aldol cyclisation

We can then conclude that, due to the development of the reductive aldol reaction, the application of this methodology to the synthesis of natural products has been possible, with inter- and also intramolecular examples. In the remaining part of this section, we will describe methodologies for intramolecular reductive aldol reactions to form carbocyclic rings, which are therefore relevant to the methodology of our project.

In 1989, Mukaiyama reported the first cobalt-catalysed cross coupling reaction of $\alpha, \beta$-unsaturated nitriles, amides and esters with aldehydes using phenylsilane as a hydride source to give the corresponding $\beta$-hydroxy nitriles, amides or esters. ${ }^{93}$ Krische, in 2001, reported the first examples of intramolecular reductive aldol reactions catalysed by cobalt. ${ }^{94}$ The optimised conditions for this transformation proved general for five-, six- and seven-membered ring formation. The aldol products were obtained in moderate to excellent yield (35-87\%) and in all cases, irrespective of yield, only the syn-diastereomer was observed (Scheme 3.15). Studies into the mechanism of this cycloreduction were also performed. ${ }^{81,95}$

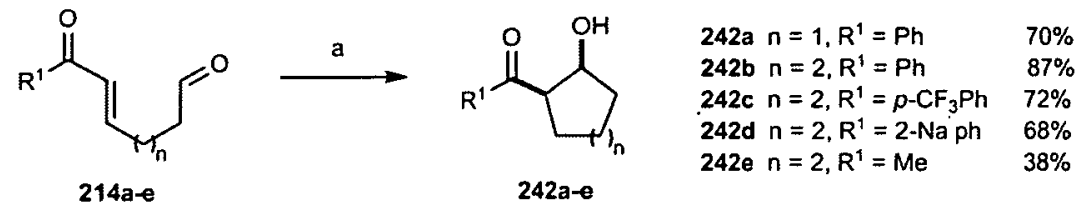

Reagents and conditions: (a) $5 \mathrm{~mol} \% \mathrm{Co}(\mathrm{dpm})_{2}, \mathrm{PhSiH}_{3}(1.2 \mathrm{eq}), \mathrm{Cl}\left(\mathrm{CH}_{2}\right)_{2} \mathrm{Cl}, 20^{\circ} \mathrm{C}$.

Scheme 3.15 First example of a Co-catalysed intramolecular reductive aldol cyclisation 
Krische played a major role in the development of $\mathrm{Rh}$ based catalyst systems in the reductive aldol reaction. In 2002, he reported the first example of inter- and intramolecular reductive aldol reaction of enones in which the enolates were generated using elemental hydrogen as reductant. ${ }^{96}$

Generally, the accepted mechanism for the Rh-catalysed alkene hydrogenation has three steps: oxidative addition of $L_{n} R h(I)$ to elemental hydrogen, followed by alkene hydrometalation forming $\mathrm{L}_{n} \mathrm{Rh}(\mathrm{III})$ (alkyl)(hydrido) intermediates and, finally, alkyl-hydrogen reductive elimination to give the saturated product and regenerate $\mathrm{L}_{n} \mathrm{Rh}(\mathrm{I})$. If the double bond is part of an enone, an enolate will be generated. Therefore they hypothesised that if there was an electrophile, the alkyl-rhodium intermediate could be trapped, in competition with alkyl-hydrogen reductive. elimination (Scheme 3.16).

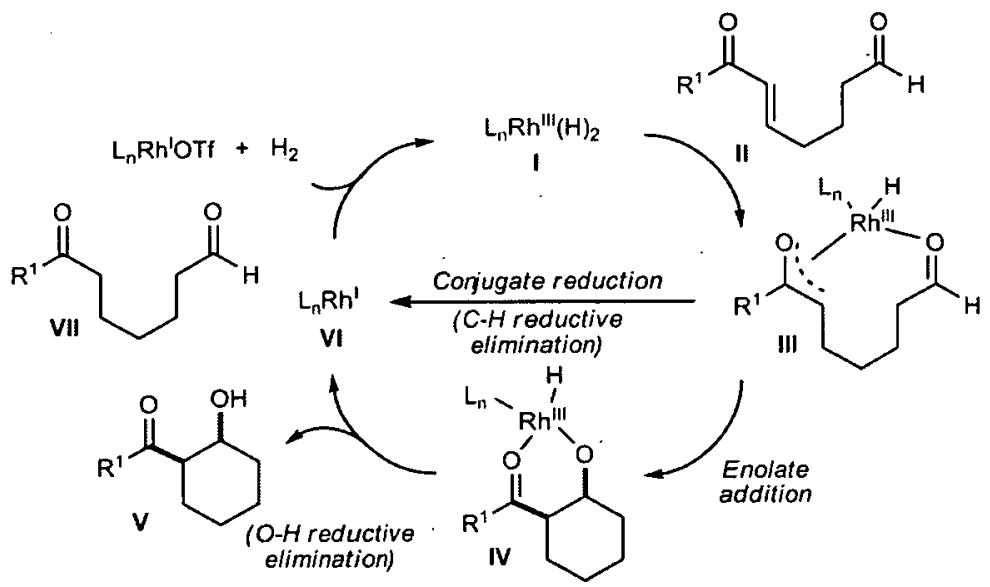

Scheme 3.16 Proposed catalytic cycle of the reductive generation of enolates under hydrogenative conditions

With this theory, they attempted the hydrogenative cycloreduction of monoenone monoaldehydes. A variety of $\mathrm{Rh}$ sources and phosphines were screened but when using $\mathrm{Rh}(\mathrm{COD})_{2} \mathrm{OTf} / \mathrm{PPh}_{3}$, equal proportions of $s y n$-aldol cycloreduction product (244) and 1,4-reduction product (245) were obtained (Scheme 3.17). Bearing in mind the proposed mechanism, they speculated that deprotonation of (hydrido)Rh species I or III would diminish the 1,4-reduction. Indeed, when the reactions were performed using potassium acetate 244 and 245 were obtained in $59 \%$ and $21 \%$ yield, respectively. A higher Lewis acidity of the metal was another important factor, since 
when $\left(p-\mathrm{CF}_{3} \mathrm{Ph}\right)_{3} \mathrm{P}$ was used as ligand, together with the base, the yield of 244 was increased to $89 \%$, obtaining only $0.1 \%$ of 245 .

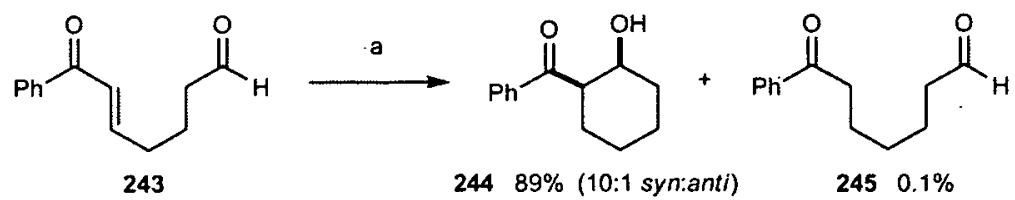

Reagents and conditions: (a) $10 \mathrm{~mol} \%$ [Rh(COD) $\left.)_{2}\right] \mathrm{OTf}, 24 \mathrm{~mol} \%$ $\left(p-\mathrm{CF}_{3} \mathrm{Ph}\right)_{3} \mathrm{P}, \mathrm{H}_{2}(1 \mathrm{~atm}), 30 \% \mathrm{KOAc}, \mathrm{Cl}\left(\mathrm{CH}_{2}\right) \mathrm{Cl}, \mathrm{rt}, 89 \%$.

\section{Scheme 3.17 Rh-catalysed hydrogenative aldol cycloreduction of 243}

Using these conditions, cycloreduction of aromatic, heteroaromatic, and aliphatic enone substrates yielded the five- and six-membered ring $\beta$-hydroxy ketone products in good to excellent yields (65-90\%) and with high syn diastereoselectivities (up to 24:1) (Table 3.3).

\begin{tabular}{|c|c|c|c|c|}
\hline & $\underbrace{}_{246 a-f}=0$ & $\begin{array}{l}{\left[\mathrm{Rh}(\mathrm{COO})_{2}\right] \mathrm{OTf}} \\
6\left(p-\mathrm{CF}_{3} \mathrm{Ph}\right)_{3} \mathrm{P} \\
\mathrm{KOAC}(30 \mathrm{~mol} \%) \\
\left.\mathrm{I}_{2}\right)_{2} \mathrm{Cl}, 25^{\circ} \mathrm{C} \\
\end{array}$ & & $\sum_{248 a-f}$ \\
\hline & Substrate & $\begin{array}{c}\text { Yield product (\%) } \\
\text { (syn:anti) }\end{array}$ & & $\begin{array}{c}\text { Yield } \\
\text { 1,4-reduction (\%) } \\
\end{array}$ \\
\hline 1 & $\mathrm{n}=2, \mathrm{R}^{\prime}=p-\mathrm{MeOPh}$ & $74(5: 1)$ & $247 a$ & 3 \\
\hline 2 & $\mathrm{n}=2, \mathrm{R}^{1}=2$-naphthyl & $90(10: 1)$ & $247 b$ & 1 \\
\hline 3 & $\mathrm{n}=2, \mathrm{R}^{1}=2$-thiophenyl & $76(19: 1)$ & $247 c$ & 2 \\
\hline 4 & $\mathrm{n}=2, \mathrm{R}^{1}=2$-furyl & $70(6: 1)$ & 247d & 10 \\
\hline 5 & $\mathrm{n}=1, \mathrm{R}^{1}=\mathrm{Ph}$ & $71(24: 1)$ & $247 e$ & 1 \\
\hline 6 & $\mathrm{n}=2, \mathrm{R}^{\prime}=\mathrm{Me}$ & $65(1: 5)$ & $247 f$ & - \\
\hline
\end{tabular}

Table 3.3 Rh-catalysed hydrogenative aldol cycloreduction of enone aldehydes

The fact that 1,4-reduction had been practically eliminated led to the formulation of another possible mechanism and explanation of the role of the base (Scheme $3.18){ }^{97}$ It was believed that Rh-monohydride was being formed via heterolytic cleavage of hydrogen (oxidative addition and reductive elimination by the base). Therefore, if enone hydrometalation occurred via a Rh-monohydride complex, the 
resulting enolate would lack an extra hydride ligand and could not go under $\mathrm{C}-\mathrm{H}$ reductive elimination (II $v s$ VI, Scheme 3.18).$^{98}$ After enolate addition, III follows the catalytic cycle, suffering oxidative addition to form $\mathrm{Rh}(\mathrm{III})$ enolate IV that liberates the desired aldol product and Rh-monohydride, which reenters the cycle.

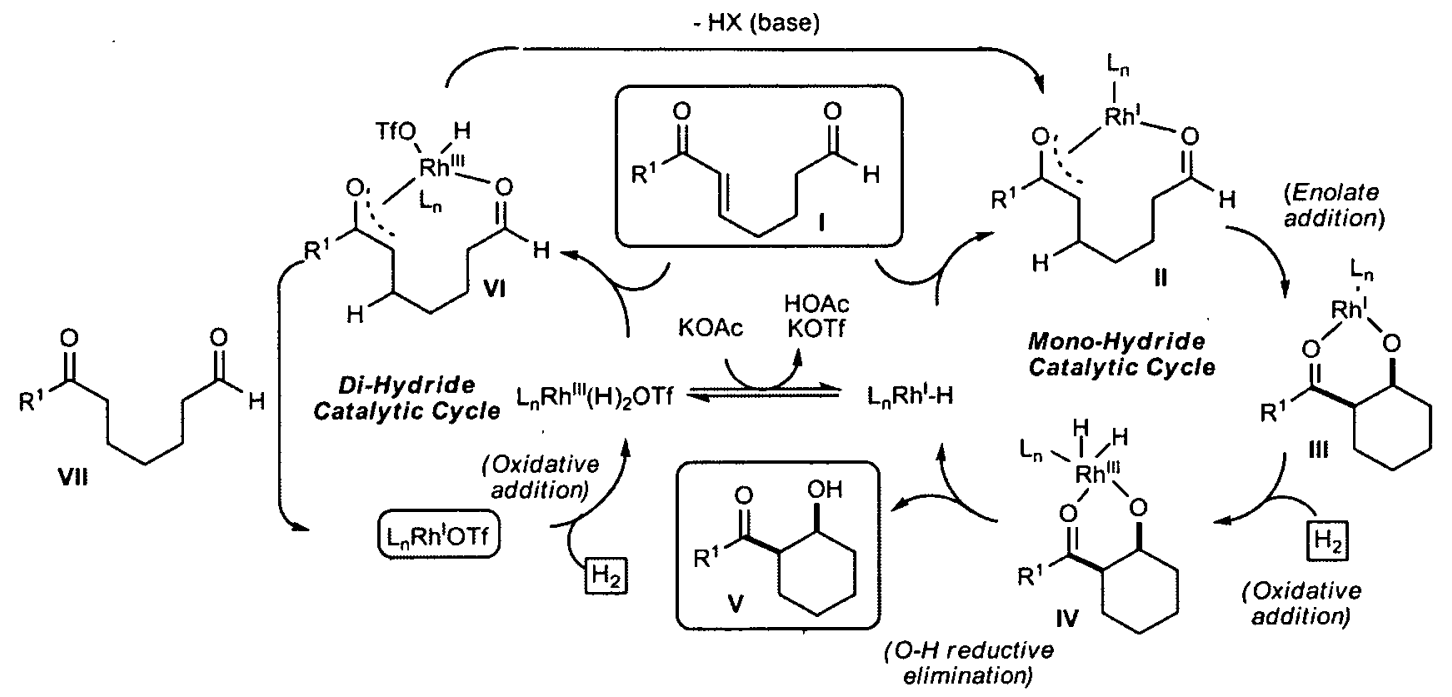

Scheme 3.18 Formal heterolytic activation of hydrogen mitigates competitive conjugate reduction by enabling monohydride catalytic cycle

Keto-enones were also cyclised using $\mathrm{Rh}(\mathrm{COD})_{2} \mathrm{OTf} / \mathrm{PPh}_{3}$ and in the presence of $\mathrm{K}_{2} \mathrm{CO}_{3}$ as base, yielding the aldol products with good yields and exclusive cis-selectivity (cis:trans $>95: 5$ ) (Scheme 3.19). ${ }^{98}$ Based on this system, there have been several other reports of Rh-catalysed reductive aldol reactions from the Krische group. ${ }^{99}$

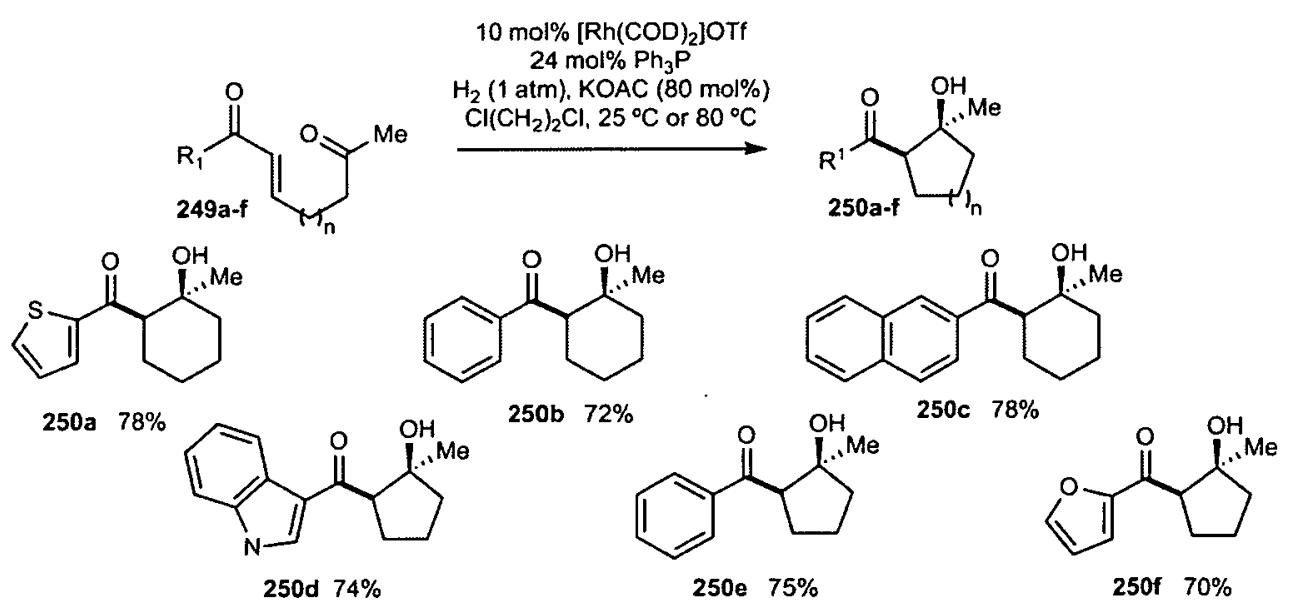

Scheme 3.19 Catalytic hydrogenative cycloreduction of keto-enones 
In 2003, Krische reported a reductive aldol cyclisation of monoenone monoketones using catecholborane. In this intramolecular reaction, the reduction of either the enone or the ketone was avoided. The desired cyclised products were obtained in good yields and high levels of syn-diastereoselectivity (>99:1) (Scheme 3.20). ${ }^{100}$

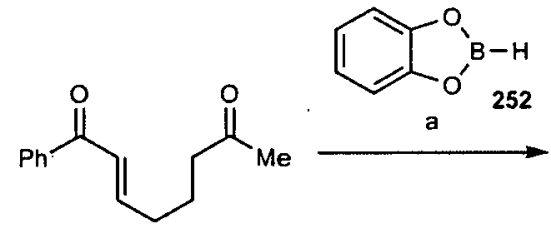

251

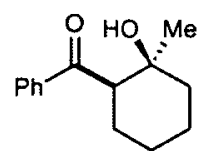

253

Reagents and conditions: (a) Catecholborane 252 (115 mol\%), THF, $25^{\circ} \mathrm{C}, 89 \%$.

Scheme 3.20 Catecholborane mediated aldol cycloreduction of monoenone monoketones

Baba and co-workers reported a reductive aldol reaction of enones with aldehydes utilising $\mathrm{InBr}_{3}$ and hydrosilanes as the hydride source. ${ }^{101}$ In 2004 Hosomi and co-workers reported a similar indium catalysed reductive aldol cyclisation of enones with ketones, employing $\mathrm{In}(\mathrm{OAc})_{3}$ and $\mathrm{PhSiH}_{3}$ (Scheme 3.21). ${ }^{102}$

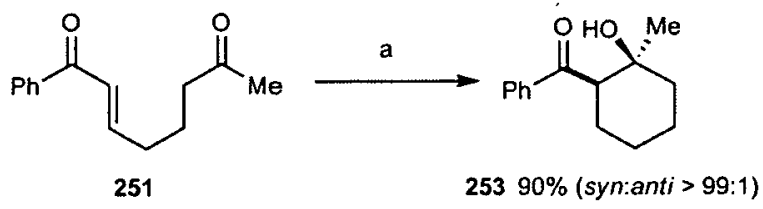

Reagents and conditions: (a) $\mathrm{In}(\mathrm{OAc})_{3}(10 \mathrm{~mol} \%), \mathrm{PhSiH}_{3}(1.2 \mathrm{eq})$, THF, EtOH, reflux.

Scheme 3.21 Indium catalysed reductive aldol reaction of keto-enones.

The Cu-catalysed reductive aldol reaction has also made a major contribution to the development of the intramolecular reductive aldol reaction forming carbocyclic rings. The copper hydride species $\left[\mathrm{Ph}_{3} \mathrm{PCuH}\right]_{6}$ (Stryker's reagent), ${ }^{103}$ is a well characterised, stoichiometric source of hydride which chemoselectively reduces conjugate systems, being inert toward isolated olefins. In 1998 Chiu and co-workers were able to develop the first example of an intramolecular reductive aldol reaction using Stryker's reagent. Working on the synthesis of pseudolaric acid A, keto-enone 
254 was successfully cyclised using $\left[\mathrm{Ph}_{3} \mathrm{PCuH}\right]_{6}$, to yield two bicyclic diastereomers, $255(66 \%)$ and $256(16 \%)$ (Scheme 3.22$){ }^{104}$

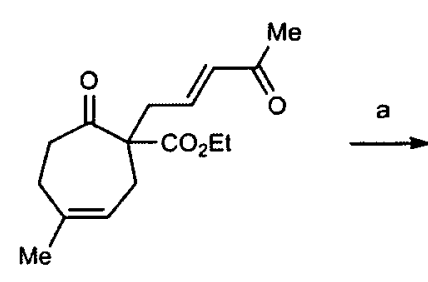

254

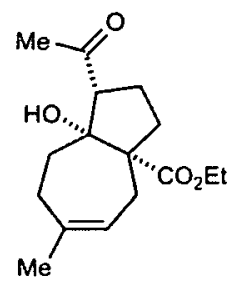

$25566 \%$

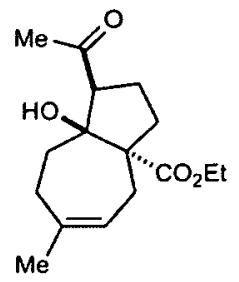

$25616 \%$

Reagents and conditions: (a) $\left[\mathrm{Ph}_{3} \mathrm{PCuH}\right]_{6}(2 \mathrm{eq})$, toluene, $-23^{\circ} \mathrm{C}$.

Scheme 3.22 First example of an intramolecular reductive aldol reaction using Stryker's reagent

In. 2001, Chiu reported a more in-depth investigation of the intramolecular reductive aldol cyclisation reaction promoted by stoichiometric Stryker's reagent (Scheme 3.23). ${ }^{105}$ When the reactions were performed at low temperatures $(-40$ to $-25^{\circ} \mathrm{C}$ ), to avoid dehydration, $\beta$-hydroxyketones were obtained with good to excellent yields and exclusive syn-selectivity.

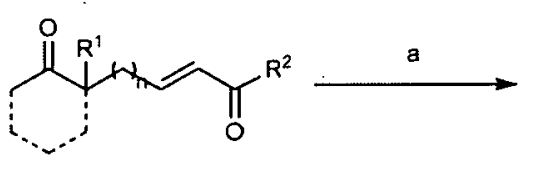

256

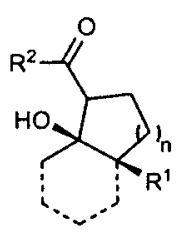

$25866-93 \%$

Reagents and conditions: (a) $\left[\mathrm{Ph}_{3} \mathrm{PCuH}\right]_{6}\left(2\right.$ eq), toluene, $-40^{\circ} \mathrm{C}$

Scheme 3.23 Reductive aldol cyclisation of enediones by Stryker's reagent

This methodology was applied to the synthesis of lucinone (261) (Scheme 3.24). ${ }^{106}$ Enedione $\mathbf{2 5 9}$ was transformed quantitatively and stereoselectively into $\mathbf{2 6 0}$ using two hydride equivalents of the Stryker's reagent in toluene at $0{ }^{\circ} \mathrm{C}$ to room temperature. 


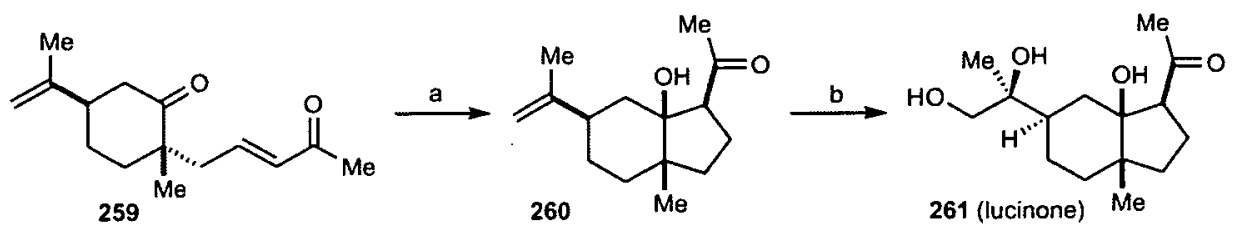

Reagents and conditions: (a) $\left[\mathrm{Ph}_{3} \mathrm{PCuH}\right]_{6}$ (2 eq), toluene, $0{ }^{\circ} \mathrm{C}$, 99\%; (b) cat $\mathrm{OsO}_{4}, \mathrm{~K}_{3} \mathrm{Fe}(\mathrm{CN})_{6}, 38 \%$.

\section{Scheme 3.24 Synthesis of lucinone}

The use of copper salts and organosilanes to form copper hydride species had been reported for conjugate reductive reactions by Mori and Hosomi in 1997. ${ }^{107}$ Buchwald ${ }^{108}$ and Lipshutz ${ }^{109}$ had been great contributors in this area, reporting several excellent methods for enantioselective conjugate reductions.

In 2000, Lipshutz reported the catalytic reductive aldol reaction of enones and aldehydes using Stryker's reagent ( $5 \mathrm{~mol} \%)$ in the presence of a silyl hydride (1.5 eq) such as dimethylphenylsilane $\left(\mathrm{Me}_{2} \mathrm{PhSiH}\right)$, tetramethyldisiloxane $\left(\mathrm{HMe}_{2} \mathrm{SiOSiMe}_{2} \mathrm{H}\right)$ or polymethylhydrosiloxane (PMHS), to regenerate it. ${ }^{110}$ This was a one pot- two step reaction and provided $\beta$-hydroxyketones in high yield. In the first step, silyl enol ethers were formed. In the second step, Lewis acids such as $\mathrm{BF}_{3}$ or $\mathrm{TiCl}_{4}$ were used to promote the aldol reaction.

Chiu reported, in 2004, the development of the intramolecular catalytic $\mathrm{Cu}$ catalysed reductive aldol reaction using Strykers reagent $(10 \mathrm{~mol} \%)$ as the $\mathrm{Cu}$ source and PMHS (2.0 eq) as a hydride source. ${ }^{83,111} \mathrm{~A}$ variety of alkynediones were cyclised using these conditions, to afford the cyclic $\beta$-hydroxy bicycloalkenones with similar or improved yields that with the stoichiometric reaction. An example is represented in Scheme 3.25.

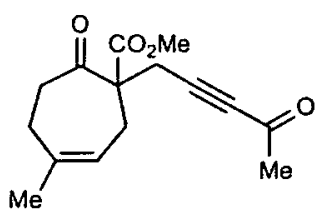

262

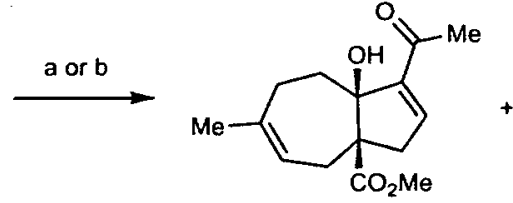

(a) Stoichiometric (b) Catalytic
$263(48 \%)$ $(65 \%)$

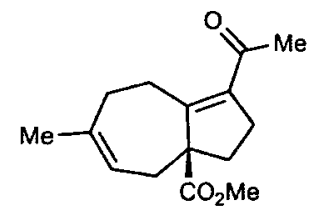

$264(16 \%)$ $(6 \%)$

Reagents and conditions: (a) $\left[\mathrm{Ph}_{3} \mathrm{PCuH}\right]_{6}(1.5 \mathrm{eq})$, toluene, $-40^{\circ} \mathrm{C}$. (b) $\left[\mathrm{Ph}_{3} \mathrm{PCuH}\right]_{6}(10 \mathrm{~mol} \%)$, PMHS (2 eq), toluene, $-40^{\circ} \mathrm{C}$.

Scheme 3.25 Reductive aldol cyclisations catalysed by Stryker's reagent 
These were the precedents in the literature on intramolecular reductive aldol cyclisations when our group became interested in developing the reductive aldol methodology for the preparation of heterocyclic products. In the next section, the development within the group on this methodology will be described.

\subsection{Development of the reductive aldol cyclisation in the}

\section{Lam group}

\subsubsection{Copper-catalysed reductive aldol cyclisation}

In 2004, with the aim of developing a methodology that ultimately allowed the synthesis of salinosporamide $\mathrm{A}$, an investigation on the reductive aldol cyclisation of $\alpha, \beta$-unsaturated amides and, although not related to our project, $\alpha, \beta$-unsaturated esters was initiated in our group. As a result of these studies, in 2005 we reported the first intramolecular copper-catalysed reductive aldol reaction of $\alpha, \beta$-unsaturated esters with ketones, affording five- and six-membered $\beta$-hydroxylactones with high yields and very high $s y n$-stereoselectivity (>95:5) ${ }^{66}$

Initial efforts of this study focused on the cyclisation of $\alpha, \beta$-unsaturated esters tethered to a ketone utilising phosphine-stabilised copper hydride complexes as catalyst system. As it was described before (Scheme 3.25), the use of substoichiometric amounts of Stryker's reagent in the presence of stoichiometric siloxane had proved successful in promoting reductive aldol cyclisations in carbocycle synthesis. ${ }^{105,111}$

In optimising the reaction, several copper salts were investigated $\left(\mathrm{CuCl}_{2} \cdot 2 \mathrm{H}_{2} \mathrm{O}\right.$, $\mathrm{CuF}_{2}, \mathrm{Cu}(\mathrm{OAc})_{2} \cdot \mathrm{H}_{2} \mathrm{O}, \mathrm{Cu}$ (2-ethylhexanoate) $)_{2}, \mathrm{Cu}(\mathrm{OBz})_{2}$, and $\left.\mathrm{Cu}(\mathrm{acac})_{2}\right)$, also several siloxanes (PMHS and TMDS) and bisphosphine ligands [DPPE, DPPB, DPPF, rac-BINAP, and (S,S)-Et-DuPhos] (Scheme 3.26). 

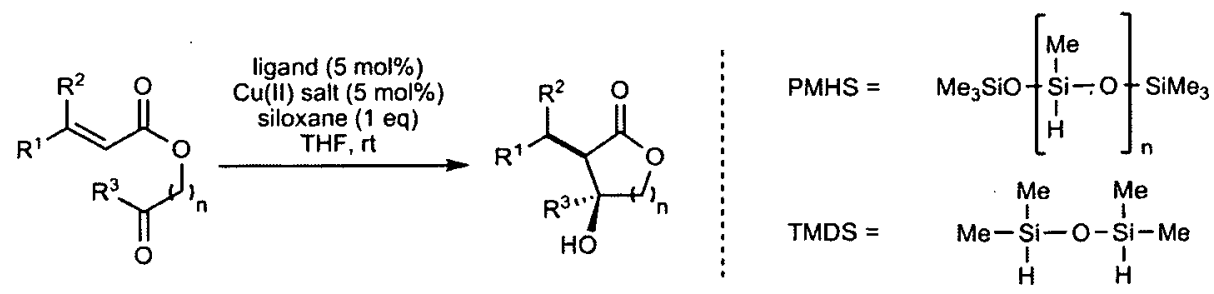
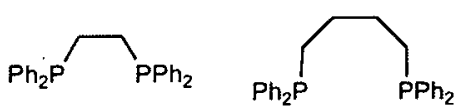<smiles>CCC1CC[C@H](CC)P1c1ccccc1P1C(CC)CC[C@H]1CC</smiles>

$267(S, S)$-Et-Duphos<smiles>Pc1cccc(-c2c(-c3ccccc3)ccc3ccccc23)c1-c1ccccc1</smiles>

(rac)-268 (rac)-BINAP

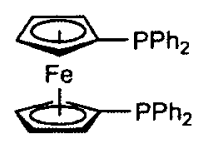

269 DPPF

Scheme 3.26 Optimisation of the Cu-catalysed reductive aldol cyclisation

Finally, it was identified that $\mathrm{Cu}(\mathrm{OAc})_{2} \cdot \mathrm{H}_{2} \mathrm{O}$, TMDS and either DPPF or rac-BINAP, in THF, promoted the cyclisation with good yields and high diastereoselectivities (>95:5). Several $\alpha, \beta$-unsaturated ester components were tolerated (Table 3.4); methyl ketone or phenyl ketone were both permitted and the process could also be applied to the formation of five-membered lactones.

Entry

Table 3.4 Copper-catalysed reductive aldol cyclisations 
The use of nonracemic chiral bisphosphines (Figure 3.4) was also investigated and asymmetric cyclisations were performed with good enantioselectivities. The Roche MeO-BIPHEP Ligands (272-274) and SEGPHOS ligand 275 afforded slightly higher enantioselectivities than (S)-BINAP 268 (Table 3.5).<smiles>c1ccc(-c2ccc3ccccc3c2-c2c(-c3ccccc3)ccc3ccccc23)cc1</smiles>

268 (S)-BINAP

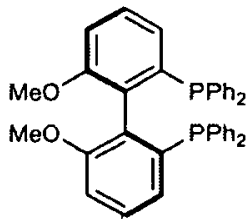

(S)-Ph-MeO-BIPHEP

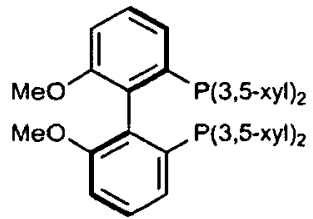

273

(R)-3,5-xyl-MeO-BIPHEP

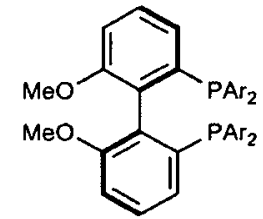

274 $\mathrm{Ar}=3,5-\mathrm{di}-\mathrm{i}-\mathrm{Pr} \mathrm{Ph}$ (R)-3,5-di-i-Pr-MeO-BIPHEP<smiles>Pc1ccccc1-c1ccc2c(c1-c1c(P)ccc3c1OCO3)OCO2</smiles>

275 (S)-SEGPHOS

Figure 3.4 Biphosphine ligands employed in asymmetric cyclisations

\begin{tabular}{|c|c|c|c|c|c|}
\hline entry & Subtrate & Ligand & Yield (\%) & & ee $\%$ \\
\hline 1 & & 268 & 72 & $277 a$ & 62 \\
\hline 2 & & 272 & 73 & $277 b$ & 73 \\
\hline 3 & & 273 & 69 & $277 \mathrm{c}$ & 70 \\
\hline 4 & & 274 & 64 & 277d & 77 \\
\hline 5 & & 275 & 62 & $277 e$ & 74 \\
\hline 6 & & 268 & 79 & $277 f$ & 73 \\
\hline 7 & & 272 & 71 & $277 \mathrm{~g}$ & 76 \\
\hline 8 & & 273 & 71 & $277 \mathrm{~h}$ & 83 \\
\hline 9 & & 275 & 73 & $277 i$ & 82 \\
\hline 10 & & 268 & 60 & $\mathbf{2 7 7 j}$ & 72 \\
\hline 11 & & 272 & 60 & $277 k$ & 72 \\
\hline 12 & & 273 & 61 & 2771 & 80 \\
\hline 13 & & 275 & 68 & $277 \mathrm{~m}$ & 80 \\
\hline
\end{tabular}

Table 3.5 Cu-catalysed asymmetric reductive aldol cyclisations

A catalytic cycle of the reaction was proposed (Scheme 3.27). This involves the generation of (bisphosphine) $\mathrm{Cu}-\mathrm{H}$ species, from the reduction of the $\mathrm{Cu}$ (II) salt in the presence of bisphosphine and TMDS. Conjugate reduction occurs to generate the copper enolate, which is followed by aldol addition and finally, $\sigma$-bond metathesis with the siloxane liberates the silylated product, deprotected upon workup. 


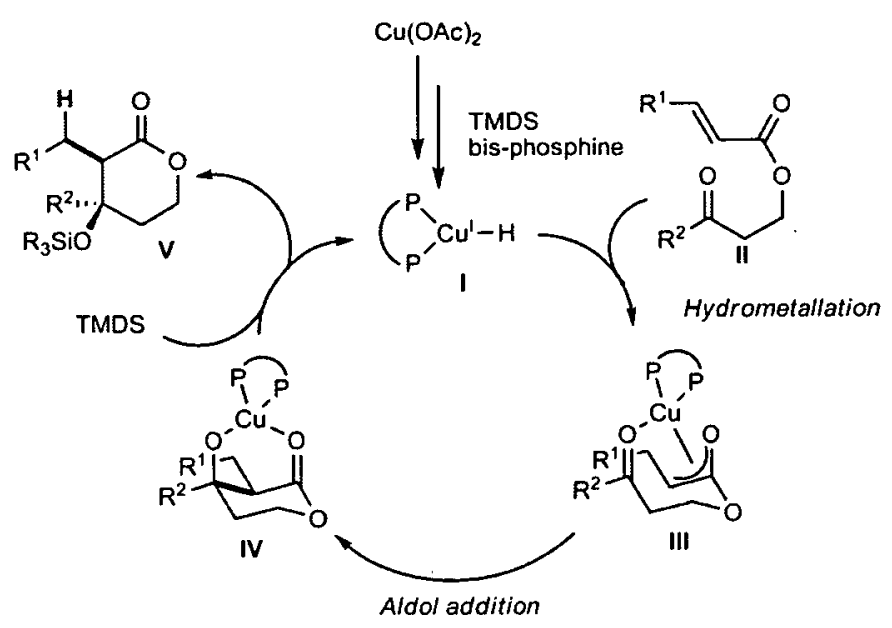

Scheme 3.27 Proposed catalytic cycle of the copper-catalysed reductive aldol cyclisation

These results made a significant contribution to the copper-catalysed reductive aldol methodology, but since we were aiming to apply this approach into the synthesis of salinosporamide $A$, our interest was centred on the reductive aldol cyclisation of $\alpha, \beta$-unsaturated amides. Therefore, we were very pleased to see that the same copper catalyst system was also successfully applied to the reductive aldol cyclisation of $\alpha, \beta$-unsaturated amides, to form 4-hydroxypiperidin-2-ones in a highly diastereoselective fashion $(>95: 5) .{ }^{65}$

The reaction was tolerant to wide variation in the ketone component, since alkyl, aromatic, and hetereoaromatic ketones were reacted in good yields (Table 3.6). However, the $\alpha, \beta$-unsaturated amide component did not allow much variety. Acryloyl amides were found to be the best substrates and crotonoyl amides were also cyclised but with lower reaction rates and conversions. Unfortunately, the formation of five-membered lactams was not possible. 


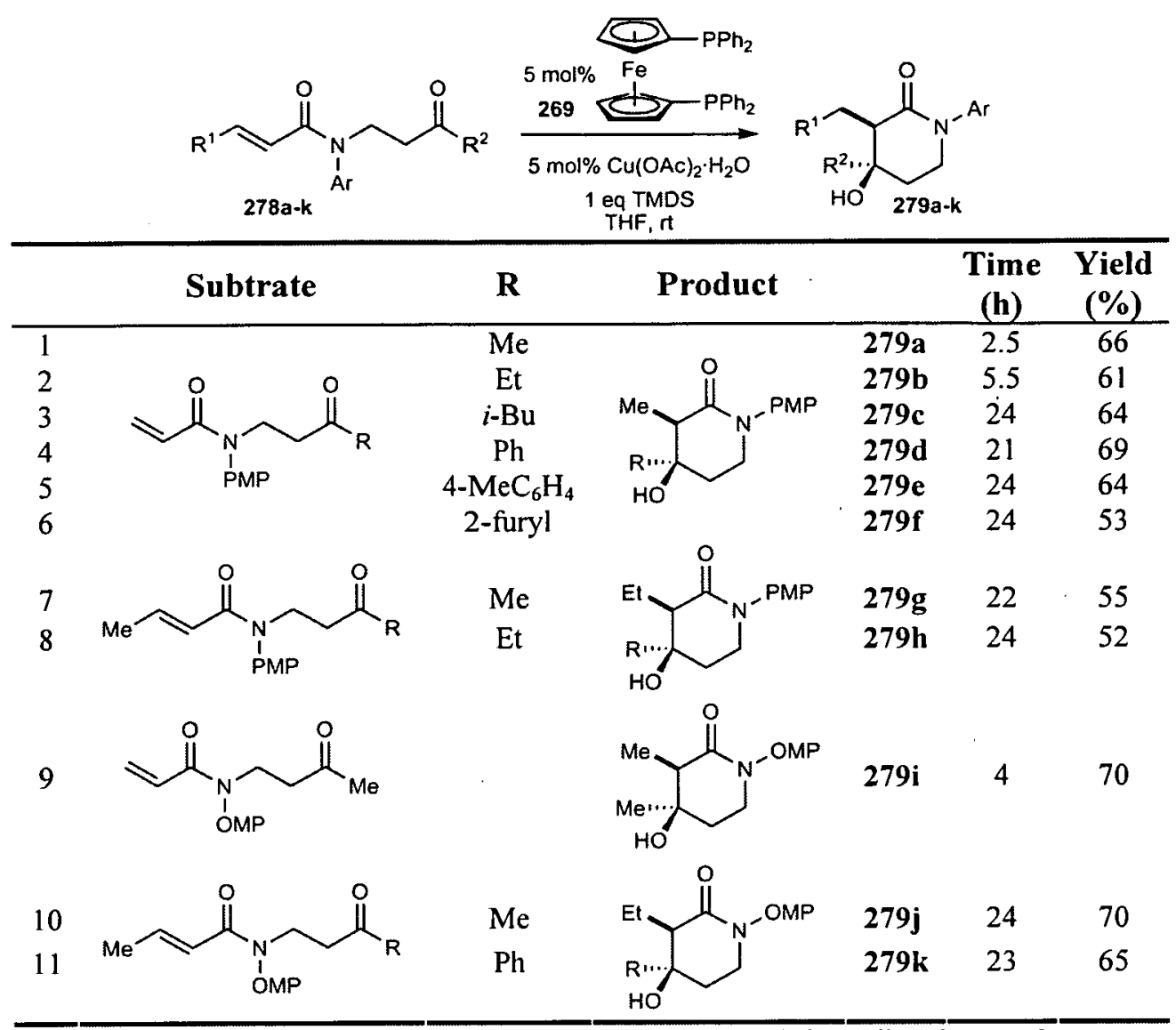

Table 3.6 Catalytic Cu-catalysed reductive aldol cyclisation to form 4-hydroxypiperidin-2-ones

The effect on the diastereoselectivity of the cyclisation of pre-existing centres in the tether linking the amide and the ketone was also studied. Formation of C5- and C6- substituted 4-hydroxypiperidi-2-ones was achieved with moderate to excellent levels of diastereoselectivity, due to the 1,2- or 1,3-asymmetric induction in the cyclisation (Table 3.7). 


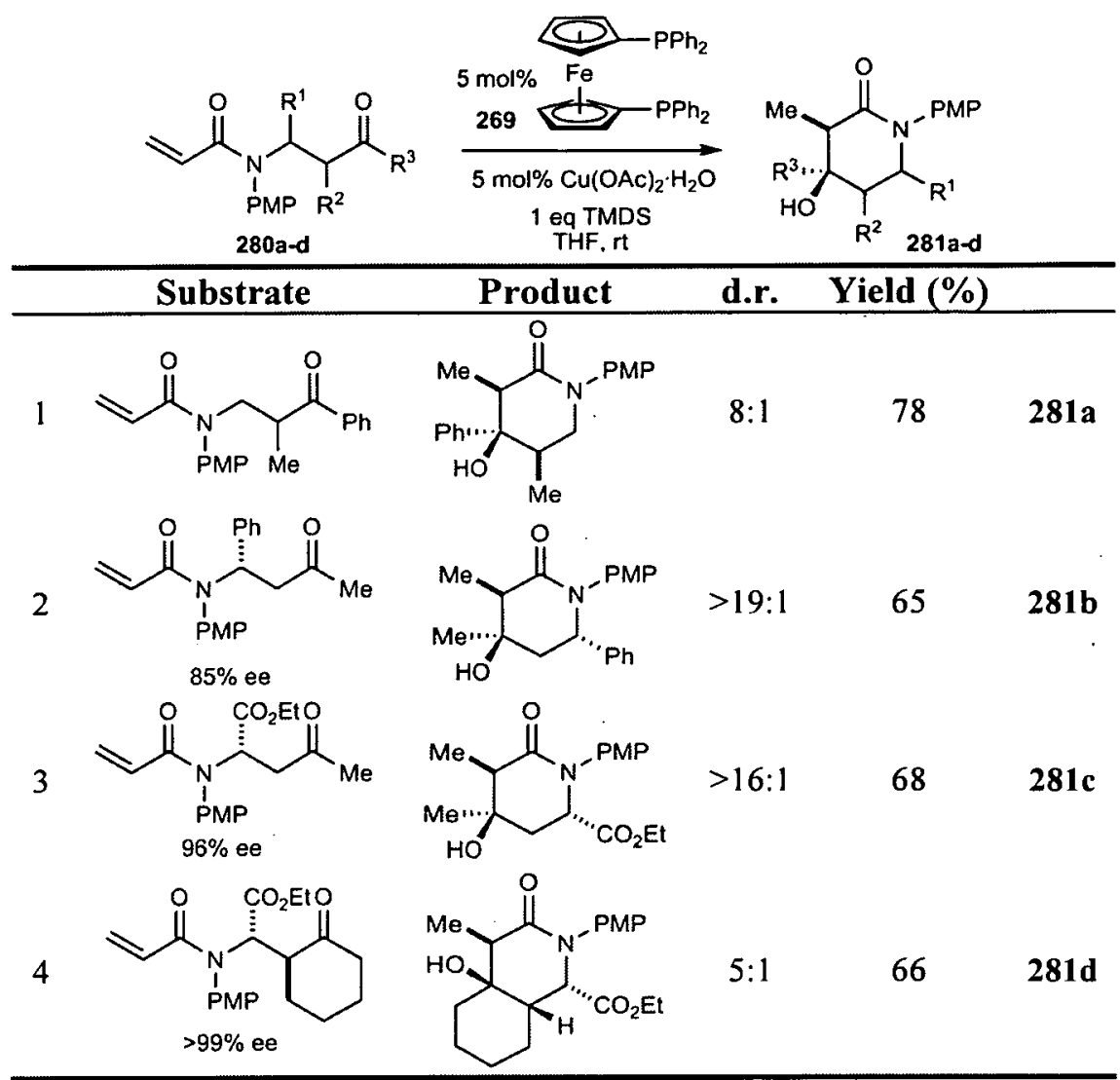

Table 3.7 Formation of C5- and C6-substituted 4-hydroxypiperidin-2-ones

\subsubsection{Cobalt-catalysed reductive aldol cyclisation}

The copper(I)-bisphosphine-catalysed reductive aldol cyclisation developed in our laboratories proved to be very efficient for the synthesis of $\beta$-hydroxylactones and $\beta$-hydroxylactams. The products were obtained with high diastereoselectivities and with moderate enantioselectivities in some cases.

However, there were two main limitations in this methodology, first, that the yields were only moderate at best $(60-70 \%)$ and second and more importantly regarding the synthesis of 1 , that due to the lower electrophilicity of $\alpha, \beta$-unsaturated amides, the range of amides permitted in this reaction was very limited, with acryloyl and crotonyl amides being the only competent substrates. Moreover, the cyclisation to five-membered lactams was not achieved. With this substrate limitation, the application of this methodology to the synthesis of salinosporamide A was not feasible. Therefore, a search for a new catalyst system that could overcome these limitations was initiated. 
After trying different combinations of $\mathrm{Cu}(\mathrm{I})$ salts, hydrosilanes and ligands without any improvement of the reaction, the use of other metals was investigated. The combination of $\mathrm{Co}$ (II) salts and hydrosilanes, combinations that had been employed in intermolecular reductive aldol reạctions, ${ }^{93-95}$ also proved ineffective.

Several methods had been reported in which organometallic reagents with $\beta$-hydrogen-containing alkyl groups were employed as stoichiometric reductants for transition metal-catalysed reductive couplings. ${ }^{112}$ Thus, the use of $\mathrm{Et}_{3} \mathrm{~B}$ and $\mathrm{Et}_{2} \mathrm{Zn}$ in the reaction was examined. In the presence of $\mathrm{Co}(\mathrm{acac})_{2} \cdot 2 \mathrm{H}_{2} \mathrm{O}(5 \mathrm{~mol} \%), \mathrm{Et}_{3} \mathrm{~B}$ resulted in no reaction, but when $\mathrm{Et}_{2} \mathrm{Zn}$ was employed, the desired product was obtained in $89 \%$ yield. $^{67 a}$

The scope of the reaction with these new conditions was next explored. It was discovered that it allowed the cyclisation of substrates with a wide range of substitution at both the $\alpha, \beta$-unsaturated amide and the ketone; to give five- and sixmembered rings $\beta$-hydroxylactams, in moderate to excellent yields and with high diastereoselectivities (Table 3.8). The standard conditions used $\mathrm{Co}(\mathrm{acac})_{2} \cdot 2 \mathrm{H}_{2} \mathrm{O}(5$ mol\%) as the precatalyst (method $\mathrm{A})$, but in some cases a combination of $\mathrm{CoCl}_{2}(5$ mol\%) and the electron-rich phosphine ligand $\mathrm{Cy}_{2} \mathrm{PPh}(5.5 \mathrm{~mol} \%)$ proved to be more efficient (method B). Most of these substrates were not cyclised under the copper conditions. 


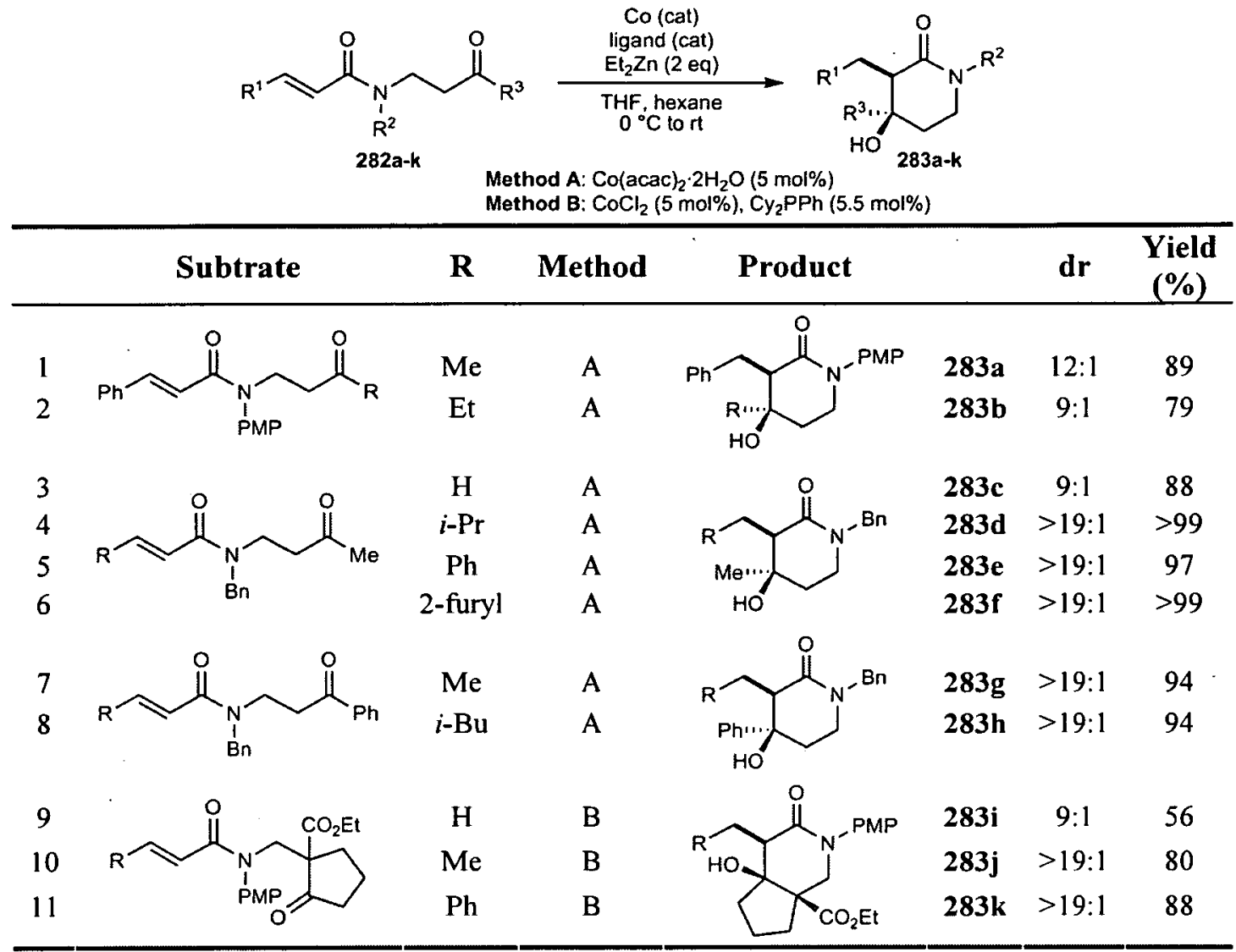

Table 3.8 Cobalt-catalysed reductive aldol cyclisations

More importantly, the cyclisation to five-membered lactams was also successfully achieved (Scheme 3.28), and although with slightly lower yields and diastereoselectivities. This was a very encouraging result towards the possible application of this methodology to form $\gamma$-lactam 103, intermediate in the route to 1 .

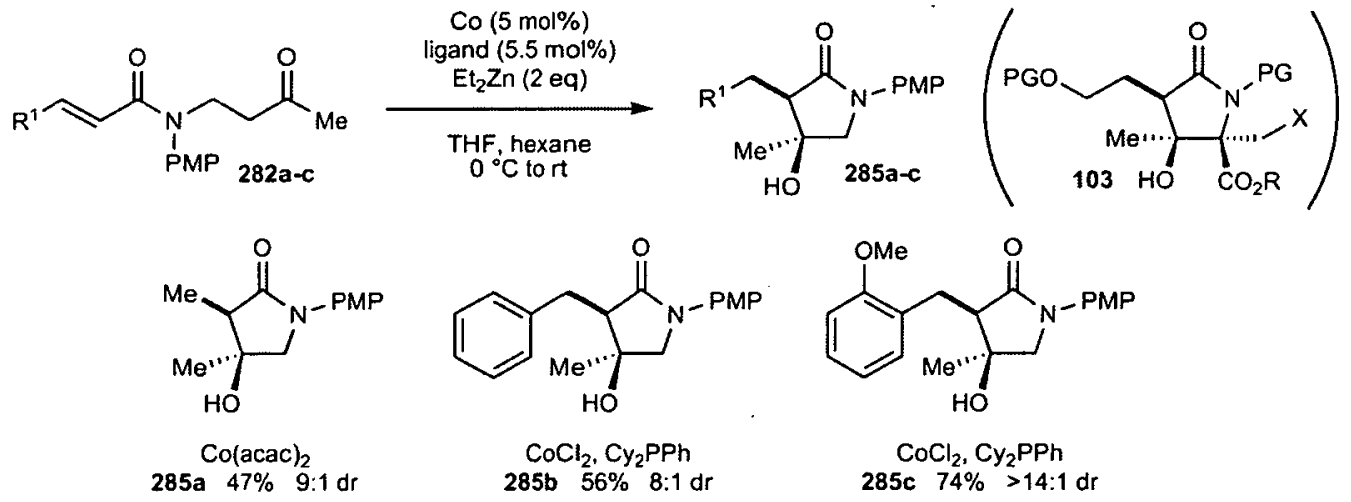

Scheme 3.28 Cobalt-catalysed reductive aldol cyclisations to 5-membered lactams 
The cyclisation to obtain five-membered lactams from $\alpha, \beta$-unsaturated amides containing phenyl ketones was very low yielding $(<20 \%)$ when using $\mathrm{Et}_{2} \mathrm{Zn}$. However, when the reaction was performed using $\mathrm{Et}_{3} \mathrm{Al}$ instead, the desired cyclised products were obtained in modest yields (Scheme 3.29).

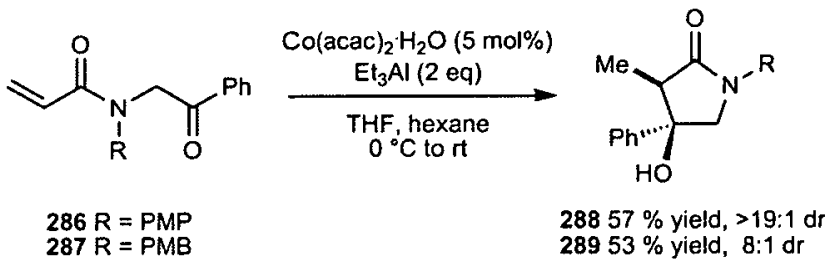

Scheme 3.29 Cyclisations to five-membered ring lactams using $\mathrm{Et}_{3} \mathrm{Al}$

The mechanism for this Co-catalysed cyclisation reaction is explained in the next section, together with the Ni-catalysed methodology.

\subsubsection{Nickel-catalysed reductive aldol cyclisation}

In the search for new, and more efficient, catalyst systems for the reductive aldol cyclisation of $\alpha, \beta$-unsaturated esters and amides with ketones, the use of nickel was also contemplated. It was surprising that there is an extended development of catalytic reductive aldol couplings and cyclisations of $\alpha, \beta$-unsaturated carbonyl substrates mediated by other metals, but not using nickel. Only recently, Montgomery reported a Ni-catalysed reductive aldol addition of acrylates and aldehydes, using $\mathrm{Et}_{3} \mathrm{~B}, \mathrm{Ni}(\mathrm{COD})_{2}$ and in the presence of an aryl iodide, which was shown to be linked to an initiation step. ${ }^{113}$

However, it has been demonstrated in our laboratories that the use of the combination of $\mathrm{Ni}(\mathrm{acac})_{2}$ with $\mathrm{Et}_{2} \mathrm{Zn}$ is not only as active as the cobalt catalyst system $^{67 a}$ but also very efficient in the reaction of substrates that were completely inert or reacted inefficiently (Table 3.9). ${ }^{67 \mathrm{~b}}$

The substrates containing the fumaric acid ethyl esters were successfully reacted to give the bicyclic products due to lactonisation of the alkoxide produced into the pendant ester (Table 3.9, entries 14 and 15). 


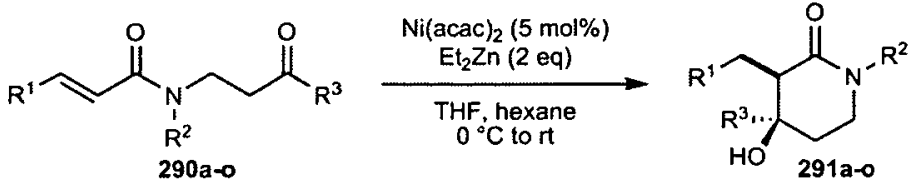

(1)

Table 3.9 Nickel-catalysed reductive aldol cyclisation to form

4-hydroxypiperidin-2-ones

Most encouragingly towards the synthesis of 1 , the reaction was also possible for the synthesis of five-membered lactams (Scheme 3.30). 

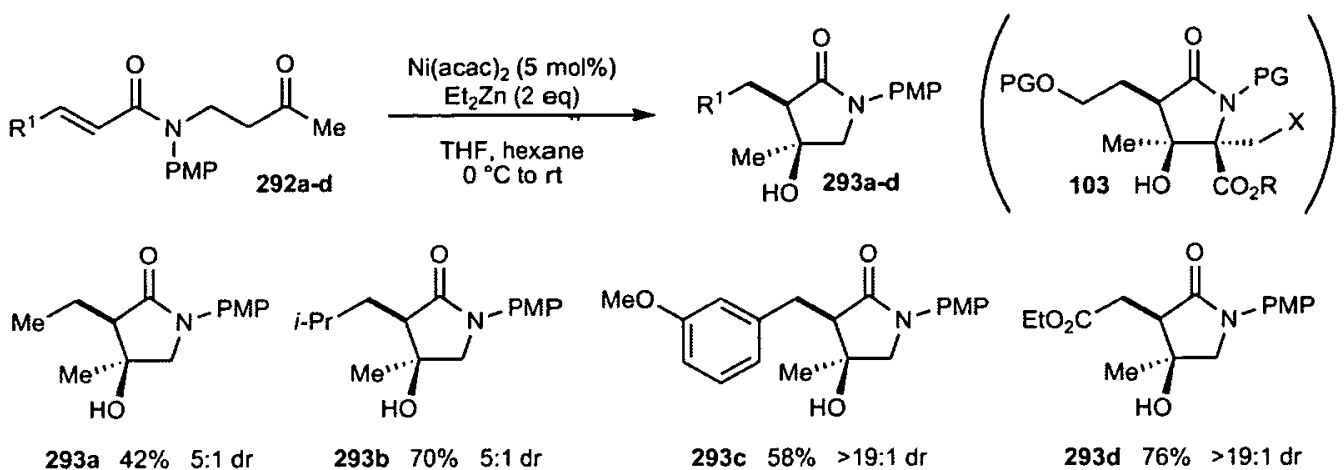

Scheme 3.30 Successful Ni-catalysed cyclisations to form $\gamma$-lactams

The cyclisations of esters was also performed using this new catalytic system. These substrates did not react under the cobalt conditions, but the $\mathrm{Ni}(\mathrm{acac})_{2} / \mathrm{Et}_{2} \mathrm{Zn}$ combination gave productive cyclisations affording a range of $\beta$-hydroxylactones in good to excellent yields and with high diastereoselectivities (Table 3.10).

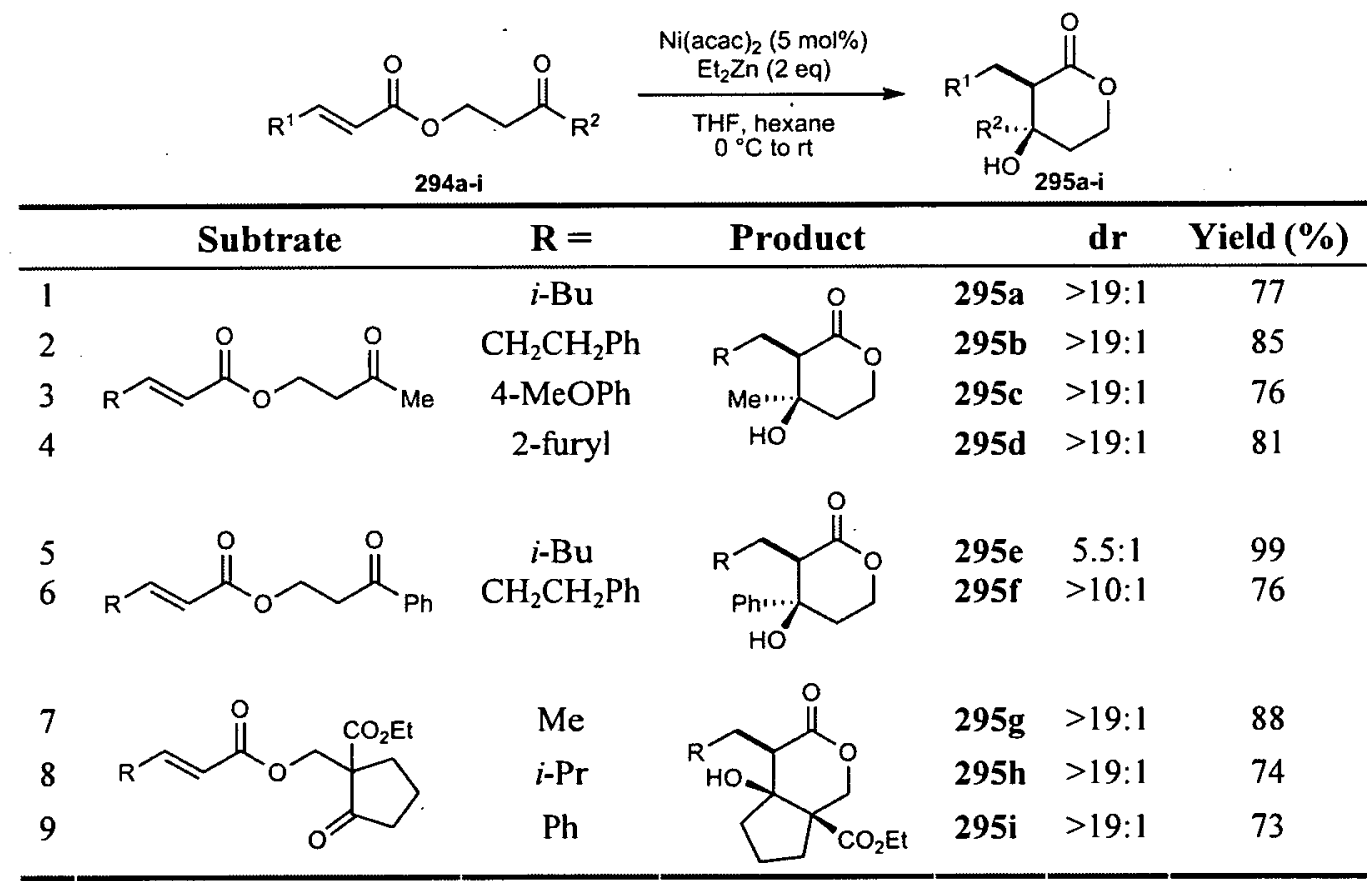

Table 3.10 Nickel-catalysed reductive aldol cyclisation to form $\beta$-hydroxylactones

Several mechanistic possibilities have been proposed for the nickel-catalysed reductive couplings and cyclisations. ${ }^{114}$ It is known that $\mathrm{Ni}(\mathrm{acac})_{2}$ in the presence of $\mathrm{Et}_{2} \mathrm{Zn}$ is reduced from $\mathrm{Ni}(\mathrm{II})$ to $\mathrm{Ni}(0)$, forming what is accepted to be the active 
catalytic species. It is believed that $\mathrm{Co}(\mathrm{acac})_{2} \cdot 2 \mathrm{H}_{2} \mathrm{O}$ or $\mathrm{CoCl}_{2}$ in the presence of $\mathrm{Et}_{2} \mathrm{Zn}$ behave in a very similar manner. Two possible reactive pathways were proposed in our group in order to explain the nickel- and cobalt-catalysed reductive aldol reaction. ${ }^{65 \mathrm{~b}}$

The oxidative cyclisation of $\mathrm{Ni}(0)$ with two $\pi$-components, forming metallacyclic intermediates, has been proposed as one of the possibles mechanisms for the three-components couplings of two $\pi$-components with a main-group organometallic complex. In this mechanism, oxidative cyclisation of $\mathrm{Ni}(0)$ with two $\pi$-components forms metallacycle II, which is followed by transmetallation with a metal alkyl MR to afford III and, finally, reductive elimination affords IV (Scheme 3.31). ${ }^{114}$

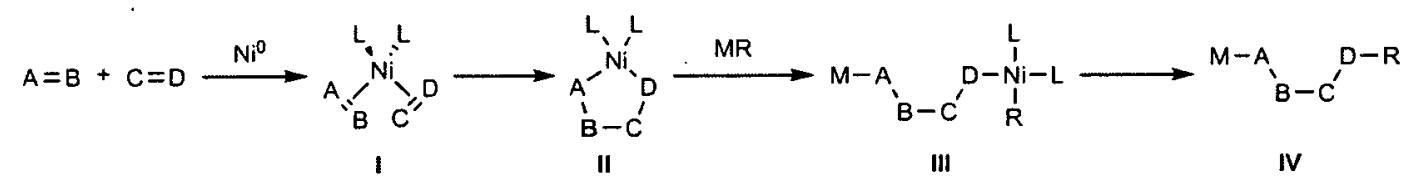

Scheme 3.31 Oxidative cyclisation of $\mathrm{Ni}(0)$ with two $\pi$-components

A possible catalytic cycle via the formation of metallacycles was proposed and is illustrated in Scheme 3.32. Oxidative cyclisation of $M(0)$ with the alkene and the ketone of $\alpha, \beta$-unsaturated substrate I would form metallacycle II. Diethylzinc would facilitate oxidative cyclisation by Lewis acid activation of the ketone through binding with zinc and by Lewis basic activation of $\mathrm{M}(0)$ through a three-center-two-electron bridging of a zinc-ethyl bond. ${ }^{115}$ Transmetallation would cleave the metallacycle and would provide metal-ethyl species III, which undergo $\beta$-hydride elimination to generate metal hydride IV. Finally, reductive elimination affords alkoxide V, ethylene and $\mathrm{M}(0)$ which would reenter the catalytic cycle. Alkoxide $\mathbf{V}$ would be protonated during workup to give the desired product.

With this reaction mechanism, the relative $s y n$-stereochemistry of the major diastereomer obtained in the reaction could be explained by the preference for the formation of the bicyclic metallacycle II, containing a cis-ring junction. 

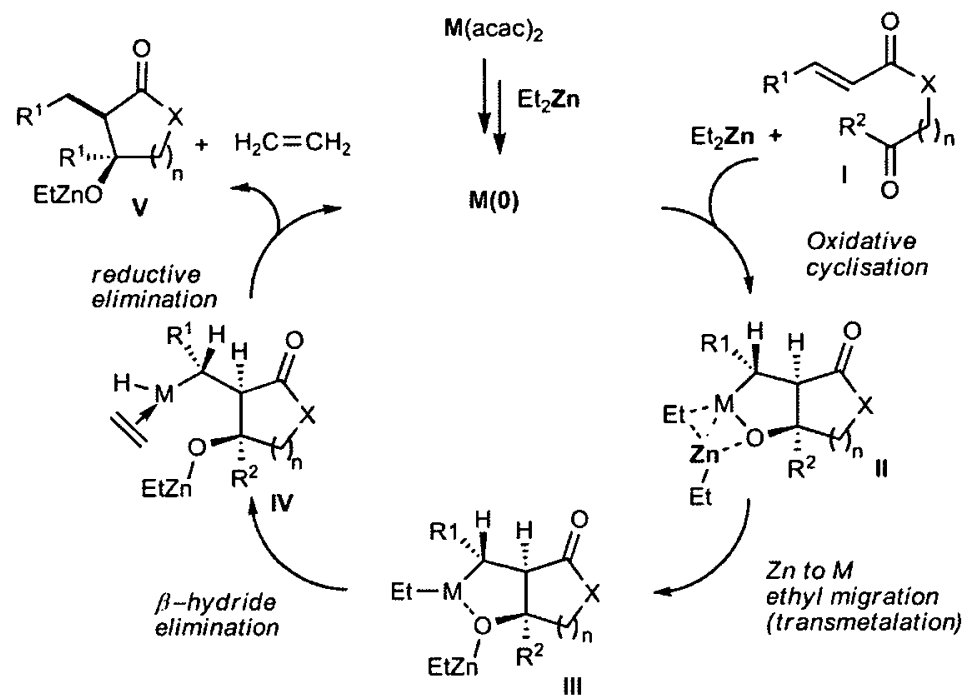

Scheme 3.32 First possible reaction mechanism: via formation of metallacycles

A second possible mechanism involving the formation of discrete enolates was proposed (Scheme 3.33). Interaction of $\mathrm{M}(0)$ and $\mathrm{Et}_{2} \mathrm{Zn}$ forms intermediate $\mathbf{I}$, a threecentre-two-electron bridging interaction, product of an oxidative addition of $\mathrm{M}(0)$ with the ethyl-zinc bond. Coordination of this intermediate to substrate II would provide III, which undergoes $\beta$-hydride elimination to form metal hydride IV. Reorganization of IV provides $\mathbf{V}$ and $\mathbf{M}(0)$, that can reenter the cycle. Enolate $\mathbf{V}$ undergoes aldol cyclisation to form VII, which is protonated during workup to give the desired product. The syn-stereochemistry of the major diastereomer can be explained by preferential formation of the $Z$-zinc enolate and the chelated Zimmerman-Traxler transition state VI. 


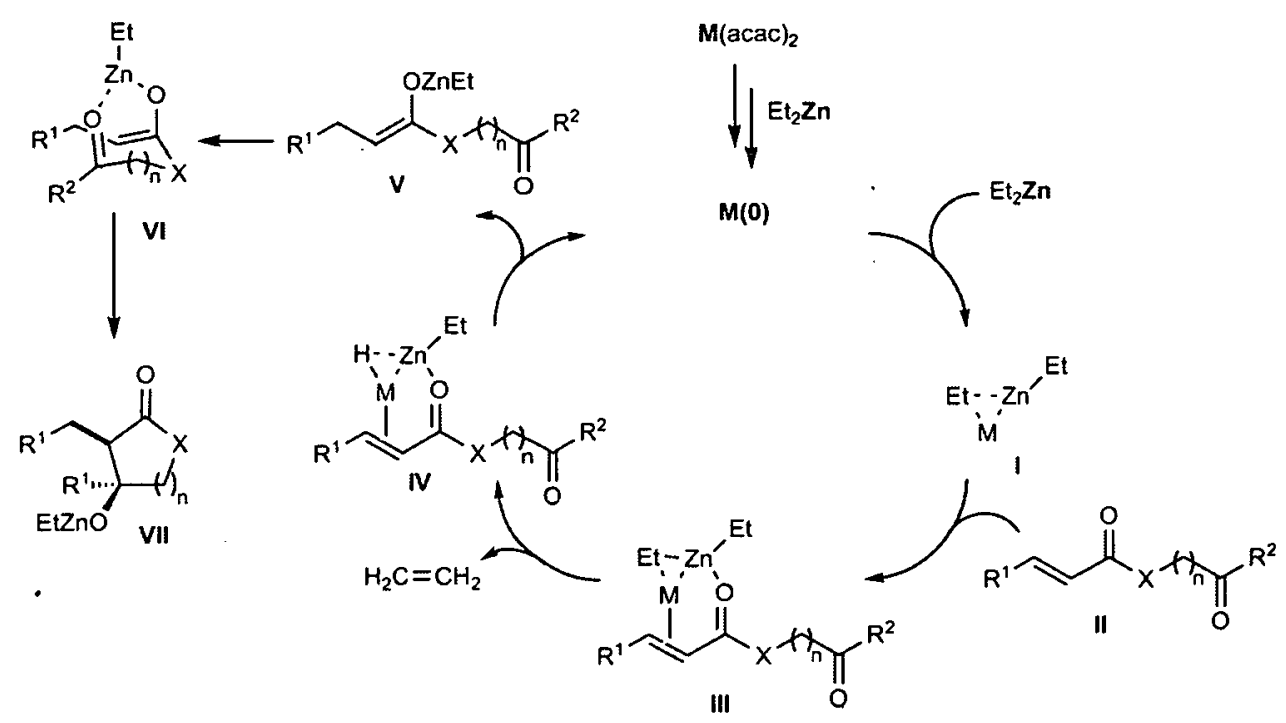

Scheme 3.33 Second possible reaction mechanism: via formation of a zinc enolate

\subsection{Retrosynthetic analysis}

The development in our group of the $\mathrm{Cu}$-, $\mathrm{Co}-$ or $\mathrm{Ni}$ - catalysed reductive aldol cyclisation of $\alpha, \beta$-unsaturated amides and esters has hugely contributed to the scope and strength of the reductive aldol methodology. ${ }^{65-67}$ Regarding the application of these methodologies to the synthesis of salinosporamide A, the copper methodology was very substrate limited, showing poor reactivity with highly functionalised $\alpha, \beta$-unsaturated amide substrates and the formation of $\gamma$-lactams was not achieved. However, the Co- and mostly the Ni-catalysed methodology had a significantly expanded substrate scope. Most importantly towards the synthesis of 1 , more examples of successful cyclisations affording 5-membered lactams were achieved (Scheme 3.28 and Scheme 3.30).

The full retrosynthetic analysis for this approach devised towards the synthesis of the natural product is outlined in Scheme 3.34. Initial disconnections on 1 lead to $\gamma$-lactam intermediate 296. The aldehyde group of 296 would arise from transformation of group $\mathrm{X}$ of $\mathbf{1 0 3}, \mathrm{X}$ representing an alcohol or an alcohol equivalent functionality. 
We envisaged that $\gamma$-lactam 103 could be synthesised from $\alpha, \beta$-unsaturated amide 102 via a reductive aldol cyclisation reaction. The objective was to find the optimal catalyst system that would allow this cyclisation with good yields and diastereoselectivities. Moreover, if the cyclisation reaction proved successful with several $\alpha, \beta$-unsaturated amides, the synthesis of derivatives of 1 would also be possible. Amide 102 could be constructed by coupling of an $\alpha, \beta$-unsaturated acid 298, having a protected hydroxyl at the $\gamma$-position, with an amine 297.

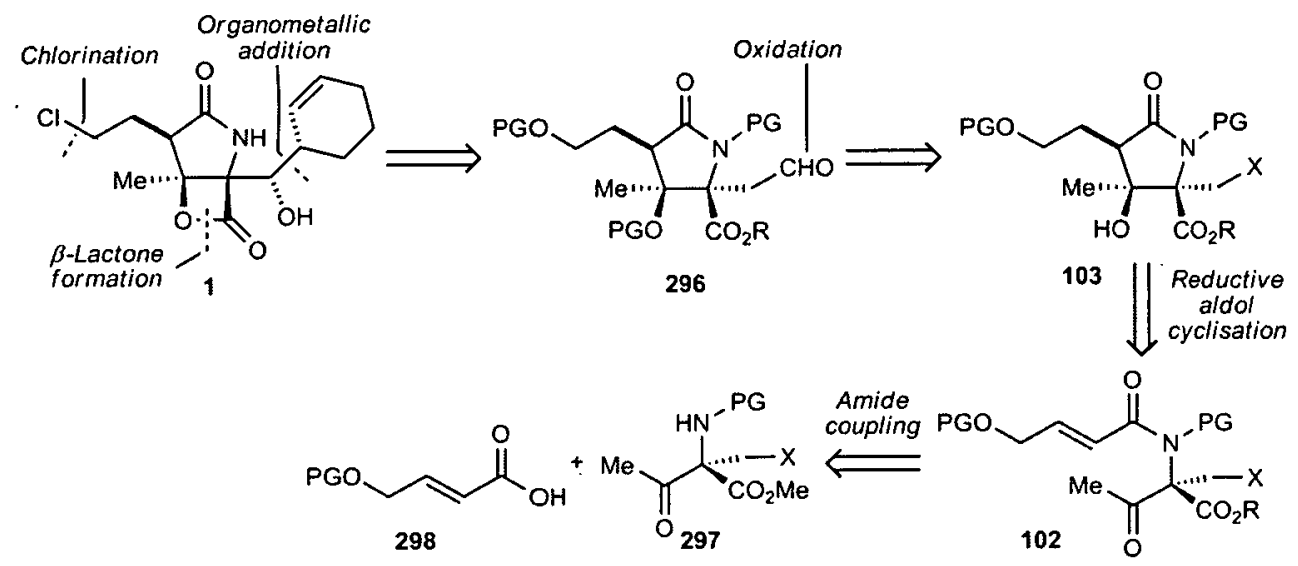

Scheme 3.34 Retrosynthetic analysis for the reductive aldol cyclisation approach

The required functionalities of $\alpha, \beta$-unsaturated amide $\mathbf{1 0 2}$ to be converted into the final target 1 were then identified. Amide 299, with a methyl ester, PMB protected amide, and a $\mathrm{CH}_{2} \mathrm{SiR}_{3}$ group, was selected as a suitable substrate for this route (Scheme 3.35). This substitution of amide 299 was also chosen in order to avoid employing similar functionality to the one employed by Corey and co-workers in their reported synthesis of $\mathbf{1}$ (Scheme 1.6). ${ }^{26} \mathrm{~A}$ synthetic route to amine 300 would be developed.

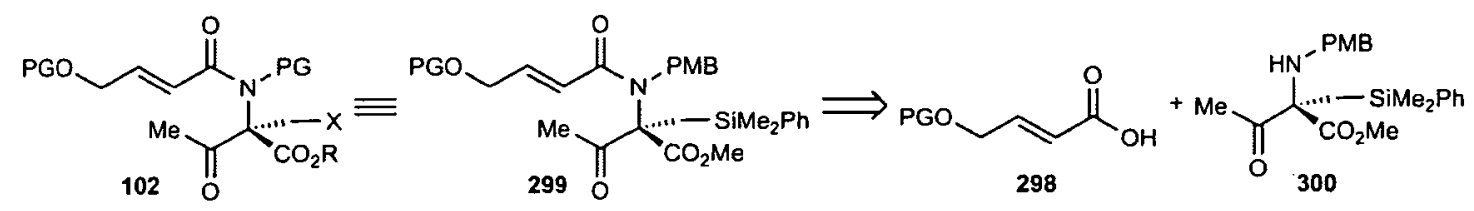

Scheme 3.35 Design of amide 102 
After cyclisation of amide 299, the silyl group would be converted into the alcohol via a Tamao-Flaming oxidation (Scheme 3.36). ${ }^{37}$ Oxidation of alcohol 302 would afford aldehyde 303 . We chose to have a PMB protecting group on the amide nitrogen because it was known that the insertion of the cyclohexenyl side chain at $\mathrm{C} 5$ following Corey's procedure ${ }^{26}$ worked best in the presence of a PMB group on that position. Ideally, it was desired to install also a PMB protecting group on the alcohol of the acid, so that deprotection of both PMB groups at a later stage could be performed simultaneously. However, the alcohol of the $\alpha, \beta$-unsaturated acid could also be protected with other groups, in order to prepare several $\alpha, \beta$-unsaturated amides and have a wider variety of substrates for the study of the reductive aldol cyclisation. If the preparation of triol $\mathbf{2 6}$ was achieved, this route would represent a formal synthesis of salinosporamide $A$, since $\mathbf{2 6}$ is a final intermediate in the synthesis of 1 reported by Corey ${ }^{26}$ and also in the one reported by Pattenden. ${ }^{29}$

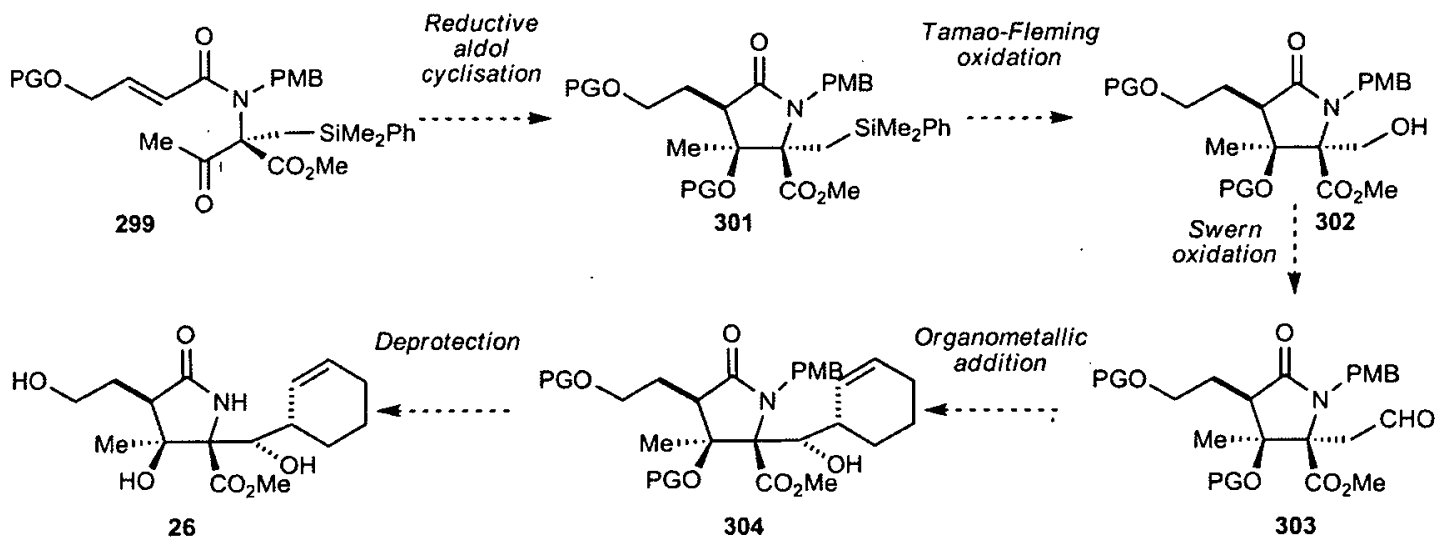

Scheme 3.36 Main key steps after the cyclisation reaction

It is worth pointing out that, with this approach, several steps to $\mathbf{2 6}$ are saved compared to Corey's synthesis. In the reported route, acrylamide $\mathbf{2 0}$ was prepared and successfully cyclised (Scheme 3.37). The $\gamma$-lactam product of the cyclisation was then silylated, affording 21. A tributyltin-mediated radical-chain cyclisation was performed on 21 to obtain cis-fused $\gamma$-lactam 22. This step introduced the ethyl chain at $\mathrm{C} 2$, setting up the desired stereochemical relationship at $\mathrm{C} 2$ and $\mathrm{C} 3$. In our route, the $\gamma$-lactam obtained in the cyclisation (301) would already contain the side chain at C2. From intermediate 22, they prepared triol $\mathbf{2 6}$ in further four steps. 


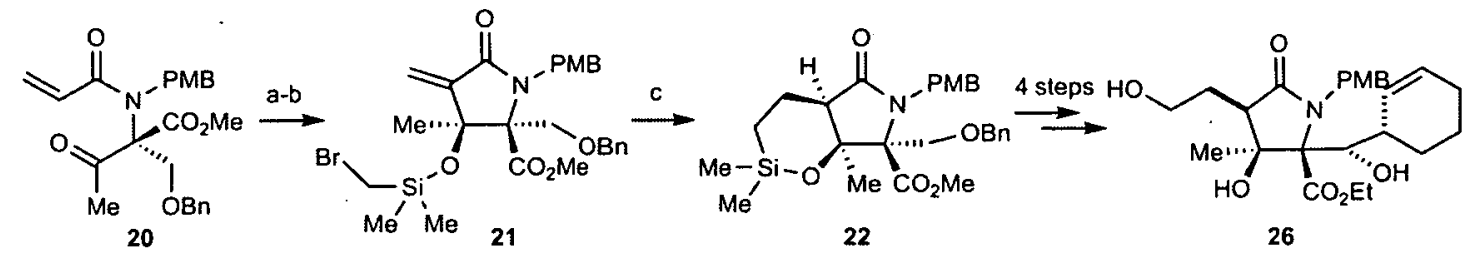

Reagents and conditions: (a) i) $\mathrm{Ti}(\mathrm{O} i \mathrm{Pr})_{4}, \mathrm{c}-\mathrm{C}_{5} \mathrm{H}_{9} \mathrm{MgCl},{ }^{\prime} \mathrm{BuOMe},-40^{\circ} \mathrm{C}, 30$ min, $\mathrm{I}_{2},-40^{\circ} \mathrm{C}, 2 \mathrm{~h}$ then $0{ }^{\circ} \mathrm{C}, 2 \mathrm{~h}$.; (ii) $\mathrm{NEt}_{3}, \mathrm{CH}_{2} \mathrm{Cl}_{2}, 30 \mathrm{~min}, 83 \%$; (b) $\mathrm{BrCH}_{2} \mathrm{Si}\left(\mathrm{CH}_{3}\right)_{2} \mathrm{Cl}, \mathrm{Et}_{3} \mathrm{~N}, \mathrm{DMAP}, \mathrm{CH}_{2} \mathrm{Cl}_{2}, 0^{\circ} \mathrm{C}, 30 \mathrm{~min}, 95 \%$; (c) $\mathrm{Bu}_{3} \mathrm{SnH}$, AIBN, Benzene, reflux, $8 \mathrm{~h}, 89 \%$.

Scheme 3.37 Extra steps for the insertion of the C2 side chain in Corey's total synthesis of 1

\subsection{Results and discussion}

Our initial efforts focused on the synthesis of amine $\mathbf{3 0 0}$ and initially a racemic synthetic route was developed. Coupling of $\mathbf{3 0 0}$ with several acids would afford a range of $\alpha, \beta$-unsaturated amides that would allow us to study the feasibility of the cyclisation reaction with highly substituted substrates. If the cyclisation proved successful, this route would lead to the synthesis of $( \pm)-1$. We would then develop an asymmetric route to amine $\mathbf{3 0 0}$ and work towards the synthesis of $\mathbf{1}$.

\subsubsection{Preparation of amine 300}

Retrosynthetic analysis of $\mathbf{3 0 0}$ leads to the unprotected amine $\mathbf{3 0 5}$ (Scheme 3.38), which might be readily prepared via hydrogenolysis of $\mathbf{3 0 6}$. This intermediate is the product of the addition of dibenzylazodicarboxylate (DBAD) into 307, which arises from the alkylation of methyl acetoacetate $\mathbf{3 0 8}$ with chloromethyldimethylphenyl silane.

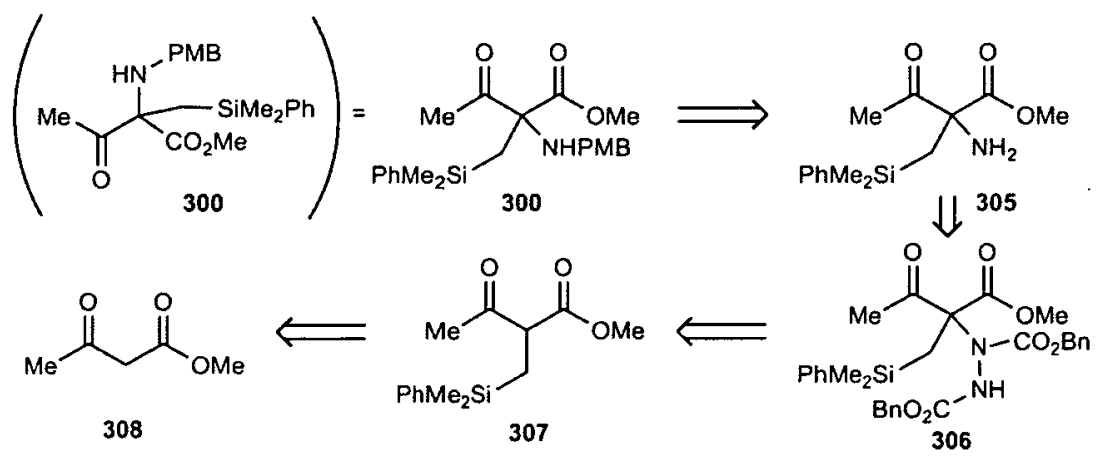

Scheme 3.38 Retrosynthetic route to amine 300 
The synthesis of amine $\mathbf{3 0 0}$ began with the alkylation of methyl acetoacetate $\mathbf{3 0 8}$ with (chloromethyl)dimethylphenyl silane. The reaction was first attempted via deprotonation of methyl acetoacetate with $\mathrm{NaH}$, followed by addition of $\mathrm{ClCH}_{2} \mathrm{SiMe}_{2} \mathrm{Ph}$. Several attempts using different solvents, such as THF, DMF or $\mathrm{Et}_{2} \mathrm{O}$, were carried out. These were conducted at a range of temperatures varying from room temperature to reflux, and for a variety of reaction times. Unfortunately, no product was observed even after heating under reflux for several days.

Formation of the desired product was only observed when an iodide source was added. Sequential addition of methyl acetoacetate and chloromethyl dimethylphenylsilane (1.1 eq) to a suspension of $\mathrm{KI}(0.6 \mathrm{eq})$ and $\mathrm{NaH}(1.1 \mathrm{eq})$ in DMF:THF (1:1) followed by heating under reflux for $14 \mathrm{~h}$ yielded the desired alkylated product in $45 \%$ yield. ${ }^{116}$ The same procedure was repeated in THF only as solvent but product 307 was not formed. When a mixture of THF with other polar solvents (acetonitrile or DMSO) was employed, traces of product were obtained but the yield could not be improved.

The number of equivalents and the source of iodide were also investigated. It was demonstrated that the optimal amount of $\mathrm{KI}$ was 0.6 equivalents. When $\mathrm{Bu}_{4} \mathrm{NI}$ was used, only traces of the desired alkylated product were formed. The use of NaI instead of KI also afforded the desired alkylated product but in slightly lower yield (40\%). The yield of the reaction could finally be improved to $65 \%$ by increasing the amount of $\mathrm{NaH}$ (1.5 equivalents) and alkylating agent (1.2 equivalents) (Scheme 3.39).

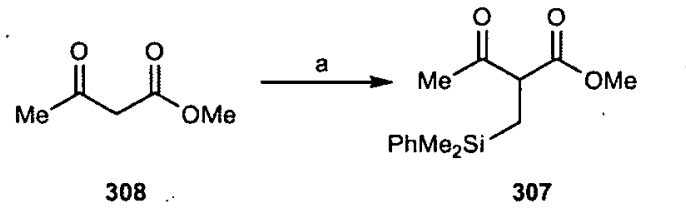

Reagents and conditions: (a) $\mathrm{NaH}$ ( $1.5 \mathrm{eq}), \mathrm{KI}(0.6 \mathrm{eq}), \mathrm{ClCH}_{2} \mathrm{SiMe}_{2} \mathrm{Ph}$ (1.2 eq), THF:DMF (1:1), reflux, 14 h, 65\%.

\section{Scheme 3.39 Alkylation of methyl acetoacetate to afford 307}

The next step involved the amination of 307. Jorgensen reported in 2003 a catalytic enantioselective $\alpha$-amination of $\alpha$-substituted $\beta$-ketoesters catalysed by a chiral copper(II)-bisoxazoline complex with azodicarboxylates as the nitrogen 
fragment source. ${ }^{117}$ With only $0.5 \mathrm{~mol} \%$ of the catalyst, the corresponding products were obtained in high yields and excellent enantioselectivities (Table 3.11).

\begin{tabular}{|c|c|c|c|c|c|c|c|c|}
\hline & \multirow[b]{2}{*}{$\mathbf{R}^{1}$} & \multirow[b]{2}{*}{$\mathbf{R}^{2}$} & \multirow[b]{2}{*}{$\mathbf{R}^{3}$} & & \multicolumn{2}{|c|}{$10 \mathrm{~mol} \%$ cat. } & \multicolumn{2}{|c|}{$0.5 \mathrm{~mol} \%$ cat. } \\
\hline & & & & & Yield (\%) & ee $(\%)$ & Yield (\%) & ee $(\%)$ \\
\hline 1 & $\mathrm{Me}$ & $\mathrm{Me}$ & Et & $312 a$ & 98 & 98 & 91 & 96 \\
\hline 2 & Et & $\mathrm{Me}$ & Et & 312b & 94 & 98 & 98 & 98 \\
\hline 3 & $\mathrm{Ph}$ & $\mathrm{Me}$ & Et & $312 c$ & 85 & 95 & 81 & 87 \\
\hline 4 & $i-\operatorname{Pr}$ & $\mathrm{Me}$ & $t$-Bu & 312d & 96 & 98 & 89 & 98 \\
\hline 5 & $\mathrm{Bn}$ & $\mathrm{Me}$ & $t-\mathrm{Bu}$ & $312 \mathrm{e}$ & 84 & 98 & 79 & 98 \\
\hline 6 & $\mathrm{Me}$ & Allyl & $t-\mathrm{Bu}$ & $312 f$ & - & - & 80 & 98 \\
\hline 7 & $\mathrm{Me}$ & $\mathrm{Me}$ & $t$-Bu & $312 \mathrm{~g}$ & 86 & 98 & - & - \\
\hline 8 & \multicolumn{2}{|c|}{$\left(\mathrm{CH}_{2}\right)_{3}$} & Et & $312 \mathrm{~h}$ & 98 & 99 & 96 & 99 \\
\hline 9 & \multicolumn{2}{|c|}{$\left(\mathrm{CH}_{2}\right)_{4}$} & Et & $312 \mathrm{i}$ & 98 & 99 & 96 & 99 \\
\hline 10 & \multicolumn{2}{|c|}{$\left(\mathrm{CH}_{2}\right)_{5}$} & Et & $312 j$ & 76 & 98 & 70 & 99 \\
\hline
\end{tabular}

The newly formed stereogenic carbon centre was proved to be of $R$ stereochemistry by X-ray crystallography of a derivative of one of the addition. products. On the basis of this result, a reaction mechanism involving the approach of dibenzyl azodicarboxylate to the $\beta$-ketoester coordinated to the catalyst was proposed (Figure 3.5).

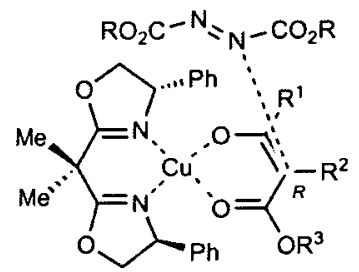

Figure 3.5 Proposed reaction mechanism 
Consequently, it was planned to apply this methodology in the synthesis of amine 300, which also had a quaternary stereocentre with $R$ stereochemistry (Scheme 3.40).

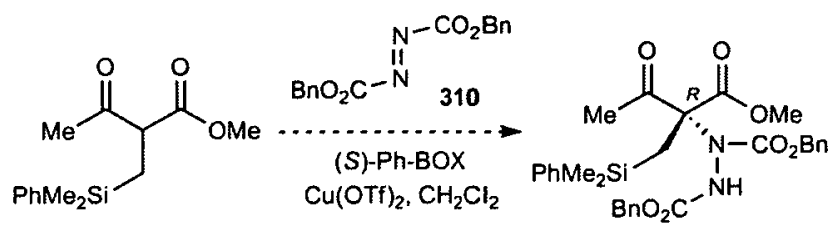

307

306

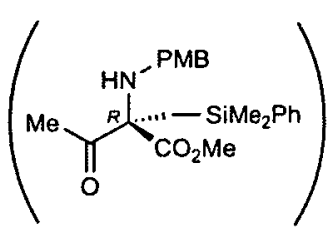

300

Scheme 3.40 Possible application of Jorgensen's methodology to our system

The need to test the feasibility of the key cyclisation step however, led us to first develop a simple and efficient route to racemic 300. Amination of $\mathbf{3 0 7}$ was carried out via a phosphine-catalysed addition of $\mathbf{3 0 7}$ to azodicarboxylates. ${ }^{118}$ We were very pleased to see that when $\beta$-keto ester $\mathbf{3 0 7}$ was reacted with a substoichiometric amount of tributylphosphine and with dibenzyl azodicarboxylate (310) in acetonitrile, the desired addition product 306 was easily prepared and isolated in $86 \%$ yield (Scheme 3.41).

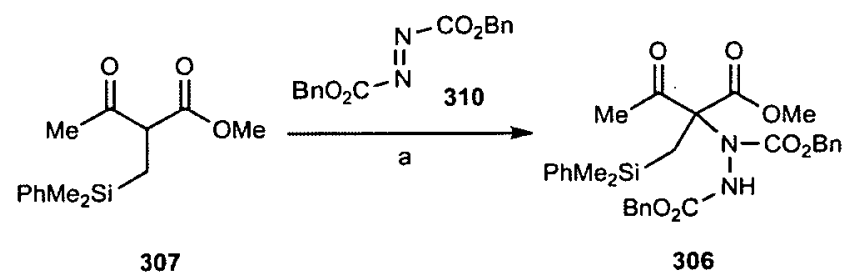

Reagents and conditions: (a) $\mathrm{PBu}_{3}(0.2 \mathrm{eq})$, DBAD (310) (1.1 eq), $\mathrm{MeCN}, \mathrm{rt}, 30 \mathrm{~min}, 86 \%$.

Scheme 3.41 Phosphine catalysed $\alpha$-amination of 307

The proposed mechanism for this reaction is outlined in Scheme 3.42. The phosphine attacks the electronically deficient double bond of the azodicarboxylate, forming a phosphonium ylide intermediate 313 that deprotonates the dicarbonyl compound 314 generating its conjugate base (316). The conjugate base can now attack the nitrogen of the phosphonium cation 315 , affording the addition product and releasing the phosphine (pathway A). Another alternative (pathway B) involves a 
cyclic mechanism with attack of the conjugate base onto the double bond of the azodicarboxylate followed by transprotonation.

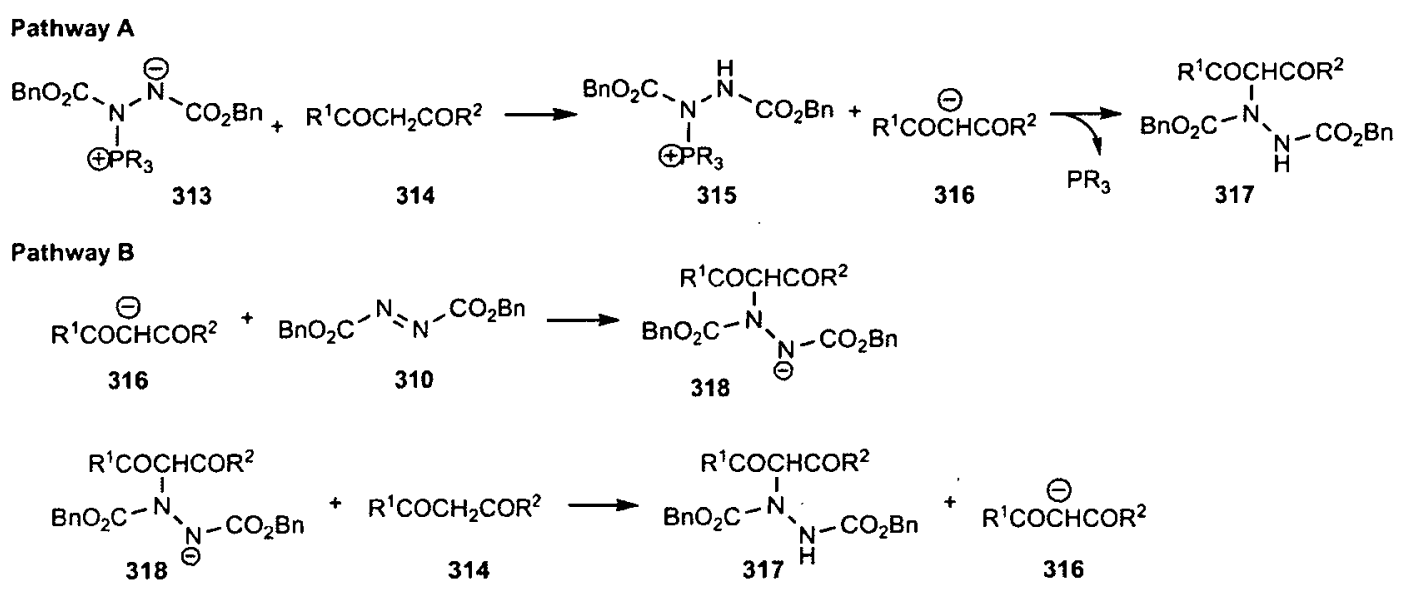

\section{Scheme 3.42 Proposed mechanism of the phosphine catalysed addition of} $\beta$-keto esters to azodicarboxylates

We next looked at the hydrogenolysis of 306 , to obtain the amine moiety. When compound 306 was treated with $\mathrm{PtO}_{2}$ or $\mathrm{Pd} / \mathrm{C}$ as catalyst under a hydrogen atmosphere, no reaction was observed. The reaction with $\mathrm{Ra}-\mathrm{Ni}$ was slow, it did not go to completion and it only afforded an inseparable mixture of products. However, the starting material was consumed when the reaction was performed in a high pressure reactor (45 bar) and using a large excess of Ra-Ni. Purification of the crude reaction mainly afforded a diastereomeric mixture of aminoalcohols 319a and 319b (approximately 2.4:1), arising from the reduction of both the $\mathrm{N}-\mathrm{N}$ bond and the ketone of 306 (Scheme 3.43). Unfortunately, the yield of this reaction was inconsistent, ranging from 50 to $70 \%$. This is a problem that would need to be addressed in the future.

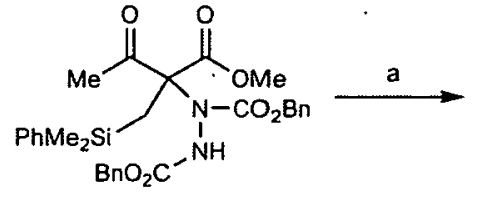

306

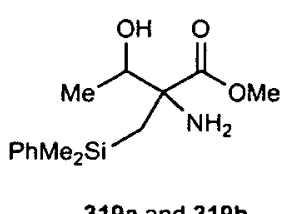

$319 a$ and $319 b$

Reagents and conditions: (a) Ra-Ni, $\mathrm{HOAc}, \mathrm{MeOH}, \mathrm{H}_{2}, 40{ }^{\circ} \mathrm{C}, 16 \mathrm{~h}, 50-70 \%$.

Scheme 3.43 Hydrogenolysis of 306 
PMB protection on the nitrogen of 319a and 319b was first attempted using $\mathrm{NaHCO}_{3}$ and $\mathrm{PMBCl}$, conditions that afforded some desired product but the yield was low and inconsistent (25-40\%). Formation of the product protected both on the oxygen and on the nitrogen was problematic. Several attempts were carried out using different bases and different number of reagent equivalents, and also using iodide additives (KI, NaI), but in none of these was the yield of the reaction significantly improved. We were pleased to find that reductive amination with $p$-anisaldehyde gave the desired product in a consistent and good yield (65-70\%) (Scheme 3.44). The reaction was performed on the mixture of both diastereomers 319a and 319b, which yielded a mixture of both diastereomers $320 \mathrm{a}$ and $320 \mathrm{~b}$. Oxidation to the aminoketone was achieved under Swern conditions. ${ }^{61}$ The yield of this oxidation was good $(>80 \%)$ when the reaction was performed on a small scale, but on scaling $(>0.75 \mathrm{mmol})$, the yield was only moderate $(60-70 \%)$.

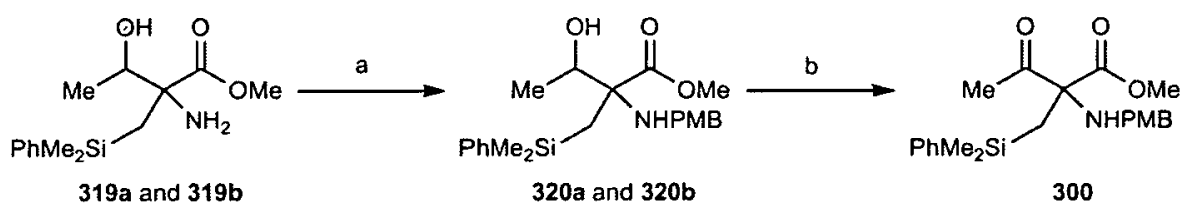

Reagents and conditions: (a) $\mathrm{NaCNBH}_{3}$ (1.5 eq), $p$-anisaldehyde (1.2 eq), HOAc (2 eq), MeOH, rt, 18 h, 65\%; (b) (COCl) 2 (2.1 eq), DMSO (4.2 eq), $\mathrm{Et}_{3} \mathrm{~N}$ (4.2 eq), $\mathrm{CH}_{2} \mathrm{Cl}_{2},-78^{\circ} \mathrm{C}, 3 \mathrm{~h}, 60-80 \%$.

\section{Scheme 3.44 Final steps of the synthesis of amine $\mathbf{3 0 0}$}

The five step route shown in Scheme 3.45 allowed us to prepare sufficient amounts of amine $\mathbf{3 0 0}$ and studies on the reductive aldol reaction could be undertaken. However, a general improvement of the synthetic route, focusing on the hydrogenolysis of $\mathbf{3 0 6}$ and on the final oxidation step, was necessary.

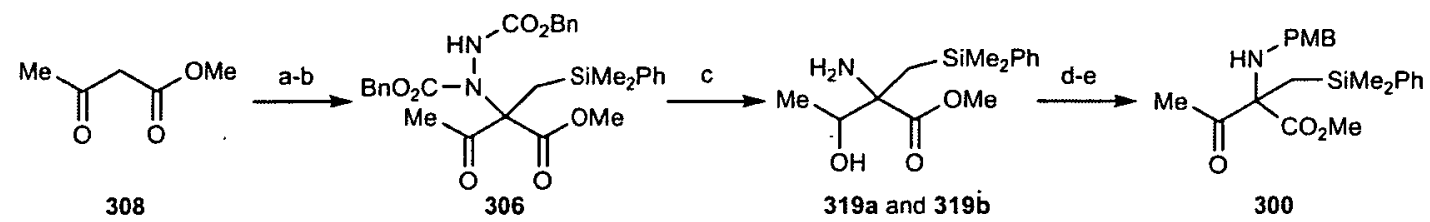

\footnotetext{
Reagents and conditions: (a) $\mathrm{NaH}, \mathrm{KI}, \mathrm{ClCH}_{2} \mathrm{SiMe}_{2} \mathrm{Ph}$, THF:DMF (1:1), reflux, 14 h, 65\%; (b) $\mathrm{PBu}_{3}$, DBAD (310), MeCN, rt, 30 min, 86\%; (c) RaNi, $\mathrm{HOAc}, \mathrm{MeOH}, \mathrm{H}_{2}, 40{ }^{\circ} \mathrm{C}, 16 \mathrm{~h}, 50-70 \%$; (d) $\mathrm{NaCNBH}_{3}$, p-anisaldehyde, $\mathrm{HOAc}$, $\mathrm{MeOH}, \mathrm{rt}, 18 \mathrm{~h}, 65 \%$; (e) $(\mathrm{COCl})_{2}, \mathrm{DMSO}, \mathrm{Et}_{3} \mathrm{~N}, \mathrm{CH}_{2} \mathrm{Cl}_{2},-78^{\circ} \mathrm{C}, 3 \mathrm{~h}, 60-80 \%$.
}

Scheme 3.45 Full synthetic route to amine 300 


\subsubsection{Studies on the amide coupling and reductive aldol cyclisation}

We next examined the preparation of the protected 4-hydroxy $\alpha, \beta$-unsaturated acid. Ideally, the 4-hydroxy group should be protected with a PMB group, so that it could be simultaneously deprotected with the PMB-protected amide nitrogen. It was envisaged that alcohol $\mathbf{3 2 2}$ could derive from ethyl trans-4-oxo-2-buteanoate 321 (Scheme 3.46). Fortunately, Luche reduction ${ }^{119}$ of 321 afforded alcohol 322 in excellent yield. PMB trichloroacetimidate 323 was next prepared ${ }^{120}$ and reacted with alcohol 322 yielding ester $\mathbf{3 2 4}$. Hydrolysis of the ester afforded acid 325 in $70 \%$ yield.

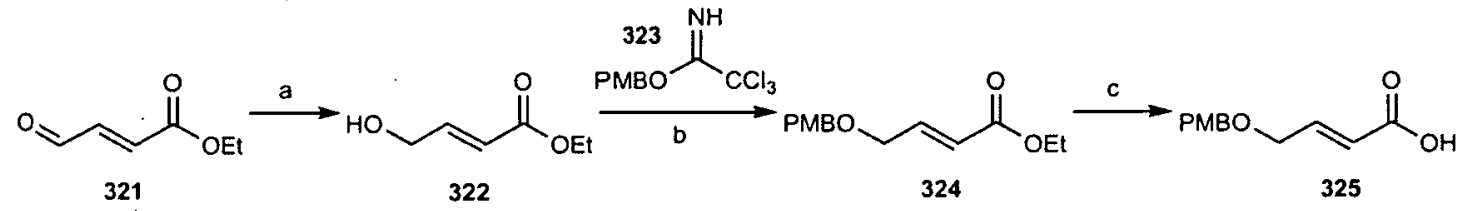

Reagents and conditions: (a) $\mathrm{NaBH}_{4}(1 \mathrm{eq}), \mathrm{CeCl}_{3}(1 \mathrm{eq}), \mathrm{EtOH}, 0{ }^{\circ} \mathrm{C}$ to $\mathrm{rt}, 14 \mathrm{~h}, 95 \%$; (b) acetimidate 323 (1.5 eq), $\mathrm{CSA}(1 \mathrm{eq}), \mathrm{CH}_{2} \mathrm{Cl}_{2}, 0^{\circ} \mathrm{C}$ to rt, 14 h, 57\%; (c) $\mathrm{NaOH}$ (1.1 eq), EtoH, rt, 14 h, 70\%.

Scheme 3.46 Preparation of PMB-protected acid 325

The formation of the amide derived from acid $\mathbf{3 2 5}$ and amine $\mathbf{3 0 0}$ was attempted mainly via two methods: 1) formation of the acid chloride followed by coupling with the amine and 2) activation of the acid with a coupling agent followed by reaction with the amine.

In the first method, the acid chloride of $\mathbf{3 2 5}$ was formed and then reacted with a solution of amine $\mathbf{3 0 0}$ in the presence of a base. Corey and co-workers have reported interesting results on the coupling reaction of structurally similar amine 327 and acid chlorides (Scheme 3.47). ${ }^{121}$ They reported a novel reaction that produced bicyclic $\beta$-lactones 328 and 329, from the reaction of acryloyl chloride 326 and amino ketone 327 with pyridine as base. However, this discovery originated from the study of the formation of the corresponding acrylamide, which proved to be unexpectedly troublesome. It was observed that the formation of the acrylamide was only achieved when highly hindered tertiary amines, like triisobutylamine or 2,6-di-tert-butylpyridine, were employed as base. Instead, in the presence of less 
hindered bases, like pyridine or triethylamine, the main products were the bicyclic $\beta$-lactones 328 and 329 (4:1 ratio). Due to the low basicity and high steric hindrance of amine 327 , the reaction rate of the coupling with the acid chloride is so slow that the side reaction that affords the $\beta$-lactones becomes dominant.

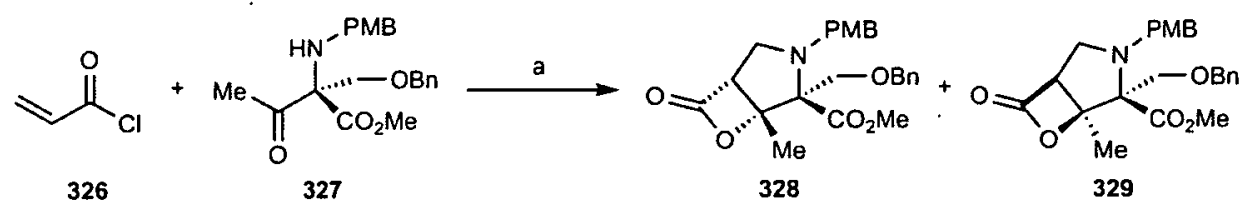

Reagents and conditions: (a) Pyridine ( $3 \mathrm{eq}), \mathrm{CH}_{2} \mathrm{Cl}_{2}, 80 \%$ (4:1).

Scheme 3.47 Formation of $\beta$-lactones 328 and 329 using a less hindered amine

It was proposed that the most likely mechanism for the $\beta$-lactone formation in the presence of pyridine involved the formation of an $\mathrm{N}$-acryloylpyridinium complex 331, which suffers an addition of the secondary amine 327 at the carbon $\beta$ to the carbonyl group instead of at the carbonyl, probably due to steric reasons. Keto ketene 332 is then formed and cyclises via an internal [2+2]-cycloaddition giving $\beta$-lactones 328 and 329 (Scheme 3.48).

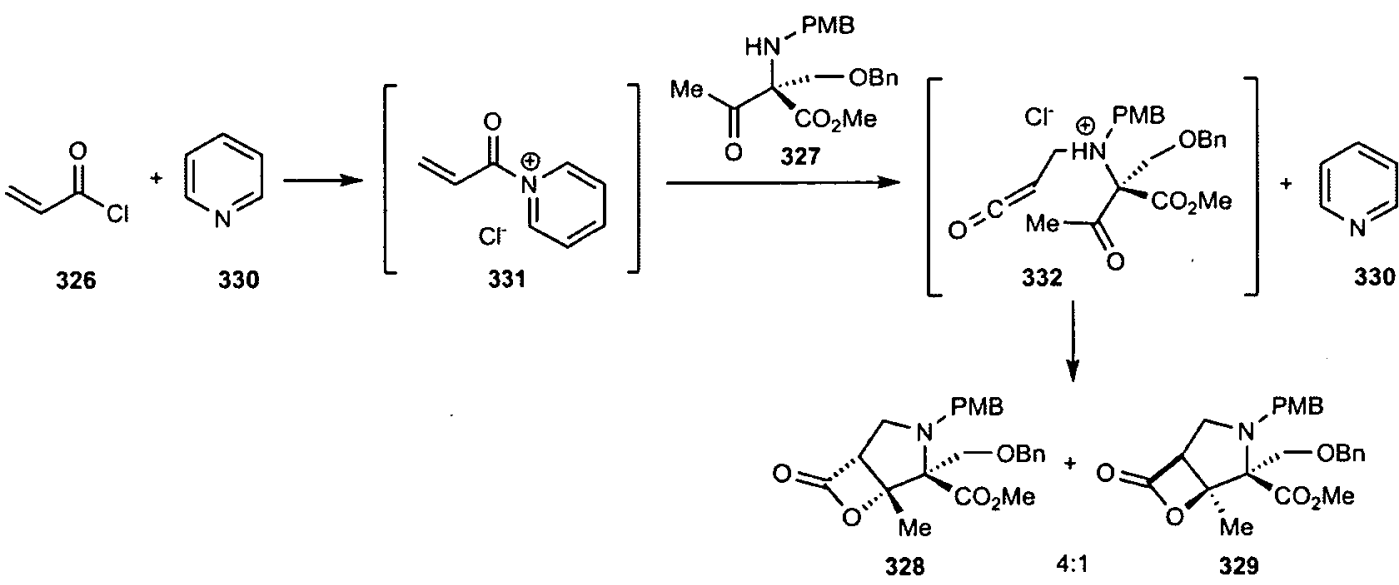

Scheme 3.48 Proposed mechanism for the formation of $\beta$-lactones 328 and 329

When more hindered bases are used, the activated $N$-acryloylammonium species is not formed and the amine is exclusively functioning as base, and $N$-acylation of amine 327 is the main reaction (Scheme 3.49). 


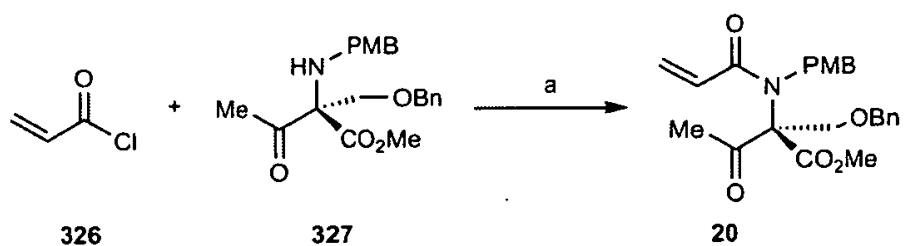

Reagents and conditions: (a) Triisobutylamine ( $3 \mathrm{eq}), \mathrm{CH}_{2} \mathrm{Cl}_{2}, 78 \%$.

Scheme 3.49 Acylation of amine 327 using hindered amines

Bearing this example in mind, we proceeded to perform the amide coupling of our substrates. Acid 325 was reacted with oxalyl chloride and a substoichiometric amount of DMF and stirred for $6 \mathrm{~h}$. The formation of the acid chloride was also carried out using thionyl chloride and allowing the reaction to stir at room temperature overnight. The solution containing the acid chloride was then concentrated in vacuo, redissolved in $\mathrm{CH}_{2} \mathrm{Cl}_{2}$ and transferred into a solution of the base and amine 300 (Scheme 3.50). This coupling reaction was initially attempted in the presence of 2,6-di-tert-butylpyridine or triisobutylamine as base, but without success. The reaction was also attempted at various temperatures, using different number of equivalents of both reagents and also using other bases $\left(\mathrm{Na}_{2} \mathrm{CO}_{3}(\mathrm{aq})\right.$ and pyridine). However, all efforts to obtain amide 334 were unsuccessful.

The main reason of the failure of this reaction was the cleavage of the PMB protecting group of the alcohol during the formation of the acid chloride of 325 , due to the acidic conditions produced in the reaction. This cleavage, combined with the low basicity and high steric hindrance of amine 300, did not allow the formation of amide 334. All reactions yielded a complex mixture of products and remaining starting amine $\mathbf{3 0 0}$, but the desired amide was not isolated.

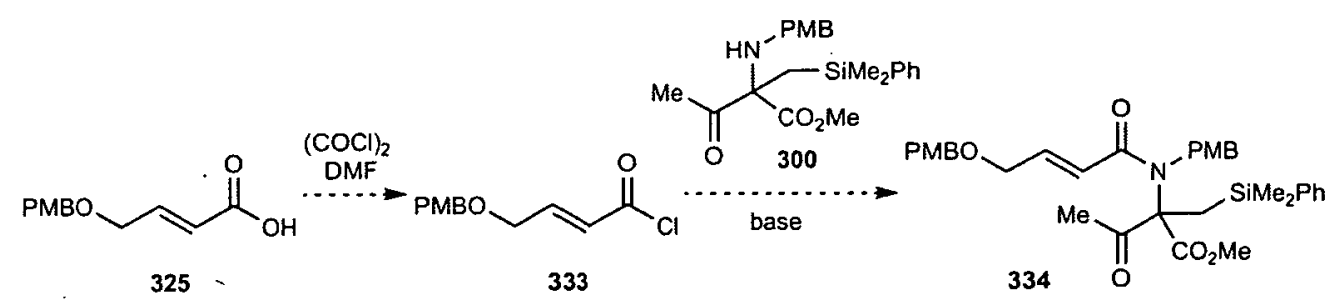

Scheme 3.50 Preparation of $\mathbf{3 3 4}$ via formation of the acid chloride of $\mathbf{3 2 5}$ 
The use of peptide coupling agents was next investigated. In a peptide coupling reaction, the carboxylic acid is typically activated by an appropriate peptide coupling reagent followed by reaction with the amine of a second amino acid. Carbodiimide coupling reagents (335-337) have been extensively used in peptide synthesis because of their moderate activity and because they are relatively inexpensive (Figure 3.6). Additives like 1-hydroxybenzotriazole (HOBt) or DMAP are successfully used to enhance the reaction rate and reduce racemisation. Another coupling reagent extensively used is the imidazolium CDI.

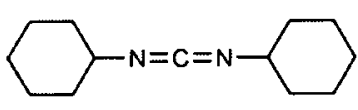

$335 \mathrm{DCC}$<smiles>On1nnc2ccccc21</smiles>

338 HOBt<smiles>CC(C)N=C=NC(C)C</smiles>

336 DIC<smiles>CN(C)c1ccncc1</smiles>

339 DMAP<smiles>CN(C)CCCN=C=NCCCl</smiles>

337 EDC

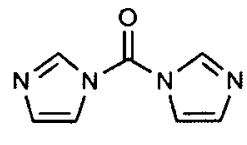

$340 \mathrm{CDI}$

Figure 3.6 Examples of coupling agents and additives

Phosphonium reagents as CloP and BroP were introduced in the early 1970s but they showed high racemisation in their reactions (Figure 3.7). After HOBt was discovered as an antiracemisation agent, a new CloP-HOBt combined reagent (BOP) was introduced. Later, reagents like PyBroP were employed, in which the dimethylamine moiety has been replaced by a pyrrolidine. These reagents avoid the formation of the toxic hexamethylphosphoramide (HMPA) in the reaction.

Uronium reagents, like HBTU or TBTU, are also extensively used in peptide chemistry (Figure 3.7). Tetrafluoroborate or hexafluorophosphate anions are generally the non-nucleophilic counteranion used in these reagents. It has been reported that the active species of these reagents is the $N$-guanidium rather than the $O$-uronium salt. 


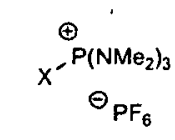

$341 \mathrm{X}=\mathrm{Cl}$ CloP $\begin{array}{lll}341 & X=C l & \text { CloP } \\ 342 & X=B r & \text { BroP }\end{array}$

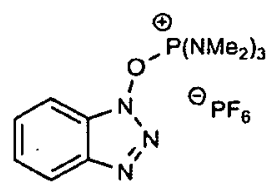

343 BOP

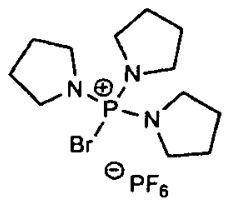

344 PyBroP

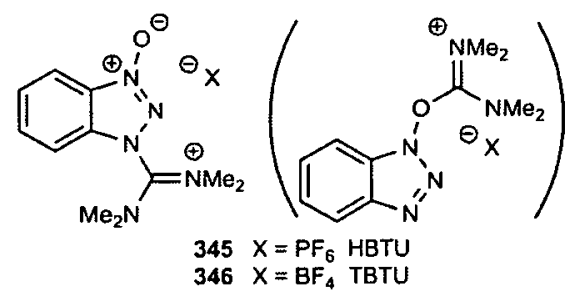

Figure 3.7 Phosphonium and uranium coupling reagents

We carried out different attempts using several coupling reagents, but all of them met with failure and no pure products could be isolated from the reactions. Table 3.12 summarises the different conditions employed for this reaction.

Attempts using DCC or EDC were performed in the presence of additives HOBt or DMAP (entries 1-4). These reactions were carried out in several solvents, under different temperatures, using different number of equivalents of the reagents and different reaction times. Even if under some conditions degradation of the starting material occurred, in every case unreacted amine $\mathbf{3 0 0}$ was recovered. An attempt was made using $\mathrm{CDI}$ in $\mathrm{CH}_{2} \mathrm{Cl}_{2}$ as solvent (entry 5), but no reaction was observed. Similarly disappointing results were obtained when HBTU, PyBrop or BOP was used in the presence of diisopropylamine (entries 6-9).

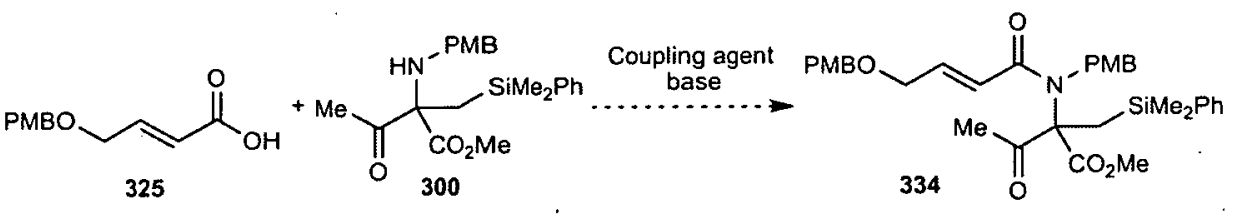

\begin{tabular}{ccc}
\hline & Coupling agent & Solvent \\
\hline 1 & DCC, DMAP & Tol \\
2 & DCC, HOBt & $\mathrm{CH}_{3} \mathrm{CN}$ \\
3 & EDC, DMAP & $\mathrm{CH}_{2} \mathrm{Cl}_{2}$ or DMF \\
4 & EDC, HOBt & $\mathrm{CH}_{2} \mathrm{Cl}_{2}$ or DMF \\
5 & CDI & $\mathrm{CH}_{2} \mathrm{Cl}_{2}$ \\
6 & HBTU, DIPEA & $\mathrm{DMF}^{2}$ \\
7 & HBTU, HOBt, DIPEA & $\mathrm{DMF}^{-}$ \\
8 & PyBrop, DIPEA & $\mathrm{CH}_{2} \mathrm{Cl}_{2}$ \\
9 & BOP, DIPEA & $\mathrm{CH}_{2} \mathrm{Cl}_{2}$ \\
\hline
\end{tabular}

Table 3.12 Attempts for the formation of amide 334 using coupling agents 
In order to confirm that amine $\mathbf{3 0 0}$ is nucleophilic enough to couple with acid chlorides, reactions were repeated using commercially available acid chlorides. Amine 300 was successfully reacted with acroloyl or crotonoyl chloride $(3 \mathrm{eq})$ in the presence of triisobutylamine, ${ }^{121}$ yielding amides 347 and 349, respectively, in good yields (Scheme 3.51). This suggests that decomposition of the acid was the main factor impeding the formation of amide 334.
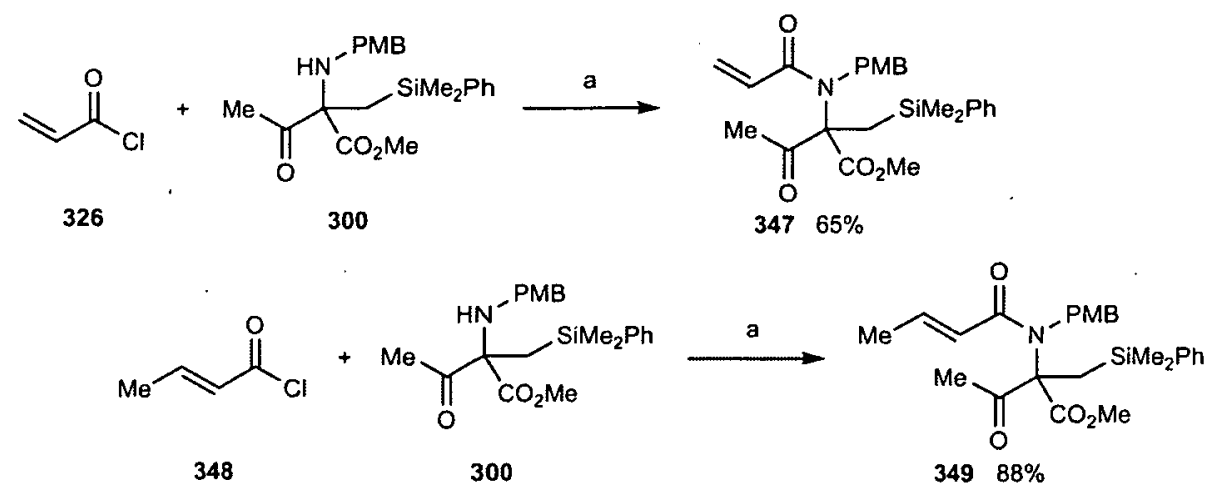

Reagents and conditions: (a) Triisobutylamine ( $3 \mathrm{eq}), \mathrm{CH}_{2} \mathrm{Cl}_{2}, 0^{\circ} \mathrm{C}$ to $\mathrm{rt}, 14 \mathrm{~h}$.

Scheme 3.51 Succesful coupling of amine $\mathbf{3 0 0}$ with commercially available acid chlorides

Consequently, we decided to prepare another $\alpha, \beta$-unsaturated acid, this time with a less acid-labile protecting group on the alcohol. We chose to use a benzyl protecting group, which is more stable under acidic conditions and should not cleave during the acid chloride formation.

Protection of alcohol 322 with a benzyl group was performed using the same method employed in the PMB protection. Benzyl trichloroacetimidate 350 was easily prepared and it was then reacted with alcohol 322 in the presence of an acid (Scheme 3.52). The first attempt was performed employing CSA but, although some desired product was formed, the reaction afforded a complex mixture of products and it did not go to completion, even when a large excess of acid was used. The use of triflic acid in a similar benzyl protection had been reported. ${ }^{122}$ We were pleased to see that under these conditions, a fast and clean reaction afforded the desired ester $\mathbf{3 5 1}$ in moderate yield $(60 \%$ to $70 \%)$. Hydrolysis of the ester using $\mathrm{LiOH}$ in $\mathrm{H}_{2} \mathrm{O} / \mathrm{THF}^{123}$ gave access to the desired protected acid 352 in $85 \%$ yield after purification (Scheme 3.52). 


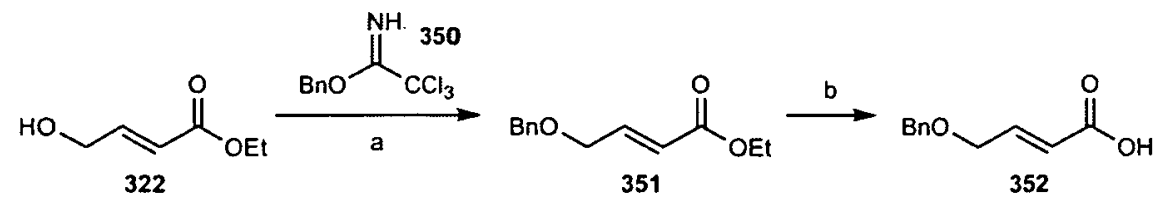

Reagents and conditions: (a) Benzyl acetimidate 350 ( $1.5 \mathrm{eq})$, TfOH ( 0.1 eq), $\mathrm{CH}_{2} \mathrm{Cl}_{2}, \mathrm{rt}, 24 \mathrm{~h}, 60-70 \%$, (b) LiOH (2 eq), $\mathrm{H}_{2} \mathrm{O} / \mathrm{THF}, \mathrm{rt}, 18 \mathrm{~h}, 85 \%$.

\section{Scheme 3.52 Synthesis of benzyl protected acid 352}

The coupling of amine $\mathbf{3 0 0}$ with acid $\mathbf{3 5 2}$ was next attempted. After some experimentation, it was found that 4.5 equivalents of acid chloride were necessary in the reaction. Therefore, 4.5 equivalents of acid $\mathbf{3 5 2}$ were reacted with oxalyl chloride for $18 \mathrm{~h}$ and this solution was concentrated, redissolved in $\mathrm{CH}_{2} \mathrm{Cl}_{2}$ and then added to a solution of amine 300 and triisobutylamine in $\mathrm{CH}_{2} \mathrm{Cl}_{2}$. Amide 354 was obtained in $60 \%$ yield, after tedious column chromatography purification (Scheme 3.53 ).

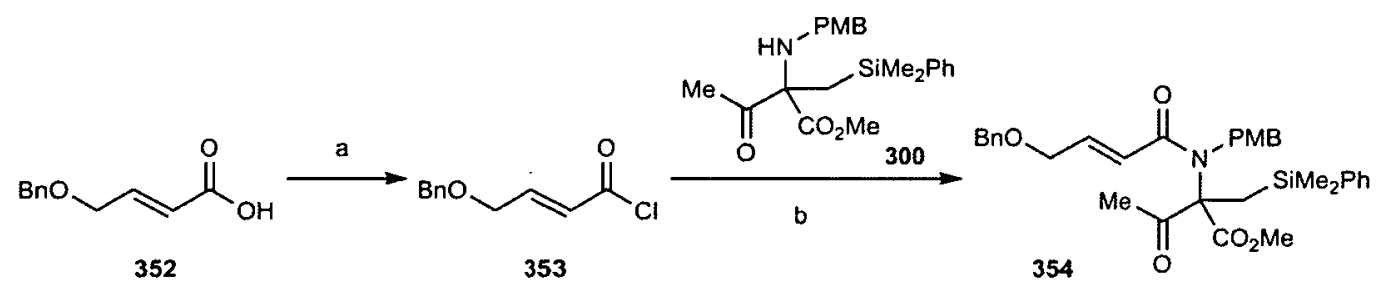

Reagents and conditions: (a) $(\mathrm{COCl})_{2}(13.5 \mathrm{eq}), \mathrm{CH}_{2} \mathrm{Cl}_{2}, 14 \mathrm{~h}$, rt; (b) amine 300, triisobutylamine ( $4.5 \mathrm{eq}$ ), $\mathrm{CH}_{2} \mathrm{Cl}_{2}, 0^{\circ} \mathrm{C}$ to reflux, $14 \mathrm{~h}, 60 \%$.

Scheme 3.53 Successful amide coupling of 300 with acid 352

We could now proceed to attempt the reductive aldol cyclisation of 354 . For these trials we would screen the most effective catalyst systems discovered within the group. Therefore, amide 354 was treated with $\mathrm{Et}_{2} \mathrm{Zn}$ as the stoichiometric reductant and either Co salts $\left(\mathrm{Co}(\mathrm{acac})_{2} \cdot 2 \mathrm{H}_{2} \mathrm{O}\right.$ or $\mathrm{CoCl}_{2}$ and $\left.\mathrm{Cy}_{2} \mathrm{PPh}\right)$ or $\mathrm{Ni}(\mathrm{acac})_{2}$ as precatalyst. Unfortunately, in all cases the reaction only afforded a complex mixture of products, together with unreacted starting material (Scheme 3.54). The analysis of the reactions proved very difficult and desired products were never isolated. The reactions were also carried out using $\mathrm{Et}_{3} \mathrm{Al}$ instead of $\mathrm{Et}_{2} \mathrm{Zn}$, but again a complex mixture of products was obtained. 


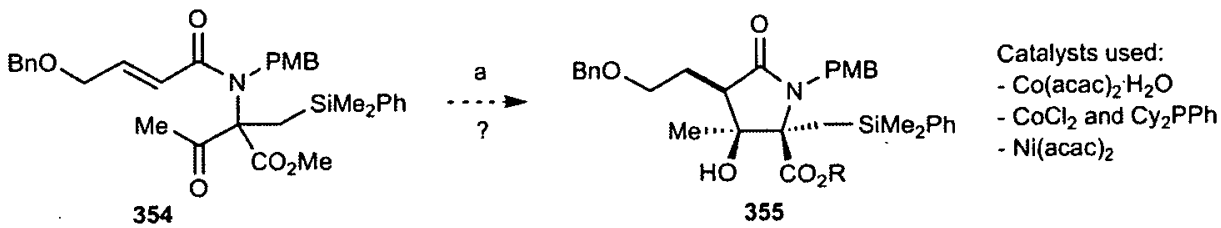

Reagents and conditions: (a) Catalyst (10 mol\%), ligand (11 mol\%), $\mathrm{Et}_{2} \mathrm{Zn}$ or $\mathrm{Et}_{3} \mathrm{Al}$ ( 2 eq), THF.

Scheme 3.54 Inconclusive cyclisation trials of amide 354

As mentioned previously, amides 347 and 349 (Scheme 3.51) were synthesised to confirm whether amine $\mathbf{3 0 0}$ was nucleophilic enough to react with an acid chloride to form an amide. These substrates do not contain the 4-hydroxy functionality in the acyl moiety of the amide required for the synthesis of 1 , but we questioned whether the cyclisation of these substrates would be possible. If these cyclisations were successful, more information about the optimal catalyst system and about the reaction conditions would be gained. Furthermore, we would learn that it was the functionalisation of the acyl part of the amide what was preventing the cyclisation of amide 354 from occurring.

Cyclisation of $\mathbf{3 4 7}$ and $\mathbf{3 4 9}$ was attempted employing the same catalyst systems as previously. These substrates were treated with $\mathrm{Et}_{2} \mathrm{Zn}$ as the stoichiometric reductant and either Co salts $\left(\mathrm{Co}(\mathrm{acac})_{2} \cdot 2 \mathrm{H}_{2} \mathrm{O}\right.$ or $\mathrm{CoCl}_{2}$ and $\left.\mathrm{Cy}_{2} \mathrm{PPh}\right)$ or $\mathrm{Ni}(\mathrm{acac})_{2}$ as precatalyst (Scheme 3.55). In all cases the reaction resulted in a complex mixture of products and the desired product was never isolated.

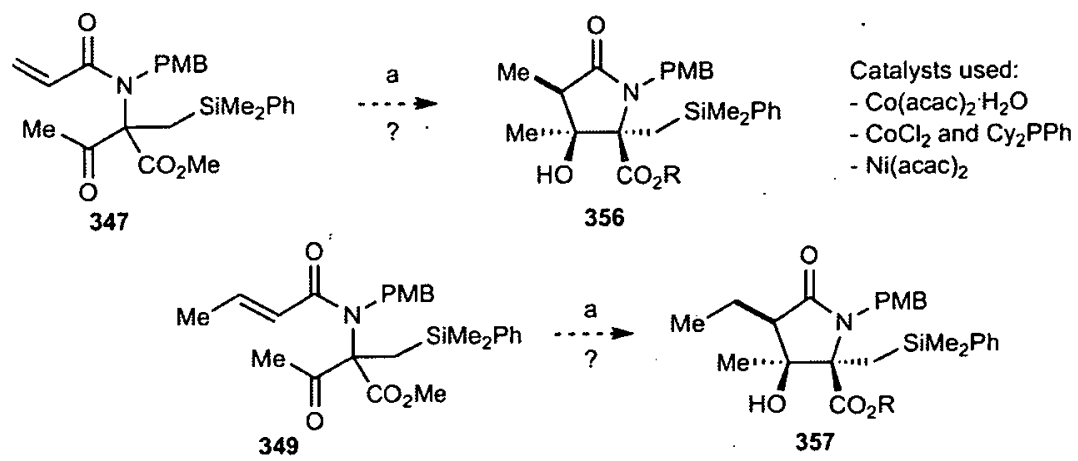

Reagents and conditions: (a) Catalyst (10 mol\%), ligand (11 mol\%), $\mathrm{Et}_{2} \mathrm{Zn}$ (2 eq), THF.

Scheme 3.55 Inconclusive attempts of cyclisation of 347 and 349 
In order to verify that the failure of the cyclisation could be attributed to the highly substituted amide, we decided to couple the simpler amine $359^{\mathrm{i}}$ with the benzyl protected acid 352 (Scheme 3.56). The formation of the acid chloride ( $2.5 \mathrm{eq}$ ) was achieved reacting acid $\mathbf{3 5 2}$ with oxalyl chloride, stirring at room temperature for $14 \mathrm{~h},{ }^{123}$ followed by the reaction with a solution of the amide and triisobutylamine in $\mathrm{CH}_{2} \mathrm{Cl}_{2}$. Amide 360 was obtained in very good yield (>94\%). The fact that the conditions needed for amide coupling with $\mathbf{3 5 9}$ were less harsh than for the coupling with amine 300 (where 4.5 equivalents of the acid chloride were needed), demonstrates the low reactivity of the latter.

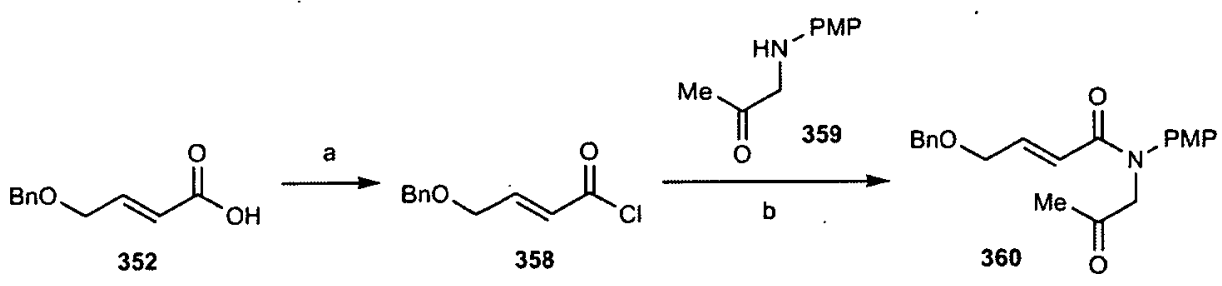

Reagents and conditions: (a) $(\mathrm{COCl})_{2}(12.5 \mathrm{eq}), \mathrm{CH}_{2} \mathrm{Cl}_{2}, 14 \mathrm{~h}$, rt; (b) amine 359, triisobutylamine ( $3 \mathrm{eq}), \mathrm{CH}_{2} \mathrm{Cl}_{2}, \mathrm{rt}, 12 \mathrm{~h}, 94 \%$.

Scheme 3.56 Preparation of amide 360

When substrate 360 was treated with $\mathrm{Et}_{2} \mathrm{Zn}$ and $\mathrm{Co}(\mathrm{acac})_{2} \cdot 2 \mathrm{H}_{2} \mathrm{O}$, some new products were formed, but most of the starting material remained unreacted. Surprisingly, exposure of 360 to $\mathrm{Et}_{2} \mathrm{Zn}$ and $\mathrm{CoCl}_{2}$ and $\mathrm{Cy}_{2} \mathrm{PPh}$ as precatalyst afforded, in a very clean reaction, two cyclised products in a total conversion of $85 \%$ and in a 2:1 ratio (Scheme 3.57). The relative stereochemistry of each product was not assigned, although due to the known syn-selectivity of the cyclisation reaction, ${ }^{67}$ we assumed that the major diastereomer would be the syn-product. The reaction using $\mathrm{Ni}(\mathrm{acac})_{2}$ also afforded the two cyclised products, but with slightly lower conversions.

i Kindly donated by Dr. G. J. Murray 


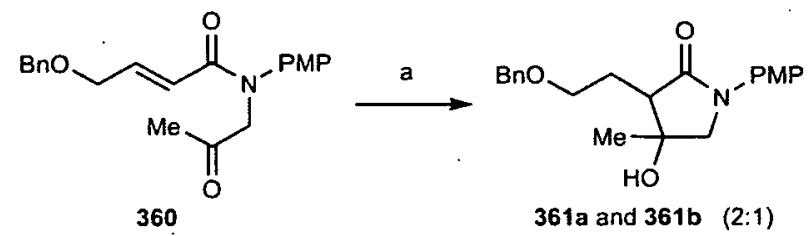

Reagents and conditions: (a) $\mathrm{CoCl}_{2}$ (10 mol\%), $\mathrm{Cy}_{2} \mathrm{PPh}$ (11 mol\%), $\mathrm{Et}_{2} \mathrm{Zn}(2 \mathrm{eq}), \mathrm{THF}, 0^{\circ} \mathrm{C}$ to rt, $18 \mathrm{~h}, 85 \%(2: 1)$.

\section{Scheme 3.57 Successful cyclisation of amide 360}

We decided to prepare yet another substrate, containing a sorbyl acyl fragment. ${ }^{124}$ A substrate with the sorbyl functionality was potentially useful since ozonolysis of the double bond followed by a reductive work-up would reveal the ethyl hydroxyl side-chain at $\mathrm{C} 2$ needed for the synthesis of $\mathbf{1}$ (Scheme 3.58).

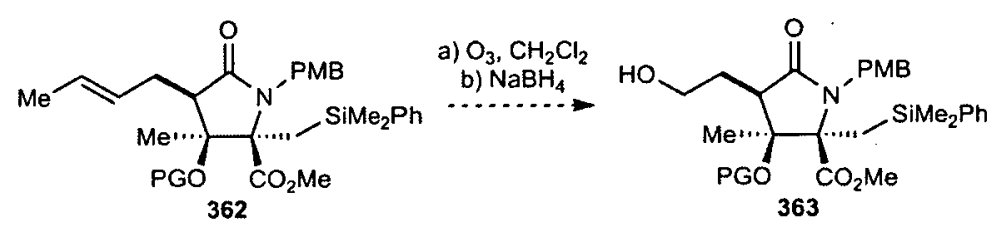

Scheme 3.58 Obtention of the ethyl hydroxyl chain at C2 via ozonolysis of 362

Therefore, sorbyl chloride was readily prepared ${ }^{125}$ and reacted in the first place with amine 359 (Scheme 3.59). The cyclisation of this simpler amide would be attempted, hoping to gain knowledge of the cyclisation reaction.

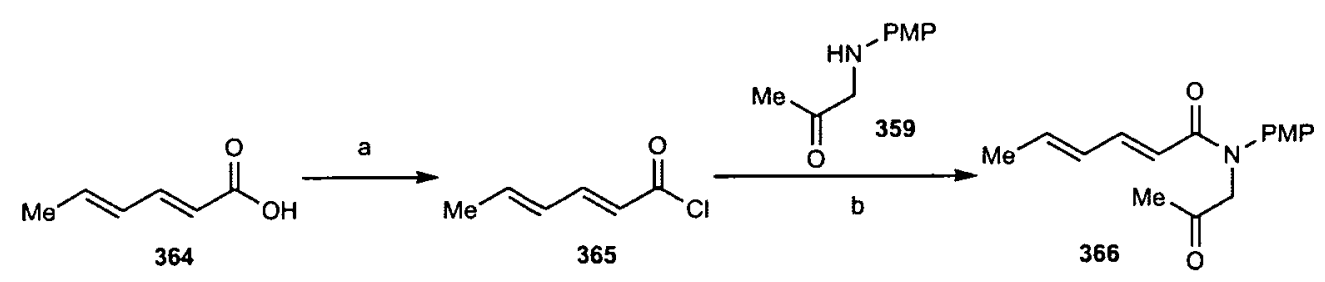

Reagents and conditions: (a) $\mathrm{SOCl}_{2}$ (2 eq), DMF (cat), $1 \mathrm{~h}$, reflux; (b) amine 359, triisobutylamine ( $3 \mathrm{eq}$ ), $\mathrm{CH}_{2} \mathrm{Cl}_{2}, \mathrm{rt}, 12 \mathrm{~h}, 62 \%$.

\section{Scheme 3.59 Preparation of sorbyl amide 366}

When substrate 366 was reacted using $\mathrm{Co}(\mathrm{acac})_{2} \cdot 2 \mathrm{H}_{2} \mathrm{O}$ and $\mathrm{Et}_{2} \mathrm{Zn}$, most of the starting material was recovered. Surprisingly, when the reaction was performed using $\mathrm{Ni}(\mathrm{acac})_{2}$ as precatalyst, the reaction went practically to completion and several 
products were obtained. The crude mixture of this reaction was purified, and the major product isolated was a cyclisation product 367 , in $20-25 \%$ yield. The relative stereochemistry of this compound was not known. Some traces of what was tentatively identified as the alternative diastereomer of $\mathbf{3 6 7}$ and also of the double bond isomer 368 were isolated. The overall conversion was approximately 50\% (Scheme 3.60). The reaction employing $\mathrm{CoCl}_{2}$ and $\mathrm{Cy}_{2} \mathrm{PPh}$ also afforded 367 and the other isomers in a very similar reaction to the one with $\mathrm{Ni}(\mathrm{acac})_{2}$.

Although the cyclisation of amide $\mathbf{3 6 6}$ had only afforded small amounts of cyclised product, this example demonstrated again that the cyclisation could take place with amides that were not prepared from amine $\mathbf{3 0 0 .}$

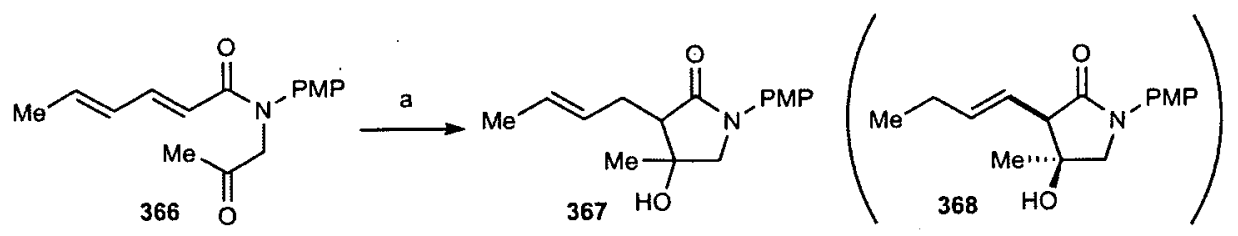

Reagents and conditions: (a) $\mathrm{Ni}(\mathrm{acac})_{2}, \mathrm{Et}_{2} \mathrm{Zn}, \mathrm{THF}, 0^{\circ} \mathrm{C}$ to $\mathrm{rt}, 18 \mathrm{~h}, 25 \%$.

\section{Scheme 3.60 Cyclisation of 366}

We then moved on to prepare amide 369 and attempt the cyclisation reaction. Sorbyl chloride was prepared and reacted with amine 300, employing the same procedure as for the coupling of amide 366 . Unfortunately, although amide 369 was formed, the complete purification was not successfully achieved and therefore we were not able to use this substrate to study the cyclisation reaction (Scheme 3.61).

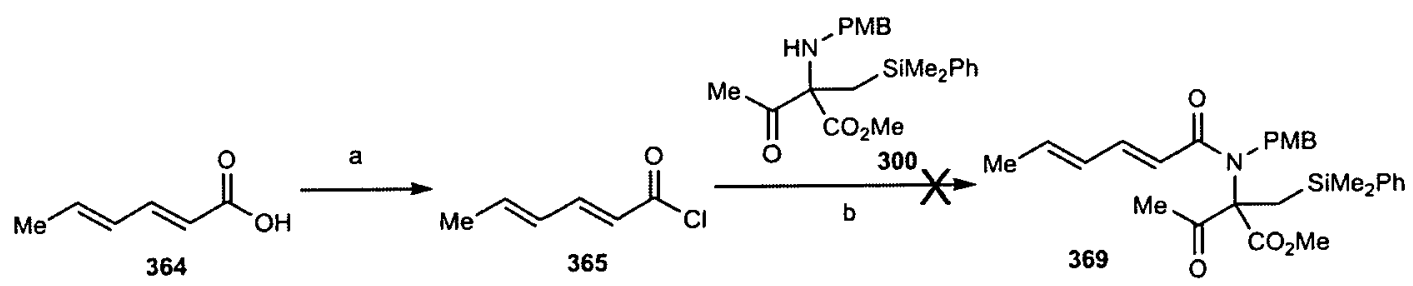

Reagents and conditions: (a) $\operatorname{SOCl}_{2}$ (2 eq), DMF (cat), $1 \mathrm{~h}$, reflux; (b) amine 300, triisobutylamine ( $3 \mathrm{eq}$ ), $\mathrm{CH}_{2} \mathrm{Cl}_{2}, \mathrm{rt}, 12 \mathrm{~h}$.

Scheme 3.61 The coupling of amine 300 with sorbyl chloride was not successful 


\subsection{Conclusions}

Due to the successful advances in the development of the reductive aldol cyclisation reaction within the group, especially some encouraging results in the synthesis of five-membered lactams, we decided to use this approach in the synthesis of salinosporamide $\mathrm{A}$.

Amide 354, containing the required substitution to be transformed into 1 , was prepared and employed in several attempts of the cyclisation reaction. Unfortunately, in all cases only a complex mixture of products, together with unreacted starting material, was obtained and the isolation of cyclisation products proved impossible. Simpler amides 360 and 366 afforded cyclised products but all cyclisation attempts of highly substituted amides 354, 347 and 349 were unsuccessful.

The failure of these cyclisations was attributed to the dense functionalisation of these substrates. Other side reactions, like conjugate reduction or reduction of the ketone, could be taking place, instead of the cyclisation reaction. Moreover, if some substrate had cyclised and the reaction was not diastereoselective, up to four cyclised products could be formed in similar ratios. All these factors could explain the formation of a complex mixture of compounds, from which the isolation of cyclisation products was not possible.

Disappointingly, due to these negative results the use of amine 300 was abandoned and a new approach towards the synthesis of the natural product was investigated. 


\section{Reformatsky reaction approach in the synthesis of salinoporamide A}

The following chapter will concentrate on the route we adopted next. On this occasion we envisaged that the $\gamma$-lactam of 1 could be constructed via an intramolecular Reformatsky reaction. It was believed that the cyclisation could be achieved with high yield and high stereoselectivity.

\subsection{Retrosynthetic analysis}

The retrosynthetic plan adopted for this approach is outlined in Scheme 4.1. As described previously, initial disconnections of 1 lead to a $\gamma$-lactam intermediate represented by 370 (Scheme 4.1). We envisaged that this $\gamma$-lactam could be constructed from an $\alpha$-halo amide such as 371, via an intramolecular Reformatsky reaction.

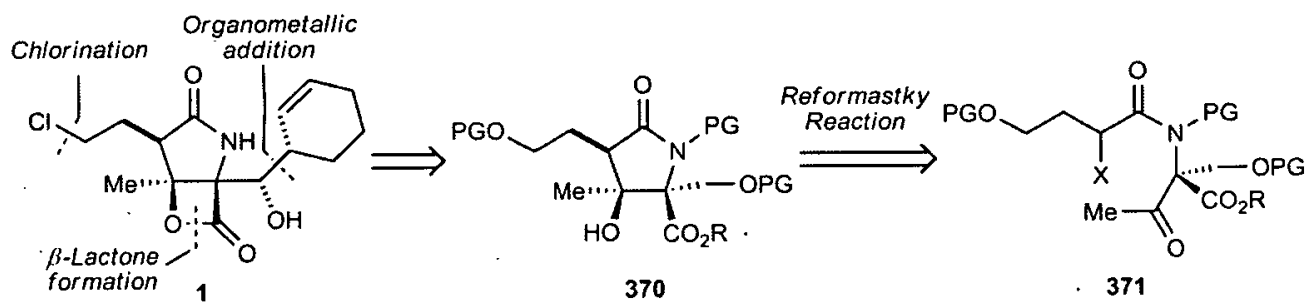

Scheme 4.1 Retrosynthetic analysis using the Reformastky reaction approach

Amide 371 could be readily prepared from the coupling of an $\alpha$-halo acid $\mathbf{3 7 3}$ with the corresponding amine (Scheme 4.2). For this route, it was decided not to employ amine 300, due to the unsuccessful results obtained when it was used in the reductive aldol approach (Chapter 3). It was decided to use amine 327 (Scheme 4.2), which had been utilised by Corey and co-workers in their synthesis of. $1{ }^{26}$ Amine 327 had proved successful in inducing diastereoselectivity in the cyclisation performed in Corey's synthesis. It was hoped that the quaternary stereocentre would also result in some internal asymmetric induction in the Reformatsky cyclisation of amide 372. Moreover, amine 327 could be prepared following reported methods and 
since it was prepared from $(L)$-threonine, we would be already working on an asymmetric route to 1 .
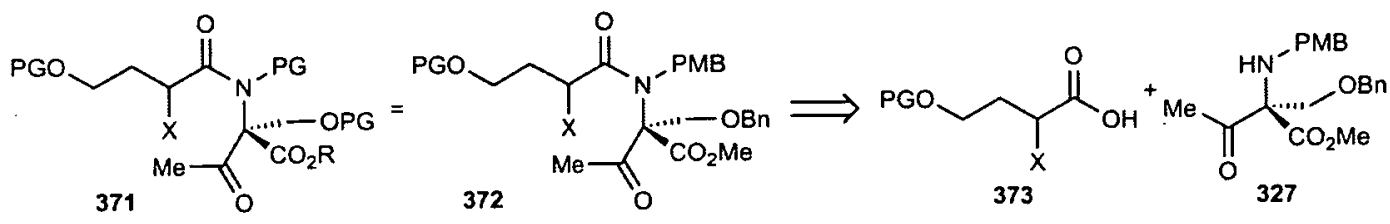

Scheme 4.2 Retrosynthetic analysis of $\alpha$-halo amide 372

Amide 372 contains all the required functionalities for the synthesis of salinosporamide A. If $\mathbf{3 7 2}$ was successfully cyclised to afford $\gamma$-lactam 374 (Scheme 4.3), only a few operations would be necessary (transformation of the protected alcohol into the aldehyde, insertion of the cyclohexenyl chain, protecting group manipulations), to yield triol 26. This intermediate is the same compound we were aiming to obtain via the reductive aldol approach (Chapter 3, Scheme 3.36), which is an intermediate in Corey's ${ }^{26}$ and also in Pattenden's ${ }^{29}$ reported syntheses of 1.

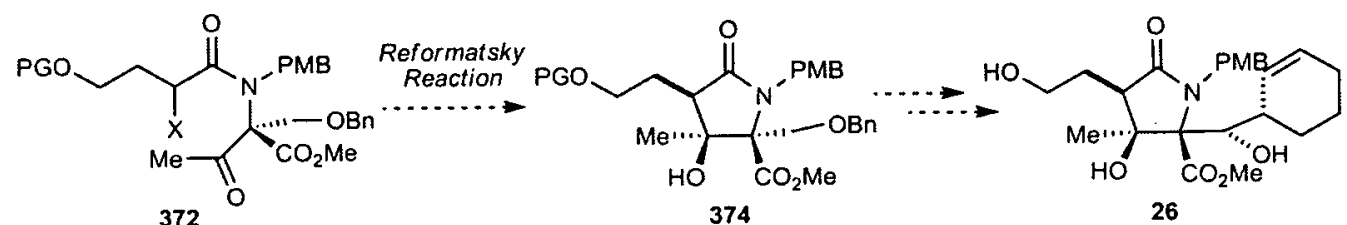

Scheme 4.3 Application of the Reformatsky reaction in the cyclisation to $\gamma$ lactam 374 and transformation to intermediate 26

If the proposed route to intermediate $\mathbf{2 6}$ was successful, we would have developed a concise and effective formal synthesis of salinosporamide A. The challenge was to find the optimal conditions for the Reformatsky cyclisation of amide 372 to obtain $\beta$-hydroxy $\gamma$-lactam 374. In the next section, we will briefly review the Reformatsky reaction focusing on methodologies that have been successfully employed in intramolecular systems. 


\subsection{The Reformatsky reaction}

The classical Reformatsky reaction, as it was reported for the first time in $1887,{ }^{126}$ consisted of a zinc-induced formation of $\beta$-hydroxy alkanoates from ethyl haloacetates and aldehydes or ketones (Scheme 4.4). However, since then the reaction has been found to be applicable to other alkyl 2-haloalkanoates, the scope of the reaction has also been extended beyond aldehydes and ketones as electrophiles and, moreover, other metals and catalysts have been found to promote the reaction in a similar way to zinc. ${ }^{127-129}$ Therefore, a more general definition for the Reformatsky reaction was needed and it is now formulated as "all reactions resulting from metal insertions into carbon-halogen bonds activated by carbonyl or carbonyl-derived groups in vicinal or vinylogous positions with practically all kinds of electrophiles". 127

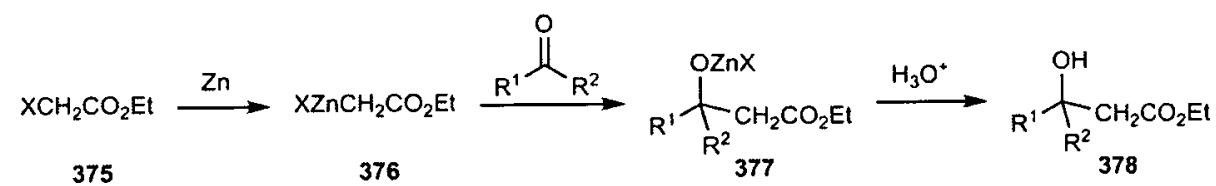

Scheme 4.4 Classical Reformatsky reaction

The Reformatsky reaction has become a very important carbon-carbon bond formation method in organic synthesis. In the field of enolate chemistry, it represents. an alternative to base-induced aldol reactions. A separate treatment is due mainly to the specific mode of metal enolate formation from halo carbonyl compounds, with the site of the reaction being strictly determined by the halogen substituent. This may be advantageous with polycarbonyl compounds, for which a regioselective enolate formation is difficult to achieve via proton abstraction with a base.

The main disadvantage attributed to the Reformatsky reaction has been the lower yields and diastereoselectivities associated with it, compared to those of the aldol reaction. However, great improvements have been made in this area and there are now a great number of examples of highly diastereo- and enantioselective Reformatsky reactions. Therefore, it has become a perfectly suitable reaction for the synthesis of complex target molecules. 
Several zinc-activation methods have been developed. ${ }^{127}$ Activation is achieved either via effective removal of the zinc oxide layer from the metal surface or via a fine distribution of the metal, most efficiently accomplished by reduction of anhydrous zinc halides (e.g., Rieke $z^{2}$ c $^{130}$ is obtained from the reduction of anhydrous zinc chloride by potassium). The most common depassivating procedures employ reagents such as iodine, 1,2-dibromoethane, copper(I) halides, chlorotrimethylsilane or simple acid washing of the zinc.

Apart from the different available methods for zinc activation, there are numerous examples of additives or inductors in the Reformatsky reaction. Yamamoto and co-workers, in 1977, reported an aldol reaction of $\alpha$-haloketones or $\alpha$-haloesters with dialkylaluminium chloride and zinc. ${ }^{131}$ An aluminium enolate was generated regiospecifically and then it was added to the carbonyl compounds affording the $\beta$-hydroxy carbonyl compounds after workup (Scheme 4.5).

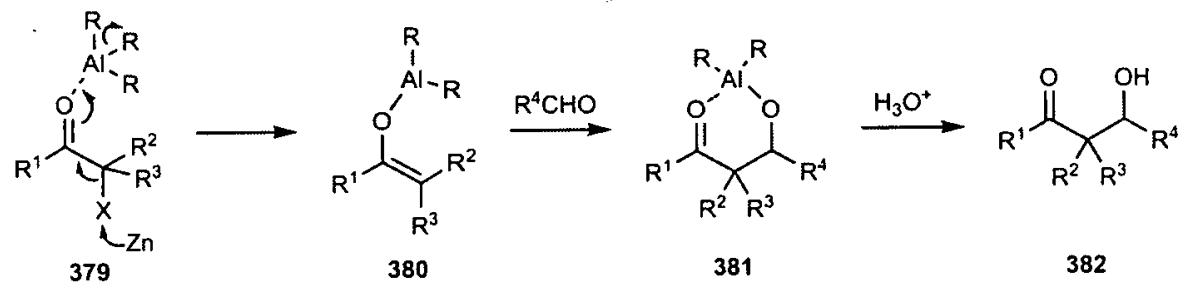

Scheme 4.5 Yamamoto dialkylaluminium chloride mediated Reformatsky reaction

This methodology was. employed in an intramolecular cyclisation of $\mathbf{3 8 3}$ which afforded diastereomer 384 as the major product (Scheme 4.6). ${ }^{132}$

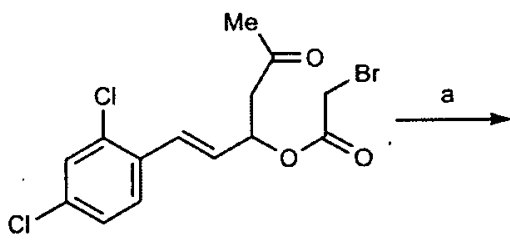

383

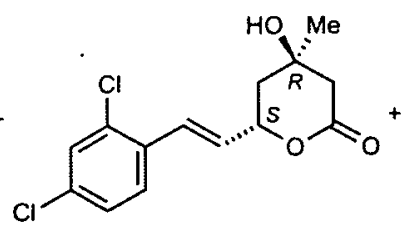

384<smiles>C[C@]1(O)CC(=O)O[C@@H](/C=C/c2ccc(Cl)cc2Cl)C1</smiles>

385

Reagents and conditions: (a) $\mathrm{Et}_{2} \mathrm{AlCl}, \mathrm{Zn}, \mathrm{THF}, 5 \mathrm{~h}, \mathrm{rt}, 50 \%$.

Scheme 4.6 Intramolecular example of Yamamoto's methodology

More recently, this procedure was applied to the synthesis of the BCD framework of richardianidins (Scheme 4.7). ${ }^{133}$ Treatment of 386 with diethylaluminium chloride 
and zinc in the presence of a substoichiometric amount of $\mathrm{CuBr}_{2} \cdot \mathrm{SMe}_{2}$ as zinc activator and at room temperature, afforded a diastereomeric mixture of syn-hydroxyester products 387 and 388 (1:20), due to the aluminium Z-enolate formation.

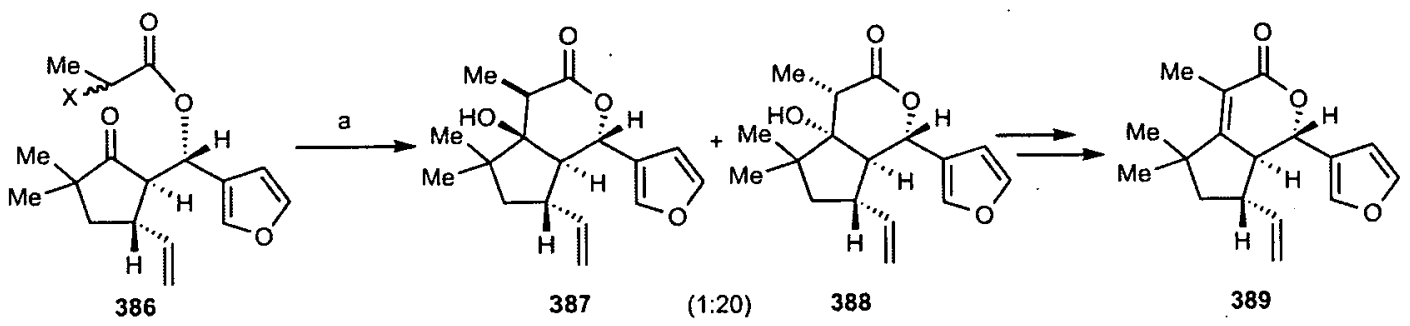

Reagents and conditions: (a) $\mathrm{Et}_{2} \mathrm{AlCl}, \mathrm{Zn}, \mathrm{CuBr}_{2} \cdot \mathrm{SMe}_{2}, \mathrm{THF}, 15 \mathrm{~h}, 57 \%$.

Scheme 4.7 Synthesis of richardianidin 389

Another relevant example of a zinc-mediated intramolecular Reformatsky reaction was reported by Heathcock and co-workers. During studies towards the synthesis of alkaloid daphnilactone A (393), ${ }^{134}$ they developed a one-pot process that involved treatment of keto amide 390 with activated zinc in THF at $0{ }^{\circ} \mathrm{C}$. This was followed by treatment with HMPA, affording lactam ether 392 in 73\% yield (Scheme 4.8). This example was highly relevant, since the structure and cyclisation of $\alpha$-halo amide 390 significantly resembles our system.

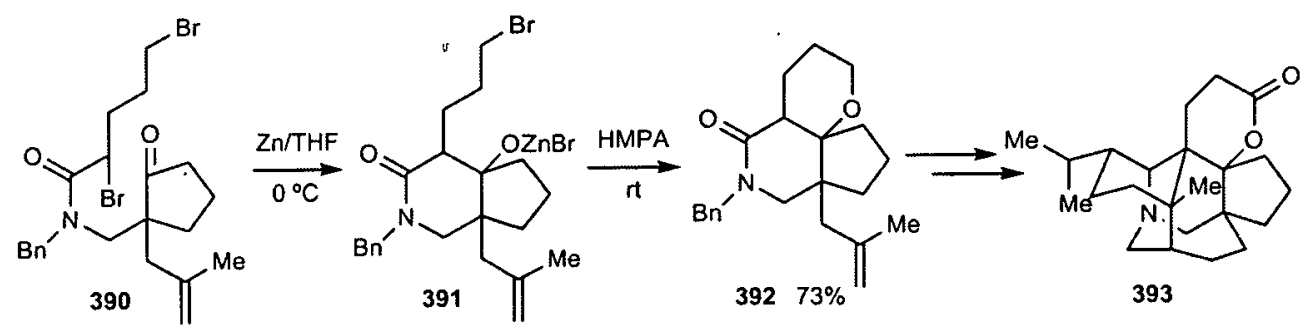

Scheme 4.8 Synthesis of polycyclic lactam ether 392 via intramolecular Reformatsky reaction

In 2000, Honda reported a rhodium-catalysed Reformatsky reaction using $\mathrm{RhCl}\left(\mathrm{PPh}_{3}\right)_{3}$ and $\mathrm{Et}_{2} \mathrm{Zn}{ }^{135}$ Surprisingly, prior to this work, there had been very few literature examples in the area of catalytic Reformatsky reactions. ${ }^{136,137}$ Honda and co-workers were especially encouraged by Ding's work, which employed zinc and a 
catalytic amount of $\mathrm{Cp}_{2} \mathrm{TiCl}_{2}{ }^{137}$, however, in this example it was thought that the active species was a titanium enolate.

Aromatic and aliphatic aldehydes or ketones were reacted successfully with ethyl bromoacetate or 2-bromopropionate in the presence of Wilkinson's catalyst and $\mathrm{Et}_{2} \mathrm{Zn}$ to afford $\beta$-hydroxy esters within 5 minutes in moderate to good yields. The formation of the ethyl adduct was not observed. More encouragingly for us, the intramolecular version of the reaction was also developed and secondary and tertiary cycloalkanols were obtained in good to excellent yields and with moderate diastereoselectivities. Some of the examples of the intramolecular reactions with methyl ketones are represented in Scheme 4.9. A transition state of the zinc enolate (400) was proposed to explain the obtention of cis-hydroxyesters.

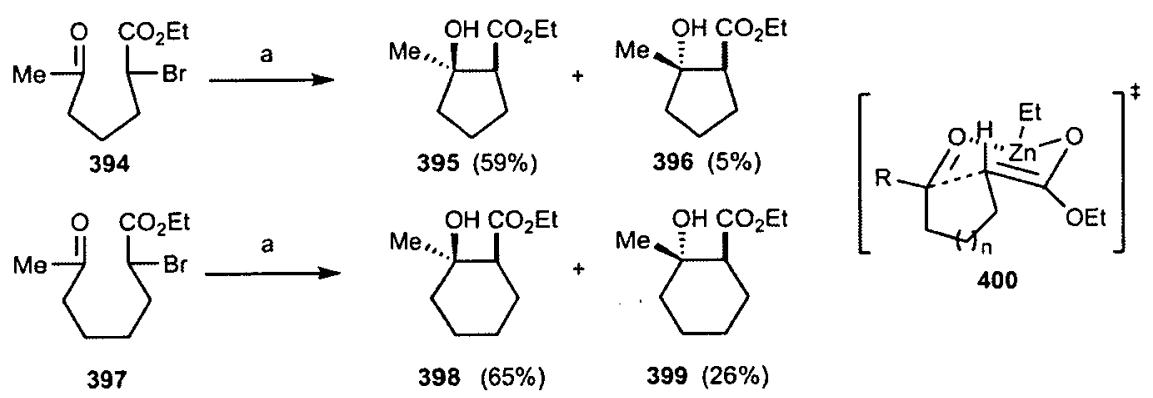

Reagents and conditions: (a) $\mathrm{Et}_{2} \mathrm{Zn}(2.2 \mathrm{eq}), \mathrm{RhCl}\left(\mathrm{PPh}_{3}\right)_{3}(5 \mathrm{~mol} \%)$, THF.

Scheme 4.9 Intermolecular $R$ h-catalysed Reformatsky-type reaction

The proposed mechanism for this reaction is shown in Scheme 4.10. Oxidative addition of $\alpha$-haloester I into $\mathrm{Rh}(\mathrm{I})$ forms a $\mathrm{Rh}(\mathrm{III})$ complex II that, after transmetallation with $\mathrm{Et}_{2} \mathrm{Zn}$, produces the ethylzinc enolate III and $\mathrm{Rh}(\mathrm{I})$, which can start the cycle again. The zinc enolate adds to the carbonyl compound forming zinc alkoxide IV that affords $\beta$-hydroxy ester $\mathbf{V}$ after work-up.

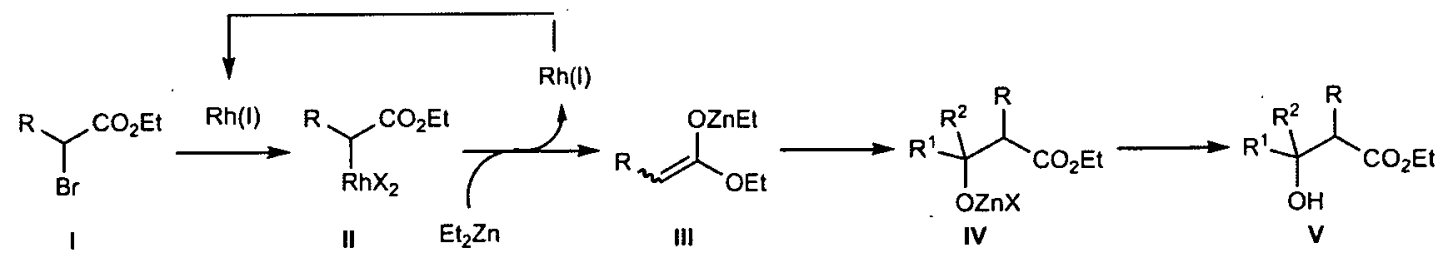

Scheme 4.10 Mechanism of the Reformatsky reaction catalysed by $\mathrm{RhCl}\left(\mathrm{PPh}_{3}\right)_{3}$ and $\mathrm{Et}_{2} \mathrm{Zn}$ 
This methodology was applied in the synthesis of 15-epi-haterumalide NA methyl ester 403, a cytotoxic macrolide from an Okinawan sponge (Scheme 4.11). ${ }^{138}$ When the cyclisation of $\mathbf{4 0 1}$ was attempted using Honda's conditions, ${ }^{135}$ only decomposition of the starting material occurred. By monitoring the reaction it was observed that the $\beta$-hydroxy lactone was being formed but it was decomposing during workup. The addition of $\mathrm{Ac}_{2} \mathrm{O}$ to trap the intermediate allowed the isolation of the desired $(3 S, 4 Z)-402$ in $9 \%$ yield, along with the $(3 R, 4 Z)$ - and $(4 E)$ - isomers.

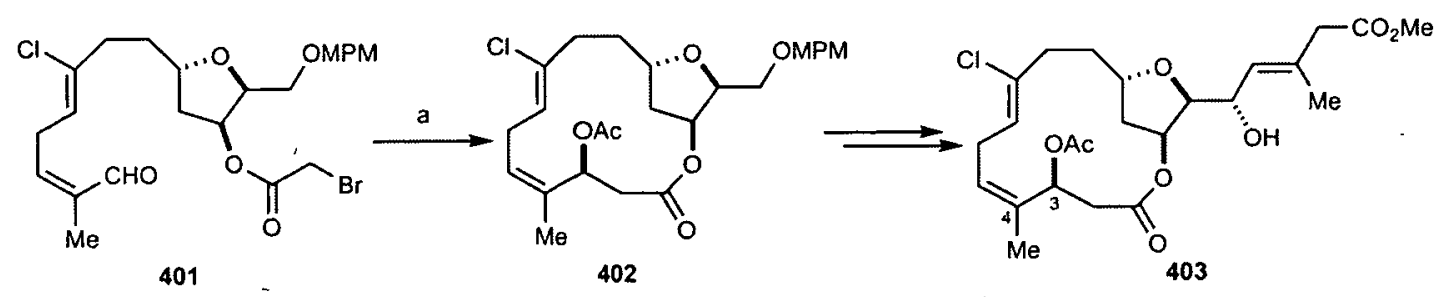

Reagents and conditions: (a) (i) $\mathrm{Et}_{2} \mathrm{Zn}, \mathrm{RhCl}\left(\mathrm{PPh}_{3}\right)_{3}$, THF-hexane; (ii) $\mathrm{Ac}_{2} \mathrm{O}, 9 \%$.

Scheme 4.11 Application of Honda's methodology in total synthesis

In 1977, Kagan introduced samarium(II) diiodide as a reagent for organic synthesis. ${ }^{139}$ Three years later, the first studies on its use in deoxygenation of epoxides and sulfoxides, the chemoselective reduction of aldehydes in the presence of ketones, dehalogenations, and coupling between ketones and alkyl halides were reported. ${ }^{140,141}$ Since then, this polyvalent reducing agent has been used in a multitude of important synthetic transformations and in the synthesis of natural products. ${ }^{142}$ One important use of this reagent is the $\mathrm{SmI}_{2}$ mediated reaction of organic halides with an electrophile, including $\mathrm{Sml}_{2}$ mediated Reformatsky reactions.

$\mathrm{SmI}_{2}$ is commercially available as a solution in THF, however it can be prepared from the metal and diiodomethane, diiodoethane or iodine, in THF. ${ }^{140,}{ }^{143} \mathrm{SmI}_{2}$ is a single electron reducing agent, with $\mathrm{Sm}$ (II) wanting to revert to the more stable $\mathrm{Sm}$ (III) oxidation state. Therefore, the $\mathrm{SmI}_{2}$ promoted reactions of organic halides are achieved using 2 molar equivalents of $\mathrm{SmI}_{2}$ and occur by stepwise one-electron transfer to the halide. The most generally accepted mechanism for these reactions proceeds via organosamarium species which react with the electrophile (Scheme 
4.12). These organosamarium species are formed via reduction of the alkyl halide to the radical by the first equivalent of $\mathrm{SmI}_{2}$ and then further reduction to alkyldiiodosamariums by the second equivalent of $\mathrm{SmI}_{2}{ }^{144}$

$$
R-x \underset{-\left[S m l_{2} X\right]}{\stackrel{S m l_{2}}{\longrightarrow}}\left|R^{\cdot}\right| \stackrel{S m l_{2}}{\longrightarrow} R-S m l_{2}
$$

\section{Scheme 4.12 Formation of organosamarium species}

Most of the reactions employing $\mathrm{SmI}_{2}$ are performed in $\mathrm{THF}$, but other solvents employed have been tetrahydropyran (THP) and alkylnitriles. ${ }^{145}$ The use of additives and cosolvents has also shown a crucial effect on the $\mathrm{SmI}_{2}$ mediated reactions. The most commonly employed additive is the Lewis base HMPA. ${ }^{146}$ It was reported that the redox potential $\left(\mathrm{E}^{\circ}\right)$ of $\mathrm{Sm}^{+2} / \mathrm{Sm}^{+3}$ in $\mathrm{THF}$ (vs. $\mathrm{Ag} / \mathrm{AgNO}_{3}$ reference electrode in THF) increased from $-1.33 \mathrm{~V}$ to $-1.95 \mathrm{~V}$ with 3 equivalents of HMPA and reached $-2.05 \mathrm{~V}$ with 4 equivalents, remaining constant with more equivalents. ${ }^{147} \mathrm{Hou}$ and co-workers ${ }^{148}$ isolated and characterised $\mathrm{SmI}_{2}(\mathrm{HMPA})_{4}$, although it was suggested that the active species in solution are more closely represented by the formula [Sm$(\mathrm{HMPA})_{4}(\mathrm{THF})_{2} \mathrm{II}_{2}{ }^{149}$ When HMPA was replaced for the less toxic HMPU, there was also an increase in the reduction potential, although more equivalents were required to achieve a similar level of activation. ${ }^{150}$ The use of TMEDA as an additive has also been reported. ${ }^{151}$

The rate of $\mathrm{SmI}_{2}$ mediated reactions can also be increased by the addition of inorganic salts like lithium chloride and bromide. ${ }^{152}$ The chemoselectivity was also altered by the addition of lithium halide salts. ${ }^{153}$ These reactions have also been markedly improved by the addition of catalytic amounts of transition metal salts. ${ }^{154}$ Ferric salts $\left(\mathrm{FeCl}_{3}, \mathrm{FeBr}_{3}, \mathrm{FeI}_{3}\right)$ have been extensively employed, Kagan, in his first paper, already observed the enhanced reactivity when substoichiometric amounts of $\mathrm{FeCl}_{3}$ were used. ${ }^{140} \mathrm{CuI}$ and $\mathrm{NiI}_{2}$ have also shown to be efficient additives. ${ }^{154,155}$

$\mathrm{SmI}_{2}$ has efficiently been used to promote the Reformatsky-type reaction of $\alpha$-halogenoesters, therefore becoming a useful alternative to the conventional Zn-mediated Reformatsky reactions. Due to the homogeneous conditions afforded by $\mathrm{SmI}_{2}$, the reaction can proceed with enhanced reactivity and reproducibility, under 
milder conditions, with even better chemoselectivity and, in intramolecular examples, with better stereoselectivity.

Intramolecular versions of the Reformatsky-like reactions have seen further developments and applications than their intermolecular versions. In fact, $\mathrm{SmI}_{2}$ is one of the most exploited reagents for intramolecular Reformatsky-like reactions, especially due to the yields and diastereoselectivities. It is believed that the reaction proceeds through a samarium (III) enolate and cyclisation occurs through a rigid cyclic transition structure enforced by chelation, where the large ionic radius, the flexible coordination and the high oxophilicity of samarium play an important role. ${ }^{156}$

Molander studied the 1,2-, 1,3-, and 1,4-asymmetric induction in intramolecular $\mathrm{SmI}_{2}$ mediated Reformatsky reactions. ${ }^{157}$ The trends of diastereoselectivity observed could be all explained through an eight membered ring samarium chelate (405) (Scheme 4.13). The products were obtained in excellent yields and very high diastereoselectivities.

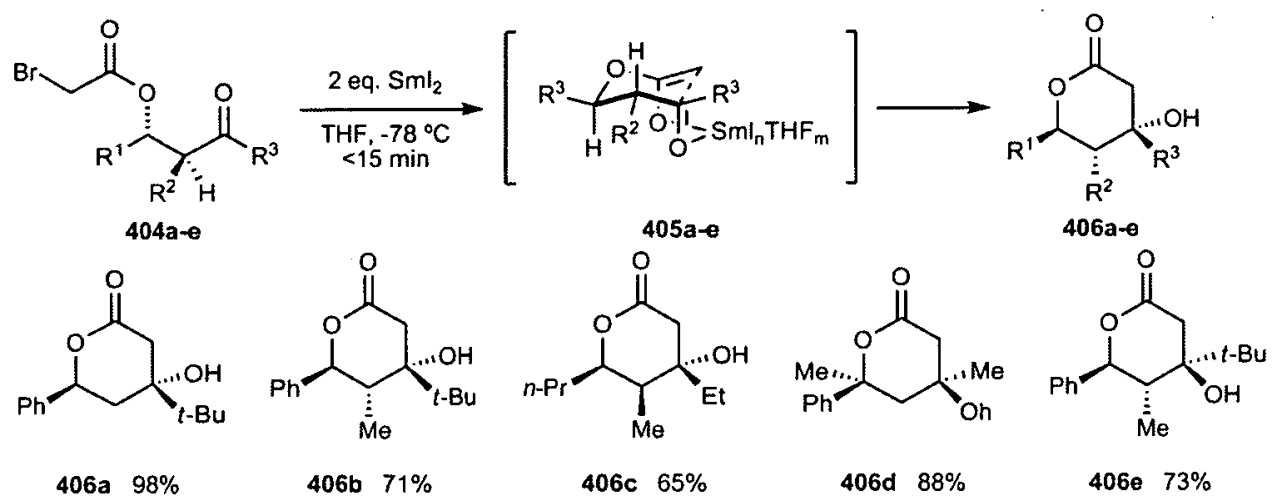

Scheme 4.13 Molander $\mathrm{Sml}_{2}$ mediated intramolecular Reformatsky reactions

The stereoselectivity of this reaction was also very high with $\gamma$-bromoacetoxy ketones which lead to the related seven-membered lactones (Scheme 4.14).

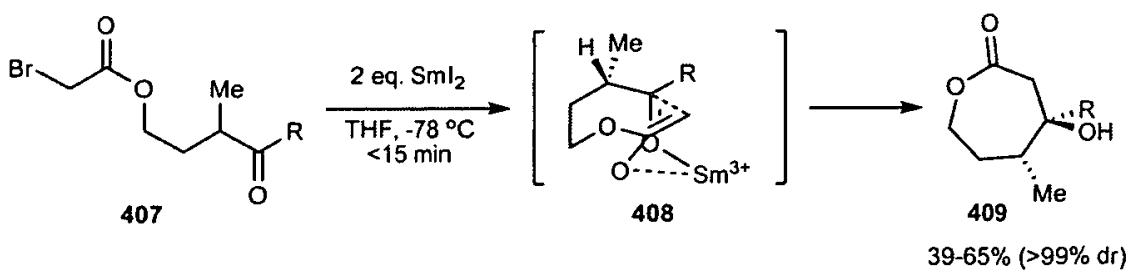

Scheme 4.14 $\mathrm{Sml}_{2}$ mediated cyclisations of $\gamma$ bromoacetoxy ketones 
Finally, it is worth highlighting a very interesting example due to the resemblance with our system, reported by Kocikowski in 1999. ${ }^{158}$ Treatment of $\mathbf{4 1 0}$ and 412 with $\mathrm{SmI}_{2}$ afforded $\gamma$-lactams 411 and 413 as single diastereomers (Scheme 4.15). No rationale was provided for the stereochemical outcomes of these reactions.

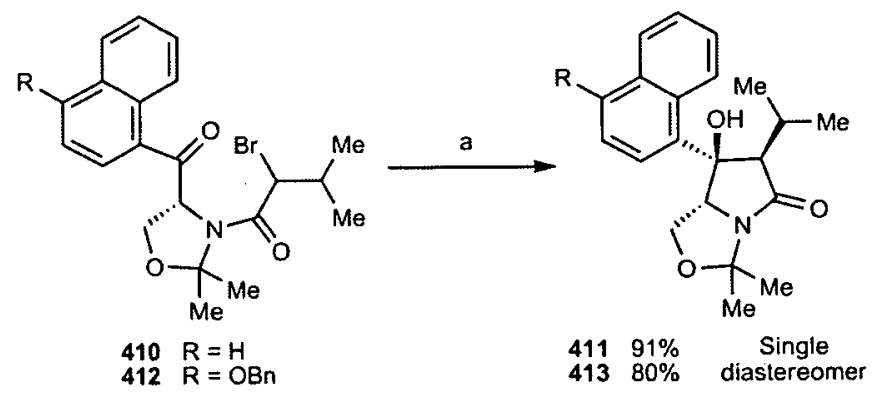

Reagents and conditions: (a) $\mathrm{SmI}_{2}, \mathrm{FeCl}_{3}$ (cat), THF-HMPA, rt.

Scheme 4.15 Kocikowski Sml $\mathrm{Sm}_{2}$ mediated $\gamma$ lactam formation

\subsection{Results and discussion}

Initial efforts centred on the preparation of amine 327, using a slightly modified synthetic route from the one previously reported by Corey and co-workers. ${ }^{26}$ Amine 327 was then successfully coupled to a number of $\alpha$-halo acids affording several substrates which were used in the investigation of the intramolecular Reformatsky reaction.

\subsubsection{Synthesis of amine 327}

Methyl ester 415 was readily prepared from reaction of $(L)$-threonine (414) with thionyl chloride in $\mathrm{MeOH}$ heated to reflux overnight, and it was used in the next step without further purification (Scheme 4.16). 4-Methoxybenzimidate hydrochloride (417) was obtained from an overnight reaction of $p$-methoxybenzonitrile (416) and acetyl chloride in $\mathrm{MeOH}$. Purification of 417 only involved several washings with $\mathrm{Et}_{2} \mathrm{O}$. 


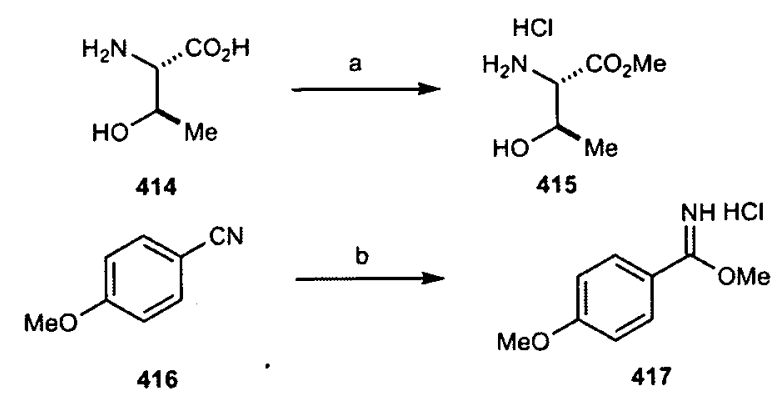

Reagents and conditions: (a) $\mathrm{SOCl}_{2}(1 \mathrm{eq}), \mathrm{MeOH}, 0^{\circ} \mathrm{C}$ to $\mathrm{rt}, 18 \mathrm{~h}$, quantitative; (b) Acetyl chloride (8 eq), $\mathrm{MeOH}, \mathrm{rt}, 18 \mathrm{~h}, 91 \%$.

\section{Scheme 4.16 Preparation of compounds 415 and 417}

Reaction of methyl ester $\mathbf{4 1 5}$ with triethyl amine and hydrochloric acid salt $\mathbf{4 1 7}$ in $\mathrm{CH}_{2} \mathrm{Cl}_{2}$ afforded oxazoline 418 (Scheme 4.17), in a moderate yield of $40 \%$, due also to a difficult purification. The yield of this step was improved to $65 \%$ when $\mathrm{K}_{2} \mathrm{CO}_{3}$ was employed as base instead of triethyl amine. ${ }^{\mathrm{i}}$ Although the yield was a little low, the procedure was simpler and more convergent than the one reported by Corey, which involved acylation of methyl ester 415 with 4-methoxybenzoyl chloride followed by acid catalysed cyclisation (Scheme 1.6$).{ }^{26}$ Moreover, it was amenable to a large scale preparation.

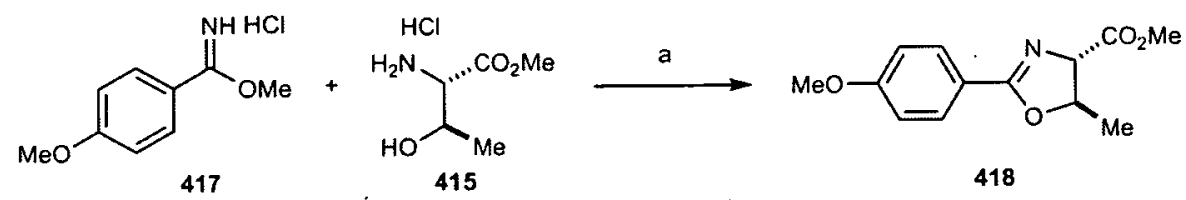

Reagents and conditions: (a) $\mathrm{Et}_{3} \mathrm{~N}$ (2 eq), $\mathrm{CH}_{2} \mathrm{Cl}_{2}$, reflux, $14 \mathrm{~h}, 40 \%$.

Scheme 4.17 Oxazoline 418 formation

We next examined the alkylation of oxazoline 418, an operation that introduces the $\mathrm{CH}_{2} \mathrm{OBn}$ group, and sets the stereochemistry of the quaternary centre $\mathrm{C} 4$ (Scheme 4.18). In Corey's procedure ${ }^{26}$ deprotonation was achieved using LDA in the presence of HMPA, and the enolate was reacted with chloromethyl benzyl ether affording the desired product in $69 \%$ yield. Using the commercially available base LHMDS instead of LDA, alkylated oxazoline 17 was obtained with an increased 75 $\%$ yield.

\footnotetext{
' Step improved by Leszek Rupnicki
} 


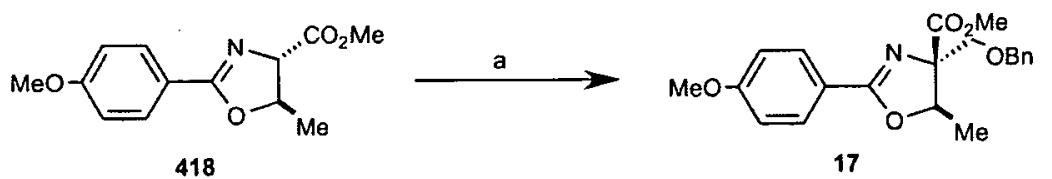

Reagents and conditions: (a) LHMDS (1.1 eq), HMPA (4.3 eq),

$\mathrm{ClCH}_{2} \mathrm{OBn}$ (1.2 eq), THF, $-78^{\circ} \mathrm{C}, 4 \mathrm{~h}, 75 \%$.

Scheme 4.18 Successful alternative procedure for the alkylation of $\mathbf{4 1 8}$

Transformation of oxazoline 17 into amine 327 was successfully performed following Corey's reported procedure (Scheme 4.19). ${ }^{26}$ Reductive opening of oxazoline 17 was achieved using sodium cyanoborohydride in acetic acid to give amino alcohol 18 in good yield (from 65 to 80\%). Finally, amine 327 was readily obtained via Swern $^{61}$ oxidation.

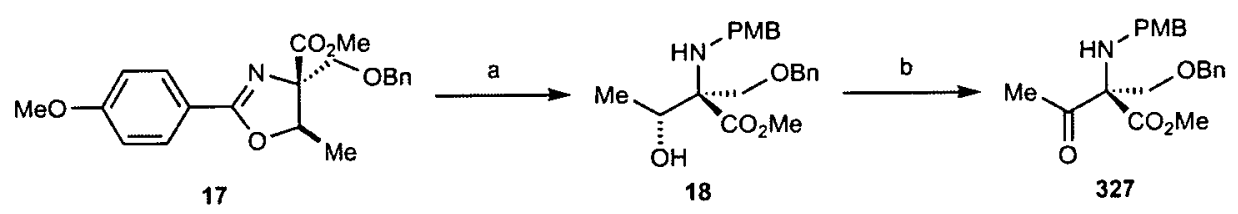

Reagents and conditions: (a) $\mathrm{NaCNBH}_{3}\left(3.3 \mathrm{eq}\right.$ ), $\mathrm{HOAc}, 50^{\circ} \mathrm{C}, 18 \mathrm{~h}, 65 \%$; (b) $(\mathrm{COCl})_{2}$ (2.1 eq), DMSO (4.2 eq), $\mathrm{Et}_{3} \mathrm{~N}(4.2 \mathrm{eq}), \mathrm{CH}_{2} \mathrm{Cl}_{2},-78^{\circ} \mathrm{C}, 3 \mathrm{~h}, 72 \%$.

Scheme 4.19 Final steps of the synthesis of amine 327

\subsubsection{Study of the Reformatsky cyclisation reaction}

To investigate the viability of the Reformatsky cyclisation in the proposed route to $\mathbf{1}$, an advanced model system was used. Ideally, this model substrate should be easily prepared and it should allow the optimisation of the reaction. This way, once the optimal conditions had been found, they could be applied to the real system, the substrate with the required functionalities to be transformed into 1 .

With this in mind, we chose to couple amine 327 to the commercially available 2-bromobutyryl bromide (419), to give $\alpha$-bromo amide 420 (Scheme 4.20). This amide was an optimal compound to investigate the reaction, since it was readily prepared from a commercially available starting material and it was structurally similar to the real substrate, only lacking the hydroxyl group in the acyl fragment. 


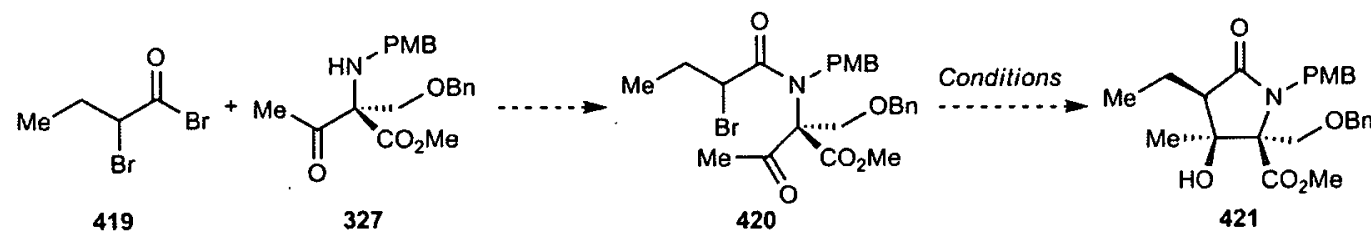

Scheme 4.20 Model studies for the Reformatsky cyclisation reaction

As expected, treatment of amine 327 with the hindered base triisobutylamine ${ }^{121}$ followed by addition of 2-bromobutyryl bromide 419 afforded the two expected diastereomers of the amide in an excellent overall yield $(9.3 \%)$ and approximately a $3: 2$ ratio (Scheme 4.21). The diastereomers were differentiated on the basis of their polarity on the silica plate: the more polar and major diastereomer (57\% yield) was referred to as $\mathbf{4 2 0 a}$ and the less polar and minor diastereomer (36\% yield) as $\mathbf{4 2 0 b}$; but the absolute stereochemistry of the bromine bearing carbon of each compound was not known. As the two diastereomers could differ in reactivity, they were separated by column chromatography and were used independently in the study of the cyclisation reaction.

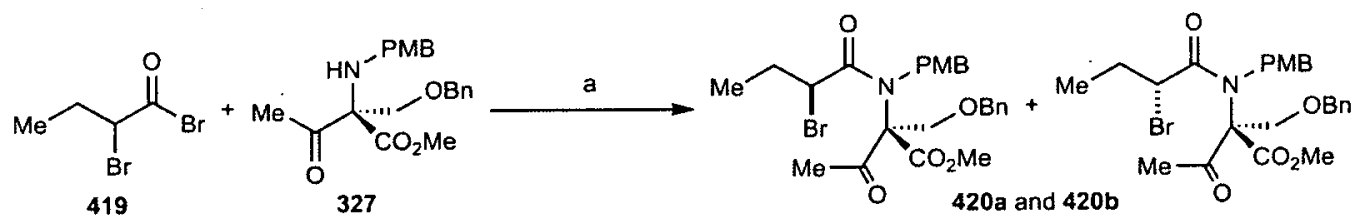

Reagents and conditions: (a) Triisobutylamine (1.3 eq), $\mathrm{CH}_{2} \mathrm{Cl}_{2}, \mathrm{rt}, 16 \mathrm{~h}$, 93\% (420a $57 \%$ and 420 b $36 \%$ ).

Scheme 4.21 Synthesis of amides 420a and $420 \mathrm{~b}$

The initial studies on the reaction focused on the application of the conditions of the "classical" Reformatsky reaction. The substrates prepared would be reacted with activated zinc on its own and also in the presence of additives. For instance, bearing in mind the examples using Yamamoto's conditions (Scheme 4.6), ${ }^{131-133}$ the reaction would also be attempted in the presence of $\mathrm{Et}_{2} \mathrm{AlCl}$.

Although being an intermolecular system, it was found in the literature another interesting example of a Reformatsky reaction. It involved the addition of azetidin-2-ones (422) to aldehydes and nitriles, and the effect of the addition of Lewis acids was also investigated (Table 4.1). ${ }^{159}$ The organozinc was prepared in 
THF and when it was reacted with benzaldehyde at room temperature the condensation product was not obtained (entry 1). However, when $\mathrm{AlMe}_{2} \mathrm{Cl}$ was added as an activator of the electrophile, both diastereomeric products, $\mathbf{4 2 3}$ and $\mathbf{4 2 4}$, were obtained within two hours and in good yield. The formation of the debrominated starting material (about 15\%) was observed. The reaction was attempted also with several other Lewis acids. It was discovered that when the reaction was activated with $\mathrm{BF}_{3} \cdot \mathrm{OEt}_{2}$ or $\mathrm{Ti}(\mathrm{O} i-\mathrm{Pr})_{4}$, the products were obtained in good yields within two hours (entries 5 and 6) and, in the case of the titaniumcatalyzed reaction, some diastereoselectivity was observed.

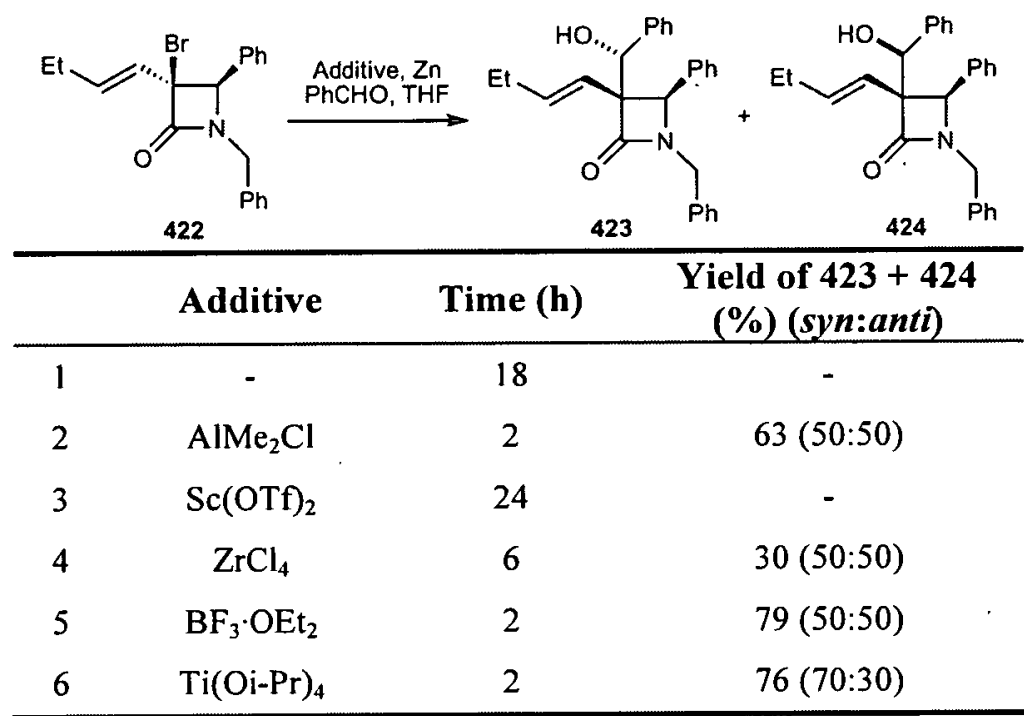

Table 4.1 Use of Lewis acids in the classical Reformatsky reaction

Several attempts of the cyclisation employing the classical Reformatsky conditions were carried out. Unfortunately, no reaction was observed when $\alpha$-bromoamides $420 \mathrm{a}$ and $\mathbf{4 2 0 b}$ were treated only with zinc, which had been activated either with $\mathrm{HCl}$ washings or with $\mathrm{I}_{2}$, and unreacted starting material 420 was recovered (Scheme 4.22).

The effect of the addition of Lewis acid additives was next investigated. Attempts were carried out in the presence of $\mathrm{Et}_{3} \mathrm{Al}^{160}$ or $\mathrm{Et}_{2} \mathrm{AlCl}$. These reactions were started at $-40^{\circ} \mathrm{C}$ and, since no reaction occurred at this temperature, they were then allowed to warm up slowly to room temperature over 4 hours, while monitoring the reaction by TLC. Disappointingly, amides 420 a or 420 b suffered quantitative debromination 
to form 425. Other Lewis acids were also evaluated: the reaction with $\mathrm{TiCl}_{4}$ or $\mathrm{BF}_{3} . \mathrm{Et}_{2} \mathrm{O}$ afforded the debrominated amide $425 ; \mathrm{Zn}(\mathrm{OTf})_{2}, \mathrm{Mg}(\mathrm{OTf})_{2}$ or $\mathrm{AgOTf}$ failed to furnish any product and starting material was recovered even after long reaction times.

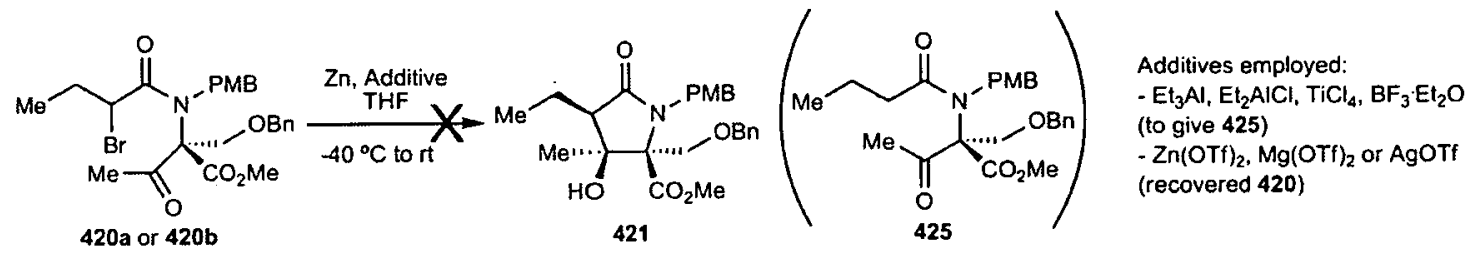

Scheme 4.22 Failure of cyclisation trials using "classical" Reformatsky conditions

Next, it was decided to attempt the cyclisation using Honda's conditions (Scheme 4.9). ${ }^{135}$ Substrates $420 \mathrm{a}$ and $420 \mathrm{~b}$ were treated with $\mathrm{Et}_{2} \mathrm{Zn}$ and $\mathrm{RhCl}\left(\mathrm{PPh}_{3}\right)_{3}$. Unfortunately, a complex mixture of products was obtained, from which it was only possible to identify unreacted starting material and debrominated 425 (Scheme 4.23).

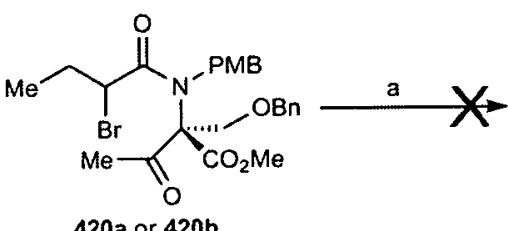

$420 \mathrm{a}$ or $420 \mathrm{~b}$

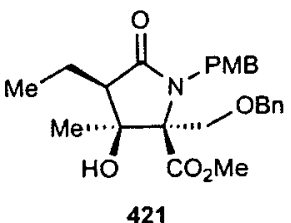

421

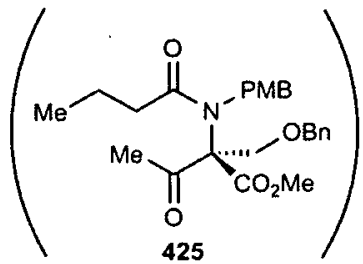

425

Reagents and conditions: (a) $\mathrm{Et}_{2} \mathrm{Zn}(2.2 \mathrm{eq}), \mathrm{RhCl}\left(\mathrm{PPh}_{3}\right)_{3}(5 \mathrm{~mol} \%)$, $-78^{\circ} \mathrm{C}$ to $0^{\circ} \mathrm{C}, 5 \mathrm{~h}$, THF.

\section{Scheme 4.23 Unsuccessful attempt of the cyclisation using Honda's} methodology

A related methodology was reported in 2003, which consisted of a Ni-catalysed Reformatsky-type three-component condensation reaction that combined an aldehyde, aniline and an $\alpha$-bromocarbonyl affording $\beta$-amino carbonyl compounds (Scheme 4.24). ${ }^{161}$ Using $\mathrm{Et}_{2} \mathrm{Zn}$, several catalysts $\left[\mathrm{RhCl}\left(\mathrm{PPh}_{3}\right)_{3}, \mathrm{PdCl}_{2}\left(\mathrm{PPh}_{3}\right)_{2}\right.$, $\mathrm{Cr}(\mathrm{acac})_{2}, \mathrm{Co}(\mathrm{acac})_{2} \cdot 2 \mathrm{H}_{2} \mathrm{O}$ ] were surveyed for the reaction before discovering that $\mathrm{Ni}$ (acac) 2 was a very effective catalyst and afforded the desired product in $95 \%$ yield. Further optimisation of the reaction revealed $\mathrm{NiCl}_{2}\left(\mathrm{PPh}_{3}\right)_{2}$ as a better catalyst and the 
use of the less nucleophilic dimethyl zinc instead of diethyl zinc avoided the formation of traces of the ethyl adduct

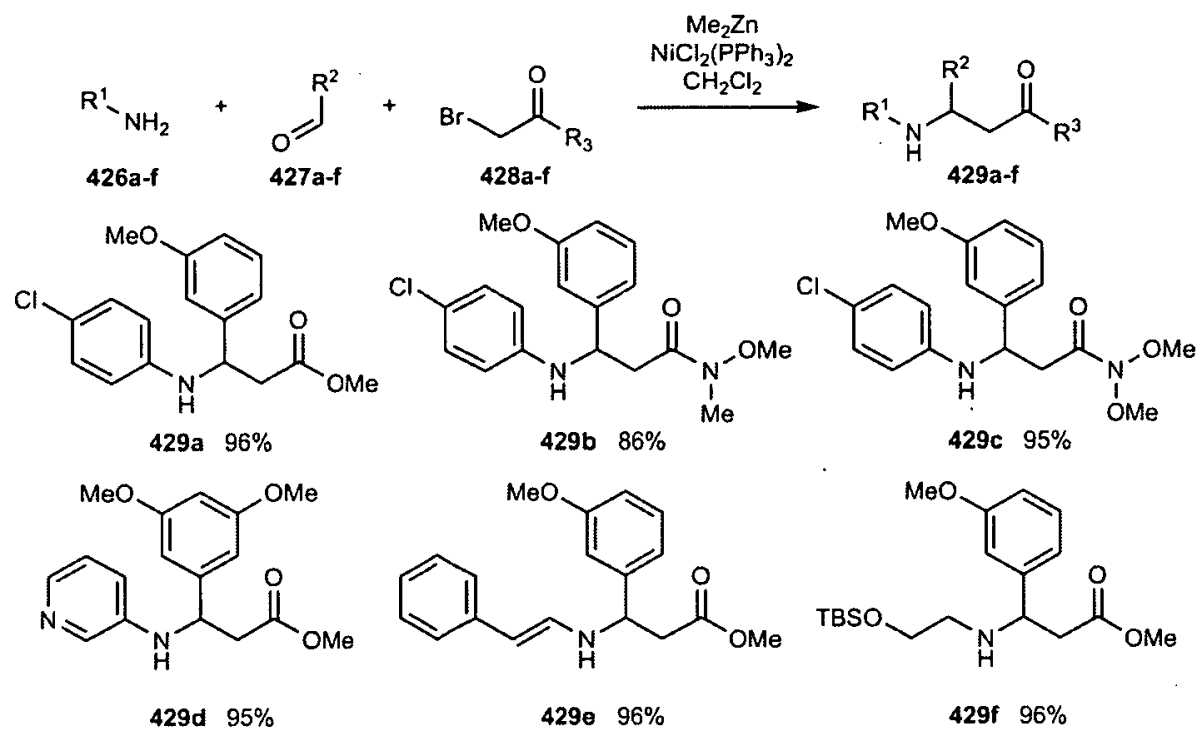

Scheme 4.24 3-Component coupling Ni-catalysed Reformatsky reaction

The proposed catalytic cycle of the reaction starts with the in situ generation of the active $\mathrm{Ni}(0)$, followed by formation of II via an oxidative addition to the $\alpha$-halo carbonyl (Scheme 4.25). Then, transmetallation with dimethylzinc forms the methylzinc enolate III and the $\mathrm{Ni}$ (II) complex IV, which undergoes reductive elimination liberating the active $\mathrm{Ni}(0)$ that can start the cycle again. Zinc enolate III reacts with the imine affording the $\beta$-amino carbonyl (VII) after workup.

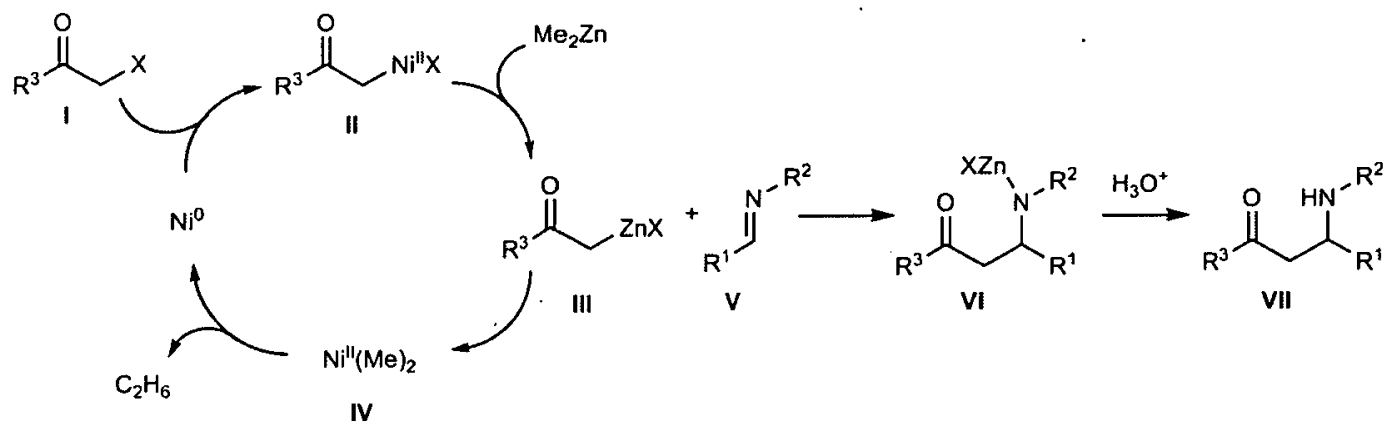

Scheme 4.25 Proposed catalytic cycle for the nickel-catalysed Reformatskytype reaction 
This intermolecular methodology was applied to our model substrates (Table 4.2). When amides 420a and $420 \mathrm{~b}$ were exposed to $\mathrm{Et}_{2} \mathrm{Zn}$ and $\mathrm{Ni}(\mathrm{acac})_{2}$, the reaction went to completion but only to form a complex mixture of products, from which it was only possible to identify the debrominated amide 425. An intractable mixture of compounds was also obtained when using $\mathrm{CoCl}_{2}$ and $\mathrm{Cy}_{2} \mathrm{PPh}$ (entry 2). When the reaction was carried out in the presence of $\mathrm{Co}(\text { acac })_{2} \cdot 2 \mathrm{H}_{2} \mathrm{O}$ (entry 3 ), no reaction occurred and starting material $420 \mathrm{a}$ or $420 \mathrm{~b}$ was recovered.

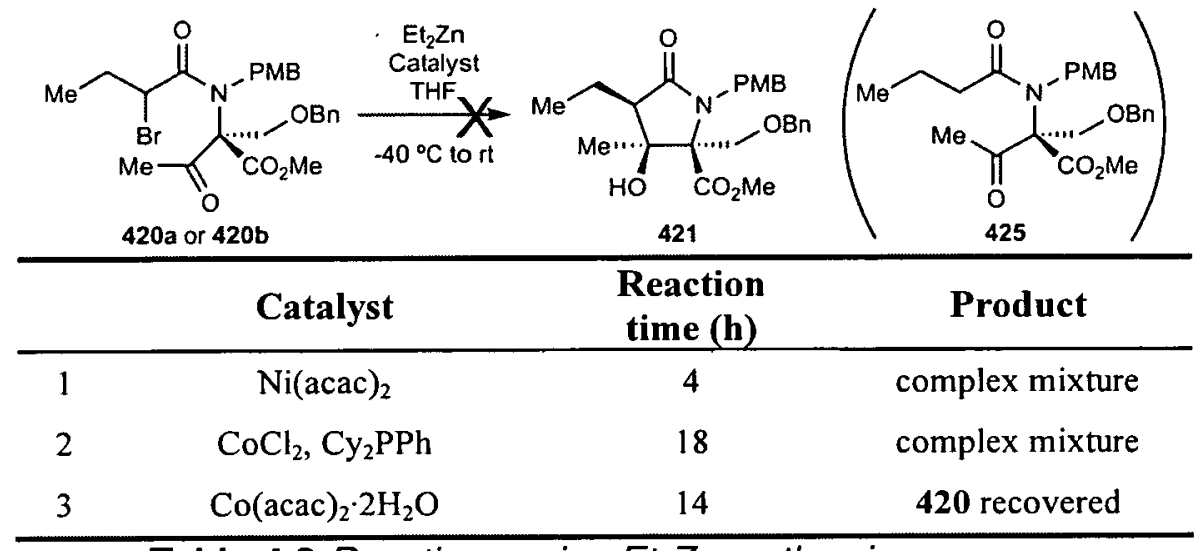

Table 4.2 Reactions using $E t_{2} Z n$ as the zinc source

The Cr-mediated Reformatsky reaction was then investigated, although this methodology had been developed on intermolecular systems. Since the 1970s, Nozaki and Hiyama were the pioneers of the use of organochronium reagents in organic synthesis. ${ }^{131}$ They demonstrated the insertion of $\mathrm{Cr}$ (II) into allyl-, alkyl-, alkynyl-, propargyl- and aryl halides or sulfonates under aprotic conditions, forming the corresponding organochromium(III) reagents that can react as nucleophiles in organic transformations. In 1986, both Kishi and Nozaki independently discovered a catalytic effect of nickel salts in the reaction, which improved the reproducibility of the reaction. ${ }^{162}$ This was explained via the mechanism represented in Scheme 4.26. $\mathrm{Ni}(\mathrm{II})$ is reduced to $\mathrm{Ni}(0)$ with 2 equivalents of $\mathrm{Cr}_{2} \mathrm{Cl}$. Then oxidative addition to the alkenyl halide occurs, followed by transmetallation with $\operatorname{Cr}(\mathrm{III})$, forming the alkenylchromium (III) reagent, which is the species that reacts with the carbonyl. 


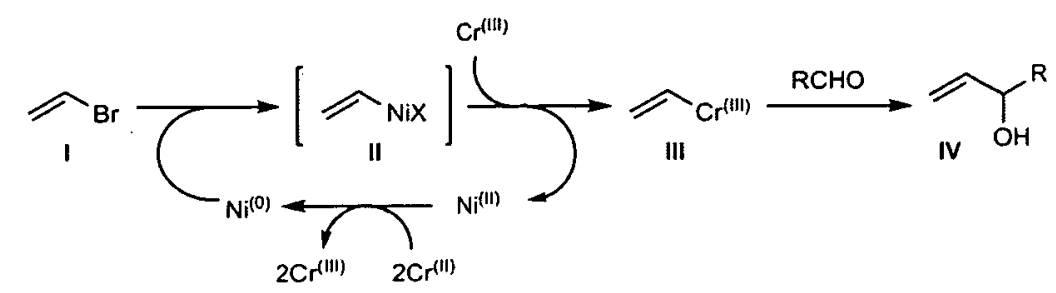

Scheme 4.26 Explanation of the role of $\mathrm{Ni}(I I)$ salts in the $\mathrm{Cr}(\mathrm{II})$-mediated coupling reaction

The major drawback of the Nozaki-Hiyama-Kishi (NHK) reaction is that, since $\mathrm{Cr}$ (II) is a one-electron donor, at least 2 mole are needed per mole of halide and, in reality, huge excesses (4-16 eq) of $\mathrm{Cr}$ (II) are used in the reaction. Because of the toxicity of $\mathrm{Cr}$ and Ni salts, a catalytic version of the reaction was highly desirable.

In 1995, Furstner and co-workers developed the Nozaki-Hiyama-Kishi (NHK) reaction catalytic in chromium (Scheme 4.27). ${ }^{163}$ They reasoned that, in the reaction, the formation of the highly stable $\mathrm{Cr}$ (III) alkoxide drives the reaction to conversion but also traps the metal, impeding catalysis. It was hypothesised that if the chromium alkoxide (III) was reacted with a chlorosilane, a exchange of ligands (via $\sigma$ bond metathesis) could occur, liberating the silyl ether of the desired product (IV) and liberating the second mole of $\mathrm{Cr}$ (III). The liberated $\mathrm{Cr}$ (III) species could be rereduced in situ by a non toxic reducing agent, this enabling the catalytic cycle. They found that use of the cheap and non-toxic Mn in combination with TMSCl and catalytic amounts of $\mathrm{CrCl}_{2}$ allowed the first NHK reactions catalytic in chromium.

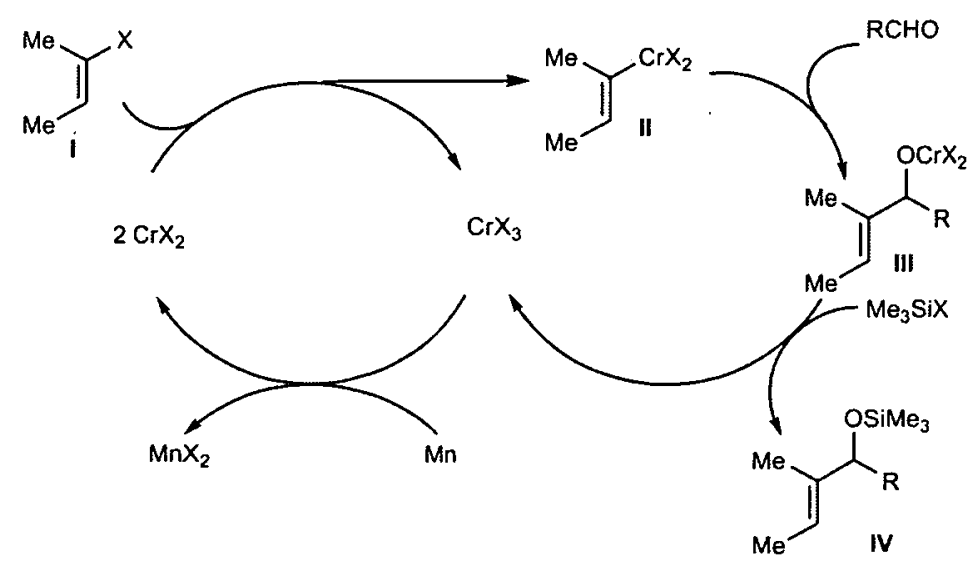

Scheme 4.27 Mechanism of the NHK reaction catalytic in chromium 
$\alpha$-Halo ketones, esters, amides, nitriles and other Reformatsky substrates ${ }^{128,} 164$ have been reacted with $\mathrm{Cr}$ (II) salts, forming $\mathrm{Cr}$ (III) enolates via oxidative addition of the low valent metal salt into the carbon-halogen bond activated by the carbonyl group. In the presence of carbonyl compounds, the aldol products are obtained. For instance, intermolecular reaction of $\alpha$-bromo ketones with aldehydes in the presence of $\mathrm{CrCl}_{2}$ yielded the aldol products in good yields and with complete syndiastereoseletivity (Scheme 4.28). ${ }^{165}$

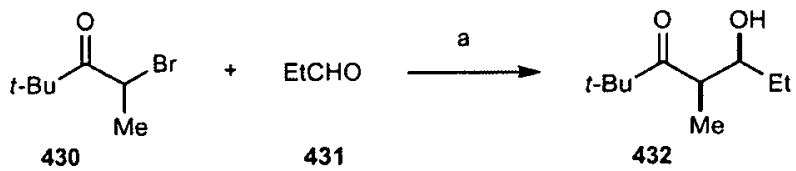

Reagents and conditions: (a) $\mathrm{CrCl}_{2}, \mathrm{THF}, 81 \%$.

\section{Scheme 4.28 Intermolecular Cr-mediated Reformatsky reaction}

The Cr-mediated Reformatsky reactions tend to proceed in THF, DMF or acetonitrile. This reaction is highly chemoselective towards aldehydes, ${ }^{166}$ although reactions with ketones as electrophiles are also possible. ${ }^{167}$ However, when $\alpha$-bromoamides $420 \mathrm{a}$ and $\mathbf{4 2 0 \mathrm { b }}$ were subjected to standard catalytic NHK reaction conditions ( $\mathrm{Mn}, \mathrm{TMSCl}$ and $\mathrm{CrCl}_{3}$ doped with $\mathrm{NiCl}_{2}$ in $\mathrm{THF}$ ), debrominated product 425 was obtained quantitatively (Scheme 4.29). The same result was obtained when the reaction was performed in DMF. Wessjohann reported increased yields in NHK reactions in the presence of a catalytic amount of LiI, due probably to a general Lewis acid effect and to its ability to solubilise and modify $\mathrm{CrCl}_{2} \cdot{ }^{168} \mathrm{~A}$ last attempt incorporating $10 \mathrm{~mol} \%$ of LiI also failed, with 425 being again the only product of the reaction. With these results we concluded that the chromium enolate was being formed but it was not reacting with the methyl ketone and instead it was being immediately quenched. 


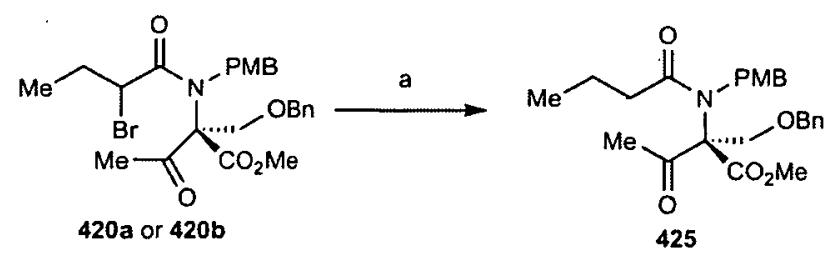

Reagents and conditions: (a) $\mathrm{Mn}(1.7 \mathrm{eq}), \mathrm{CrCl}_{3}(0.15 \mathrm{eq}), \mathrm{TMSCl}(2.4$ eq), $\mathrm{NiCl}_{2}(0.03 \mathrm{eq})$, THF or DMF, $\mathrm{rt}, 2 \mathrm{~h}$.

\section{Scheme 4.29 Unsuccessful Cr-mediated Reformatsky reaction}

At this point, the Reformatsky reaction had been attempted with several different metals but these were not successful in the formation of the cyclisation product and in most cases the debrominated starting material was obtained. The investigation next focused on the $\mathrm{SmI}_{2}$ mediated Reformatsky reaction. It was hoped that, in this case, the substrates would cyclise into the ketone due to the known chelating ability of $\mathrm{Sm}^{3+}$.

Initially, both diastereomers $420 \mathrm{a}$ and $420 \mathrm{~b}$ were treated with 2.2 equivalents of $\mathrm{Sml}_{2}$ (commercially available $0.1 \mathrm{M}$ solution) in THF. The reaction did not proceed at $-78^{\circ} \mathrm{C}$, but it quickly went to completion at room temperature (Scheme 4.30). We were very pleased to find that this reaction afforded the desired cyclised product $\mathbf{4 2 1}$ (30\% yield), although it was accompanied by amide 433, obtained in approximately $40 \%$ yield and debrominated amide 425 (10\% yield).

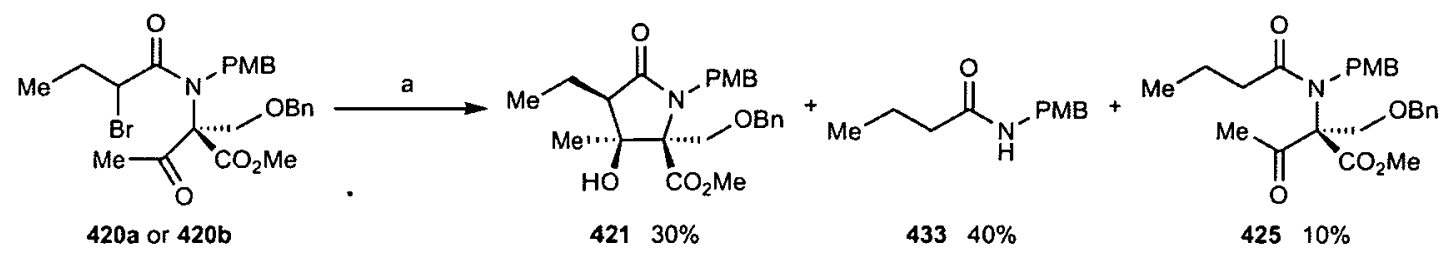

Reagents and conditions: (a) $\mathrm{SmI}_{2}(2.2 \mathrm{eq}), \mathrm{THF}, \mathrm{rt}, 2 \mathrm{~h}$.

Scheme 4.30 Preliminary result for the $\mathrm{Sml}_{2}$ mediated Reformatsky reaction

Although 421 was obtained in low yield, the good news was also that it was obtained as a single diastereomer (only one diastereomer of product was observed by ${ }^{1} \mathrm{H}$ NMR spectroscopy). Furthermore, the relative configuration was confirmed by a 2D NOESY experiment (421 was not crystalline), which indicated that 421 was the desired diastereomer. Two cross peaks were observed to support this fact: the $\mathrm{CH}_{3}$ at C3 with $\mathrm{CH}_{2} \mathrm{OBn}$ at $\mathrm{C} 4$ and the same $\mathrm{CH}_{3}$ at $\mathrm{C} 3$ with the $\mathrm{CH}$ at $\mathrm{C} 2$ (Figure 4.1). 


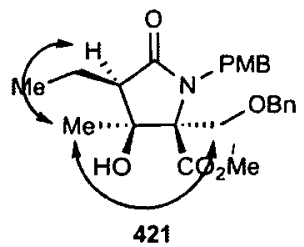

Figure 4.1 2D NOESY experiment

A proposed mechanism for the cyclisation to $\gamma$-lactam 421 is represented in Scheme 4.31. A first equivalent of $\mathrm{SmI}_{2}$ reduces $\alpha$-bromo amide $\mathbf{4 2 0}$ to form 434, which then receives another electron from a second equivalent of $\mathrm{SmI}_{2}$, to form enolate 435. Finally, this enolate is trapped by the methyl ketone, forming $\gamma$-lactam 421. A proposed transition state to explain the stereoselectivity of the reaction is represented by 436 .

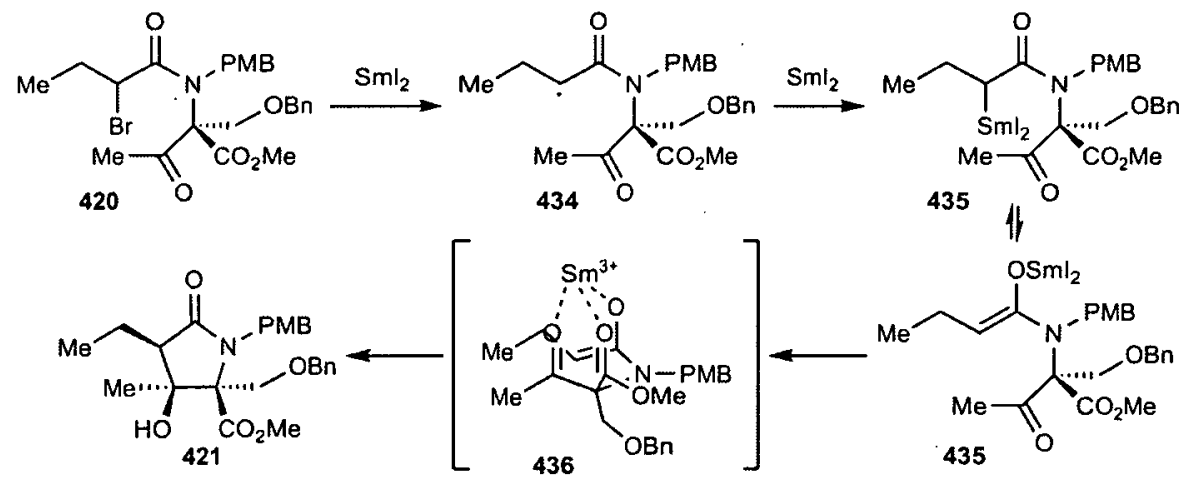

Scheme 4.31 Proposed mechanism of the formation of 421

A similar reductive mechanism is proposed for the formation of amide 433 (Scheme 4.32). Probably after loss of the bromine atom of $\mathbf{4 2 1}$, the carbonyl of the ketone is reduced by a first equivalent of $\mathrm{SmI}_{2}$. Then, a second equivalent forms anion 438 which rearranges, facilitated by the anion-stabilising effect of the ester, causing the C-N bond cleavage and yielding 433 .

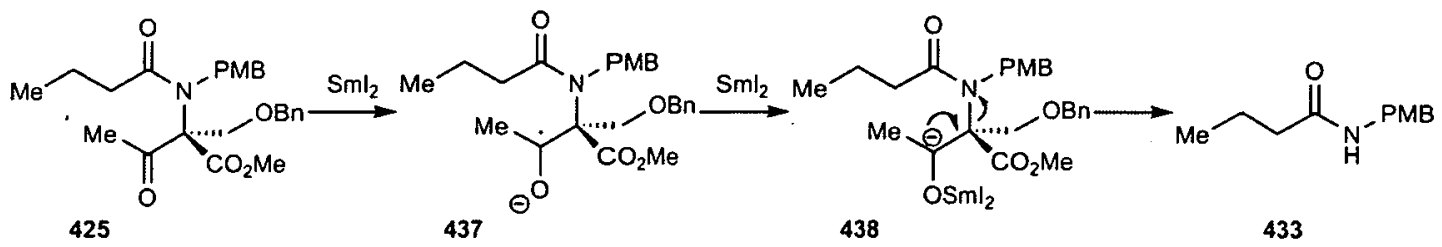

Scheme 4.32 Proposed mechanism of the formation of $\mathbf{4 3 3}$ 
Similar observations were made by Matsuda and co-workers, in an interesting example of an intramolecular Reformatsky cyclisation of 3-ketouridine derivatives promoted by $\mathrm{SmI}_{2}{ }^{169}$ When 439 was reacted with 2.0 equivalents of $\mathrm{SmI}_{2}$ in THF at room temperature, the Reformatsky reaction proceeded giving 440 in $71 \%$ yield (Scheme 4.33). The reaction performed at $0{ }^{\circ} \mathrm{C}$ afforded 440 in $75 \%$ yield and at -78 ${ }^{\circ} \mathrm{C}$ the yield was $90 \%$. When the reaction was performed at $-78^{\circ} \mathrm{C}$ but using HMPA as an additive, the yield of 440 was decreased to $76 \%$ and 441 was obtained in $11 \%$ yield.
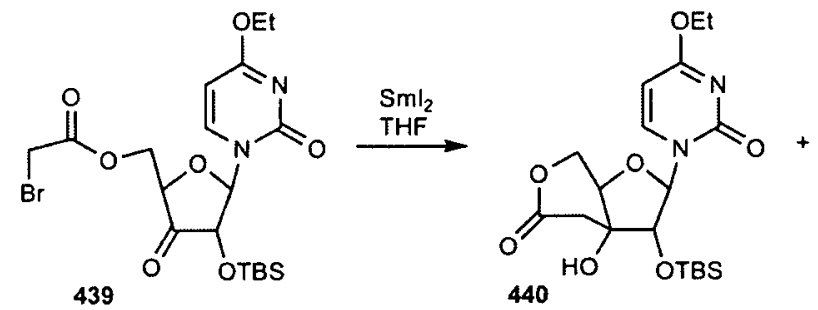<smiles>CCOc1cc[nH]c(=O)n1</smiles>

Scheme 4.33 Matsuda intramolecular cyclisation to 439

When 439 was reacted with zinc as a reductant in toluene, 440 was not formed and only debrominated starting material (442) was isolated. To study the mechanism of the formation of 441,442 was treated with $\mathrm{SmI}_{2}$ and HMPA in THF at room temperature, which produced 441 in 64\% yield (Scheme 4.34). This could be explained via an electron transfer from $\mathrm{SmI}_{2}$ to the $3^{\prime}$-carbonyl of the 3 '-ketouridine derivative, followed by reductive cleavage of the carbon-oxygen bond at the $4^{\prime}$-position.

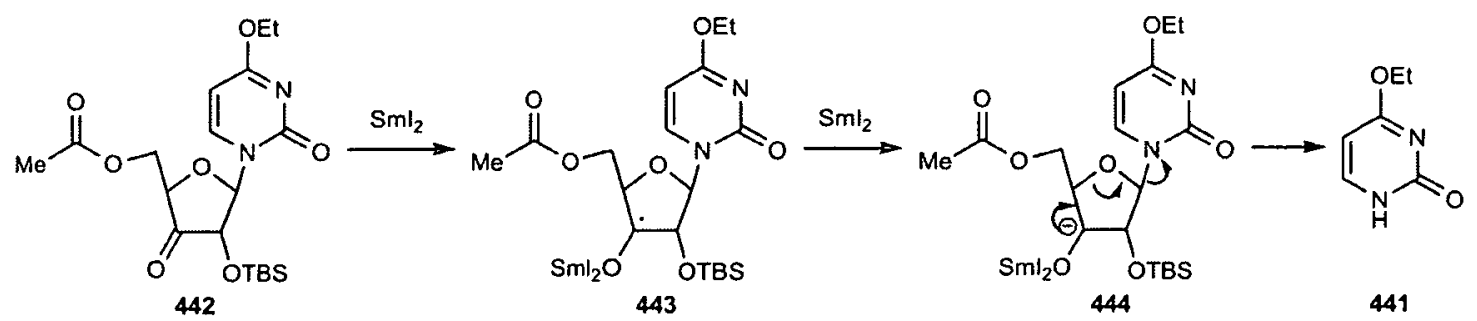

Scheme 4.34 Proposed mechanism form the formation of 441

They also reasoned that the ability of $\mathrm{SmI}_{2}$ in promoting the reaction was due to the ability of $\mathrm{Sm}^{3+}$ to chelate to the $5^{\prime}$-enolate and the $3^{\circ}$-carbonyl (Figure 4.2). It was because of the formation of this chelate that the enolate addition to the carbonyl 
occurred. That was the reason why, in the reaction with zinc as a reductant, the 5 -enolate was generated, but the addition into the 3 '-carbonyl did not proceed, due to the lack of chelating ability of zinc.

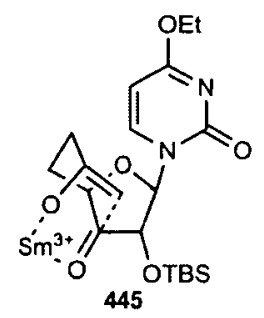

Figure 4.2 Chelating ability of $\mathrm{Sm}^{+3}$

Based on the encouraging preliminary result of the desired cyclised product, work proceeded on the optimisation of the reaction. When the reaction was performed using 2.2 equivalents of $\mathrm{SmI}_{2}$, but at $0{ }^{\circ} \mathrm{C}$ instead of at room temperature, the reaction was slowed down and did not go to completion; the main product obtained was debrominated amide 425. The reaction was repeated in other solvents, for example $\mathrm{CH}_{3} \mathrm{CN}$ and toluene, but THF remained the solvent of choice since it gave cleaner and better conversions.

The use of additives did not afford any improvement in the reaction. In the presence of HMPA or DMPU the reaction was again slower and would not go to completion. The same result was obtained with the addition of substoichiometric amounts of $\mathrm{FeCl}_{3}$. Performing the reaction in the presence of $\mathrm{Et}_{2} \mathrm{AlCl}$ afforded debrominated amide $\mathbf{4 2 5}$ only, in a very fast reaction. This example resembled to the attempts of the cyclisation using other metals than $\mathrm{Sm}$, where presumably chelation with the ketone was not occurring and debrominated amide $\mathbf{4 2 5}$ was the only product obtained.

The amount of samarium iodide required was next revised. The number of equivalents employed was slowly raised from 2.2 to 12 equivalents. We discovered that when the reaction was performed using a large excess of $\mathrm{SmI}_{2}(10-12$ equivalents) at $0{ }^{\circ} \mathrm{C}$, only traces of debrominated product 421 were obtained. The large excess of $\mathrm{SmI}_{2}$ resulted in a fast reaction (completed in a matter of minutes) which seemed to be beneficial since the debromination had been practically eradicated and, by TLC, the desired cyclised product seemed to be the major product. 
However, when the crude reaction was purified and the products isolated by column chromatography, amide $\mathbf{4 3 3}$ was again obtained in $40 \%$ yield and the yield of $\mathbf{4 2 1}$ had only increased slightly from $30 \%$ to $40 \%$ (Scheme 4.35 ). Under these conditions, the use of additives like HMPA, DMPU or $\mathrm{FeCl}_{3}$, also resulted in lower conversions and more debrominated $\mathbf{4 2 5}$ was formed. There was no reaction when the temperature was lowered further $\left(-40^{\circ} \mathrm{C}\right.$ or $\left.-20^{\circ} \mathrm{C}\right)$.

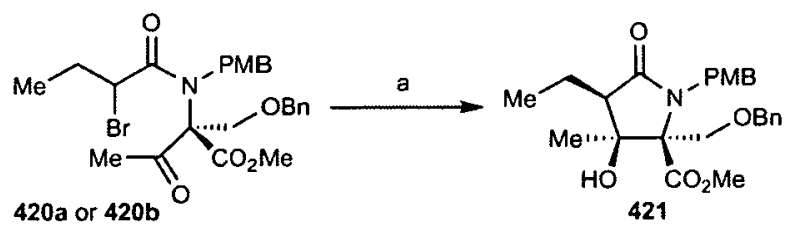

Reagents and conditions: (a) $\mathrm{SmI}_{2}(10 \mathrm{eq})$, $\mathrm{THF}, 0^{\circ} \mathrm{C}, 40 \%$.

Scheme 4.35 Improved yield towards $\gamma$-lactam 421

A catalytic $\mathrm{SmI}_{2}$-mediated Reformatsky reaction had been reported in the literature, using $\mathrm{Mg}$ metal to carry out the reduction of $\mathrm{Sm}$ (III) to $\mathrm{Sm}$ (II). ${ }^{170} \mathrm{We}$ speculated whether a catalytic procedure could favour the cyclisation at the expense of the $\mathrm{C}-\mathrm{N}$ bond cleavage. However, little reaction occurred under these conditions.

It is important to note that the formation of the cyclised product only occurred when reacting the major diastereomer 420a. Diastereomer 420b reacted slightly faster and at lower temperatures than 420a did, but to yield mainly debrominated amide $\mathbf{4 2 5}$ and amide $\mathbf{4 3 3}$, and only traces of the desired product. The fact that $\mathbf{4 2 0 a}$ was slightly less reactive turned out to be beneficial. However, being able to use only one diastereomer worsened the overall yield of a possible route to 1 .

In the next stages of the investigation, the $\alpha$-iodo derivative was prepared, in order to determine if the nature of the halide had an effect on the cyclisation reaction. The synthesis of 2-iodo butanoic acid (448) was easily achieved from commercially available butanal (446) (Scheme 4.36). $\alpha$-Iodo butanal (447) was prepared via a $D L$-proline catalysed $\alpha$-iodination of 446 with NIS, ${ }^{171}$ which afforded 447 as a racemic mixture. Oxidation of $\mathbf{4 4 7}$ to acid $\mathbf{4 4 8}$ was successfully performed using $\mathrm{KMnO}_{4}$, in $t-\mathrm{BuOH}$ and a $\mathrm{NaH}_{2} \mathrm{PO}_{4}$ buffer to adjust the $\mathrm{pH}^{172}$ In a similar manner to that of the bromo-derivative preparation, from the racemic $\alpha$-iodo carboxylic acid 
both diastereomers of the amide would be obtained, they would be separated and the cyclisation reaction of each diastereomer would be studied independently. If one of the diastereomers were to react more efficiently, it could be selectively prepared with the same methodology but employing the chiral non-racemic organocatalyst.

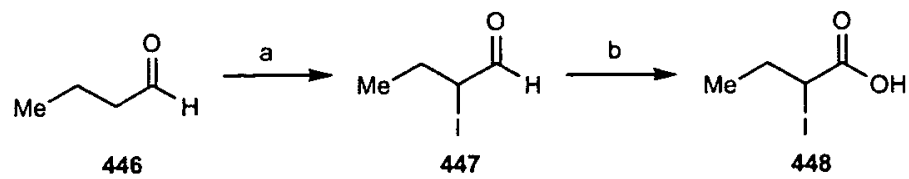

Reagents and conditions: (a) $D L$-Proline ( $0.3 \mathrm{eq}$ ), NIS (1.3 eq), $\mathrm{CH}_{2} \mathrm{Cl}_{2}$, $0{ }^{\circ} \mathrm{C}$ to rt, $5 \mathrm{~h}, 42 \%$; (b) $\mathrm{KMnO}_{4}$ (6 eq), $5 \% \mathrm{NaH}_{2} \mathrm{PO} 4, t-\mathrm{BuOH}, \mathrm{rt}, 3 \mathrm{~h}, 95 \%$.

\section{Scheme 4.36 Successful preparation of 2-iodo butanoic acid 448}

The amide coupling of amine 327 and 2-iodo butanoic acid 448 was successfully achieved using triisobutylamine ${ }^{121}$ as base, which yielded two diastereomers of the amide (449a and $449 \mathrm{~b}$ ) in $75 \%$ overall yield (Scheme 4.37 ). In this case both diastereomers were obtained in practically the same proportion (1.2:1 ratio).

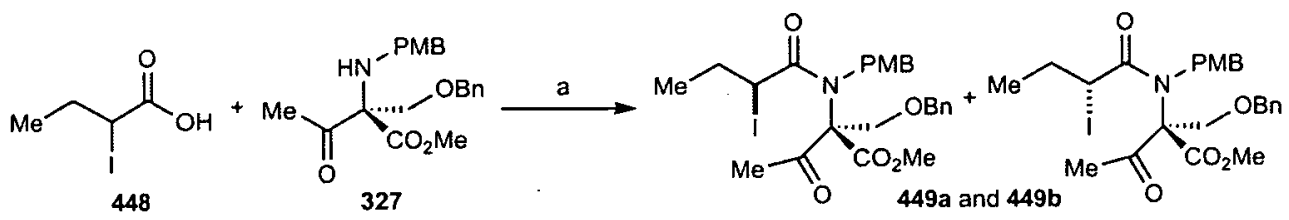

Reagents and conditions: (a) Oxalyl Chloride (1l eq), DMF (cat), $\mathrm{CH}_{2} \mathrm{Cl}_{2}$, rt, $1 \mathrm{~h}$; (b) amine 327 , triisobutylamine ( $1.2 \mathrm{eq}), \mathrm{CH}_{2} \mathrm{Cl}_{2}, 0^{\circ} \mathrm{C}$ to $\mathrm{rt}, 14 \mathrm{~h}, 75 \%$.

Scheme 4.37 Successful amide coupling to $\alpha$-iodo amides 449

The Reformatsky reaction with the iodo-derivatives was investigated, using from 2.2 to 12 equivalents of $\mathrm{SmI}_{2}$ at different temperatures (from $-40{ }^{\circ} \mathrm{C}$ to room temperature). In this case, neither the amount of $\mathrm{SmI}_{2}$ nor the temperature led to any significant variation in the reaction. In all cases, amide 433 was the main product, obtained in 40-50\% yield, followed by the debrominated amide 425 in 15-30\% yield. $\gamma$-Lactam 421 was isolated only in a maximum yield of $10-15 \%$ (Scheme 4.38). The use of additives ( $\mathrm{HMPA}, \mathrm{FeCl}_{3}$ or $\mathrm{NiI}_{2}$ ) was again not beneficial in the reaction. 


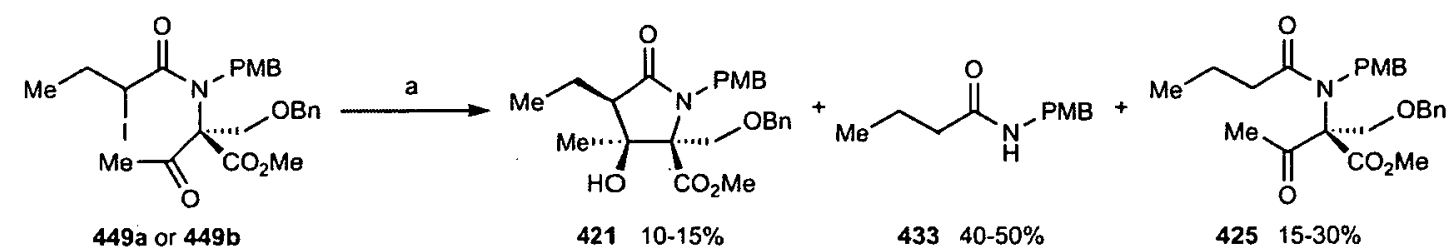

Reagents and conditions: (a) $\mathrm{SmI}_{2}(12 \mathrm{eq}), \mathrm{THF}, 0^{\circ} \mathrm{C}, 2 \mathrm{~h}$.

Scheme 4.38 Cyclisation reaction of iodo-derivative 449

It was concluded that the iodo-derivative had a similar reactivity to the bromo-diastereomer $420 \mathrm{~b}$, which only afforded traces of the desired product. The reaction of $420 \mathrm{~b}$ and the iodo derivatives 449 was faster and less controllable, and the cyclised product was formed in a lesser proportion than with the bromide diastereomer 420a. Consequently, only $\alpha$-bromo amides were employed for the rest of the investigation.

After this thorough study, an increase in the yield of the desired product had been achieved by reduction of the debromination of the starting material, but amide $\mathbf{4 3 3}$ was still being formed as a major product. An ideal set of conditions were required that, without completely stopping the reaction, would favour the formation of the desired cyclised product $\mathbf{4 2 1}$ at the expense of the formation of $\mathbf{4 3 3}$.

Since $\gamma$-lactam 421 would only lead to the synthesis of salinosporamide B (Scheme 4.39), it was next decided to investigate further the optimisation of the reaction employing a substrate that would allow the synthesis of salinosporamide A.

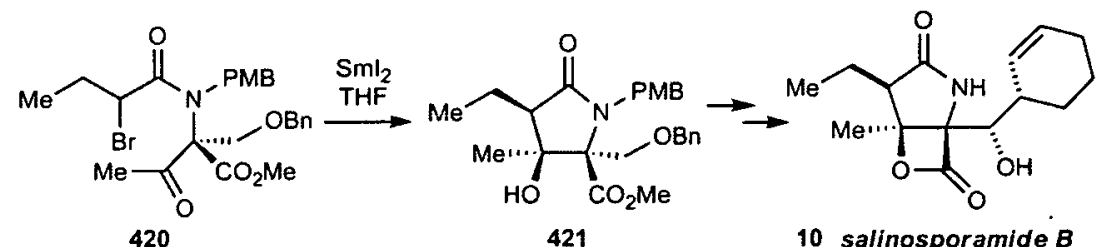

Scheme 4.39 Cyclisation of $\mathbf{4 2 0}$ allows the formation of Salinosporamide B

For this purpose, amine 327 would be coupled to an $\alpha$-bromo acid such as $\mathbf{4 5 0}$, which contained a protected alcohol on the $\gamma$-position (Scheme 4.40). If the amide obtained was successfully cyclised, the desired $\gamma$-lactam 374 would be formed. 


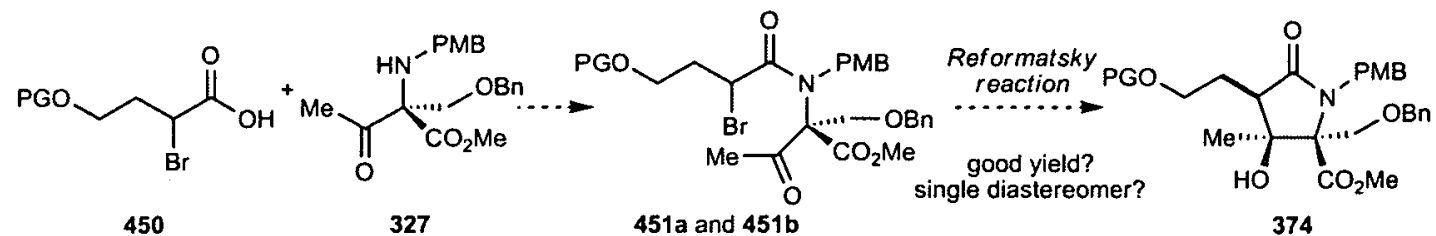

Scheme 4.40 Synthesis and cyclisation reaction of the real substrate

The formation of the amide was initially attempted via the ring opening of $\alpha$-bromo- $\gamma$-butyrolactone with amine 327 (Scheme 4.41). Amine 327 and butyrolactone 452 were reacted under reflux, ${ }^{173}$ but there was no reaction after 5 days. The reaction was also attempted in the presence of several bases (triisobutylamine, pyridine, KHMDS), in several solvents $\left(\mathrm{CH}_{3} \mathrm{CN}\right.$, xylene, THF) and varying reaction times, but there was either no reaction or only a complex mixture of products was obtained.

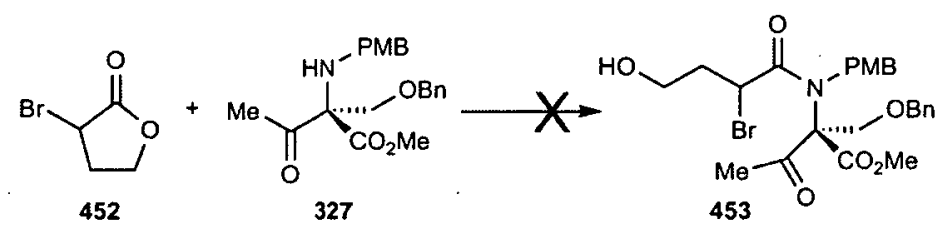

Scheme 4.41 Failure of the ring opening of $\alpha$-bromo- $\gamma$ butyrolactone 452

We centred then on the preparation of an $\alpha$-bromo acid for acylation of amine 327. As described previously in the reductive aldol approach (Scheme 3.36), ideally the alcohol would be protected with a PMB group. The PMB protected acid 456 was easily prepared in good yield from 1,4 butanediol (Scheme 4.42). ${ }^{174}$

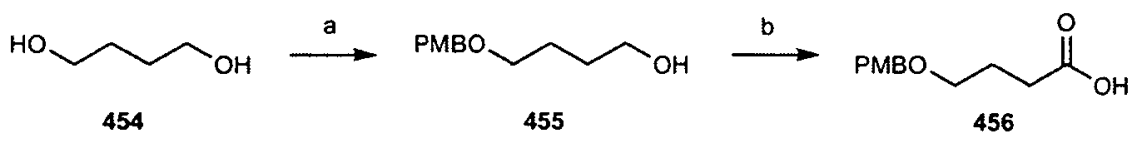

Reagents and conditions: (a) PMBCl (0.49 eq), $\mathrm{KOH}$ (1.05 eq), DMSO, $0{ }^{\circ} \mathrm{C}$ to $\mathrm{rt}, 14 \mathrm{~h}, 85 \%$; (b) PDC (3.5 eq), DMF, rt, $14 \mathrm{~h}, 71 \%$.

Scheme 4.42 Preparation of PMB protected acid 456

Treatment of acid 456 with bromine ${ }^{175}$ or bromine and $\mathrm{PCl}_{3}{ }^{176}$ did not afford the desired $\alpha$-brominated acid (457). The cleavage of the PMB group occurred instead, 
due to the $\mathrm{HBr}$ released in the reaction. $\alpha$-Bromination of carboxylic acids can also be achieved via the formation of the acid chloride. ${ }^{177,178}$ However, when acid 456 was treated with thionyl chloride, followed by the addition of bromine, the cleavage of the PMB group also occurred. The use of acid 456 was therefore abandoned (Scheme 4.43).

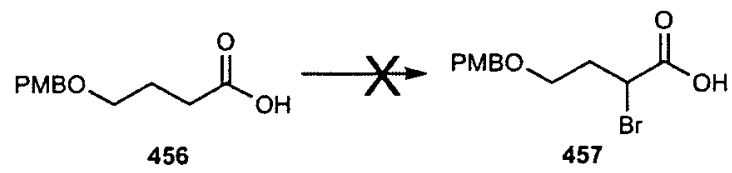

\section{Scheme 4.43 Failure of the $\alpha$-bromination of acid 456}

Acid 459, containing a TBDPS protecting group on the alcohol, was next prepared (Scheme 4.44). ${ }^{179}$ Monoprotection of 1,4-butanediol (454) was followed by oxidation of 458 with PDC, which afforded acid 459 in moderate yield. The bromination was attempted employing the same procedures: acid 459 was reacted with bromine or bromine and $\mathrm{PCl}_{3}$ in $\mathrm{Et}_{2} \mathrm{O}$ or $\mathrm{CCl}_{4}$ and also via the formation of the acid chloride, followed by reaction with bromine or NBS. ${ }^{177}$ All attempts to brominate on the $\alpha$-position of the acid failed to furnish the desired acid $\mathbf{4 6 0}$.

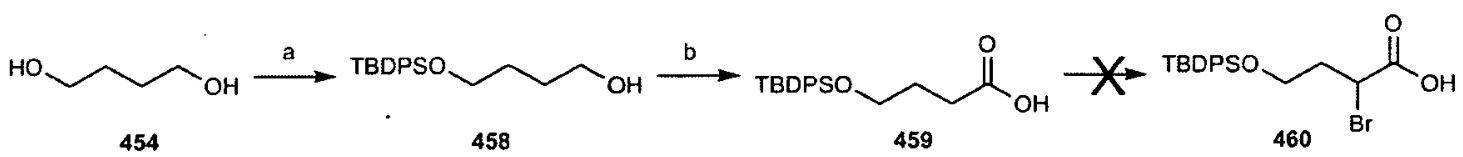

Reagents and conditions: (a) $n$-BuLi ( $0.33 \mathrm{eq})$, TBDPSCl ( $0.33 \mathrm{eq})$, THF, $-78^{\circ} \mathrm{C}$ to rt, $2 \mathrm{~h}, 97 \%$; (b) PDC (3.5 eq), DMF, $0{ }^{\circ} \mathrm{C}$ to rt, $14 \mathrm{~h}, 51 \%$.

Scheme 4.44 Unsuccessful preparation of brominated acid 460

Bromination of the acid was not being achieved; therefore, we decided to attempt the bromination on the aldehyde. Aldehyde 461 was prepared in good yield from alcohol 458 via a Swern oxidation (Scheme 4.45$)^{61}$

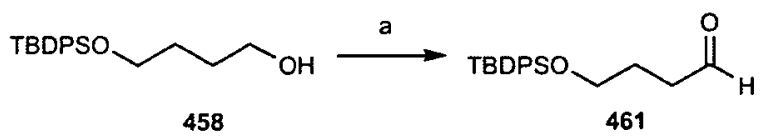

Reagents and conditions: (a) $(\mathrm{COCl})_{2}(2.1 \mathrm{eq})$, DMSO (4.2 eq), $\mathrm{Et}_{3} \mathrm{~N}$ (4.2 eq), $\mathrm{CH}_{2} \mathrm{Cl}_{2},-78^{\circ} \mathrm{C}, 3 \mathrm{~h}, 79 \%$.

Scheme 4.45 Swern oxidation of alcohol 458 
Aldehyde 461 was reacted with bromine in $\mathrm{Et}_{2} \mathrm{O}, \mathrm{CCl}_{4}$ or dioxane, but no reaction occurred. However, when the reaction was carried out in a mixture of $\mathrm{Et}_{2} \mathrm{O}$ and dioxane, ${ }^{180}$ brominated aldehyde 463 was successfully obtained (Scheme 4.46). This successful bromination procedure was also carried out with acid 459 , so that the oxidation step would be avoided, but with no success.

After bromination of 461, the crude product was employed without further purification in the oxidation reaction to acid 463, using $\mathrm{KMnO}_{4}$, in $t-\mathrm{BuOH}$ and a $\mathrm{NaH}_{2} \mathrm{PO}_{4}$ buffer. ${ }^{172}$ The yield of the oxidation was low due to partial cleavage of the TBDPS group. Consequently, the overall yield for the two-step sequence was $38 \%$.

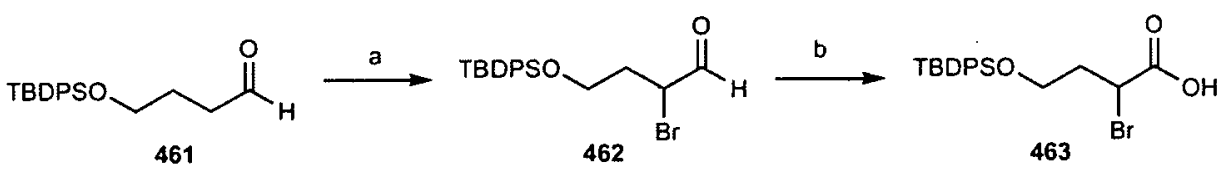

Reagents and conditions: (a) $\mathrm{Br}_{2}(1 \mathrm{eq})$, dioxane, $\mathrm{Et}_{2} \mathrm{O}, 0^{\circ} \mathrm{C}$ to $\mathrm{rt}, 2 \mathrm{~h}$;

(b) $\mathrm{KMnO}_{4}(6 \mathrm{eq}), 5 \% \mathrm{NaH}_{2} \mathrm{PO} 4, t$-BuOH, rt, $3 \mathrm{~h}, 38 \%$ in two steps.

\section{Scheme 4.46 Bromination and oxidation of aldehyde 461}

With acid 463 in hand, we next centred on the coupling with amine 327 . The acid chloride of 463 was prepared and transferred into a solution of amine 327 and triisobutylamine (Scheme 4.47). ${ }^{121}$ The two expected diastereomers of the amide (464a and 464b) were obtained in an overall $73 \%$ yield, and in approximately a 1.2:1 ratio.

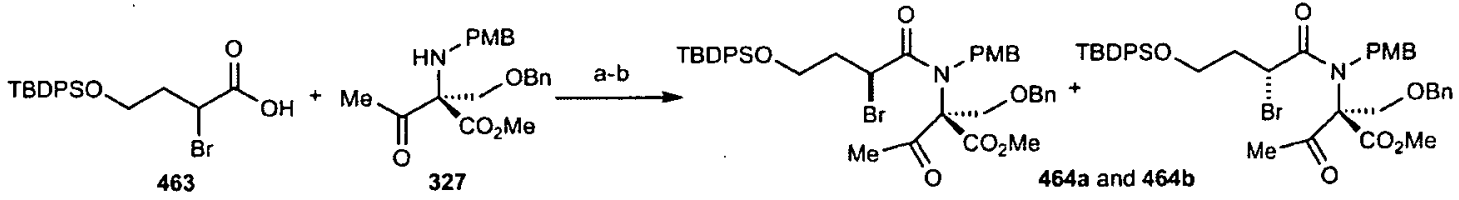

Reagents and conditions: (a) $(\mathrm{COCl})_{2}(11 \mathrm{eq}), \mathrm{DMF}$ (cat), $\mathrm{CH}_{2} \mathrm{Cl}_{2}, 0^{\circ} \mathrm{C}$ to $\mathrm{rt}, 1 \mathrm{~h}$; (b) amine 327, triisobutylamine (1.2 eq), $\mathrm{CH}_{2} \mathrm{Cl}_{2}, 0{ }^{\circ} \mathrm{C}$ to $\mathrm{rt}, 14$ h, $73 \%$ ( $40 \%$ and $33 \%)$.

Scheme 4.47 Synthesis of amides $464 a$ and $464 b$

The $\alpha$-bromo carboxylic acid containing an acetate protecting group (468) was also prepared, and it would be used in the study of the cyclisation reaction. The 
acetate monoprotection of $\mathbf{4 5 4}$ was achieved, although in low yield, using $\mathrm{Ac}_{2} \mathrm{O}$ and $\mathrm{CeCl}_{3}{ }^{181}$ The yield of this reaction was not optimised. A PCC oxidation of 465 afforded aldehyde 466 in $65 \%$ yield (Scheme 4.48 ).

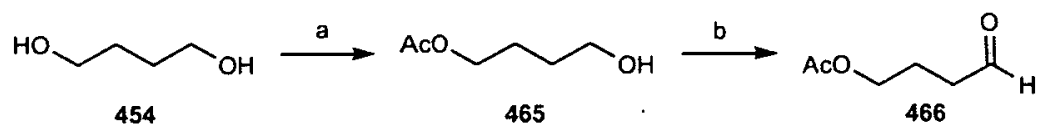

Reagents and conditions: (a) $\mathrm{Ac}_{2} \mathrm{O}(10 \mathrm{eq}), \mathrm{CeCl}_{3}(0.1 \mathrm{eq})$, THF, rt, $16 \mathrm{~h}, 43 \%$; (b) PCC (1.5 eq), $\mathrm{CH}_{2} \mathrm{Cl}_{2}, \mathrm{rt}, 1.5 \mathrm{~h}, 65 \%$.

Scheme 4.48 Synthesis of aldehyde 466

Aldehyde 466 was successfully brominated using $D L$-Proline and $\mathrm{NBS}$ in $\mathrm{CH}_{2} \mathrm{Cl}_{2}$ (Scheme 4.49). Oxidation to the carboxylic acid (468) was achieved in good yield using the same $\mathrm{KMnO}_{4}$ protocol.

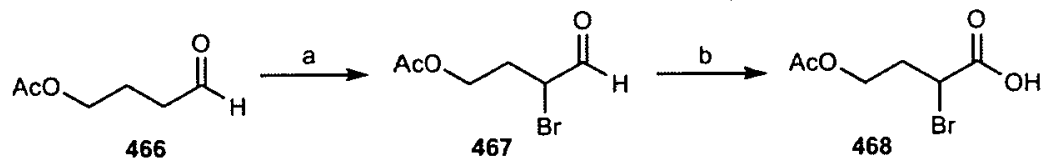

Reagents and conditions: (a) DL-Proline ( $0.3 \mathrm{eq}$ ), NBS (1.3 eq), $\mathrm{CH}_{2} \mathrm{Cl}_{2}$, rt, 4 h, 75\%; (b) $\mathrm{KMnO}_{4}$ (6 eq), 5\% $\mathrm{NaH}_{2} \mathrm{PO}_{4}, t-\mathrm{BuOH}, \mathrm{rt}, 3 \mathrm{~h}, 70 \%$.

\section{Scheme 4.49 Bromination and oxidation of 466}

The amide coupling was performed under the same conditions employed for the rest of amide couplings (Scheme 4.50). However, this reaction proved troublesome, because although amides 469 were obtained, complete purification of the two diastereomers was not achieved. Therefore, it was not possible to attempt the cyclisation reaction on these substrates.

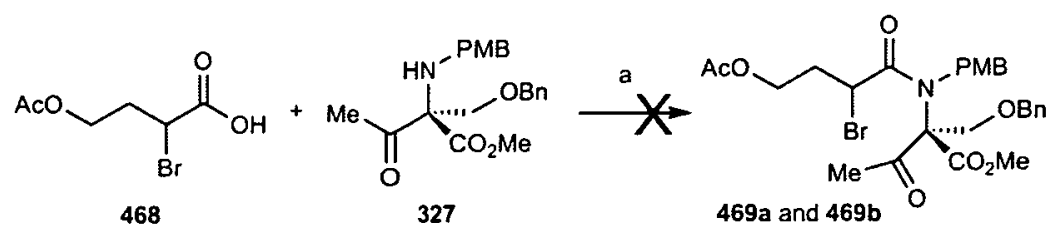

Reagents and conditions: (a) $(\mathrm{COCl})_{2}(11 \mathrm{eq}), \mathrm{DMF}$ (cat), $\mathrm{CH}_{2} \mathrm{Cl}_{2}, 0^{\circ} \mathrm{C}$ to $\mathrm{rt}, 1 \mathrm{~h}$; (b) amine 327 , triisobutylamine (1.2 eq), $\mathrm{CH}_{2} \mathrm{Cl}_{2}, 0^{\circ} \mathrm{C}$ to $\mathrm{rt}, 14 \mathrm{~h}$.

Scheme 4.50 Unsuccessful amide coupling 
The cyclisation reactions with the TDBPS derivatives $464 \mathrm{a}$ and $464 \mathrm{~b}$ were then investigated. Each diastereomer was treated independently with $\mathrm{SmI}_{2}$ in $\mathrm{THF}$, using from 2.2 to 12 equivalents, and at different temperatures (room temperature, $0{ }^{\circ} \mathrm{C}$ or $-40^{\circ} \mathrm{C}$ ). Both diastereomers had a similar and a reasonably high reactivity, since both substrates reacted at $0{ }^{\circ} \mathrm{C}$ and also at lower temperatures $\left(-20^{\circ} \mathrm{C}\right)$. However, the cyclisation product was not obtained and the only isolated product was amide $\mathbf{4 7 0}$ $(40 \%)$, as well as traces of a compound that was tentatively characterised by ${ }^{1} \mathrm{H}$ NMR analysis as the debrominated starting material (471) (Scheme 4.51).

The reactivity of these compounds was comparable to the most reactive diastereomer of the model substrate $\mathbf{4 2 0 b}$ or to the iodide derivatives, which reacted at lower temperatures than $\mathbf{4 2 0 a}$ but forming only traces of the cyclised product. Therefore, this example also contributed to the idea that a higher reactivity meant a faster and more uncontrollable reaction, which did not allow the cyclisation to the $\gamma$-lactam.

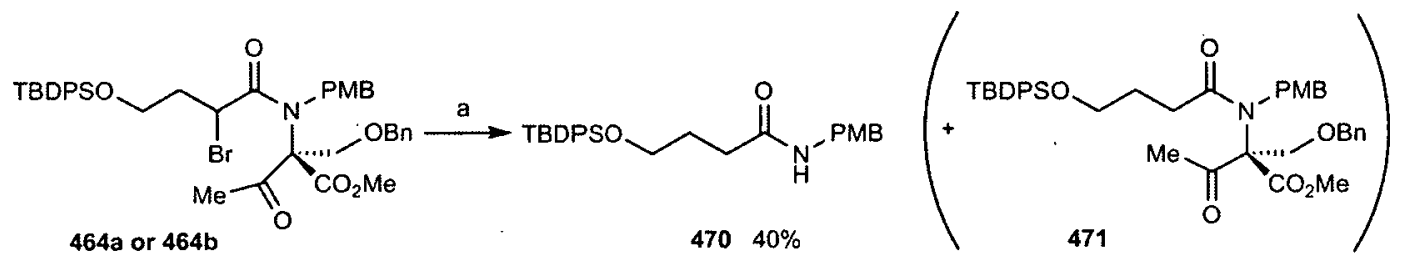

Reagents and conditions: (a) $\mathrm{SmI}_{2}(12 \mathrm{eq}), \mathrm{THF}, 0^{\circ} \mathrm{C}, 1 \mathrm{~h}, 40 \%$.

Scheme 4.51 Unsuccessful cyclisation of amides 464a and 464b

\subsection{Conclusions}

In this approach we aimed to utilise a Reformatsky cyclisation of an $\alpha$-halo amide to obtain the $\gamma$-lactam of 1 , having the side chain at $\mathrm{C} 2$ already in place. Initially, a model system of the $\alpha$-bromo amide substrate was prepared and employed in the investigation of the reaction. The Reformatsky cyclisation employing different metals ( $\mathrm{Zn}, \mathrm{Rh}, \mathrm{Ni}, \mathrm{Co}$ and $\mathrm{Cr}$ ) was attempted but the cyclised product was not obtained. As the precedent in the literature indicated, $\mathrm{SmI}_{2}$ turned out to be the best reagent for this reaction, affording the desired cyclised product and as a single diastereomer, although in low yield. The fact that the cyclised product was formed only under the $\mathrm{SmI}_{2}$ conditions was attributed to the known chelating ability of $\mathrm{Sm}^{3+}$, 
which would make possible the enolate addition into the carbonyl of the methyl ketone.

After a thorough optimisation study, the yield was slightly improved to $40 \%$, but the formation of the main by-product was not avoided. From the two diastereomers of the substrate, only one afforded the cyclisation product. The other one reacted faster and at lower temperatures, but to yield only traces of the cyclised product. The effect of the nature of the halide was also investigated, but the iodo- derivatives were slightly more reactive and therefore afforded the cyclised product in lower yield.

The feasibility of the reaction with a substrate containing the required functionalities to obtain 1 was next studied. Efforts were made in the synthesis of several $\alpha$-bromo amides. The cyclisation of the TBDPS derivative 464 was attempted, but the desired cyclised product was not obtained, and the amide resulting from a $\mathrm{C}-\mathrm{N}$ bond cleavage was the only isolated product.

We concluded that the $\mathrm{SmI}_{2}$ mediated Reformatsky reaction would either not progress at all at low temperatures or it would be too fast, yielding mainly the undesired by-product. At this stage, this approach was abandoned and work began on the next strategy. 


\section{Formal synthesis of salinosporamide $A^{i}$}

\subsection{Aims}

As described previously, our desire was to incorporate the reductive aldol methodology developed within our group ${ }^{65-67}$ into a synthetic route to our natural product. We envisaged that a precursor such as 102 could be cyclised employing our methodology into $\gamma$-lactam 178, and this intermediate could be converted into our final target $\mathbf{1}$ in only a few straightforward transformations.

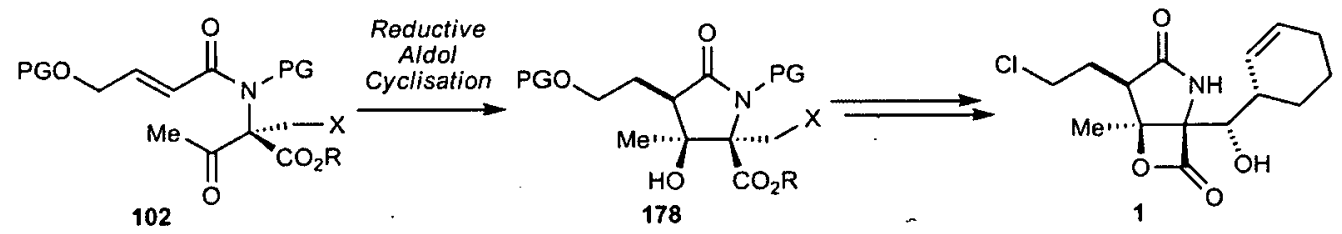

Scheme 5.1 Application of the reductive aldol reaction into the synthesis of 1

We had already undertaken investigations into the application of this approach into our synthetic route, which were described in Chapter 3. In these first studies, the $\alpha, \beta$-unsaturated amide substrates for the cyclisation reaction were prepared from racemic amine $\mathbf{3 0 0}$ (Scheme 3.35). All attempts at the cyclisation reaction afforded a complex mixture of products and its analysis proved to be very difficult.

It was decided to reinvestigate the reductive aldol approach. On this occasion, we would utilise amine 327 to prepare the $\alpha, \beta$-unsaturated substrates. This amine was utilised by Corey and co-workers in the synthesis of 1 , forming part of the acrylamide substrate of the cyclisation step that successfully afforded the $\gamma$-lactam core of 1 , with very good yield and diastereoselectivity. ${ }^{22,}{ }^{26} \mathrm{We}$ had also employed amine 327 during the investigation of the Reformatsky cyclisation approach (Chapter 4). In this study a positive influence of the stereocentre of amine 327 was also observed, in that the cyclised product of the model substrate was achieved as a single diastereomer.

\footnotetext{
${ }^{i}$ Work done in collaboration with Leszek Rupnicki, who developed the reductive aldol cyclisation-lactonisation reaction (Section 5.3).
} 
We aimed to find out whether the use of amine 327 could also be beneficial in the reductive aldol methodology, facilitating the cyclisation and hopefully inducing diastereoselectivity in the reaction. In addition, unlike when this approach was first investigated, the $\mathrm{Co}$ - and Ni-catalysed methodologies were now completely developed, having been applied to a broader range of substrates. ${ }^{65-67}$ Therefore we hoped to successfully utilise this reaction in our route to salinosporamide A.

The Co- and Ni-catalysed methodologies have afforded promising results in the formation of 5-membered lactams. In particular we should highlight the cyclisation of amide 292d, containing the fumaric acid ethyl ester, which afforded $\gamma$-lactam 293d in good yield and high syn-diastereoselectivity (Scheme 5.2). This example proved the high chemoselectivity of this methodology, because product 472 , resulting from cyclisation $\alpha$ - to the ester instead of $\alpha$ - to the amide was not observed. The formation of 293d was especially encouraging, since the ethyl ester side chain could potentially be converted into the chloroethyl side chain at $\mathrm{C} 2$ of salinosporamide $\mathrm{A}$.
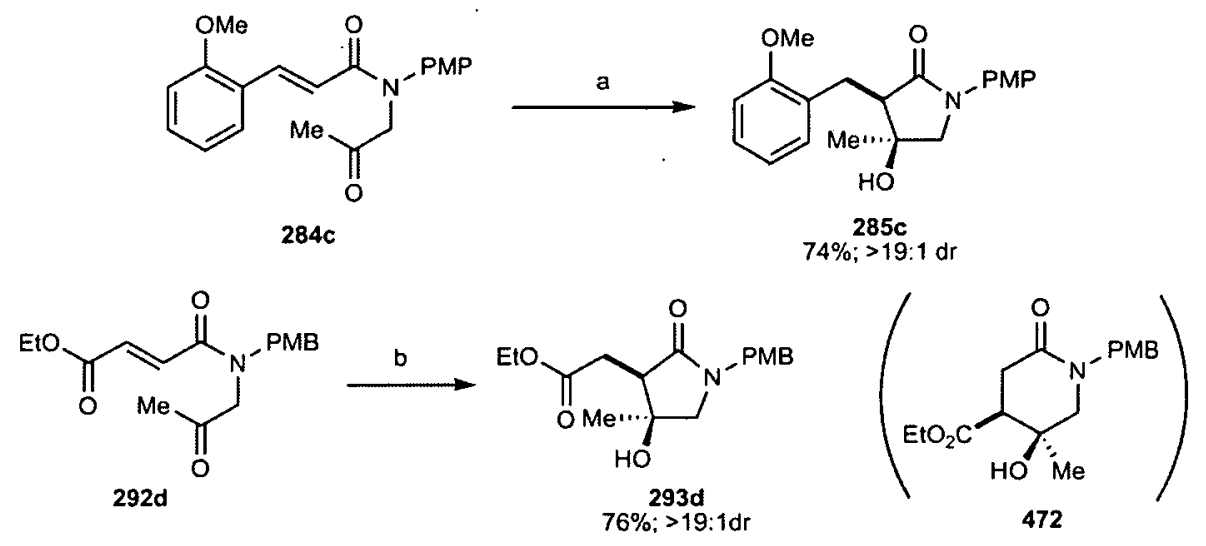

Reagents and conditions: (a) $\mathrm{Co}(\mathrm{acac})_{2} \cdot 2 \mathrm{H}_{2} \mathrm{O}$ (5 mol\%), $\mathrm{Et}_{2} \mathrm{Zn}$ (2 eq), $\mathrm{THF} /$ hexane; (b) Ni(acac) $)_{2}(5 \mathrm{~mol} \%), \mathrm{Et}_{2} \mathrm{Zn}(2 \mathrm{eq}), \mathrm{THF} /$ hexane.

Scheme 5.2 Relevant examples of successful cyclisations to 5-membered ring rlactams

In conclusion, the reductive aldol approach was re-examined in order to employ in this occasion an $\alpha, \beta$-unsaturated amide prepared from amine 327. It was hoped that we could find the optimal substrate and catalyst which would allow cyclisation, 
and hence provide the $\gamma$-lactam core of our natural product in good yield and with the desired relative stereochemistry.

\subsection{Retrosynthetic analysis}

The retrosynthetic analysis in this new study incorporates amine $\mathbf{3 2 7}$ into the retrosynthetic analysis outlined in Chapter 3 (Scheme 3.34) and it is briefly outlined in Scheme 5.3. $\gamma$-Lactam 374 represents the desired product of the reductive aldol cyclisation of $\alpha, \beta$-unsaturated amide 473. This amide could be obtained from the coupling of amine 327 with an $\alpha, \beta$-unsaturated acid represented by 298 .

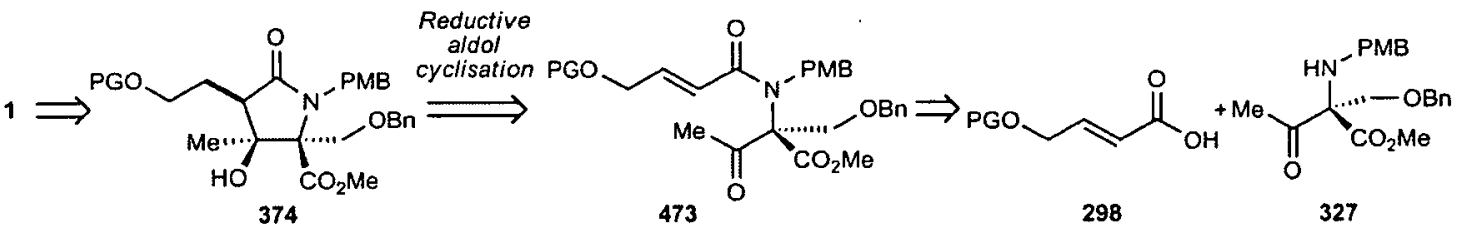

Scheme 5.3 Retrosynthetic analysis of the reductive aldol cyclisation approach using amine 327

If amide 473 cyclises to yield the desired $\gamma$-lactam 374, this intermediate could be easily transformed into triol $\mathbf{2 6}$, the same intermediate we were aiming to obtain during the first investigation of the reductive aldol approach (Chapter 3, Scheme 3.36), which is an advanced intermediate in Corey's synthesis ${ }^{2}$ and also in Pattenden's synthesis. ${ }^{29}$ Therefore, if the proposed route was successful, we would have developed a formal total synthesis of 1 .

\subsection{Preliminary studies on the reductive aldol cyclisation reaction.}

Initial work focused on the synthesis of the amide represented by 473 (Scheme 5.3). This amide would allow a thorough study of the reductive aldol cyclisation reaction, investigation that was carried out by another $\mathrm{PhD}$ student of the Lam group, Leszek Rupnicki and it is explained in this section. 
Amine 327 was prepared following the method described in Chapter 4. The coupling of this amine with acid 325, containing a PMB protecting group on the alcohol (for synthesis see Chapter 3, Scheme 3.52) was attempted, but all efforts were unsuccessful. The attempts of formation of the acid chloride of $\mathbf{3 2 5}$ followed by coupling with amine 327 also resulted in cleavage of the PMB group. Investigations into the use of coupling reagents also proved fruitless, presumably due to the low nucleophilicity and bulkiness of amine 327 (Scheme 5.4).

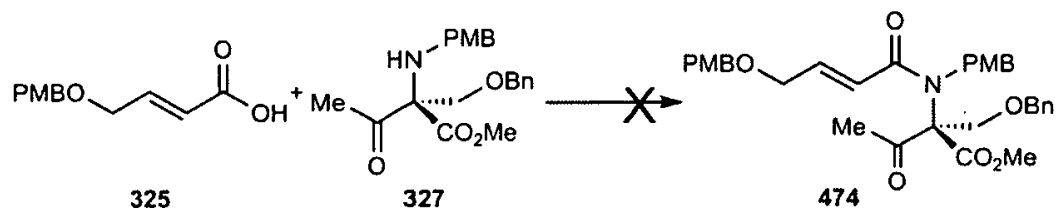

Scheme 5.4 Unsuccessful preparation of amide 474

Bearing in mind the successful cyclisation of 292d (Scheme 5.2), it was next decided to couple amine $\mathbf{3 2 7}$ to the commercially available fumaric acid monoethyl ester 479. However a potential problem was whether the ethyl ester on the $\mathrm{C} 2$ side chain of $\mathbf{4 7 6}$ could be, later in the synthesis, selectively reduced over the methyl ester at C4 (Scheme 5.5).

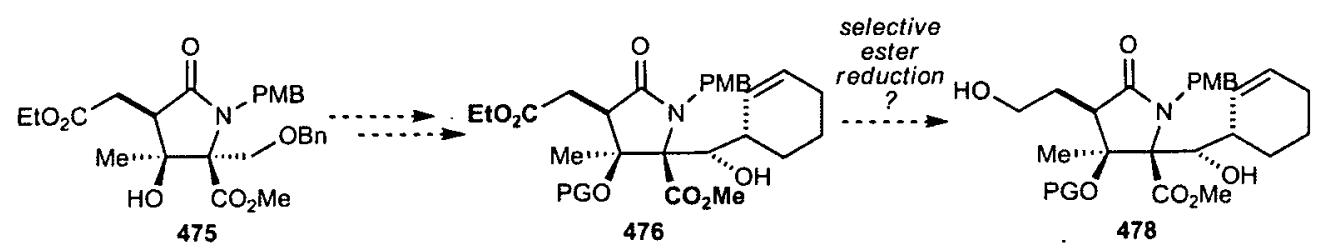

Scheme 5.5 Possibly problematic selective ethyl ester reduction

In spite of this, it was decided to prepare the substrate and investigate the cyclisation reaction. Amide $\mathbf{4 8 1}$ was successfully obtained via addition of a solution of acid chloride $\mathbf{4 8 0}$ into a solution of amine 327 and diisopropylethyl amine in $\mathrm{CH}_{2} \mathrm{Cl}_{2}$ (Scheme 5.6). 


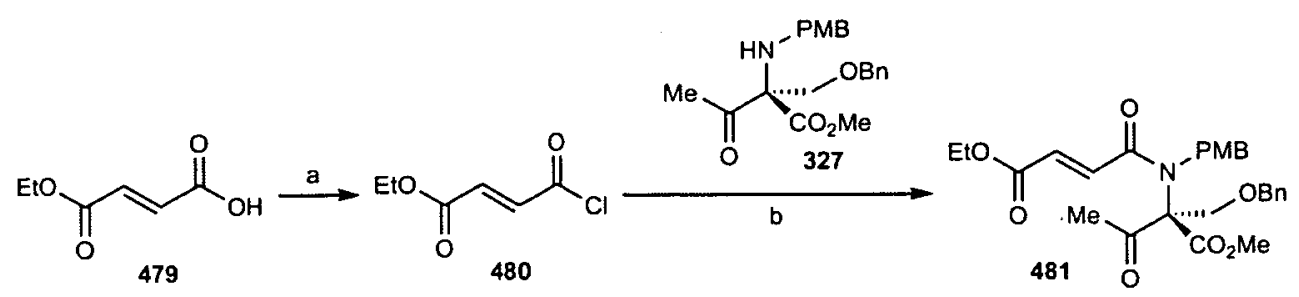

Reagents and conditions: (a) $(\mathrm{COCl})_{2}(1.2 \mathrm{eq}), \mathrm{DMF}$ (cat), $\mathrm{CH}_{2} \mathrm{Cl}_{2}, 0^{\circ} \mathrm{C}$ to rt, $2 \mathrm{~h}$; (b) 327, $i-\mathrm{Pr}_{2} \mathrm{EtN}$ (1.7 eq), $\mathrm{CH}_{2} \mathrm{Cl}_{2}, 0^{\circ} \mathrm{C}$ to rt, $14 \mathrm{~h}, 95 \%$.

Scheme 5.6 Preparation of amide 481

The cyclisation of amide 481 employing the reductive aldol methodology developed within the group was then investigated. The observed stereochemical outcome (syn-selectivity) of this methodology can be explained by preferential formation of the Z-enolate, along with a chelated chairlike Zimmerman-Traxler ${ }^{182}$ transition state. ${ }^{65-67}$ It was expected to observe a high syn-diastereoselectivity in the cyclisation of $\mathbf{4 8 1}$ and also to find out whether the quaternary stereocentre had an effect on the absolute configuration of the two newly formed stereocentres (Scheme 5.7).

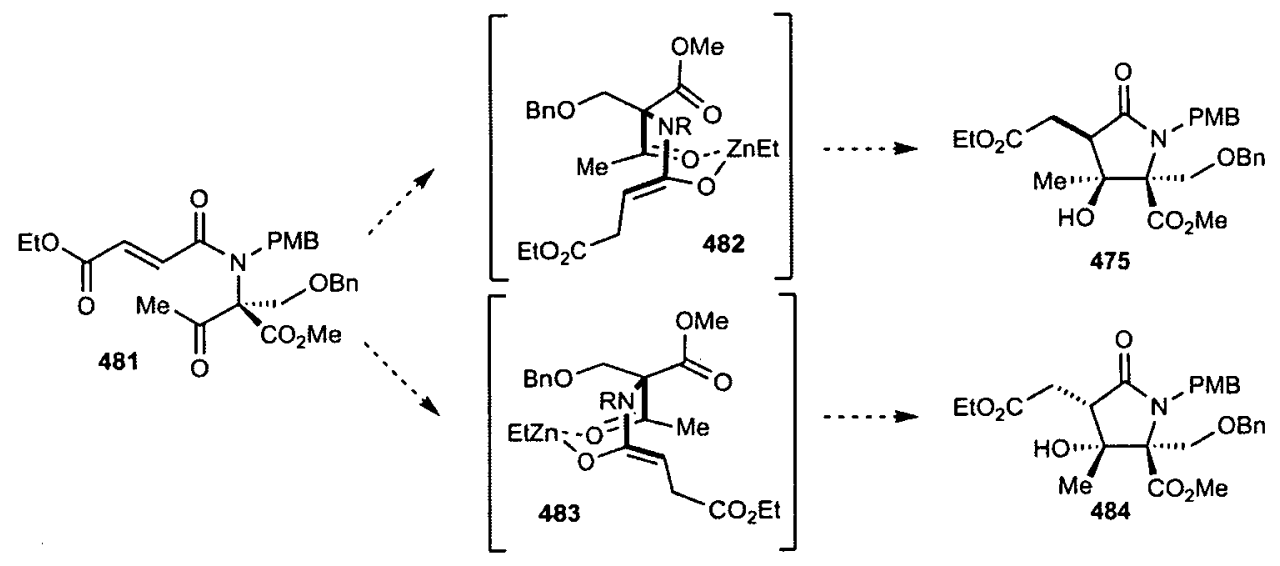

Scheme 5.7 Desired transition state of the cyclisation reaction

The catalyst could potentially bind, as well as to the enolate and the methyl ketone oxygens, to the carbonyl of the methyl ester forming 485 , which would yield the desired diastereomer 475 (Scheme 5.8). However, it could also bind to the benzyl ether (486) and therefore form the undesired diastereomer 484. Ideally, formation of chelate 485 would be preferred and therefore product 475 would be obtained as the 
major diastereomer, at the expense of the alternative syn-diastereomer 484 . However, the diastereoselectivity of this reaction was difficult to predict, since both proposed transition states could be formed in competition, or $\mathbf{4 8 6}$ could be the preferred one, and also neither of them could be occurring.

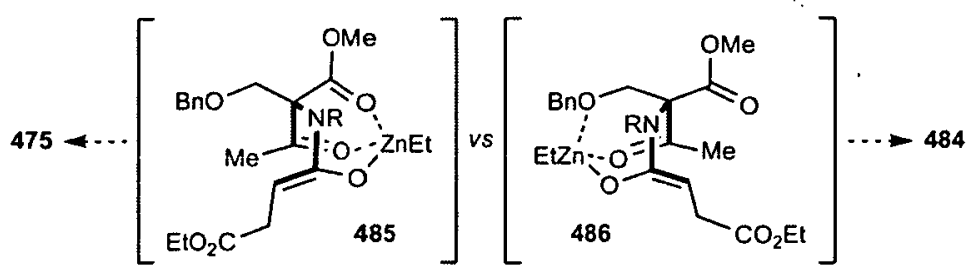

Scheme 5.8 Influence of the quaternary stereocentre

Initially, the cyclisation reaction was attempted using the standard $\mathrm{Co}$ and $\mathrm{Ni}$ conditions, but when 481 was exposed to $\mathrm{Et}_{2} \mathrm{Zn}$ and $\mathrm{Ni}(\mathrm{acac})_{2}$ or $\mathrm{CoCl}_{2}$ and $\mathrm{Cy}_{2} \mathrm{PPh}$ only an inseparable mixture of compounds was obtained (Table 5.1, entry 1 and 2). When $\mathrm{Co}(\mathrm{acac})_{2} \cdot 2 \mathrm{H}_{2} \mathrm{O}$ was used, hardly any reaction was observed at all (entry 3 ). $\mathrm{Et}_{2} \mathrm{Zn}$ was replaced with $\mathrm{Et}_{3} \mathrm{Al}$ but again, a very complex mixture of compounds was obtained (entry 4 and 5).

The influence of phosphine ligands was next investigated. The use of $\mathrm{CoCl}_{2}$ and $\mathrm{PPh}_{3}$ proved unsuccessful, and unreacted starting material was recovered (entry 6). The combination of $\mathrm{CoCl}_{2}$ and DPPF offered no improvement, since it afforded a complex mixture of compounds (entry 7). However, it was found that commercially available nickel-phosphine complexes were effective precatalysts. Complex $\left(\mathrm{Ph}_{3} \mathrm{P}\right)_{2} \mathrm{NiBr}_{2}$ was able to promote the reductive cyclisation of 481 under dilute conditions to give bicyclic fused $\gamma$-lactone- $\gamma$-lactam 489 containing the desired stereochemistry in $35 \%$ yield. Unfortunately, this compound was accompanied by a comparable amount of the alternative diastereoisomer 490 (entry 8). This result was slightly improved when using $\left(\mathrm{Me}_{3} \mathrm{P}\right)_{2} \mathrm{NiCl}_{2}$ and performing the reaction at an initial temperature of $-15^{\circ} \mathrm{C}$. In this case, the yield of 489 increased to $42 \%$ (entry 9 ). 


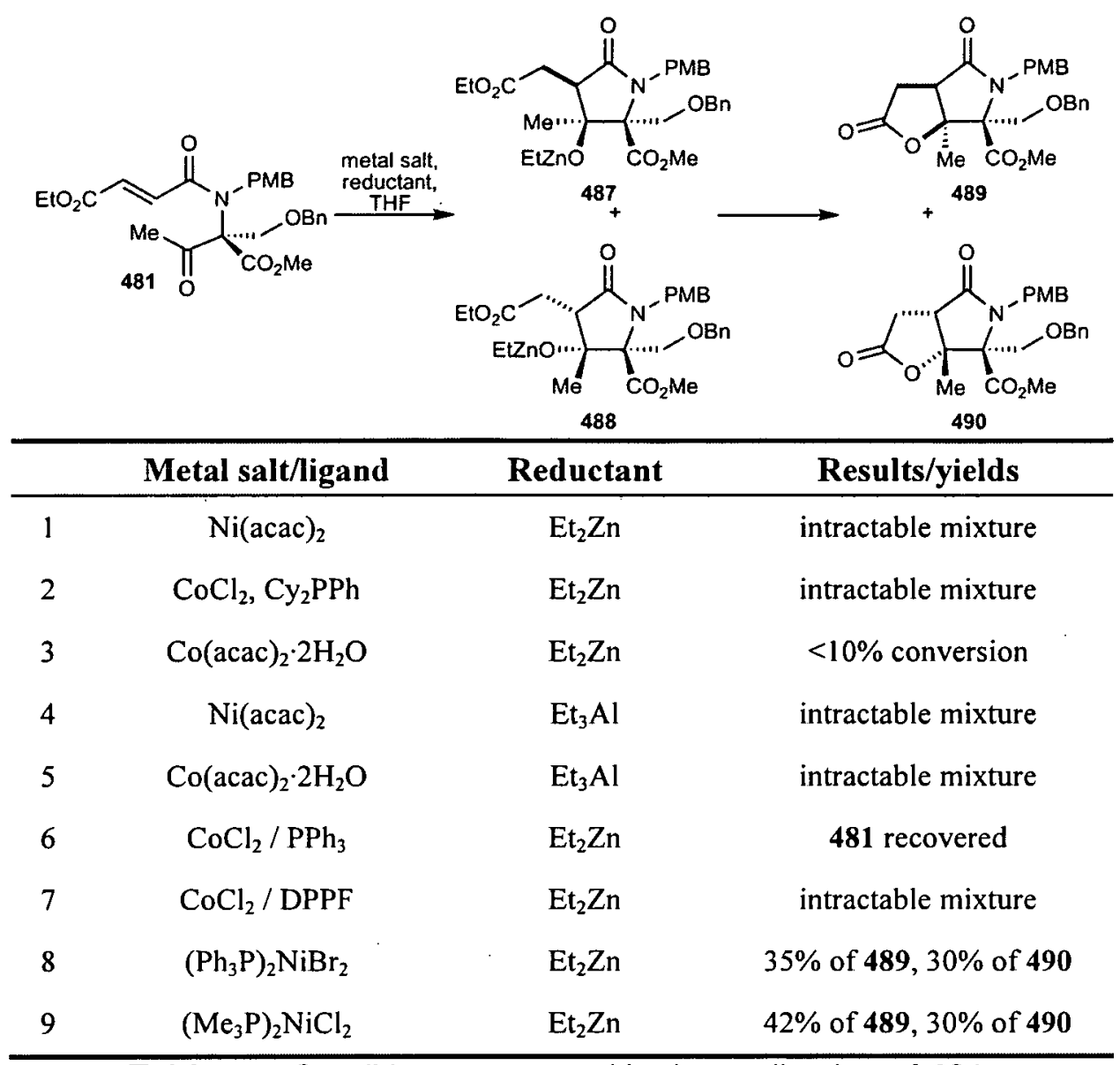

Table 5.1 Conditions attempted in the cyclisation of 481

The fact that both syn-diastereomers were obtained indicated that under these conditions, the pre-existing stereocentre in 327 was not effective in controlling the absolute configuration of the two new stereocentres formed in the reaction. The stereochemistry of diastereomer $\mathbf{4 9 0}$ was established by X-ray crystallography (Figure 5.1).

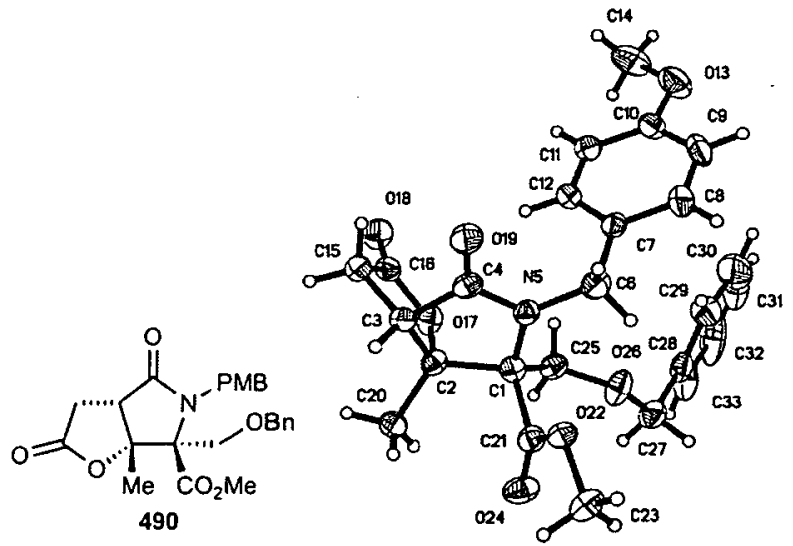

Figure $5.1 X$-ray crystallography of 490 
The lactones of $\mathbf{4 8 9}$ and $\mathbf{4 9 0}$ were formed as a result of the tertiary zinc alkoxides formed in the aldol reaction cyclising with the pendant ethyl esters. This lactonisation occurred in the $\mathrm{Ni}$-catalysed cyclisation of 6-membered lactams $290 \mathrm{n}$ and 2900 (Scheme 5.9), but this cyclisation had been observed only as a minor product $(<10 \%)$ in the cyclisation to the $\gamma$-lactam 292d (Scheme 5.2). Therefore, the lactonisation was unexpected in the cyclisation of our substrate 481. However, it turned out to be a useful bonus because it protected the tertiary alcohol of 489 towards subsequent transformations.

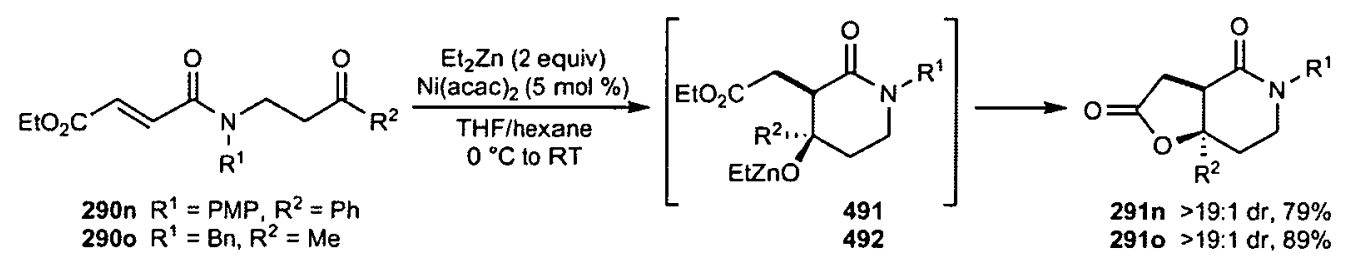

Scheme 5.9 Sequential reductive cyclisation-lactonisation

\subsection{Completion of the formal synthesis}

Once the reductive aldol cyclisation of $\mathbf{4 8 1}$ to afford the desired cyclised product 489 had been developed, we only needed to perform a few transformations to obtain intermediate 26. After separation of diastereomers 489 and 490 by column chromatography, the next step was the deprotection of the alcohol. This was achieved via a palladium-catalysed debenzylation, which afforded alcohol 493 in good yield (Scheme 5.10). This alcohol was crystalline and X-ray crystallography ${ }^{\mathrm{i}}$ of this compound allowed confirmation of its stereochemistry and therefore that of $\mathbf{4 8 9}$.

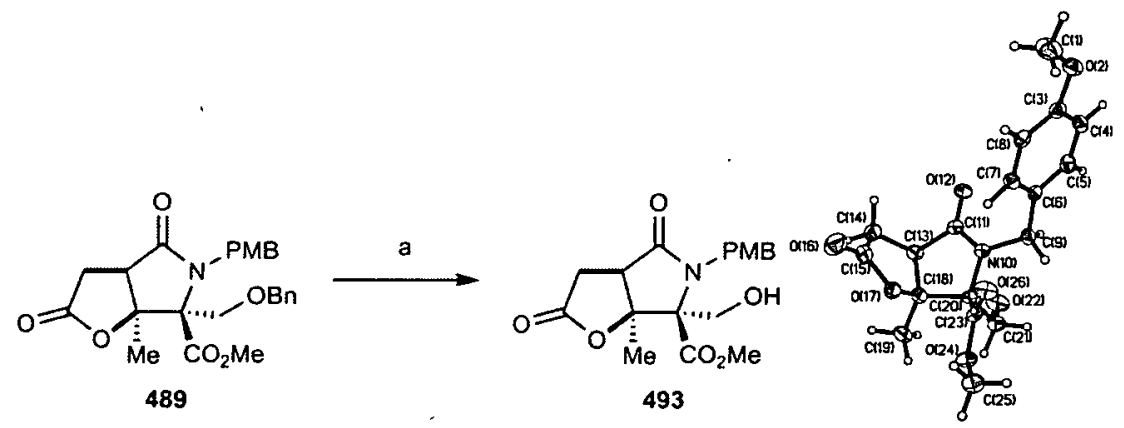

Reagents and conditions: (a) $\mathrm{Pd} / \mathrm{C}(10 \%)$, EtOH, $23^{\circ} \mathrm{C}, 12 \mathrm{~h}, 84 \%$

Scheme 5.10 Obtention of alcohol 493 and confirmation of its stereochemistry

'Crystal of 493 was obtained by Leszek Rupnicki 
Oxidation of alcohol 493 to aldehyde 494 was performed using Dess-Martin periodinane reagent. ${ }^{35}$ This aldehyde proved to be very unstable and therefore it had to be immediately reacted with 2-cyclohexenylzinc chloride, following Corey's procedure. $^{26}$ This step was readily performed, since we were already familiar with this transformation, which had been carried out during the first route we investigated for the synthesis of salinosporamide A (Chapter 2). Therefore, homoallylic alcohol 495 was successfully obtained with high diastereoselectivity (only one observable diastereoisomer by $1 \mathrm{H}$ NMR analysis). The overall yield for this two-step sequence was $61 \%$ (Scheme 5.11).
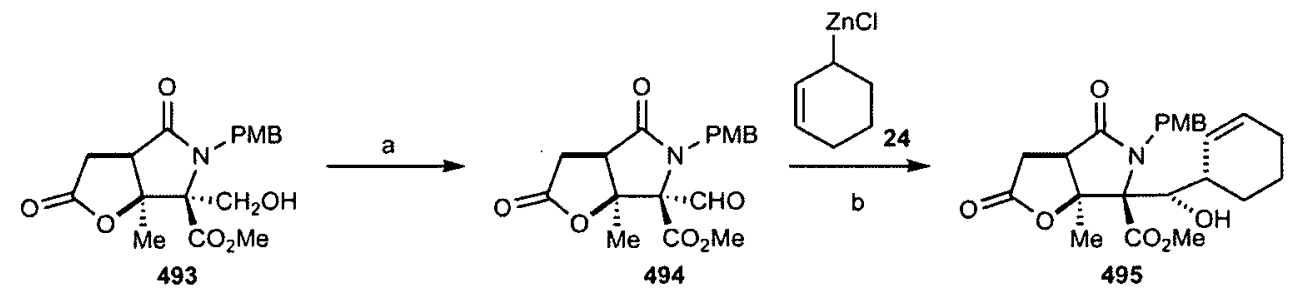

Reagents and conditions: (a) Dess-Martin periodinane (1.2 eq), $\mathrm{CH}_{2} \mathrm{Cl}_{2}$, rt, $1.5 \mathrm{~h}$; (b) 24 (2.5 eq), THF, $-78^{\circ} \mathrm{C}, 5 \mathrm{~h}, 61 \%$.

Scheme 5.11 Oxidation to the aldehyde and immediate insertion of the cyclohexenyl chain

Alcohol 495 was also crystalline and X-ray crystallography showed that the desired stereochemistry had been achieved in the insertion of the side chain (Figure 5.2).

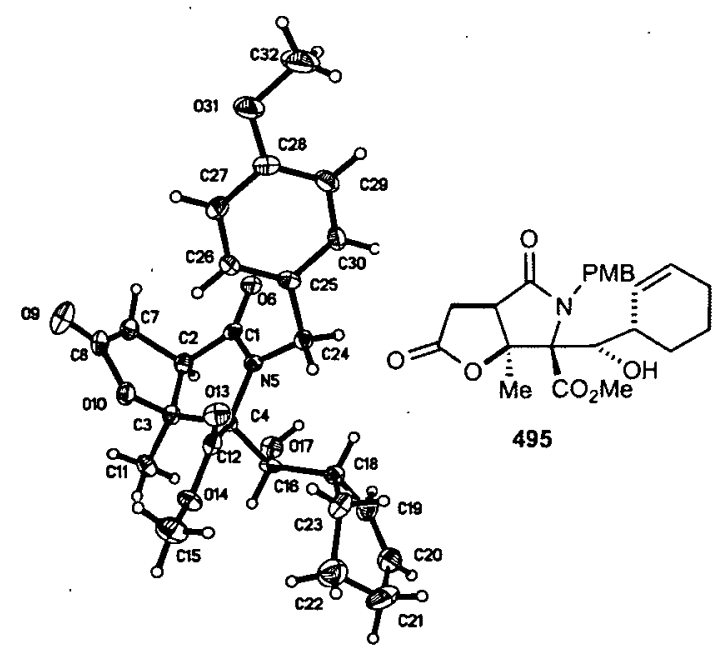

Figure $5.2 X$-ray crystallography of 495 
The last step to complete the formal synthesis involved the selective reduction of the lactone over reduction of the methyl ester. We were pleased to find out that the lactone of 495 was selectively reduced with $\mathrm{NaBH}_{4}$ to give triol 26 (Scheme 5.12), intermediate in the synthesis of Corey ${ }^{26}$ and Pattenden ${ }^{29}$ groups. A vast excess of $\mathrm{NaBH}_{4}$ was necessary to obtain a satisfactory yield, otherwise, a small fraction of unreacted starting material was recovered. The conversion of $\mathbf{2 6}$ into salinosporamide $\mathrm{A}$ is performed in four steps and has already been described by these groups.

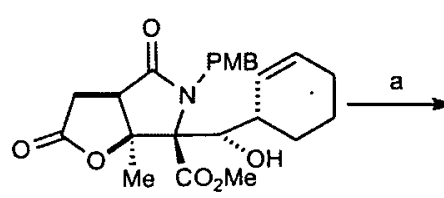

495

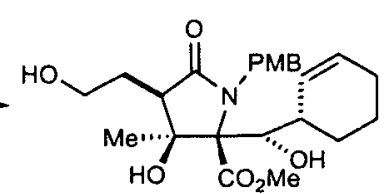

26

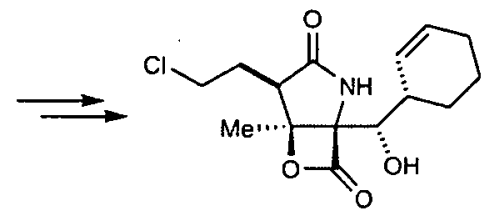

Reagents and conditions: (a) $\mathrm{NaBH}_{4}$ (45 eq), EtOH, $\mathrm{rt}, 14 \mathrm{~h}, 60 \%$.

Scheme 5.12 Final reductive ring-opening to triol 26

Interestingly, the NMR data we obtained for triol $\mathbf{2 6}$ displayed subtle but appreciable differences to those reported by Corey and co-workers for the same compound, ${ }^{26}$ most notably in the ${ }^{13} \mathrm{C}$ NMR chemical shifts. ${ }^{\mathrm{i}, \mathrm{ii}}$ However, comparison of our spectra with that obtained by Pattenden for the same compound ${ }^{29}$ showed good agreement. The most likely explanation for this observation is that the values of the ${ }^{13} \mathrm{C}$ NMR chemical shifts for triol 26 are concentration-dependent; both our and that obtained by Pattenden group ${ }^{13} \mathrm{C}$ NMR spectra were run at relatively low concentration, whereas the spectrum obtained by Corey was run at relatively high concentration.

\footnotetext{
${ }^{\mathrm{i}}$ Both the Corey group and the Pattenden group had kindly supplied us with a copy of their ${ }^{13} \mathrm{C}$ NMR spectrum

${ }^{i i}$ Both ${ }^{13} \mathrm{C}$ NMR data and spectrum included in the experimental section. The higher concentration of Corey's sample can also be observed.
} 


\subsection{Conclusions}

In this chapter the reductive aldol approach was re-examined. On this occasion, we employed for the preparation of the $\alpha, \beta$-unsaturated amides the amine used by Corey and co-workers (327) in the synthesis of 1. $\alpha, \beta$-Unsaturated amide 481 was prepared from monoethyl fumarate and amine 327, and it was discovered that it successfully cyclised when treated with $\left(\mathrm{Me}_{3} \mathrm{P}\right)_{2} \mathrm{NiCl}_{2}$ and $\mathrm{Et}_{2} \mathrm{Zn}$. The cyclisation reaction was followed by lactonisation due to the tertiary zinc alkoxide formed in the aldol reaction. The desired cyclised product was obtained in $42 \%$ yield and it was accompanied by a $30 \%$ yield of the alternative diastereomer. The lack of diastereoselectivity meant that the quaternary stereocentre of the amine was not effective in inducing stereoselection in the reaction.

The lactonisation step turned out to be beneficial, because an extra step for the protection of the tertiary alcohol formed was not necessary. This approach achieved the formation of the $\gamma$-lactam, installing the side-chain at C2 and with protection of the oxygen at C5 simultaneously, therefore saving several steps over alternative routes. The desired cyclised product was successfully transformed in four steps to an intermediate in the synthesis of $\mathbf{1}$ by Corey and co-workers, which had been converted into salinosporamide $\mathrm{A}$ in a further four steps. Consequently, a formal synthesis of 1 had been achieved.

Although very pleased with having been able to use the reductive aldol methodology developed in the group and with the success of the formal synthesis, the diastereoselectivity of the cyclisation reaction remains to be improved. Future work would involve more investigation to uncover alternative catalyst systems/reaction conditions that are able to promote the sequential reductive aldol cyclisation-lactonisation of 481 , in the hope of obtaining a highly diastereoselective reaction to give 489 at the expense of 490 .

At this point, our synthetic route to intermediate 26 was achieved in a $2.5 \%$ overall yield and over eleven steps, which represents a total synthesis of salinosporamide A with an overall yield of $1.4 \%$ over fiften steps. The comparison of synthetic routes is not completely obvious, since the starting materials employed and the compounds obtained in the reported syntheses can differ significantly. 
However, if our route is compared to the other enantioselective syntheses reported (Danishefsky, Langlois or Macherla's), it is clear that our route is already more efficient, since it achieves the synthesis of 1 over less steps and with a better overall yield (Table 5.2, entries 3, 5, 7 and 8).

If we compare our route with Corey's synthesis, which is the best reported synthesis of 1 so far, we observe that our synthetic route is 4 steps shorter, although the overall yield is notably lower (Table 5.2, entries 2 and 8). Nevertheless, if the diastereoselectivity of the key cyclisation step is improved, the overall yield of our route will increase significantly, and our synthesis of salinosporamide A will become as powerful as, if not more than, the one reported by Corey and co-workers.

\begin{tabular}{|c|c|c|c|c|}
\hline & Synthesis & Number of steps & Overall yield & $\begin{array}{c}\text { Asymmetric } \\
\text { /racemic }\end{array}$ \\
\hline 1 & $\begin{array}{c}\text { Corey } \\
\text { (Baylis-Hillman) }^{26}\end{array}$ & 18 & $8.7 \%$ & asymmetric \\
\hline 2 & 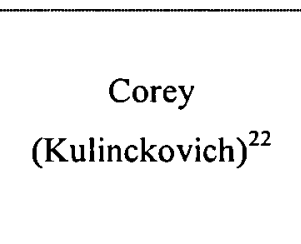 & $\begin{array}{c}18 \\
\text { (8 steps to } 29) \\
(14 \text { steps to } 26) \\
\text { (10 steps from } 18 \text { to } 26)\end{array}$ & $\begin{array}{c}9.1 \% \\
(27.0 \% \text { to } 29) \\
(16.8 \% \text { to } 26) \\
(47.7 \% \text { from } 18 \text { - to } 26)\end{array}$ & asymmetric \\
\hline 3 & Danishefsky $^{27}$ & 28 (from 37 ) & $1.8 \%($ from 37$)$ & asymmetric \\
\hline 4 & Pattenden $^{29}$ & 14 (from 52) & $11.5 \%$ (from 52) & racemic \\
\hline 5 & $\begin{array}{l}\text { Langlois (formal } \\
\text { synthesis) }\end{array}$ & $\begin{array}{c}8 \text { (from } 67 \text { to } 29) \\
\text { (18 steps from } 67 \text { to } 1)\end{array}$ & $\begin{array}{c}11.8 \% \text { (from } 67 \text { to } 29) \\
(4.0 \% \text { from } 67 \text { to } 1)\end{array}$ & asymmetric \\
\hline 6 & Romo $^{30}$ & 9 steps & $1.2 \%$ & racemic \\
\hline 7 & Macherla $^{28}$ & 24 steps & $0.2 \%$ & asymmetric \\
\hline 8 & $\begin{array}{l}\text { HWL (formal } \\
\text { synthesis) }\end{array}$ & $\begin{array}{c}11 \text { (to } 26) \\
\text { (15 steps to } 1) \\
\text { (7 steps from } 18 \text { to } 26 \text { ) }\end{array}$ & $\begin{array}{c}2.5 \% \text { (to } 26) \\
(1.4 \% \text { to } 1) \\
(8.8 \% \text { from } 18 \text { to } 26)\end{array}$ & asymmetric \\
\hline
\end{tabular}

Table 5.2 Comparison of the reported sintesis of salinosporamide $A$ with the HWL synthetic route

Another step in the synthesis we wish to modify is the installation of the cyclohexenyl side-chain. Practically all total syntheses of salinosporamide $A^{22,26,27}$, 29-32 reported so far employ the allylzinc reagent, as described by Corey and coworkers. ${ }^{26}$ Although this chemistry works very well, the preparation of this reagent 
requires access to an allylstannane, which is then subjected to a tin-lithium-zinc transmetallation sequence. In addition to employing a toxic reagent, this chemistry is atom-inefficient and somewhat experimentally inconvenient. Therefore, we intend to seek alternative methods to this chemistry involving more user-friendly precursors and reaction conditions. Particularly, we are interested in investigating the application to our system of the methodology reported by Tamaru and co-workers. ${ }^{183}$ They developed a regio- and stereoselective Ni-catalysed homoallylation of aromatic and aliphatic aldehydes using $\mathrm{Et}_{3} \mathrm{~B}$. The use of $\mathrm{Et}_{2} \mathrm{Zn}$ instead of $\mathrm{Et}_{3} \mathrm{~B}$ in the reaction with sterically congested aliphatic aldehydes and ketones afforded improved yields and similarly high regio- and stereoselectivities (Scheme 5.13).

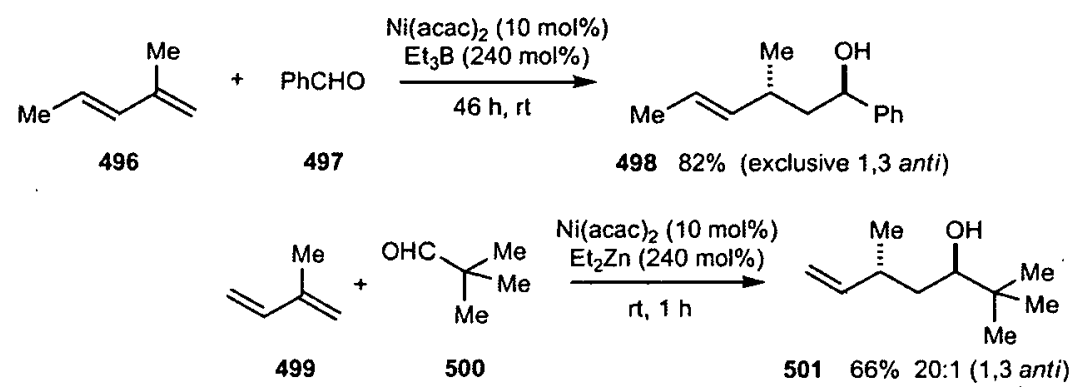

Scheme 5.13 Ni-Catalysed homoallylation of aldehydes with 1,3-dienes

The reaction with 1,3-cyclohexadiene was the only exception that resulted in an allylation, to yield $\mathbf{5 0 2}$ diastereoselectively and in good yield (Scheme 5.14). We therefore would like to investigate the application of this methodology to the insertion of the cyclohexenyl chain into aldehyde 494 .

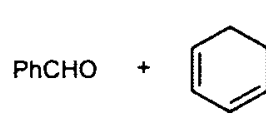

497<smiles>CO[C@]1(C=O)N(P)C(=O)C2CC(=O)O[C@@]21C</smiles>

494

142

142

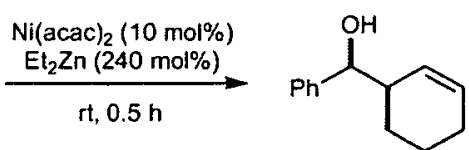

$50261 \% \quad 4: 1(1,2$ anti)<smiles>C1=CCCC=C1</smiles>

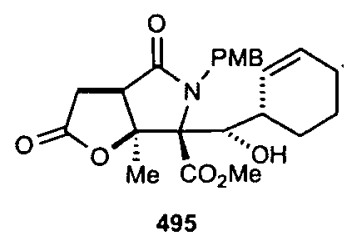

Scheme 5.14 Application of Tamaru's methodology 


\section{Experimental}

\subsection{General Information}

All reactions were carried out under a nitrogen atmosphere in oven-dried apparatus. Only in section 6.2.1, $\mathrm{CH}_{2} \mathrm{Cl}_{2}$ and $\mathrm{MeCN}$ were distilled from $\mathrm{CaH}_{2}$ and $\mathrm{Et}_{2} \mathrm{O}$ and $\mathrm{THF}$ were distilled from sodium with benzophenone as an indicator. In the rest of sections, $\mathrm{CH}_{2} \mathrm{Cl}_{2}, \mathrm{THF}, \mathrm{MeCN}$ and $\mathrm{MeOH}$ were dried and purified by passage through activated alumina columns using a solvent purification system from www.glasscontour.com. 'Petrol' refers to that fraction of light petroleum ether boiling in the range $40-60{ }^{\circ} \mathrm{C}$. Anhydrous DMF was purchased from Aldrich. Pyridine, $\mathrm{Et}_{3} \mathrm{~N}$ and diisopropylamine were distilled from $\mathrm{CaH}_{2}$. All other commercially available reagents were used as received. Thin layer chromatography (TLC) was performed on Merck DF-Alufoilien $60 \mathrm{~F}_{254} 0.2 \mathrm{~mm}$ precoated plates. Product spots were visualized by UV light at $254 \mathrm{~nm}$, and subsequently developed using vanillin, potassium permanganate or ceric ammonium molybdate solution as appropriate. The compounds were purified by wet flash column chromatography using silica gel (Fisher Scientific $60 \AA$ particle size $35-70$ micron) under a positive pressure. ${ }^{184}$ Melting points were recorded on a Gallenkamp melting point apparatus and are uncorrected. Infra-red spectra were recorded on a Jasco FT/IR-460 Plus instrument as a thin film on sodium chloride plates. The wavelengths of maximum absorbance $\left(v_{\max }\right)$ are quoted in $\mathrm{cm}^{-1} .{ }^{1} \mathrm{H}$ NMR spectra were recorded on a Bruker DPX360 (360 MHz) spectrometer or a Bruker ARX250 (250 MHz) spectrometer. Chemical shifts $(\delta)$ are quoted in parts per million (ppm) downfield of tetramethylsilane, using residual protonated solvent as internal standard $\left(\mathrm{CDCl}_{3}\right.$ at $7.27 \mathrm{ppm}$ ). Abbreviations used in the description of resonances are: s (singlet), $\mathrm{d}$ (doublet), $\mathrm{t}$ (triplet), q, (quartet), app (apparent), b (broad), bs (broad singlet). Coupling constants $(J)$ are quoted to the nearest $0.1 \mathrm{~Hz}$. Proton-decoupled ${ }^{13} \mathrm{C}$ NMR spectra were recorded on a Bruker DPX360 (90.6 MHz) spectrometer or a Bruker ARX250 $(62.9 \mathrm{MHz})$ spectrometer. Chemical shifts $(\delta)$ are quoted in parts per million (ppm) downfield of tetramethylsilane, using deuterated solvent as internal standard $\left(\mathrm{CDCl}_{3}\right.$ at $\left.77.0 \mathrm{ppm}\right)$. Assignments were made using the DEPT sequence 
with secondary pulses at $90^{\circ}$ and $135^{\circ}$. High resolution mass spectra were recorded on a Finnigan MAT 900 XLT spectrometer using the electrospray (ES) positive ion mode at the EPSRC National Mass Spectrometry Service Centre, University of Wales Swansea, or on a Kratos MS50TC spectrometer using the electron ionization (EI) technique in the mass spectrometry laboratory at the School of Chemistry, University of Edinburgh. Stated calculated mass values refer to that of the ion (i.e. the actual species being detected), not that of the neutral parent compound. Optical rotations were performed on an Optical Activity POLAAR 20 polarimeter with a path length of $0.5 \mathrm{dm}$ at the sodium D line $(589 \mathrm{~nm})$.

\subsection{Experimental Procedures}

\subsubsection{Racemic approaches based on amidomalonate aldol cyclisations}

Diethyl 2-(4-methoxyphenylamino)malonate $(109)^{51}$

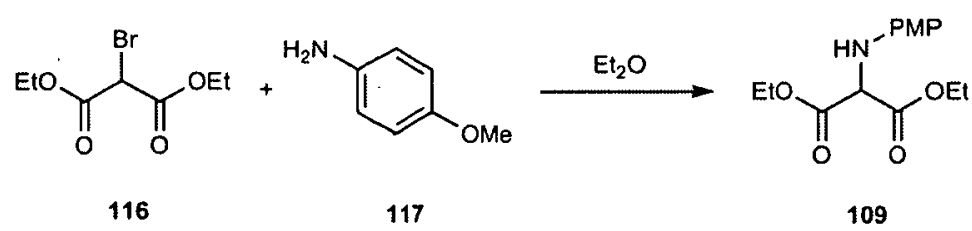

To a stirred solution of $p$-anisidine $117(26.9 \mathrm{~g}, 218 \mathrm{mmol})$ in THF (120 mL) at room temperature was added 3-diethyl-2-bromomalonate $116(20 \mathrm{~mL}, 109 \mathrm{mmol})$ via syringe. The reaction was heated to reflux for $2 \mathrm{~h}$ and stirred for $14 \mathrm{~h}$ at room temperature. The solution was then concentrated in vacuo and then redissolved in $\mathrm{Et}_{2} \mathrm{O}$. The reaction mixture was filtered, washing the solids with $\mathrm{Et}_{2} \mathrm{O}$ and this solution was again concentrated in vacuo. This concentrate was now redissolved in EtOAc, filtered washing with EtOAc and concentrated in vacuo. A crystalline solid was formed and washed with petrol, obtaining pure amine $109(26.2 \mathrm{~g}, 85 \%)$ as brown crystals. $\mathrm{R}_{\mathrm{f}}(30 \%$ EtOAc in hexane $)=0.63$; m.p. $65-67{ }^{\circ} \mathrm{C}$, lit. ${ }^{51} 64-65{ }^{\circ} \mathrm{C} ;{ }^{1} \mathrm{H}$ NMR $\left(360 \mathrm{MHz}, \mathrm{CDCl}_{3}\right) \delta 6.78(2 \mathrm{H}, \mathrm{d}, J=9.1 \mathrm{~Hz}, \operatorname{ArH}), 6.65(2 \mathrm{H}, \mathrm{d}, J=9.1 \mathrm{~Hz}$, $\mathrm{ArH}), 4.69\left(1 \mathrm{H}, \mathrm{s}, \mathrm{CH}\left(\mathrm{CO}_{2} \mathrm{Et}\right)_{2}\right), 4.58(1 \mathrm{H}, \mathrm{bs}, \mathrm{NH}), 4.27(4 \mathrm{H}, \mathrm{q}, J=7.1 \mathrm{~Hz}, 2 \mathrm{x}$ $\left.\mathrm{CO}_{2} \mathrm{CH}_{2} \mathrm{CH}_{3}\right), 3.75\left(3 \mathrm{H}, \mathrm{s}, \mathrm{OCH}_{3}\right), 1.28\left(6 \mathrm{H}, \mathrm{t}, J=7.1 \mathrm{~Hz}, 2 \times \mathrm{CO}_{2} \mathrm{CH}_{2} \mathrm{CH}_{3}\right) ;{ }^{13} \mathrm{C}$ 
NMR (90 MHz, CDCl 3 ) $\delta 167.9(2 \times \mathrm{C}), 153.1(\mathrm{C}), 139.4(\mathrm{C}), 115.1(2 \times \mathrm{CH}), 114.8$ $(2 \times \mathrm{CH}), 62.2\left(2 \times \mathrm{CH}_{2}\right), 61.8(\mathrm{CH}), 55.6\left(\mathrm{CH}_{3}\right), 14.0\left(2 \times \mathrm{CH}_{3}\right)$.

${ }^{1} \mathrm{H}$ spectroscopic data in good agreement with the literature. ${ }^{51}$

\section{Diethyl 2-(4-methoxybenzylamino)malonate (119)}<smiles>CCOC(=O)C(Br)C(=O)OCCN(C)CCCCCN</smiles>

To a stirred solution of 4-methoxy benzylamine $118(3.30 \mathrm{~mL}, 25.0 \mathrm{mmol})$ in $\operatorname{MeCN}(100 \mathrm{~mL})$ at $0{ }^{\circ} \mathrm{C}$, was added triethylamine $(3: 50 \mathrm{~mL}, 25.0 \mathrm{mmol})$, followed by the addition of 3-diethyl-2-bromomalonate $116(4.60 \mathrm{~mL}, 25.0 \mathrm{mmol})$. The reaction was stirred for $14 \mathrm{~h}$, allowed to warm up to room temperature, concentrated in vacuo and then redissolved in $\mathrm{Et}_{2} \mathrm{O}$. It was then washed with water $(2 \times 20 \mathrm{~mL})$ and with saturated aqueous $\mathrm{NaHCO}_{3}$ solution $(2 \times 20 \mathrm{~mL})$. The combined aqueous layers were extracted with $\mathrm{Et}_{2} \mathrm{O}(1 \times 20 \mathrm{~mL})$, and the organic layers were combined, dried $\left(\mathrm{MgSO}_{4}\right)$, filtered and concentrated in vacuo. Purification of the residue by column chromatography (10\% to $20 \%$ EtOAc/petrol) gave amine 119 (3.73 g, 52\%) as a yellow oil. $R_{f}(20 \%$ EtOAc in hexane $)=0.33$; IR $($ film $) 2981(\mathrm{NH}), 1752(\mathrm{C}=\mathrm{O})$, $1734(\mathrm{C}=\mathrm{O}), 1612,1513,1465,1369,1302,1248,1031 \mathrm{~cm}^{-1} ;{ }^{1} \mathrm{H}$ NMR $(360 \mathrm{MHz}$, $\left.\mathrm{CDCl}_{3}\right) \delta 7.14(2 \mathrm{H}, \mathrm{d}, J=8.5 \mathrm{~Hz}, \mathrm{ArH}), 6.74(2 \mathrm{H}, \mathrm{d}, J=8.5 \mathrm{~Hz}, \mathrm{ArH}), 4.10(4 \mathrm{H}, \mathrm{q}$, $\left.J=7.1 \mathrm{~Hz}, 2 \times \mathrm{CO}_{2} \mathrm{CH}_{2} \mathrm{CH}_{3}\right), 3.92\left(1 \mathrm{H}, \mathrm{s}, \mathrm{CH}\left(\mathrm{CO}_{2} \mathrm{Et}\right)_{2}\right), 3.67\left(3 \mathrm{H}, \mathrm{s}, \mathrm{OCH}_{3}\right), 3.63$ $\left(2 \mathrm{H}, \mathrm{s}, \mathrm{CH}_{2} \mathrm{Ar}\right), 2.33(1 \mathrm{H}, \mathrm{bs}, \mathrm{NH}), 1.15\left(6 \mathrm{H}, \mathrm{t}, J=7.1 \mathrm{~Hz}, 2 \times \mathrm{CO}_{2} \mathrm{CH}_{2} \mathrm{CH}_{3}\right) ;{ }^{13} \mathrm{C}$ NMR (90 MHz, $\left.\mathrm{CDCl}_{3}\right) \delta 166.4(2 \times \mathrm{C}), 158.8(\mathrm{C}), 130.7$ (C), $129.6(2 \times \mathrm{CH}), 113.7$ $(2 \times \mathrm{CH}), 63.8(\mathrm{CH}), 61.6\left(2 \times \mathrm{CH}_{2}\right), 55.1\left(\mathrm{CH}_{3}\right), 50.9\left(\mathrm{CH}_{2}\right), 13.9\left(2 \times \mathrm{CH}_{3}\right) ; \mathrm{HRMS}$ (ES) Exact mass calcd for $\mathrm{C}_{15} \mathrm{H}_{21} \mathrm{NO}_{5}[\mathrm{M}+\mathrm{H}]^{+}: 296.1492$, found: 296.1494 .

\section{Dimethyl 2-(4-methoxybenzylamino)malonate $(121)^{51}$}

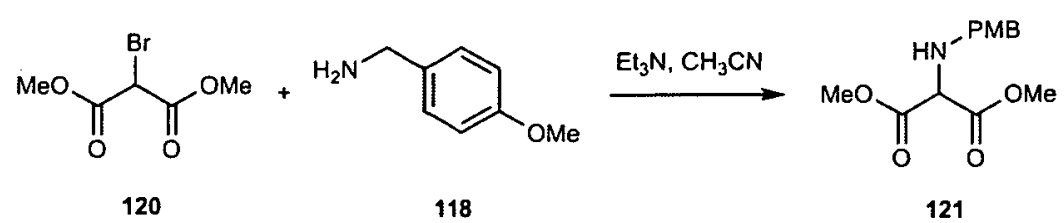


To a stirred solution of 4-methoxybenzylamine $118(8.00 \mathrm{~g}, 60.0 \mathrm{mmol})$ in $\mathrm{MeCN}(60 \mathrm{~mL})$ at $0{ }^{\circ} \mathrm{C}$ was was added triethylamine $(8.40 \mathrm{~mL}, 60.0 \mathrm{mmol})$, followed by the addition of 3-diethyl-2-bromomalonate $120(8.80 \mathrm{~mL}, 60.0 \mathrm{mmol})$. The reaction was stirred for $14 \mathrm{~h}$ at room temperature, concentrated in vacuo and then redissolved in $\mathrm{Et}_{2} \mathrm{O}$ and saturated aqueous $\mathrm{NaHCO}_{3}$ solution. This mixture was stirred for $30 \mathrm{~min}$. The aqueous layer was separated and extracted with $\mathrm{Et}_{2} \mathrm{O}(3 \times 60$ $\mathrm{mL})$ and the combined organic layers were dried $\left(\mathrm{MgSO}_{4}\right)$, filtered and concentrated in vacuo. Purification of the residue by column chromatography (petrol/EtOAc, $10 \%$ to $30 \%$ in EtOAc) gave amine $121(5.52 \mathrm{~g}, 35 \%)$ as a yellow oil. $\mathrm{R}_{\mathrm{f}}(40 \% \mathrm{EtOAc}$ in hexane $)=0.50 ;{ }^{1} \mathrm{H}$ NMR $\left(360 \mathrm{MHz}, \mathrm{CDCl}_{3}\right) \delta 7.25(2 \mathrm{H}, \mathrm{d}, J=8.7 \mathrm{~Hz}, \mathrm{ArH}), 6.86$ $(2 \mathrm{H}, \mathrm{d}, J=8.7 \mathrm{~Hz}, \mathrm{ArH}), 4.08\left(1 \mathrm{H}, \mathrm{s}, \mathrm{CH}\left(\mathrm{CO}_{2} \mathrm{Me}\right)_{2}\right), 3.80\left(3 \mathrm{H}, \mathrm{s}, \mathrm{OCH}_{3}\right), 3.77(6 \mathrm{H}$, s, $\left.2 \times \mathrm{CO}_{2} \mathrm{CH}_{3}\right), 3.75\left(2 \mathrm{H}, \mathrm{s}, \mathrm{CH}_{2} \mathrm{Ar}\right), 2.32(1 \mathrm{H}, \mathrm{bs}, \mathrm{NH}) ;{ }^{13} \mathrm{C}$ NMR $(90 \mathrm{MHz}$, $\left.\mathrm{CDCl}_{3}\right) \delta 168.9(2 \times \mathrm{C}), 158.9(\mathrm{C}), 130.6(\mathrm{C}), 129.6(2 \times \mathrm{CH}), 113.8(2 \times \mathrm{CH}), 63.6$ $(\mathrm{CH}), 55.2\left(\mathrm{CH}_{3}\right), 52.7\left(\mathrm{CH}_{3}\right), 51.0\left(\mathrm{CH}_{2}\right)$.

${ }^{1} \mathrm{H}$ spectroscopic data in good agreement with the literature. ${ }^{51}$

\section{1-(4-Methoxybenzyl)-3-hydroxy-3-methyl-5-oxopyrrolidine-2,2-dicarboxylic} acid methyl ester (123)
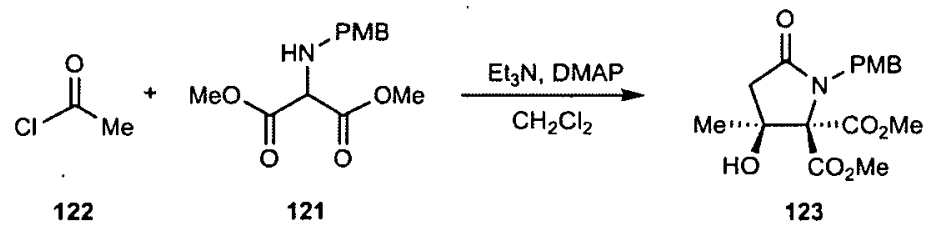

123

To a stirred solution of acetyl chloride $122(2.75 \mathrm{~mL}, 38.4 \mathrm{mmol})$ in $\mathrm{CH}_{2} \mathrm{Cl}_{2}$ (30 $\mathrm{mL})$ at $0^{\circ} \mathrm{C}$, was added $\mathrm{Et}_{3} \mathrm{~N}(5.70 \mathrm{~mL}, 40.8 \mathrm{mmol})$ dropwise. After stirring for $1 \mathrm{~h}$ at $0{ }^{\circ} \mathrm{C}$, a solution of amine $121(3.26 \mathrm{~g}, 12.0 \mathrm{mmol})$ and DMAP (293 $\mathrm{mg}, 2.40 \mathrm{mmol}$ ) in $\mathrm{CH}_{2} \mathrm{Cl}_{2}(30 \mathrm{~mL})$ was added via cannula. The reaction was stirred for $14 \mathrm{~h}$ at room temperature and then quenched with $\mathrm{pH} 7$ phosphate buffer $(20 \mathrm{~mL})$. The aqueous layer was separated and extracted with $\mathrm{CH}_{2} \mathrm{Cl}_{2}(3 \times 20 \mathrm{~mL})$ and the combined organic layers were dried $\left(\mathrm{MgSO}_{4}\right)$, filtered and concentrated in vacuo. Purification of the residue by column chromatography (20\% to $80 \%$ EtOAc/petrol) gave $\gamma$-lactam $123(2.95 \mathrm{~g}, 70 \%)$ as a yellow powder. $R_{f}(40 \%$ EtOAc in hexane $)=0.23$; m.p. 125-128 ${ }^{\circ} \mathrm{C}$; IR (film) $2954(\mathrm{OH}), 2360,1742(\mathrm{C}=\mathrm{O}), 1682(\mathrm{C}=\mathrm{O}), 1612(\mathrm{C}=\mathrm{O})$, 
1513, 1438, 1244, $1067 \mathrm{~cm}^{-1} ;{ }^{1} \mathrm{H}$ NMR $\left(360 \mathrm{MHz}, \mathrm{CDCl}_{3}\right) \delta 7.16(2 \mathrm{H}, \mathrm{d}, J=8.7 \mathrm{~Hz}$, $\operatorname{ArH}), 6.79(2 \mathrm{H}, \mathrm{d}, J=8.7 \mathrm{~Hz}, \mathrm{ArH}), 4.79\left(2 \mathrm{H}, \mathrm{s}, \mathrm{CH}_{2} \mathrm{Ar}\right), 3.76\left(3 \mathrm{H}, \mathrm{s}, \mathrm{OCH}_{3}\right), 3.62$ $\left(3 \mathrm{H}, \mathrm{s}, \mathrm{CO}_{2} \mathrm{CH}_{3}\right), 3.44\left(3 \mathrm{H}, \mathrm{s}, \mathrm{CO}_{2} \mathrm{CH}_{3}\right), 2.74\left(1 \mathrm{H}, \mathrm{d}, J=16.8 \mathrm{~Hz}, \mathrm{CH}_{\mathrm{A}} \mathrm{H}_{\mathrm{B}} \mathrm{C}=\mathrm{O}\right)$, $2.68\left(1 \mathrm{H}, \mathrm{d}, J=16.8 \mathrm{~Hz}, \mathrm{CH}_{\mathrm{A}} \mathbf{H}_{\mathrm{B}} \mathrm{C}=\mathrm{O}\right), 1.49\left(3 \mathrm{H}, \mathrm{s}, \mathrm{COHCH}_{3}\right) ;{ }^{13} \mathrm{C} \mathrm{NMR}(90 \mathrm{MHz}$, $\left.\mathrm{CDCl}_{3}\right) \delta 174.8(\mathrm{C}), 168.1(2 \times \mathrm{C}), 167.7(\mathrm{C}), 159.1(\mathrm{C}), 130.2(2 \times \mathrm{CH}), 128.9(\mathrm{C})$, $113.9(2 \times \mathrm{CH}), 79.5(\mathrm{C}), 55.6\left(\mathrm{CH}_{3}\right), 53.1\left(2 \times \mathrm{CH}_{3}\right), 45.5\left(\mathrm{CH}_{2}\right), 45.3\left(\mathrm{CH}_{2}\right), 23.8$ $\left(\mathrm{CH}_{3}\right)$; HRMS (ES) Mass calcd. for $\mathrm{C}_{17} \mathrm{H}_{22} \mathrm{NO}_{7}[\mathrm{M}+\mathrm{H}]^{+}:$352.1391, found: 352.1393 .

\section{3-Hydroxy-1-(4-methoxyphenyl)-3-methyl-5-oxopyrrolidine-2,2-dicarboxylic} acid ethyl ester (110)<smiles>CC1=CC(=O)OC(C)(C)O1</smiles>

129<smiles>CCOC(=O)C(NP)C(=O)OCC</smiles>

109

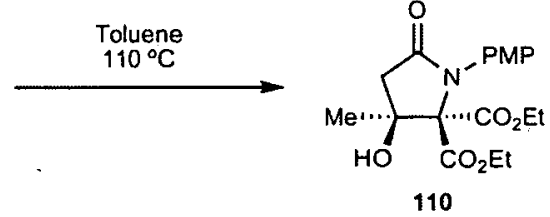

110

A stirred solution of dioxinone $129(4.80 \mathrm{~mL}, 33.0 \mathrm{mmol})$ and amine $109(8.44 \mathrm{~g}$, $30.0 \mathrm{mmol})$ in toluene $(25 \mathrm{~mL})$ in a round bottom flask with an air condenser was placed in an oil bath preheated at $150^{\circ} \mathrm{C}$. The reaction was heated to reflux for $6 \mathrm{~h}$ and then concentrated in vacuo. Purification of the residue by column chromatography (25\% to $45 \%$ EtOAc/petrol) gave $\gamma$-lactam $110(8.72 \mathrm{~g}, 80 \%)$ as a yellow solid. $\mathrm{R}_{\mathrm{f}}(50 \%$ EtOAc in hexane $)=0.17$; m.p. $127-130^{\circ} \mathrm{C}$, lit ${ }^{51} 136-137{ }^{\circ} \mathrm{C}$; IR (film) $2983(\mathrm{OH}), 1754(\mathrm{C}=\mathrm{O}), 1727(\mathrm{C}=\mathrm{O}), 1697(\mathrm{C}=\mathrm{O}), 1512,1366,1297$, 1250, $1031 \mathrm{~cm}^{-1} ;{ }^{1} \mathrm{H}$ NMR (360 MHz, $\left.\mathrm{CDCl}_{3}\right) 7.12(2 \mathrm{H}, \mathrm{d}, J=9.1 \mathrm{~Hz}, \mathrm{ArH}), 6.86$ $(2 \mathrm{H}, \mathrm{d}, J=9.1 \mathrm{~Hz}, \mathrm{ArH}), 4.29\left(2 \mathrm{H}, \mathrm{m}, \mathrm{CO}_{2} \mathrm{CH}_{2} \mathrm{CH}_{3}\right), 4.13\left(2 \mathrm{H}, \mathrm{m}, \mathrm{CO}_{2} \mathrm{CH}_{2} \mathrm{CH}_{3}\right)$, $3.88(1 \mathrm{H}, \mathrm{bs}, \mathrm{COH}), 3.78\left(3 \mathrm{H}, \mathrm{s}, \mathrm{OCH}_{3}\right), 2.82\left(1 \mathrm{H}, \mathrm{d}, J=16.7 \mathrm{~Hz}, \mathrm{CH}_{\mathrm{A}} \mathrm{H}_{\mathrm{B}} \mathrm{C}=\mathrm{O}\right)$, $2.73\left(1 \mathrm{H}, \mathrm{d}, J=16.7 \mathrm{~Hz}, \mathrm{CH}_{\mathrm{A}} \mathbf{H}_{\mathrm{B}} \mathrm{C}=\mathrm{O}\right), 1.58\left(3 \mathrm{H}, \mathrm{s}, \mathrm{COHCH}_{3}\right), 1.27(3 \mathrm{H}, \mathrm{t}, J=7.1$ $\left.\mathrm{Hz}, \mathrm{CO}_{2} \mathrm{CH}_{2} \mathrm{CH}_{3}\right), 1.07\left(3 \mathrm{H}, \mathrm{t}, J=7.1 \mathrm{~Hz}, \mathrm{CO}_{2} \mathrm{CH}_{2} \mathrm{CH}_{3}\right) ;{ }^{13} \mathrm{C}$ NMR $(90 \mathrm{MHz}$, $\left.\mathrm{CDCl}_{3}\right) \delta 174.2(\mathrm{C}), 167.6(2 \times \mathrm{C}), 166.5(\mathrm{C}), 158.7(\mathrm{C}), 130.1(\mathrm{C}), 128.8(2 \times \mathrm{CH})$, $113.8(2 \times \mathrm{CH}), 80.9(\mathrm{C}), 62.4\left(\mathrm{CH}_{2}\right), 62.0\left(\mathrm{CH}_{2}\right), 55.2\left(\mathrm{CH}_{3}\right), 44.6\left(\mathrm{CH}_{2}\right), 23.6$ $\left(\mathrm{CH}_{3}\right), 13.8\left(\mathrm{CH}_{3}\right), 14.4\left(\mathrm{CH}_{3}\right)$.

${ }^{1} \mathrm{H}$ spectroscopic data in good agreement with the literature. ${ }^{51}$ 
3-Hydroxy-1-(4-methoxybenzyl)-3-methyl-5-oxopyrrolidine-2,2-dicarboxylic acid ethyl ester (131)

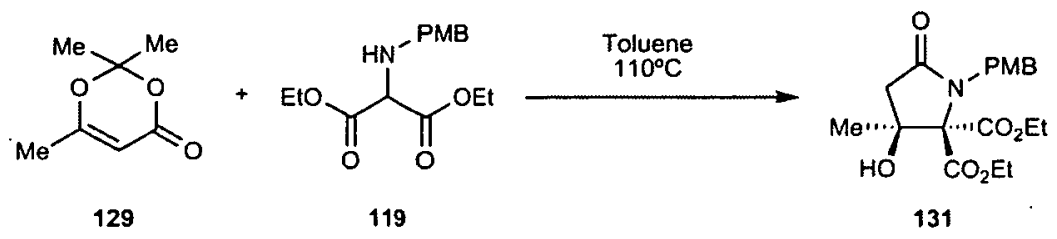

A stirred solution of dioxinone $129(1.30 \mathrm{~mL}, 8.80 \mathrm{mmol})$ and amine 119 (2.30 g, $8.00 \mathrm{mmol})$ in toluene $(25 \mathrm{~mL})$ in a round bottom flask with an air condenser was placed in an oil bath preheated at $150^{\circ} \mathrm{C}$. The mixture was heated to reflux $14 \mathrm{~h}$ and then concentrated in vacuo. Purification of the residue by column chromatography (15\% to 25\% EtOAc/petrol) gave $\gamma$-lactam $131(2.65 \mathrm{~g}, 86 \%$ ) as a brown oil. $\mathrm{R}_{\mathrm{f}}(40 \%$ EtOAc in hexane $)=0.24$; IR (film) $3392(\mathrm{OH}), 1738(\mathrm{C}=\mathrm{O}), 1683(\mathrm{C}=\mathrm{O})$, $1612(\mathrm{C}=\mathrm{O}), 1513,1397,1244,1176,1032 \mathrm{~cm}^{-1} ;{ }^{1} \mathrm{H} \mathrm{NMR}\left(360 \mathrm{MHz}, \mathrm{CDCl}_{3}\right) \delta 7.16$ $(2 \mathrm{H}, \mathrm{d}, J=8.7 \mathrm{~Hz}, \operatorname{ArH}), 6.78(2 \mathrm{H}, \mathrm{d}, J=8.7 \mathrm{~Hz}, \operatorname{ArH}), 4.81(1 \mathrm{H}, \mathrm{d}, J=14.9 \mathrm{~Hz}$, $\left.\mathrm{CH}_{\mathrm{A}} \mathrm{H}_{\mathrm{B}} \mathrm{Ar}\right), 4.74\left(1 \mathrm{H}, \mathrm{d}, J=14.9 \mathrm{~Hz}, \mathrm{CH}_{\mathrm{A}} \mathrm{H}_{\mathrm{B}} \mathrm{Ar}\right), 4.20-4.08(1 \mathrm{H}, \mathrm{m}$, $\left.\mathrm{CO}_{2} \mathrm{CH}_{\mathrm{C}} \mathrm{H}_{\mathrm{D}} \mathrm{CH}_{3}\right), \quad 4.05-3.95\left(1 \mathrm{H}, \mathrm{m}, \mathrm{CO}_{2} \mathrm{CH}_{\mathrm{C}}, \mathrm{H}_{\mathrm{D}}, \mathrm{CH}_{3}\right), 3.95-3.86(1 \mathrm{H}, \mathrm{m}$, $\left.\mathrm{CO}_{2} \mathrm{CH}_{\mathrm{C}} \mathbf{H}_{\mathbf{D}} \mathrm{CH}_{3}\right), 3.86-3.77\left(1 \mathrm{H}, \mathrm{m}, \mathrm{CO}_{2} \mathrm{CH}_{\mathrm{C}}, \mathbf{H}_{\mathbf{D}}, \mathrm{CH}_{3}\right), 3.75\left(3 \mathrm{H}, \mathrm{s}, \mathrm{OCH}_{3}\right), 2.72$ $\left(1 \mathrm{H}, \mathrm{d}, J=16.5 \mathrm{~Hz}, \mathrm{CH}_{\mathrm{A}} \mathrm{H}_{\mathrm{B}} \mathrm{C}=\mathrm{O}\right), 2.65\left(1 \mathrm{H}, \mathrm{d}, J=16.5 \mathrm{~Hz}, \mathrm{CH}_{\mathrm{A}} \mathbf{H}_{\mathbf{B}} \mathrm{C}=\mathrm{O}\right), 1.51(3 \mathrm{H}$, $\left.\mathrm{s}, \mathrm{COHCH}_{3}\right), 1.17\left(3 \mathrm{H}, \mathrm{t}, J=7.1 \mathrm{~Hz}, \mathrm{CO}_{2} \mathrm{CH}_{2} \mathrm{CH}_{3}\right), 1.14(3 \mathrm{H}, \mathrm{t}, J=7.1 \mathrm{~Hz}$, $\left.\mathrm{CO}_{2} \mathrm{CH}_{2} \mathrm{CH}_{3}\right) ;{ }^{13} \mathrm{C} \mathrm{NMR}\left(62.9 \mathrm{MHz}, \mathrm{CDCl}_{3}\right) \delta 174.5(\mathrm{C}), 167.2(\mathrm{C}), 166.8(\mathrm{C})$, $158.6(\mathrm{C}), 129.4(2 \times \mathrm{CH}), 128.8(\mathrm{C}), 113.4(2 \times \mathrm{CH}), 79.1(\mathrm{C}), 76.4(\mathrm{C}), 62.2\left(\mathrm{CH}_{2}\right)$, $62.1\left(\mathrm{CH}_{2}\right), 55.2\left(\mathrm{CH}_{3}\right), 45.1\left(\mathrm{CH}_{2}\right), 44.9\left(\mathrm{CH}_{2}\right), 23.4\left(\mathrm{CH}_{3}\right), 13.7\left(2 \times \mathrm{CH}_{3}\right) ; \mathrm{HRMS}$ (ES) Exact mass calcd for $\mathrm{C}_{19} \mathrm{H}_{26} \mathrm{NO}_{7}[\mathrm{M}+\mathrm{H}]^{+}: 380.1704$, found: 380.1703 .

\section{1-(4-Methoxy-phenyl)-3-methyl-5-oxo-3-trimethylsilanyloxy-pyrrolidine-2,2-} dicarboxylic acid ethyl ester (137)

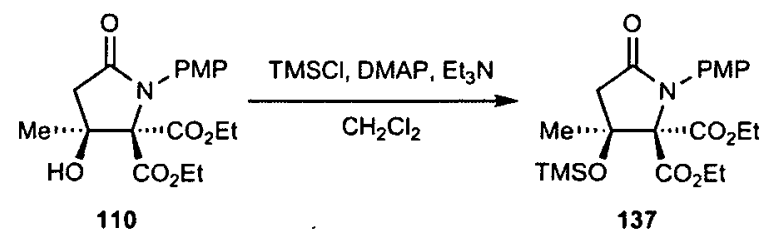

To a stirred solution of lactam $110(4.00 \mathrm{~g}, 10.9 \mathrm{mmol})$ and DMAP (540 $\mathrm{mg}$, $4.38 \mathrm{mmol})$ in $\mathrm{CH}_{2} \mathrm{Cl}_{2}(30 \mathrm{~mL})$ at $0{ }^{\circ} \mathrm{C}$, was added $\mathrm{Et}_{3} \mathrm{~N}(4.60 \mathrm{~mL}, 33 \mathrm{mmol})$ dropwise, followed by the addition of $\mathrm{TMSCl}(2.15 \mathrm{~mL}, 16.4 \mathrm{mmol})$. The reaction 
was stirred for $30 \mathrm{~min}$ at $0{ }^{\circ} \mathrm{C}$ and then quenched with saturated aqueous $\mathrm{NaHCO}_{3}$ solution $(20 \mathrm{~mL})$. The aqueous layer was separated and extracted with $\mathrm{CH}_{2} \mathrm{Cl}_{2}(3 \mathrm{x}$ $30 \mathrm{~mL})$ and the combined organic layers were dried $\left(\mathrm{MgSO}_{4}\right)$, filtered and concentrated in vacuo. Purification of the residue by column chromatography $(50 \%$ EtOAc/petrol) gave $\gamma$-lactam $137(4.27 \mathrm{~g}, 92 \%)$ as colourless crystals. $R_{\mathrm{f}}(50 \%$ EtOAc in hexane) $=0.80$; m.p. 85-87 ${ }^{\circ} \mathrm{C}$; IR (film) 2982, $1759(\mathrm{C}=\mathrm{O}), 1746(\mathrm{C}=\mathrm{O})$, $1721(\mathrm{C}=\mathrm{O}), 1512,1250,1063,845 \mathrm{~cm}^{-1} ;{ }^{1} \mathrm{H}$ NMR $\left(360 \mathrm{MHz}, \mathrm{CDCl}_{3}\right) \delta 7.13(2 \mathrm{H}, \mathrm{d}$, $J=8.9 \mathrm{~Hz}, \mathrm{ArH}), 6.87(2 \mathrm{H}, \mathrm{d}, J=8.9 \mathrm{~Hz}, \mathrm{ArH}), 4.27\left(2 \mathrm{H}, \mathrm{m}, \mathrm{CO}_{2} \mathrm{CH}_{2} \mathrm{CH}_{3}\right), 4.17$ $\left(2 \mathrm{H}, \mathrm{m}, \mathrm{CO}_{2} \mathrm{CH}_{2} \mathrm{CH}_{3}\right), 3.79\left(3 \mathrm{H}, \mathrm{s}, \mathrm{OCH}_{3}\right), 2.97\left(1 \mathrm{H}, \mathrm{d}, J=16.7 \mathrm{~Hz}, \mathrm{CH}_{\mathrm{A}} \mathrm{H}_{\mathrm{B}} \mathrm{C}=\mathrm{O}\right)$, $2.77\left(1 \mathrm{H}, \mathrm{d}, J=16.7 \mathrm{~Hz}, \mathrm{CH}_{\mathrm{A}} \mathbf{H}_{\mathrm{B}} \mathrm{C}=\mathrm{O}\right), 1.67\left(3 \mathrm{H}, \mathrm{s}, \operatorname{COTMSCH}_{3}\right), 1.27(3 \mathrm{H}, \mathrm{t}, J=$ $\left.7.1 \mathrm{~Hz}, \mathrm{CO}_{2} \mathrm{CH}_{2} \mathrm{CH}_{3}\right), 1.24\left(3 \mathrm{H}, \mathrm{t}, J=7.1 \mathrm{~Hz}, \mathrm{CO}_{2} \mathrm{CH}_{2} \mathrm{CH}_{3}\right), 0.17(9 \mathrm{H}, \mathrm{s}, 3 \mathrm{x}$ $\mathrm{SiCH}_{3}$ ); ${ }^{13} \mathrm{C}$ NMR (90 MHz, $\left.\mathrm{CDCl}_{3}\right) \delta 173.5$ (C), 167.1 (2 x C), 164.9 (C), 157.8 (C), $129.8(\mathrm{C}), 127.6(2 \times \mathrm{CH}), 113.2(2 \times \mathrm{CH}), 78.8(\mathrm{C}), 61.6\left(\mathrm{CH}_{2}\right), 60.8\left(\mathrm{CH}_{2}\right)$, $54.7\left(\mathrm{CH}_{3}\right), 45.2\left(\mathrm{CH}_{2}\right), 23.3\left(\mathrm{CH}_{3}\right), 13.3\left(2 \times \mathrm{CH}_{3}\right), 1.2\left(3 \times \mathrm{CH}_{3}\right) ; \mathrm{HRMS}(\mathrm{ES})$ Exact mass calcd. for $\mathrm{C}_{21} \mathrm{H}_{32} \mathrm{NO}_{7} \mathrm{Si}[\mathrm{M}+\mathrm{H}]^{+}: 438.1943$, found: 438.1948 .

\section{1-(4-Methoxybenzyl)-3-methyl-5-oxo-3-(trimethylsilyloxy)pyrrolidine-2,2-} dicarboxylic acid ethyl ester (138)

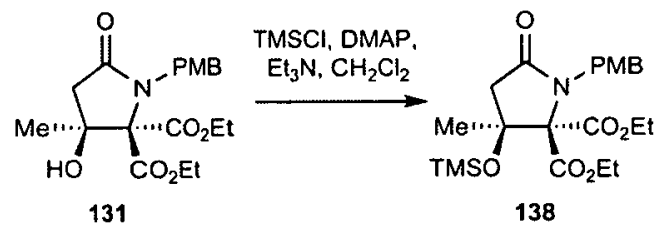

To a stirred solution of lactam $131(2.50 \mathrm{~g}, 6.58 \mathrm{mmol})$ and DMAP (160 mg, $1.31 \mathrm{mmol})$ in $\mathrm{CH}_{2} \mathrm{Cl}_{2}(120 \mathrm{~mL})$ at $0{ }^{\circ} \mathrm{C}$, was added $\mathrm{Et}_{3} \mathrm{~N}(2.80 \mathrm{~mL}, 19.8 \mathrm{mmol})$ dropwise, followed by the addition of $\mathrm{TMSCl}(1.30 \mathrm{~mL}, 9.90 \mathrm{mmol})$ via syringe. The reaction was stirred for $14 \mathrm{~h}$ at room temperature and then quenched with saturated aqueous $\mathrm{NaHCO}_{3}$ solution $(40 \mathrm{~mL})$. The aqueous layer was separated and extracted with $\mathrm{CH}_{2} \mathrm{Cl}_{2}(3 \times 30 \mathrm{~mL})$ and the combined organic layers were dried $\left(\mathrm{MgSO}_{4}\right)$, filtered and concentrated in vacuo. Purification of the residue by column chromatography (50\% EtOAc/petrol) gave $\gamma$-lactam $138(2.50 \mathrm{~g}, 85 \%)$ as a brown solid. $\mathrm{R}_{\mathrm{f}}(40 \%$ EtOAc in hexane $)=0.67$; m.p. $68-71^{\circ} \mathrm{C}$; IR (film) $2958,1736(\mathrm{C}=\mathrm{O})$, $1707(\mathrm{C}=\mathrm{O}), 1613(\mathrm{C}=\mathrm{O}), 1513,1387,1302,1248,1064,844 \mathrm{~cm}^{-1} ;{ }^{1} \mathrm{H}$ NMR $(360$ 
$\left.\mathrm{MHz} \mathrm{CDCl}_{3}\right) \delta 7.19(2 \mathrm{H}, \mathrm{d}, J=8.7 \mathrm{~Hz}, \mathrm{ArH}), 6.76(2 \mathrm{H}, \mathrm{d}, J=8.7 \mathrm{~Hz}, \operatorname{ArH}), 5.01$ $\left(1 \mathrm{H}, \mathrm{d}, J=14.9 \mathrm{~Hz}, \mathrm{CH}_{\mathrm{A}} \mathrm{H}_{\mathrm{B}} \mathrm{Ar}\right), 4.64\left(1 \mathrm{H}, \mathrm{d}, J=14.9 \mathrm{~Hz}, \mathrm{CH}_{\mathrm{A}} \mathbf{H}_{\mathrm{B}} \mathrm{Ar}\right), 4.28-4.19$ $\left(1 \mathrm{H}, \mathrm{m}, \mathrm{CO}_{2} \mathrm{CH}_{\mathrm{C}} \mathrm{H}_{\mathrm{D}} \mathrm{CH}_{3}\right), 4.14-4.06\left(1 \mathrm{H}, \mathrm{m}, \mathrm{CO}_{2} \mathrm{CH}_{\mathrm{C}} \mathrm{H}_{\mathrm{D}} \mathrm{CH}_{3}\right), 3.75\left(3 \mathrm{H}, \mathrm{s}, \mathrm{OCH}_{3}\right)$, 3.68-3.59 (2H, m, CO $\left.\mathrm{CH}_{2} \mathrm{CH}_{3}\right), 2.80\left(1 \mathrm{H}, \mathrm{d}, J=16.5 \mathrm{~Hz}, \mathrm{CH}_{\mathrm{A}} \mathrm{H}_{\mathrm{B}} \mathrm{C}=\mathrm{O}\right), 2.61(1 \mathrm{H}$, $\left.\mathrm{d}, J=16.5 \mathrm{~Hz}, \mathrm{CH}_{\mathrm{A}} \mathbf{H}_{\mathrm{B}} \mathrm{C}=\mathrm{O}\right), 1.57\left(3 \mathrm{H}, \mathrm{s}, \mathrm{COHCH}_{3}\right), 1.27(3 \mathrm{H}, \mathrm{t}, J=7.1 \mathrm{~Hz}$, $\left.\mathrm{CO}_{2} \mathrm{CH}_{2} \mathrm{CH}_{3}\right), 1.07\left(3 \mathrm{H}, \mathrm{t}, J=7.1 \mathrm{~Hz}, \mathrm{CO}_{2} \mathrm{CH}_{2} \mathrm{CH}_{3}\right), 0.12\left(9 \mathrm{H}, \mathrm{s}, \mathrm{Si}\left(\mathrm{CH}_{3}\right)_{3}\right) ;{ }^{13} \mathrm{C}$ $\mathrm{NMR}\left(62.9 \mathrm{MHz}, \mathrm{CDCl}_{3}\right) \delta 174.2(\mathrm{C}), 167.3$ (C), 166.1 (C), 158.7 (C), $130.3(2 \mathrm{x}$ $\mathrm{CH}), 128.6(\mathrm{C}), 113.2(2 \times \mathrm{CH}), 80.7(\mathrm{C}), 79.4(\mathrm{C}), 61.8\left(\mathrm{CH}_{2}\right), 61.4\left(\mathrm{CH}_{2}\right), 55.2$ $\left(\mathrm{CH}_{3}\right), 45.7\left(\mathrm{CH}_{2}\right), 44.9\left(\mathrm{CH}_{2}\right), 23.5\left(\mathrm{CH}_{3}\right), 13.9\left(\mathrm{CH}_{3}\right), 13.6\left(\mathrm{CH}_{3}\right), 1.9\left(3 \times \mathrm{CH}_{3}\right)$; HRMS (ES) Exact mass calcd for $\mathrm{C}_{22} \mathrm{H}_{33} \mathrm{NO}_{7} \mathrm{SiNa}[\mathrm{M}+\mathrm{Na}]^{+}: 474.1918$, found: 474.1916.

(2RS,3SR)-2-Formyl-1-(4-methoxy-phenyl)-3-methyl-5-oxo-3trimethylsilanyloxy-pyrrolidine-2-carboxylic acid ethyl ester (144)

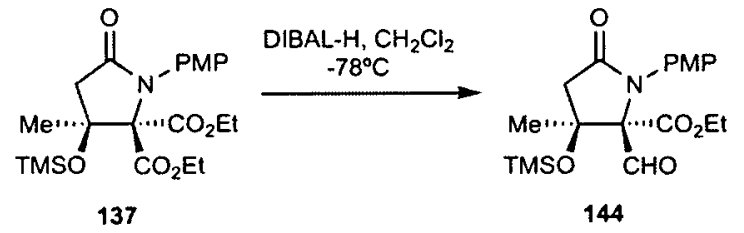

To a stirred solution of lactam $137(3.53 \mathrm{~g}, 9.00 \mathrm{mmol})$ in $\mathrm{CH}_{2} \mathrm{Cl}_{2}(36 \mathrm{~mL})$ at $-78^{\circ} \mathrm{C}$, was added DIBAL-H $\left(36 \mathrm{~mL}, 1 \mathrm{M}\right.$ in $\left.\mathrm{CH}_{2} \mathrm{Cl}_{2}, 36 \mathrm{mmol}\right)$ dropwise over 40 min. The reaction was stirred for $1.5 \mathrm{~h}$ at $-78^{\circ} \mathrm{C}$ and then quenched with $\mathrm{EtOH}(3$ $\mathrm{mL}$ ), added dropwise. The mixture was stirred for $15 \mathrm{~min}$ and then it was poured into a solution of saturated Rochelle's salt $(70 \mathrm{~mL})$ and $\mathrm{CH}_{2} \mathrm{Cl}_{2}(70 \mathrm{~mL})$. The resulting solution was stirred for $1 \mathrm{~h}$. The aqueous phase was then extracted with $\mathrm{CH}_{2} \mathrm{Cl}_{2}(3 \mathrm{x}$ $30 \mathrm{~mL})$ and the combined organic layers were dried $\left(\mathrm{MgSO}_{4}\right)$, filtered and concentrated in vacuo. Purification of the residue by column chromatography $(10 \%$ to $40 \% \mathrm{EtOAc} /$ petrol) gave aldehyde $144(2.16 \mathrm{~g}, 69 \%)$ as a yellow solid. $\mathrm{R}_{\mathrm{f}}(40 \%$ EtOAc in hexane) $=0.65$; m.p. 69-72 ${ }^{\circ} \mathrm{C}$; IR (film) 2959, $1754(\mathrm{C}=\mathrm{O}), 1719(\mathrm{C}=\mathrm{O})$, $1643(\mathrm{C}=\mathrm{O}), 1512,1351,1250,1028,844 \mathrm{~cm}^{-1} ;{ }^{1} \mathrm{H} \mathrm{NMR}\left(360 \mathrm{MHz}, \mathrm{CDCl}_{3}\right) \delta 10.01$ $(1 \mathrm{H}, \mathrm{s}, \mathrm{CHO}), 7.11(2 \mathrm{H}, \mathrm{d}, J=9.0 \mathrm{~Hz}, \mathrm{ArH}), 6.81(2 \mathrm{H}, \mathrm{d}, J=9.0 \mathrm{~Hz}, \operatorname{ArH}), 4.23$ $\left(2 \mathrm{H}, \mathrm{m}, \mathrm{CO}_{2} \mathrm{CH}_{2} \mathrm{CH}_{3}\right), 3.74\left(3 \mathrm{H}, \mathrm{s}, \mathrm{OCH}_{3}\right), 2.77\left(1 \mathrm{H}, \mathrm{d}, J=16.4 \mathrm{~Hz}, \mathrm{COCH}_{\mathbf{A}} \mathrm{H}_{\mathrm{B}}\right)$, $2.65\left(1 \mathrm{H}, \mathrm{d}, J=16.4 \mathrm{~Hz}, \operatorname{COCH}_{\mathrm{A}} \mathbf{H}_{\mathrm{B}}\right), 1.57\left(3 \mathrm{H}, \mathrm{s}, \operatorname{COTMSCH}_{3}\right), 1.19(3 \mathrm{H}, \mathrm{t}, J=7.1$ 
$\mathrm{Hz}, \mathrm{CO}_{2} \mathrm{CH}_{2} \mathrm{CH}_{3}$ ), 0.15 (9H, s, $3 \times \mathrm{SiCH}_{3}$ ); ${ }^{13} \mathrm{C} \mathrm{NMR}\left(90 \mathrm{MHz}, \mathrm{CDCl}_{3}\right) \delta 195.7$ $(\mathrm{CH}), 172.3(\mathrm{C}), 166.8(\mathrm{C}), 157.9(\mathrm{C}), 129.6(\mathrm{C}), 126.9(2 \times \mathrm{CH}), 113.6(2 \times \mathrm{CH})$, $81.9(\mathrm{C}), 80.8(\mathrm{C}), 62.0\left(\mathrm{CH}_{2}\right), 55.1\left(\mathrm{CH}_{3}\right), 45.3\left(\mathrm{CH}_{2}\right), 25.9\left(\mathrm{CH}_{3}\right), 13.7\left(\mathrm{CH}_{3}\right), 1.5$ (3 x CH $\mathrm{CH}_{3}$ ); HRMS (ES) Exact mass calcd. for $\mathrm{C}_{19} \mathrm{H}_{28} \mathrm{NO}_{6} \mathrm{Si}[\mathrm{M}+\mathrm{H}]^{+}$: 394.1680, found: 394.1678 .

Tributyl(cyclohex-2-enyl)stannane (143) ${ }^{185}$

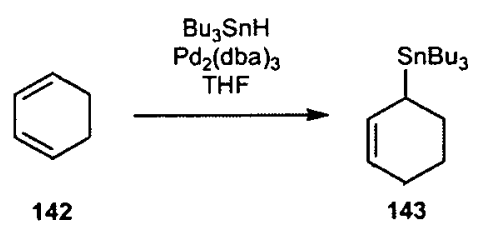

To a stirred solution of $\mathrm{Pd}_{2}(\mathrm{dba})_{3}(65.2 \mathrm{mg}, 71 \mu \mathrm{mol})$ and $\mathrm{PPh}_{3}(150 \mathrm{mg}, 0.56$ mmol) in THF ( $45 \mathrm{~mL})$, was added 1,3 cyclohexadiene (142) $(4.2 \mathrm{~mL}, 42.7 \mathrm{mmol})$, followed by the slow addition of $\mathrm{Bu}_{3} \mathrm{SnH}(3.95 \mathrm{~mL}, 14.2 \mathrm{mmol})$ at room temperature. The reaction was stirred for $14 \mathrm{~h}$ and then the solvent was concentrated in vacuo. The catalyst was filtered off through silica plug, obtaining a cloudy colourless oil ( $4.7 \mathrm{~g})$ that was distilled in vacuo to afford 2-cyclohexenyltributyltin 143 (3.2 g, 85\% purity assessed by ${ }^{1} \mathrm{H}$ NMR spectroscopy, 52\%). $\mathrm{R}_{\mathrm{f}}\left(1 \% \mathrm{Et}_{3} \mathrm{~N}\right.$ in hexane $)=0.75 ;{ }^{1} \mathrm{H}$ NMR $\left(360 \mathrm{MHz}, \mathrm{CDCl}_{3}\right) \delta 5.76(1 \mathrm{H}, \mathrm{dm}, J=9.8 \mathrm{~Hz}, \mathrm{CH}=\mathrm{CH})$, $5.41(1 \mathrm{H}, \mathrm{dm}, J=9.8 \mathrm{~Hz}, \mathrm{CH}=\mathrm{CH}), 2.16\left(1 \mathrm{H}, \mathrm{bs}, \mathrm{SnCHCH}_{2}\right), 2.09-1.96(3 \mathrm{H}, \mathrm{m}$, $\left.\mathrm{CH}_{2} \mathrm{CH}_{2} \mathrm{CH}_{2}\right), 1.85-1.76\left(1 \mathrm{H}, \mathrm{m}, \mathrm{CH}_{2} \mathrm{CH}_{2} \mathrm{CH}_{2}\right), 1.72-1.63\left(1 \mathrm{H}, \mathrm{m}, \mathrm{CH}_{2} \mathrm{CH}_{2} \mathrm{CH}_{2}\right)$, 1.60-1.42 $\left(6 \mathrm{H}+1 \mathrm{H}, \mathrm{m}, 3 \times \mathrm{SnCH}_{2} \mathrm{CH}_{2} \mathrm{CH}_{2} \mathrm{CH}_{3}+\mathrm{CH}_{2} \mathrm{CH}_{2} \mathrm{CH}_{2}\right), 1.31(6 \mathrm{H}, \mathrm{m}, 3 \times$ $\left.\mathrm{SnCH}_{2} \mathrm{CH}_{2} \mathrm{CH}_{2} \mathrm{CH}_{3}\right), 0.88\left(15 \mathrm{H}, \mathrm{m}, 3 \times \mathrm{SnCH}_{2} \mathrm{CH}_{2} \mathrm{CH}_{2} \mathrm{CH}_{3}\right) ;{ }^{13} \mathrm{C} \mathrm{NMR}(90 \mathrm{MHz}$, $\left.\mathrm{CDCl}_{3}\right) \delta 131.6(\mathrm{CH}), 121.4(\mathrm{CH}), 29.2\left(3 \times \mathrm{CH}_{2}\right), 27.5\left(3 \times \mathrm{CH}_{2}\right), 26.8\left(\mathrm{CH}_{2}\right), 25.0$ $\left(\mathrm{CH}_{2}\right), 23.2\left(\mathrm{CH}_{2}\right), 13.7\left(3 \times \mathrm{CH}_{3}\right), 9.0\left(3 \times \mathrm{CH}_{2}\right)$.

${ }^{1} \mathrm{H}$ spectroscopic data in good agreement with the literature. ${ }^{185}$ 
(2SR,3SR)-2-[(66R)-(7R)-Cyclohex-2-enylhydroxymethyl]-1-(4-methoxy-

phenyl)-3-methyl-5-oxo-3-trimethylsilanyloxy-pyrrolidine-2-carboxylic

acid ethyl ester (145)

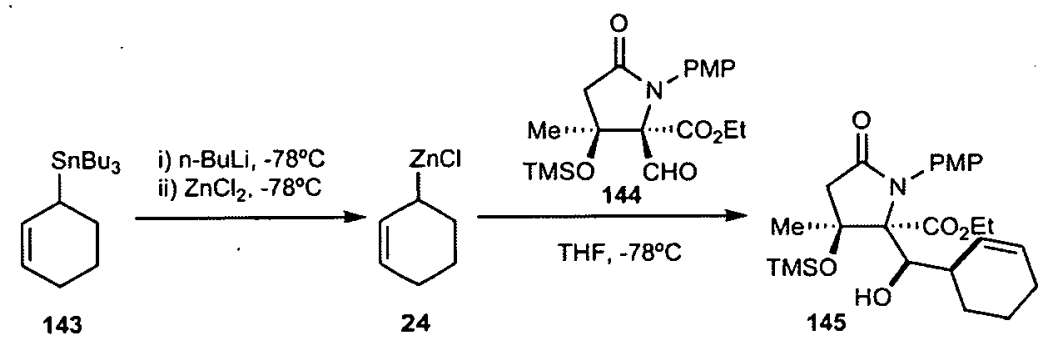

To a stirred solution of cyclohexenyltributyltin $143(3.40 \mathrm{~mL}, 6.37 \mathrm{mmol})$ in THF $(12 \mathrm{~mL})$ at $-78^{\circ} \mathrm{C}$ was added $n$-BuLi $(3.10 \mathrm{~mL}, 2.1 \mathrm{M}$ solution in hexanes, 6.37 $\mathrm{mmol})$. The reaction was stirred for 30 min stirring, $\mathrm{ZnCl}_{2}(12.8 \mathrm{~mL}, 0.5 \mathrm{M}$ solution in THF, $6.37 \mathrm{mmol}$ ) was added and stirring was continued for $30 \mathrm{~min}$ at $-78^{\circ} \mathrm{C}$. To this solution was added a solution of aldehyde $144(1.01 \mathrm{~g}, 2.55 \mathrm{mmol})$ in THF (20 $\mathrm{mL}$ ) at $-78^{\circ} \mathrm{C}$ via cannula. After stirring for $2.5 \mathrm{~h}$ at $-78^{\circ} \mathrm{C}$ the reaction mixture was quenched with water $(20 \mathrm{~mL})$, extracted with EtOAc $(3 \times 30 \mathrm{~mL})$ and the combined organic layers were dried $\left(\mathrm{MgSO}_{4}\right)$, filtered and concentrated in vacuo. Purification of the residue by column chromatography (20\% to $40 \%$ EtOAc/petrol) gave homoallylic alcohol $145(1.1 \mathrm{~g}, 83 \%)$ as a colourless solid. $\mathrm{R}_{\mathrm{f}}(40 \%$ EtOAc in hexane $)=0.69$; m.p. $124-126^{\circ} \mathrm{C}$; IR (film) $2953(\mathrm{OH}), 1688(\mathrm{C}=\mathrm{O}), 1644(\mathrm{C}=\mathrm{O})$, $1510,1375,1295,1250,1148,1028,844 \mathrm{~cm}^{-1} ;{ }^{1} \mathrm{H}$ NMR $\left(360 \mathrm{MHz}, \mathrm{CDCl}_{3}\right) \delta 7.06$ $(2 \mathrm{H}, \mathrm{d}, J=9.0 \mathrm{~Hz}, \mathrm{ArH}), 6.61(2 \mathrm{H}, \mathrm{d}, J=9.0 \mathrm{~Hz}, \mathrm{ArH}), 5.28(1 \mathrm{H}, \mathrm{dm}, J=10.4 \mathrm{~Hz}$, $\mathrm{CH}=\mathrm{CH}), 4.57(1 \mathrm{H}, \mathrm{dm}, J=10.4 \mathrm{~Hz}, \mathrm{CH}=\mathrm{CH}), 4.03(2 \mathrm{H}, \mathrm{q}, J=7.1 \mathrm{~Hz}$, $\left.\mathrm{CO}_{2} \mathrm{CH}_{2} \mathrm{CH}_{3}\right), 3.88(1 \mathrm{H}, \mathrm{t}, J=3.9 \mathrm{~Hz}, \mathrm{CHOH}), 3.52\left(3 \mathrm{H}, \mathrm{s}, \mathrm{OCH}_{3}\right), 3.21(1 \mathrm{H}, \mathrm{s}, J=$ $3.9 \mathrm{~Hz}, \mathrm{CHOH}), 2.45\left(1 \mathrm{H}, \mathrm{d}, J=16.7 \mathrm{~Hz}, \mathrm{CH}_{\mathrm{A}} \mathrm{H}_{\mathrm{B}} \mathrm{C}=\mathrm{O}\right), 2.38(1 \mathrm{H}, \mathrm{d}, J=16.7 \mathrm{~Hz}$, $\left.\mathrm{CH}_{\mathrm{A}} \mathrm{H}_{\mathrm{B}} \mathrm{C}=\mathrm{O}\right), 2.02-1.96(1 \mathrm{H}, \mathrm{m}, \mathrm{CHOHCH}), 1.65-1.53\left(2 \mathrm{H}, \mathrm{m}, \mathrm{CHCH}_{2} \mathrm{CH}_{2}\right), 1.36-$ $1.28\left(2 \mathrm{H}, \mathrm{m}, \mathrm{CH}_{2} \mathrm{CH}_{2} \mathrm{CH}_{2}\right), 1.25\left(3 \mathrm{H}, \mathrm{s}, \mathrm{COTMSCH}_{3}\right), 1.21-1.13\left(2 \mathrm{H}, \mathrm{m}, \mathrm{CH}_{2} \mathrm{CH}_{2}\right)$, $1.10\left(3 \mathrm{H}, \mathrm{t}, J=7.1 \mathrm{~Hz}, \mathrm{CO}_{2} \mathrm{CH}_{2} \mathrm{CH}_{3}\right),-0.01\left(9 \mathrm{H}, \mathrm{s}, 3 \times \mathrm{SiCH}_{3}\right) ;{ }^{13} \mathrm{C} \mathrm{NMR}(90 \mathrm{MHz}$, $\left.\mathrm{CDCl}_{3}\right) \delta 172.1(\mathrm{C}), 169.0(\mathrm{C}), 156.9(\mathrm{C}), 128.8(\mathrm{CH}), 128.2(\mathrm{C}), 126.2(\mathrm{CH}), 125.4$ $(2 \times \mathrm{CH}), 112.1(2 \times \mathrm{CH}), 80.9(\mathrm{C}), 79.5(\mathrm{C}), 73.8(\mathrm{CH}), 59.7\left(\mathrm{CH}_{2}\right), 53.3\left(\mathrm{CH}_{3}\right)$, $43.9\left(\mathrm{CH}_{2}\right), 36.4(\mathrm{CH}), 28.1\left(\mathrm{CH}_{2}\right), 23.4\left(\mathrm{CH}_{3}\right), 22.9\left(\mathrm{CH}_{2}\right), 19.1\left(\mathrm{CH}_{2}\right), 11.9\left(\mathrm{CH}_{3}\right)$, 0.00 (3 x SiCH 3 ); HRMS (ES) Mass calcd. for $\mathrm{C}_{25} \mathrm{H}_{38} \mathrm{NO}_{6} \mathrm{Si}[\mathrm{M}+\mathrm{H}]^{+}: 476.2463$, found: 476.2466 . 
(2RS,3SR)-2-(Hydroxymethyl)-1-(4-methoxyphenyl)-3-methyl-5-oxo-3-

(trimethylsilyloxy)pyrrolidine-2-carboxylic acid ethyl ester (147)

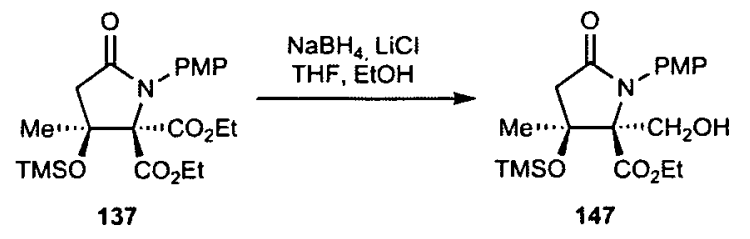

To a stirred solution of diester $137(106 \mathrm{mg}, 0.242 \mathrm{mmol})$ in THF ( $1 \mathrm{~mL})$ at $0{ }^{\circ} \mathrm{C}$ was added anhydrous lithium chloride $(57.3 \mathrm{mg}, 1.33 \mathrm{mmol})$ and sodium borohydride $(51.4 \mathrm{mg}, 1.33 \mathrm{mmol})$, followed by the addition of EtOH $(2 \mathrm{~mL})$. The reaction was stirred for $14 \mathrm{~h}$ at room temperature, then quenched with saturated aqueous $\mathrm{NH}_{4} \mathrm{Cl}$ solution $(1 \mathrm{~mL})$ and the THF removed in vacuo. The solution was extracted with $\mathrm{CH}_{2} \mathrm{Cl}_{2}(3 \times 10 \mathrm{~mL})$ and the combined organic layers were dried $\left(\mathrm{MgSO}_{4}\right)$, filtered and concentrated in vacuo. Purification of the residue by column chromatography (50\% EtOAc/petrol) gave alcohol $147(86.2 \mathrm{mg}, 90 \%)$ as a colourless solid. $\mathrm{R}_{\mathrm{f}}(50 \%$ EtOAc in hexane $)=0.56 ; \mathrm{m} . \mathrm{p} ; 143-144{ }^{\circ} \mathrm{C}$; IR (film) 3389 $(\mathrm{OH}), 2958,1756(\mathrm{C}=\mathrm{O}), 1685(\mathrm{C}=\mathrm{O}), 1511,1392,1250,1102,1047,916 \mathrm{~cm}^{-1} ;{ }^{1} \mathrm{H}$ NMR $\left(360 \mathrm{MHz}, \mathrm{CDCl}_{3}\right) \delta 7.08(2 \mathrm{H}, \mathrm{d}, J=8.9 \mathrm{~Hz}, \mathrm{ArH}), 6.89(2 \mathrm{H}, \mathrm{d}, J=8.9 \mathrm{~Hz}$, ArH), $4.29\left(2 \mathrm{H}, \mathrm{q}, J=7.1 \mathrm{~Hz}, \mathrm{CO}_{2} \mathrm{CH}_{2} \mathrm{CH}_{3}\right), 3.96(1 \mathrm{H}, \mathrm{d}, J=11.6 \mathrm{~Hz}$, $\left.\mathrm{CH}_{\mathrm{A}} \mathrm{CH}_{\mathrm{B}} \mathrm{OH}\right), 3.79\left(4 \mathrm{H}, \mathrm{s}+\mathrm{d}, J=11.6 \mathrm{~Hz}, \mathrm{OCH}_{3}+\mathrm{CH}_{\mathrm{A}} \mathrm{CH}_{\mathrm{B}} \mathrm{OH}\right), 3.04(1 \mathrm{H}, \mathrm{d}, J=$ $\left.16.1 \mathrm{~Hz}, \mathrm{CH}_{\mathrm{C}} \mathrm{H}_{\mathrm{D}} \mathrm{C}=\mathrm{O}\right), 2.56\left(1 \mathrm{H}, \mathrm{d}, J=16.1 \mathrm{~Hz}, \mathrm{CH}_{\mathrm{C}} \mathbf{H}_{\mathrm{D}} \mathrm{C}=\mathrm{O}\right), 1.36(3 \mathrm{H}, \mathrm{s}$, $\left.\mathrm{COTMSCH}_{3}\right), 1.36\left(3 \mathrm{H}, \mathrm{t}, J=7.1 \mathrm{~Hz}, \mathrm{CO}_{2} \mathrm{CH}_{2} \mathrm{CH}_{3}\right), 0.16\left(9 \mathrm{H}, \mathrm{s}, 3 \times \mathrm{SiCH}_{3}\right) ;{ }^{13} \mathrm{C}$ NMR (90 MHz, $\mathrm{CDCl}_{3}$ ) $\delta 172.3$ (C), 169.5 (C), 157.2 (C), 127.3 (2 x CH), 126.7 (C), $112.5(2 \times \mathrm{CH}), 76.6(\mathrm{C}), 75.2(\mathrm{C}), 60.3\left(\mathrm{CH}_{2}\right), 59,7\left(\mathrm{CH}_{2}\right), 53.4\left(\mathrm{CH}_{3}\right), 44.9$ $\left(\mathrm{CH}_{2}\right), 22.8\left(\mathrm{CH}_{3}\right), 12.3\left(\mathrm{CH}_{3}\right), 0.0\left(3 \times \mathrm{CH}_{3}\right)$; HRMS (ES) Mass calcd. for $\mathrm{C}_{19} \mathrm{H}_{30} \mathrm{NO}_{6} \mathrm{Si}[\mathrm{M}+\mathrm{H}]^{+}:$396.1837, found: 396.1837 .

\section{(2SR,3SR)-2-Formyl-1-(4-methoxy-phenyl)-3-methyl-5-oxo-3-}

trimethylsilanyloxy-pyrrolidine-2-carboxylic acid ethyl ester (148)

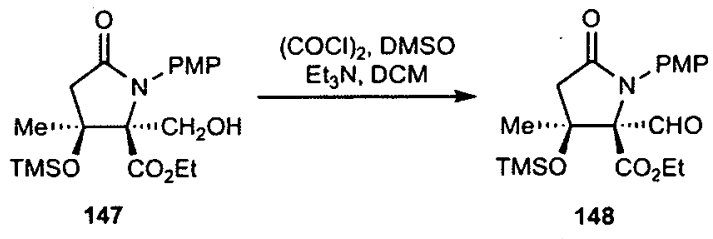


To a stirred solution of oxalyl chloride $(0.047 \mathrm{~mL}, 0.52 \mathrm{mmol})$ in $\mathrm{CH}_{2} \mathrm{Cl}_{2}(2 \mathrm{~mL})$ at $-78{ }^{\circ} \mathrm{C}$ was added DMSO $(0.075 \mathrm{~mL}, 1.1 \mathrm{mmol})$ dropwise over $1 \mathrm{~min}$. After stirring for 15 minutes a solution of alcohol $147(99.0 \mathrm{mg}, 0.25 \mathrm{mmol})$ in $\mathrm{CH}_{2} \mathrm{Cl}_{2}$ $(1.5 \mathrm{~mL})$ was added via cannula. This reaction was stirred at $-78{ }^{\circ} \mathrm{C}$ for $1 \mathrm{~h}$ and $\mathrm{Et}_{3} \mathrm{~N}$ $(0.15 \mathrm{~mL}, 1.1 \mathrm{mmol})$ was then added. The reaction was stirred at $-78^{\circ} \mathrm{C}$ for $2 \mathrm{~h}$ and then was quenched with saturated aqueous $\mathrm{NH}_{4} \mathrm{Cl}$ solution $(3 \mathrm{~mL})$. The aqueous layer was separated and extracted with $\mathrm{CH}_{2} \mathrm{Cl}_{2}(3 \times 10 \mathrm{~mL})$, and the combined organic layers were dried $\left(\mathrm{MgSO}_{4}\right)$, filtered and concentrated in vacuo. Purification of the residue by column chromatography (30\% EtOAc/petrol) gave aldehyde 148 $(88 \mathrm{mg}, 90 \%)$ as a yellow oil. $\mathrm{R}_{\mathrm{f}}(40 \%$ EtOAc in hexane) $=0.54$; IR (film) 3418 , 2959, $1722(\mathrm{C}=\mathrm{O}), 1644(\mathrm{C}=\mathrm{O}), 1512,1351,1249,1159,1024,844 \mathrm{~cm}^{-1} ;{ }^{1} \mathrm{H}$ NMR $\left(360 \mathrm{MHz}, \mathrm{CDCl}_{3}\right) \delta 10.18(1 \mathrm{H}, \mathrm{s}, \mathrm{CHO}), 7.12(2 \mathrm{H}, \mathrm{d}, J=9.1 \mathrm{~Hz}, \mathrm{ArH}), 6.84(2 \mathrm{H}$, d, $J=9.1 \mathrm{~Hz}, \mathrm{ArH}), 4.31-4.23\left(2 \mathrm{H}, \mathrm{m}, \mathrm{CO}_{2} \mathrm{CH}_{2} \mathrm{CH}_{3}\right), 3.79\left(3 \mathrm{H}, \mathrm{s}, \mathrm{OCH}_{3}\right), 2.94(1 \mathrm{H}$, $\left.\mathrm{d}, J=16.4 \mathrm{~Hz}, \mathrm{CH}_{\mathrm{A}} \mathrm{H}_{\mathrm{B}} \mathrm{C}=\mathrm{O}\right), 2.60\left(1 \mathrm{H}, \mathrm{d}, J=16.4 \mathrm{~Hz}, \mathrm{CH}_{\mathrm{A}} \mathbf{H}_{\mathrm{B}} \mathrm{C}=\mathrm{O}\right), 1.52(3 \mathrm{H}, \mathrm{s}$, $\left.\mathrm{COTMSCH}_{3}\right), 1.26\left(3 \mathrm{H}, \mathrm{t}, J=7.2 \mathrm{~Hz}, \mathrm{CO}_{2} \mathrm{CH}_{2} \mathrm{CH}_{3}\right), 0.19\left(9 \mathrm{H}, \mathrm{s}, 3 \times \mathrm{SiCH}_{3}\right) ;{ }^{13} \mathrm{C}$ NMR (90 MHz, $\left.\mathrm{CDCl}_{3}\right) \delta 195.3(\mathrm{CH}), 171.0(\mathrm{C}), 165.5(\mathrm{C}), 156.3(\mathrm{C}), 128.2(\mathrm{C})$, $125.6(2 \times \mathrm{CH}), 112.1(2 \times \mathrm{CH}), 82.8(\mathrm{C}), 78.2(\mathrm{C}), 60.4\left(\mathrm{CH}_{2}\right), 53.5\left(\mathrm{CH}_{3}\right), 43.7$ $\left(\mathrm{CH}_{2}\right), 23.5\left(\mathrm{CH}_{3}\right), 12.2\left(\mathrm{CH}_{3}\right), 0.0\left(3 \times \mathrm{CH}_{3}\right)$; HRMS (ES) Mass calcd. for $\mathrm{C}_{19} \mathrm{H}_{28} \mathrm{NO}_{6} \mathrm{Si}[\mathrm{M}+\mathrm{H}]^{+}: 394.1680$, found: 394.1682 .

(2RS,3SR)-2-[(6S)-(7S)-Cyclohex-2-enylhydroxymethyl]-1-(4-methoxy-phenyl)3-methyl-5-oxo-3-trimethylsilanyloxy-pyrrolidine-2-carboxylic acid ethyl ester (149)

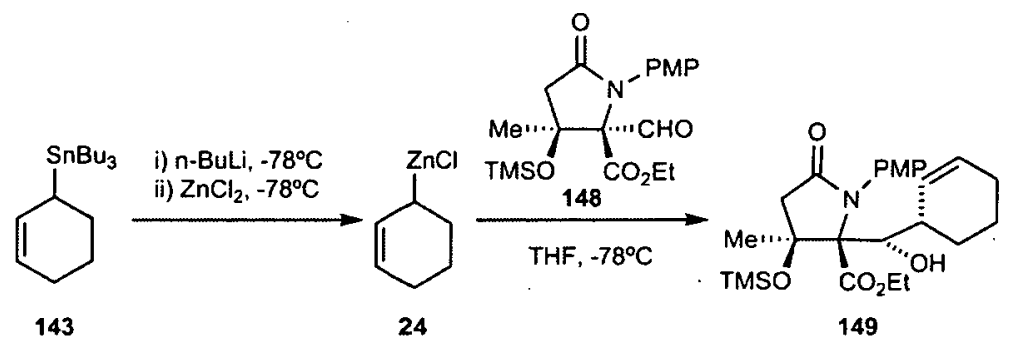

To a stirred solution of cyclohexenyltributyltin $143(1.30 \mathrm{~g}, 3.35 \mathrm{mmol})$ in THF $(7 \mathrm{~mL})$ at $-78{ }^{\circ} \mathrm{C}$ was added $n$-BuLi $(1.3 \mathrm{~mL}, 2.5 \mathrm{M}$ solution in hexanes, $3.2 \mathrm{mmol})$. The reaction was stirred for $30 \mathrm{~min}, \mathrm{ZnCl}_{2}(6.70 \mathrm{~mL}, 0.5 \mathrm{M}$ solution in THF, 3.35 mmol) was added and stirring was continued for $30 \mathrm{~min}$ at $-78{ }^{\circ} \mathrm{C}$. To this solution 
was added a $-78^{\circ} \mathrm{C}$ solution of the aldehyde $148(0.53 \mathrm{~g}, 1.34 \mathrm{mmol})$ in THF $(6 \mathrm{~mL})$ via cannula. After stirring for $2.5 \mathrm{~h}$ at $-78^{\circ} \mathrm{C}$ the reaction mixture was quenched with water $(20 \mathrm{~mL})$, extracted with $\mathrm{Et}_{2} \mathrm{O}(3 \times 30 \mathrm{~mL})$ and the combined organic layers were dried $\left(\mathrm{MgSO}_{4}\right)$, filtered and concentrated in vacuo. Purification of the residue by column chromatography (30\% to $40 \% \mathrm{EtOAc} / \mathrm{petrol})$ gave homoallylic alcohol 149 (543 mg, 84\%) as a colourless solid. $\mathrm{R}_{\mathrm{f}}(40 \%$ EtOAc in hexane $)=0.16$; m.p. 175-177 ${ }^{\circ} \mathrm{C}$; IR (film) $3406(\mathrm{OH}), 2932,1755(\mathrm{C}=\mathrm{O}), 1683(\mathrm{C}=\mathrm{O}), 1509,1456$, $1382,1250,1137,841 \mathrm{~cm}^{-1} ;{ }^{1} \mathrm{H}$ NMR $\left(360 \mathrm{MHz}, \mathrm{CDCl}_{3}\right) \delta 7.53(2 \mathrm{H}, \mathrm{d}, J=9.0 \mathrm{~Hz}$, $\operatorname{ArH}), 6.87(2 \mathrm{H}, \mathrm{d}, J=9.0 \mathrm{~Hz}, \mathrm{ArH}), 5.79(1 \mathrm{H}, \mathrm{dm}, J=10.2 \mathrm{~Hz}, \mathrm{CH}=\mathrm{CH}), 5.35(1 \mathrm{H}$, $\mathrm{dm}, J=10.2 \mathrm{~Hz}, \mathrm{CH}=\mathrm{CH}), 4.25-4.13\left(2 \mathrm{H}, \mathrm{m}, \mathrm{CO}_{2} \mathrm{CH}_{2} \mathrm{CH}_{3}\right), 4.01(1 \mathrm{H}, \mathrm{t}, J=5.7 \mathrm{~Hz}$, $\mathrm{CHOH}), 3.80\left(3 \mathrm{H}, \mathrm{s}, \mathrm{OCH}_{3}\right), 2.84\left(1 \mathrm{H}, \mathrm{d}, J=16.5 \mathrm{~Hz}, \mathrm{CH}_{\mathrm{A}} \mathrm{H}_{\mathrm{B}} \mathrm{C}=\mathrm{O}\right), 2.67(1 \mathrm{H}, \mathrm{d}, J$ $\left.=16.5, \mathrm{CH}_{\mathrm{A}} \mathbf{H}_{\mathrm{B}} \mathrm{C}=\mathrm{O}\right), 2.25-2.19(1 \mathrm{H}, \mathrm{bs}, \mathrm{CHOHCH}), 1.94-1.84(3 \mathrm{H}, \mathrm{bs}$, $\left.\mathrm{CHCH}_{2} \mathrm{CH}_{2}\right), 1.70(3 \mathrm{H}, \mathrm{s}, \mathrm{COTMSCH}), 1.63-1.59\left(2 \mathrm{H}, \mathrm{m}, \mathrm{CH}_{2} \mathrm{CH}_{2} \mathrm{CH}_{2}\right), 1.55-1.47$ $\left(1 \mathrm{H}, \mathrm{m}, \mathrm{CH}_{2} \mathrm{CH}_{2}\right), 1.34\left(3 \mathrm{H}, \mathrm{t}, J=7.2 \mathrm{~Hz}, \mathrm{CO}_{2} \mathrm{CH}_{2} \mathrm{CH}_{3}\right), 0.16\left(9 \mathrm{H}, \mathrm{s}, 3 \times \mathrm{SiCH}_{3}\right)$; ${ }^{13} \mathrm{C} \mathrm{NMR}\left(90 \mathrm{MHz}, \mathrm{CDCl}_{3}\right) \delta 172.2(\mathrm{C}), 167.4(\mathrm{C}), 156.8(\mathrm{C}), 130.1(\mathrm{CH}), 128.9$ (C), $128.5(\mathrm{CH}), 124.8(2 \times \mathrm{CH}), 111.5(2 \times \mathrm{CH}), 81.9(\mathrm{C}), 78.7(\mathrm{C}), 73.9(\mathrm{CH}), 58.7$ $\left(\mathrm{CH}_{2}\right), 53.3\left(\mathrm{CH}_{3}\right), 44.4\left(\mathrm{CH}_{2}\right), 36.5(\mathrm{CH}), 24.8\left(\mathrm{CH}_{2}\right), 22.9\left(\mathrm{CH}_{2}\right), 22.2\left(\mathrm{CH}_{3}\right), 18.3$ $\left(\mathrm{CH}_{2}\right), 11.9\left(\mathrm{CH}_{3}\right), 0.0\left(3 \times \mathrm{CH}_{3}\right)$; LRMS (ES) Mass calcd. for $\mathrm{C}_{25} \mathrm{H}_{38} \mathrm{NO}_{6} \mathrm{Si}[\mathrm{M}+\mathrm{H}]^{+}$: 476.2463, found: 476.2465 .

(2RS,3SR)-2-(Hydroxymethyl)-1-(4-methoxybenzyl)-3-methyl-5-0x0-3(trimethylsilyloxy)pyrrolidine-2-carboxylic acid ethyl ester (503)

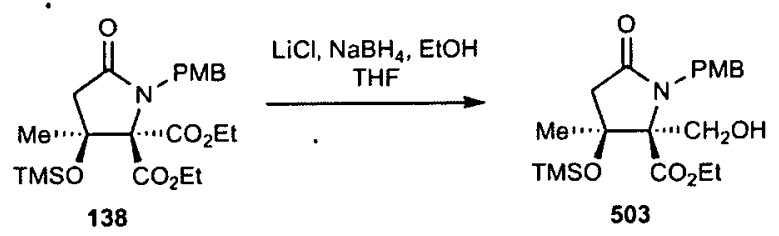

To a stirred solution of diester $138(6.16 \mathrm{~g}, 13.6 \mathrm{mmol})$ in THF $(38 \mathrm{~mL})$ at $0{ }^{\circ} \mathrm{C}$, was added anhydrous lithium chloride (1.20 g, $27.3 \mathrm{mmol})$ and sodium borohydride $(1.03 \mathrm{~g}, 27.3 \mathrm{mmol})$, followed by the addition of EtOH $(76 \mathrm{~mL})$. The reaction was stirred for $14 \mathrm{~h}$ at room temperature, then quenched with saturated aqueous $\mathrm{NH}_{4} \mathrm{Cl}$ solution $(40 \mathrm{~mL})$ and the THF removed in vacuo. The solution was extracted with $\mathrm{CH}_{2} \mathrm{Cl}_{2}(3 \times 50 \mathrm{~mL})$ and the combined organic layers were dried $\left(\mathrm{MgSO}_{4}\right)$, filtered 
and concentrated in vacuo. Purification of the residue by column chromatography (30\% EtOAc/petrol) gave alcohol $503(3.82 \mathrm{~g}, 68 \%)$ as a colourless solid. $\mathrm{R}_{\mathrm{f}}(40 \%$ EtOAc in hexane $)=0.20$; m.p. $112-114^{\circ} \mathrm{C}$; IR (film) $3453(\mathrm{OH}), 2957,2348,1734$ $(\mathrm{C}=\mathrm{O}), 1678(\mathrm{C}=\mathrm{O}), 1513,1248,1176,1034,841 \mathrm{~cm}^{-1} ;{ }^{1} \mathrm{H} \mathrm{NMR}\left(360 \mathrm{MHz}, \mathrm{CDCl}_{3}\right)$ $\delta 7.29(2 \mathrm{H}, \mathrm{d}, J=8.9 \mathrm{~Hz}, \operatorname{ArH}), 6.80(2 \mathrm{H}, \mathrm{d}, J=8.9 \mathrm{~Hz}, \operatorname{ArH}), 4.73(1 \mathrm{H}, \mathrm{d}, J=15.0$ $\left.\mathrm{Hz}, \mathrm{CH}_{\mathrm{A}} \mathrm{H}_{\mathrm{B}} \mathrm{OH}\right), 4.38\left(1 \mathrm{H}, \mathrm{d}, J=15.0 \mathrm{~Hz}, \mathrm{CH}_{\mathrm{A}} \mathbf{H}_{\mathrm{B}} \mathrm{OH}\right), 4.12-4.02(1 \mathrm{H}, \mathrm{m}$, $\left.\mathrm{CO}_{2} \mathrm{CH}_{\mathrm{C}} \mathrm{H}_{\mathrm{D}} \mathrm{CH}_{3}\right), 3.96-3.88\left(1 \mathrm{H}, \mathrm{m}, \mathrm{CO}_{2} \mathrm{CH}_{\mathrm{C}} \mathbf{H}_{\mathbf{D}} \mathrm{CH}_{3}\right), 3.87(1 \mathrm{H}, \mathrm{d}, J=12.5 \mathrm{~Hz}$, $\left.\mathrm{CH}_{\mathbf{E}} \mathrm{H}_{\mathrm{F}} \mathrm{Ar}\right), 3.77\left(3 \mathrm{H}, \mathrm{s}, \mathrm{OCH}_{3}\right), 3.72\left(1 \mathrm{H}, \mathrm{d}, J=12.5 \mathrm{~Hz}, \mathrm{CH}_{\mathrm{E}} \mathbf{H}_{\mathrm{F}} \mathrm{Ar}\right), 2.76(1 \mathrm{H}, \mathrm{d}, J$ $\left.=16.2 \mathrm{~Hz}, \mathrm{CH}_{\mathrm{A}} \mathrm{H}_{\mathrm{B}} \mathrm{C}=\mathrm{O}\right), 2.47\left(1 \mathrm{H}, \mathrm{d}, J=16.2 \mathrm{~Hz}, \mathrm{CH}_{\mathrm{A}} \mathbf{H}_{\mathrm{B}} \mathrm{C}=\mathrm{O}\right), 2.25(1 \mathrm{H}, \mathrm{bs}, \mathrm{OH})$, $1.47\left(3 \mathrm{H}, \mathrm{s}, \mathrm{COTMSCH}{ }_{3}\right), 1.19\left(3 \mathrm{H}, \mathrm{td}, J=7.1,2.6 \mathrm{~Hz}, \mathrm{CO}_{2} \mathrm{CH}_{2} \mathrm{CH}_{3}\right), 0.09(9 \mathrm{H}, \mathrm{s}$, $\left.\mathrm{Si}\left(\mathrm{CH}_{3}\right)_{3}\right) ;{ }^{13} \mathrm{C}$ NMR (62.9 $\left.\mathrm{MHz}_{,} \mathrm{CDCl}_{3}\right) \delta 174.2$ (C), 170.4 (C), 158.9 (C), 129.8 (C), $129.7(2 \times \mathrm{CH}), 114.0(\mathrm{C}), 113.8(2 \times \mathrm{CH}), 78.2(\mathrm{C}), 62.1\left(\mathrm{CH}_{2}\right), 61.3\left(\mathrm{CH}_{2}\right)$, $55.2\left(\mathrm{CH}_{3}\right), 46.6\left(\mathrm{CH}_{2}\right), 44.1\left(\mathrm{CH}_{2}\right), 24.3\left(\mathrm{CH}_{3}\right), 14.0\left(\mathrm{CH}_{3}\right), 1.8\left(3 \times \mathrm{CH}_{3}\right)$; HRMS (ES) Exact mass calcd for $\mathrm{C}_{20} \mathrm{H}_{32} \mathrm{NO}_{6} \mathrm{Si}[\mathrm{M}+\mathrm{H}]^{+}: 410.1993$, found: 410.1994 .

\section{(2SR,3SR)-2-Formyl-1-(4-methoxybenzyl)-3-methyl-5-oxo-3-}

(trimethylsilyloxy)pyrrolidine-2-carboxylic acid ethyl ester (150)

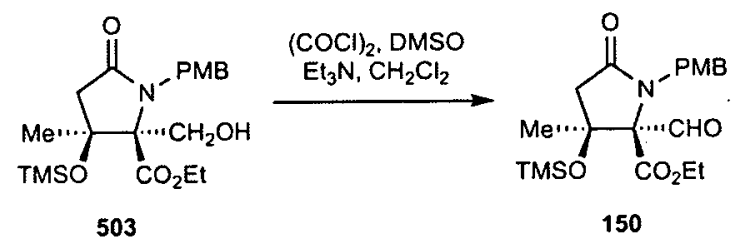

To a stirred solution of oxalyl chloride $(0.74 \mathrm{~mL}, 8.46 \mathrm{mmol})$ in $\mathrm{CH}_{2} \mathrm{Cl}_{2}(40 \mathrm{~mL})$ at $-78{ }^{\circ} \mathrm{C}$ was added DMSO $(1.20 \mathrm{~mL}, 16.9 \mathrm{mmol})$ dropwise over $1 \mathrm{~min}$ and this solution was stirred for $15 \mathrm{~min}$. A solution of alcohol 503 (1.65 g, $4.03 \mathrm{mmol})$ in $\mathrm{CH}_{2} \mathrm{Cl}_{2}(40 \mathrm{~mL})$ at $-78^{\circ} \mathrm{C}$, was treated with $\mathrm{Et}_{3} \mathrm{~N}(2.40 \mathrm{~mL}, 16.9 \mathrm{mmol})$, followed by the addition of the Swern reagent via cannula. The reaction was stirred at $-78{ }^{\circ} \mathrm{C}$ for $3 \mathrm{~h}$ and then quenched with $\mathrm{NH}_{4} \mathrm{Cl}(30 \mathrm{~mL})$. The aqueous layer was separated and extracted with $\mathrm{CH}_{2} \mathrm{Cl}_{2}(3 \times 20 \mathrm{~mL})$ and the combined organic layers were washed with saturated aqueous $\mathrm{NaHCO}_{3}$ solution $(2 \times 30 \mathrm{~mL})$, dried $\left(\mathrm{MgSO}_{4}\right)$, filtered and concentrated in vacuo. Purification of the residue by column chromatography $(30 \%$ EtOAc/petrol) gave aldehyde $150(1.28 \mathrm{~g}, 78 \%)$ as a yellow oil. $\mathrm{R}_{\mathrm{f}}(30 \%$ EtOAc in hexane $)=0.45$; IR (film) 2957, $1756(\mathrm{C}=\mathrm{O}), 1724(\mathrm{C}=\mathrm{O}), 1612(\mathrm{C}=\mathrm{O}), 1513,1378$, 
$1282,1250,1025,845 \mathrm{~cm}^{-1} ;{ }^{1} \mathrm{H}$ NMR $\left(360 \mathrm{MHz}, \mathrm{CDCl}_{3}\right) \delta 9.91(1 \mathrm{H}, \mathrm{s}, \mathrm{CHO}), 7.09$ $(2 \mathrm{H}, \mathrm{d}, J=8.6 \mathrm{~Hz}, \operatorname{ArH}), 6.75(2 \mathrm{H}, \mathrm{d}, J=8.6 \mathrm{~Hz}, \operatorname{ArH}), 4.89(1 \mathrm{H}, \mathrm{d}, J=14.4 \mathrm{~Hz}$, $\left.\mathrm{CH}_{\mathrm{A}} \mathrm{H}_{\mathrm{B}} \mathrm{Ar}\right), 4.34\left(1 \mathrm{H}, \mathrm{d}, J=14.4 \mathrm{~Hz}, \mathrm{CH}_{\mathrm{A}} \mathbf{H}_{\mathrm{B}} \mathrm{Ar}\right), 3.96-3.87(1 \mathrm{H}, \mathrm{m}$, $\left.\mathrm{CO}_{2} \mathrm{CH}_{\mathrm{A}} \mathrm{H}_{\mathrm{B}} \mathrm{CH}_{3}\right), 3.82-3.75\left(1 \mathrm{H}, \mathrm{m}, \mathrm{CO}_{2} \mathrm{CH}_{\mathrm{A}} \mathbf{H}_{\mathrm{B}} \mathrm{CH}_{3}\right), 3.74\left(3 \mathrm{H}, \mathrm{s}, \mathrm{OCH}_{3}\right), 2.89(1 \mathrm{H}$, $\left.\mathrm{d}, J=16.0 \mathrm{~Hz}, \mathrm{CH}_{\mathrm{A}} \mathrm{H}_{\mathrm{B}} \mathrm{C}=\mathrm{O}\right), 2.41\left(1 \mathrm{H}, \mathrm{d}, J=16.2 \mathrm{~Hz}, \mathrm{CH}_{\mathrm{A}} \mathbf{H}_{\mathbf{B}} \mathrm{C}=\mathrm{O}\right), 1.35(3 \mathrm{H}, \mathrm{s}$, $\left.\mathrm{COTMSCH}_{3}\right), 1.11\left(3 \mathrm{H}, \mathrm{t}, J=7.1 \mathrm{~Hz}, \mathrm{CO}_{2} \mathrm{CH}_{2} \mathrm{CH}_{3}\right), 0.10\left(9 \mathrm{H}, \mathrm{s}, \mathrm{Si}\left(\mathrm{CH}_{3}\right)_{3}\right) ;{ }^{13} \mathrm{C}$ $\operatorname{NMR}\left(62.9 \mathrm{MHz}, \mathrm{CDCl}_{3}\right) \delta 196.6(\mathrm{CH}), 172.6(\mathrm{C}), 167.0(\mathrm{C}), 158.4(\mathrm{C}), 130.5(2 \mathrm{x}$ $\mathrm{CH}), 126.9(\mathrm{C}), 112.9(2 \times \mathrm{CH})^{\prime}, 80.5(\mathrm{C}), 78.9(\mathrm{C}), 61.2\left(\mathrm{CH}_{2}\right), 54.6\left(\mathrm{CH}_{3}\right), 45.3$ $\left(\mathrm{CH}_{2}\right), 43.9\left(\mathrm{CH}_{2}\right), 25.1\left(\mathrm{CH}_{3}\right), 13.3\left(\mathrm{CH}_{3}\right), 1.2\left(3 \times \mathrm{CH}_{3}\right)$; HRMS (ES) Exact mass calcd for $\mathrm{C}_{20} \mathrm{H}_{30} \mathrm{NO}_{6} \mathrm{Si}[\mathrm{M}+\mathrm{H}]^{+}: 408.1837$, found: 408.1836 .

(2RS,3SR)-2-[(6S)-(7S)-Cyclohex-2-enylhydroxymethyl]-1-(4-methoxybenzyl)-3methyl-5-oxo-3-(trimethylsilyloxy)pyrrolidine-2-carboxylic acid ethyl ester (151)

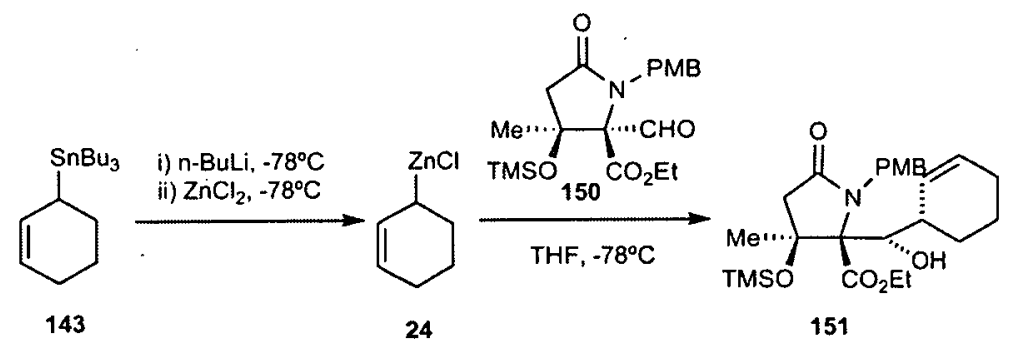

To a stirred solution of cyclohexenyltributyltin $143(2.78 \mathrm{~g}, 6.44 \mathrm{mmol}, 85 \%)$ in THF $(5 \mathrm{~mL})$ at $-78{ }^{\circ} \mathrm{C}$ was added $n$-BuLi $(2.50 \mathrm{~mL}, 2.5 \mathrm{M}$ solution in hexanes, 6.31 $\mathrm{mmol})$. The reaction was stirred for $30 \mathrm{~min}, \mathrm{ZnCl}_{2}(12.8 \mathrm{~mL}, 0.5 \mathrm{M}$ solution in THF, $6.44 \mathrm{mmol}$ ) was added and stirring was continued for $30 \mathrm{~min}$ at $-78{ }^{\circ} \mathrm{C}$. To this solution was added a $-78^{\circ} \mathrm{C}$ solution of aldehyde 150 (1.05 g, $\left.2.57 \mathrm{mmol}\right)$ in THF (6 $\mathrm{mL}$ ) via cannula. After stirring for $1.5 \mathrm{~h}$ at $-78^{\circ} \mathrm{C}$ the reaction mixture was quenched with water $(15 \mathrm{~mL})$, extracted with $\mathrm{Et}_{2} \mathrm{O}(3 \times 20 \mathrm{~mL})$ and the combined organic layers were dried $\left(\mathrm{MgSO}_{4}\right)$, filtered and concentrated in vacuo. Purification of the residue by column chromatography (20\% to $30 \%$ EtOAc/petrol) gave homoallylic alcohol 151 (892 $\mathrm{mg}, 71 \%)$ as a colourless solid. $\mathrm{R}_{\mathrm{f}}(40 \%$ EtOAc in hexane $)=0.31$; m.p. 169-171 ${ }^{\circ} \mathrm{C}$; IR (film) $3429(\mathrm{OH}), 2923,2666,1748(\mathrm{C}=\mathrm{O}), 1681(\mathrm{C}=\mathrm{O}), 1513$, 1442, 1247, 1034, $841 \mathrm{~cm}^{-1} ;{ }^{1} \mathrm{H}$ NMR $\left(360 \mathrm{MHz}, \mathrm{CDCl}_{3}\right) \delta 7.29(2 \mathrm{H}, \mathrm{d}, J=8.8 \mathrm{~Hz}$, ArH), $6.81(2 \mathrm{H}, \mathrm{d}, J=8.8 \mathrm{~Hz}, \mathrm{ArH}), 5.95(1 \mathrm{H}, \mathrm{dm}, J=10.4 \mathrm{~Hz}, \mathrm{CH}=\mathrm{CH}), 5.69(1 \mathrm{H}$, $\mathrm{dm}, J=10.4 \mathrm{~Hz}, \mathrm{CH}=\mathrm{CH}), 4.82\left(1 \mathrm{H}, \mathrm{d}, J=15.2 \mathrm{~Hz}, \mathrm{CH}_{\mathrm{A}} \mathrm{H}_{\mathrm{B}} \mathrm{Ar}\right), 4.47(1 \mathrm{H}, \mathrm{d}, J=$ 
15.2 Hz, $\mathrm{CH}_{\mathrm{A}} \mathbf{H}_{\mathrm{B}} \mathrm{Ar}$ ), 4.11-4.05 (2H, m, $\left.\mathrm{CO}_{2} \mathrm{CH}_{2} \mathrm{CH}_{3}+\mathrm{CHOH}\right), 3.78\left(3 \mathrm{H}, \mathrm{s}, \mathrm{OCH}_{3}\right)$, $2.85\left(1 \mathrm{H}, \mathrm{d}, J=16.6 \mathrm{~Hz}, \mathrm{CH}_{\mathrm{A}} \mathrm{H}_{\mathrm{B}} \mathrm{C}=\mathrm{O}\right), 2.45(1 \mathrm{H}, \mathrm{bs}, \mathrm{CHOH}), 2.39(1 \mathrm{H}, \mathrm{d}, J=16.6$ $\left.\mathrm{Hz}, \mathrm{CH}_{\mathrm{A}} \mathbf{H}_{\mathrm{B}} \mathrm{C}=\mathrm{O}\right), 2.32-2.26(1 \mathrm{H}, \mathrm{m}, \mathrm{CHOHCH}), 2.03-1.98\left(2 \mathrm{H}, \mathrm{m}, \mathrm{CHCH}_{2} \mathrm{CH}_{2}\right)$, 1.75-1.68 (3H + 2H, m, COTMSCH $\left.3+\mathrm{CH}_{2} \mathrm{CH}_{2} \mathrm{CH}_{2}\right), 1.57-1.44\left(2 \mathrm{H}, \mathrm{m}, \mathrm{CH}_{2} \mathrm{CH}_{2}\right)$, $1.11\left(3 \mathrm{H}, \mathrm{t}, J=7.0 \mathrm{~Hz}, \mathrm{CO}_{2} \mathrm{CH}_{2} \mathrm{CH}_{3}\right), 0.16\left(9 \mathrm{H}, \mathrm{s}, \mathrm{Si}\left(\mathrm{CH}_{3}\right)_{3}\right) ;{ }^{13} \mathrm{C} \mathrm{NMR}(90 \mathrm{MHz}$, $\left.\mathrm{CDCl}_{3}\right) \delta 175.1(\mathrm{C}), 168.9(\mathrm{C}), 157.9(\mathrm{C}), 133.7(\mathrm{CH}), 130.6(\mathrm{C}), 127.6(2 \times \mathrm{CH})$, $124.4(\mathrm{CH}), 113.2(2 \times \mathrm{CH}), 82.8(\mathrm{C}), 82.0(\mathrm{C}), 76.5(\mathrm{CH}), 60.9\left(\mathrm{CH}_{2}\right), 55.2\left(\mathrm{CH}_{3}\right)$, $47.5\left(\mathrm{CH}_{2}\right), 46.7\left(\mathrm{CH}_{2}\right), 38.0(\mathrm{CH}), 29.0\left(\mathrm{CH}_{2}\right), 25.0\left(\mathrm{CH}_{2}\right), 22.6\left(\mathrm{CH}_{3}\right), 20.5\left(\mathrm{CH}_{2}\right)$, $13.7\left(\mathrm{CH}_{3}\right), 2.1\left(3 \times \mathrm{CH}_{3}\right)$; HRMS (ES) Exact mass calcd for $\mathrm{C}_{26} \mathrm{H}_{40} \mathrm{NO}_{6} \mathrm{Si}[\mathrm{M}+\mathrm{H}]^{+}$: 490.2619 , found: 490.2616 .

(2RS,3SR)-2-[(6S)-(7S)-Cyclohex-2-enylhydroxymethyl]-3-hydroxy-1-(4methoxyphenyl)-3-methyl-5-oxopyrrolidine-2-carboxylic acid ethyl ester (156)

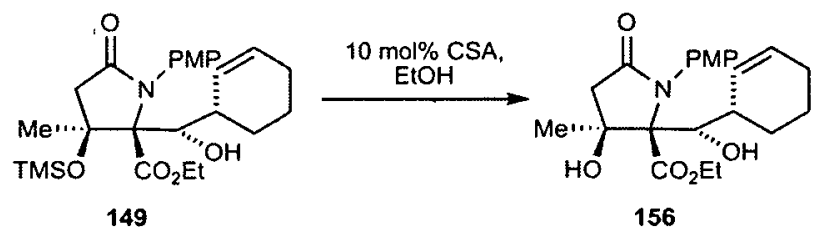

To a stirred solution of alcohol $149(2.00 \mathrm{~g}, 4.80 \mathrm{mmol})$ in EtOH $(10 \mathrm{~mL})$ was added camphor sulfonic acid $(245 \mathrm{mg}, 1.03 \mathrm{mmol}$ ) at room temperature. This solution was stirred for $3 \mathrm{~h}$ and then quenched with $\mathrm{Et}_{3} \mathrm{~N}(0.14 \mathrm{~mL}, 1.04 \mathrm{mmol})$. The solution was concentrated in vacuo. Purification of the residue by column chromatography (60\% to $80 \%$ EtOAc/petrol) gave diol $156(1.92 \mathrm{~g}, 95 \%)$ as a colourless solid. $\mathrm{R}_{\mathrm{f}}(50 \%$ EtOAc in hexane $)=0.16$; m.p. $184-186^{\circ} \mathrm{C}$; IR (film) 3393 $(\mathrm{OH}), 2933,2359,2341,1753(\mathrm{C}=\mathrm{O}), 1682(\mathrm{C}=\mathrm{O}), 1509,1372,1249,1030 \mathrm{~cm}^{-1} ;{ }^{1} \mathrm{H}$ NMR $\left(360 \mathrm{MHz}, \mathrm{CDCl}_{3}\right) \delta 7.57(2 \mathrm{H}, \mathrm{d}, J=9.0 \mathrm{~Hz}, \operatorname{ArH}), 6.86(2 \mathrm{H}, \mathrm{d}, J=9.0 \mathrm{~Hz}$, $\operatorname{ArH}), 5.77(1 \mathrm{H}, \mathrm{dm}, J=10.3 \mathrm{~Hz}, \mathbf{C H}=\mathrm{CH}), 5.41(1 \mathrm{H}, \mathrm{dm}, J=10.4 \mathrm{~Hz}, \mathrm{CH}=\mathrm{CH})$, 4.41-4.28 (1H, m, $\left.\mathrm{CO}_{2} \mathrm{CH}_{\mathrm{A}} \mathrm{H}_{\mathrm{B}} \mathrm{CH}_{3}\right), 4.24-4.12\left(2 \mathrm{H}, \mathrm{m}, \mathrm{CO}_{2} \mathrm{CH}_{\mathrm{A}} \mathrm{H}_{\mathbf{B}} \mathrm{CH}_{3}\right), 4.12-4.06$ $(1 \mathrm{H}, \mathrm{m}, \mathrm{CHOH}), 3.81\left(3 \mathrm{H}, \mathrm{s}, \mathrm{OCH}_{3}\right), 2.95\left(1 \mathrm{H}, \mathrm{d}, J=16.8 \mathrm{~Hz}, \mathrm{CH}_{\mathrm{C}} \mathrm{H}_{\mathrm{D}} \mathrm{C}=\mathrm{O}\right), 2.93$ $(1 \mathrm{H}, \mathrm{s}, \mathrm{CHOH}), 2.55\left(1 \mathrm{H}, \mathrm{d}, J=16.8 \mathrm{~Hz}, \mathrm{CH}_{\mathrm{C}} \mathrm{H}_{\mathrm{D}} \mathrm{C}=\mathrm{O}\right), 2.26-2.14(2 \mathrm{H}, \mathrm{m}$, $\left.\mathrm{CHCH}_{2} \mathrm{CH}_{2}\right), 1.91-1.82\left(2 \mathrm{H}, \mathrm{m}, \mathrm{CHCH}_{2} \mathrm{CH}_{2}\right), 1.65\left(3 \mathrm{H}, \mathrm{s}, \mathrm{COHCH}_{3}\right), 1.52-1.43$ $(1 \mathrm{H}, \mathrm{m}, \mathrm{CHOHCH}), 1.39\left(3 \mathrm{H}, \mathrm{t}, J=7.1 \mathrm{~Hz}, \mathrm{CO}_{2} \mathrm{CH}_{2} \mathrm{CH}_{3}\right), 1.19-1.07(2 \mathrm{H}, \mathrm{m}$, $\left.\mathrm{CHCH}_{2} \mathrm{CH}_{2}\right) ;{ }^{13} \mathrm{C}$ NMR $\left(90 \mathrm{MHz}, \mathrm{CDCl}_{3}\right) \delta 175.9(\mathrm{C}), 170.0(\mathrm{C}), 158.8(\mathrm{C}), 131.5$ 
$(2 \times \mathrm{CH}), 130.9(\mathrm{CH}), 130.6(\mathrm{C}), 126.2(\mathrm{CH}), 113.6(2 \times \mathrm{CH}), 82.9(\mathrm{C}), 77.5(\mathrm{C})$, $75.9(\mathrm{CH}), 61.4\left(\mathrm{CH}_{2}\right), 55.3\left(\mathrm{CH}_{3}\right), 46.6\left(\mathrm{CH}_{2}\right), 38.3(\mathrm{CH}), 26.9\left(\mathrm{CH}_{2}\right), 25.3\left(\mathrm{CH}_{2}\right)$, $23.2\left(\mathrm{CH}_{3}\right), 20.7\left(\mathrm{CH}_{2}\right), 14.4\left(\mathrm{CH}_{3}\right)$; HRMS (ES) Exact mass calcd for $\mathrm{C}_{22} \mathrm{H}_{30} \mathrm{NO}_{6}$ $[\mathrm{M}+\mathrm{H}]^{+}:$404.2068, found: 404.2063 .

\section{$(2 R S, 3 S R)-2-\{(6 S)$-Acetoxy-[(7S)-cyclohex-2-enyl]methyl $\}-1-(4-m e t h o x y b e n z y l)-$} 3-methyl-5-oxo-3-(trimethylsilyloxy)pyrrolidine-2-carboxylic acid ethyl ester (158)

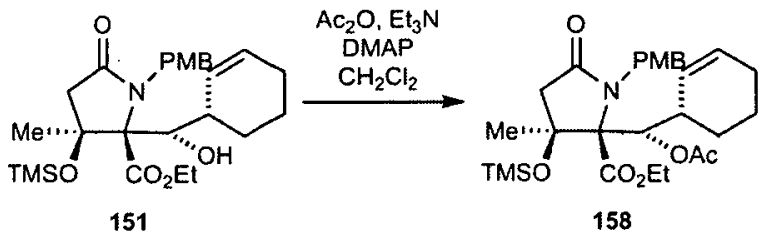

To a stirred solution of alcohol $151(100 \mathrm{mg}, 0.2 \mathrm{mmol})$ and DMAP (20.0 $\mathrm{mg}$, $0.16 \mathrm{mmol})$ in $\mathrm{CH}_{2} \mathrm{Cl}_{2}(1.5 \mathrm{~mL})$ at $0{ }^{\circ} \mathrm{C}$, was added $\mathrm{Et}_{3} \mathrm{~N}(0.14 \mathrm{~mL}, 1.02 \mathrm{mmol})$ dropwise, followed by the addition of $\mathrm{Ac}_{2} \mathrm{O}(0.058 \mathrm{~mL}, 0.61 \mathrm{mmol})$ via syringe. The reaction was stirred for $14 \mathrm{~h}$ and then quenched with saturated aqueous $\mathrm{NaHCO}_{3}$ solution $(3 \mathrm{~mL})$. The aqueous layer was separated and extracted with $\mathrm{CH}_{2} \mathrm{Cl}_{2}(3 \times 10$ $\mathrm{mL})$ and the combined organic layers were dried $\left(\mathrm{MgSO}_{4}\right)$, filtered and concentrated in vacuo. Purification of the residue by column chromatography (30\% EtOAc/petrol) gave acetate protected homoallylic alcohol $158(96 \mathrm{mg}, 90 \%)$ as a pale yellow solid. $\mathrm{R}_{\mathrm{f}}\left(50 \%\right.$ EtOAc in hexane) $=0.63$; m.p. 146-148 ${ }^{\circ} \mathrm{C}$; IR (film) 2938, $1748(\mathrm{C}=\mathrm{O})$, $1708(\mathrm{C}=\mathrm{O}), 1613(\mathrm{C}=\mathrm{O}), 1513,1247,1221,1175,1032,842 \mathrm{~cm}^{-1} ;{ }^{1} \mathrm{H}$ NMR $(360$ $\left.\mathrm{MHz} \mathrm{CDCl}_{3}\right) \delta 7.31(2 \mathrm{H}, \mathrm{d}, J=8.6 \mathrm{~Hz}, \mathrm{ArH}), 6.81(2 \mathrm{H}, \mathrm{d}, J=8.6 \mathrm{~Hz}, \operatorname{ArH}), 5.76$ $(1 \mathrm{H}, \mathrm{dm}, J=10.4 \mathrm{~Hz}, \mathrm{CH}=\mathrm{CH}), 5.43(1 \mathrm{H}, \mathrm{dm}, J=10.4 \mathrm{~Hz}, \mathrm{CH}=\mathrm{CH}), 5.36(1 \mathrm{H}, \mathrm{d}, J$ $=3.8 \mathrm{~Hz}, \mathrm{CHOAc}), 4.79\left(1 \mathrm{H}, \mathrm{d}, J=15.3 \mathrm{~Hz}, \mathrm{CH}_{\mathrm{A}} \mathrm{H}_{\mathrm{B}} \mathrm{Ar}\right), 4.56(1 \mathrm{H}, \mathrm{d}, J=15.3 \mathrm{~Hz}$, $\left.\mathrm{CH}_{\mathrm{A}} \mathrm{H}_{\mathrm{B}} \mathrm{Ar}\right), 4.19-4.10\left(2 \mathrm{H}, \mathrm{m}, \mathrm{CO}_{2} \mathrm{CH}_{2} \mathrm{CH}_{3}\right), 3.78\left(3 \mathrm{H}, \mathrm{s}, \mathrm{OCH}_{3}\right), 2.53-2.47(1 \mathrm{H}, \mathrm{m}$, CHOHCH), $2.47\left(1 \mathrm{H}, \mathrm{d}, J=16.8 \mathrm{~Hz}, \mathrm{CH}_{\mathrm{C}} \mathrm{H}_{\mathrm{D}} \mathrm{C}=\mathrm{O}\right), 2.41(1 \mathrm{H}, \mathrm{d}, J=16.8 \mathrm{~Hz}$, $\left.\mathrm{CH}_{\mathrm{C}} \mathbf{H}_{\mathrm{D}} \mathrm{C}=\mathrm{O}\right), 2.05\left(3 \mathrm{H}, \mathrm{s}, \mathrm{C}=\mathrm{OCH}_{3}\right), 1.99-1.92\left(2 \mathrm{H}, \mathrm{m}, \mathrm{CHCH}_{2} \mathrm{CH}_{2}\right), 1.80-1.72(2 \mathrm{H}$ $\left.+1 \mathrm{H}, \mathrm{m}, \mathrm{CH}_{2} \mathrm{CH}_{2} \mathrm{CH}_{2}\right), 1.57\left(3 \mathrm{H}, \mathrm{s}, \mathrm{COTMSCH}_{3}\right), 1.52-1.39\left(1 \mathrm{H}, \mathrm{m}, \mathrm{CH}_{2} \mathrm{CH}_{2}\right)$, $1.18\left(3 \mathrm{H}, \mathrm{t}, J=7.0 \mathrm{~Hz}, \mathrm{CO}_{2} \mathrm{CH}_{2} \mathrm{CH}_{3}\right), 0.16\left(9 \mathrm{H}, \mathrm{s}, \mathrm{Si}\left(\mathrm{CH}_{3}\right)_{3}\right) ;{ }^{13} \mathrm{C} \mathrm{NMR}(90 \mathrm{MHz}$, $\left.\mathrm{CDCl}_{3}\right) \delta 173.9(\mathrm{C}), 169.5(\mathrm{C}), 167.9(\mathrm{C}), 157.9(\mathrm{C}), 130.4(\mathrm{C}), 130.1(\mathrm{CH}), 127.7$ $(2 \times \mathrm{CH}), 124.4(\mathrm{CH}), 113.2(2 \times \mathrm{CH}), 82.0(\mathrm{C}), 81.1(\mathrm{C}), 77.1(\mathrm{CH}), 61.4\left(\mathrm{CH}_{2}\right)$, 
$55.2\left(\mathrm{CH}_{3}\right), 47.6\left(\mathrm{CH}_{2}\right), 46.2\left(\mathrm{CH}_{2}\right), 37.8(\mathrm{CH}), 29.4\left(\mathrm{CH}_{2}\right), 24.5\left(\mathrm{CH}_{2}\right), 22.6\left(\mathrm{CH}_{3}\right)$, $21.4\left(\mathrm{CH}_{2}\right), 21.1\left(\mathrm{CH}_{3}\right), 13.8\left(\mathrm{CH}_{3}\right), 2.0\left(3 \times \mathrm{CH}_{3}\right)$; HRMS (ES) Exact mass calcd for $\mathrm{C}_{28} \mathrm{H}_{42} \mathrm{NO}_{7} \mathrm{Si}[\mathrm{M}+\mathrm{H}]^{+}: 532.2725$, found: 532.2721 .

\section{(2RS,3SR)-2-\{(6S)-Acetoxy-[(7S)-cyclohex-2-enyl] methyl $\}-3-h y d r o x y-1-(4-$} methoxybenzyl)-3-methyl-5-oxopyrrolidine-2-carboxylic acid ethyl ester (159)

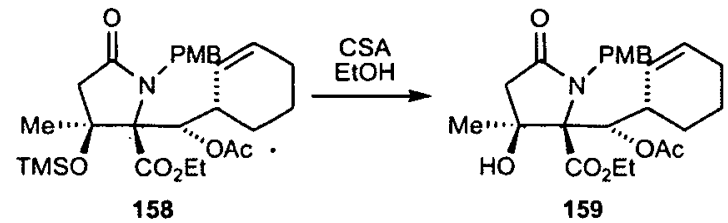

To a stirred solution of the lactam $158(253 \mathrm{mg}, 0: 48 \mathrm{mmol})$ in EtOH $(5 \mathrm{~mL})$ was added camphor sulfonic acid $(220 \mathrm{mg}, 0.95 \mathrm{mmol})$. The reaction was stirred for 30 min and it was then quenched with $\mathrm{Et}_{3} \mathrm{~N}(0.13 \mathrm{~mL}, 0.95 \mathrm{mmol})$. The solvent was concentrated in vacuo and purification of the residue by column chromatography (80\% EtOAc/petrol) gave $\beta$-hydroxy- $\gamma$-lactam 159 (195 $\mathrm{mg}, 91 \%$ ) as a colourless solid. $\mathrm{R}_{\mathrm{f}}(50 \%$ EtOAc in hexane $)=0.22$; m.p. 182-184 ${ }^{\circ} \mathrm{C}$; IR (film) $3468(\mathrm{OH})$, 2922, $1748(\mathrm{C}=\mathrm{O}), 1703(\mathrm{C}=\mathrm{O}), 1687(\mathrm{C}=\mathrm{O}), 1513,1220,1032,522 \mathrm{~cm}^{-1} ;{ }^{1} \mathrm{H}$ NMR $\left(360 \mathrm{MHz} \mathrm{CDCl}_{3}\right) \delta 7.36(2 \mathrm{H}, \mathrm{d}, J=8.5 \mathrm{~Hz}, \mathrm{ArH}), 6.84(2 \mathrm{H}, \mathrm{d}, J=8.5 \mathrm{~Hz}, \operatorname{ArH})$, $5.74(1 \mathrm{H}, \mathrm{dm}, J=10.3 \mathrm{~Hz}, \mathrm{CH}=\mathrm{CH}), 5.38-5.31(2 \mathrm{H}, \mathrm{m}, \mathrm{CH}=\mathrm{CH}+\mathrm{CHOAc}), 4.77$ $\left(1 \mathrm{H}, \mathrm{d}, J=15.1 \mathrm{~Hz}, \mathrm{CH}_{\mathrm{A}} \mathrm{H}_{\mathrm{B}} \mathrm{Ar}\right), 4.57\left(1 \mathrm{H}, \mathrm{d}, J=15.1 \mathrm{~Hz}, \dot{C}_{\mathrm{A}} \mathbf{H}_{\mathrm{B}} \mathrm{Ar}\right), 4.32-4.29$ $\left(1 \mathrm{H}, \mathrm{m}, \mathrm{CO}_{2} \mathrm{CH}_{\mathrm{C}} \mathrm{H}_{\mathrm{D}} \mathrm{CH}_{3}\right), 4.21-4.11\left(1 \mathrm{H}, \mathrm{m}, \mathrm{CO}_{2} \mathrm{CH}_{\mathrm{C}} \mathbf{H}_{\mathrm{D}} \mathrm{CH}_{3}\right), 3.78\left(3 \mathrm{H}, \mathrm{s}, \mathrm{OCH}_{3}\right)$, $2.69(1 \mathrm{H}, \mathrm{bs}, \mathrm{OH})$ 2.53-2.48 (1H, m, CHOHCH), $2.47(1 \mathrm{H}, \mathrm{d}, J=17.2 \mathrm{~Hz}$, $\left.\mathrm{CH}_{\mathrm{E}} \mathrm{H}_{\mathrm{F}} \mathrm{C}=\mathrm{O}\right), 2.39\left(1 \mathrm{H}, \mathrm{d}, J=17.2 \mathrm{~Hz}, \mathrm{CH}_{\mathrm{E}} \mathbf{H}_{\mathrm{F}} \mathrm{C}=\mathrm{O}\right), 2.07\left(3 \mathrm{H}, \mathrm{s}, \mathrm{C}=\mathrm{OCH}_{3}\right), 1.99-$ $1.92\left(2 \mathrm{H}, \mathrm{m}, \mathrm{CHCH}_{2} \mathrm{CH}_{2}\right), 1.79-1.69\left(2 \mathrm{H}+1 \mathrm{H}, \mathrm{m}, \mathrm{CH}_{2} \mathrm{CH}_{2} \mathrm{CH}_{2}\right), 1.49(3 \mathrm{H}, \mathrm{s}$, $\left.\mathrm{COTMSCH}_{3}\right), 1.49-1.41\left(1 \mathrm{H}, \mathrm{m}, \mathrm{CH}_{2} \mathrm{CH}_{2}\right), 1.23\left(3 \mathrm{H}, \mathrm{t}, J=7.1 \mathrm{~Hz}, \mathrm{CO}_{2} \mathrm{CH}_{2} \mathrm{CH}_{3}\right)$; ${ }^{13} \mathrm{C} \mathrm{NMR}\left(90 \mathrm{MHz}, \mathrm{CDCl}_{3}\right) \delta 174.2(\mathrm{C}), 169.4(\mathrm{C}), 168.2(\mathrm{C}), 158.2(\mathrm{C}), 130.2$ (CH), $130.1(\mathrm{C}), 128.3(2 \times \mathrm{CH}), 128.2(\mathrm{C}), 124.3(\mathrm{CH}), 113.4(2 \times \mathrm{CH}), 80.9(\mathrm{C})$, $77.1(\mathrm{CH}), 61.8\left(\mathrm{CH}_{2}\right), 55.2\left(\mathrm{CH}_{3}\right), 47.6\left(\mathrm{CH}_{2}\right), 46.1\left(\mathrm{CH}_{2}\right), 37.7(\mathrm{CH}), 29.1\left(\mathrm{CH}_{2}\right)$, $24.5\left(\mathrm{CH}_{2}\right), 22.1\left(\mathrm{CH}_{3}\right), 21.2\left(\mathrm{CH}_{2}\right), 21.1\left(\mathrm{CH}_{3}\right), 13.9\left(\mathrm{CH}_{3}\right)$; HRMS (ES) Exact mass calcd for $\mathrm{C}_{25} \mathrm{H}_{34} \mathrm{NO}_{7}[\mathrm{M}+\mathrm{H}]^{+}: 460.2330$, found: 460.2329 . 
(2RS,3SR)-2-[(1S)-Hydroxy-2-methylallyl]-1-(4-methoxybenzyl)-3-methyl-5-oxo-

\section{3-(trimethylsilyloxy)pyrrolidine-2-carboxylic acid ethyl ester (164)}

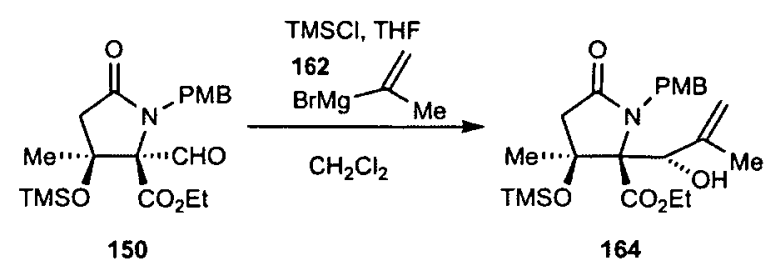

To a stirred solution of aldehyde $150(1.26 \mathrm{mg}, 3.10 \mathrm{mmol})$, in THF (150 mL) at $-78{ }^{\circ} \mathrm{C}$ was added TMSCl $(2.0 \mathrm{~mL}, 15.5 \mathrm{mmol})$, followed by the addition of isopropenylmagnesium bromide $(18.6 \mathrm{~mL}, 0.5 \mathrm{M}$ solution in THF, $9.31 \mathrm{mmol}$ ) via syringe. The reaction was stirred at $-78^{\circ} \mathrm{C}$ for $3 \mathrm{~h}$ and then slowly quenched with saturated aqueous $\mathrm{NH}_{4} \mathrm{Cl}$ solution $(30 \mathrm{~mL})$. After warming up to room temperature and stirring for $30 \mathrm{~min}$, the aqueous layer was extracted with EtOAc ( $2 \times 30 \mathrm{~mL})$ and the combined organic layers were washed with water $(1 \times 30 \mathrm{~mL})$, saturated $\mathrm{NaCl}$ aqueous solution $(1 \times 30 \mathrm{~mL})$ and then were dried $\left(\mathrm{Na}_{2} \mathrm{SO}_{4}\right)$, filtered and concentrated in vacuo. Purification of the residue by column chromatography ( $25 \%$ EtOAc/petrol) gave alcohol $164(1.06 \mathrm{~g}, 76 \%)$ as a colourless solid. $\mathrm{R}_{\mathrm{f}}(40 \%$ EtOAc in hexane $)=$ 0.14; m.p. 171-173 ${ }^{\circ} \mathrm{C}$; IR (film) $3318(\mathrm{OH}), 2957,2359,1751(\mathrm{C}=\mathrm{O}), 1680(\mathrm{C}=\mathrm{O})$, $1513,1246,1174,1034,841 \mathrm{~cm}^{-1} ;{ }^{1} \mathrm{H}$ NMR $\left(360 \mathrm{MHz}, \mathrm{CDCl}_{3}\right) \delta 7.21(2 \mathrm{H}, \mathrm{d}, J=$ $8.8 \mathrm{~Hz}, \operatorname{ArH}), 6.78(2 \mathrm{H}, \mathrm{d}, J=8.8 \mathrm{~Hz}, \mathrm{ArH}), 5.24(1 \mathrm{H}, \mathrm{s}, \mathrm{CHOH}), 5.24(1 \mathrm{H}, \mathrm{s}$, $\left.\mathrm{C}=\mathrm{CH}_{\mathrm{A}} \mathrm{H}_{\mathrm{B}}\right), 5.08\left(1 \mathrm{H}, \mathrm{s}, \mathrm{C}=\mathrm{CH}_{\mathrm{A}} \mathbf{H}_{\mathbf{B}}\right), 4.63\left(1 \mathrm{H}, \mathrm{d}, J=15.7 \mathrm{~Hz}, \mathrm{CH}_{\mathrm{A}} \mathrm{H}_{\mathrm{B}} \mathrm{Ar}\right), 4.31$ $\left(1 \mathrm{H}, \mathrm{d}, J=15.7 \mathrm{~Hz}, \mathrm{CH}_{\mathrm{A}} \mathbf{H}_{\mathrm{B}} \mathrm{Ar}\right), 4.14-4.00\left(2 \mathrm{H}, \mathrm{m}, \mathrm{CO}_{2} \mathrm{CH}_{2} \mathrm{CH}_{3}\right), 3.77(3 \mathrm{H}, \mathrm{s}$, $\left.\mathrm{OCH}_{3}\right), 2.91\left(1 \mathrm{H}, \mathrm{d}, J=16.6 \mathrm{~Hz}, \mathrm{CH}_{\mathrm{A}} \mathrm{H}_{\mathrm{B}} \mathrm{C}=\mathrm{O}\right), 2.40\left(1 \mathrm{H}, \mathrm{d}, J=16.5 \mathrm{~Hz}, \mathrm{CH}_{\mathrm{A}} \mathbf{H}_{\mathbf{B}}\right.$ $\mathrm{C}=\mathrm{O}), 2.23(1 \mathrm{H}, \mathrm{bs}, \mathrm{CHOH}), 1.81\left(3 \mathrm{H}, \mathrm{s}, \mathrm{CCH}_{3}\right), 1.76\left(3 \mathrm{H}, \mathrm{s}, \mathrm{COTMSCH}_{3}\right), 1.12-$ $1.06\left(3 \mathrm{H}, \mathrm{m}, \mathrm{CO}_{2} \mathrm{CH}_{2} \mathrm{CH}_{3}\right), 0.16\left(9 \mathrm{H}, \mathrm{s}, \mathrm{Si}\left(\mathrm{CH}_{3}\right)_{3}\right) ;{ }^{13} \mathrm{C} \mathrm{NMR}\left(90 \mathrm{MHz}, \mathrm{CDCl}_{3}\right) \delta$ 174.9 (C), 168.7 (C), 157.8 (C), 144.5 (C), 131.1 (C), 127.5 (2 x CH), $115.1\left(\mathrm{CH}_{2}\right)$, $113.1(2 \times \mathrm{CH}), 82.1(\mathrm{C}), 82.0(\mathrm{C}), 75.7(\mathrm{CH}), 61.0\left(\mathrm{CH}_{2}\right), 55.2\left(\mathrm{CH}_{3}\right), 48.5\left(\mathrm{CH}_{2}\right)$, $46.8\left(\mathrm{CH}_{2}\right), 22.3\left(\mathrm{CH}_{3}\right), 21.2\left(\mathrm{CH}_{3}\right), 13.6\left(\mathrm{CH}_{3}\right), 2.1\left(3 \times \mathrm{CH}_{3}\right)$; HRMS (ES) Exact mass calcd for $\mathrm{C}_{23} \mathrm{H}_{36} \mathrm{NO}_{6} \mathrm{Si}[\mathrm{M}+\mathrm{H}]^{+}: 450.2306$, found: 450.2310 . 
(2RS,3SR)-2-[(1S)-Hydroxy-2-methylallyl]-1-(4-methoxybenzyl)-3-methyl-5-oxo-

3-(trimethylsilyloxy)pyrrolidine-2-carboxylic acid ethyl ester (165)

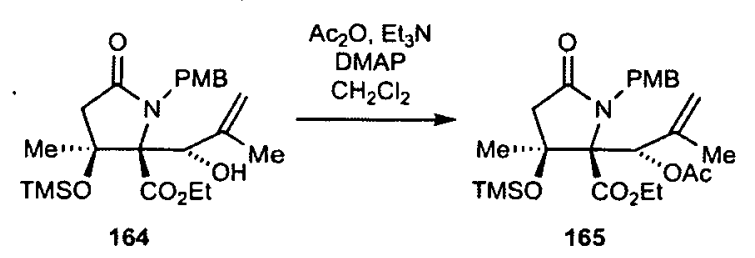

To a stirred solution of alcohol $164(1.32 \mathrm{~g}, 2.92 \mathrm{mmol})$ and DMAP (71 $\mathrm{mg}, 0.58$ mmol) in $\mathrm{CH}_{2} \mathrm{Cl}_{2}(10 \mathrm{~mL})$ at $0{ }^{\circ} \mathrm{C}$, was added pyridine $(0.83 \mathrm{~mL}, 10.2 \mathrm{mmol})$ dropwise, followed by $\mathrm{Ac}_{2} \mathrm{O}(1.70 \mathrm{~mL}, 17.6 \mathrm{mmol})$ via syringe. The reaction was stirred for $14 \mathrm{~h}$ at room temperature and then quenched with saturated aqueous $\mathrm{NH}_{4} \mathrm{Cl}$ solution $(10 \mathrm{~mL})$. The aqueous layer was separated and extracted with $\mathrm{CH}_{2} \mathrm{Cl}_{2}(3 \times 10 \mathrm{~mL})$ and the combined organic layers were dried $\left(\mathrm{MgSO}_{4}\right)$, filtered and concentrated in vacuo. Purification of the residue by column chromatography (40\% EtOAc/petrol) gave acetate protected allylic alcohol 165 (1.32 g, 92\%) as a pale yellow oil. $\mathrm{R}_{\mathrm{f}}(40 \%$ EtOAc in hexane $)=0.38$; IR (film) 2957, 2359, 1749 $(\mathrm{C}=\mathrm{O}), 1707(\mathrm{C}=\mathrm{O}), 1614(\mathrm{C}=\mathrm{O}), 1513,1247,1222,1175,1033,912 \mathrm{~cm}^{-1} ;{ }^{1} \mathrm{H}$ NMR $\left(360 \mathrm{MHz}, \mathrm{CDCl}_{3}\right) \delta 7.23(2 \mathrm{H}, \mathrm{d}, J=8.8 \mathrm{~Hz}, \mathrm{ArH}), 6.79(2 \mathrm{H}, \mathrm{d}, J=8.8 \mathrm{~Hz}, \mathrm{ArH})$, $5.65(1 \mathrm{H}, \mathrm{s}, \mathrm{CHOAc}), 5.09\left(2 \mathrm{H}, \mathrm{s}, \mathrm{C}=\mathrm{CH}_{2}\right), 4.68\left(1 \mathrm{H}, \mathrm{d}, J=15.7 \mathrm{~Hz}, \mathrm{CH}_{\mathrm{A}} \mathrm{H}_{\mathrm{B}} \mathrm{Ar}\right)$, $4.42\left(1 \mathrm{H}, \mathrm{d}, J=15.7 \mathrm{~Hz}, \mathrm{CH}_{\mathrm{A}} \mathbf{H}_{\mathrm{B}} \mathrm{Ar}\right), 4.22-4.01\left(2 \mathrm{H}, \mathrm{m}, \mathrm{CO}_{2} \mathrm{CH}_{2} \mathrm{CH}_{3}\right), 3.77(3 \mathrm{H}, \mathrm{s}$, $\left.\mathrm{OCH}_{3}\right), 2.68\left(1 \mathrm{H}, \mathrm{d}, J=16.7 \mathrm{~Hz}, \mathrm{CH}_{\mathrm{C}} \mathrm{H}_{\mathrm{D}} \mathrm{C}=\mathrm{O}\right), 2.49(1 \mathrm{H}, \mathrm{d}, J=16.7 \mathrm{~Hz}$, $\left.\mathrm{CH}_{\mathrm{C}} \mathrm{H}_{\mathrm{D}} \mathrm{C}=\mathrm{O}\right), 2.13\left(3 \mathrm{H}, \mathrm{s}, \mathrm{C}=\mathrm{OCH}_{3}\right), 1.77$ (3H, s, CH $\left.\mathbf{C H}_{3} \mathrm{COTM}\right), 1.60$ (3H, s, $\left.\mathrm{CCH}_{3}\right), 1.14\left(3 \mathrm{H}, \mathrm{t}, J=7.1 \mathrm{~Hz}, \mathrm{CO}_{2} \mathrm{CH}_{2} \mathrm{CH}_{3}\right), 0.18\left(9 \mathrm{H}, \mathrm{s}, \mathrm{Si}\left(\mathrm{CH}_{3}\right)_{3}\right) ;{ }^{13} \mathrm{C} \mathrm{NMR}(90$ $\left.\mathrm{MHz} \mathrm{CDCl}_{3}\right) \delta 179.9(\mathrm{C}), 168.8(\mathrm{C}), 167.6(\mathrm{C}), 157.8(\mathrm{C}), 139.8(\mathrm{C}), 130.6(\mathrm{C})$, $127.4(2 \times \mathrm{CH}), 116.1\left(\mathrm{CH}_{2}\right), 113.1(2 \times \mathrm{CH}), 81.4(\mathrm{C}), 81.1(\mathrm{C}), 77.2(\mathrm{CH}), 61.4$ $\left(\mathrm{CH}_{2}\right), 55.1\left(\mathrm{CH}_{3}\right), 48.6\left(\mathrm{CH}_{2}\right), 46.4\left(\mathrm{CH}_{2}\right), 22.1\left(\mathrm{CH}_{3}\right), 21.2\left(\mathrm{CH}_{3}\right), 20.9\left(\mathrm{CH}_{3}\right), 13.6$ $\left(\mathrm{CH}_{3}\right), 2.0$ (3 x CH$)$; HRMS (ES) Exact mass calcd for $\mathrm{C}_{25} \mathrm{H}_{38} \mathrm{NO}_{7} \mathrm{Si}[\mathrm{M}+\mathrm{H}]^{+}$: 492.2412, found: 492.2407 . 
(2RS,3SR)-2-[(1S)-Hydroxy-2-methylallyl]-3-hydroxy-1-(4-methoxybenzyl)-3methyl-5-oxopyrrolidine-2-carboxylic acid ethyl ester (166)

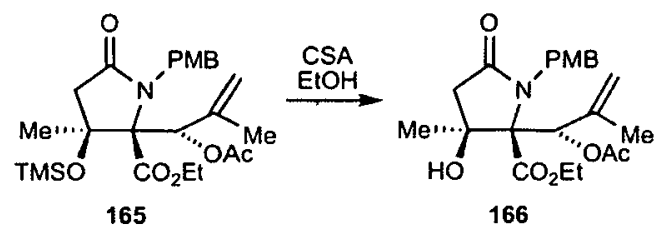

To a stirred solution of $165(710 \mathrm{mg}, 1.43 \mathrm{mmol})$ in $\mathrm{EtOH}(10 \mathrm{~mL})$ was added camphor sulfonic acid $(1.30 \mathrm{~g}, 5.70 \mathrm{mmol})$. The reaction was stirred for $14 \mathrm{~h}$ and then quenched with $\mathrm{Et}_{3} \mathrm{~N}(0.80 \mathrm{~mL}, 5.70 \mathrm{mmol})$ and extracted with $\mathrm{CH}_{2} \mathrm{Cl}_{2}(3 \times 15$ $\mathrm{mL}$ ). The combined organic layers were concentrated in vacuo and purification of the residue by column chromatography $(50 \% \mathrm{EtOAc} /$ petrol) gave $\beta$-hydroxy- $\gamma$ lactam $166(536 \mathrm{mg}, 83 \%)$ as a colourless solid. $\mathrm{R}_{\mathrm{f}}(50 \%$ EtOAc in hexane $)=0.22$; m.p. 194-196 ${ }^{\circ} \mathrm{C}$; IR (film) $3386(\mathrm{OH}), 2922,2663,2347,1749(\mathrm{C}=\mathrm{O}), 1711(\mathrm{C}=\mathrm{O}), 1682$ $(\mathrm{C}=\mathrm{O}), 1224,1032 \mathrm{~cm}^{-1}$; ${ }^{1} \mathrm{H}$ NMR $\left(360 \mathrm{MHz}, \mathrm{CDCl}_{3}\right) \delta 7.28(2 \mathrm{H}, \mathrm{d}, J=8.7 \mathrm{~Hz}$, ArH), $6.81(2 \mathrm{H}, \mathrm{d}, J=8.7 \mathrm{~Hz}, \operatorname{ArH}), 5.65(1 \mathrm{H}, \mathrm{s}, \mathrm{CHOAc}), 5.08\left(2 \mathrm{H}, \mathrm{s}, \mathrm{C}=\mathrm{CH}_{2}\right)$, $4.67\left(1 \mathrm{H}, \mathrm{d}, J=15.4 \mathrm{~Hz}, \mathrm{CH}_{\mathrm{A}} \mathrm{H}_{\mathrm{B}} \mathrm{Ar}\right), 4.38\left(1 \mathrm{H}, \mathrm{d}, J=15.4 \mathrm{~Hz}, \mathrm{CH}_{\mathrm{A}} \mathbf{H}_{\mathrm{B}} \mathrm{Ar}\right), 4.28-$ $4.19\left(1 \mathrm{H}, \mathrm{m}, \mathrm{CO}_{2} \mathrm{CH}_{\mathrm{C}} \mathrm{H}_{\mathrm{D}} \mathrm{CH}_{3}\right), 4.15-4.06\left(1 \mathrm{H}, \mathrm{m}, \mathrm{CO}_{2} \mathrm{CH}_{\mathrm{C}} \mathbf{H}_{\mathrm{D}} \mathrm{CH}_{3}\right), 3.76(3 \mathrm{H}, \dot{\mathrm{s}}$, $\left.\mathrm{OCH}_{3}\right), 2.64\left(1 \mathrm{H}, \mathrm{d}, J=17.1 \mathrm{~Hz}, \mathrm{CH}_{\mathrm{E}} \mathrm{H}_{\mathrm{F}} \mathrm{C}=\mathrm{O}\right), 2.38(1 \mathrm{H}, \mathrm{d}, J=17.1 \mathrm{~Hz}$, $\left.\mathrm{CH}_{\mathrm{E}} \mathbf{H}_{\mathrm{F}} \mathrm{C}=\mathrm{O}\right), 2.14\left(3 \mathrm{H}, \mathrm{s}, \mathrm{C}=\mathrm{OCH}_{3}\right), 1.78\left(3 \mathrm{H}, \mathrm{s}, \mathrm{CCH}_{3}\right), 1.49\left(3 \mathrm{H}, \mathrm{s}, \mathrm{CH}_{3} \mathrm{COH}\right)$; $1.17\left(3 \mathrm{H}, \mathrm{t}, J=7.1 \mathrm{~Hz}, \mathrm{CO}_{2} \mathrm{CH}_{2} \mathrm{CH}_{3}\right) ;{ }^{13} \mathrm{C} \mathrm{NMR}\left(90 \mathrm{MHz}, \mathrm{CDCl}_{3}\right) \delta 174.1$ (C), 168.7 (C), 167.9 (C), 158.0 (C), 139.7 (C), 130.5 (C), 127.9 (2 x CH), $116.1\left(\mathrm{CH}_{2}\right)$, $113.3(2 \times \mathrm{CH}), 80.0(\mathrm{C}), 77.8(\mathrm{CH}), 77.2(\mathrm{C}), 61.8\left(\mathrm{CH}_{2}\right), 55.1\left(\mathrm{CH}_{3}\right), 48.6\left(\mathrm{CH}_{2}\right)$, $46.2\left(\mathrm{CH}_{2}\right), 21.9\left(\mathrm{CH}_{3}\right), 21.1\left(\mathrm{CH}_{3}\right), 21.0\left(\mathrm{CH}_{3}\right), 13.7\left(\mathrm{CH}_{3}\right)$; HRMS (ES) Exact mass calcd for $\mathrm{C}_{22} \mathrm{H}_{29} \mathrm{NO}_{7} \mathrm{Na}[\mathrm{M}+\mathrm{Na}]^{+}: 442.1836$, found: 442.1837 .

(2RS,3SR)-2-[(1S)-Acetoxy-2-methylpropyl]-3-hydroxy-1-(4-methoxybenzyl)-3methyl-5-oxopyrrolidine-2-carboxylic acid ethyl ester (167)

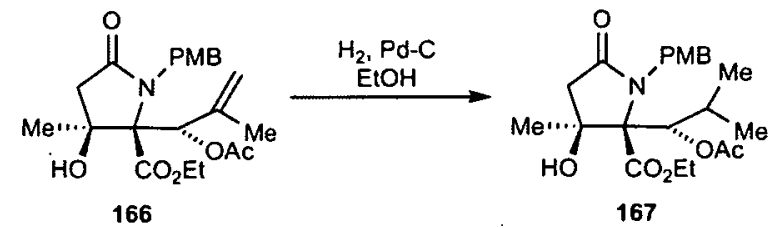

A mixture of alcohol 166 (180 mg, $0.43 \mathrm{mmol})$ and 10\% $\mathrm{Pd} / \mathrm{C}(23.0 \mathrm{mg}, 0.22$ $\mathrm{mmol}$ ) in $\mathrm{EtOH}(4 \mathrm{~mL})$ at room temperature was evacuated and flushed with $\mathrm{H}_{2}$ (3 
times) and then stirred vigorously under an atmosphere of $\mathrm{H}_{2}$ (1 atm, $\mathrm{H}_{2}$ balloon) at room temperature for $18 \mathrm{~h}$. The reaction mixture was filtered through celite and . concentrated in vacuo. Purification of the residue by column chromatography $(50 \%$. EtOAc/petrol) gave alcohol $167(91 \mathrm{mg}, 50 \%)$ as a white powder. $\mathrm{R}_{\mathrm{f}}(50 \%$ EtOAc in hexane $)=0.36$; m.p. $182-184{ }^{\circ} \mathrm{C}$; IR (film) $3365(\mathrm{OH}), 2359,2341,1749(\mathrm{C}=\mathrm{O})$, $1702(\mathrm{C}=\mathrm{O}), 1682(\mathrm{C}=\mathrm{O}), 1512,1223,1175,1025 \mathrm{~cm}^{-1} ;{ }^{1} \mathrm{H}$ NMR $(360 \mathrm{MHz}$, $\left.\mathrm{CDCl}_{3}\right) \delta 7.34(2 \mathrm{H}, \mathrm{d}, J=8.7 \mathrm{~Hz}, \mathrm{ArH}), 6.83(2 \mathrm{H}, \mathrm{d}, J=8.7 \mathrm{~Hz}, \mathrm{ArH}), 5.30(1 \mathrm{H}, \mathrm{d}$, $J=6.5 \mathrm{~Hz}$, CHOAc), $4.74\left(1 \mathrm{H}, \mathrm{d}, J=15.2 \mathrm{~Hz}, \mathrm{CH}_{\mathrm{A}} \mathrm{H}_{\mathrm{B}} \mathrm{Ar}\right), 4.62(1 \mathrm{H}, \mathrm{d}, J=15.2 \mathrm{~Hz}$, $\left.\mathrm{CH}_{\mathrm{A}} \mathbf{H}_{\mathrm{B}} \mathrm{Ar}\right), \quad 4.31-4.21 \quad\left(1 \mathrm{H}, \quad \mathrm{m}, \mathrm{CO}_{2} \mathrm{CH}_{\mathrm{C}} \mathrm{H}_{\mathrm{D}} \mathrm{CH}_{3}\right), \quad 4.18-4.09 \quad(1 \mathrm{H}, \mathrm{m}$, $\left.\mathrm{CO}_{2} \mathrm{CH}_{\mathbf{C}} \mathbf{H}_{\mathbf{D}} \mathrm{CH}_{3}\right), 3.77\left(3 \mathrm{H}, \mathrm{s}, \mathrm{OCH}_{3}\right), 2.47\left(1 \mathrm{H}, \mathrm{d}, J=17.1 \mathrm{~Hz}, \mathrm{CH}_{\mathbf{E}} \mathrm{H}_{\mathrm{F}} \mathrm{C}=\mathrm{O}\right), 2.36$ $\left(1 \mathrm{H}, \mathrm{d}, J=17.1 \mathrm{~Hz}, \mathrm{CH}_{\mathrm{E}} \mathbf{H}_{\mathrm{F}} \mathrm{C}=\mathrm{O}\right), 2.11\left(3 \mathrm{H}, \mathrm{s}, \mathrm{C}=\mathrm{OCH}_{3}\right), 2.04-1.94(1 \mathrm{H}, \mathrm{m}$, $\mathrm{CHOHCH}), 1.47\left(3 \mathrm{H}, \mathrm{s}, \mathrm{CH}_{3} \mathrm{COH}\right), 1.23\left(3 \mathrm{H}, \mathrm{t}, J=7.1 \mathrm{~Hz}, \mathrm{CO}_{2} \mathrm{CH}_{2} \mathrm{CH}_{3}\right), 1.05(3 \mathrm{H}$, $\left.\mathrm{d}, J=6.7 \mathrm{~Hz}, \mathrm{CHCH}_{3}\right), 0.76\left(3 \mathrm{H}, \mathrm{d}, J=6.7 \mathrm{~Hz}, \mathrm{CHCH}_{3}\right) ;{ }^{13} \mathrm{C} \mathrm{NMR}(90 \mathrm{MHz}$, $\left.\mathrm{CDCl}_{3}\right) \delta 174.4(\mathrm{C}), 169.9(\mathrm{C}), 168.4(\mathrm{C}), 158.2(\mathrm{C}), 130.1(\mathrm{C}), 128.3(2 \times \mathrm{CH})$, $113.4(2 \times \mathrm{CH}), 80.6(\mathrm{C}), 78.6(\mathrm{CH}), 77.6(\mathrm{C}), 61.7\left(\mathrm{CH}_{2}\right), 55.1\left(\mathrm{CH}_{3}\right), 47.7\left(\mathrm{CH}_{2}\right)$, $46.1\left(\mathrm{CH}_{2}\right), 30.6(\mathrm{CH}), 22.0\left(\mathrm{CH}_{3}\right), 21.9\left(\mathrm{CH}_{3}\right), 21.0\left(\mathrm{CH}_{3}\right), 18.7\left(\mathrm{CH}_{3}\right), 13.8\left(\mathrm{CH}_{3}\right)$; HRMS (ES) Exact mass calcd for $\mathrm{C}_{22} \mathrm{H}_{31} \mathrm{NO}_{7} \mathrm{Na}[\mathrm{M}+\mathrm{Na}]^{+}: 444.1993$, found: 444.1989.

\subsubsection{Reductive aldol cyclisation approach}

Methyl 2-[(dimethylphenylsilyl)methyl]-3-oxobutanoate (307)

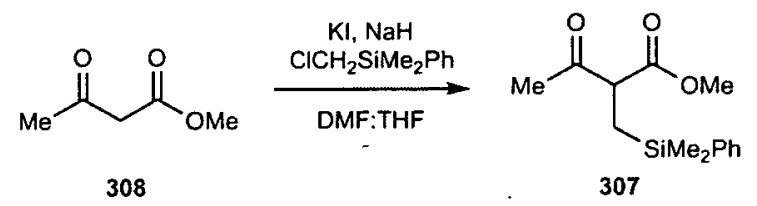

To a suspension of KI $(9.90 \mathrm{~g}, 60.0 \mathrm{mmol})$ and $\mathrm{NaH}(6.00 \mathrm{~g}, 60 \%$ dispersion in mineral oil, $150 \mathrm{mmol})$ in a mixture of DMF:THF $(1: 1,200 \mathrm{~mL})$ at room temperature, was added methyl acetoacetate (308) $(11.5 \mathrm{~mL}, 100 \mathrm{mmol})$ dropwise over $25 \mathrm{~min}$. This solution was stirred for $30 \mathrm{~min}$, until the effervescence stopped, when chloromethyl dimethylphenyl silane $(22.0 \mathrm{~mL}, 120 \mathrm{mmol})$ was added and the solution was heated to reflux for $14 \mathrm{~h}$. The reaction mixture was then diluted with $\mathrm{Et}_{2} \mathrm{O}$, washed with $2 \mathrm{M} \mathrm{HCl}(2 \times 50 \mathrm{~mL})$ and with saturated $\mathrm{NaCl}$ aqueous solution 
$(2 \times 50 \mathrm{~mL})$, dried $\left(\mathrm{MgSO}_{4}\right)$, filtered and concentrated in vacuo. Purification of the residue by column chromatography ( $2 \%$ to $6 \% \mathrm{EtOAc/petrol)} \mathrm{gave} \mathrm{alkylated} \mathrm{product}$ $307(17 \mathrm{~g}, 65 \%)$ as a colourless oil. $\mathrm{R}_{\mathrm{f}}(40 \%$ EtOAc in hexane) $=0.50$; IR (film) 3070, 2953, $1744(\mathrm{C}=\mathrm{O}), 1717(\mathrm{C}=\mathrm{O}), 1428,1357,1249,1113,836,701 \mathrm{~cm}^{-1} ;{ }^{1} \mathrm{H}$

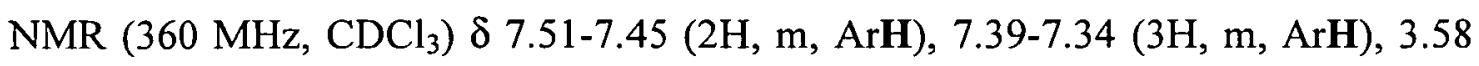
$\left(3 \mathrm{H}, \mathrm{s}, \mathrm{CO}_{2} \mathrm{CH}_{3}\right), 3.41\left(1 \mathrm{H}, \mathrm{dd}, J=9.1,6.0 \mathrm{~Hz}, \mathrm{CHCH}_{2} \mathrm{Si}\right), 2.11\left(3 \mathrm{H}, \mathrm{s}, \mathrm{CH}_{3} \mathrm{C}=\mathrm{O}\right)$, 1.43-1.27 (2H, m, $\left.\mathrm{SiCH}_{2}\right), 0.31\left(3 \mathrm{H}, \mathrm{s}, \mathrm{CH}_{3} \mathrm{Si}\right), 0.30\left(3 \mathrm{H}, \mathrm{s}, \mathrm{CH}_{3} \mathrm{Si}\right) ;{ }^{13} \mathrm{C}$ NMR (62.9 $\left.\mathrm{MHz}, \mathrm{CDCl}_{3}\right) \delta 203.4(\mathrm{C}), 171.0(\mathrm{C}), 137.5(\mathrm{C}), 133.6(2 \times \mathrm{CH}), 129.2(\mathrm{CH}), 127.8$ $(2 \times \mathrm{CH}), 55.3(\mathrm{CH}), 52.2\left(\mathrm{CH}_{3}\right), 27.9\left(\mathrm{CH}_{3}\right), 14.5\left(\mathrm{CH}_{2}\right),-2.9\left(\mathrm{CH}_{3}\right),-3.0\left(\mathrm{CH}_{3}\right)$; HRMS (ES) Exact mass calcd for $\mathrm{C}_{14} \mathrm{H}_{20} \mathrm{O}_{2} \mathrm{SiNa}[\mathrm{M}+\mathrm{Na}]^{+}: 287.1074$, found: 287.1077.

\section{Dibenzyl 1-\{-2-[(dimethylphenylsilyl)methyl]-1-methoxy-1,3-dioxobutan-2-yl\}} hydrazine-1,2-dicarboxylate (306)

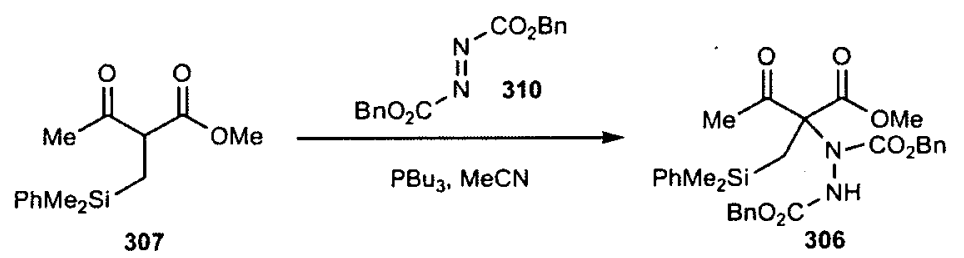

To a stirred solution of $\beta$-ketoester $307(2.28 \mathrm{~g}, 8.62 \mathrm{mmol})$ in dry acetonitrile $(60 \mathrm{~mL})$ at room temperature was added tributylphosphine $(0.12 \mathrm{~mL}, 1.72 \mathrm{mmol})$, followed by the addition of dibenzyl azodicarboxylate $(3.15 \mathrm{~g}, 9.48 \mathrm{mmol})$. The solution was stirred for $30 \mathrm{~min}$ and then concentrated in vacuo. Purification of the residue by column chromatography (20\% EtOAc/petrol) gave addition product 306 $(4.16 \mathrm{~g}, 86 \%)$ as a colourless oil. $\mathrm{R}_{\mathrm{f}}(30 \%$ EtOAc in hexane $)=0.43$; IR (film) 3297 (NH), 3033, 1722 (4 x C=O), 1497, 1394, 1338, 1216, 1111, 979, $830 \mathrm{~cm}^{-1}$; ${ }^{1} \mathrm{H}$ NMR $\left(360 \mathrm{MHz}, \mathrm{CDCl}_{3}\right) \delta 7.51(2 \mathrm{H}, \mathrm{bs}, \mathrm{ArH}), 7.37(10 \mathrm{H}, \mathrm{bs}, 2 \times \mathrm{ArH}), 7.30(3 \mathrm{H}, \mathrm{bs}$, ArH), $6.53(1 \mathrm{H}$, bs, $\mathrm{NH}), 5.17\left(4 \mathrm{H}, \mathrm{bs}, 2 \times \mathrm{CO}_{2} \mathrm{CH}_{2} \mathrm{Ar}\right), 3.57\left(3 \mathrm{H}, \mathrm{bs}, \mathrm{OCH}_{3}\right), 2.38$ ( $3 \mathrm{H}, \mathrm{s}, \mathrm{CH}_{3} \mathrm{C}=0$ ), $1.66\left(2 \mathrm{H}, \mathrm{bs}, \mathrm{SiCH}_{2}\right), 0.35\left(3 \mathrm{H}, \mathrm{bs}, \mathrm{CH}_{3} \mathrm{Si}\right), 0.31$ ( $\left.3 \mathrm{H}, \mathrm{bs}, \mathrm{CH}_{3} \mathrm{Si}\right)$; ${ }^{13} \mathrm{C}$ NMR (62.9 MHz, $\left.\mathrm{CDCl}_{3}\right) \delta 199.7$ (C), 169.4 (C), $156.1(2 \times \mathrm{C}), 138.8(2 \times \mathrm{C})$, $135.2(2 \times \mathrm{C}), 133.48(2 \times \mathrm{CH}), 129.0(2 \times \mathrm{CH}), 128.6(4 \times \mathrm{CH}), 128.5(4 \times \mathrm{CH})$, $128.1(\mathrm{CH}), 127.8(2 \times \mathrm{CH}), 68.7\left(\mathrm{CH}_{2}\right), 68.1\left(\mathrm{CH}_{2}\right), 52.4\left(\mathrm{CH}_{3}\right), 24.7\left(\mathrm{CH}_{3}\right), 20.6$ 
$\left(\mathrm{CH}_{2}\right),-0.9\left(\mathrm{CH}_{3}\right),-2.0\left(\mathrm{CH}_{3}\right)$; HRMS (ES) Exact mass calcd for $\mathrm{C}_{30} \mathrm{H}_{38} \mathrm{~N}_{3} \mathrm{O}_{7} \mathrm{Si}$ $\left[\mathrm{M}+\mathrm{NH}_{4}\right]^{+}: 580.2474$, found: 580.2475 .

\section{Methyl 2-amino-2-[(dimethylphenylsilyl)methyl]-3-hydroxybutanoate (319a and} 319b)

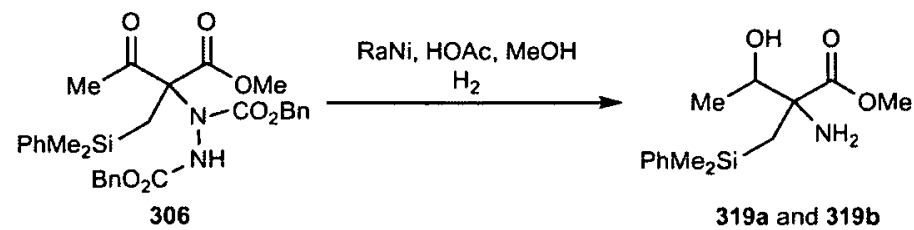

Raney nickel ( $17 \mathrm{~g}$, suspension in $\mathrm{MeOH})$ was pre-washed with methanol $(3 \times 10$ $\mathrm{mL}) 306(2.10 \mathrm{~g}, 3.72 \mathrm{mmol})$ was placed in the reaction vessel of the parr hydrogenator and then it was added a suspension of the catalyst in $\mathrm{MeOH}(100 \mathrm{~mL})$, followed by $\mathrm{HOAc}$ (40 drops). The reaction mixture was stirred at $40{ }^{\circ} \mathrm{C}$ under hydrogen ( 45 bar) for $16 \mathrm{~h}$. The reaction mixture was filtered over celite, the solids were washed with EtOAc and the combined organic layers were concentrated in vacuo. Purification of the residue by column chromatography (20\% to $30 \%$ EtOAc/petrol) afforded the two aminoalcohols 319a (386 mg, 40\%) and 319b (182 $\mathrm{mg}, 17 \%)$ as colourless oils.

Data for 319a: $\mathrm{R}_{\mathrm{f}}(40 \%$ EtOAc in hexane $)=0.15$; IR (film) $3404(\mathrm{NH}+\mathrm{OH})$, 2359, 2341, $1637(\mathrm{C}=\mathrm{O}), 669,524,509 \mathrm{~cm}^{-1} ;{ }^{1} \mathrm{H}$ NMR $\left(360 \mathrm{MHz}, \mathrm{CDCl}_{3}\right) \delta 7.53-$ $7.50(2 \mathrm{H}, \mathrm{m}, \mathrm{ArH}), 7.38-7.35(3 \mathrm{H}, \mathrm{m}, \mathrm{ArH}), 3.68\left(1 \mathrm{H}, \mathrm{q}, J=6.4 \mathrm{~Hz}, \mathrm{CH}_{3} \mathrm{CHOH}\right)$, $3.51\left(3 \mathrm{H}, \mathrm{s}, \mathrm{CO}_{2} \mathrm{CH}_{3}\right), 2.41(2 \mathrm{H}, \mathrm{bs}, \mathrm{OH}+\mathbf{N H}), 1.52(1 \mathrm{H}, \mathrm{d}, J=14.9 \mathrm{~Hz}$, $\left.\operatorname{SiCH}_{\mathbf{A}} \mathrm{H}_{\mathrm{B}}\right), 1.41\left(1 \mathrm{H}, \mathrm{d}, J=14.9 \mathrm{~Hz}, \operatorname{SiCH}_{\mathbf{A}} \mathrm{H}_{\mathrm{B}}\right), 0.97(3 \mathrm{H}, \mathrm{d}, J=6.4 \mathrm{~Hz}$, $\left.\mathrm{CH}_{3} \mathrm{CHOH}\right), 0.33\left(3 \mathrm{H}, \mathrm{s}, \mathrm{CH}_{3} \mathrm{Si}\right), 0.32\left(3 \mathrm{H}, \mathrm{s}, \mathrm{CH}_{3} \mathrm{Si}\right) ;{ }^{13} \mathrm{C}$ NMR $(62.9 \mathrm{MHz}$, $\left.\mathrm{CDCl}_{3}\right) \delta 176.3(\mathrm{C}), 138.3(\mathrm{C}), 133.6(2 \times \mathrm{CH}), 129.2(\mathrm{CH}), 127.9(2 \times \mathrm{CH}), 73.1$ $(\mathrm{CH}), 63.3(\mathrm{C}), 51.9\left(\mathrm{CH}_{3}\right), 24.6\left(\mathrm{CH}_{2}\right), 18.1\left(\mathrm{CH}_{3}\right),-1.8\left(\mathrm{CH}_{3}\right),-2.4\left(\mathrm{CH}_{3}\right)$; HRMS (ES) Exact mass calcd for $\mathrm{C}_{14} \mathrm{H}_{24} \mathrm{NO}_{3} \mathrm{Si}[\mathrm{M}+\mathrm{H}]^{+}: 282.1520$, found: 282.1520 .

Data for 319b: $\mathrm{R}_{\mathrm{f}}(40 \%$ EtOAc in hexane) $=0.10$; IR (film) $3382(\mathrm{NH}+\mathrm{OH})$, $1730(\mathrm{C}=\mathrm{O}), 1587,1427,1249,1112,835,701,521 \mathrm{~cm}^{-1} ;{ }^{1} \mathrm{H}$ NMR $(360 \mathrm{MHz}$, $\left.\mathrm{CDCl}_{3}\right) \delta$ 7.52-7.48 (2H, m, ArH), 7.37-7.34 (3H, m, $\left.\mathrm{ArH}\right), 3.83(1 \mathrm{H}, \mathrm{q}, J=6.3 \mathrm{~Hz}$, $\left.\mathrm{CH}_{3} \mathrm{CHOH}\right), 3.47\left(3 \mathrm{H}, \mathrm{s}, \mathrm{CO}_{2} \mathrm{CH}_{3}\right), 2.20(2 \mathrm{H}, \mathrm{bs}, \mathrm{OH}+\mathrm{NH}), 1.33(1 \mathrm{H}, \mathrm{d}, J=14.7$ $\left.\mathrm{Hz}, \mathrm{SiCH}_{\mathrm{A}} \mathrm{H}_{\mathrm{B}}\right), 1.14\left(3 \mathrm{H}, \mathrm{d}, J=6.3 \mathrm{~Hz}, \mathrm{CH}_{3} \mathrm{CHOH}\right), 1.13(1 \mathrm{H}, \mathrm{d}, J=14.7 \mathrm{~Hz}$, 
$\left.\mathrm{SiCH}_{\mathrm{A}} \mathbf{H}_{\mathrm{B}}\right), 0.33\left(6 \mathrm{H}, \mathrm{s},\left(\mathrm{CH}_{3}\right)_{2} \mathrm{Si}\right) ;{ }^{13} \mathrm{C}$ NMR $\left(62.9 \mathrm{MHz}, \mathrm{CDCl}_{3}\right) \delta 176.5(\mathrm{C}), 138.3$ (C), $133.5(2 \times \mathrm{CH}), 129.1(\mathrm{CH}), 127.8(2 \times \mathrm{CH}), 73.0(\mathrm{CH}), 63.5(\mathrm{C}), 51.9\left(\mathrm{CH}_{3}\right)$, $23.6\left(\mathrm{CH}_{2}\right), 16.6\left(\mathrm{CH}_{3}\right),-1.9\left(\mathrm{CH}_{3}\right),-2.1\left(\mathrm{CH}_{3}\right)$; HRMS (ES) Exact mass calcd for $\mathrm{C}_{14} \mathrm{H}_{24} \mathrm{NO}_{3} \mathrm{Si}[\mathrm{M}+\mathrm{H}]^{+}: 282.1520$, found: 282.1521 .

\section{Methyl 2-(4-methoxybenzylamino)-2-[(dimethylphenylsilyl)methyl]-3-} hydroxybutanoate (320a and 320b)

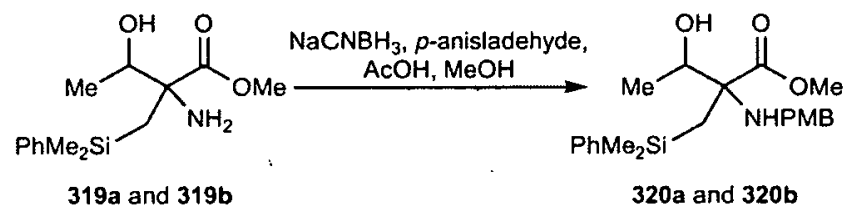

To a solution of a mixture of alcohols $319 \mathrm{a}$ and $319 \mathrm{~b}(1.27 \mathrm{~g}, 4.50 \mathrm{mmol})$ in $\mathrm{MeOH}(40 \mathrm{~mL})$ at room temperature was added HOAc $(0.52 \mathrm{~mL}, 9.00 \mathrm{mmol})$, and the reaction flask was placed in a water bath. $\mathrm{NaBH}_{3} \mathrm{CN}(450 \mathrm{mg}, 6.77 \mathrm{mmol})$ was then added, followed by $p$-anisaldehyde $(0.70 \mathrm{~mL}, 5.40 \mathrm{mmol})$. The reaction was stirred at room temperature for $18 \mathrm{~h}$ and it was then quenched with saturated aqueous $\mathrm{NaHCO}_{3}$ solution. The suspension was concentrated in vacuo, and the crude mixture was partitioned between $\mathrm{H}_{2} \mathrm{O}$ and $\mathrm{CH}_{2} \mathrm{Cl}_{2}$. The aqueous layer was extracted with $\mathrm{CH}_{2} \mathrm{Cl}_{2}$, and the combined organic extracts were dried $\left(\mathrm{Na}_{2} \mathrm{SO}_{4}\right)$, filtered and concentrated. Purification of the residue by column chromatography $(10 \%$ EtOAc/petrol) gave the mixture of aminoalcohols 320a and 320b (1.16 g, mixture of both diastereomers, $65 \%$ ) as a colourless oil.

This reaction was typically performed on a mixture of both diastereomers $319 \mathrm{a}$ and $319 \mathrm{~b}$ and it was isolated a mixture of both diastereomers of 320a and 320b. Only on one occasion was the reaction performed individually on each diastereomer for characterisation purposes

Data for 320a: $R_{f}(30 \%$ EtOAc in hexane) $=0.48$; IR (film) $2950(\mathrm{NH}+\mathrm{OH})$, 2348, $1727(\mathrm{C}=\mathrm{O}), 1612,1512,1462,1247,1111,1033,833 \mathrm{~cm}^{-1} ;{ }^{1} \mathrm{H}$ NMR (360 $\left.\mathrm{MHz}, \mathrm{CDCl}_{3}\right) \delta$ 7.56-7.53 (2H, m, ArH), 7.36-7.34 (3H, m, ArH), $7.12(2 \mathrm{H}, \mathrm{d}, J=$ $8.6 \mathrm{~Hz}, \mathrm{ArH}), 6.84(2 \mathrm{H}, \mathrm{d}, J=8.6 \mathrm{~Hz}, \mathrm{ArH}), 3.99\left(1 \mathrm{H}, \mathrm{q}, J=6.4 \mathrm{~Hz}, \mathrm{CH}_{3} \mathrm{CHOH}\right)$, $\left.3.81(3 \mathrm{H}, \mathrm{s}, \operatorname{ArOCH})_{3}\right), 3.58\left(3 \mathrm{H}, \mathrm{s}, \mathrm{CO}_{2} \mathrm{CH}_{3}\right), 3.55(1 \mathrm{H}, \mathrm{d}, J=11.6 \mathrm{~Hz}$, $\left.\mathrm{NHCH}_{\mathrm{A}} \mathrm{CH}_{\mathrm{B}} \mathrm{Ar}\right), 3.46\left(1 \mathrm{H}, \mathrm{d}, J=11.6 \mathrm{~Hz}, \mathrm{NHCH}_{\mathrm{A}} \mathrm{CH}_{\mathrm{B}} \mathrm{Ar}\right), 3.23(1 \mathrm{H}, \mathrm{bs}, \mathrm{NH}), 1.92$ $(1 \mathrm{H}, \mathrm{bs}, \mathrm{OH}), 1.57\left(1 \mathrm{H}, \mathrm{d}, J=15.2 \mathrm{~Hz}, \mathrm{SiCH}_{\mathrm{A}} \mathrm{CH}_{\mathrm{B}}\right), 1.33(1 \mathrm{H}, \mathrm{d}, J=15.2 \mathrm{~Hz}$, 
$\left.\mathrm{SiCH}_{\mathrm{A}} \mathrm{CH}_{\mathrm{B}}\right), 1.17\left(3 \mathrm{H}, \mathrm{d}, J=6.4 \mathrm{~Hz}, \mathrm{CH}_{3} \mathrm{CH}\right), 0.39\left(6 \mathrm{H}, \mathrm{s},\left(\mathrm{CH}_{3}\right)_{2} \mathrm{Si}\right) ;{ }^{13} \mathrm{C} \mathrm{NMR}$ (62.9 MHz, $\left.\mathrm{CDCl}_{3}\right) \delta 175.9(\mathrm{C}), 158.7(\mathrm{C}), 139.4(\mathrm{C}), 133.6$ (2 x CH), 131.9 (C), $129.2(2 \times \mathrm{CH}), 128.9(\mathrm{CH}), 127.8(2 \times \mathrm{CH}), 113.8(2 \times \mathrm{CH}), 70.2(\mathrm{CH}), 68.1(\mathrm{C})$, $55.2\left(\mathrm{CH}_{3}\right), 51.6\left(\mathrm{CH}_{3}\right), 47.1\left(\mathrm{CH}_{2}\right), 20.2\left(\mathrm{CH}_{2}\right), 17.3\left(\mathrm{CH}_{3}\right),-1.2\left(\mathrm{CH}_{3}\right),-1.4\left(\mathrm{CH}_{3}\right)$; HRMS (ES) Exact mass calcd for $\mathrm{C}_{22} \mathrm{H}_{32} \mathrm{NO}_{4} \mathrm{Si}[\mathrm{M}+\mathrm{H}]^{+}: 402.2095$, found: 402.2096 .

Data for 320b: $\mathrm{R}_{\mathrm{f}}(30 \%$ EtOAc in hexane) $=0.48$; IR (film) $2951(\mathrm{NH}+\mathrm{OH})$, 2348, $1729(\mathrm{C}=\mathrm{O}), 1612,1512,1427,1246,1173,1111,832 \mathrm{~cm}^{-1} ;{ }^{1} \mathrm{H}$ NMR $(360$ $\left.\mathrm{MHz}, \mathrm{CDCl}_{3}\right) \delta$ 7.55-7.51 (2H, m, ArH), 7.36-7.32 (3H, m, ArH), $7.07(2 \mathrm{H}, \mathrm{d}, J=$ $8.6 \mathrm{~Hz}, \mathrm{ArH}), 6.81(2 \mathrm{H}, \mathrm{d}, J=8.6 \mathrm{~Hz}, \mathrm{ArH}), 4.04\left(1 \mathrm{H}, \mathrm{q}, J=6.4 \mathrm{~Hz}, \mathrm{CH}_{3} \mathrm{CHOH}\right)$, $3.80\left(3 \mathrm{H}, \mathrm{s}, \mathrm{ArOCH}_{3}\right), 3.61\left(1 \mathrm{H}, \mathrm{d}, J=11.6 \mathrm{~Hz}, \mathrm{NHCH}_{\mathbf{A}} \mathrm{CH}_{\mathrm{B}} \mathrm{Ar}\right), 3.58-3.52(3 \mathrm{H}, \mathrm{s}$, $\left.\mathrm{CO}_{2} \mathrm{CH}_{3}+1 \mathrm{H}, \mathrm{d}, J=11.6 \mathrm{~Hz}, \mathrm{NHCH}_{\mathrm{A}} \mathrm{CH}_{\mathrm{B}} \mathrm{Ar}\right), 3.31(1 \mathrm{H}, \mathrm{bs}, \mathrm{NH}), 1.60(1 \mathrm{H}, \mathrm{bs}$, $\mathrm{OH}), 1.43\left(1 \mathrm{H}, \mathrm{d}, J=15.0 \mathrm{~Hz}, \mathrm{SiCH}_{\mathrm{A}} \mathrm{CH}_{\mathrm{B}}\right), 1.33\left(1 \mathrm{H}, \mathrm{d}, J=15.0 \mathrm{~Hz}, \mathrm{SiCH}_{\mathrm{A}} \mathrm{CH}_{\mathrm{B}}\right)$, $1.23\left(3 \mathrm{H}, \mathrm{d}, J=6.3 \mathrm{~Hz}, \mathrm{CH}_{3} \mathrm{CHOH}\right), 0.38\left(3 \mathrm{H}, \mathrm{s}, \mathrm{CH}_{3} \mathrm{Si}\right), 0.36\left(3 \mathrm{H}, \mathrm{s}, \mathrm{CH}_{3} \mathrm{Si}\right) ;{ }^{13} \mathrm{C}$ NMR (62.9 MHz, $\left.\mathrm{CDCl}_{3}\right) \delta 175.5(\mathrm{C}), 158.7$ (C), $138.9(\mathrm{C}), 133.6(2 \times \mathrm{CH}), 131.8$ (C), $129.2(2 \times \mathrm{CH}), 129.0(\mathrm{CH}), 127.8(2 \times \mathrm{CH}), 113.8(2 \times \mathrm{CH}), 70.2(\mathrm{CH}), 68.0$ (C), $55.3\left(\mathrm{CH}_{3}\right), 51.7\left(\mathrm{CH}_{3}\right), 47.5\left(\mathrm{CH}_{2}\right), 20.5\left(\mathrm{CH}_{2}\right), 17.3\left(\mathrm{CH}_{3}\right),-1.2\left(\mathrm{CH}_{3}\right),-1.3$ $\left(\mathrm{CH}_{3}\right)$; HRMS (ES) Exact mass calcd for $\mathrm{C}_{22} \mathrm{H}_{32} \mathrm{NO}_{4} \mathrm{Si}[\mathrm{M}+\mathrm{H}]^{+}: 402.2095$, found: 402.2095 .

\section{Methyl 2-(4-methoxybenzylamino)-2-[(dimethylphenylsilyl)methyl]-3-} oxobutanoate (300)

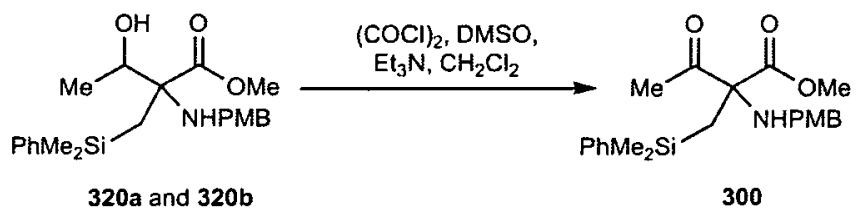

To a stirred solution of oxalyl chloride $(0.51 \mathrm{~mL}, 5.76 \mathrm{mmol})$ in $\mathrm{CH}_{2} \mathrm{Cl}_{2}(12 \mathrm{~mL})$ at $-78{ }^{\circ} \mathrm{C}$ was added DMSO $(0.82 \mathrm{~mL}, 11.5 \mathrm{mmol})$ dropwise. After stirring for 15 minutes, a solution of the mixture of alcohols $320 \mathrm{a}$ and $320 \mathrm{~b}(1.10 \mathrm{~g}, 2.74 \mathrm{mmol})$ in $\mathrm{CH}_{2} \mathrm{Cl}_{2}(12 \mathrm{~mL})$ was added via cannula. The reaction was stirred at $-78^{\circ} \mathrm{C}$ for $1 \mathrm{~h}$ and $\mathrm{Et}_{3} \mathrm{~N}(1.61 \mathrm{~mL}, 11.5 \mathrm{mmol})$ was then added. The reaction was stirred at $-78{ }^{\circ} \mathrm{C}$ for $1 \mathrm{~h}$, allowed to warm up to $-40{ }^{\circ} \mathrm{C}$ over $2 \mathrm{~h}$ and then quenched with saturated aqueous $\mathrm{NH}_{4} \mathrm{Cl}$ solution $(10 \mathrm{~mL})$. The aqueous layer was separated and extracted 
with $\mathrm{CH}_{2} \mathrm{Cl}_{2}(3 \times 20 \mathrm{~mL})$ and the combined organic layers were dried $\left(\mathrm{MgSO}_{4}\right)$, filtered and concentrated in vacuo. Purification of the residue by column chromatography (10\% to $20 \%$ EtOAc/petrol) gave amino ketone 300 (886 $\mathrm{mg}, 81 \%$ ) as a yellow oil. $\mathrm{R}_{\mathrm{f}}(30 \%$ EtOAc in hexane $)=0.53$; IR (film) $2952(\mathrm{NH}), 2359,1739$ $(\mathrm{C}=\mathrm{O}), 1717(\mathrm{C}=\mathrm{O}), 1612,1512,1247,1172,1035,832 \mathrm{~cm}^{-1} ;{ }^{1} \mathrm{H}$ NMR $(360 \mathrm{MHz}$, $\left.\mathrm{CDCl}_{3}\right) \delta$ 7.56-7.52 (2H, m, ArH), 7.36-7.33 (3H, m, ArH), 7.09 (2H, d, J=8.5 Hz, $\operatorname{ArH}), 6.83(2 \mathrm{H}, \mathrm{d}, J=8.5 \mathrm{~Hz}, \operatorname{ArH}), 3.80\left(3 \mathrm{H}, \mathrm{s}, \operatorname{ArOCH}_{3}\right), 3.52\left(3 \mathrm{H}, \mathrm{s}, \mathrm{CO}_{2} \mathrm{CH}_{3}\right)$, $3.40\left(1 \mathrm{H}, \mathrm{d}, J=12.0 \mathrm{~Hz}, \mathrm{NHCH}_{\mathrm{A}} \mathrm{CH}_{\mathrm{B}} \mathrm{Ar}\right), 3.32(1 \mathrm{H}, \mathrm{d}, J=12.0 \mathrm{~Hz}$, $\left.\mathrm{NHCH}_{\mathrm{A}} \mathrm{CH}_{\mathrm{B}} \mathrm{Ar}\right), 2.28(1 \mathrm{H}, \mathrm{bs}, \mathrm{NH}), 2.11\left(3 \mathrm{H}, \mathrm{s}, \mathrm{CH}_{3} \mathrm{C}=\mathrm{O}\right), 1.73(1 \mathrm{H}, \mathrm{d}, J=15.4$ $\left.\mathrm{Hz}, \mathrm{SiCH}_{\mathrm{A}} \mathrm{CH}_{\mathrm{B}}\right), 1.63\left(1 \mathrm{H}, \mathrm{d}, J=15.4 \mathrm{~Hz}, \mathrm{SiCH}_{\mathrm{A}} \mathrm{CH}_{\mathbf{B}}\right), 0.35\left(3 \mathrm{H}, \mathrm{s}, \mathrm{CH}_{3} \mathrm{Si}\right), 0.34$ (3H, s, $\left.\mathrm{CH}_{3} \mathrm{Si}\right) ;{ }^{13} \mathrm{C}$ NMR (62.9 MHz, $\left.\mathrm{CDCl}_{3}\right) \delta 204.6$ (C), 172.1 (C), 158.7 (C), $138.7(\mathrm{C}), 133.7(2 \times \mathrm{CH}), 131.5(\mathrm{C}), 129.2(2 \times \mathrm{CH}), 129.0(\mathrm{CH}), 127.7(2 \times \mathrm{CH})$, $113.7(2 \times \mathrm{CH}), 73.6(\mathrm{C}), 55.3\left(\mathrm{CH}_{3}\right), 52.3\left(\mathrm{CH}_{3}\right), 46.8\left(\mathrm{CH}_{2}\right), 24.9\left(\mathrm{CH}_{3}\right), 19.2$ $\left(\mathrm{CH}_{2}\right),-2.0\left(\mathrm{CH}_{3}\right),-2.1\left(\mathrm{CH}_{3}\right)$; HRMS (ES) Exact mass calcd for $\mathrm{C}_{22} \mathrm{H}_{30} \mathrm{NO}_{4} \mathrm{SiNa}$ $[\mathrm{M}+\mathrm{H}]^{+}: 400.1939$, found: 400.1933 .

\section{(E)-Ethyl 4-hydroxybut-2-enoate (322) ${ }^{186}$}

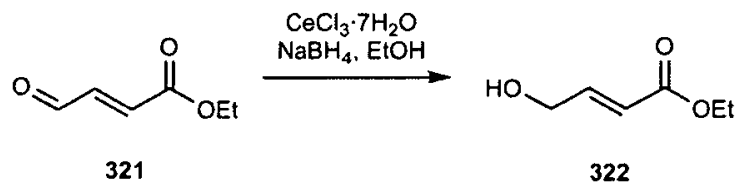

To a stirred solution of aldehyde $321(5.60 \mathrm{~mL}, 44.6 \mathrm{mmol})$ in $\mathrm{EtOH}(200 \mathrm{~mL})$ at $0{ }^{\circ} \mathrm{C}$ was added $\mathrm{CeCl}_{3} \cdot 7 \mathrm{H}_{2} \mathrm{O}(16.6 \mathrm{~g}, 44.6 \mathrm{mmol})$ followed by $\mathrm{NaBH}_{4}(1.72 \mathrm{~g}, 44.6$ mmol), added portionwise over 20 minutes. The reaction was allowed to warm up to room temperature and stirred for $14 \mathrm{~h}$ and then quenched with saturated aqueous $\mathrm{NH}_{4} \mathrm{Cl}$ solution. This mixture was concentrated in vacuo and the remaining mixture was taken up with $\mathrm{Et}_{2} \mathrm{O}$. The ether phase was washed with water $(2 \times 40 \mathrm{~mL})$ and the combined aqueous layers were extracted with $\mathrm{Et}_{2} \mathrm{O}(2 \times 30 \mathrm{~mL})$. The combined organic layers were dried $\left(\mathrm{MgSO}_{4}\right)$, filtered and concentrated in vacuo to afford alcohol $322(5.5 \mathrm{~g}, 95 \%)$ as a colourless oil which was used in the next step without further purification. $\mathrm{R}_{\mathrm{f}}(30 \%$ EtOAc in hexane $)=0.26 ;{ }^{1} \mathrm{H} \mathrm{NMR}\left(250 \mathrm{MHz}, \mathrm{CDCl}_{3}\right)$ $\delta 6.99(1 \mathrm{H}, \mathrm{dt}, J=3.9,15.7 \mathrm{~Hz}, \mathrm{CH}=\mathrm{CHC}=\mathrm{O}), 6.05(1 \mathrm{H}, \mathrm{dt}, J=2.1,15.7 \mathrm{~Hz}$, $\mathrm{CH}=\mathrm{CHC}=\mathrm{O}), 4.29\left(2 \mathrm{H}\right.$, bs, $\left.\mathrm{HOCH}_{2}\right), 4.16\left(2 \mathrm{H}, \mathrm{q}, J=7.1 \mathrm{~Hz}, \mathrm{CH}_{2} \mathrm{CH}_{3}\right), 3.06(1 \mathrm{H}$, 
bs, OH), $1.25\left(3 \mathrm{H}, \mathrm{t}, J=7.1 \mathrm{~Hz}, \mathrm{CH}_{2} \mathrm{CH}_{3}\right) ;{ }^{13} \mathrm{C} \mathrm{NMR}\left(62.9 \mathrm{MHz}, \mathrm{CDCl}_{3}\right) \delta 166.6$

(C), $147.2(\mathrm{CH}), 119.8(\mathrm{C}), 61.5\left(\mathrm{CH}_{2}\right), 60.4\left(\mathrm{CH}_{2}\right), 14.1\left(\mathrm{CH}_{3}\right)$.

${ }^{1} \mathrm{H}$ spectroscopic data in good agreement with the literature. ${ }^{186}$

(E)-Ethyl 4-(4-methoxybenzyloxy)but-2-enoate $(324)^{187}$

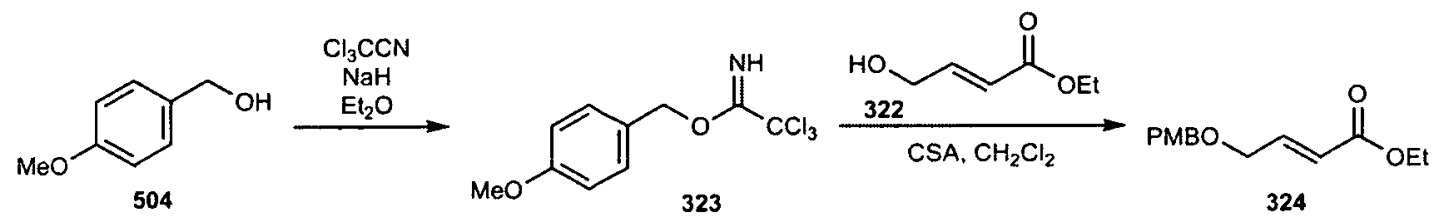

To a stirred suspension of sodium hydride $(80 \mathrm{mg}, 60 \%$ dispersion in mineral oil, $2.0 \mathrm{mmol})$ in $\mathrm{Et}_{2} \mathrm{O}(25.0 \mathrm{~mL})$ at $0{ }^{\circ} \mathrm{C}$ was added 4-methoxybenzyl alcohol $504(2.50$ $\mathrm{mL}, 20.0 \mathrm{mmol}$ ) dropwise over $3 \mathrm{~min}$. The reaction was stirred at $0{ }^{\circ} \mathrm{C}$ for $5 \mathrm{~min}$ and trichloroacetonitrile $(2.00 \mathrm{~mL}, 21.0 \mathrm{mmol})$ was added over $2 \mathrm{~min}$. The reaction was allowed to warm up to room temperature, stirred for $12 \mathrm{~h}$ and then concentrated in vacuo to give crude $p$-methoxybenzyl trichloroacetimidate 323 (6.10 g), which was used in the next step without further purification. ${ }^{120}$

To a stirred solution of alcohol $322(4.35 \mathrm{~g}, 33.3 \mathrm{mmol})$ in $\mathrm{CH}_{2} \mathrm{Cl}_{2}(80 \mathrm{~mL})$ at $0{ }^{\circ} \mathrm{C}$ was added a solution of $p$-methoxybenzyl trichloroacetimidate $(14.1 \mathrm{~g}, 49.9$ $\mathrm{mmol})$ in $\mathrm{CH}_{2} \mathrm{Cl}_{2}(30 \mathrm{~mL})$ via cannula, followed by the addition of CSA $(780 \mathrm{mg}$, $3.33 \mathrm{mmol}$ ) in one portion. The reaction was allowed to warm up to room temperature, stirred for $14 \mathrm{~h}$ and then filtered through celite. The solids were washed with $\mathrm{CH}_{2} \mathrm{Cl}_{2}(3 \times 30 \mathrm{~mL})$ and the combined organic layer was washed with $\mathrm{NaHCO}_{3}$ $(2 \times 30 \mathrm{~mL})$, dried $\left(\mathrm{MgSO}_{4}\right)$, filtered and concentrated in vacuo. Purification of the residue by column chromatography (10\% to $20 \% \mathrm{EtOAc} /$ petrol) gave ester 324 (4.73 $\mathrm{g}, 57 \%$, reaction not optimised) as a yellow oil. $\mathrm{R}_{\mathrm{f}}(10 \%$ EtOAc in hexane $)=0.26$; 'H NMR (250 MHz, $\left.\mathrm{CDCl}_{3}\right) \delta 7.20(2 \mathrm{H}, \mathrm{d}, J=8.3 \mathrm{~Hz}, \mathrm{ArH}), 6.94(1 \mathrm{H}, \mathrm{dt}, J=15.5$, $4.2 \mathrm{~Hz}, \mathrm{CH}=\mathrm{CHC}=\mathrm{O}), 6.85(2 \mathrm{H}, \mathrm{d}, J=8.3 \mathrm{~Hz}, \mathrm{ArH}), 6.10(1 \mathrm{H}, \mathrm{dt}, J=15.5,2.3 \mathrm{~Hz}$, $\mathrm{CH}=\mathrm{CHC}=\mathrm{O}), 4.50\left(2 \mathrm{H}, \mathrm{s}, \mathrm{ArCH}_{2} \mathrm{O}\right), 4.18\left(2 \mathrm{H}, \mathrm{q}, J=7.1 \mathrm{~Hz}, \mathrm{CH}_{2} \mathrm{CH}_{3}\right), 4.10(2 \mathrm{H}$, dd, $\left.J=4.2,2.3 \mathrm{~Hz}, \mathrm{CH}_{2} \mathrm{CH}=\mathrm{CH}\right), 3.8\left(3 \mathrm{H}, \mathrm{s}, \mathrm{CH}_{3} \mathrm{O}\right), 1.28(3 \mathrm{H}, \mathrm{t}, J=7.1 \mathrm{~Hz}$, $\mathrm{CH}_{2} \mathrm{CH}_{3}$ ).

${ }^{1} \mathrm{H}$ spectroscopic data in good agreement with the literature. ${ }^{187}$ 
(E)-4-(4-Methoxybenzyloxy)but-2-enoic acid (325)

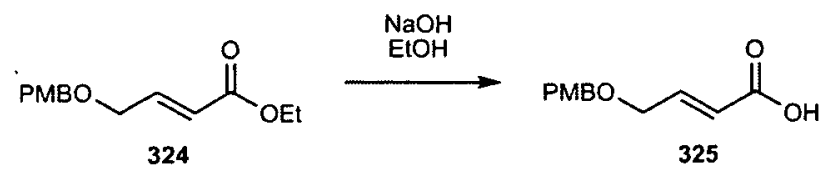

To a stirred solution of ester $324(5.17 \mathrm{~g}, 20.6 \mathrm{mmol})$ in EtOH $(100 \mathrm{~mL})$ was added a $0.5 \mathrm{M} \mathrm{NaOH}$ aqueous solution $(45 \mathrm{~mL})$. The reaction was stirred at room temperature for $14 \mathrm{~h}$, concentrated in vacuo, dissolved in water $(40 \mathrm{~mL})$ and washed with $\mathrm{CH}_{2} \mathrm{Cl}_{2}(1 \times 30 \mathrm{~mL})$. The $\mathrm{pH}$ of the aqueous layer was.taken to $\mathrm{pH}=4$ and it was then extracted with $\mathrm{CH}_{2} \mathrm{Cl}_{2}(3 \times 30 \mathrm{~mL})$. The combined organic layers were dried $\left(\mathrm{MgSO}_{4}\right)$, filtered and concentrated in vacuo, to give acid $\mathbf{3 2 5}$ as a colourless solid $(3.1 \mathrm{~g}, 70 \%)$, which was used in the next step without further purification. $R_{\mathrm{f}}$ $(30 \%$ EtOAc in hexane $)=0.23 ;$ m.p. $=83-85{ }^{\circ} \mathrm{C}$; IR (film) $2838(\mathrm{OH}), 2360,1685$ $(\mathrm{C}=\mathrm{O}), 1515,1425,1311,1251,1180,1029,821 \mathrm{~cm}^{-1} ;{ }^{1} \mathrm{H}$ NMR $\left(250 \mathrm{MHz}, \mathrm{CDCl}_{3}\right)$ $\delta 7.43(2 \mathrm{H}, \mathrm{d}, J=8.1 \mathrm{~Hz}, \mathrm{ArH}), 7.24(1 \mathrm{H}, \mathrm{dt}, J=15.7,4.1 \mathrm{~Hz}, \mathrm{CH}=\mathrm{CHC}=\mathrm{O}), 7.05$ $(2 \mathrm{H}, \mathrm{d}, J=8.1 \mathrm{~Hz}, \mathrm{ArH}), 6.29(1 \mathrm{H}, \mathrm{dt}, J=15.7,2.0 \mathrm{~Hz}, \mathrm{CH}=\mathrm{CHC}=\mathrm{O}), 4.67(2 \mathrm{H}, \mathrm{s}$, $\left.\operatorname{ArCH}_{2} \mathrm{O}\right), 4.33\left(2 \mathrm{H}, \mathrm{dd}, J=4.1,2.0 \mathrm{~Hz}, \mathrm{CH}_{2} \mathrm{CH}=\mathrm{CH}\right), 3.97\left(3 \mathrm{H}, \mathrm{s}, \mathrm{OCH}_{3}\right) ;{ }^{13} \mathrm{C}$ NMR (62.9 MHz, $\left.\mathrm{CDCl}_{3}\right) \delta 171.4(\mathrm{C}), 159.3(\mathrm{C}), 147.1(\mathrm{CH}), 129.5(\mathrm{C}), 129.3(2 \mathrm{x}$ $\mathrm{CH}), 120.3(\mathrm{CH}), 113.8(2 \times \mathrm{CH}), 72.5\left(\mathrm{CH}_{2}\right), 68.1\left(\mathrm{CH}_{2}\right), 55.2\left(\mathrm{CH}_{3}\right)$; HRMS (ES) Exact mass calcd for $\mathrm{C}_{12} \mathrm{H}_{18} \mathrm{NO}_{4}\left[\mathrm{M}+\mathrm{NH}_{4}\right]^{+}: 240.1230$, found: 240.1232 .

Methyl 2-[ $N$-(4-methoxybenzyl)acrylamido]-2-[(dimethylphenylsilyl)methyl]-3oxobutanoate (347)

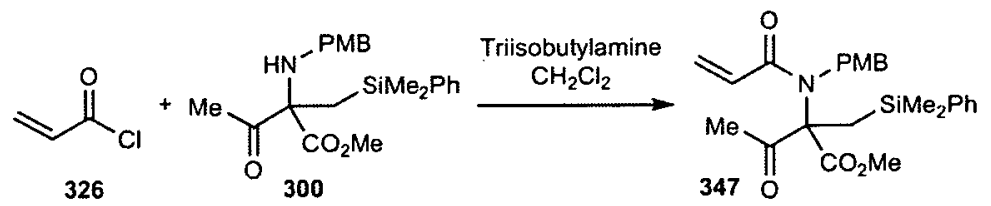

To a stirred solution of amine $300(652 \mathrm{mg}, 1.63 \mathrm{mmol})$ in $\mathrm{CH}_{2} \mathrm{Cl}_{2}(10 \mathrm{~mL})$ at $0{ }^{\circ} \mathrm{C}$, was added triisobutylamine $(1.20 \mathrm{~mL}, 4.89 \mathrm{mmol})$. The reaction was stirred at $0{ }^{\circ} \mathrm{C}$ for $10 \mathrm{~min}$ and acryloyl chloride $(0.44 \mathrm{~mL}, 4.89 \mathrm{mmol})$ was then added. The reaction was stirred for $14 \mathrm{~h}$ and then quenched with $1 \mathrm{M} \mathrm{HCl}(10 \mathrm{~mL})$. The aqueous layer was separated and extracted with $\mathrm{CH}_{2} \mathrm{Cl}_{2}(2 \times 10 \mathrm{~mL})$ and the combined organic layers were dried $\left(\mathrm{MgSO}_{4}\right)$, filtered and concentrated in vacuo. Purification of the residue by column chromatography (10\% to $20 \%$ EtOAc/petrol) gave amide 
$347(452 \mathrm{mg}, 65 \%)$ as a pale yellow oil. $\mathrm{R}_{\mathrm{f}}(30 \%$ EtOAc in hexane $)=0.46$; IR (film) 2952, $1741(\mathrm{C}=\mathrm{O}), 1716(\mathrm{C}=\mathrm{O}), 1652(\mathrm{C}=\mathrm{O}), 1513,1415,1249,1174,829,734$ $\mathrm{cm}^{-1} ;{ }^{1} \mathrm{H}$ NMR $\left(360 \mathrm{MHz}, \mathrm{CDCl}_{3}\right) \delta$ 7.48-7.44 $(2 \mathrm{H}, \mathrm{m}, \operatorname{ArH}), 7.37-7.33(3 \mathrm{H}, \mathrm{m}$, ArH), $7.30(2 \mathrm{H}, \mathrm{d}, J=8.4 \mathrm{~Hz}, \operatorname{ArH}), 6.95(2 \mathrm{H}, \mathrm{d}, J=8.4 \mathrm{~Hz}, \operatorname{ArH}), 6.37(1 \mathrm{H}, \mathrm{dd}, J$ $\left.=3.2,16.7 \mathrm{~Hz}, \mathrm{CH}_{\mathrm{A}} \mathrm{H}_{\mathrm{B}}=\mathrm{CH}\right), 6.34\left(1 \mathrm{H}, \mathrm{dd}, J=9.1,16.7 \mathrm{~Hz}, \mathrm{CH}_{2}=\mathrm{CHC}=\mathrm{O}\right), 5.67$ $\left(1 \mathrm{H}, \mathrm{dd}, J=9.1,3.2 \mathrm{~Hz}, \mathrm{CH}_{\mathrm{A}} \mathbf{H}_{\mathrm{B}}=\mathrm{CH}\right), 4.62\left(1 \mathrm{H}, \mathrm{d}, J=18.3 \mathrm{~Hz}, \mathrm{NHCH}_{\mathrm{A}} \mathrm{H}_{\mathrm{B}} \mathrm{Ar}\right)$, $4.53\left(1 \mathrm{H}, \mathrm{d}, J=18.3 \mathrm{~Hz}, \mathrm{NHCH}_{\mathrm{A}} \mathbf{H}_{\mathrm{B}} \mathrm{Ar}\right), 3.86\left(3 \mathrm{H}, \mathrm{s}, \operatorname{ArOCH}_{3}\right), 3.42(3 \mathrm{H}, \mathrm{s}$, $\left.\mathrm{CO}_{2} \mathrm{CH}_{3}\right), 2.38\left(3 \mathrm{H}, \mathrm{s}, \mathrm{CH}_{3} \mathrm{C}=\mathrm{O}\right), 1.79\left(1 \mathrm{H}, \mathrm{d}, J=13.7 \mathrm{~Hz}, \mathrm{SiCH}_{\mathrm{C}} \mathrm{H}_{\mathrm{D}}\right), 1.47(1 \mathrm{H}, \mathrm{d}$, $\left.J=13.7 \mathrm{~Hz}, \mathrm{SiCH}_{\mathrm{C}} \mathbf{H}_{\mathbf{D}}\right), 0.30\left(6 \mathrm{H}, \mathrm{s},\left(\mathrm{CH}_{3}\right)_{2} \mathrm{Si}\right) ;{ }^{13} \mathrm{C} \mathrm{NMR}\left(62.9 \mathrm{MHz}, \mathrm{CDCl}_{3}\right) \delta$ 201.9 (C), 169.9 (C), 168.2 (C), 158.9 (C), 138.9 (C), $133.7(2 \times \mathrm{CH}), 130.1\left(\mathrm{CH}_{2}\right)$, $129.7(\mathrm{C}), 128.8(\mathrm{CH}), 128.2(\mathrm{CH}), 127.6(2 \times \mathrm{CH}), 127.2(2 \times \mathrm{CH}), 114.1(2 \times \mathrm{CH})$, $73.9(\mathrm{C}), 55.3\left(\mathrm{CH}_{3}\right), 52.1\left(\mathrm{CH}_{3}\right), 47.9\left(\mathrm{CH}_{2}\right), 28.0\left(\mathrm{CH}_{3}\right), 21.3\left(\mathrm{CH}_{2}\right),-1.2\left(\mathrm{CH}_{3}\right)$, $-2.0\left(\mathrm{CH}_{3}\right)$; HRMS (ES) Exact mass calcd for $\mathrm{C}_{25} \mathrm{H}_{32} \mathrm{NO}_{5} \mathrm{Si}[\mathrm{M}+\mathrm{H}]^{+}: 454.2044$, found: 454.2044 .

\section{(E)-Methyl 2-[N-(4-methoxybenzyl)but-2-enamido]-2-}

[(dimethylphenylsilyl)methyl]-3-oxobutanoate (349)

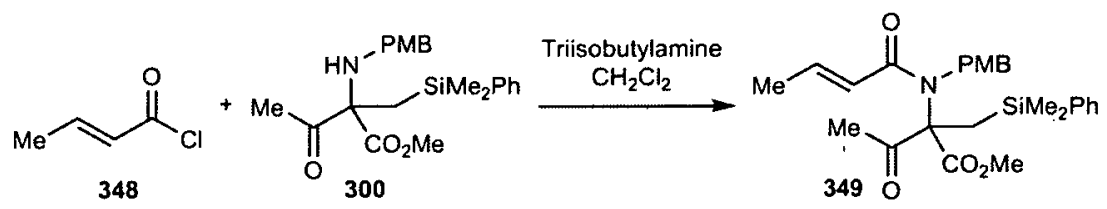

To a stirred solution of amine $300(400 \mathrm{mg}, 1.00 \mathrm{mmol})$ in $\mathrm{CH}_{2} \mathrm{Cl}_{2}(10 \mathrm{~mL})$ at $0^{\circ} \mathrm{C}$, was added triisobutylamine $(0.73 \mathrm{~mL}, 3.00 \mathrm{mmol})$. The reaction was stirred at $0{ }^{\circ} \mathrm{C}$ for $10 \mathrm{~min}$ and crotonoyl chloride $(0.29 \mathrm{~mL}, 3.00 \mathrm{mmol})$ was then added. The reaction was stirred for $15 \mathrm{~h}$ and then quenched with $1 \mathrm{M} \mathrm{HCl}(10 \mathrm{~mL})$. The aqueous layer was separated and extracted with $\mathrm{CH}_{2} \mathrm{Cl}_{2}(2 \times 10 \mathrm{~mL})$ and the combined organic layers were dried $\left(\mathrm{MgSO}_{4}\right)$, filtered and concentrated in vacuo. Purification of the residue by column chromatography (10\% to $20 \% \mathrm{EtOAc/petrol)}$ gave amide $349(413 \mathrm{mg}, 88 \%)$ as a yellow oil. $\mathrm{R}_{\mathrm{f}}(30 \%$ EtOAc in hexane $)=0.50$; IR (film) 2952, $1740(\mathrm{C}=\mathrm{O}), 1715(\mathrm{C}=\mathrm{O}), 1660(\mathrm{C}=\mathrm{O}), 1616,1512,1398,1248,1173,829$ $\mathrm{cm}^{-1} ;{ }^{1} \mathrm{H}$ NMR $\left(360 \mathrm{MHz}, \mathrm{CDCl}_{3}\right) \delta$ 7.44-7.40 $(2 \mathrm{H}, \mathrm{m}, \operatorname{ArH}), 7.32-7.28(3 \mathrm{H}, \mathrm{m}$, $\operatorname{ArH}), 7.26(2 \mathrm{H}, \mathrm{d}, J=8.5 \mathrm{~Hz}, \mathrm{ArH}), 6.98-6.88(1 \mathrm{H}, \mathrm{m}, \mathrm{CH}=\mathrm{CHC}=\mathrm{O}), 6.92(2 \mathrm{H}, \mathrm{d}, J$ $=8.5 \mathrm{~Hz}, \mathrm{ArH}), 6.00(1 \mathrm{H}, \mathrm{dd}, J=15.0,1.6 \mathrm{~Hz}, \mathrm{CH}=\mathrm{CHC}=\mathrm{O}), 4.56(1 \mathrm{H}, \mathrm{d}, J=18.3$ 
$\left.\mathrm{Hz}, \mathrm{NHCH}_{\mathrm{A}} \mathrm{H}_{\mathrm{B}} \mathrm{Ar}\right), 4.49\left(1 \mathrm{H}, \mathrm{d}, J=18.3 \mathrm{~Hz}, \mathrm{NHCH}_{\mathrm{A}} \mathbf{H}_{\mathrm{B}} \mathrm{Ar}\right), 3.83\left(3 \mathrm{H}, \mathrm{s}, \operatorname{ArOCH}_{3}\right)$, $3.36\left(3 \mathrm{H}, \mathrm{s}, \mathrm{CO}_{2} \mathrm{CH}_{3}\right), 2.33\left(3 \mathrm{H}, \mathrm{s}, \mathrm{CH}_{3} \mathrm{CO}\right), 1.79(3 \mathrm{H}, \mathrm{dd}, J=6.9,1.6 \mathrm{~Hz}$, $\left.\mathrm{CH}_{3} \mathrm{CH}=\mathrm{CH}\right), 1.73\left(1 \mathrm{H}, \mathrm{d}, J=13.8 \mathrm{~Hz}, \operatorname{SiCH}_{\mathrm{C}} \mathrm{H}_{\mathrm{D}}\right), 1.42(1 \mathrm{H}, \mathrm{d}, J=13.8 \mathrm{~Hz}$, $\left.\mathrm{SiCH}_{\mathrm{C}} \mathbf{H}_{\mathrm{D}}\right), 0.26\left(3 \mathrm{H}, \mathrm{s}, \mathrm{CH}_{3} \mathrm{Si}\right), 0.25\left(3 \mathrm{H}, \mathrm{s}, \mathrm{CH}_{3} \mathrm{Si}\right) ;{ }^{13} \mathrm{C} \mathrm{NMR}\left(62.9 \mathrm{MHz}, \mathrm{CDCl}_{3}\right)$ $\delta 201.8(\mathrm{C}), 170.0(\mathrm{C}), 168.4(\mathrm{C}), 158.8$ (C), 144.3 (CH), $139.0(\mathrm{C}), 133.7$ (2x CH), $129.9(\mathrm{C}), 128.7(\mathrm{CH}), 127.5(2 \times \mathrm{CH}), 127.3(2 \times \mathrm{CH}), 122.3(\mathrm{CH}), 114.1(2 \times \mathrm{CH})$, $73.8(\mathrm{C}), 55.3\left(\mathrm{CH}_{3}\right), 52.0\left(\mathrm{CH}_{3}\right), 47.9\left(\mathrm{CH}_{2}\right), 28.0\left(\mathrm{CH}_{3}\right), 21.3\left(\mathrm{CH}_{2}\right), 18.2\left(\mathrm{CH}_{3}\right)$, -1.2 $\left(\mathrm{CH}_{3}\right),-1.9\left(\mathrm{CH}_{3}\right)$; HRMS (ES) Exact mass calcd for $\mathrm{C}_{26} \mathrm{H}_{34} \mathrm{NO}_{5} \mathrm{Si}[\mathrm{M}+\mathrm{H}]^{+}$: 468.2201, found: 468.2204 .

\section{(E)-Ethyl 4-(benzyloxy)but-2-enoate $(351)^{188}$}

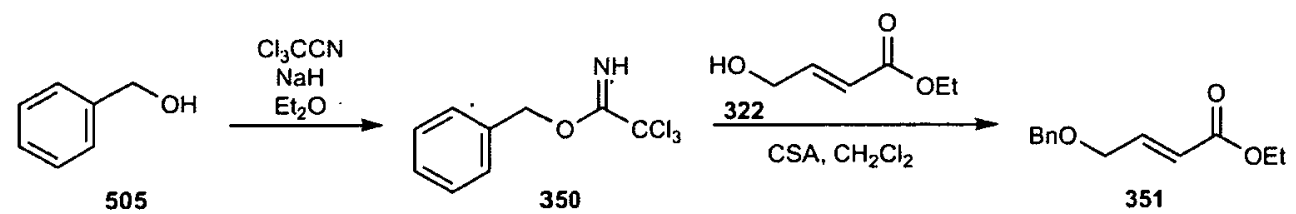

To a stirred suspension of sodium hydride $(256 \mathrm{mg}, 60 \%$ dispersion in mineral oil, $6.40 \mathrm{mmol})$ in $\mathrm{Et}_{2} \mathrm{O}(40.0 \mathrm{~mL})$ at $0{ }^{\circ} \mathrm{C}$ was added benzyl alcohol $505(6.6 \mathrm{~mL}, 64$ mmol) dropwise over $4 \mathrm{~min}$. The reaction was stirred at $0{ }^{\circ} \mathrm{C}$ for $5 \mathrm{~min}$ and trichloroacetonitrile $(13.2 \mathrm{~mL}, 128 \mathrm{mmol})$ was added over $3 \mathrm{~min}$. The reaction was allowed to warm up to room temperature, stirred for $12 \mathrm{~h}$ and concentrated in vacuo to give crude benzyl trichloroacetimidate (350) (15.5 g, 95\%), which was used in the next step without further purification. ${ }^{120}$

To a stirred solution of alcohol $322(2.40 \mathrm{~g}, 18.4 \mathrm{mmol})$ in $\mathrm{CH}_{2} \mathrm{Cl}_{2}(40 \mathrm{~mL})$ and toluene $(20 \mathrm{~mL})$ at room temperature was added a solution of benzyl trichloroacetimidate (350) $(7.80 \mathrm{~g}, 27.6 \mathrm{mmol})$ in $\mathrm{CH}_{2} \mathrm{Cl}_{2}(20 \mathrm{~mL})$ and toluene (10 $\mathrm{mL}$ ), followed by addition of a catalytic amount of triflouromethanesulfonic acid $(0.16 \mathrm{~mL}, 1.84 \mathrm{mmol})$. The reaction was stirred for $24 \mathrm{~h}$ and then filtered. The filtrates were washed with $\mathrm{CH}_{2} \mathrm{Cl}_{2}$ and the combined organic layers were washed with saturated aqueous $\mathrm{NaHCO}_{3}$ solution $(2 \times 30 \mathrm{~mL})$, water $(1 \times 30 \mathrm{~mL})$ and then were dried $\left(\mathrm{MgSO}_{4}\right)$, filtered and concentrated in vacuo. Purification of the residue by column chromatography (12\% EtOAc/petrol) gave ester 351 (2.5 g, 63\%) as a pale yellow oil. $\mathrm{R}_{\mathrm{f}}(30 \%$ EtOAc in hexane $)=0.57 ;{ }^{1} \mathrm{H}$ NMR $\left(250 \mathrm{MHz}, \mathrm{CDCl}_{3}\right) \delta$ 7.46-7.40 (5H, m, ArH), $7.06(1 \mathrm{H}, \mathrm{dt}, J=15.7,4.3 \mathrm{~Hz}, \mathrm{CH}=\mathrm{CHC}=\mathrm{O}), 6.22(1 \mathrm{H}, \mathrm{dt}$, 
$J=15.7,1.9 \mathrm{~Hz}, \mathrm{CH}=\mathrm{CHC}=\mathrm{O}), 5.09\left(2 \mathrm{H}, \mathrm{dd}, J=4.3,1.9 \mathrm{~Hz}, \mathrm{CH}_{2} \mathrm{CH}=\mathrm{CH}\right), 4.65$ $\left(2 \mathrm{H}, \mathrm{s}, \operatorname{ArCH}_{2} \mathrm{O}\right), 4.32\left(2 \mathrm{H}, \mathrm{q}, J=7.1 \mathrm{~Hz}, \mathrm{CH}_{2} \mathrm{CH}_{3}\right), 1.38(3 \mathrm{H}, \mathrm{t}, J=7.1 \mathrm{~Hz}$, $\left.\mathrm{CH}_{2} \mathrm{CH}_{3}\right) ;{ }^{13} \mathrm{C}$ NMR $\left(62.9 \mathrm{MHz}, \mathrm{CDCl}_{3}\right) \delta 166.2(\mathrm{C}), 144.1(\mathrm{CH}), 137.7(\mathrm{C}), 128.4$ $(2 \times \mathrm{CH}), 127.8(\mathrm{CH}), 127.6(2 \times \mathrm{CH}), 121.4(\mathrm{CH}), 72.7\left(\mathrm{CH}_{2}\right), 68.6\left(\mathrm{CH}_{2}\right), 66.6$ $\left(\mathrm{CH}_{2}\right), 14.2\left(\mathrm{CH}_{3}\right)$.

${ }^{1} \mathrm{H}$ and ${ }^{13} \mathrm{C}$ spectroscopic data in good agreement with the literature. ${ }^{188}$

(E)-4-(Benzyloxy)but-2-enoic acid $(352)^{123}$

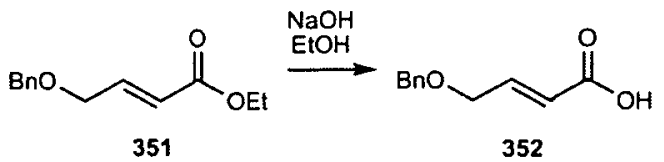

To a stirred solution of ester $351(9.20 \mathrm{mg}, 4.17 \mathrm{mmol})$ in THF $(12 \mathrm{~mL})$ and water $(2 \mathrm{~mL})$, was added $\mathrm{LiOH}(351 \mathrm{mg}, 8.34 \mathrm{mmol})$. The reaction was stirred at room temperature for $18 \mathrm{~h}$, diluted in water $(6 \mathrm{~mL})$ and concentrated in vacuo. This mixture was dissolved in water $(40 \mathrm{~mL})$ and washed with $\mathrm{Et}_{2} \mathrm{O}(2 \times 10 \mathrm{~mL})$. The $\mathrm{pH}$ of the aqueous was taken to $\mathrm{pH}=4$ and it was then extracted with $\mathrm{Et}_{2} \mathrm{O}(3 \times 15 \mathrm{~mL})$. The combined organic layers were dried $\left(\mathrm{MgSO}_{4}\right)$, filtered and concentrated in vacuo. Purification of the residue by column chromatography ( $20 \%$ EtOAc/petrol) gave acid 352 (690 mg, 85\%) as a pale yellow solid, which was used in the next step without further purification. $\mathrm{R}_{\mathrm{f}}(30 \% \mathrm{EtOAc}$ in hexane $)=0.17$; m.p. $46-48^{\circ} \mathrm{C}$, lit. ${ }^{123}$ 46-47 ${ }^{\circ} \mathrm{C} ;{ }^{1} \mathrm{H}$ NMR $\left(360 \mathrm{MHz}, \mathrm{CDCl}_{3}\right) \delta$ 7.39-7.34 (5H, m, ArH), $7.12(1 \mathrm{H}, \mathrm{dt}, J=$ 15.7, $\left.4.0 \mathrm{~Hz}, \quad \mathrm{OCH}_{2} \mathrm{CH}=\mathrm{CHC}=\mathrm{O}\right), \quad 6.18(1 \mathrm{H}, \quad \mathrm{dt}, J=15.7,2.1 \mathrm{~Hz}$, $\left.\mathrm{OCH}_{2} \mathrm{CH}=\mathrm{CHC}=\mathrm{O}\right), 4.60\left(2 \mathrm{H}, \mathrm{s}, \quad \mathrm{ArCH}_{2} \mathrm{O}\right), 4.24(1 \mathrm{H}, \mathrm{d}, J=2.1 \mathrm{~Hz}$, $\left.\mathrm{OCH}_{\mathrm{A}} \mathrm{H}_{\mathrm{B}} \mathrm{CH}=\mathrm{CHC}=\mathrm{O}\right), 4.24\left(1 \mathrm{H}, \mathrm{d}, J=2.1 \mathrm{~Hz}, \mathrm{OCH}_{\mathrm{A}} \mathbf{H}_{\mathbf{B}} \mathrm{CH}=\mathrm{CHC}=\mathrm{O}\right) ;{ }^{13} \mathrm{C} \mathrm{NMR}$ $\left(62.9 \mathrm{MHz}, \mathrm{CDCl}_{3}\right) \delta 171.6(\mathrm{C}), 147.1(\mathrm{CH}), 137.5(\mathrm{C}), 128.5(2 \times \mathrm{CH}), 127.9(\mathrm{CH})$, $127.6(2 \times \mathrm{CH}), 120.4(\mathrm{CH}), 72.8\left(\mathrm{CH}_{2}\right), 68.4\left(\mathrm{CH}_{2}\right)$.

${ }^{1} \mathrm{H}$ and ${ }^{13} \mathrm{C}$ spectroscopic data in good agreement with the literature. ${ }^{123}$ 


\section{(E)-Methyl 2-[4-(benzyloxy)- $N$-(4-methoxybenzyl)but-2-enamido]-2-}

\section{[(dimethylphenylsilyl)methyl]-3-oxobutanoate (354)}

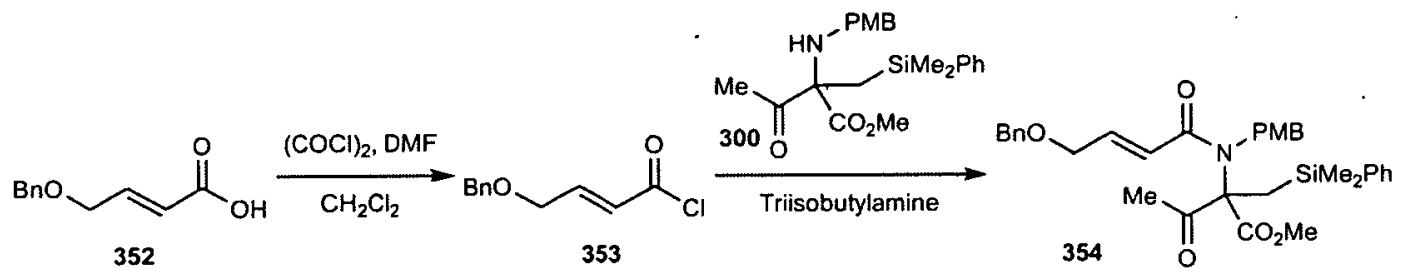

To a stirred solution of acid $352(865 \mathrm{mg}, 4.50 \mathrm{mmol})$ in $\mathrm{CH}_{2} \mathrm{Cl}_{2}(10 \mathrm{~mL})$ was added oxalyl chloride $(1.16 \mathrm{~mL}, 13.5 \mathrm{mmol})$ at room temperature. The reaction was stirred for $14 \mathrm{~h}$, concentrated in vacuo, redissolved in $\mathrm{CH}_{2} \mathrm{Cl}_{2}(8 \mathrm{~mL})$ and then transferred via cannula into a stirred solution of amine $300(400 \mathrm{mg}, 1.00 \mathrm{mmol})$ in $\mathrm{CH}_{2} \mathrm{Cl}_{2}(10 \mathrm{~mL})$ and triisobutylamine $(1.10 \mathrm{~mL}, 4.50 \mathrm{mmol})$ at $0{ }^{\circ} \mathrm{C}$. The reaction mixture was stirred for $1 \mathrm{~h}$ at room temperature, heated to reflux for $14 \mathrm{~h}$, and then quenched with saturated aqueous $\mathrm{NH}_{4} \mathrm{Cl}$ solution $(10 \mathrm{~mL})$. The aqueous layer was separated and extracted with $\mathrm{CH}_{2} \mathrm{Cl}_{2}(3 \times 15 \mathrm{~mL})$ and the combined organic layers were dried $\left(\mathrm{MgSO}_{4}\right)$, filtered and concentrated in vacuo. Purification of the residue by column chromatography (20\% EtOAc/petrol) gave amide $354(331 \mathrm{mg}, 60 \%)$ as a pale yellow oil. $\mathrm{R}_{\mathrm{f}}(30 \%$ EtOAc in hexane $)=0.25$; $\operatorname{IR}($ film $) 2952,1741,1718,1654$, $1513,1249,1174,1033,829 \mathrm{~cm}^{-1} ;{ }^{1} \mathrm{H}$ NMR $\left(360 \mathrm{MHz}, \mathrm{CDCl}_{3}\right) \delta 7.46-7.42(2 \mathrm{H}, \mathrm{m}$, ArH), 7.33-7.26 (8H, m, ArH), 7.17-7.14 (2H, m, ArH), 6.98-6.90 (2H + 1H, m, $\mathrm{ArH}+\mathrm{CH}=\mathrm{CHC}=\mathrm{O}), 6.34(1 \mathrm{H}, \mathrm{d}, J=15.1 \mathrm{~Hz}, \mathrm{CH}=\mathrm{CHC}=\mathrm{O}), 4.58(1 \mathrm{H}, \mathrm{d}, J=18.2$ $\left.\mathrm{Hz}, \mathrm{NHCH}_{\mathrm{A}} \mathrm{H}_{\mathrm{B}} \mathrm{Ar}\right), 4.52\left(1 \mathrm{H}, \mathrm{d}, J=18.2 \mathrm{~Hz}, \mathrm{NHCH}_{\mathrm{A}} \mathbf{H}_{\mathbf{B}} \mathrm{Ar}\right), 4.42\left(2 \mathrm{H}, \mathrm{s}, \operatorname{ArCH} \mathbf{H}_{2} \mathrm{O}\right)$, 4.12-4.09 (2H, m, OCH$\left.{ }_{2} \mathrm{CH}=\mathrm{CHC}=\mathrm{O}\right), 3.82\left(3 \mathrm{H}, \mathrm{s}, \operatorname{ArOCH}_{3}\right), 3.38(3 \mathrm{H}, \mathrm{s}$, $\left.\mathrm{CO}_{2} \mathrm{CH}_{3}\right), 2.34\left(3 \mathrm{H}, \mathrm{s}, \mathrm{CH}_{3} \mathrm{C}=\mathrm{O}\right), 1.75\left(1 \mathrm{H}, \mathrm{d}, J=13.7 \mathrm{~Hz}, \mathrm{SiCH}_{\mathrm{C}} \mathrm{H}_{\mathrm{D}}\right), 1.45(1 \mathrm{H}, \mathrm{d}$, $\left.J=13.7 \mathrm{~Hz}, \mathrm{SiCH}_{\mathrm{C}} \mathbf{H}_{\mathbf{D}}\right), 0.27\left(6 \mathrm{H}, \mathrm{s},\left(\mathrm{CH}_{3}\right)_{2} \mathrm{Si}\right) ;{ }^{13} \mathrm{C} \mathrm{NMR}\left(62.9 \mathrm{MHz}, \mathrm{CDCl}_{3}\right) \delta$ 201.8 (C), 169.9 (C), 168.1 (C), 158.8 (C), 144.0 (CH), 138.9 (C), 137.6 (C), 133.7 $(2 \times \mathrm{CH}), 129.8(\mathrm{C}), 128.8(\mathrm{CH}), 128.3(2 \times \mathrm{CH}), 127.6(\mathrm{CH}), 127.5(2 \times \mathrm{CH}), 127.4$ $(2 \times \mathrm{CH}), 127.3(2 \times \mathrm{CH}), 120.7(\mathrm{CH}), 114.0(2 \times \mathrm{CH}), 73.9(\mathrm{C}), 72.5\left(\mathrm{CH}_{2}\right), 68.8$ $\left(\mathrm{CH}_{2}\right), 55.2\left(\mathrm{CH}_{3}\right), 52.0\left(\mathrm{CH}_{3}\right), 47.9\left(\mathrm{CH}_{2}\right), 28.0\left(\mathrm{CH}_{3}\right), 21.3\left(\mathrm{CH}_{2}\right),-1.2\left(\mathrm{CH}_{3}\right),-1.9$ $\left(\mathrm{CH}_{3}\right)$; HRMS (ES) Exact mass calcd for $\mathrm{C}_{33} \mathrm{H}_{40} \mathrm{NO}_{6} \mathrm{SiNa}[\mathrm{M}+\mathrm{H}]^{+}: 574.2619$, found: 574.2619 . 
(E)-4-(Benzyloxy)- $N$-(4-methoxyphenyl)- $N$-(2-oxopropyl)but-2-enamide (360)

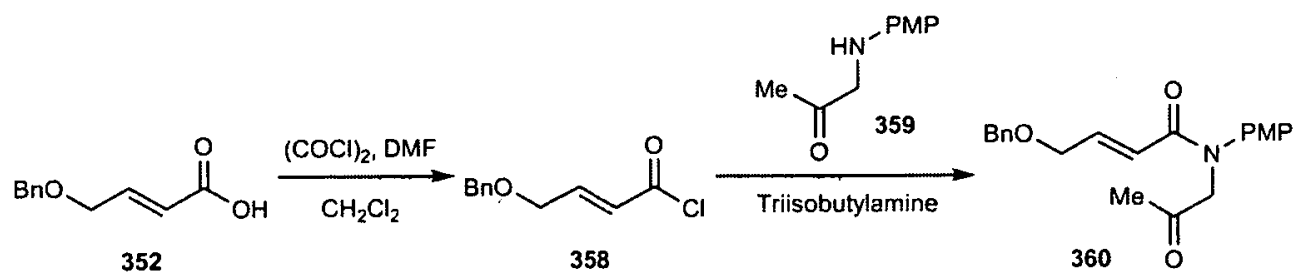

To a stirred solution of acid $352(723 \mathrm{mg}, 3.76 \mathrm{mmol})$ in $\mathrm{CH}_{2} \mathrm{Cl}_{2}(10 \mathrm{~mL})$, was added oxalyl chloride $(1.70 \mathrm{~mL}, 18.8 \mathrm{mmol})$ at room temperature. The reaction was stirred for $14 \mathrm{~h}$, concentrated in vacuo, redissolved in $\mathrm{CH}_{2} \mathrm{Cl}_{2}(8 \mathrm{~mL})$ and then transferred via cannula into a stirred solution of amine $359(270 \mathrm{mg}, 1.50 \mathrm{mmol})$ in $\mathrm{CH}_{2} \mathrm{Cl}_{2}(10 \mathrm{~mL})$ and triisobutylamine $(1.10 \mathrm{~mL}, 4.51 \mathrm{mmol})$ at $0{ }^{\circ} \mathrm{C}$. The reaction mixture was stirred for $12 \mathrm{~h}$ at room temperature, and then quenched with saturated aqueous $\mathrm{NH}_{4} \mathrm{Cl}$ solution $(10 \mathrm{~mL})$. The aqueous layer was separated and extracted with $\mathrm{CH}_{2} \mathrm{Cl}_{2}(3 \times 15 \mathrm{~mL})$ and the combined organic layers were dried $\left(\mathrm{MgSO}_{4}\right)$, filtered and concentrated in vacuo. Purification of the residue by column chromatography (20\% EtOAc/petrol) gave amide 360 (497 mg, 94\%) as a pale yellow solid. $\mathrm{R}_{\mathrm{f}}(30 \%$ EtOAc in hexane $)=0.19$; m.p. $=94-96{ }^{\circ} \mathrm{C}$; IR (film) 2989 , $2867,1731(\mathrm{C}=\mathrm{O}), 1666(\mathrm{C}=\mathrm{O}), 1511,1380,1249,1170,1029 \mathrm{~cm}^{-1} ;{ }^{1} \mathrm{H}$ NMR $(360$ $\left.\mathrm{MHz}, \mathrm{CDCl}_{3}\right) \delta$ 7.24-7.06 (7H, m, ArH), $6.86(1 \mathrm{H}, \mathrm{dt}, J=15.3,4.4 \mathrm{~Hz}$, $\mathrm{CH}=\mathrm{CHC}=\mathrm{O}), 6.82(2 \mathrm{H}, \mathrm{d}, J=8.8 \mathrm{~Hz}, \mathrm{ArH}), 6.04(1 \mathrm{H}, \mathrm{dt}, J=15.3,1.9 \mathrm{~Hz}$, $\mathrm{CH}=\mathrm{CHC}=\mathrm{O}), 4.40\left(2 \mathrm{H}, \mathrm{s}, \mathrm{CH}_{2}\right), 4.35\left(2 \mathrm{H}, \mathrm{s}, \mathrm{CH}_{2}\right), 4.02(2 \mathrm{H}, \mathrm{dd}, J=4.4,1.9 \mathrm{~Hz}$, $\left.\mathrm{OCH}_{2} \mathrm{CH}=\mathrm{CH}\right), 3.74\left(3 \mathrm{H}, \mathrm{s}, \mathrm{OCH}_{3}\right), 2.11\left(3 \mathrm{H}, \mathrm{s}, \mathrm{CH}_{3} \mathrm{C}=\mathrm{O}\right) ;{ }^{13} \mathrm{C} \mathrm{NMR}(62.9 \mathrm{MHz}$, $\left.\mathrm{CDCl}_{3}\right) \delta 202.4(\mathrm{C}), 165.8(\mathrm{C}), 159.0(\mathrm{C}), 141.9(\mathrm{CH}), 137.7(\mathrm{C}), 135.1(\mathrm{C}), 129.2(2$ $\mathrm{x} \mathrm{CH}), 128.3(2 \times \mathrm{CH}), 127.6(\mathrm{CH}), 127.4(2 \times \mathrm{CH}), 120.6(\mathrm{CH}), 114.6(2 \times \mathrm{CH})$, $72.3\left(\mathrm{CH}_{2}\right), 68.8\left(\mathrm{CH}_{2}\right), 59.7\left(\mathrm{CH}_{2}\right), 55.4\left(\mathrm{CH}_{3}\right), 27.2\left(\mathrm{CH}_{3}\right)$; HRMS (ES) Exact mass calcd for $\mathrm{C}_{21} \mathrm{H}_{24} \mathrm{NO}_{4}[\mathrm{M}+\mathrm{H}]^{+}: 354.1700$, found: 354.1703 .

3-(2-Benzyloxyethyl)-4-hydroxy-1-(4-methoxyphenyl)-4-methylpyrrolidin-2-one (361a and 361b)

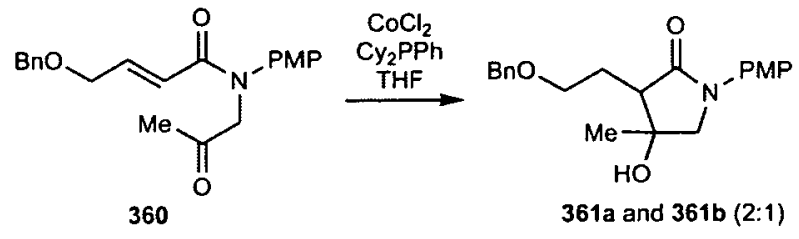


A solution of $\alpha, \beta$-unsaturated amide $360(53 \mathrm{mg}, 0.15 \mathrm{mmol}), \mathrm{CoCl}_{2}(2.0 \mathrm{mg}, 15$ $\mu \mathrm{mol})$ and $\mathrm{Cy}_{2} \mathrm{PPh}(4.5 \mathrm{mg}, 16 \mu \mathrm{mol})$ in $\mathrm{THF}(1 \mathrm{~mL})$ was stirred at room temperature for $30 \mathrm{~min}$ and then cooled to $0{ }^{\circ} \mathrm{C}$. $\mathrm{Et}_{2} \mathrm{Zn}(0.3 \mathrm{~mL}, 1 \mathrm{M}$ solution in THF, $0.3 \mathrm{mmol}$ ) was then added. The reaction was allowed to warm slowly to room temperature over $18 \mathrm{~h}$, and then quenched carefully with saturated aqueous $\mathrm{NH}_{4} \mathrm{Cl}$ solution $(2 \mathrm{~mL})$ and extracted with EtOAc $(3 \times 30 \mathrm{~mL})$. The combined organic layers were dried $\left(\mathrm{MgSO}_{4}\right)$, filtered and concentrated in vacuo. Purification of the residue by column chromatography ( $10 \%$ to $20 \% \mathrm{EtOAc/petrol)}$ gave the two diastereomers of the cyclised products $361 \mathrm{a}(14.8 \mathrm{mg}, 29 \%)$. and $361 \mathrm{~b}(29.8 \mathrm{mg}, 55 \%)$ as pale yellow oils.

Data for 361a: $R_{f}(30 \%$ EtOAc in hexane) $=0.50$; IR (film) $3434(\mathrm{OH}), 2929,2360$, $1694(\mathrm{C}=\mathrm{O}), 1511,1397,1246,1102,1030,829 \mathrm{~cm}^{-1} ;{ }^{1} \mathrm{H} \mathrm{NMR}\left(360 \mathrm{MHz}, \mathrm{CDCl}_{3}\right) \delta$ $7.46(2 \mathrm{H}, \mathrm{d}, J=9.1 \mathrm{~Hz}, \operatorname{ArH}), 7.39-7.32(5 \mathrm{H}, \mathrm{m}, \operatorname{ArH}), 6.90(2 \mathrm{H}, \mathrm{d}, J=9.1 \mathrm{~Hz}$, $\operatorname{ArH}), 4.62\left(1 \mathrm{H}, \mathrm{d}, J=11.5 \mathrm{~Hz}, \mathrm{CH}_{\mathrm{A}} \mathrm{H}_{\mathrm{B}} \mathrm{Ph}\right), 4.57\left(1 \mathrm{H}, \mathrm{d}, J=11.5 \mathrm{~Hz}, \mathrm{CH}_{\mathrm{A}} \mathrm{H}_{\mathrm{B}} \mathrm{Ph}\right)$, 3.89-3.72 (1H, m, OCH $\left.\mathrm{CH}_{\mathrm{D}} \mathrm{CH}_{2}\right), 3.80\left(3 \mathrm{H}, \mathrm{s}, \mathrm{OCH}_{3}\right), 3.79(1 \mathrm{H}, \mathrm{d}, J=9.0 \mathrm{~Hz}$, $\left.\mathrm{NCH}_{\mathrm{E}} \mathrm{H}_{\mathrm{F}}\right), 3.63-3.52\left(1 \mathrm{H}, \mathrm{m}, \mathrm{OCH}_{\mathrm{C}} \mathbf{H}_{\mathrm{D}} \mathrm{CH}_{2}\right), 3.79\left(1 \mathrm{H}, \mathrm{d}, J=9.0 \mathrm{~Hz}, \mathrm{NCH}_{\mathrm{E}} \mathbf{H}_{\mathrm{F}}\right)$, $2.68\left(1 \mathrm{H}, \mathrm{dd}, J=10.8,2.4 \mathrm{~Hz}, \mathrm{CH}_{2} \mathrm{CHC}=\mathrm{O}\right), 2.25(1 \mathrm{H}, \mathrm{dm}, J=15.3 \mathrm{~Hz}$, $\left.\mathrm{CH}_{2} \mathrm{CH}_{\mathrm{G}} \mathrm{H}_{\mathrm{H}} \mathrm{CH}\right), 2.05-1.8\left(1 \mathrm{H}, \mathrm{m}, \mathrm{CH}_{2} \mathrm{CH}_{\mathrm{G}} \mathrm{H}_{\mathrm{H}} \mathrm{CH}\right), 1.34\left(3 \mathrm{H}, \mathrm{s}, \mathrm{CH}_{3} \mathrm{COH}\right) ; 2 \mathrm{D}$ COSY experiment showed cross peak between: $\mathrm{CH}_{\mathbf{E}} \mathbf{H}_{\mathbf{F}}$ and $\mathrm{CH}_{\mathbf{G}} \mathbf{H}_{\mathbf{H}} ; \mathrm{CH}_{\mathbf{G}} \mathrm{H}_{\mathrm{H}}$ and CHCO; ${ }^{13} \mathrm{C}$ NMR (62.9 MHz, $\mathrm{CDCl}_{3}$ ) $\delta 172.1$ (C), 156.5 (C), 136.9 (C), 132.4 (C), $128.6(2 \times \mathrm{CH}), 128.1(\mathrm{CH}), 127.9(2 \times \mathrm{CH}), 121.5(2 \times \mathrm{CH}), 114.0(2 \times \mathrm{CH}), 73.7$ $\left(\mathrm{CH}_{2}\right), 73.1(\mathrm{C}), 70.7\left(\mathrm{CH}_{2}\right), 59.6\left(\mathrm{CH}_{2}\right), 55.6(\mathrm{CH}), 55.4\left(\mathrm{CH}_{3}\right), 24.5\left(\mathrm{CH}_{2}\right), 23.7$ $\left(\mathrm{CH}_{3}\right)$; HRMS (EI) Exact mass calcd for $\mathrm{C}_{21} \mathrm{H}_{25} \mathrm{NO}_{4}[\mathrm{M}]^{+}: 355.1778$, found: 355.1780 .

Data for 361b: $R_{f}(30 \%$ EtOAc in hexane $)=0.38$; IR (film) $3425(\mathrm{OH}), 2931,2868$, $1682(\mathrm{C}=\mathrm{O}), 1511,1397,1247,1103,829,699 \mathrm{~cm}^{-1} ;{ }^{1} \mathrm{H}$ NMR $\left(360 \mathrm{MHz}, \mathrm{CDCl}_{3}\right) \delta$ $7.48(2 \mathrm{H}, \mathrm{d}, J=9.2 \mathrm{~Hz}, \operatorname{ArH}), 7.36-7.29(5 \mathrm{H}, \mathrm{m}, \mathrm{ArH}), 6.89(2 \mathrm{H}, \mathrm{d}, J=9.2 \mathrm{~Hz}$, $\operatorname{ArH}), 4.58\left(1 \mathrm{H}, \mathrm{d}, J=11.6 \mathrm{~Hz}, \mathrm{CH}_{\mathrm{A}} \mathrm{H}_{\mathrm{B}} \mathrm{Ph}\right), 4.57\left(1 \mathrm{H}, \mathrm{d}, J=11.6 \mathrm{~Hz}, \mathrm{CH}_{\mathrm{A}} \mathbf{H}_{\mathrm{B}} \mathrm{Ph}\right)$, 3.87-3.79 (1H, m, $\left.\mathrm{OCH}_{\mathrm{C}} \mathrm{H}_{\mathrm{D}} \mathrm{CH}_{2}\right), 3.80\left(3 \mathrm{H}, \mathrm{s}, \mathrm{OCH}_{3}\right), 3.71(1 \mathrm{H}, \mathrm{d}, J=10.2 \mathrm{~Hz}$, $\left.\mathrm{NCH}_{\mathrm{E}} \mathrm{H}_{\mathrm{F}}\right), 3.66\left(1 \mathrm{H}, \mathrm{d}, J=10.2 \mathrm{~Hz}, \mathrm{NCH}_{\mathrm{E}} \mathrm{H}_{\mathrm{F}}\right), 3.69-3.60\left(1 \mathrm{H}, \mathrm{m}, \mathrm{OCH}_{\mathrm{C}} \mathrm{H}_{\mathrm{D}} \mathrm{CH}_{2}\right)$, $2.61\left(1 \mathrm{H}, \mathrm{dd}, J=8.7,4.3 \mathrm{~Hz}, \mathrm{CH}_{2} \mathrm{CHC}=\mathrm{O}\right), 2.30-2.09\left(2 \mathrm{H}, \mathrm{m}, \mathrm{CH}_{2} \mathrm{CH}_{2} \mathrm{CH}\right), 2.05$ $\left(3 \mathrm{H}, \mathrm{s}, \mathrm{CH}_{3} \mathrm{COH}\right) ; 2 \mathrm{D}$ COSY experiment showed cross peak between: $\mathbf{C H}_{\mathbf{C}} \mathbf{H}_{\mathbf{D}} \mathrm{CH}_{2}$ 
and $\mathrm{CH}_{2} \mathrm{CH} ; \mathrm{CH}_{2} \mathrm{CH}$ and $\mathrm{CH}_{2} \mathrm{CH} ;{ }^{13} \mathrm{C} \mathrm{NMR}\left(62.9 \mathrm{MHz}, \mathrm{CDCl}_{3}\right) \delta 172.1(\mathrm{C}), 156.5$ (C), 136.9 (C), $132.4(\mathrm{C}), 128.6(2 \times \mathrm{CH}), 128.1(\mathrm{CH}), 127.9$ (2 x CH), 121.5 (2 x $\mathrm{CH}), 114.0(2 \times \mathrm{CH}), 73.7\left(\mathrm{CH}_{2}\right), 73.1(\mathrm{C}), 70.7\left(\mathrm{CH}_{2}\right), 59.6\left(\mathrm{CH}_{2}\right), 55.6(\mathrm{CH}), 55.4$ $\left(\mathrm{CH}_{3}\right), 24.5\left(\mathrm{CH}_{2}\right), 23.7\left(\mathrm{CH}_{3}\right)$; HRMS (EI) Exact mass calcd for $\mathrm{C}_{21} \mathrm{H}_{25} \mathrm{NO}_{4}[\mathrm{M}]^{+}$: 355.178 , found: 355.1778 .

$(2 E, 4 E)-N$-(4-Methoxyphenyl)- $N$-(2-oxopropyl)hexa-2,4-dienamide (366)

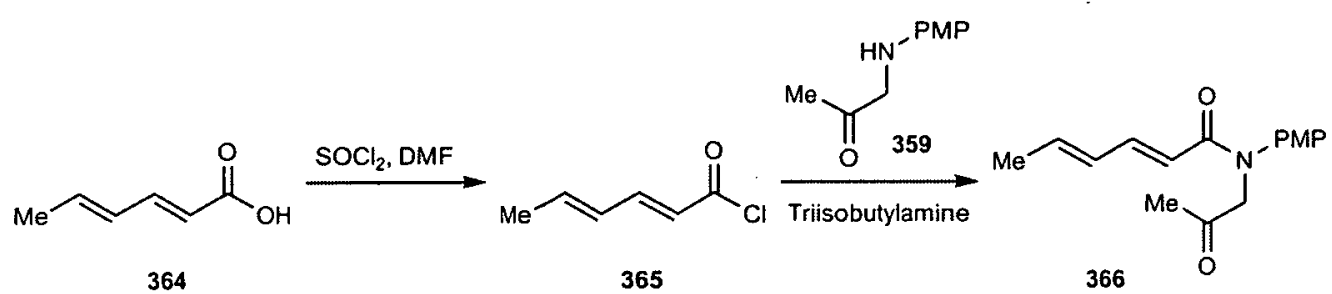

Sorbic acid $(5.00 \mathrm{~g}, 44.6 \mathrm{mmol})$ was heated to reflux with thionyl chloride $(6.50$ $\mathrm{mL}, 89.2 \mathrm{mmol})$ and DMF $(15 \mu \mathrm{L}, 0.13 \mathrm{mmol})$ for $1 \mathrm{~h}$. The reaction mixture was distilled under reduced pressure to give acid chloride 365 in quantitative yield. ${ }^{125}$

To a stirred solution of amine $359(500 \mathrm{mg}, 2.79 \mathrm{mmol})$ and triisobutylamine $(2.00 \mathrm{~mL}, 8.37 \mathrm{mmol})$ in $\mathrm{CH}_{2} \mathrm{Cl}_{2}(15 \mathrm{~mL})$ at $0{ }^{\circ} \mathrm{C}$ was transferred via cannula a solution of sorbyl chloride $(1.1 \mathrm{~g}, 8.37 \mathrm{mmol})$ in $\mathrm{CH}_{2} \mathrm{Cl}_{2}(10 \mathrm{~mL})$ at $0{ }^{\circ} \mathrm{C}$. The reaction mixture was stirred for $1 \mathrm{~h}$ at $0{ }^{\circ} \mathrm{C}$, allowed to warm up to room temperature, stirred for $12 \mathrm{~h}$ and then quenched with saturated aqueous $\mathrm{NH}_{4} \mathrm{Cl}$ solution $(15 \mathrm{~mL})$. The aqueous layer was separated and extracted with $\mathrm{CH}_{2} \mathrm{Cl}_{2}(3 \times 15 \mathrm{~mL})$ and the combined organic layers were dried $\left(\mathrm{MgSO}_{4}\right)$, filtered and concentrated in vacuo. Purification of the residue by column chromatography (20\% to $45 \%$ EtOAc/petrol) gave amide $366(470 \mathrm{mg}, 62 \%)$ as a brown oil. $\mathrm{R}_{\mathrm{f}}(40 \% \mathrm{EtOAc}$ in hexane $)=0.32$; IR (film) 2975, $1729(\mathrm{C}=\mathrm{O}), 1652(\mathrm{C}=\mathrm{O}), 1511,1384,1249,1170,1029,840,561 \mathrm{~cm}^{-1}$; ${ }^{1} \mathrm{H}$ NMR $\left(250 \mathrm{MHz}, \mathrm{CDCl}_{3}\right) \delta 7.21(2 \mathrm{H}, \mathrm{d}, J=8.9 \mathrm{~Hz}, \operatorname{ArH}), 6.89(2 \mathrm{H}, \mathrm{d}, J=8.9$ $\mathrm{Hz}, \mathrm{ArH}), 6.02(2 \mathrm{H}, \mathrm{m}, \mathrm{CH}=\mathrm{CH}), 5.73(2 \mathrm{H}, \mathrm{m}, \mathrm{CH}=\mathrm{CH}), 4.46\left(2 \mathrm{H}, \mathrm{s}, \mathrm{CH}_{2}\right), 3.83$ $\left(3 \mathrm{H}, \mathrm{s}, \mathrm{OCH}_{3}\right), 2.17\left(3 \mathrm{H}, \mathrm{s}, \mathrm{CH}_{3} \mathrm{C}=\mathrm{O}\right), 1.77\left(3 \mathrm{H}, \mathrm{d}, J=5.4 \mathrm{~Hz}, \mathrm{CH}_{3} \mathrm{CH}\right) ;{ }^{13} \mathrm{C} \mathrm{NMR}$ $\left(62.9 \mathrm{MHz} \mathrm{CDCl}_{3}\right) \delta 202.7(\mathrm{C}), 192.4(\mathrm{C}), 166.8(\mathrm{C}), 158.9(\mathrm{C}), 142.9(\mathrm{CH}), 133.0$ $(\mathrm{CH}), 130.1(\mathrm{CH}), 129.2(2 \times \mathrm{CH}), 118.6(\mathrm{CH}), 114.5(2 \times \mathrm{CH}), 59.7\left(\mathrm{CH}_{2}\right) 55.4$ $\left(\mathrm{CH}_{3}\right), 27.2\left(\mathrm{CH}_{3}\right), 18.5\left(\mathrm{CH}_{3}\right)$; HRMS (ES) Exact mass calcd for $\mathrm{C}_{16} \mathrm{H}_{20} \mathrm{NO}_{3}$ $[\mathrm{M}+\mathrm{H}]^{+}:$274.1438, found: 274.1440 . 


\section{(E)-3-(But-2-enyl)-4-hydroxy-1-(4-methoxyphenyl)-4-methylpyrrolidin-2-one} (367)

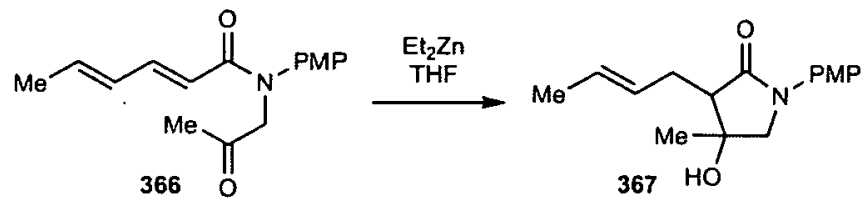

A solution of $\alpha, \beta$-unsaturated amide $366(50 \mathrm{mg}, 0.18 \mathrm{mmol})$ and $\mathrm{Ni}(\mathrm{acac})_{2}(4.8 \mathrm{mg}$, $18 \mu \mathrm{mol})$ in THF ( $1 \mathrm{~mL}$ ) was stirred at room temperature for $30 \mathrm{~min}$ and then cooled to $0{ }^{\circ} \mathrm{C}$. $\mathrm{Et}_{2} \mathrm{Zn}(0.37 \mathrm{~mL}, 1 \mathrm{M}$ solution in $\mathrm{THF}, 0.36 \mathrm{mmol})$ was then added. The reaction was allowed to warm slowly to room temperature over $18 \mathrm{~h}$, and then quenched carefully with saturated aqueous $\mathrm{NH}_{4} \mathrm{Cl}$ solution $(2 \mathrm{~mL})$ and extracted with EtOAc $(3 \times 30 \mathrm{~mL})$. The combined organic layers were dried $\left(\mathrm{MgSO}_{4}\right)$, filtered and concentrated in vacuo. Purification of the residue by column chromatography (5\% to $10 \% \mathrm{EtOAc/petrol)}$ gave cyclised product $367(13.5 \mathrm{mg}, 25 \%)$ as a yellow oil. $\mathrm{R}_{\mathrm{f}}$ $(30 \%$ EtOAc in hexane $)=0.52$; IR (film) $3404(\mathrm{OH}), 2934,1674(\mathrm{C}=\mathrm{O}), 1511$, $1400,1248,1180,1033,829 \mathrm{~cm}^{-1} ;{ }^{1} \mathrm{H}$ NMR $\left(250 \mathrm{MHz}, \mathrm{CDCl}_{3}\right) \delta 7.45(2 \mathrm{H}, \mathrm{d}, J=$ $9.2 \mathrm{~Hz}, \operatorname{ArH}), 6.89(2 \mathrm{H}, \mathrm{d}, J=9.2 \mathrm{~Hz}, \mathrm{ArH}), 5.79-5.37(2 \mathrm{H}, \mathrm{m}, \mathbf{C H}=\mathbf{C H}), 3.79(3 \mathrm{H}$, s, $\left.\mathrm{OCH}_{3}\right), 3.72\left(1 \mathrm{H}, \mathrm{d}, J=9.6 \mathrm{~Hz}, \mathrm{NCH}_{\mathrm{A}} \mathrm{H}_{\mathrm{B}}\right), 3.55\left(1 \mathrm{H}, \mathrm{d}, J=9.6 \mathrm{~Hz}, \mathrm{NCH}_{\mathrm{A}} \mathbf{H}_{\mathrm{B}}\right)$, $2.67(1 \mathrm{H}, \mathrm{dd}, J=9.0,5.0 \mathrm{~Hz}, \mathrm{CHC}=\mathrm{O}), 2.62-2.48\left(1 \mathrm{H}, \mathrm{m}, \mathrm{CH}_{\mathrm{C}} \mathrm{H}_{\mathrm{D}} \mathrm{CHC}=\mathrm{O}\right), 2.40-$ $2.22\left(1 \mathrm{H}, \mathrm{m}, \mathrm{CH}_{\mathrm{C}} \mathbf{H}_{\mathrm{D}} \mathrm{CHC}=\mathrm{O}\right), 1.67\left(3 \mathrm{H}, \mathrm{d}, J=5.3 \mathrm{~Hz}, \mathrm{CH}_{3} \mathrm{CH}\right), 1.42(3 \mathrm{H}, \mathrm{s}$, $\mathrm{CH}_{3} \mathrm{COH}$ ); 2D COSY experiment showed cross peak of $\mathrm{CH}=\mathrm{CH}$ protons with $\mathrm{CH}_{\mathrm{A}} \mathrm{CH}_{\mathrm{B}}, \mathrm{CH}_{2} \mathrm{CH}$ and $\mathrm{CH}_{3} ; 2 \mathrm{D}$ NOESY experiment showed cross peak of $\mathrm{CH}_{3}$ at $\mathrm{C} 3$ with $\mathrm{CHC}=\mathrm{O}$ proton and with $\mathrm{CH}_{\mathrm{C}} \mathbf{H}_{\mathrm{D}} \mathrm{CHC}=\mathrm{O}$ protons; ${ }^{13} \mathrm{C}$ NMR $(62.9 \mathrm{MHz}$, $\left.\mathrm{CDCl}_{3}\right) \delta 172.9(\mathrm{C}), 156.6(\mathrm{C}), 132.2(\mathrm{C}), 127.9(\mathrm{CH}), 127.8(\mathrm{CH}), 121.7(2 \times \mathrm{CH})$, $114.0(2 \times \mathrm{CH}), 74.3(\mathrm{C}), 60.5\left(\mathrm{CH}_{2}\right), 55.4(\mathrm{CH}), 54.7\left(\mathrm{CH}_{3}\right), 29.4\left(\mathrm{CH}_{2}\right), 22.8\left(\mathrm{CH}_{3}\right)$, $17.9\left(\mathrm{CH}_{3}\right)$; HRMS (EI) Exact mass calcd for $\mathrm{C}_{16} \mathrm{H}_{21} \mathrm{NO}_{3}[\mathrm{M}]^{+}:$275.1516, found: 275.1519 . 


\subsubsection{Reformatsky reaction approach}

\section{$(L)$-Threonine methyl ester $(415)^{189}$}

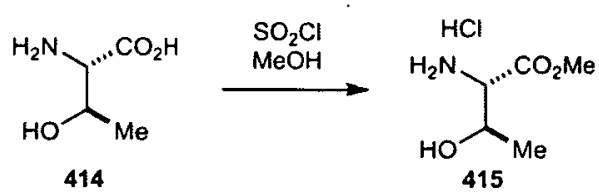

To a stirred solution of $\mathrm{SOCl}_{2}(11.0 \mathrm{~mL}, 150 \mathrm{mmol})$ in $\mathrm{MeOH}(115 \mathrm{~mL})$ at $0{ }^{\circ} \mathrm{C}$ was added $(L)$-threonine $(18.2 \mathrm{~g}, 150 \mathrm{mmol})$ in portions. After stirring for $30 \mathrm{~min}$ at room temperature, the reaction was heated to reflux for $14 \mathrm{~h}$ and then concentrated in vacuo, to obtain a thick colourless oil. Methyl ester 415 was used in the next step without further purification. ${ }^{1} \mathrm{H} \mathrm{NMR}\left(360 \mathrm{MHz}, \mathrm{CD}_{3} \mathrm{OD}\right) \delta 5.11\left(3 \mathrm{H}, \mathrm{bs}, \mathrm{NH}_{2}+\right.$ $\mathrm{OH}), 4.52\left(1 \mathrm{H}, \mathrm{dq}, J=6.5,4.2 \mathrm{~Hz}, \mathrm{CHCH}_{3}\right), 4.19(1 \mathrm{H}, \mathrm{d}, J=4.2 \mathrm{~Hz}, \mathrm{CHCHOH})$, $4.08\left(3 \mathrm{H}, \mathrm{s}, \mathrm{OCH}_{3}\right), 1.56\left(3 \mathrm{H}, \mathrm{d}, J=6.5 \mathrm{~Hz}, \mathrm{CH}_{3} \mathrm{CH}\right) ;{ }^{13} \mathrm{C} \mathrm{NMR}(62.9 \mathrm{MHz}$, $\left.\mathrm{CD}_{3} \mathrm{OD}\right) \delta 170.1(\mathrm{C}), 66.8(\mathrm{CH}), 60.3(\mathrm{CH}), 54.2\left(\mathrm{CH}_{3}\right), 20.9\left(\mathrm{CH}_{3}\right)$.

${ }^{1} \mathrm{H}$ and ${ }^{13} \mathrm{C}$ spectroscopic data in good agreement with the literature. ${ }^{189}$

\section{4-Methoxybenzimidate hydrochloride $(\mathbf{4 1 7})^{190}$}
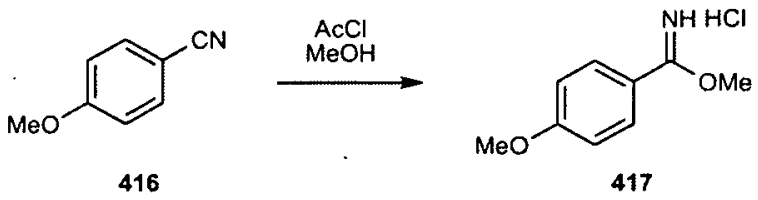

To a stirred solution of $p$-methoxybenzonitrile $416(22.4 \mathrm{~g}, 168.6 \mathrm{mmol})$ in $\mathrm{MeOH}(85 \mathrm{~mL}, 2.0 \mathrm{~mol})$ at $0{ }^{\circ} \mathrm{C}$ was added acetyl chloride $(96.0 \mathrm{~mL}, 1.34 \mathrm{~mol})$ slowly via syringe. The solution was stirred for $14 \mathrm{~h}$ at room temperature and then concentrated in vacuo which afforded 417 as a colourless solid. This solid was washed with $\mathrm{Et}_{2} \mathrm{O}(5 \times 30 \mathrm{~mL})$, dried and used without further purification in the next step (30.5 g, 91\%). m.p. $165-167{ }^{\circ} \mathrm{C} ;{ }^{1} \mathrm{H}$ NMR $\left(360 \mathrm{MHz}, \mathrm{CDCl}_{3}\right) \delta 11.60(1 \mathrm{H}$, bs, NH), 8.39 (2H, d, $J=9.0 \mathrm{~Hz}, \operatorname{ArH}), 7.02(2 \mathrm{H}, \mathrm{d}, J=9.0 \mathrm{~Hz}, \operatorname{ArH}), 4.51(3 \mathrm{H}, \mathrm{s}$, $\left.\mathrm{OCH}_{3}\right), 3.88\left(3 \mathrm{H}, \mathrm{s}, \mathrm{OCH}_{3}\right) ;{ }^{13} \mathrm{C} \mathrm{NMR}\left(62.9 \mathrm{MHz}, \mathrm{CDCl}_{3}\right) \delta 171.1(\mathrm{C}), 165.5(\mathrm{C})$, $132.1(2 \times \mathrm{CH}), 116.7(\mathrm{C}), 114.6(2 \times \mathrm{CH}), 60.8\left(\mathrm{CH}_{3}\right), 55.6\left(\mathrm{CH}_{3}\right)$.

${ }^{1} \mathrm{H}$ spectroscopic data in good agreement with the literature. ${ }^{190}$ 
(4S,5R)-Methyl 4,5-dihydro-2 (4-methoxyphenyl)-5-methyloxazole-4carboxylate $(418)^{26}$

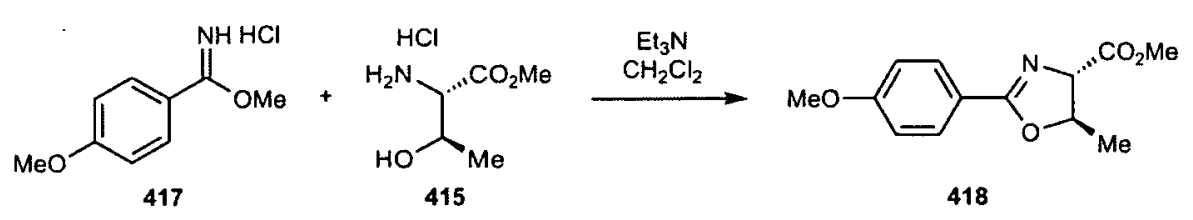

To a stirred solution of threonine methyl ester 415 (25.5 g, $150 \mathrm{mmol})$ in $\mathrm{CH}_{2} \mathrm{Cl}_{2}$ $(175 \mathrm{~mL})$ at room temperature was added $\mathrm{Et}_{3} \mathrm{~N}(41.0 \mathrm{~mL}, 300 \mathrm{mmol})$ slowly via syringe, followed by the addition of $\mathbf{4 1 7}$ in one portion. The reaction was stirred for $1 \mathrm{~h}$ at room temperature, heated to reflux for $14 \mathrm{~h}$, then it was quenched with saturated aqueous $\mathrm{NH}_{4} \mathrm{Cl}$ solution $(150 \mathrm{~mL})$ and extracted with $\mathrm{CH}_{2} \mathrm{Cl}_{2}(3 \times 70 \mathrm{~mL})$. The combined organic layers were dried $\left(\mathrm{MgSO}_{4}\right)$, filtered and concentrated in vacuo. Purification of the residue by column chromatography (15\% to $40 \%$ EtOAc/petrol) afforded oxazoline $418(12.8 \mathrm{~g}, 40 \%)$ as a pale yellow solid. $\mathrm{R}_{\mathrm{f}}(30 \%$ EtOAc in hexane $)=0.29$; m.p. $87-88^{\circ} \mathrm{C}$, lit. $^{26} 86-87^{\circ} \mathrm{C} ;[\alpha]_{\mathrm{D}}^{22}+76.5\left(c 1.98, \mathrm{CHCl}_{3}\right)$, lit. $^{26}+69.4\left(c\right.$ 2.0, $\left.\mathrm{CHCl}_{3}\right) ;{ }^{1} \mathrm{H}$ NMR $\left(360 \mathrm{MHz}, \mathrm{CDCl}_{3}\right) \delta 7.86(2 \mathrm{H}, \mathrm{d}, J=8.9 \mathrm{~Hz}$, ArH), $6.83(2 \mathrm{H}, \mathrm{d}, J=8.9 \mathrm{~Hz}, \mathrm{ArH}), 4.89\left(1 \mathrm{H}, \mathrm{dq}, J=7.4,6.3 \mathrm{~Hz}, \mathrm{OCHCH}_{3}\right), 4.38$ $(1 \mathrm{H}, \mathrm{d}, J=7.4 \mathrm{~Hz}, \mathrm{NCH}), 3.75\left(3 \mathrm{H}, \mathrm{s}, \mathrm{OCH}_{3}\right), 3.73\left(3 \mathrm{H}, \mathrm{s}, \mathrm{OCH}_{3}\right), 1.45(3 \mathrm{H}, \mathrm{d}, J=$ $\left.6.2 \mathrm{~Hz}, \mathrm{CHCH}_{3}\right) ;{ }^{13} \mathrm{C}$ NMR (62.9 MHz, $\left.\mathrm{CDCl}_{3}\right) \delta 171.4(\mathrm{C}), 165.0(\mathrm{C}), 162.1$ (C), $130.0(2 \times \mathrm{CH}), 119.4(\mathrm{C}), 113.4(2 \times \mathrm{CH}), 78.4(\mathrm{CH}), 74.8(\mathrm{CH}), 55.0\left(\mathrm{CH}_{3}\right), 52.2$ $\left(\mathrm{CH}_{3}\right), 20.7\left(\mathrm{CH}_{3}\right)$.

${ }^{1} \mathrm{H}$ and ${ }^{13} \mathrm{C}$ spectroscopic data in good agreement with the literature. ${ }^{26}$

(4R,5R)-Methyl 4-[(benzyloxy)methyl]-4,5-dihydro-2-(4-methoxyphenyl)-5methyloxazole-4-carboxylate $(17)^{26}$

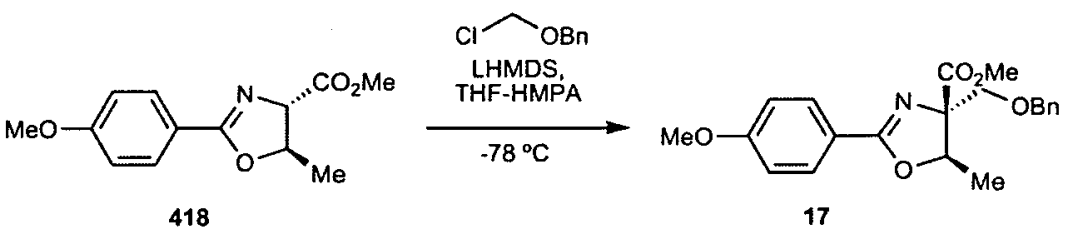

To a stirred solution of LHMDS (56.6 mL, $1 \mathrm{M}$ solution in THF, $56.5 \mathrm{mmol}$ ), in THF $(200 \mathrm{~mL})$ at $-78^{\circ} \mathrm{C}$ was added HMPA $(30.0 \mathrm{~mL}, 221 \mathrm{mmol})$, followed by the transfer of a solution of oxazoline $418(12.8 \mathrm{~g}, 51.3 \mathrm{mmol})$ in THF (100 mL) via 
cannula. This solution was stirred at $-78^{\circ} \mathrm{C}$ for $1 \mathrm{~h}$ to allow enolate formation. Benzyloxy chloromethyl ether $(14.3 \mathrm{~mL}, 61.7 \mathrm{mmol})$ was then added and the reaction was stirred at $-78^{\circ} \mathrm{C}$ for $3 \mathrm{~h}$, when it was quenched with saturated aqueous $\mathrm{NH}_{4} \mathrm{Cl}$ solution $(150 \mathrm{~mL})$ and warmed up to room temperature for 30 minutes. The aqueous layer was extracted with EtOAc $(4 \times 80 \mathrm{~mL})$ and the combined organic layers were dried $\left(\mathrm{MgSO}_{4}\right)$, filtered and concentrated in vacuo. Purification of the residue by column chromatography (15\% to $20 \%$ EtOAc/petrol) afforded alkylated oxazoline $17(14.8 \mathrm{~g}, 75 \%)$ as a pale yellow oil. $\mathrm{R}_{\mathrm{f}}(30 \%$ EtOAc in hexane $)=0.53$; $[\alpha]_{\mathrm{D}}^{22}-6.0\left(c\right.$ 1.0, $\left.\mathrm{CHCl}_{3}\right)$, lit. $^{26}-6.3\left(c\right.$ 1.0, $\left.\mathrm{CHCl}_{3}\right) ;{ }^{1} \mathrm{H} \mathrm{NMR}\left(360 \mathrm{MHz}, \mathrm{CDCl}_{3}\right) \delta$ $7.97(2 \mathrm{H}, \mathrm{d}, J=9.0 \mathrm{~Hz}, \operatorname{ArH}), 7.29-7.24(5 \mathrm{H}, \mathrm{m}, \operatorname{ArH}), 6.90(2 \mathrm{H}, \mathrm{d}, J=9.0 \mathrm{~Hz}$, $\operatorname{ArH}), 4.78\left(1 \mathrm{H}, \mathrm{q}, J=6.6 \mathrm{~Hz}, \mathrm{OCHCH}_{3}\right), 4.61\left(2 \mathrm{H}, \mathrm{s}, \mathrm{OCH}_{2} \mathrm{Ar}\right), 3.87(1 \mathrm{H}, \mathrm{d}, J=$ $\left.9.9 \mathrm{~Hz}, \mathrm{CH}_{\mathrm{A}} \mathrm{H}_{\mathrm{B}} \mathrm{O}\right), 3.81\left(1 \mathrm{H}, \mathrm{d}, J=9.9 \mathrm{~Hz}, \mathrm{CH}_{\mathrm{A}} \mathbf{H}_{\mathbf{B}} \mathrm{O}\right), 3.80\left(3 \mathrm{H}, \mathrm{s}, \mathrm{OCH}_{3}\right), 3.73$ $\left(3 \mathrm{H}, \mathrm{s}, \mathrm{OCH}_{3}\right), 1.35\left(3 \mathrm{H}, \mathrm{d}, J=6.6 \mathrm{~Hz}, \mathrm{CHCH}_{3}\right) ;{ }^{13} \mathrm{C} \mathrm{NMR}\left(62.9 \mathrm{MHz}, \mathrm{CDCl}_{3}\right) \delta$ 170.8 (C), 165.0 (C), 162.2 (C), 137.8 (C), 130.2 (2 x CH), $128.0(2 \times \mathrm{CH}), 127.4(2$ x CH), $127.3(\mathrm{CH}), 119.7(\mathrm{C}), 113.4(2 \times \mathrm{CH}), 81.0(\mathrm{C}), 79.5(\mathrm{CH}), 73.5\left(\mathrm{CH}_{2}\right), 73.0$ $\left(\mathrm{CH}_{2}\right), 55.1\left(\mathrm{CH}_{3}\right), 52.0\left(\mathrm{CH}_{3}\right), 16.5\left(\mathrm{CH}_{3}\right)$.

${ }^{1} \mathrm{H}$ and ${ }^{13} \mathrm{C}$ spectroscopic data in good agreement with the literature. ${ }^{26}$

\section{(2R,3R)-Methyl 2-benzyloxymethyl-2-(4-methoxybenzylamino)-3-} hydroxybutanoate $(18)^{26}$
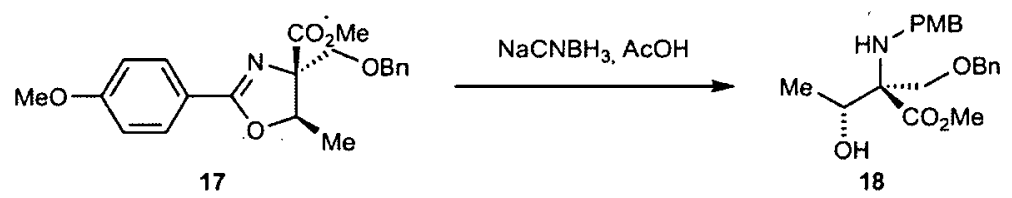

To a stirred solution of oxazoline $17(14.8 \mathrm{~g}, 40.0 \mathrm{mmol})$ in HOAc $(50 \mathrm{~mL})$ at room temperature was added $\mathrm{NaCNBH}_{3}(8.80 \mathrm{~g}, 132 \mathrm{mmol})$ in small portions over $2 \mathrm{~h}$. The reaction mixture was stirred for $16 \mathrm{~h}$ at $50^{\circ} \mathrm{C}$, when it was diluted in water $(100 \mathrm{~mL})$, neutralised with solid $\mathrm{Na}_{2} \mathrm{CO}_{3}$ and the aqueous layer was extracted with ethyl acetate $(5 \times 60 \mathrm{~mL})$. The combined organic layers were dried $\left(\mathrm{MgSO}_{4}\right)$, filtered and concentrated in vacuo. Purification of the residue by column chromatography (10\% to $25 \%$ EtOAc/petrol) afforded aminoalcohol $18(9.3 \mathrm{~g}, 65 \%)$ as a pale yellow oil. $\mathrm{R}_{\mathrm{f}}(30 \%$ EtOAc in hexane $)=0.21 ;[\alpha]_{\mathrm{D}}^{22}-5.8$ (c 1.03, $\left.\mathrm{CHCl}_{3}\right)$, lit. $^{26}-9.1$ (c 1.0, $\left.\mathrm{CHCl}_{3}\right) ;{ }^{1} \mathrm{H}$ NMR $\left(360 \mathrm{MHz}, \mathrm{CDCl}_{3}\right) \delta 7.54-7.44(5 \mathrm{H}, \mathrm{m}, \mathrm{ArH}), 7.43(2 \mathrm{H}, \mathrm{d}, J=8.7$ 
$\mathrm{Hz}, \mathrm{ArH}), 7.02(2 \mathrm{H}, \mathrm{d}, J=8.7 \mathrm{~Hz}, \mathrm{ArH}), 4.71\left(2 \mathrm{H}, \mathrm{s}, \mathrm{OCH}_{2} \mathrm{Ph}\right), 4.15(1 \mathrm{H}, \mathrm{q}, J=6.4$ $\left.\mathrm{Hz}, \mathrm{HOCHCH}_{3}\right), 4.03\left(1 \mathrm{H}, \mathrm{d}, J=9.8 \mathrm{~Hz}, \mathrm{NCH}_{\mathrm{A}} \mathrm{H}_{\mathrm{B}} \mathrm{Ar}\right), 3.98(1 \mathrm{H}, \mathrm{d}, J=9.8 \mathrm{~Hz}$, $\left.\mathrm{NCH}_{\mathrm{A}} \mathbf{H}_{\mathrm{B}} \mathrm{Ar}\right), 3.95\left(3 \mathrm{H}, \mathrm{s}, \mathrm{OCH}_{3}\right), 3.92\left(3 \mathrm{H}, \mathrm{s}, \mathrm{OCH}_{3}\right), 3.87(1 \mathrm{H}, \mathrm{d}, J=11.8 \mathrm{~Hz}$,

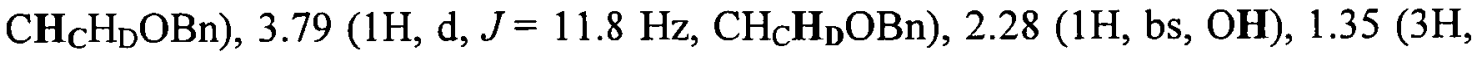
d, $\left.J=6.6 \mathrm{~Hz}, \mathrm{CHCH}_{3}\right) ;{ }^{13} \mathrm{C} \mathrm{NMR}\left(62.9 \mathrm{MHz}, \mathrm{CDCl}_{3}\right) \delta 172.8(\mathrm{C}), 158.8(\mathrm{C}), 137.5$ (C), $131.7(\mathrm{C}), 129.5(2 \times \mathrm{CH}), 128.4(2 \times \mathrm{CH}), 127.8(\mathrm{CH}), 127.7(2 \times \mathrm{CH}), 113.8(2$ x CH), $73.5\left(\mathrm{CH}_{2}\right), 69.8(\mathrm{C}), 69.5\left(\mathrm{CH}_{2}\right), 69.4(\mathrm{CH}), 55.2\left(\mathrm{CH}_{3}\right), 52.1\left(\mathrm{CH}_{3}\right), 47.3$ $\left(\mathrm{CH}_{2}\right), 17.9\left(\mathrm{CH}_{3}\right)$.

${ }^{1} \mathrm{H}$ and ${ }^{13} \mathrm{C}$ spectroscopic data in good agreement with the literature. ${ }^{26}$

(R)-Methyl 2-benzyloxymethyl-2-(4-methoxybenzylamino)-3-oxobutanoate (327)

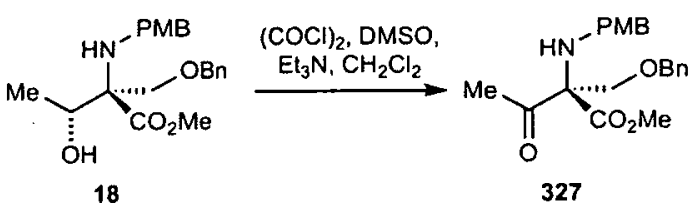

To a stirred solution of oxalyl chloride $(1.70 \mathrm{~mL}, 18.8 \mathrm{mmol})$ in $\mathrm{CH}_{2} \mathrm{Cl}_{2}(40 \mathrm{~mL})$ at $-78{ }^{\circ} \mathrm{C}$, was added DMSO $(2.10 \mathrm{~mL}, 37.6 \mathrm{mmol})$ dropwise over $3 \mathrm{~min}$. After stirring for $15 \mathrm{~min}$, a solution of alcohol 18 (3.34 g, $8.94 \mathrm{mmol})$ in $\mathrm{CH}_{2} \mathrm{Cl}_{2}(30 \mathrm{~mL})$ was added via cannula over $5 \mathrm{~min}$. The reaction stirred at $-78^{\circ} \mathrm{C}$ for $1 \mathrm{~h}$ and $\mathrm{Et}_{3} \mathrm{~N}$ $(5.25 \mathrm{~mL}, 37.6 \mathrm{mmol})$ was then added over $1 \mathrm{~min}$. The reaction was stirred at $-78^{\circ} \mathrm{C}$ for $1 \mathrm{~h}$, allowed to warm up to $-40{ }^{\circ} \mathrm{C}$ over $2 \mathrm{~h}$, and then quenched with saturated aqueous $\mathrm{NH}_{4} \mathrm{Cl}$ solution $(40 \mathrm{~mL})$. The aqueous layer was separated and extracted with $\mathrm{CH}_{2} \mathrm{Cl}_{2}(3 \times 20 \mathrm{~mL})$ and the combined organic layers were dried $\left(\mathrm{MgSO}_{4}\right)$, filtered and concentrated in vacuo. Purification of the residue by column chromatography (10\% to 20\% EtOAc/petrol) gave amino ketone 327 (2.39 g, 72\%) as a pale yellow solid. $\mathrm{R}_{\mathrm{f}}(30 \%$ EtOAc in hexane $)=0.55$; m.p. $=60-62{ }^{\circ} \mathrm{C}$; $[\alpha]_{\mathrm{D}}^{22}-12.7$ (c 1.02, $\mathrm{CHCl}_{3}$ ); IR (film) $3347(\mathrm{NH}), 2952,2836,1741(\mathrm{C}=\mathrm{O}), 1718$ $(\mathrm{C}=\mathrm{O}), 1511,1245,1178,1033,700 \mathrm{~cm}^{-1} ;{ }^{1} \mathrm{H}$ NMR $\left(250 \mathrm{MHz}, \mathrm{CDCl}_{3}\right) \delta$ 7.39-7.28 $(5 \mathrm{H}, \mathrm{m}, \operatorname{ArH}), 7.25(2 \mathrm{H}, \mathrm{d}, J=8.7 \mathrm{~Hz}, \operatorname{ArH}), 6.85(2 \mathrm{H}, \mathrm{d}, J=8.7 \mathrm{~Hz}, \operatorname{ArH}), 4.59$ $\left(1 \mathrm{H}, \mathrm{d}, J=12.4 \mathrm{~Hz}, \mathrm{OCH}_{\mathrm{A}} \mathrm{H}_{\mathrm{B}} \mathrm{Ph}\right), 4.53\left(1 \mathrm{H}, \mathrm{d}, J=12.4 \mathrm{~Hz}, \mathrm{OCH}_{\mathrm{A}} \mathbf{H}_{\mathrm{B}} \mathrm{Ph}\right), 4.02(1 \mathrm{H}$, $\left.\mathrm{d}, J=10.1 \mathrm{~Hz}, \mathrm{NHCH}_{\mathbf{C}} \mathrm{H}_{\mathrm{D}}\right), 3.90\left(1 \mathrm{H}, \mathrm{d}, J=10.1 \mathrm{~Hz}, \mathrm{NHCH}_{\mathrm{C}} \mathbf{H}_{\mathbf{D}}\right), 3.80(3 \mathrm{H}, \mathrm{s}$, $\left.\mathrm{OCH}_{3}\right), 3.74\left(3 \mathrm{H}, \mathrm{s}, \mathrm{OCH}_{3}\right), 3.59\left(1 \mathrm{H}, \mathrm{d}, J=12.3 \mathrm{~Hz}, \mathrm{CH}_{\mathbf{E}} \mathrm{H}_{\mathrm{F}} \mathrm{OBn}\right), 3.46(1 \mathrm{H}, \mathrm{d}, J=$ 
$\left.12.3 \mathrm{~Hz}, \mathrm{CH}_{\mathrm{E}} \mathrm{H}_{\mathrm{F}} \mathrm{OBn}\right), 2.53\left(1 \mathrm{H}\right.$, br s, NH), $2.21\left(3 \mathrm{H}, \mathrm{s}, \mathrm{CH}_{3} \mathrm{C}=\mathrm{O}\right) ;{ }^{13} \mathrm{C} \mathrm{NMR}(62.9$ $\left.\mathrm{MHz}_{,} \mathrm{CDCl}_{3}\right) \delta 203.3$ (C), 169.8 (C), 158.7 (C), 137.6 (C), 131.7 (C), $129.3(2 \mathrm{x}$ $\mathrm{CH}), 128.4(2 \times \mathrm{CH}), 127.8(3 \times \mathrm{CH}), 113.7(2 \times \mathrm{CH}), 74.8(\mathrm{C}), 73.4\left(\mathrm{CH}_{2}\right), 68.1$ $\left(\mathrm{CH}_{2}\right), 55.3\left(\mathrm{CH}_{3}\right), 52.7\left(\mathrm{CH}_{3}\right), 46.7\left(\mathrm{CH}_{2}\right), 25.7\left(\mathrm{CH}_{3}\right)$; HRMS (ES) Exact mass calcd for $\mathrm{C}_{21} \mathrm{H}_{26} \mathrm{NO}_{5}[\mathrm{M}+\mathrm{H}]^{+}: 372.1805$, found: 372.1804 .

(R)-Methyl 2-benzyloxymethyl-2-[2-bromo- $N$-(4-methoxybenzyl)butanamido]-3oxobutanoate $(420 \mathrm{a}$ and $420 \mathrm{~b})$

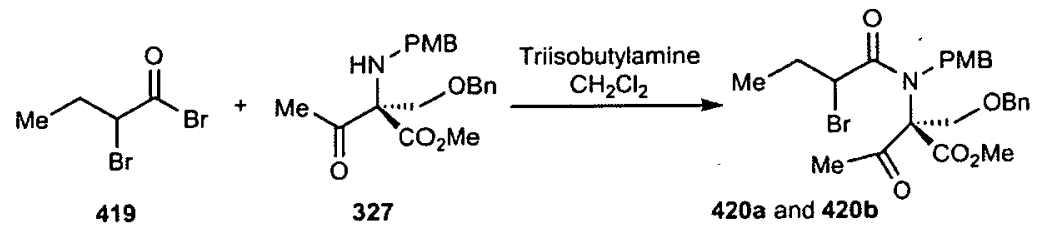

To a stirred solution of amine $327(1.25 \mathrm{~g}, 3.36 \mathrm{mmol})$ in $\mathrm{CH}_{2} \mathrm{Cl}_{2}(15 \mathrm{~mL})$ at room temperature was added triisobutylamine $(1.10 \mathrm{~mL}, 4.37 \mathrm{mmol})$. The reaction was stirred for $10 \mathrm{~min}$ and 2-bromobutyryl bromide $(0.55 \mathrm{~mL}, 4.04 \mathrm{mmol})$ was then added. The reaction was stirred for $16 \mathrm{~h}$ and then quenched with saturated aqueous $\mathrm{NH}_{4} \mathrm{Cl}$ solution $(10 \mathrm{~mL})$. The aqueous layer was separated and extracted with $\mathrm{CH}_{2} \mathrm{Cl}_{2}(2 \times 15 \mathrm{~mL})$ and the combined organic layers were dried $\left(\mathrm{MgSO}_{4}\right)$, filtered and concentrated in vacuo. Purification of the residue by column chromatography ( $10 \%$ to $15 \% \mathrm{EtOAc} /$ petrol) gave the two diastereomers of the amide, the more polar diastereomer 420a (990 mg, 57\%) and the less polar diastereomer 420b (627 mg, $36 \%$ ), as yellow oils.

Data for 420b: $\mathrm{R}_{\mathrm{f}}(30 \%$ EtOAc in hexane $)=0.46 ;[\alpha]_{\mathrm{D}}^{22}-63.3\left(c 1.03, \mathrm{CHCl}_{3}\right)$; IR (film) 2972, 1741 (C=O), 1709 (C=O), $1657(\mathrm{C}=0), 1513,1433,1246,1177,741$, $510(\mathrm{C}-\mathrm{Br}) \mathrm{cm}^{-1}$; ${ }^{1} \mathrm{H}$ NMR $\left(250 \mathrm{MHz}, \mathrm{CDCl}_{3}\right) \delta 7.31(2 \mathrm{H}, \mathrm{d}, J=8.7 \mathrm{~Hz}, \mathrm{ArH}), 7.22-$ $7.17(3 \mathrm{H}, \mathrm{m}, \operatorname{ArH}), 7.07-7.01(2 \mathrm{H}, \mathrm{m}, \operatorname{ArH}), 6.84(2 \mathrm{H}, \mathrm{d}, J=8.7 \mathrm{~Hz}, \operatorname{ArH}), 5.01$ $\left(1 \mathrm{H}, \mathrm{d}, J=18.7 \mathrm{~Hz}, \mathrm{OCH}_{\mathrm{A}} \mathrm{H}_{\mathrm{B}} \mathrm{Ph}\right), 4.75\left(1 \mathrm{H}, \mathrm{d}, J=18.7 \mathrm{~Hz}, \mathrm{OCH}_{\mathrm{A}} \mathbf{H}_{\mathrm{B}} \mathrm{Ph}\right), 4.27(1 \mathrm{H}$, $\left.\mathrm{d}, J=11.8 \mathrm{~Hz}, \mathrm{NHCH}_{\mathbf{C}} \mathrm{H}_{\mathrm{D}}\right), 4.19\left(1 \mathrm{H}, \mathrm{d}, J=11.8 \mathrm{~Hz}, \mathrm{NHCH}_{\mathrm{C}} \mathbf{H}_{\mathrm{D}}\right), 4.00(1 \mathrm{H}, \mathrm{dd}, J=$ 8.6, 6.1 Hz, CHBr), $3.76\left(3 \mathrm{H}, \mathrm{s}, \mathrm{OCH}_{3}\right), 3.75\left(3 \mathrm{H}, \mathrm{s}, \mathrm{OCH}_{3}\right), 3.63\left(2 \mathrm{H}, \mathrm{s}, \mathrm{CH}_{2} \mathrm{OBn}\right)$, $2.42\left(3 \mathrm{H}, \mathrm{s}, \mathrm{CH}_{3} \mathrm{C}=\mathrm{O}\right), 2.16-1.96\left(1 \mathrm{H}, \mathrm{m}, \mathrm{CHBrCH}_{\mathbf{E}} \mathrm{H}_{\mathrm{F}} \mathrm{CH}_{3}\right), 1.90-1.76(1 \mathrm{H}, \mathrm{m}$, $\left.\mathrm{CHBrCH}_{\mathrm{E}} \mathbf{H}_{\mathrm{F}} \mathrm{CH}_{3}\right), 0.71\left(3 \mathrm{H}, \mathrm{t}, J=7.3 \mathrm{~Hz}, \mathrm{CH}_{\mathrm{A}} \mathrm{H}_{\mathrm{B}} \mathrm{CH}_{3}\right) ;{ }^{13} \mathrm{C} \mathrm{NMR}(62.9 \mathrm{MHz}$, $\left.\mathrm{CDCl}_{3}\right) \delta 198.1(\mathrm{C}), 171.4(\mathrm{C}), 168.6(\mathrm{C}), 158.8(\mathrm{C}), 136.6(\mathrm{C}), 130.2(\mathrm{C}), 128.3(2 \mathrm{x}$ 
$\mathrm{CH}), 127.9(\mathrm{CH}), 127.4(2 \times \mathrm{CH}), 126.7(2 \times \mathrm{CH}), 114.2(2 \times \mathrm{CH}), 77.2(\mathrm{C}), 73.8$ $\left(\mathrm{CH}_{2}\right), 70.3\left(\mathrm{CH}_{2}\right), 55.3\left(\mathrm{CH}_{3}\right), 52.9\left(\mathrm{CH}_{3}\right), 48.5\left(\mathrm{CH}_{2}\right), 44.5(\mathrm{CH}), 28.6\left(\mathrm{CH}_{2}\right), 28.1$ $\left(\mathrm{CH}_{3}\right), 12.0\left(\mathrm{CH}_{3}\right)$; HRMS (ES) Exact mass calcd for $\mathrm{C}_{25} \mathrm{H}_{30}{ }^{79} \mathrm{BrNO}_{6} \mathrm{Na}[\mathrm{M}+\mathrm{Na}]^{+}$: 542.1149, found: 542.1155 .

Data for 420a: $\mathrm{R}_{\mathrm{f}}(30 \%$ EtOAc in hexane $)=0.35 ;[\alpha]_{\mathrm{D}}^{22}+41.0\left(c 0.925, \mathrm{CHCl}_{3}\right)$; IR (film) 2971, 2359, $1744(\mathrm{C}=\mathrm{O}), 1735(\mathrm{C}=\mathrm{O}) 1660(\mathrm{C}=\mathrm{O}), 1513,1413,1247$, 1176, 1095, 740, $503(\mathrm{C}-\mathrm{Br}) \mathrm{cm}^{-1} ;{ }^{1} \mathrm{H} \mathrm{NMR}\left(250 \mathrm{MHz}, \mathrm{CDCl}_{3}\right) \delta 7.38(2 \mathrm{H}, \mathrm{d}, J=$ 8.7 Hz, ArH), 7.34-7.30 (3H, m, ArH), 7.21-7.18 (2H, m, ArH), 6.95 (2H, d, $J=8.7$ $\mathrm{Hz}, \operatorname{ArH}), 4.95\left(1 \mathrm{H}, \mathrm{d}, J=18.4 \mathrm{~Hz}, \mathrm{OCH}_{\mathrm{A}} \mathrm{H}_{\mathrm{B}} \mathrm{Ph}\right), 4.86(1 \mathrm{H}, \mathrm{d}, J=18.4 \mathrm{~Hz}$, $\left.\mathrm{OCH}_{\mathrm{A}} \mathbf{H}_{\mathrm{B}} \mathrm{Ph}\right), 4.42\left(1 \mathrm{H}, \mathrm{d}, J=11.8 \mathrm{~Hz}, \mathrm{NHCH}_{\mathrm{C}} \mathrm{H}_{\mathrm{D}}\right), 4.38(1 \mathrm{H}, \mathrm{d}, J=11.8 \mathrm{~Hz}$, $\left.\mathrm{NHCH}_{\mathrm{C}} \mathbf{H}_{\mathrm{D}}\right), 4.22(1 \mathrm{H}, \mathrm{dd}, J=8.5,6.1 \mathrm{~Hz}, \mathrm{CHBr}), 3.87(1 \mathrm{H}, \mathrm{d}, J=10.5 \mathrm{~Hz}$, $\left.\mathrm{CH}_{\mathrm{E}} \mathrm{H}_{\mathrm{F}} \mathrm{OBn}\right), 3.93\left(1 \mathrm{H}, \mathrm{d}, J=10.5 \mathrm{~Hz}, \mathrm{CH}_{\mathrm{E}} \mathbf{H}_{\mathrm{F}} \mathrm{OBn}\right), 3.87\left(3 \mathrm{H}, \mathrm{s}, \mathrm{OCH}_{3}\right), 3.80(3 \mathrm{H}$, $\left.\mathrm{s}, \mathrm{OCH}_{3}\right), 2.43\left(3 \mathrm{H}, \mathrm{s}, \mathrm{CH}_{3} \mathrm{C}=\mathrm{O}\right), 2.28-2.15\left(1 \mathrm{H}, \mathrm{m}, \mathrm{CHBrCH}_{\mathrm{G}} \mathrm{H}_{\mathrm{H}} \mathrm{CH}_{3}\right), 2.00-1.88$ $\left(1 \mathrm{H}, \mathrm{m}, \mathrm{CHBrCH}_{\mathrm{G}} \mathbf{H}_{\mathrm{H}} \mathrm{CH}_{3}\right), 0.88\left(3 \mathrm{H}, \mathrm{t}, J=7.3 \mathrm{~Hz}, \mathrm{CH}_{\mathrm{G}} \mathrm{H}_{\mathrm{H}} \mathrm{CH}_{3}\right) ;{ }^{13} \mathrm{C}$ NMR (62.9 $\left.\mathrm{MHz}, \mathrm{CDCl}_{3}\right) \delta 197.5(\mathrm{C}), 171.6(\mathrm{C}), 167.6(\mathrm{C}), 158.8$ (C), $136.6(\mathrm{C}), 130.0$ (C), $128.4(2 \times \mathrm{CH}), 127.9(\mathrm{CH}), 127.6(2 \times \mathrm{CH}), 126.9(2 \times \mathrm{CH}), 114.1(2 \times \mathrm{CH}), 77.8$ (C), $73.8\left(\mathrm{CH}_{2}\right), 70.2\left(\mathrm{CH}_{2}\right), 55.3\left(\mathrm{CH}_{3}\right), 52.8\left(\mathrm{CH}_{3}\right), 49.7\left(\mathrm{CH}_{2}\right), 44.7(\mathrm{CH}), 28.6$ $\left(\mathrm{CH}_{2}\right), 27.5\left(\mathrm{CH}_{3}\right), 12.0\left(\mathrm{CH}_{3}\right)$; HRMS (ES) Exact mass calcd for $\mathrm{C}_{25} \mathrm{H}_{30}{ }^{79} \mathrm{BrNO}_{6} \mathrm{Na}$ $[\mathrm{M}+\mathrm{Na}]^{+}:$:542.1149, found: 542.1153 .

\section{$(2 R, 3 S, 4 R)-2-B e n z y l o x y m e t h y l-4-e t h y l-3-h y d r o x y-1-(4-m e t h o x y b e n z y l)-3-$} methyl-5-oxopyrrolidine-2-carboxylic acid methyl ester (421), $\mathrm{N}$-(4methoxybenzyl)butyramide (433) and $(R)$-methyl 2-benzyloxymethyl-2-[ $N$-(4methoxybenzyl)butyramido]-3-oxobutanoate (425)

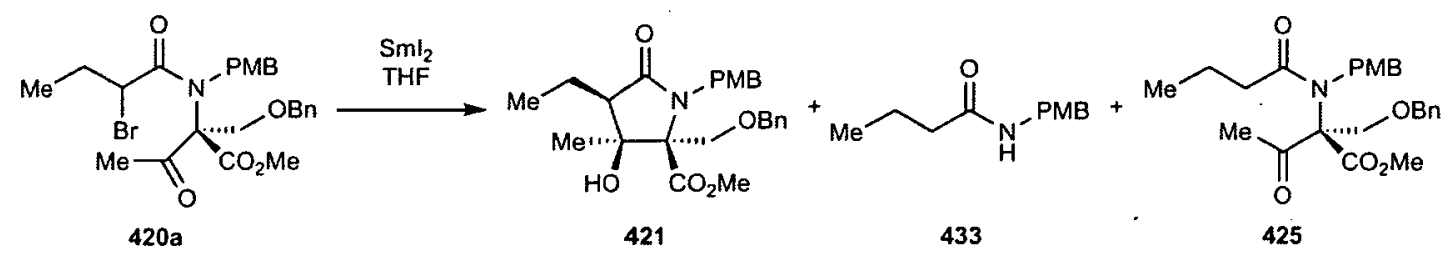

To a stirred solution of amide $\mathbf{4 2 0 a}$ (more polar diastereomer, $30 \mathrm{mg}, 57 \mu \mathrm{mol}$ ) in THF $(3 \mathrm{~mL})$ at $0{ }^{\circ} \mathrm{C}$ was added $\mathrm{SmI}_{2}(0.1 \mathrm{M}$ solution in hexanes, $6.90 \mathrm{~mL}, 0.69$ mmol). The reaction was stirred at $0{ }^{\circ} \mathrm{C}$ for $1 \mathrm{~h}$ and then quenched with saturated aqueous $\mathrm{NH}_{4} \mathrm{Cl}$ solution ( $5 \mathrm{~mL}$ ). The aqueous layer was separated and extracted with 
EtOAc $(2 \times 15 \mathrm{~mL})$ and the combined organic layers were dried $\left(\mathrm{Na}_{2} \mathrm{SO}_{4}\right)$, filtered and concentrated in vacuo. Purification of the residue by column chromatography (10\% to $15 \%$ EtOAc/petrol) gave $\gamma$ lactam 421 (9.5 mg, 38\%), amide 433 (4.5 mg, $40 \%$ ) and debrominated amide 425 (2.6 mg, 10\%).

Data for 421: $\mathrm{R}_{\mathrm{f}}(30 \%$ EtOAc in hexane $)=0.10 ;[\alpha]_{\mathrm{D}}^{22}-24.6\left(c 0.73, \mathrm{CHCl}_{3}\right)$; IR (film) $3424(\mathrm{OH}), 2956,1749(\mathrm{C}=\mathrm{O}), 1670(\mathrm{C}=\mathrm{O}), 1511,1454,1245,1176,1058$, 736, $511(\mathrm{C}-\mathrm{Br}) \mathrm{cm}^{-1}$; ${ }^{1} \mathrm{H}$ NMR $\left(360 \mathrm{MHz} \mathrm{CDCl}_{3}\right) \delta 7.30-7.20(5 \mathrm{H}, \mathrm{m}, \mathrm{ArH}), 7.07$ $(2 \mathrm{H}, \mathrm{d}, J=8.6 \mathrm{~Hz}, \mathrm{ArH}), 6.69(2 \mathrm{H}, \mathrm{d}, J=8.6 \mathrm{~Hz}, \operatorname{ArH}), 4.97(1 \mathrm{H}, \mathrm{d}, J=15.2 \mathrm{~Hz}$, $\left.\mathrm{OCH}_{\mathrm{A}} \mathrm{H}_{\mathrm{B}} \mathrm{Ph}\right), 4.19\left(1 \mathrm{H}, \mathrm{d}, J=15.2 \mathrm{~Hz}, \mathrm{OCH}_{\mathrm{A}} \mathbf{H}_{\mathbf{B}} \mathrm{Ph}\right), 3.89(1 \mathrm{H}, \mathrm{d}, J=11.8 \mathrm{~Hz}$, $\left.\mathrm{NHCH}_{\mathrm{C}} \mathrm{H}_{\mathrm{D}}\right), 3.83\left(1 \mathrm{H}, \mathrm{d}, J=11.8 \mathrm{~Hz}, \mathrm{NHCH}_{\mathrm{C}} \mathbf{H}_{\mathbf{D}}\right), 3.73(1 \mathrm{H}, \mathrm{d}, J=10.3 \mathrm{~Hz}$, $\left.\mathrm{CH}_{\mathrm{E}} \mathrm{H}_{\mathrm{F}} \mathrm{OBn}\right), 3.70\left(3 \mathrm{H}, \mathrm{s}, \mathrm{OCH}_{3}\right), 3.69\left(3 \mathrm{H}, \mathrm{s}, \mathrm{OCH}_{3}\right), 3.17(1 \mathrm{H}, \mathrm{d}, J=10.3 \mathrm{~Hz}$, $\left.\mathrm{CH}_{\mathrm{E}} \mathbf{H}_{\mathrm{F}} \mathrm{OBn}\right), 2.44\left(1 \mathrm{H}, \mathrm{t}, J=6.9 \mathrm{~Hz}, \mathrm{CH}_{\mathrm{G}} \mathrm{H}_{\mathrm{H}} \mathrm{CH}\right), 1.83-1.71\left(1 \mathrm{H}, \mathrm{m}, \mathrm{CH}_{\mathrm{G}} \mathrm{H}_{\mathrm{H}} \mathrm{CH}\right)$, 1.60-1.48 (1H, m, $\left.\mathrm{CH}_{\mathrm{G}} \mathbf{H}_{\mathrm{H}} \mathrm{CH}\right), 1.40\left(3 \mathrm{H}, \mathrm{s}, \mathrm{CCH}_{3} \mathrm{OH}\right), 1.11(3 \mathrm{H}, \mathrm{t}, J=7.5 \mathrm{~Hz}$, $\mathrm{CH}_{3} \mathrm{CH}_{\mathrm{G}} \mathrm{H}_{\mathrm{H}}$ ); 2D NOESY experiment showed cross peak between: $\mathrm{CCH}_{3} \mathrm{OH}$ and $\mathrm{CH}_{\mathrm{E}} \mathrm{H}_{\mathrm{F}} \mathrm{OBn}$ and between $\mathrm{CCH}_{3} \mathrm{OH}$ and $\mathrm{CH}_{\mathrm{G}} \mathrm{H}_{\mathrm{H}} \mathrm{CH} ;{ }^{13} \mathrm{C} \mathrm{NMR}\left(62.9 \mathrm{MHz}, \mathrm{CDCl}_{3}\right) \delta$ 176.5 (C), 169.3 (C), 158.7 (C), 137.2 (C), 130.4 (C), 130.0 (2 x CH), 128.3 (2 x $\mathrm{CH}), 127.7(2 \times \mathrm{CH}), 127.2(\mathrm{CH}), 113.5(2 \times \mathrm{CH}), 78.4(\mathrm{C}), 77.2(\mathrm{C}), 72.8\left(\mathrm{CH}_{2}\right)$, $68.5\left(\mathrm{CH}_{2}\right), 55.2\left(\mathrm{CH}_{3}\right), 52.7(\mathrm{CH}), 52.4\left(\mathrm{CH}_{3}\right), 44.9\left(\mathrm{CH}_{2}\right), 21.1\left(\mathrm{CH}_{3}\right), 17.8\left(\mathrm{CH}_{2}\right)$, $13.4\left(\mathrm{CH}_{3}\right)$; HRMS (ES) Exact mass calcd for $\mathrm{C}_{25} \mathrm{H}_{32} \mathrm{NO}_{6}[\mathrm{M}+\mathrm{H}]^{+}: 442.2224$, found: 442.2227 .

Data for 433: $\mathrm{R}_{\mathrm{f}}(30 \%$ EtOAc in hexane $)=0.13$; IR (film) $3289(\mathrm{NH}), 2930,1632$ $(\mathrm{C}=\mathrm{O}), 1556,1512,1434,1253,1029,813,754 \mathrm{~cm}^{-1} ;{ }^{1} \mathrm{H} \mathrm{NMR}\left(250 \mathrm{MHz}, \mathrm{CDCl}_{3}\right) \delta$ $7.20(2 \mathrm{H}, \mathrm{d}, J=8.7 \mathrm{~Hz}, \operatorname{ArH}), 6.86(2 \mathrm{H}, \mathrm{d}, J=8.7 \mathrm{~Hz}, \operatorname{ArH}), 5.75(1 \mathrm{H}, \mathrm{bs}, \mathrm{NH})$, $4.36\left(2 \mathrm{H}, \mathrm{d}, J=5.6 \mathrm{~Hz}, \mathrm{OCH}_{2} \mathrm{Ph}\right), 3.79\left(3 \mathrm{H}, \mathrm{s}, \mathrm{OCH}_{3}\right), 2.17(2 \mathrm{H}, \mathrm{t}, J=7.6 \mathrm{~Hz}$, $\left.\mathrm{CH}_{2} \mathrm{C}=\mathrm{O}\right), 1.68\left(2 \mathrm{H}, \mathrm{qt}, J=7.6,7.3 \mathrm{~Hz}, \mathrm{CH}_{3} \mathrm{CH}_{2} \mathrm{CH}_{2}\right), 0.95(2 \mathrm{H}, \mathrm{t}, J=7.3 \mathrm{~Hz}$, $\mathrm{CH}_{3} \mathrm{CH}_{2} \mathrm{CH}_{2}$ ); ${ }^{13} \mathrm{C}$ NMR (62.9 MHz, $\left.\mathrm{CDCl}_{3}\right) \delta 172.6$ (C), 158.9 (C), 130.5 (C), $129.1(2 \times \mathrm{CH}), 114.0(2 \times \mathrm{CH}), 55.2\left(\mathrm{CH}_{3}\right), 43.0\left(\mathrm{CH}_{2}\right), 38.6\left(\mathrm{CH}_{2}\right), 19.1\left(\mathrm{CH}_{2}\right)$, $13.7\left(\mathrm{CH}_{3}\right)$; HRMS (EI) Exact mass calcd for $\mathrm{C}_{12} \mathrm{H}_{17} \mathrm{NO}_{2}[\mathrm{M}]^{+}: 207.1254$, found: 207.1253.

Data for 425: $R_{f}(30 \%$ EtOAc in hexane $)=0.32$; IR (film) $2959,2924,2853,1740$ $(\mathrm{C}=\mathrm{O}), 1716(\mathrm{C}=\mathrm{O}), 1651(\mathrm{C}=\mathrm{O}), 1513,1247,1096,1029 \mathrm{~cm}^{-1} ;{ }^{1} \mathrm{H}$ NMR $(250 \mathrm{MHz}$ 
$\left.\mathrm{CDCl}_{3}\right) \delta 7.34(2 \mathrm{H}, \mathrm{d}, J=8.7 \mathrm{~Hz}, \mathrm{ArH}), 7.30-7.26(3 \mathrm{H}, \mathrm{m}, \mathrm{ArH}), 7.15-7.10(2 \mathrm{H}, \mathrm{m}$, $\operatorname{ArH}), 6.91(2 \mathrm{H}, \mathrm{d}, J=8.7 \mathrm{~Hz}, \operatorname{ArH}), 4.89\left(1 \mathrm{H}, \mathrm{d}, J=18.3 \mathrm{~Hz}, \mathrm{OCH}_{\mathrm{A}} \mathrm{H}_{\mathrm{B}} \mathrm{Ph}\right), 4.71$ $\left(1 \mathrm{H}, \mathrm{d}, J=18.3 \mathrm{~Hz}, \mathrm{OCH}_{\mathrm{A}} \mathbf{H}_{\mathrm{B}} \mathrm{Ph}\right), 4.32\left(1 \mathrm{H}, \mathrm{d}, J=11.9 \mathrm{~Hz}, \mathrm{NHCH}_{\mathrm{C}} \mathrm{H}_{\mathrm{D}}\right), 4.26(1 \mathrm{H}$, $\left.\mathrm{d}, J=11.9 \mathrm{~Hz}, \mathrm{NHCH}_{\mathrm{C}} \mathbf{H}_{\mathrm{D}}\right), 3.83\left(3 \mathrm{H}, \mathrm{s}, \mathrm{OCH}_{3}\right), 3.79\left(3 \mathrm{H}, \mathrm{s}, \mathrm{OCH}_{3}\right), 3.73(2 \mathrm{H}, \mathrm{s}$, $\left.\mathrm{CH}_{2} \mathrm{OBn}\right), 2.42\left(3 \mathrm{H}, \mathrm{s}, \mathrm{CH}_{3} \mathrm{C}=\mathrm{O}\right), 2.26\left(2 \mathrm{H}, \mathrm{t}, J=11.9 \mathrm{~Hz}, \mathrm{CH}_{2} \mathrm{C}=\mathrm{O}\right), 1.62(2 \mathrm{H}, \mathrm{m}$, $\left.\mathrm{CH}_{3} \mathrm{CH}_{2} \mathrm{CH}_{2}\right), 0.85\left(3 \mathrm{H}, \mathrm{t}, J=7.4 \mathrm{~Hz}, \mathrm{CH}_{3} \mathrm{CH}_{2}\right) ;{ }^{13} \mathrm{C} \mathrm{NMR}\left(62.9 \mathrm{MHz}, \mathrm{CDCl}_{3}\right) \delta$ 197.9 (C), 176.0 (C), 168.7 (C), 158.6 (C), 136.7 (C), 130.5 (C), 128.3 (2 x CH), $127.8(\mathrm{CH}), 127.4(2 \times \mathrm{CH}), 126.9(2 \times \mathrm{CH}), 114.0(2 \times \mathrm{CH}), 77.2(\mathrm{C}), 73.7\left(\mathrm{CH}_{2}\right)$, $70.3\left(\mathrm{CH}_{2}\right), 55.2\left(\mathrm{CH}_{3}\right), 52.7\left(\mathrm{CH}_{3}\right), 48.6\left(\mathrm{CH}_{2}\right), 34.9\left(\mathrm{CH}_{2}\right), 28.0\left(\mathrm{CH}_{3}\right), 18.3\left(\mathrm{CH}_{2}\right)$, $13.6\left(\mathrm{CH}_{3}\right)$; HRMS (EI) Exact mass calcd for $\mathrm{C}_{25} \mathrm{H}_{31} \mathrm{NO}_{6}[\mathrm{M}]^{+}:$441.2146, found: 441.2146 .

\section{2-Iodobutanal (447)}

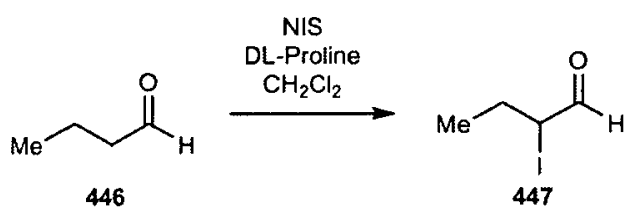

To a stirred solution of butanal $446(1.80 \mathrm{~mL}, 20.0 \mathrm{mmol})$ in $\mathrm{CH}_{2} \mathrm{Cl}_{2}(20 \mathrm{~mL})$ at $0{ }^{\circ} \mathrm{C}$ was added $D L$-proline $(690 \mathrm{mg}, 6.00 \mathrm{mmol})$, followed by the addition of NIS $(5.80 \mathrm{~g}, 10.1 \mathrm{mmol})$. This reaction mixture was stirred for 30 minutes at $0{ }^{\circ} \mathrm{C}$, allowed to warm up to room temperature and then stirred for another $5 \mathrm{~h}$. Hexane was added to the reaction mixture and the precipitated NIS, succinimide and catalyst were filtered off. The solvents were concentrated in vacuo and purification of the residue by column chromatography (10\% EtOAc/hexane) gave $\alpha$-iodoaldehyde 446 $(1.66 \mathrm{~g}, 42 \%)$ as a light pink oil. $\mathrm{R}_{\mathrm{f}}(30 \%$ EtOAc in hexane $)=0.55 ;{ }^{1} \mathrm{H}$ NMR $(250$ $\left.\mathrm{MHz} \mathrm{CDCl}_{3}\right) \delta 9.27$ (1H, s, CHO), 4.46-4.37 (1H, m, CHICHO), 2.09-1.86 (2H, m, $\left.\mathrm{CH}_{3} \mathrm{CH}_{2} \mathrm{CHI}\right), 1.09-1.00\left(3 \mathrm{H}, \mathrm{m}, \mathrm{CH}_{3} \mathrm{CH}_{2}\right) ;{ }^{13} \mathrm{C} \mathrm{NMR}\left(62.9 \mathrm{MHz}, \mathrm{CDCl}_{3}\right) \delta 191.7$ (C), $38.6(\mathrm{CH}), 25.6\left(\mathrm{CH}_{2}\right), 14.0\left(\mathrm{CH}_{3}\right)$.

${ }^{1} \mathrm{H}$ and ${ }^{13} \mathrm{C}$ spectroscopic data in good agreement with the literature. ${ }^{191}$ 


\section{2-Iodobutanoic acid (448)}

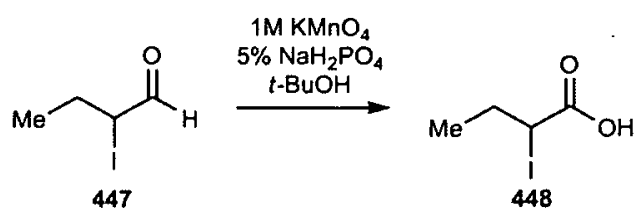

To a solution of the $\alpha$-iodoaldehyde $447(1.27 \mathrm{~g}, 6.40 \mathrm{mmol})$ in $t$-BuOH (38 mL) and $5 \% \mathrm{NaH}_{2} \mathrm{PO}_{4}$ solution $(26 \mathrm{~mL})$ at room temperature was added $\mathrm{KMNO}_{4}(38 \mathrm{~mL}$, $1 \mathrm{M}$ solution). The reaction mixture was stirred for $3 \mathrm{~h}$ and then quenched by the addition of solid $\mathrm{Na}_{2} \mathrm{SO}_{3}$ until the solution turned dark brown from the original purple colour. $1 \mathrm{M} \mathrm{HCl}(30 \mathrm{~mL})$ was added to the reaction mixture until acidic $\mathrm{pH}$. The reaction was then extracted with $\mathrm{Et}_{2} \mathrm{O}(4 \times 40 \mathrm{~mL})$ and the combined organic layers were dried $\left(\mathrm{MgSO}_{4}\right)$, filtered and concentrated in vacuo. Purification of the residue by column chromatography (50\% EtOAc/petrol) gave $\alpha$-iodoacid 448 (1.31 $\mathrm{mg}, 95 \%)$ as a light pink oil. $\mathrm{R}_{\mathrm{f}}(30 \%$ EtOAc in hexane) $=0.26$; IR (film) $2970(\mathrm{OH})$, $1705(\mathrm{C}=\mathrm{O}), 1456,1417,1271,1218,1143,930,783,506(\mathrm{C}-\mathrm{I}) \mathrm{cm}^{-1}$; ${ }^{1} \mathrm{H}$ NMR (250 $\left.\mathrm{MHz} \mathrm{CDCl}_{3}\right) \delta 10.3(1 \mathrm{H}, \mathrm{bs}, \mathrm{OH}), 4.27\left(1 \mathrm{H}, \mathrm{t}, J=7.5 \mathrm{~Hz}, \mathrm{CHICO}_{2} \mathrm{H}\right), 2.00(2 \mathrm{H}, \mathrm{dq}$, $\left.J=7.5,7.3 \mathrm{~Hz}, \mathrm{CH}_{3} \mathrm{CH}_{2} \mathrm{CHI}\right), 1.01\left(3 \mathrm{H}, \mathrm{t}, J=7.2 \mathrm{~Hz}, \mathrm{CH}_{3} \mathrm{CH}_{2}\right) ;{ }^{13} \mathrm{C} \mathrm{NMR}(62.9$ $\left.\mathrm{MHz}, \mathrm{CDCl}_{3}\right) \delta 177.6(\mathrm{C}), 29.2(\mathrm{CH}), 21.9\left(\mathrm{CH}_{2}\right), 13.9\left(\mathrm{CH}_{3}\right)$; HRMS (EI) Exact mass calcd for $\mathrm{C}_{4} \mathrm{H}_{7} \mathrm{O}_{2} \mathrm{I}[\mathrm{M}]^{+}: 213.9485$, found: 213.9489 .

\section{(2R)-Methyl 2-benzyloxymethyl-2-[2-iodo-N-(4-methoxybenzyl)butanamido]-3-} oxobutanoate (449a and $449 b)$

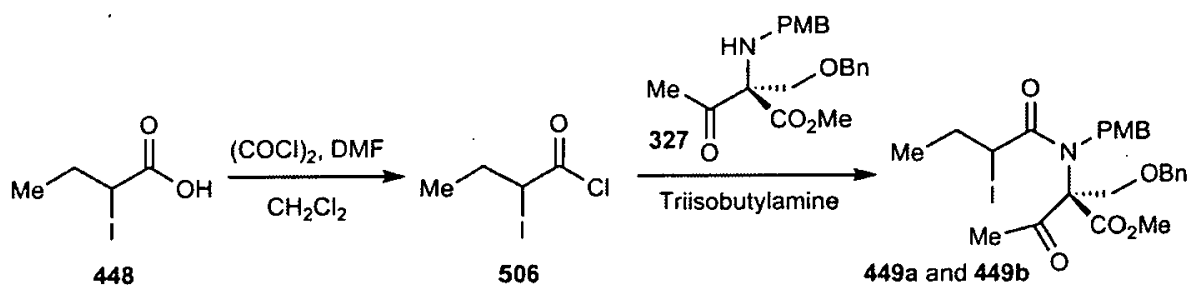

To a stirred solution of acid $448(615 \mathrm{mg}, 1.46 \mathrm{mmol})$ in $\mathrm{CH}_{2} \mathrm{Cl}_{2}(8 \mathrm{~mL})$ at $0{ }^{\circ} \mathrm{C}$, was added oxalyl chloride $(0.74 \mathrm{~mL}, 8.30 \mathrm{mmol})$ followed by DMF (1 drop). The reaction was stirred $5 \mathrm{~min}$ at $0^{\circ} \mathrm{C}$ and then allowed to warm up to room temperature and stirred for another $30 \mathrm{~min}$. The solution containing the acid chloride was concentrated in vacuo, redissolved in $\mathrm{CH}_{2} \mathrm{Cl}_{2}(8 \mathrm{~mL})$ and then transferred via cannula into a stirred solution of amine $327(270 \mathrm{mg}, 0.73 \mathrm{mmol})$ in $\mathrm{CH}_{2} \mathrm{Cl}_{2}(10 \mathrm{~mL})$ and 
triisobutylamine $(0.22 \mathrm{~mL}, 0.87 \mathrm{mmol})$ at $0^{\circ} \mathrm{C}$. The reaction mixture was allowed to warm up to room temperature, stirred for $14 \mathrm{~h}$ and then quenched with saturated aqueous $\mathrm{NH}_{4} \mathrm{Cl}$ solution $(10 \mathrm{~mL})$. The aqueous layer was separated and extracted with $\mathrm{CH}_{2} \mathrm{Cl}_{2}(3 \times 15 \mathrm{~mL})$ and the combined organic layers were dried $\left(\mathrm{MgSO}_{4}\right)$, filtered and concentrated in vacuo. Purification of the residue by column chromatography (10\% EtOAc/petrol) afforded the two diastereomers of amide, the more polar diastereomer 449a (226 $\mathrm{mg}, 40 \%$ ) and the less polar diastereomer $449 \mathrm{~b}$ (189 $\mathrm{mg}, 35 \%$ ), as yellow oils.

Data for 449b: $\mathrm{R}_{\mathrm{f}}(30 \%$ EtOAc in hexane $)=0.62 ;[\alpha]_{\mathrm{D}}^{22}-62.9\left(c 0.985, \mathrm{CHCl}_{3}\right)$; IR (film) 2952, 2360, $1741(\mathrm{C}=0), 1708(\mathrm{C}=\mathrm{O}), 1650(\mathrm{C}=\mathrm{O}), 1513,1358,1246$, 1097, 738, $504(\mathrm{C}-\mathrm{I}) \mathrm{cm}^{-1} ;{ }^{1} \mathrm{H}$ NMR $\left(250 \mathrm{MHz}, \mathrm{CDCl}_{3}\right) \delta 7.34(2 \mathrm{H}, \mathrm{d}, J=8.6 \mathrm{~Hz}$, ArH), 7.25-7.19 (3H, m, ArH), 7.10-7.04 (2H, m, ArH), $6.87(2 \mathrm{H}, \mathrm{d}, J=8.6 \mathrm{~Hz}$, ArH), $5.03\left(1 \mathrm{H}, \mathrm{d}, J=18.7 \mathrm{~Hz}, \mathrm{OCH}_{\mathrm{A}} \mathrm{H}_{\mathrm{B}} \mathrm{Ph}\right), 4.59(1 \mathrm{H}, \mathrm{d}, J=18.7 \mathrm{~Hz}$, $\left.\mathrm{OCH}_{\mathrm{A}} \mathbf{H}_{\mathrm{B}} \mathrm{Ph}\right), 4.31\left(1 \mathrm{H}, \mathrm{d}, J=11.7 \mathrm{~Hz}, \mathrm{NCH}_{\mathrm{C}} \mathrm{H}_{\mathrm{D}}\right), 4.22(1 \mathrm{H}, \mathrm{d}, J=11.7 \mathrm{~Hz}$, $\left.\mathrm{NCH}_{\mathrm{C}} \mathbf{H}_{\mathbf{D}}\right), 4.03(1 \mathrm{H}, \mathrm{dd}, J=8.9,5.9 \mathrm{~Hz}, \mathrm{CHI}), 3.80\left(3 \mathrm{H}, \mathrm{s}, \mathrm{OCH}_{3}\right), 3.78(3 \mathrm{H}, \mathrm{s}$, $\left.\mathrm{OCH}_{3}\right), 3.65\left(2 \mathrm{H}, \mathrm{s}, \mathrm{CH}_{2} \mathrm{OBn}\right), 2.45\left(3 \mathrm{H}, \mathrm{s}, \mathrm{CH}_{3} \mathrm{C}=\mathrm{O}\right), 2.15-1.95(1 \mathrm{H}, \mathrm{m}$, $\left.\mathrm{CHICH}_{\mathrm{E}} \mathrm{H}_{\mathrm{F}} \mathrm{CH}_{3}\right), 1.95-1.75\left(1 \mathrm{H}, \mathrm{m}, \mathrm{CHICH}_{\mathrm{E}} \mathbf{H}_{\mathrm{F}} \mathrm{CH}_{3}\right), 0.67(3 \mathrm{H}, \mathrm{t}, J=7.2 \mathrm{~Hz}$, $\left.\mathrm{CH}_{\mathrm{E}} \mathrm{H}_{\mathrm{F}} \mathrm{CH}_{3}\right) ;{ }^{13} \mathrm{C} \mathrm{NMR}\left(62.9 \mathrm{MHz}, \mathrm{CDCl}_{3}\right) \delta 198.4(\mathrm{C}), 173.0$ (C), 169.4 (C), 159.3 (C), $137.0(\mathrm{C}), 130.6(\mathrm{C}), 128.8(2 \times \mathrm{CH}), 128.3(\mathrm{CH}), 127.9(2 \times \mathrm{CH}), 127.1(2 \times$ $\mathrm{CH}), 114.6(2 \times \mathrm{CH}), 77.6(\mathrm{C}), 74.3\left(\mathrm{CH}_{2}\right), 70.8\left(\mathrm{CH}_{2}\right), 55.7\left(\mathrm{CH}_{3}\right), 53.2\left(\mathrm{CH}_{3}\right), 49.7$ $\left(\mathrm{CH}_{2}\right), 30.9\left(\mathrm{CH}_{2}\right), 28.5\left(\mathrm{CH}_{3}\right), 22.3(\mathrm{CH}), 14.3\left(\mathrm{CH}_{3}\right)$; HRMS (ES) Exact mass calcd for $\mathrm{C}_{25} \mathrm{H}_{31} \mathrm{INO}_{6}[\mathrm{M}+\mathrm{H}]^{+}:$: 568.1191, found: 568.1194 .

Data for 449a: $\mathrm{R}_{\mathrm{f}}(30 \%$ EtOAc in hexane $)=0.52 ;[\alpha]_{\mathrm{D}}^{22}+28.0\left(c 0.57, \mathrm{CHCl}_{3}\right)$; IR (film) $2953(\mathrm{OH}), 2360,1743(\mathrm{C}=\mathrm{O}), 1730(\mathrm{C}=\mathrm{O}), 1653(\mathrm{C}=\mathrm{O}), 1513,1410$, $1247,1094,736,503(\mathrm{C}-\mathrm{I}) \mathrm{cm}^{-1} ;{ }^{1} \mathrm{H}$ NMR $\left(250 \mathrm{MHz}, \mathrm{CDCl}_{3}\right) \delta 7.29(2 \mathrm{H}, \mathrm{d}, J=8.6$ $\mathrm{Hz}, \operatorname{ArH}), 7.27-7.20$ (3H, m, ArH), 7.14-7.08 (2H, m, ArH), $6.86(2 \mathrm{H}, \mathrm{d}, J=8.6 \mathrm{~Hz}$, ArH), $4.78\left(1 \mathrm{H}, \mathrm{d}, J=18.4 \mathrm{~Hz}, \mathrm{OCH}_{\mathrm{A}} \mathrm{H}_{\mathrm{B}} \mathrm{Ph}\right), 4.67(1 \mathrm{H}, \mathrm{d}, J=18.4 \mathrm{~Hz}$, $\left.\mathrm{OCH}_{\mathrm{A}} \mathbf{H}_{\mathrm{B}} \mathrm{Ph}\right), 4.36\left(1 \mathrm{H}, \mathrm{d}, J=11.8 \mathrm{~Hz}, \mathrm{NCH}_{\mathrm{C}} \mathrm{H}_{\mathrm{D}}\right), 4.30(1 \mathrm{H}, \mathrm{d}, J=11.8 \mathrm{~Hz}$, $\left.\mathrm{NCH}_{\mathrm{C}} \mathbf{H}_{\mathbf{D}}\right), 4.13(1 \mathrm{H}, \mathrm{dd}, J=8.9,5.8 \mathrm{~Hz}, \mathrm{CHI}), 3.93(1 \mathrm{H}, \mathrm{d}, J=10.4 \mathrm{~Hz}$, $\left.\mathrm{CH}_{\mathbf{E}} \mathrm{H}_{\mathrm{F}} \mathrm{OBn}\right), 3.85\left(1 \mathrm{H}, \mathrm{d}, J=10.4 \mathrm{~Hz}, \mathrm{CH}_{\mathrm{E}} \mathbf{H}_{\mathrm{F}} \mathrm{OBn}\right), 3.77\left(3 \mathrm{H}, \mathrm{s}, \mathrm{OCH}_{3}\right), 3.70(3 \mathrm{H}$, $\left.\mathrm{s}, \mathrm{OCH}_{3}\right), 2.33\left(3 \mathrm{H}, \mathrm{s}, \mathrm{CH}_{3} \mathrm{C}=\mathrm{O}\right), 2.17-1.97\left(1 \mathrm{H}, \mathrm{m}, \mathrm{CHICH}_{\mathrm{G}} \mathrm{H}_{\mathrm{H}} \mathrm{CH}_{3}\right), 1.96-1.75$ 
$\left(1 \mathrm{H}, \mathrm{m}, \mathrm{CHICH}_{\mathrm{G}} \mathbf{H}_{\mathrm{H}} \mathrm{CH}_{3}\right), 0.70\left(3 \mathrm{H}, \mathrm{t}, J=7.2 \mathrm{~Hz}, \mathrm{CH}_{\mathrm{G}} \mathrm{H}_{\mathrm{H}} \mathrm{CH}_{3}\right) ;{ }^{13} \mathrm{C} \mathrm{NMR}(62.9$ $\left.\mathrm{MHz}, \mathrm{CDCl}_{3}\right) \delta 197.5$ (C), 172.8 (C), 167.5 (C), 158.8 (C), 136.7 (C), 130.1 (C), $128.3(2 \times \mathrm{CH}), 127.8(\mathrm{CH}), 127.6(2 \times \mathrm{CH}), 126.9(2 \times \mathrm{CH}), 114.2(2 \times \mathrm{CH}), 78.0$ (C), $73.8\left(\mathrm{CH}_{2}\right), 70.1\left(\mathrm{CH}_{2}\right), 55.3\left(\mathrm{CH}_{3}\right), 52.8\left(\mathrm{CH}_{3}\right), 50.6\left(\mathrm{CH}_{2}\right), 30.5\left(\mathrm{CH}_{2}\right), 27.5$ $\left(\mathrm{CH}_{3}\right), 22.0(\mathrm{CH}) 13.9\left(\mathrm{CH}_{3}\right)$; HRMS (ES) Exact mass calcd for $\mathrm{C}_{25} \mathrm{H}_{31} \mathrm{INO}_{6}$ $[\mathrm{M}+\mathrm{H}]^{+}:$568.1191, found: 568.1191 .

\section{4-(4-Methoxybenzyloxy)butan-1-ol (455) ${ }^{174}$}

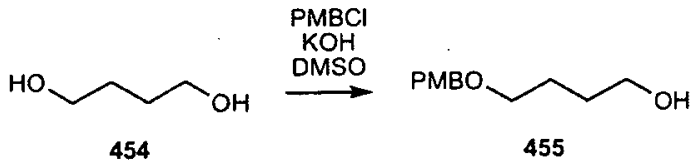

To a stirred solution of 1,4-butanediol $(55.0 \mathrm{~mL}, 61.4 \mathrm{mmol})$ and $\mathrm{KOH}$ (powder, $4.16 \mathrm{~g}, 63.0 \mathrm{mmol})$ in DMSO $(12 \mathrm{~mL})$ at $0^{\circ} \mathrm{C}$ was added $p$-(methoxy)benzyl chloride $(4.15 \mathrm{~mL}, 30.0 \mathrm{mmol})$. The reaction was stirred at $0{ }^{\circ} \mathrm{C}$ for $2 \mathrm{~h}$, allowed to warm up to room temperature, stirred then for $12 \mathrm{~h}$ and quenched with saturated aqueous $\mathrm{NH}_{4} \mathrm{Cl}$ solution $(30 \mathrm{~mL})$. The aqueous layer was extracted with $\mathrm{Et}_{2} \mathrm{O}(4 \times 40 \mathrm{~mL})$ and the combined organic layers were washed with saturated aqueous $\mathrm{NaCl}$ solution $(1 \mathrm{x}$ $60 \mathrm{~mL})$, dried $\left(\mathrm{MgSO}_{4}\right)$, filtered and concentrated in vacuo: Purification of the residue by column chromatography ( $30 \%$ to $40 \%$ EtOAc/petrol) gave monoalcohol $455(5.32 \mathrm{~g}, 85 \%)$ as a colourless oil. $\mathrm{R}_{\mathrm{f}}(30 \%$ EtOAc in hexane $)=0.50 ;{ }^{1} \mathrm{H}$ NMR $\left(360 \mathrm{MHz} \mathrm{CDCl}_{3}\right) \delta 7.25(2 \mathrm{H}, \mathrm{d}, J=8.6 \mathrm{~Hz}, \mathrm{ArH}), 6.73(2 \mathrm{H}, \mathrm{d}, J=8.6 \mathrm{~Hz}, \mathrm{ArH})$, $4.41\left(2 \mathrm{H}, \mathrm{s}, \mathrm{ArCH}_{2} \mathrm{O}\right), 3.75\left(3 \mathrm{H}, \mathrm{s}, \mathrm{OCH}_{3}\right), 3.59\left(2 \mathrm{H}, \mathrm{t}, J=5.1 \mathrm{~Hz}, \mathrm{OCH}_{2} \mathrm{CH}_{2}\right), 3.48$ $\left(2 \mathrm{H}, \mathrm{t}, J=5.3 \mathrm{~Hz}, \mathrm{CH}_{2} \mathrm{CH}_{2} \mathrm{OH}\right), 2.30(1 \mathrm{H}, \mathrm{bs}, \mathrm{OH}), 1.70-1.58\left(4 \mathrm{H}, \mathrm{m}, \mathrm{CH}_{2} \mathrm{CH}_{2}\right) ;{ }^{13} \mathrm{C}$ NMR (62.9 MHz, $\left.\mathrm{CDCl}_{3}\right) \delta 159.1$ (C), 130.1 (C), $129.2(2 \times \mathrm{CH}), 113.7(2 \times \mathrm{CH})$, $72.5\left(\mathrm{CH}_{2}\right), 69.9\left(\mathrm{CH}_{2}\right), 62.4\left(\mathrm{CH}_{2}\right), 55.1\left(\mathrm{CH}_{3}\right), 29.9\left(\mathrm{CH}_{2}\right), 26.5\left(\mathrm{CH}_{2}\right)$.

${ }^{1} \mathrm{H}$ and ${ }^{13} \mathrm{C}$ spectroscopic data in good agreement with the literature. ${ }^{174}$

\section{4-(4-Methoybenzyloxy)butyric acid (456) ${ }^{174}$}

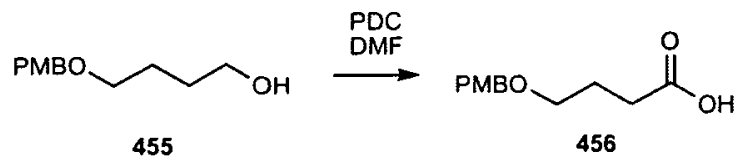


To a stirred solution of alcohol $455(5.32 \mathrm{~g}, 25.3 \mathrm{mmol})$ in DMF (125 mL) at room temperature was added PDC $(34.0 \mathrm{~g}, 88.5 \mathrm{mmol})$ portionwise. The reaction was stirred for $14 \mathrm{~h}$ and then quenched with water $(250 \mathrm{~mL})$ and extracted with $\mathrm{Et}_{2} \mathrm{O}$ $(5 \times 50 \mathrm{~mL})$. The combined organic layers were dried $\left(\mathrm{MgSO}_{4}\right)$, filtered and concentrated in vacuo. Purification of the residue by column chromatography $(10 \%$ EtOAc/petrol) afforded acid $456(4.0 \mathrm{~g}, 71 \%)$ as a colourless oil. $\mathrm{R}_{\mathrm{f}}(30 \% \mathrm{EtOAc}$ in hexane $)=0.25 ;{ }^{1} \mathrm{H} \mathrm{NMR}\left(360 \mathrm{MHz}, \mathrm{CDCl}_{3}\right) \delta 10.5(1 \mathrm{H}, \mathrm{bs}, \mathrm{OH}), 7.22(2 \mathrm{H}, \mathrm{d}, J=$ $8.7 \mathrm{~Hz}, \operatorname{ArH}), 6.84(2 \mathrm{H}, \mathrm{d}, J=5.6 \mathrm{~Hz}, \operatorname{ArH}), 4.40\left(2 \mathrm{H}, \mathrm{s}, \operatorname{ArCH}_{2} \mathrm{O}\right), 3.76(3 \mathrm{H}, \mathrm{s}$, $\left.\mathrm{OCH}_{3}\right), 3.46\left(2 \mathrm{H}, \mathrm{t}, J=6.1 \mathrm{~Hz}, \mathrm{OCH}_{2} \mathrm{CH}_{2}\right), 2.43\left(2 \mathrm{H}, \mathrm{t}, J=7.3 \mathrm{~Hz}, \mathrm{CH}_{2} \mathrm{C}=\mathrm{O}\right), 1.89$ (2H, quint, $\left.J=7.3,6.1 \mathrm{~Hz}, \mathrm{CH}_{2} \mathrm{CH}_{2} \mathrm{CH}_{2}\right) ;{ }^{13} \mathrm{C} \mathrm{NMR}\left(62.9 \mathrm{MHz}, \mathrm{CDCl}_{3}\right) \delta 178.9(\mathrm{C})$, $159.0(\mathrm{C}), 130.2(\mathrm{C}), 129.1(2 \times \mathrm{CH}), 113.7(2 \times \mathrm{CH}), 72.4\left(\mathrm{CH}_{2}\right), 68.6\left(\mathrm{CH}_{2}\right), 55.1$ $\left(\mathrm{CH}_{3}\right), 30.9\left(\mathrm{CH}_{2}\right), 24.7\left(\mathrm{CH}_{2}\right)$.

${ }^{1} \mathrm{H}$ and ${ }^{13} \mathrm{C}$ spectroscopic data in good agreement with the literature. ${ }^{174}$

\section{4-(tert-Butyldiphenylsilyloxy)butan-1-ol (458) ${ }^{179}$}

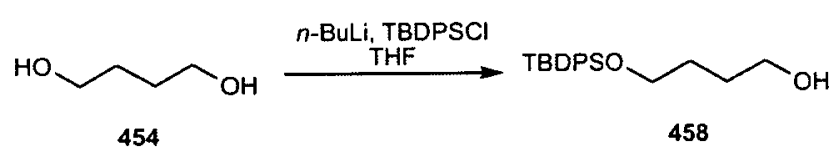

To a vigorously stirred solution of 1,4-butanediol $(4.0 \mathrm{~mL}, 45 \mathrm{mmol})$ in THF (24 $\mathrm{mL})$ at $-78{ }^{\circ} \mathrm{C}$ was added $n$-BuLi $(9.4 \mathrm{~mL}, 1.6 \mathrm{M}$ in hexanes, $15 \mathrm{mmol})$ dropwise over $10 \mathrm{~min}$. The reaction was stirred for $5 \mathrm{~min}$ and TBDPSCl $(4.0 \mathrm{~mL}, 15 \mathrm{mmol})$ was then added dropwise. The reaction was allowed to warm up to room temperature (a white suspension was formed), stirred for $1.5 \mathrm{~h}$ and then quenched with water (20 $\mathrm{mL}$ ) and saturated aqueous $\mathrm{NH}_{4} \mathrm{Cl}$ solution $(20 \mathrm{~mL})$. The aqueous layer was separated and extracted with $\mathrm{Et}_{2} \mathrm{O}(3 \times 30 \mathrm{~mL})$ and the combined organic layers were dried $\left(\mathrm{MgSO}_{4}\right)$, filtered and concentrated in vacuo. Purification of the residue by column chromatography ( $10 \%$ to $20 \% \mathrm{EtOAc/petrol)} \mathrm{gave} \mathrm{mono-protected} \mathrm{alcohol}$ $458(4.78 \mathrm{~g}, 97 \%)$ as a colourless oil. $\mathrm{R}_{\mathrm{f}}(30 \%$ EtOAc in hexane $)=0.50 ;{ }^{1} \mathrm{H}$ NMR $\left(360 \mathrm{MHz} \mathrm{CDCl}_{3}\right) \delta$ 7.72-7.68 (4H, m, ArH), 7.48-7.38 (6H, m, ArH), $3.73(2 \mathrm{H}, \mathrm{t}, J$ $\left.=5.6 \mathrm{~Hz}, \mathrm{CH}_{2} \mathrm{OH}\right), 3.68\left(2 \mathrm{H}, \mathrm{t}, J=5.7 \mathrm{~Hz}, \operatorname{TBDPSOCH}_{2}\right), 1.74-1.65(4 \mathrm{H}, \mathrm{m}$, $\left.\mathrm{CH}_{2} \mathrm{CH}_{2}\right), 1.09\left(9 \mathrm{H}, \mathrm{s}, \mathrm{C}\left(\mathrm{CH}_{3}\right)_{3}\right) ;{ }^{13} \mathrm{C} \mathrm{NMR}\left(62.9 \mathrm{MHz}, \mathrm{CDCl}_{3}\right) \delta 135.5(4 \mathrm{x} \mathrm{CH})$, 
$133.6(2 \times \mathrm{C}), 129.6(2 \times \mathrm{CH}), 127.6(4 \times \mathrm{CH}), 63.9\left(\mathrm{CH}_{2}\right), 62.7\left(\mathrm{CH}_{2}\right), 29.7\left(\mathrm{CH}_{2}\right)$, $29.2\left(\mathrm{CH}_{2}\right), 26.8\left(3 \times \mathrm{CH}_{3}\right), 19.1(\mathrm{C})$.

${ }^{1} \mathrm{H}$ spectroscopic data in good agreement with the literature. ${ }^{179}$

\section{4-(tert-Butyldiphenylsilyl)oxy butyric acid (459) ${ }^{179}$}

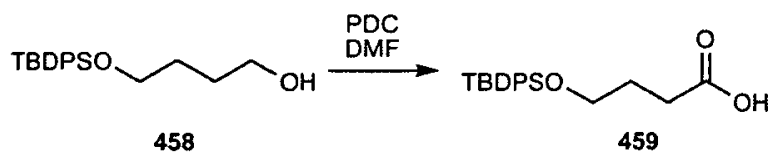

To a stirred solution of alcohol $458(4.40 \mathrm{~g}, 13.3 \mathrm{mmol})$ in DMF $(35 \mathrm{~mL})$ at $0{ }^{\circ} \mathrm{C}$ was added PDC ( $18.0 \mathrm{~g}, 46.8 \mathrm{mmol})$ portionwise. The reaction was allowed to warm to room temperature, stirred for $14 \mathrm{~h}$ and then quenched with water $(250 \mathrm{~mL})$. The aqueous layer was extracted with $\mathrm{Et}_{2} \mathrm{O}(5 \times 50 \mathrm{~mL})$ and the combined organic layers were dried $\left(\mathrm{MgSO}_{4}\right)$, filtered and concentrated in vacuo. Purification of the residue by column chromatography (10\% EtOAc/petrol) afforded acid $459(2.4 \mathrm{~g}, 51 \%)$ as a colourless solid. $\mathrm{R}_{\mathrm{f}}(30 \%$ EtOAc in hexane $)=0.20$; m.p. $68-70{ }^{\circ} \mathrm{C} ;{ }^{1} \mathrm{H}$ NMR $(360$ $\left.\mathrm{MHz}, \mathrm{CDCl}_{3}\right) \delta$ 7.70-7.65 (4H, m, ArH), 7.45-7.36 (6H, m, ArH), $3.71(2 \mathrm{H}, \mathrm{t}, J=$ $\left.5.9 \mathrm{~Hz}, \mathrm{OCH}_{2}\right), 2.53\left(2 \mathrm{H}, \mathrm{t}, J=7.3 \mathrm{~Hz}, \mathrm{CH}_{2} \mathrm{CO}_{2} \mathrm{H}\right), 1.90(2 \mathrm{H}, \mathrm{q}, J=7.3,5.9 \mathrm{~Hz}$, $\left.\mathrm{CH}_{2} \mathrm{CH}_{2} \mathrm{CH}_{2}\right), 1.06\left(9 \mathrm{H}, \mathrm{s}, \mathrm{C}\left(\mathrm{CH}_{3}\right)_{3}\right) ;{ }^{13} \mathrm{C}$ NMR $\left(62.9 \mathrm{MHz}, \mathrm{CDCl}_{3}\right) \delta 179.4(\mathrm{C})$, $135.5(4 \times \mathrm{CH}), 133.5(2 \times \mathrm{C}), 129.6(2 \times \mathrm{CH}), 127.6(4 \times \mathrm{CH}), 62.7\left(\mathrm{CH}_{2}\right), 30.7$ $\left(\mathrm{CH}_{2}\right), 27.4\left(\mathrm{CH}_{2}\right), 26.8\left(3 \times \mathrm{CH}_{3}\right), 19.1(\mathrm{C})$.

${ }^{1} \mathrm{H}$ and ${ }^{13} \mathrm{C}$ spectroscopic data in good agreement with the literature. ${ }^{179}$

\section{4-(tert-Butyldiphenylsilyl)oxy-1-butanal (461) $)^{192}$}

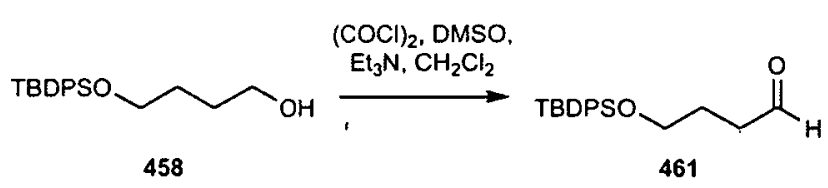

To a stirred solution of oxalyl chloride $(1.10 \mathrm{~mL}, 12.7 \mathrm{mmol})$ in $\mathrm{CH}_{2} \mathrm{Cl}_{2}(30 \mathrm{~mL})$ at $-78{ }^{\circ} \mathrm{C}$, was added DMSO $(1.80 \mathrm{~mL}, 25.6 \mathrm{mmol})$ dropwise. After stirring for 15 minutes, a solution of alcohol $458(2.0 \mathrm{~g}, 6.1 \mathrm{mmol})$ in $\mathrm{CH}_{2} \mathrm{Cl}_{2}(30 \mathrm{~mL})$ was added via cannula. The reaction stirred at $-78{ }^{\circ} \mathrm{C}$ for $1 \mathrm{~h}$ and then $\mathrm{Et}_{3} \mathrm{~N}(3.60 \mathrm{~mL}, 25.6$ mmol) was added. The reaction was stirred at $-78^{\circ} \mathrm{C}$ for $1 \mathrm{~h}$, allowed to warm up to $-40{ }^{\circ} \mathrm{C}$ for $2 \mathrm{~h}$ and then quenched with $\mathrm{NH}_{4} \mathrm{Cl}(40 \mathrm{~mL})$. The aqueous layer was 
separated and extracted with $\mathrm{CH}_{2} \mathrm{Cl}_{2}(3 \times 20 \mathrm{~mL})$ and the combined organic layers were dried $\left(\mathrm{MgSO}_{4}\right)$, filtered and concentrated in vacuo. Purification of the residue by column chromatography (20\% EtOAc/petrol) gave aldehyde $461(1.57 \mathrm{~g}, 79 \%)$ as a colourless oil. $\mathrm{R}_{\mathrm{f}}(30 \%$ EtOAc in hexane $)=0.70 ;{ }^{1} \mathrm{H}$ NMR $\left(360 \mathrm{MHz}, \mathrm{CDCl}_{3}\right) \delta$ $9.80(1 \mathrm{H}, \mathrm{d}, J=1.6 \mathrm{~Hz}, \mathrm{CHO}), 7.67(4 \mathrm{H}, \mathrm{d}, J=7.9 \mathrm{~Hz}, \mathrm{ArH}), 7.45-7.37$ (6H, m, $\operatorname{ArH}), 3.71\left(2 \mathrm{H}, \mathrm{t}, J=6.0 \mathrm{~Hz}, \mathrm{OCH}_{2} \mathrm{CH}_{2}\right), 2.56\left(2 \mathrm{H}, \mathrm{dt}, J=7.1,1.6 \mathrm{~Hz}, \mathrm{CH}_{2} \mathrm{CHO}\right)$, 1.94-1.87 (2H, m, $\left.\mathrm{CH}_{2} \mathrm{CH}_{2} \mathrm{CH}_{2}\right), 1.07\left(9 \mathrm{H}, \mathrm{s}, \mathrm{C}\left(\mathrm{CH}_{3}\right)_{3}\right) ;{ }^{13} \mathrm{C} \mathrm{NMR}(62.9 \mathrm{MHz}$, $\left.\mathrm{CDCl}_{3}\right) \delta 202.5(\mathrm{C}), 135.5(2 \times \mathrm{CH}), 135.2(\mathrm{C}), 134.8(4 \times \mathrm{CH}), 133.6(\mathrm{C}), 129.7$ $(\mathrm{CH}), 127.7(4 \times \mathrm{CH}), 62.9\left(\mathrm{CH}_{2}\right), 40.7\left(\mathrm{CH}_{2}\right), 26.6\left(3 \times \mathrm{CH}_{3}\right), 25.2\left(\mathrm{CH}_{2}\right), 19.2(\mathrm{C})$.

${ }^{1} \mathrm{H}$ and ${ }^{13} \mathrm{C}$ spectroscopic data in good agreement with the literature. ${ }^{192}$

\section{2-Bromo-4-(tert-butyldiphenylsilyl)oxy-1-butanoic acid (463)}

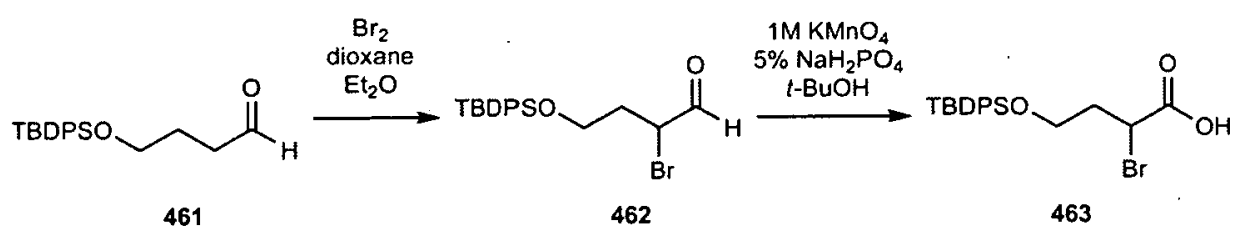

To a stirred solution of aldehyde $461(1.47 \mathrm{~g}, 4.52 \mathrm{mmol})$ and dioxane $(1.20 \mathrm{~mL})$ in dry $\mathrm{Et}_{2} \mathrm{O}(18 \mathrm{~mL})$ at $0{ }^{\circ} \mathrm{C}$, was added bromine $(0.23 \mathrm{~mL}, 4.52 \mathrm{mmol})$ dropwise over $1 \mathrm{~min}$. The reaction was stirred for 30 minutes at $0^{\circ} \mathrm{C}$ and then $1 \mathrm{~h}$ at $\mathrm{rt}$ when it was quenched with saturated aqueous $\mathrm{NaHCO}_{3}$ solution. The aqueous layer was extracted with $\mathrm{Et}_{2} \mathrm{O}(2 \times 20 \mathrm{~mL})$ and the combined organic layers were dried $\left(\mathrm{MgSO}_{4}\right)$, filtered and concentrated in vacuo. The crude residue was used in the oxidation step without further purification.

To a stirred solution of the $\alpha$-bromoaldehyde 462 in $t-\mathrm{BuOH}(29 \mathrm{~mL})$ and $5 \%$ $\mathrm{NaH}_{2} \mathrm{PO}_{4}$ solution $(19.5 \mathrm{~mL})$ at room temperature was added $\mathrm{KMNO}_{4}(1 \mathrm{M}$ solution, $29 \mathrm{~mL}$ ). The reaction was stirred for $3 \mathrm{~h}$ and then quenched by the addition of solid $\mathrm{Na}_{2} \mathrm{SO}_{3}$ until the solution turned dark brown from the original purple colour. $1 \mathrm{M}$ $\mathrm{HCl}(30 \mathrm{~mL})$ was added to the reaction mixture until acidic $\mathrm{pH}$. The reaction was then extracted with $\mathrm{Et}_{2} \mathrm{O}(4 \times 40 \mathrm{~mL})$ and the combined organic layers were dried $\left(\mathrm{MgSO}_{4}\right)$, filtered and concentrated in vacuo. Purification of the residue by column chromatography (15\% to $20 \%$ EtOAc/petrol) gave $\alpha$-bromoacid $463(0.72 \mathrm{~g}, 38 \%$ in two steps $)$ as a colourless solid. $\mathrm{R}_{\mathrm{f}}(30 \%$ EtOAc in hexane $)=0.20 ;$ m.p. $=73-75^{\circ} \mathrm{C}$; 
IR (film) $2957(\mathrm{OH}), 2931,2857,1718(\mathrm{C}=\mathrm{O}), 1428,1277,1111,737,701,505$ (C$\mathrm{Br}) \mathrm{cm}^{-1} ;{ }^{1} \mathrm{H} \mathrm{NMR}\left(360 \mathrm{MHz}, \mathrm{CDCl}_{3}\right) \delta$ 7.72-7.66 (4H, m, ArH), 7.49-7.39 (6H, m, ArH), $4.66(1 \mathrm{H}, \mathrm{dd}, J=8.1,6.1 \mathrm{~Hz}, \mathrm{CHBr}), 3.87-3.77\left(2 \mathrm{H}, \mathrm{m}, \mathrm{OCH}_{2} \mathrm{CH}_{\mathrm{A}} \mathrm{H}_{\mathrm{B}}\right), 2.46-$ $2.37\left(1 \mathrm{H}, \mathrm{m}, \mathrm{OCH}_{2} \mathrm{CH}_{\mathrm{A}} \mathrm{H}_{\mathrm{B}}\right), 2.22-2.12\left(1 \mathrm{H}, \mathrm{m}, \mathrm{OCH}_{2} \mathrm{CH}_{\mathrm{A}} \mathbf{H}_{\mathbf{B}}\right), 1.08(9 \mathrm{H}, \mathrm{s}$, $\left.\mathrm{C}\left(\mathrm{CH}_{3}\right)_{3}\right) ;{ }^{13} \mathrm{C} \mathrm{NMR}\left(62.9 \mathrm{MHz}, \mathrm{CDCl}_{3}\right) \delta 175.6(\mathrm{C}), 135.5(4 \times \mathrm{CH}), 133.1(2 \times \mathrm{C})$, $129.8(2 \times \mathrm{CH}), 127.7(4 \times \mathrm{CH}), 60.6\left(\mathrm{CH}_{2}\right), 42.6(\mathrm{CH}), 37.2\left(\mathrm{CH}_{2}\right), 26.8\left(3 \times \mathrm{CH}_{3}\right)$, 19.1 (C); HRMS (ES) Exact mass calcd for $\mathrm{C}_{20} \mathrm{H}_{29}{ }^{79} \mathrm{BrNO}_{3} \mathrm{Si}\left[\mathrm{M}+\mathrm{NH}_{4}\right]^{+}: 438.1095$, found: 438.1093 .

\section{(R)-Methyl 2-benzyloxymethyl-2-[2-bromo-4-(tert-butyldiphenylsilyloxy)- $N$-(4-} methoxybenzyl)butanamido]-3-oxobutanoate (463a and $463 \mathrm{~b}$ )

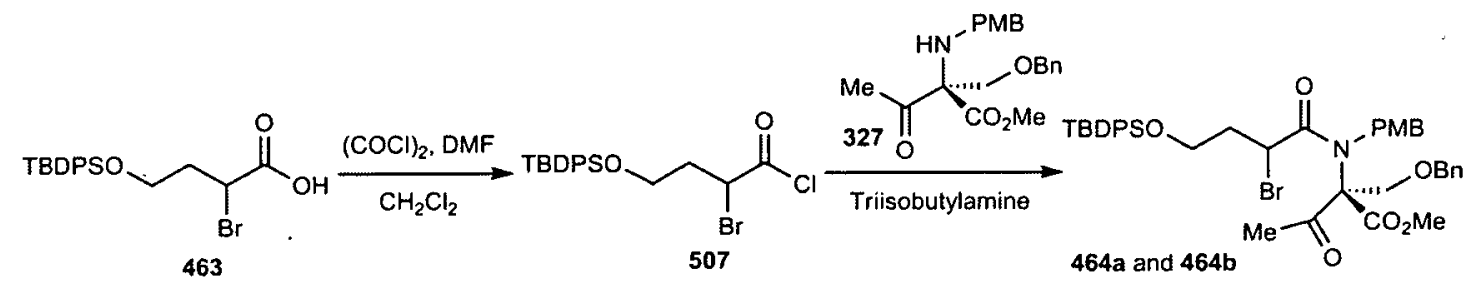

To a stirred solution of acid $463(615 \mathrm{mg}, 1.46 \mathrm{mmol})$ in $\mathrm{CH}_{2} \mathrm{Cl}_{2}(8 \mathrm{~mL})$ at $0{ }^{\circ} \mathrm{C}$, was added oxalyl chloride $(0.74 \mathrm{~mL}, 8.30 \mathrm{mmol})$ followed by DMF (1 drop). The reaction was stirred $5 \mathrm{~min}$ at $0{ }^{\circ} \mathrm{C}$ and then allowed to warm up to room temperature and stirred for another $30 \mathrm{~min}$. The solution containing the acid chloride was concentrated in vacuo, redissolved in $\mathrm{CH}_{2} \mathrm{Cl}_{2}(8 \mathrm{~mL})$ and then transferred via cannula into a stirred solution of amine $327(271 \mathrm{mg}, 0.73 \mathrm{mmol})$ in $\mathrm{CH}_{2} \mathrm{Cl}_{2}(10 \mathrm{~mL})$ and triisobutylamine $(0.22 \mathrm{~mL}, 0.87 \mathrm{mmol})$ at $0{ }^{\circ} \mathrm{C}$. The reaction mixture was stirred for $14 \mathrm{~h}$ at room temperature and then quenched with saturated aqueous $\mathrm{NH}_{4} \mathrm{Cl}$ solution $(10 \mathrm{~mL})$. The aqueous layer was separated and extracted with $\mathrm{CH}_{2} \mathrm{Cl}_{2}(3 \times 15 \mathrm{~mL})$ and the combined organic layers were dried $\left(\mathrm{MgSO}_{4}\right)$, filtered and concentrated in vacuo. Purification of the residue by column chromatography (10\% EtOAc/petrol) afforded the two diastereomers of amide, the more polar diastereomer 464a (226 mg, $40 \%$ ) and the less polar diastereomer $464 \mathrm{~b}$ (189 $\mathrm{mg}, 33 \%$ ), as yellow oils.

Data for 464b: $\mathrm{R}_{\mathrm{f}}(30 \%$ EtOAc in hexane $)=0.48 ;[\alpha]_{\mathrm{D}}^{22}-19.8\left(c \quad 0.98, \mathrm{CHCl}_{3}\right)$; IR (film) 2929, 2856, 2360, 1741 (C=O), $1708(\mathrm{C}=\mathrm{O}), 1654(\mathrm{C}=\mathrm{O}), 1513,1247$, $1106,701,505$ (C-Br) cm ${ }^{-1} ;{ }^{1} \mathrm{H}$ NMR $\left(250 \mathrm{MHz} \mathrm{CDCl}_{3}\right) \delta 7.87-7.81(4 \mathrm{H}, \mathrm{m}, \mathrm{ArH})$, 
7.70-7.52 (11H, m; ArH), $7.40(2 \mathrm{H}, \mathrm{d}, J=8.5 \mathrm{~Hz}, \operatorname{ArH}), 7.11(2 \mathrm{H}, \mathrm{d}, J=8.5 \mathrm{~Hz}$, ArH), $5.38\left(1 \mathrm{H}, \mathrm{d}, J=18.7 \mathrm{~Hz}, \mathrm{OCH}_{\mathrm{A}} \mathrm{H}_{\mathrm{B}} \mathrm{Ph}\right), 5.21(1 \mathrm{H}, \mathrm{d}, J=18.7 \mathrm{~Hz}$, $\left.\mathrm{OCH}_{\mathrm{A}} \mathbf{H}_{\mathrm{B}} \mathrm{Ph}\right), 4.99(1 \mathrm{H}, \mathrm{dd}, J=8.3,5.8 \mathrm{~Hz}, \mathrm{CHBr}), 4.61(1 \mathrm{H}, \mathrm{d}, J=11.7 \mathrm{~Hz}$, $\left.\mathrm{NHCH}_{\mathrm{C}} \mathrm{H}_{\mathrm{D}}\right), 4.54\left(1 \mathrm{H}, \mathrm{d}, J=11.7 \mathrm{~Hz}, \mathrm{NHCH}_{\mathrm{C}} \mathbf{H}_{\mathrm{D}}\right), 4.11\left(3 \mathrm{H}, \mathrm{s}, \mathrm{OCH}_{3}\right), 4.01-3.98$ $\left(3 \mathrm{H}+2 \mathrm{H}+2 \mathrm{H}, \mathrm{m}, \mathrm{OCH}_{3}+\mathrm{CH}_{2} \mathrm{OBn}+\mathrm{OCH}_{2} \mathrm{CH}_{2}\right), 2.76\left(3 \mathrm{H}, \mathrm{s}, \mathrm{CH}_{3} \mathrm{C}=\mathrm{O}\right), 2.50-2.40$ $(2 \mathrm{H}, \mathrm{m}, \mathrm{CHBrCH}), 1.13\left(9 \mathrm{H}, \mathrm{s}, \mathrm{C}\left(\mathrm{CH}_{3}\right)_{3}\right) ;{ }^{13} \mathrm{C} \mathrm{NMR}\left(62.9 \mathrm{MHz}, \mathrm{CDCl}_{3}\right) \delta 197.8$ (C), 171.5 (C), 168.5 (C), 158.7 (C), 136.5 (C), 135.3 (4 x CH), 133.3 (C), 133.2 (C), $129.5(2 \times \mathrm{CH}), 128.3(2 \times \mathrm{CH}), 127.8(\mathrm{CH}), 127.6(2 \times \mathrm{C}), 127.5(4 \times \mathrm{CH}), 127.4(2$ $\mathrm{x} \mathrm{CH}), 126.5(2 \times \mathrm{CH}), 114.2(2 \times \mathrm{CH}), 73.8\left(\mathrm{CH}_{2}\right), 70.1\left(\mathrm{CH}_{2}\right), 60.3\left(\mathrm{CH}_{2}\right), 55.1$ $\left(\mathrm{CH}_{3}\right), 52.8\left(\mathrm{CH}_{3}\right), 48.7\left(\mathrm{CH}_{2}\right), 41.5(\mathrm{CH}), 37.3\left(\mathrm{CH}_{2}\right), 28.0\left(\mathrm{CH}_{3}\right), 26.6\left(3 \times \mathrm{CH}_{3}\right)$, 18.9 (C); HRMS (ES) Exact mass calcd for $\mathrm{C}_{41} \mathrm{H}_{49}{ }^{79} \mathrm{BrNO}_{7} \mathrm{Si}[\mathrm{M}+\mathrm{H}]^{+}:$774.2456, found: 774.2460 .

Data for 464a: $\mathrm{R}_{\mathrm{f}}(30 \%$ EtOAc in hexane $)=0.35 ;[\alpha]_{\mathrm{D}}^{22}+4.4\left(c 0.905, \mathrm{CHCl}_{3}\right)$; IR (film) 2931, 2857, 2360, $1745(\mathrm{C}=\mathrm{O}), 1721(\mathrm{C}=\mathrm{O}), 1660(\mathrm{C}=\mathrm{O}), 1513,1247$, $1108,701,505$ (C-Br) cm ${ }^{-1} ;{ }^{1} \mathrm{H}$ NMR (250 MHz, $\left.\mathrm{CDCl}_{3}\right) \delta$ 7.70-7.64 (4H, m, ArH), $7.51-7.35(11 \mathrm{H}, \mathrm{m}, \mathrm{ArH}), 7.24(2 \mathrm{H}, \mathrm{d}, J=8.6 \mathrm{~Hz}, \operatorname{ArH}), 6.94(2 \mathrm{H}, \mathrm{d}, J=8.6 \mathrm{~Hz}$, $\mathrm{ArH}), 5.12\left(2 \mathrm{H}, \mathrm{d}, J=18.7 \mathrm{~Hz}, \mathrm{OCH}_{2} \mathrm{Ph}\right), 4.98(1 \mathrm{H}, \mathrm{dd}, J=8.7,5.6 \mathrm{~Hz}, \mathrm{CHBr})$, $4.23\left(2 \mathrm{H}, \mathrm{s}, \mathrm{NHCH}_{2} \mathrm{Ar}\right), 3.93\left(2 \mathrm{H}, \mathrm{s}, \mathrm{CH}_{2} \mathrm{OBn}\right), 3.88-3.84\left(2 \mathrm{H}, \mathrm{m}, \mathrm{OCH}_{2} \mathrm{CH}_{2}\right), 3.83$ $\left(3 \mathrm{H}, \mathrm{s}, \mathrm{OCH}_{3}\right), 3.81\left(3 \mathrm{H}, \mathrm{s}, \mathrm{OCH}_{3}\right), 2.49\left(3 \mathrm{H}, \mathrm{s}, \mathrm{CH}_{3} \mathrm{C}=\mathrm{O}\right), 2.42-2.23(2 \mathrm{H}, \mathrm{m}$, $\left.\mathrm{CHBrCH}_{2}\right), 0.99\left(9 \mathrm{H}, \mathrm{s}, \mathrm{C}\left(\mathrm{CH}_{3}\right)_{3}\right) ;{ }^{13} \mathrm{C} \mathrm{NMR}\left(62.9 \mathrm{MHz}, \mathrm{CDCl}_{3}\right) \delta 197.5(\mathrm{C}), 171.7$ (C), 167.6 (C), 158.7 (C), 136.5 (C), 135.3 (4 x CH), 133.3 (C), 133.1 (C), 129.5 (2 x $\mathrm{CH}), 129.3(2 \times \mathrm{C}), 128.3(2 \times \mathrm{CH}), 127.8(\mathrm{CH}), 127.6(4 \times \mathrm{CH}), 127.5(2 \times \mathrm{CH})$, $126.7(2 \times \mathrm{CH}), 114.1(2 \times \mathrm{CH}), 73.7\left(\mathrm{CH}_{2}\right), 70.1\left(\mathrm{CH}_{2}\right), 60.4\left(\mathrm{CH}_{2}\right), 55.1\left(\mathrm{CH}_{3}\right)$, $52.7\left(\mathrm{CH}_{3}\right), 49.2\left(\mathrm{CH}_{2}\right), 41.6(\mathrm{CH}), 37.7\left(\mathrm{CH}_{2}\right), 27.7\left(\mathrm{CH}_{3}\right), 26.6\left(3 \times \mathrm{CH}_{3}\right), 18.9(\mathrm{C})$; HRMS (ES) Exact mass calcd for $\mathrm{C}_{41} \mathrm{H}_{49}{ }^{79} \mathrm{BrNO}_{7} \mathrm{Si}[\mathrm{M}+\mathrm{H}]^{+}:$774.2456, found: 774.2457 .

4-Hydroxybutyl acetate $(465)^{193}$

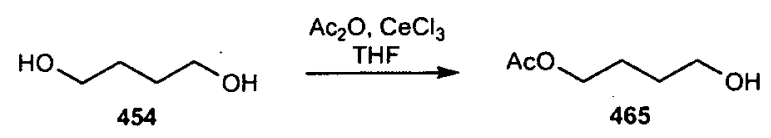


To a stirred solution of 1,4-butanediol $(5.30 \mathrm{~mL}, 60.0 \mathrm{mmol})$ and $\mathrm{CeCl}_{3} \cdot 7 \mathrm{H}_{2} \mathrm{O}$ $(2.2 \mathrm{~g}, 6.0 \mathrm{mmol})$ in THF $(170 \mathrm{~mL})$ at room temperature was added acetic anhydride $(57.0 \mathrm{~mL}, 600 \mathrm{mmol})$. The reaction was stirred for $16 \mathrm{~h}$, diluted with $\mathrm{Et}_{2} \mathrm{O}(100 \mathrm{~mL})$ and then washed with a saturated aqueous $\mathrm{NaHCO}_{3}$ solution $(2 \times 60 \mathrm{~mL})$ and with saturated $\mathrm{NaCl}$ aqueous solution $(1 \times 60 \mathrm{~mL})$. The combined organic layers were dried $\left(\mathrm{MgSO}_{4}\right)$, filtered and concentrated in vacuo. Purification of the residue by column chromatography (30\% EtOAc/hexane) gave monoacetate 465 (3.35 g, 43\%) as a colourless oil. $\mathrm{R}_{\mathrm{f}}(30 \%$ EtOAc in hexane $)=0.20 ;{ }^{1} \mathrm{H} \mathrm{NMR}\left(250 \mathrm{MHz}, \mathrm{CDCl}_{3}\right) \delta$ $4.09\left(2 \mathrm{H}, \mathrm{d}, J=6.3 \mathrm{~Hz}, \mathrm{CH}_{2} \mathrm{OAc}\right), 3.67\left(2 \mathrm{H}, \mathrm{d}, J=6.3 \mathrm{~Hz}, \mathrm{CH}_{2} \mathrm{OH}\right), 2.04(3 \mathrm{H}, \mathrm{s}$, $\left.\mathrm{CH}_{3} \mathrm{C}=\mathrm{O}\right), 1.79(1 \mathrm{H}, \mathrm{bs}, \mathrm{OH}), 1.78-1.58\left(4 \mathrm{H}, \mathrm{m}, \mathrm{CH}_{2} \mathrm{CH}_{2}\right) ;{ }^{13} \mathrm{C} \mathrm{NMR}(62.9 \mathrm{MHz}$, $\left.\mathrm{CDCl}_{3}\right) \delta 171.2(\mathrm{C}), 64.2\left(\mathrm{CH}_{2}\right), 62.3\left(\mathrm{CH}_{2}\right), 29.1\left(\mathrm{CH}_{2}\right), 25.0\left(\mathrm{CH}_{2}\right), 21.0\left(\mathrm{CH}_{3}\right)$.

${ }^{1} \mathrm{H}$ and ${ }^{13} \mathrm{C}$ spectroscopic data in good agreement with the literature. ${ }^{193}$

\section{4-Oxobutyl acetate $(466)^{194}$}

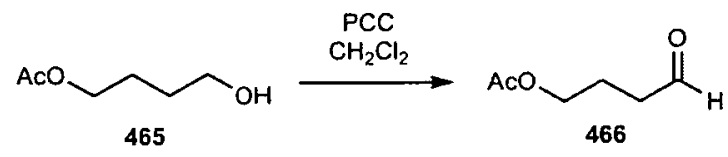

To a stirred solution of PCC $(8.20 \mathrm{~g}, 38.0 \mathrm{mmol})$ in $\mathrm{CH}_{2} \mathrm{Cl}_{2}(70 \mathrm{~mL})$ at room temperature was added a solution of monoalcohol acetate $465(3.35 \mathrm{~g}, 25.3 \mathrm{mmol})$ in $\mathrm{CH}_{2} \mathrm{Cl}_{2}(7 \mathrm{~mL})$ via cannula. The reaction was stirred for $1.5 \mathrm{~h}$, when $\mathrm{Et}_{2} \mathrm{O}(70 \mathrm{~mL})$ was added. The solution was filtered through celite and the organics were concentrated in vacuo. Purification of the residue by column chromatography $(10 \%$ to $30 \%$ EtOAc/hexane) gave aldehyde $466(2.13 \mathrm{~g}, 65 \%)$ as a colourless oil. $\mathrm{R}_{\mathrm{f}}(30 \%$ EtOAc in hexane $)=0.47 ;{ }^{1} \mathrm{H}$ NMR $\left(360 \mathrm{MHz}, \mathrm{CDCl}_{3}\right) \delta 9.77(1 \mathrm{H}, \mathrm{t}, J=1.3 \mathrm{~Hz}$, CHO), $4.10\left(2 \mathrm{H}, \mathrm{dt}, J=6.3,4.8 \mathrm{~Hz}, \mathrm{AcOCH}_{2}\right), 2.54(1 \mathrm{H}, \mathrm{td}, J=7.1,1.3 \mathrm{~Hz}$, $\mathrm{CH}_{2} \mathrm{CH}_{\mathrm{A}} \mathrm{H}_{\mathrm{B}} \mathrm{CHO}$ ), 2.43 (1H, td, $J=14.0,7.1 \mathrm{~Hz}, \mathrm{CH}_{2} \mathrm{CH}_{\mathrm{A}} \mathbf{H}_{\mathrm{B}} \mathrm{CHO}$ ), 2.18 (3H, s, $\left.\mathrm{CH}_{3} \mathrm{C}=\mathrm{O}\right), 2.17-2.05\left(2 \mathrm{H}, \mathrm{m}, \mathrm{CH}_{2} \mathrm{CH}_{2} \mathrm{CH}_{2}\right) ;{ }^{13} \mathrm{C} \mathrm{NMR}\left(62.9 \mathrm{MHz}, \mathrm{CDCl}_{3}\right) \delta 201.2$ $(\mathrm{CH}), 170.9(\mathrm{C}), 63.4\left(\mathrm{CH}_{2}\right), 40.4\left(\mathrm{CH}_{2}\right), 21.3\left(\mathrm{CH}_{2}\right), 20.8\left(\mathrm{CH}_{3}\right)$.

${ }^{1} \mathrm{H}$ spectroscopic data in good agreement with the literature. ${ }^{194}$ 


\section{4-Acetyloxy-2-bromobutanal (467) ${ }^{195}$}

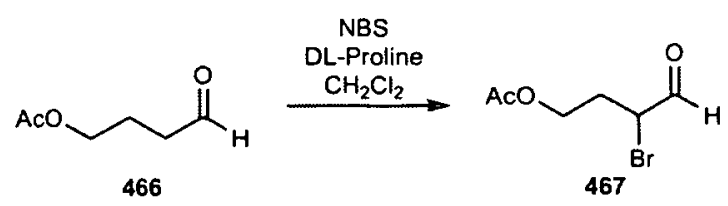

To a stirred solution of aldehyde $466(2.40 \mathrm{~g}, 18.4 \mathrm{mmol})$ in $\mathrm{CH}_{2} \mathrm{Cl}_{2}(25 \mathrm{~mL})$ at $0{ }^{\circ} \mathrm{C}$ was added $D L$-proline $(640 \mathrm{mg}, 5.53 \mathrm{mmol})$, followed by the addition of NBS $(4.60 \mathrm{~g}, 25.8 \mathrm{mmol})$. The reaction was stirred for 30 minutes at $0^{\circ} \mathrm{C}$ and then allowed to warm up to room temperature and stirred for another $4 \mathrm{~h}$. Hexane was added to the reaction mixture and the precipitated NBS, succinimide and catalyst were filtered off. The solvents were concentrated in vacuo and purification of the residue by column chromatography (15\% to $20 \%$ EtOAc/hexane) gave $\alpha$-bromoaldehyde 467 (2.92 g, $75 \%)$ as a colourless oil. $\mathrm{R}_{\mathrm{f}}(30 \%$ EtOAc in hexane $)=0.30 ;{ }^{1} \mathrm{H}$ NMR $(360 \mathrm{MHz}$, $\left.\mathrm{CDCl}_{3}\right) \delta 9.47(1 \mathrm{H}, \mathrm{d}, J=2.0 \mathrm{~Hz}, \mathrm{CHO}), 4.42-4.34(1 \mathrm{H}, \mathrm{m}, \mathrm{CHBrCHO}), 4.31-3.98$ $\left(2 \mathrm{H}, \mathrm{m}, \mathrm{OCH}_{2} \mathrm{CH}_{2}\right), 2.48-2.31\left(1 \mathrm{H}, \mathrm{m}, \mathrm{CH}_{2} \mathrm{CH}_{\mathrm{A}} \mathrm{H}_{\mathrm{B}} \mathrm{CHBr}\right), 2.24-2.07(1 \mathrm{H}, \mathrm{m}$, $\left.\mathrm{CH}_{2} \mathrm{CH}_{\mathrm{A}} \mathbf{H}_{\mathrm{B}} \mathrm{CHBr}\right), 2.04\left(3 \mathrm{H}, \mathrm{s}, \mathrm{CH}_{3} \mathrm{C}=\mathrm{O}\right) ;{ }^{13} \mathrm{C}$ NMR $\left(62.9 \mathrm{MHz}, \mathrm{CDCl}_{3}\right) \delta 191.7$ $(\mathrm{CH}), 176.3(\mathrm{C}), 61.0\left(\mathrm{CH}_{2}\right), 51.3(\mathrm{CH}), 30.5\left(\mathrm{CH}_{2}\right), 20.6\left(\mathrm{CH}_{3}\right)$.

${ }^{1} \mathrm{H}$ spectroscopic data in good agreement with the literature. ${ }^{195}$

\section{4-Acetyloxy-2-bromobutanoic acid (468)}

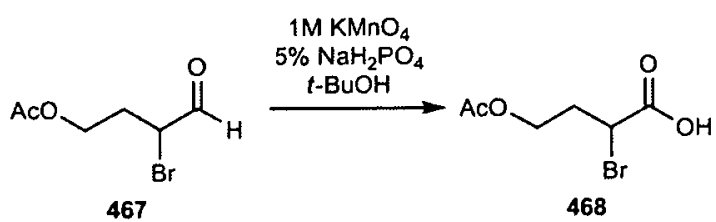

To a solution of $\alpha$-bromoaldehyde $467(500 \mathrm{mg}, 2.38 \mathrm{mmol})$ in $t$-BuOH (14 mL) and $5 \% \mathrm{NaH}_{2} \mathrm{PO}_{4}$ solution $(9.5 \mathrm{~mL})$ at room temperature was added $\mathrm{KMNO}_{4}(14$ $\mathrm{mL}, 1 \mathrm{M}$ solution). The reaction was stirred for $3 \mathrm{~h}$ and then quenched by the addition of solid $\mathrm{Na}_{2} \mathrm{SO}_{3}$ until the solution turned dark brown from the original purple colour. $1 \mathrm{M} \mathrm{HCl}(10 \mathrm{~mL})$ was added to the reaction mixture until acidic $\mathrm{pH}$. The reaction was then extracted with $\mathrm{Et}_{2} \mathrm{O}(4 \times 20 \mathrm{~mL})$ and the combined organic layers were dried $\left(\mathrm{MgSO}_{4}\right)$, filtered and concentrated in vacuo. Purification of the residue by column chromatography ( $15 \%$ to $20 \%$ EtOAc/petrol) gave $\alpha$-bromoacid $468(386 \mathrm{mg}, 70 \%)$ as a colourless oil. $\mathrm{R}_{\mathrm{f}}(30 \%$ EtOAc in hexane $)=0.12 ; \mathrm{IR}$ (film) 
$2967(\mathrm{OH}), 1738(2 \times \mathrm{C}=\mathrm{O}), 1428,1390,1368,1244,1040,806,666,607,503$ $(\mathrm{C}-\mathrm{Br}) \mathrm{cm}^{-1} ;{ }^{1} \mathrm{H}$ NMR $\left(360 \mathrm{MHz}, \mathrm{CDCl}_{3}\right) \delta 10.3(1 \mathrm{H}, \mathrm{bs}, \mathrm{OH}), 4.40(1 \mathrm{H}, \mathrm{dd}, J=8.3$, $\left.6.1 \mathrm{~Hz}, \mathrm{CHBrCO}_{2} \mathrm{H}\right), 4.29\left(1 \mathrm{H}, \mathrm{td}, J=10.9,5.4 \mathrm{~Hz}, \mathrm{AcOCH}_{\mathrm{A}} \mathrm{H}_{\mathrm{B}}\right), 4.20(1 \mathrm{H}$, ddd, $J$ $\left.=11.6,8.1,4.7 \mathrm{~Hz}, \mathrm{AcOCH}_{\mathrm{A}} \mathbf{H}_{\mathbf{B}}\right), 2.51-2.41\left(1 \mathrm{H}, \mathrm{m}, \mathrm{CH}_{2} \mathrm{CH}_{\mathrm{A}} \mathrm{H}_{\mathrm{B}} \mathrm{CHBr}\right), 2.34-2.25$ $\left(1 \mathrm{H}, \mathrm{m}, \mathrm{CH}_{2} \mathrm{CH}_{\mathrm{A}} \mathbf{H}_{\mathbf{B}} \mathrm{CHBr}\right), 2.07\left(3 \mathrm{H}, \mathrm{s}, \mathrm{CH}_{3} \mathrm{C}=\mathrm{O}\right) ;{ }^{13} \mathrm{C} \mathrm{NMR}\left(62.9 \mathrm{MHz}, \mathrm{CDCl}_{3}\right) \delta$ $173.9(\mathrm{C}), 171.2(\mathrm{C}), 61.4\left(\mathrm{CH}_{2}\right), 41.5(\mathrm{CH}), 33.5\left(\mathrm{CH}_{2}\right), 20.7\left(\mathrm{CH}_{3}\right) ; \mathrm{HRMS}(\mathrm{EI})$ Exact mass calcd for $\mathrm{C}_{6} \mathrm{H}_{9}{ }^{79} \mathrm{BrO}_{4}[\mathrm{M}]^{+}:$223.9679, found: 223.9677 .

\section{4-(tert-Butyldiphenylsilyl)oxy- $N$-(4-methoxybenzyl)butanamide (470)}

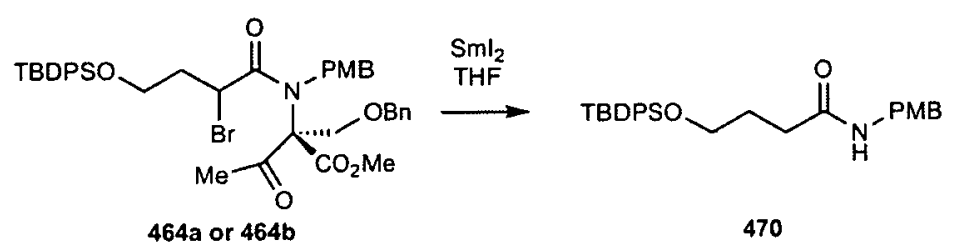

To a stirred solution of amide $464 \mathrm{a}$ or $464 \mathrm{~b}(28 \mathrm{mg}, 36 \mu \mathrm{mol})$ in THF $(1 \mathrm{~mL})$ at $0{ }^{\circ} \mathrm{C}$, was added $\mathrm{SmI}_{2}(3.6 \mathrm{~mL}, 0.1 \mathrm{M}$ solution in hexanes, $0.36 \mathrm{mmol})$. The reaction was stirred at $0{ }^{\circ} \mathrm{C}$ for $1 \mathrm{~h}$ and then quenched with saturated aqueous $\mathrm{NH}_{4} \mathrm{Cl}$ solution $(3 \mathrm{~mL})$. The aqueous layer was separated and extracted with EtOAc $(2 \times 5 \mathrm{~mL})$ and the combined organic layers were dried $\left(\mathrm{Na}_{2} \mathrm{SO}_{4}\right)$, filtered and concentrated in vacuo. Purification of the residue by column chromatography (10\% to $15 \%$ EtOAc/petrol) gave amide $470(10 \mathrm{mg}, 40 \%)$ as a pale yellow oil. $\mathrm{R}_{\mathrm{f}}(30 \%$ EtOAc in hexane $)=$ 0.39; IR (film) 3287 (NH), 2930, 2857, 1644 (C=O), 1512, 1428, 1248, 1110, 822, $701 \mathrm{~cm}^{-1}$; ' $\mathrm{H}$ NMR $\left(250 \mathrm{MHz}, \mathrm{CDCl}_{3}\right) \delta$ 7.67-7.61 (4H, m, ArH), 7.44-7.33 (6H, m, $\mathrm{ArH}), 7.18(2 \mathrm{H}, \mathrm{d}, J=8.7 \mathrm{~Hz}, \operatorname{ArH}), 6.85(2 \mathrm{H}, \mathrm{d}, J=8.7 \mathrm{~Hz}, \operatorname{ArH}), 5.77(1 \mathrm{H}, \mathrm{bs}$, $\mathrm{NH}), 4.34\left(2 \mathrm{H}, \mathrm{d}, J=5.6 \mathrm{~Hz}, \mathrm{NHCH}_{2} \mathrm{Ar}\right), 3.83\left(3 \mathrm{H}, \mathrm{s}, \mathrm{OCH}_{3}\right), 3.71(2 \mathrm{H}, \mathrm{t}, J=5.9$ $\left.\mathrm{Hz}, \mathrm{OCH}_{2} \mathrm{CH}_{2}\right), 2.35\left(2 \mathrm{H}, \mathrm{t}, J=7.3 \mathrm{~Hz}, \mathrm{CH}_{2} \mathrm{C}=\mathrm{O}\right), 1.96-1.85\left(2 \mathrm{H}, \mathrm{m}, \mathrm{CH}_{2} \mathrm{CH}_{2} \mathrm{CH}_{2}\right)$; ${ }^{13} \mathrm{C}$ NMR (62.9 MHz, $\left.\mathrm{CDCl}_{3}\right) \delta 172.5$ (C), 158.9 (C), 135.4 (4 x CH), 133.6 (2 x C), $130.4(\mathrm{C}), 129.6(2 \times \mathrm{CH}), 129.1(2 \times \mathrm{CH}), 127.6(4 \times \mathrm{CH}), 114.0(2 \times \mathrm{CH}), 63.0$ $\left(\mathrm{CH}_{2}\right), 55.2\left(\mathrm{CH}_{3}\right), 43.0\left(\mathrm{CH}_{2}\right), 33.1\left(\mathrm{CH}_{2}\right), 28.4\left(\mathrm{CH}_{2}\right), 26.8\left(3 \times \mathrm{CH}_{3}\right), 19.2(\mathrm{C})$; HRMS (EI) Exact mass calcd for $\mathrm{C}_{28} \mathrm{H}_{35} \mathrm{NO}_{3} \mathrm{Si}[\mathrm{M}]^{+}: 461.2381$, found: 461.2382 . 


\subsubsection{Formal synthesis}

$(R)$-Ethyl (E)-3-[N-(1-benzyloxymethyl-1-carbomethoxy-2-oxopropyl)- $N$-(4methoxybenzyl)carbamoyl]acrylate (481)

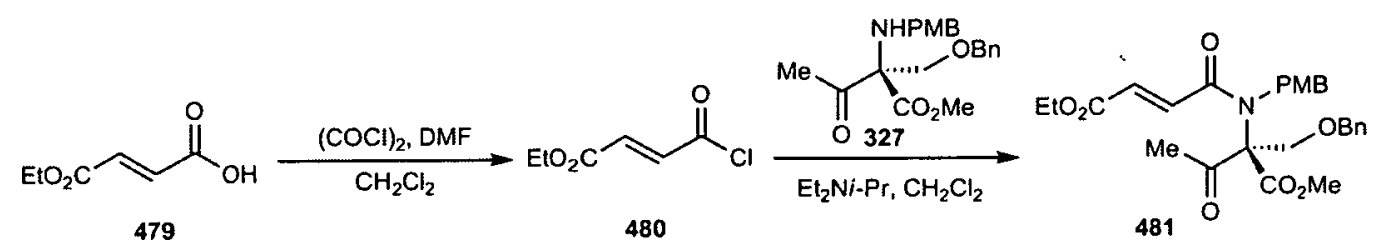

To a stirred solution of fumaric acid monoethyl ester $(858 \mathrm{mg}, 5.65 \mathrm{mmol}) \mathrm{in}$ $\mathrm{CH}_{2} \mathrm{Cl}_{2}(10 \mathrm{~mL})$ at $0{ }^{\circ} \mathrm{C}$ was added oxalyl chloride $(0.56 \mathrm{~mL}, 6.40 \mathrm{mmol})$ followed by DMF (one drop). This solution was stirred at room temperature for $1 \mathrm{~h}$, and then transferred via cannula to a solution of the amine $327(1.34 \mathrm{~g}, 3.60 \mathrm{mmol})$ and diisopropylethyl amine $(1.10 \mathrm{~mL}, 6.40 \mathrm{mmol})$ in $\mathrm{CH}_{2} \mathrm{Cl}_{2}(10 \mathrm{~mL})$. The reaction mixture was stirred at $0{ }^{\circ} \mathrm{C}$ for $10 \mathrm{~min}$ and then at room temperature for $18 \mathrm{~h}$, when it was quenched with saturated aqueous $\mathrm{NH}_{4} \mathrm{Cl}$ solution $(20 \mathrm{~mL})$. The aqueous layer was separated and extracted with $\mathrm{CH}_{2} \mathrm{Cl}_{2}(3 \times 20 \mathrm{~mL})$, and the combined organic layers were dried $\left(\mathrm{MgSO}_{4}\right)$, filtered and concentrated in vacuo. Purification of the residue by column chromatography (20\% EtOAc/petrol) gave amide 481 (1.7 g, $95 \%)$ as a pale yellow oil. $\mathrm{R}_{\mathrm{f}}(30 \%$ EtOAc in hexane $)=0.45 ;[\alpha]_{\mathrm{D}}^{22}-18.2(c 1.18$, $\mathrm{CHCl}_{3}$ ); IR (film) 2953, 2837, $1722(\mathrm{C}=\mathrm{O}), 1658(\mathrm{C}=\mathrm{O}), 1513,1408,1294,1175$, 1032, $974 \mathrm{~cm}^{-1}$; ${ }^{1} \mathrm{H}$ NMR (360 MHz, CDCl3) $\delta$ 7.35-7.27 (5H, m, ArH), $7.22(1 \mathrm{H}, \mathrm{d}$, $J=15.3 \mathrm{~Hz},=\mathrm{CH}), 7.17-7.12(2 \mathrm{H}, \mathrm{m}, \mathrm{ArH}), 6.95-6.89(2 \mathrm{H}, \mathrm{dm}, J=8.8 \mathrm{~Hz}, \operatorname{ArH})$, $6.80(1 \mathrm{H}, \mathrm{d}, J=15.3 \mathrm{~Hz},=\mathrm{CH}), 4.95\left(1 \mathrm{H}, \mathrm{d}, J=18.4 \mathrm{~Hz}, \mathrm{OCH}_{\mathrm{A}} \mathrm{H}_{\mathrm{B}} \mathrm{Ar}\right), 4.81(1 \mathrm{H}, \mathrm{d}$, $\left.J=18.4 \mathrm{~Hz}, \mathrm{OCH}_{\mathrm{A}} \mathrm{H}_{\mathrm{B}} \mathrm{Ar}\right), 4.32\left(1 \mathrm{H}, \mathrm{d}, J=11.9 \mathrm{~Hz}, \mathrm{NCH}_{\mathrm{C}} \mathrm{H}_{\mathrm{D}} \mathrm{Ar}\right), 4.28(1 \mathrm{H}, \mathrm{d}, J=$ $\left.11.9 \mathrm{~Hz}, \mathrm{NCH}_{\mathrm{C}} \mathbf{H}_{\mathbf{D}} \mathrm{Ar}\right), 4.19\left(2 \mathrm{H}, \mathrm{q}, J=7.1 \mathrm{~Hz}, \mathrm{OCH}_{2} \mathrm{CH}_{3}\right), 3.83\left(3 \mathrm{H}, \mathrm{s}, \mathrm{OCH}_{3}\right), 3.80$ (3H, s, OCH $), 3.78\left(2 \mathrm{H}, \mathrm{s}, \mathrm{CH}_{2} \mathrm{OBn}\right), 2.45\left(3 \mathrm{H}, \mathrm{s}, \mathrm{CH}_{3} \mathrm{C}=\mathrm{O}\right), 1.26(3 \mathrm{H}, \mathrm{t}, J=7.1$ $\left.\mathrm{Hz}, \mathrm{OCH}_{2} \mathrm{CH}_{3}\right) ;{ }^{13} \mathrm{C} \mathrm{NMR}\left(62.9 \mathrm{MHz}, \mathrm{CDCl}_{3}\right) \delta 197.6(\mathrm{C}), 168.0(\mathrm{C}), 167.5(\mathrm{C})$, $165.2(\mathrm{C}), 158.9(\mathrm{C}), 136.5(\mathrm{C}), 133.3(\mathrm{CH}), 133.1(\mathrm{CH}), 129.7(\mathrm{C}), 128.4(2 \times \mathrm{CH})$, $127.9(\mathrm{CH}), 127.5(2 \times \mathrm{CH}), 127.1(2 \times \mathrm{CH}) 114.2(2 \times \mathrm{CH}), 77.5(\mathrm{C}), 73.7\left(\mathrm{CH}_{2}\right)$, $70.1\left(\mathrm{CH}_{2}\right), 61.1\left(\mathrm{CH}_{2}\right), 55.2\left(\mathrm{CH}_{3}\right), 52.9\left(\mathrm{CH}_{3}\right), 48.9\left(\mathrm{CH}_{2}\right), 27.9\left(\mathrm{CH}_{3}\right), 14.0\left(\mathrm{CH}_{3}\right)$; HRMS (ES) Exact mass calcd for $\mathrm{C}_{27} \mathrm{H}_{32} \mathrm{NO}_{8}[\mathrm{M}+\mathrm{H}]^{+}: 498.2122$, found: 498.2123 . 
(3a $R, 6 R, 6 \mathrm{a} S)-6-B e n z y l o x y m e t h y l-5-(4-m e t h o x y b e n z y l)-6 a-m e t h y l-2,4-$ dioxohexahydrofuro[2,3-c]pyrrole-6-carboxylic acid methyl ester (489) and (3aS,6R,6aR)-6-benzyloxymethyl-5-(4-methoxybenzyl)-6a-methyl-2,4dioxohexahydrofuro[2,3-c]pyrrole-6-carboxylic acid methyl ester (490)

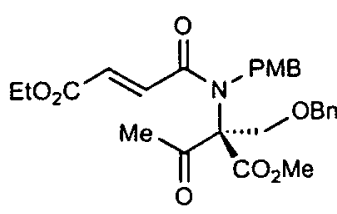

481

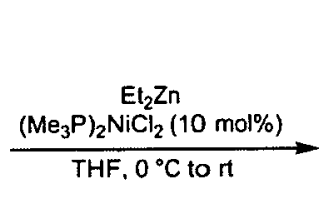

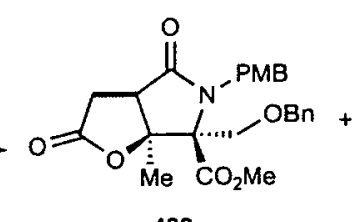

489

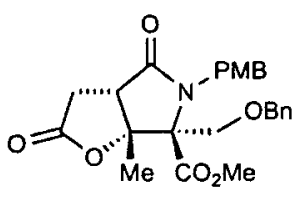

490

A solution of amide $481(875 \mathrm{mg}, 1.75 \mathrm{mmol})$ and $\left(\mathrm{Me}_{3} \mathrm{P}\right)_{2} \mathrm{NiCl}_{2}(115 \mathrm{mg}, 0.175$ $\mathrm{mmol})$ in THF $(75 \mathrm{~mL})$ was stirred at room temperature for $30 \mathrm{~min}$ and then cooled to $-15^{\circ} \mathrm{C}$ (ice-salt bath). $\mathrm{Et}_{2} \mathrm{Zn}(3.52 \mathrm{~mL}, 1 \mathrm{M}$ solution in THF, $3.52 \mathrm{mmol}$ ) was then added via syringe. The reaction was allowed to warm slowly to room temperature over $18 \mathrm{~h}$, and then quenched carefully with saturated aqueous $\mathrm{NH}_{4} \mathrm{Cl}$ solution (50 $\mathrm{mL})$ and extracted with EtOAc $(3 \times 30 \mathrm{~mL})$. The combined organic layers were dried $\left(\mathrm{MgSO}_{4}\right)$ and concentrated in vacuo. Purification of the residue by column chromatography (20\% to $35 \%$ EtOAc/petrol) gave the double cyclization product 489 (354 $\mathrm{mg}, 42 \%$ ) as a pale yellow oil, followed by the double cyclization product 490 (253 mg, 30\%) as a colourless solid. Recrystallization of 490 from EtOAc/petrol gave colourless crystals that were suitable for X-ray crystallography.

Data for desired 489: $\mathrm{R}_{\mathrm{f}}\left(30 \% \mathrm{EtOAc}\right.$ in $\left.\mathrm{CHCl}_{3}\right)=0.19 ;[\alpha]_{\mathrm{D}}^{22}-33.3\left(c 1.02, \mathrm{CHCl}_{3}\right)$; IR (film) 2955, $1789(\mathrm{C}=\mathrm{O}), 1733(\mathrm{C}=\mathrm{O}), 1699(\mathrm{C}=\mathrm{O}), 1612,1513,1439,1404$, $1248,1178 \mathrm{~cm}^{-1} ;{ }^{1} \mathrm{H}$ NMR $\left(250 \mathrm{MHz} \mathrm{CDCl}_{3}\right) \delta 7.27-7.18(3 \mathrm{H}+2 \mathrm{H}, \mathrm{m}, \mathrm{ArH}), 7.04-$ $6.99(2 \mathrm{H}, \mathrm{m}, \operatorname{ArH}), 6.69(2 \mathrm{H}, \mathrm{d}, J=8.7 \mathrm{~Hz}, \operatorname{ArH}), 4.97(1 \mathrm{H}, \mathrm{d}, J=15.2 \mathrm{~Hz}$, $\left.\mathrm{OCH}_{\mathrm{A}} \mathrm{H}_{\mathrm{B}} \mathrm{Ph}\right), 4.21\left(1 \mathrm{H}, \mathrm{d}, J=15.2 \mathrm{~Hz}, \mathrm{OCH}_{\mathrm{A}} \mathbf{H}_{\mathrm{B}} \mathrm{Ph}\right), 3.83(1 \mathrm{H}, \mathrm{d}, J=11.5 \mathrm{~Hz}$, $\left.\mathrm{NCH}_{\mathrm{C}} \mathrm{H}_{\mathrm{D}} \mathrm{Ar}\right), 3.75\left(1 \mathrm{H}, \mathrm{d}, J=10.4 \mathrm{~Hz}, \mathrm{CH}_{\mathrm{E}} \mathrm{H}_{\mathrm{F}} \mathrm{OBn}\right), 3.73(1 \mathrm{H}, \mathrm{d}, J=11.5 \mathrm{~Hz}$, $\left.\mathrm{NCH}_{\mathrm{C}} \mathbf{H}_{\mathrm{D}} \mathrm{Ar}\right), 3.73\left(3 \mathrm{H}, \mathrm{s}, \mathrm{OCH}_{3}\right), 3.66\left(3 \mathrm{H}, \mathrm{s}, \mathrm{OCH}_{3}\right), 3.13(1 \mathrm{H}, \mathrm{d}, J=10.4 \mathrm{~Hz}$, $\left.\mathrm{CH}_{\mathrm{E}} \mathrm{H}_{\mathbf{F}} \mathrm{OBn}\right), 2.97\left(1 \mathrm{H}, \mathrm{dd}, J=9.3,1.5 \mathrm{~Hz}, \mathrm{CH}_{\mathrm{G}} \mathrm{H}_{\mathrm{H}} \mathrm{CH}\right), 2.88(1 \mathrm{H}, \mathrm{dd}, J=18.3,1.5$ $\left.\mathrm{Hz}, \mathrm{CH}_{\mathbf{G}} \mathrm{H}_{\mathrm{H}} \mathrm{CH}\right), 2.72\left(1 \mathrm{H}, \mathrm{dd}, J=18.3,9.3 \mathrm{~Hz}, \mathrm{CH}_{\mathrm{G}} \mathbf{H}_{\mathrm{H}} \mathrm{CH}\right), 1.53\left(3 \mathrm{H}, \mathrm{s}, \mathrm{CH}_{3}\right) ;{ }^{13} \mathrm{C}$ NMR (62.9 MHz, $\left.\mathrm{CDCl}_{3}\right) \delta 173.5$ (C), 172.9 (C), 166.9 (C), 159.0 (C), 136.7 (C) $130.0(2 \times \mathrm{CH}), 129.4(\mathrm{C}), 128.4(2 \times \mathrm{CH}), 127.9(\mathrm{CH}), 127.3(2 \times \mathrm{CH}), 113.6(2 \times$ $\mathrm{CH}), 88.4(\mathrm{C}), 75.8(\mathrm{C}), 72.8\left(\mathrm{CH}_{2}\right), 67.7\left(\mathrm{CH}_{2}\right), 55.2\left(\mathrm{CH}_{3}\right), 52.8\left(\mathrm{CH}_{3}\right), 47.5(\mathrm{CH})$, 
$45.1\left(\mathrm{CH}_{2}\right), 30.7\left(\mathrm{CH}_{2}\right), 19.4\left(\mathrm{CH}_{3}\right)$; HRMS (ES) Exact mass calcd for $\mathrm{C}_{25} \mathrm{H}_{28} \mathrm{NO}_{7}$ $[\mathrm{M}+\mathrm{H}]^{+}: 454.1860$, found: 454.1856 .

Data for undesired 490: $\mathrm{R}_{\mathrm{f}}\left(30 \%\right.$ EtOAc in $\left.\mathrm{CHCl}_{3}\right)=0.08 ; \mathrm{m} . \mathrm{p} .=105^{\circ} \mathrm{C} ;[\alpha]_{\mathrm{D}}^{22}-17.9$ (c 1.01, $\left.\mathrm{CHCl}_{3}\right)$; IR (film) 2952, 2870, $1789(\mathrm{C}=\mathrm{O}), 1743(\mathrm{C}=\mathrm{O}), 1703(\mathrm{C}=\mathrm{O}), 1613$, 1512, 1434, 1246, $1128 \mathrm{~cm}-1$; ' $\mathrm{H}$ NMR $\left(250 \mathrm{MHz}, \mathrm{CDCl}_{3}\right) \delta 7.38-7.21(5 \mathrm{H}, \mathrm{m}$, ArH), $7.12(2 \mathrm{H}, \mathrm{d}, J=8.7 \mathrm{~Hz}, \operatorname{ArH}), 6.82(2 \mathrm{H}, \mathrm{d}, J=8.7 \mathrm{~Hz}, \operatorname{ArH}), 4.72(1 \mathrm{H}, \mathrm{d}, J=$ $\left.15.2 \mathrm{~Hz}, \mathrm{OCH}_{\mathrm{A}} \mathrm{H}_{\mathrm{B}} \mathrm{Ph}\right), 4.40\left(1 \mathrm{H}, \mathrm{d}, J=15.2 \mathrm{~Hz}, \mathrm{OCH}_{\mathrm{A}} \mathbf{H}_{\mathrm{B}} \mathrm{Ph}\right), 4.34(1 \mathrm{H}, \mathrm{d}, J=11.7$ $\left.\mathrm{Hz}, \mathrm{NCH}_{\mathrm{C}} \mathrm{H}_{\mathrm{D}} \mathrm{Ar}\right), 4.27\left(1 \mathrm{H}, \mathrm{d}, J=11.7 \mathrm{~Hz}, \mathrm{NCH}_{\mathrm{C}} \mathbf{H}_{\mathbf{D}} \mathrm{Ar}\right), 3.94(1 \mathrm{H}, \mathrm{d}, J=10.1 \mathrm{~Hz}$, $\left.\mathrm{CH}_{\mathbf{E}} \mathrm{H}_{\mathrm{F}} \mathrm{OBn}\right), 3.82\left(3 \mathrm{H}, \mathrm{s}, \mathrm{OCH}_{3}\right), 3.74\left(1 \mathrm{H}, \mathrm{d}, J=10.1 \mathrm{~Hz}, \mathrm{CH}_{\mathrm{E}} \mathbf{H}_{\mathbf{F}} \mathrm{OBn}\right), 3.67(3 \mathrm{H}$, $\left.\mathrm{s}, \mathrm{OCH}_{3}\right), 3.07\left(1 \mathrm{H}, \mathrm{dd}, J=9.7,1.8 \mathrm{~Hz}, \mathrm{CH}_{\mathrm{G}} \mathrm{H}_{\mathrm{H}} \mathrm{CH}\right), 3.04(1 \mathrm{H}, \mathrm{dd}, J=18.3,1.9 \mathrm{~Hz}$, $\left.\mathrm{CH}_{\mathrm{G}} \mathrm{H}_{\mathrm{H}} \mathrm{CH}\right), 2.88\left(1 \mathrm{H}, \mathrm{dd}, J=18.3,9.7 \mathrm{~Hz}, \mathrm{CH}_{\mathrm{G}} \mathbf{H}_{\mathbf{H}} \mathrm{CH}\right), 1.51\left(3 \mathrm{H}, \mathrm{s}, \mathrm{CH}_{3}\right) ;{ }^{13} \mathrm{C}$ NMR (62.9 MHz, $\left.\mathrm{CDCl}_{3}\right) \delta 173.4$ (C), 173.2 (C), 169.2 (C) 158.7 (C) 137.1 (C) $129.2(2 \times \mathrm{CH}), 129.0(\mathrm{C}), 128.3(2 \times \mathrm{CH}), 127.7(3 \times \mathrm{CH}), 113.6(2 \times \mathrm{CH}), 86.6(\mathrm{C})$, $75.3(\mathrm{C}), 73.6\left(\mathrm{CH}_{2}\right), 68.7\left(\mathrm{CH}_{2}\right), 55.2\left(\mathrm{CH}_{3}\right), 52.8\left(\mathrm{CH}_{3}\right), 46.9(\mathrm{CH}), 45.1\left(\mathrm{CH}_{2}\right)$, $30.9\left(\mathrm{CH}_{2}\right), 21.3\left(\mathrm{CH}_{3}\right)$; HRMS (ES) Exact mass calcd for $\mathrm{C}_{25} \mathrm{H}_{28} \mathrm{NO}_{7}[\mathrm{M}+\mathrm{H}]^{+}$: 454.1860, found: 454.1858 .

(3aR,6R,6aS)-6-Hydroxymethyl-5-(4-methoxybenzyl)-6a-methyl-2,4- . dioxohexahydrofuro[2,3-c]pyrrole-6-carboxylic acid methyl ester (493)

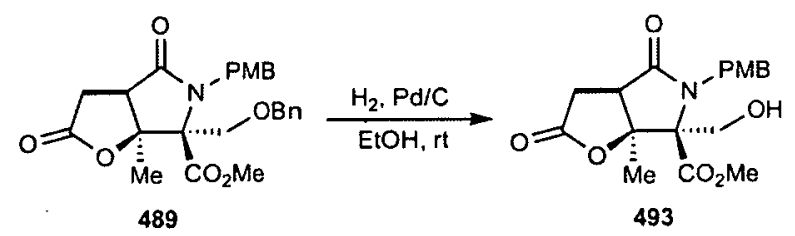

A mixture of benzyl ether $489(354 \mathrm{mg}, 0.78 \mathrm{mmol})$ and $10 \% \mathrm{Pd} / \mathrm{C}(99 \mathrm{mg}, 0.093$ mmol) in EtOH (5 mL) at room temperature was evacuated and flushed with $\mathrm{H}_{2}$ (3 times) and then stirred vigorously under an atmosphere of $\mathrm{H}_{2}\left(1 \mathrm{~atm}, \mathrm{H}_{2}\right.$ balloon) at room temperature for $18 \mathrm{~h}$. The reaction mixture was filtered through celite and concentrated in vacuo. Purification of the residue by column chromatography $(50 \%$ EtOAc/petrol) gave alcohol $493(240 \mathrm{mg}, 84 \%)$ as a white powder. $\mathrm{R}_{\mathrm{f}}(100 \%$ EtOAc) $=0.52$; m.p. $144-146^{\circ} \mathrm{C} ;[\alpha]_{\mathrm{D}}^{22}-30.1\left(c 0.95, \mathrm{CHCl}_{3}\right) ; \mathrm{IR}($ film) $3437(\mathrm{OH})$, 2955, 2253, $1787(\mathrm{C}=\mathrm{O}), 1757(\mathrm{C}=\mathrm{O}), 1692(\mathrm{C}=\mathrm{O}), 1613,1513,1247,1035$, $951 \mathrm{~cm}^{-1} ;{ }^{1} \mathrm{H}$ NMR $\left(250 \mathrm{MHz}, \mathrm{CDCl}_{3}\right) \delta 7.37(2 \mathrm{H}, \mathrm{d}, J=8.6 \mathrm{~Hz}, \operatorname{ArH}), 6.87(2 \mathrm{H}, \mathrm{d}, J$ 
$=8.6 \mathrm{~Hz}, \mathrm{ArH}), 5.22\left(1 \mathrm{H}, \mathrm{d}, J=15.2 \mathrm{~Hz}, \mathrm{NCH}_{\mathrm{A}} \mathrm{H}_{\mathrm{B}} \mathrm{Ar}\right), 4.24(1 \mathrm{H}, \mathrm{d}, J=15.2 \mathrm{~Hz}$, $\left.\mathrm{NCH}_{\mathrm{A}} \mathrm{H}_{\mathrm{B}} \mathrm{Ar}\right), 3.95\left(1 \mathrm{H}, \mathrm{dd}, J=13.0,8.9 \mathrm{~Hz}, \mathrm{CH}_{\mathrm{C}} \mathrm{H}_{\mathrm{D}} \mathrm{OH}\right), 3.83\left(3 \mathrm{H}, \mathrm{s}, \mathrm{OCH}_{3}\right), 3.80$ $\left(3 \mathrm{H}, \mathrm{s}, \mathrm{OCH}_{3}\right), 3.43\left(1 \mathrm{H}, \mathrm{dd}, J=13.0,5.5 \mathrm{~Hz}, \mathrm{CH}_{\mathrm{C}} \mathbf{H}_{\mathbf{D}} \mathrm{OH}\right), 3.04(1 \mathrm{H}, \mathrm{dd}, J=9.1,1.7$ $\left.\mathrm{Hz}, \mathrm{CH}_{\mathrm{E}} \mathrm{H}_{\mathrm{F}} \mathrm{CH}\right), 2.96\left(1 \mathrm{H}, \mathrm{dd}, J=18.1,1.7 \mathrm{~Hz}, \mathrm{CH}_{\mathrm{E}} \mathrm{H}_{\mathrm{F}} \mathrm{CH}\right), 2.85(1 \mathrm{H}, \mathrm{dd}, J=18.1$, 9.1 Hz, $\left.\mathrm{CH}_{\mathrm{E}} \mathbf{H}_{\mathrm{F}} \mathrm{CH}\right), 1.69\left(3 \mathrm{H}, \mathrm{s}, \mathrm{CH}_{3}\right) ;{ }^{13} \mathrm{C} \mathrm{NMR}\left(62.9 \mathrm{MHz}, \mathrm{CDCl}_{3}\right) \delta 173.7$ (C), 172.7 (C), 167.1 (C), 159.5 (C), $129.6(2 \times \mathrm{CH}+\mathrm{C}), 114.6(2 \times \mathrm{CH}), 88.3(\mathrm{C}), 77.3$ (C), $60.9\left(\mathrm{CH}_{2}\right), 55.3\left(\mathrm{CH}_{3}\right), 52.8\left(\mathrm{CH}_{3}\right), 47.6(\mathrm{CH}), 44.9\left(\mathrm{CH}_{2}\right), 30.5\left(\mathrm{CH}_{2}\right), 19.8$ $\left(\mathrm{CH}_{3}\right)$; HRMS (ES) Exact mass calcd for $\mathrm{C}_{18} \mathrm{H}_{25} \mathrm{~N}_{2} \mathrm{O}_{7}\left[\mathrm{M}+\mathrm{NH}_{4}\right]^{+}$: 381.1656 , found: 381.1660 .

\section{(3aR,6R,6aS)-6-Formyl-5-(4-methoxybenzyl)-6a-methyl-2,4-}

dioxohexahydrofuro[2,3-c]pyrrole-6-carboxylic acid methyl ester (494)

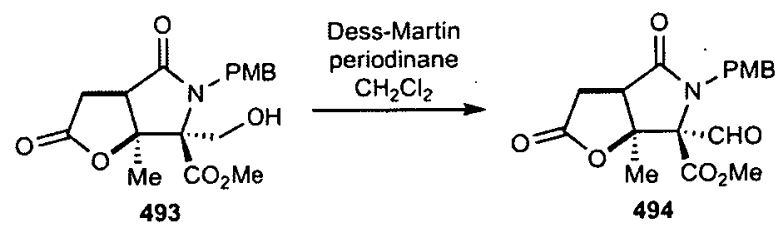

To a solution of alcohol $493(30 \mathrm{mg}, 0.082 \mathrm{mmol})$ in $\mathrm{CH}_{2} \mathrm{Cl}_{2}(0.8 \mathrm{~mL})$ at room temperature was added Dess-Martin periodinane $(43 \mathrm{mg}, 0.099 \mathrm{mmol})$. The reaction mixture was stirred for $1.5 \mathrm{~h}$, quenched with water and extracted with EtOAc $(3 \mathrm{x}$ $\mathrm{mL})$. The combined organic layers were dried $\left(\mathrm{Na}_{2} \mathrm{SO}_{4}\right)$ and concentrated in vacuo as an oil. This aldehyde proved to be unstable and was generally used immediately in the next step without purification. On one occasion, purification of a small amount of crude product by column chromatography ( $50 \% \mathrm{EtOAc} /$ petrol) was performed for characterisation purposes. $\mathrm{R}_{\mathrm{f}}(100 \%$ EtOAc $)=0.34$; IR (film) 2956, $1788(\mathrm{C}=\mathrm{O})$, $1760(\mathrm{C}=\mathrm{O}), 1728(\mathrm{C}=\mathrm{O}), 1687(\mathrm{C}=\mathrm{O}), 1513,1438,1246,1176,949 \mathrm{~cm}^{-1} ;{ }^{1} \mathrm{H}$ NMR $\left(250 \mathrm{MHz}, \mathrm{CDCl}_{3}\right) \delta 9.47(1 \mathrm{H}, \mathrm{s}, \mathrm{CHO}), 7.05(2, \mathrm{~d}, J=8.7 \mathrm{~Hz}, \mathrm{ArH}), 6.81(2 \mathrm{H}, \mathrm{d}, J$ $=8.7 \mathrm{~Hz}, \mathrm{ArH}), 4.86\left(1 \mathrm{H}, \mathrm{d}, J=14.4 \mathrm{~Hz}, \mathrm{NCH}_{\mathrm{A}} \mathrm{H}_{\mathrm{B}} \mathrm{Ar}\right), 4.39(1 \mathrm{H}, \mathrm{d}, J=14.4 \mathrm{~Hz}$, $\left.\mathrm{NCH}_{\mathrm{A}} \mathbf{H}_{\mathrm{B}} \mathrm{Ar}\right), 3.90\left(3 \mathrm{H}, \mathrm{s}, \mathrm{OCH}_{3}\right), 3.78\left(3 \mathrm{H}, \mathrm{s}, \mathrm{OCH}_{3}\right), 3.08(1 \mathrm{H}, \mathrm{dd}, J=9.7,1.8 \mathrm{~Hz}$, $\left.\mathrm{CH}_{\mathrm{C}} \mathrm{H}_{\mathrm{D}} \mathrm{CH}\right), 2.99\left(1 \mathrm{H}, \mathrm{dd}, J=18.4,1.8 \mathrm{~Hz}, \mathrm{CH}_{\mathrm{C}} \mathrm{H}_{\mathrm{D}} \mathrm{CH}\right), 2.85(1 \mathrm{H}, \mathrm{dd}, J=18.4,9.7$ $\left.\mathrm{Hz}, \mathrm{CH}_{\mathrm{C}} \mathbf{H}_{\mathbf{D}} \mathrm{CH}\right), 1.52\left(3 \mathrm{H}, \mathrm{s}, \mathrm{CH}_{3}\right) ;{ }^{13} \mathrm{C}$ NMR $\left(62.9 \mathrm{MHz}, \mathrm{CDCl}_{3}\right) \delta 195.5(\mathrm{CH})$, 172.7 (C), 172.0 (C), 165.7 (C), $159.6(\mathrm{C}), 131.1(2 \times \mathrm{CH}), 126.8(\mathrm{C}), 114.2(2 \mathrm{x}$ $\mathrm{CH}), 87.3(\mathrm{C}), 79.0(\mathrm{C}), 55.2\left(\mathrm{CH}_{3}\right), 53.4\left(\mathrm{CH}_{3}\right), 46.8(\mathrm{CH}), 45.6\left(\mathrm{CH}_{2}\right), 30.6\left(\mathrm{CH}_{2}\right)$, 
$20.5\left(\mathrm{CH}_{3}\right)$; LRMS $\left(\mathrm{EI}^{+}\right)$Mass calcd. for $\mathrm{C}_{18} \mathrm{H}_{19} \mathrm{NO}_{7} \mathrm{Na}[\mathrm{M}+\mathrm{Na}]^{+}: 384.3$, found: 383.7 .

\section{$(3 a R, 6 R, 6 a S)-6-[(7 S)-(8 S)-C y c l o h e x-2-e n y l h y d r o x y m e t h y l]-5-(4-$} methoxybenzyl)-6a-methyl-2,4-dioxohexahydrofuro[2,3-c]pyrrole-6-carboxylic acid methyl ester (495)

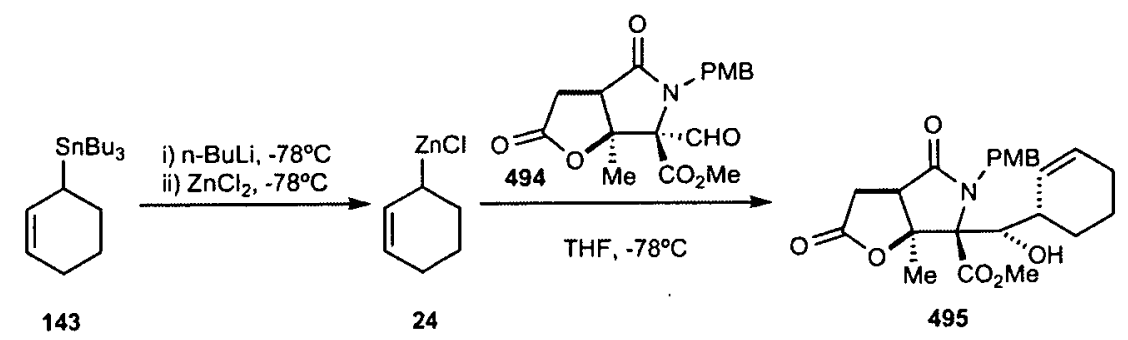

To a solution of cyclohexenyltributyltin $(\mathbf{1 4 3})^{36}(89.6 \mathrm{mg}, 0.206 \mathrm{mmol})$ in THF $(0.6 \mathrm{~mL})$ at $-78{ }^{\circ} \mathrm{C}$ was added $n$-BuLi $(130 \mu \mathrm{L} \mathrm{mL}, 1.6 \mathrm{M}$ solution in hexanes, 0.206 mmol). After stirring at $-78^{\circ} \mathrm{C}$ for $1 \mathrm{~h}, \mathrm{ZnCl}_{2}(0.42 \mathrm{~mL}, 0.5 \mathrm{M}$ solution in THF, 0.21 mmol) was added and stirring was continued at this temperature for another $1 \mathrm{~h}$. A solution of the crude aldehyde 494 from the above experiment (theoretically 0.082 $\mathrm{mmol})$ in THF (0.4 $\mathrm{mL})$ was then added via cannula, and after stirring for $3 \mathrm{~h}$ at -78 ${ }^{\circ} \mathrm{C}$, the reaction was quenched with $\mathrm{H}_{2} \mathrm{O}(5 \mathrm{~mL})$ and extracted with EtOAc $(3 \times 5$ $\mathrm{mL})$. The combined organic layers were dried $\left(\mathrm{MgSO}_{4}\right)$ and concentrated in vacuo, and purification of the residue by column chromatography (50\% EtOAc/petrol) gave the homoallylic alcohol 495 (22.4 mg, 61\% over two steps) as a colourless solid. $\mathrm{R}_{\mathbf{f}}$ $(100 \%$ EtOAc $)=0.61 ; \mathrm{R}_{\mathrm{f}}\left(70 \%\right.$ EtOAc in $\left.\mathrm{CHCl}_{3}\right)=0.45 ;$ m.p. $=114-116{ }^{\circ} \mathrm{C}$; $[\alpha]_{\mathrm{D}}^{22}+3.0\left(c\right.$ 1.47, $\left.\mathrm{CHCl}_{3}\right) ; \mathrm{IR}($ film) $3438(\mathrm{OH}), 2931,2359,1790(\mathrm{C}=\mathrm{O}), 1755$ $(\mathrm{C}=\mathrm{O}) 1687(\mathrm{C}=\mathrm{O}), 1512,1440,1246,1175,807 \mathrm{~cm}^{-1} ;{ }^{1} \mathrm{H}$ NMR $\left(250 \mathrm{MHz}, \mathrm{CDCl}_{3}\right)$ $\delta 7.18(2 \mathrm{H}, \mathrm{d}, J=8.7 \mathrm{~Hz}, \operatorname{ArH}), 6.84(2 \mathrm{H}, \mathrm{d}, J=8.7 \mathrm{~Hz}, \operatorname{ArH}), 5.91(1 \mathrm{H}, \mathrm{dm}, J=$ $10.2 \mathrm{~Hz}, \mathrm{CH}=\mathrm{CH}), 5.68(1 \mathrm{H}, \mathrm{dm}, J=10.2 \mathrm{~Hz}, \mathrm{CH}=\mathrm{CH}), 4.79(1 \mathrm{H}, \mathrm{d}, J=14.9 \mathrm{~Hz}$, $\left.\mathrm{NCH}_{\mathbf{A}} \mathrm{H}_{\mathrm{B}} \mathrm{Ar}\right), 4.54\left(1 \mathrm{H}, \mathrm{d}, J=14.9 \mathrm{~Hz}, \mathrm{NCH}_{\mathrm{A}} \mathbf{H}_{\mathbf{B}} \mathrm{Ar}\right), 4.12(1 \mathrm{H}, \mathrm{t}, J=7.1 \mathrm{~Hz}$, $\mathrm{CHOH}), 3.78\left(6 \mathrm{H}, \mathrm{s}, 2 \times \mathrm{OCH}_{3}\right), 3.13\left(1 \mathrm{H}, \mathrm{dd}, J=6.8,3.3 \mathrm{~Hz}, \mathrm{CH}_{2} \mathrm{CHC}=\mathrm{O}\right), 2.92$ (1H, br s, OH), 2.82-2.79 $\left(2 \mathrm{H}, \mathrm{m}, \mathrm{CH}_{2} \mathrm{CHC}=\mathrm{O}\right), 2.32(1 \mathrm{H}, \mathrm{bs}, \mathrm{CHOHCH}), 2.02(2 \mathrm{H}$, bs, $\left.\mathrm{CH}_{2}\right), 1.81\left(3 \mathrm{H}, \mathrm{s}, \mathrm{CH}_{3} \mathrm{CO}\right), 1.76-1-66\left(2 \mathrm{H}, \mathrm{m}, \mathrm{CH}_{2}\right), 1.57-1.46\left(1 \mathrm{H}, \mathrm{m}, \mathrm{CH}_{2}\right)$, 1.44-1.33 (1H, m, CH$) ;{ }^{13} \mathrm{C}$ NMR (62.9 MHz, $\left.\mathrm{CDCl}_{3}\right) \delta 174.8(\mathrm{C}), 173.0(\mathrm{C}), 167.8$ (C), $158.3(\mathrm{C}), 131.8(\mathrm{CH}), 129.3(\mathrm{C}), 127.6(2 \times \mathrm{CH}), 125.2(\mathrm{CH}), 113.7(2 \times \mathrm{CH})$, 
$91.1(\mathrm{C}), 79.0(\mathrm{C}), 76.9(\mathrm{CH}), 55.2\left(\mathrm{CH}_{3}\right), 52.4\left(\mathrm{CH}_{3}\right), 47.9\left(\mathrm{CH}_{2}\right), 47.8(\mathrm{CH}), 38.3$ $(\mathrm{CH}), 30.9\left(\mathrm{CH}_{2}\right), 27.0\left(\mathrm{CH}_{2}\right), 24.9\left(\mathrm{CH}_{2}\right), 20.6\left(\mathrm{CH}_{2}\right), 19.6\left(\mathrm{CH}_{3}\right) ; \mathrm{HRMS}(\mathrm{ES})$ Exact mass calcd for $\mathrm{C}_{24} \mathrm{H}_{33} \mathrm{~N}_{2} \mathrm{O}_{7}\left[\mathrm{M}+\mathrm{NH}_{4}\right]^{+}: 461.2282$, found: 461.2283 .

\section{$(2 R, 3 S, 4 R)-2-[(6 S)-(7 S)-C y c l o h e x-2-e n y l h y d r o x y m e t h y l]-3-h y d r o x y-4-(2-$} hydroxyethyl)-1-(4-methoxybenzyl)-3-methyl-5-oxopyrrolidine-2-carboxylic acid methyl ester (26)

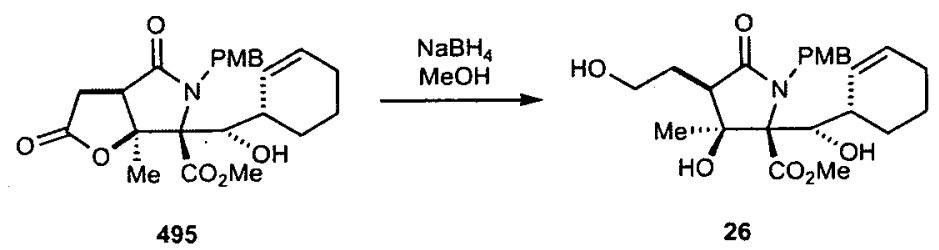

To a solution of the lactone $495(19.3 \mathrm{mg}, 0.043 \mathrm{mmol})$ in $\mathrm{MeOH}(0.6 \mathrm{~mL})$ at room temperature was added $\mathrm{NaBH}_{4}\left(75 \mathrm{mg}, 2.0^{\circ} \mathrm{mmol}\right)$ portionwise over $2 \mathrm{~min}$. The solution was stirred for $18 \mathrm{~h}$, quenched with $\mathrm{H}_{2} \mathrm{O}(4 \mathrm{~mL})$ and extracted with EtOAc ( $3 \times 4 \mathrm{~mL}$ ). The combined organic layers were dried $\left(\mathrm{Na}_{2} \mathrm{SO}_{4}\right)$ and concentrated in vacuo. Purification of the residue by column chromatography (70\% EtOAc/petrol) gave triol $26(11.7 \mathrm{mg}, 60 \%)$ as a white foam. $\mathrm{R}_{\mathrm{f}}\left(70 \%\right.$ EtOAc in $\left.\mathrm{CHCl}_{3}\right)=0.12$; m.p. $=82-83{ }^{\circ} \mathrm{C}$, lit. $^{26} 83-84{ }^{\circ} \mathrm{C} ;[\alpha]_{\mathrm{D}}^{22}+3.5$ (c $\left.0.56, \mathrm{CHCl}_{3}\right)$, lit. $^{26}+5.2$ (c 0.60 , $\mathrm{CHCl}_{3}$ ); IR (film) 3331(OH), 2925, 2853, $1752(\mathrm{C}=\mathrm{O}), 1670(\mathrm{C}=\mathrm{O}), 1512,1441$, $1245,1175,1034 \mathrm{~cm}^{-1}$; ${ }^{1} \mathrm{H}$ NMR $\left(250 \mathrm{MHz} \mathrm{CDCl}_{3}\right) \delta 7.29(2 \mathrm{H}, \mathrm{d}, J=8.7 \mathrm{~Hz}, \mathrm{ArH})$, $6.85(2 \mathrm{H}, \mathrm{d}, J=8.7 \mathrm{~Hz}, \mathrm{ArH}), 5.93(1 \mathrm{H}, \mathrm{dm}, J=10.2 \mathrm{~Hz}, \mathrm{CH}=\mathrm{CH}), 5.62(1 \mathrm{H}, \mathrm{dm}, J$ $=10.2 \mathrm{~Hz}, \mathrm{CH}=\mathrm{CH}), 4.76\left(1 \mathrm{H}, \mathrm{d}, J=15.2 \mathrm{~Hz}, \mathrm{NCH}_{\mathrm{A}} \mathrm{H}_{\mathrm{B}} \mathrm{Ar}\right), 4.39(1 \mathrm{H}, \mathrm{d}, J=15.2$ $\left.\mathrm{Hz}, \mathrm{NCH}_{\mathrm{A}} \mathbf{H}_{\mathrm{B}} \mathrm{Ar}\right), 4.15(1 \mathrm{H}, \mathrm{t}, J=6.3 \mathrm{~Hz}, \mathrm{CHOH}), 3.87-3.81(1 \mathrm{H}, \mathrm{m}$, $\left.\mathrm{HOCH}_{\mathbf{C}} \mathrm{H}_{\mathrm{D}} \mathrm{CH}_{2}\right), 3.80-3.75\left(1 \mathrm{H}, \mathrm{m}, \mathrm{HOCH}_{\mathrm{C}} \mathbf{H}_{\mathbf{D}} \mathrm{CH}_{2}\right), 3.79\left(3 \mathrm{H}, \mathrm{s}, \mathrm{OCH}_{3}\right), 3.71(3 \mathrm{H}$, $\left.\mathrm{s}, \mathrm{OCH}_{3}\right), 3.02(1 \mathrm{H}, \mathrm{dd}, J=8.1,4.8 \mathrm{~Hz}, \mathrm{COCH}), 2.20(1 \mathrm{H}, \mathrm{bs}, \mathrm{CHOHCH}), 2.01(2 \mathrm{H}$, bs, $\left.\mathrm{CH}_{2}\right), 1.93-1.78\left(2 \mathrm{H}, \mathrm{m}, \mathrm{CH}_{2}\right), 1.76-1.65\left(2 \mathrm{H}, \mathrm{m}, \mathrm{CH}_{2}\right), 1.64\left(3 \mathrm{H}, \mathrm{s}, \mathrm{CH}_{3} \mathrm{CO}\right)$, 1.54-1.41 (2H, m, CH$) ;{ }^{13} \mathrm{C}$ NMR (62.9 $\left.\mathrm{MHz} \mathrm{CDCl}_{3}\right) \delta 178.5(\mathrm{C}), 169.7$ (C), 158.2 (C), $132.9(\mathrm{CH}), 130.2(\mathrm{C}), 128.0(2 \times \mathrm{CH}), 124.8(\mathrm{CH}), 113.6(2 \times \mathrm{CH}), 81.8(\mathrm{C})$, $80.6(\mathrm{C}), 77.2(\mathrm{CH}), 61.7\left(\mathrm{CH}_{2}\right), 55.2\left(\mathrm{CH}_{3}\right), 51.9(\mathrm{CH}), 51.3\left(\mathrm{CH}_{3}\right), 47.8\left(\mathrm{CH}_{2}\right), 38.6$ $(\mathrm{CH}), 28.2\left(\mathrm{CH}_{2}\right), 26.8\left(\mathrm{CH}_{2}\right), 25.0\left(\mathrm{CH}_{2}\right), 21.2\left(\mathrm{CH}_{3}\right), 20.6\left(\mathrm{CH}_{2}\right) ;$ HRMS (ES) Exact mass calcd for $\mathrm{C}_{24} \mathrm{H}_{33} \mathrm{NO}_{7} \mathrm{Na}[\mathrm{M}+\mathrm{Na}]^{+}: 470.2149$, found: 470.2150 . 
Comparison of ${ }^{13} \mathrm{C}$ NMR data (HWL, Corey and Pattenden's) for 26

\begin{tabular}{|c|c|c|c|c|}
\hline $\begin{array}{l}\mathrm{HWL}{ }^{13} \mathrm{C} \mathrm{NMR} \\
\text { data }\left(\mathrm{CDCl}_{3}\right. \\
\text { referenced at } \\
77.0 \mathrm{ppm})\end{array}$ & $\begin{array}{c}\text { Corey }{ }^{13} \mathrm{C} \text { NMR data with } \\
\mathrm{CDCl}_{3} \text { referenced at } 77.38 \\
\text { ppm (data with } \mathrm{CDCl}_{3} \\
\text { referenced at } 77.0 \mathrm{ppm} \text { ) }\end{array}$ & $\Delta \mathrm{ppm}$ & $\begin{array}{c}\text { Pattenden } \\
{ }^{13} \mathrm{C} \mathrm{NMR} \text { data with } \\
\mathrm{CDCl}_{3} \text { referenced } \\
\text { at } 77.03 \mathrm{ppm}\end{array}$ & $\Delta \mathrm{ppm}$ \\
\hline 178.5 & $178.99(178.61)$ & -0.11 & 178.8 & -0.3 \\
\hline 169.7 & $170.12(169.74)$ & -0.04 & 169.7 & 0 \\
\hline 158.2 & $158.27(157.89)$ & +0.31 & 158.2 & 0 \\
\hline 132.9 & $131.30(130.92)$ & +1.98 & 132.6 & +0.3 \\
\hline 130.2 & $130.55(130.17)$ & +0.03 & 130.7 & -0.5 \\
\hline 128.0 & $128.13(127.75)$ & +0.25 & 128.2 & -0.2 \\
\hline 124.8 & $126.39(126.01)$ & -1.21 & 125.1 & -0.3 \\
\hline 113.7 & $113.74(113.36)$ & +0.34 & 113.7 & 0 \\
\hline 81.8 & $81.93(81.55)$ & +0.25 & 81.8 & 0 \\
\hline 80.6 & $80.75(80.37)$ & +0.23 & 80.6 & 0 \\
\hline 77.2 & $76.87(76.49)$ & +0.71 & 77.2 & 0 \\
\hline 61.7 & $61.61(61.23)$ & +0.47 & 61.6 & +0.1 \\
\hline 55.2 & $55.45(55.07)$ & +0.13 & 55.2 & 0 \\
\hline 51.9 & $51.97(51.59)$ & +0.31 & 51.9 & 0 \\
\hline 51.3 & $51.32(50.94)$ & +0.36 & 51.3 & 0 \\
\hline 47.8 & $48.07(47.69)$ & +0.11 & 47.9 & -0.1 \\
\hline 38.6 & $39.17(38.22)$ & +0.38 & 38.7 & -0.1 \\
\hline 28.2 & $27.71(27.82)$ & +0.38 & 28.0 & +0.2 \\
\hline 26.8 & $27.13(26.75)$ & +0.05 & 26.8 & 0 \\
\hline 25.0 & $25.22(24.84)$ & +0.16 & 25.0 & 0 \\
\hline 21.2 & $21.35(20.97)$ & +0.23 & 21.1 & +0.1 \\
\hline 20.6 & $21.22(20.84)$ & -0.62 & 20.7 & -0.1 \\
\hline
\end{tabular}




\subsubsection{NMR spectra}

$(R)$-Ethyl $(E)-3-[N$-(1-benzyloxymethyl-1-carbomethoxy-2-oxopropyl)- $N$-(4methoxybenzyl)carbamoyl]acrylate (481)

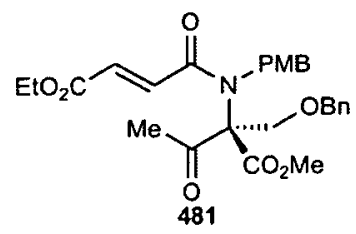

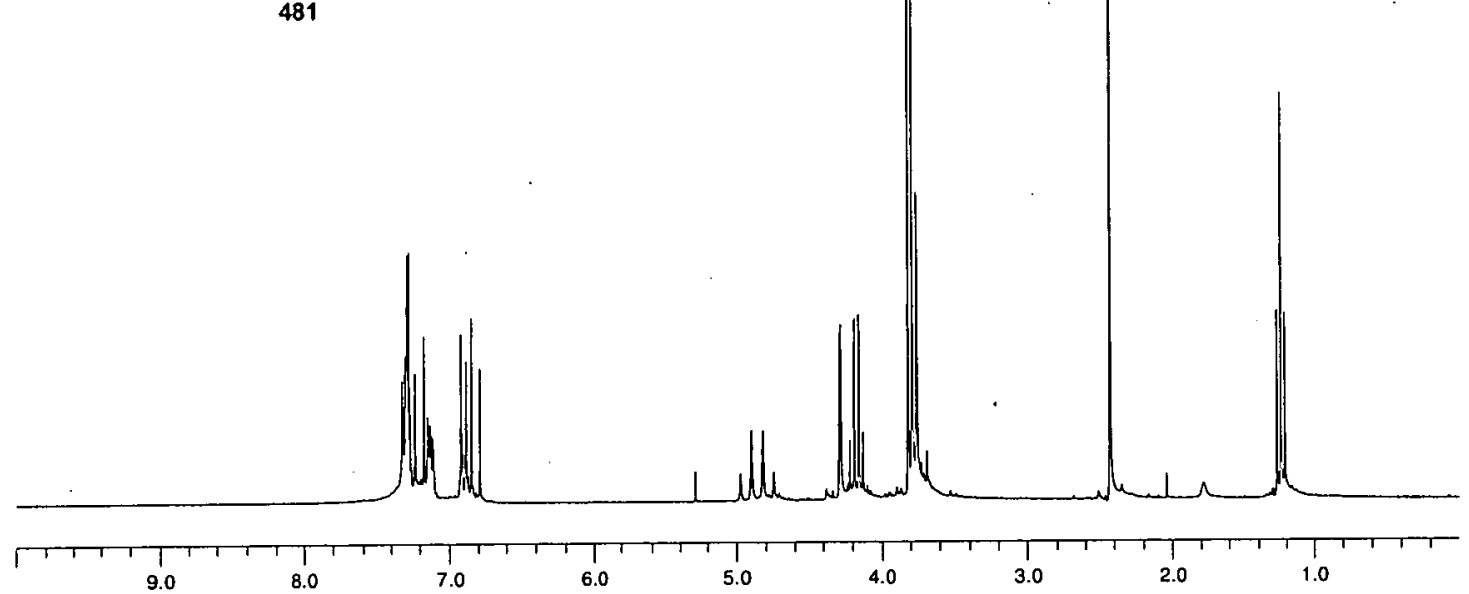

ppm (f1)
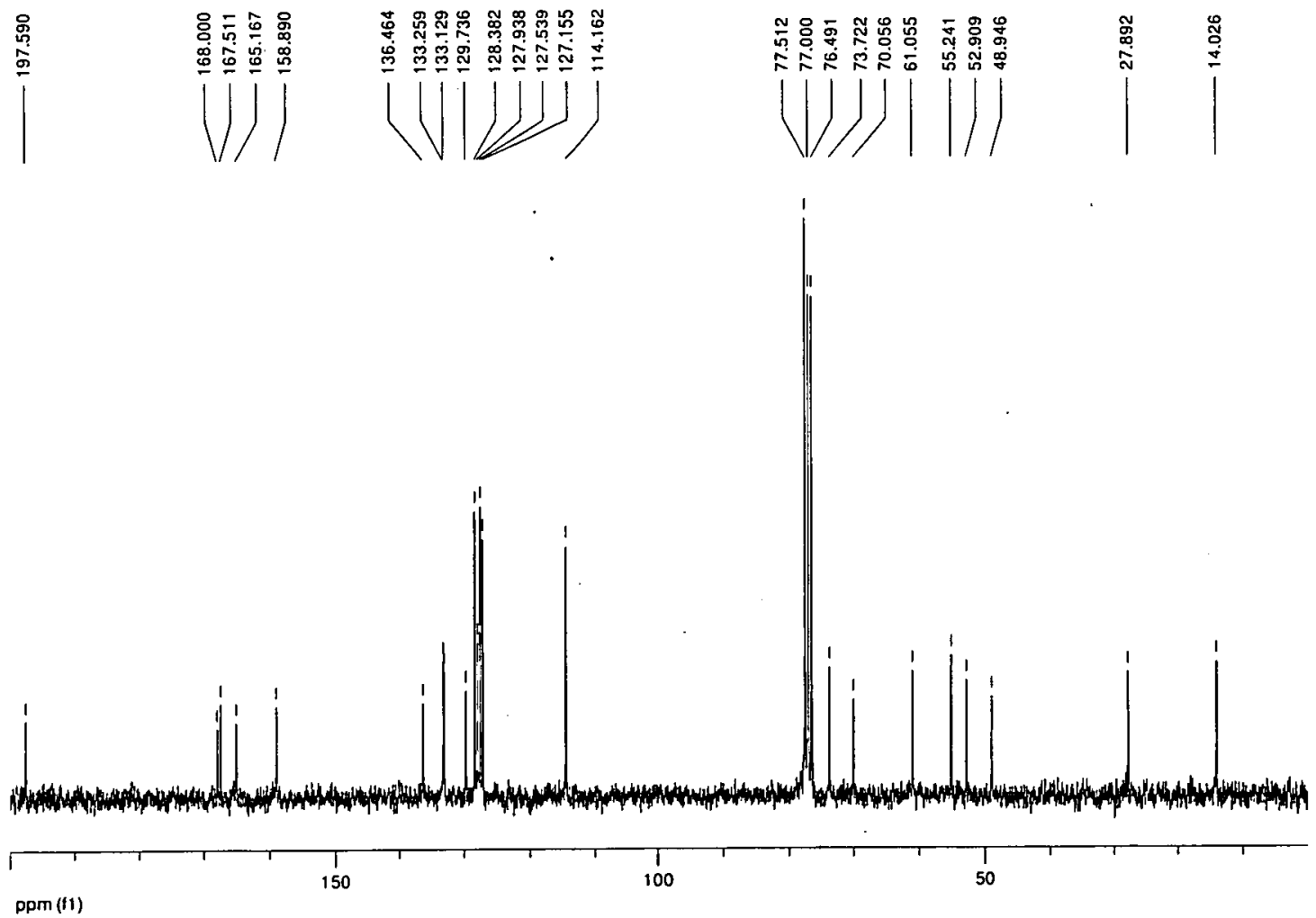
(3aR,6R,6aS)-6-Benzyloxymethyl-5-(4-methoxybenzyl)-6a-methyl-2,4dioxohexahydrofuro[2,3-c]pyrrole-6-carboxylic acid methyl ester (489)

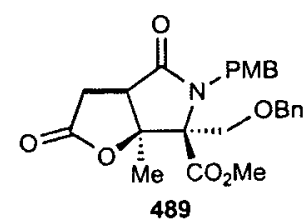

489

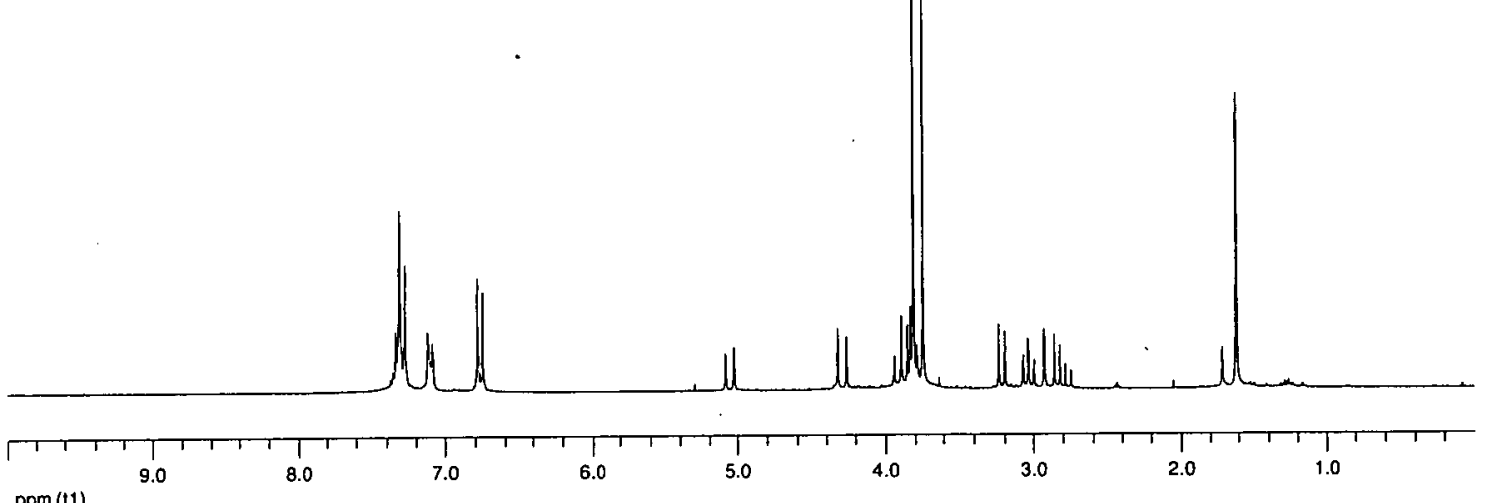
$\operatorname{ppm}(t)$
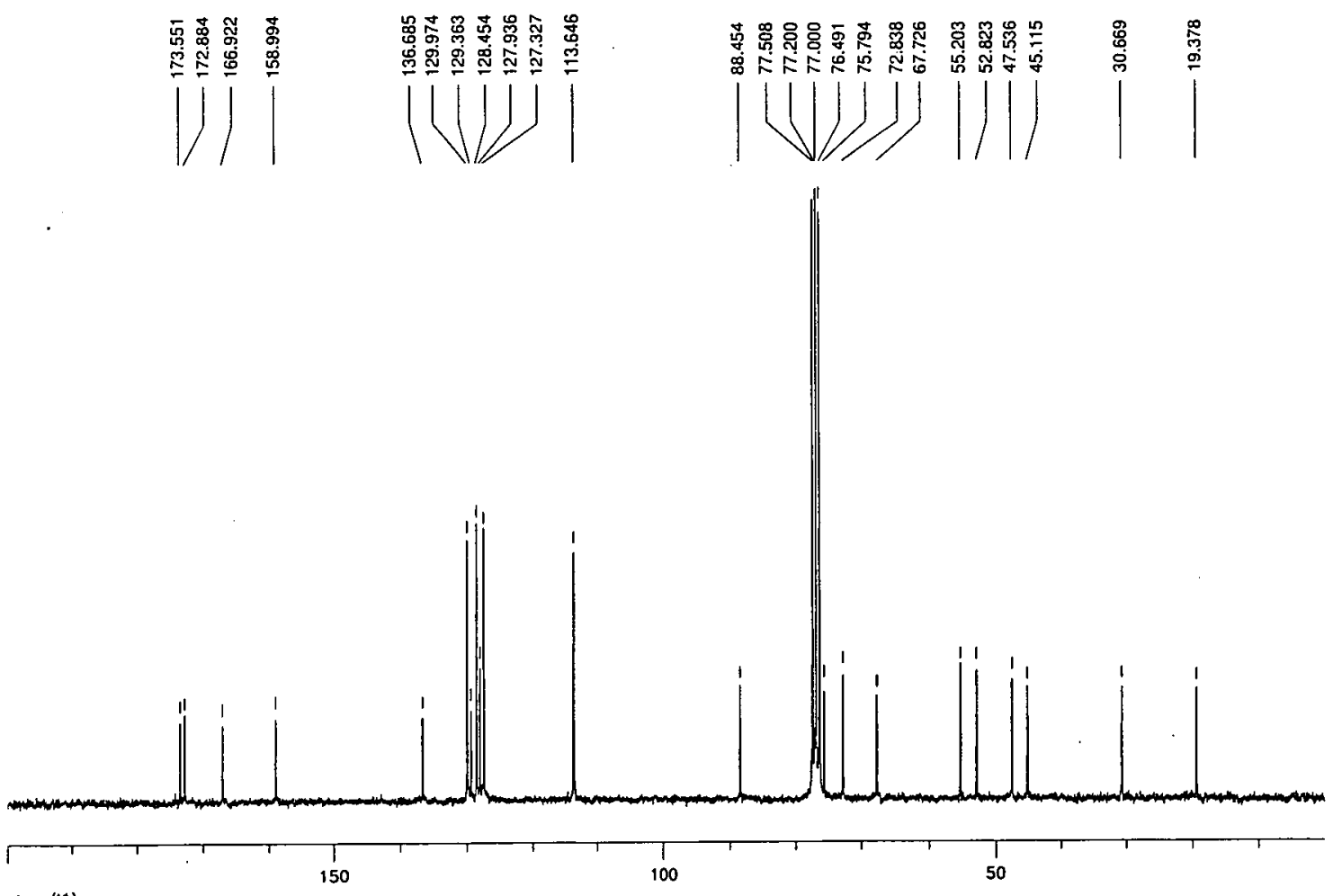

$\operatorname{ppm}(11)$ 
(3aS,6R,6aR)-6-benzyloxymethyl-5-(4-methoxybenzyl)-6a-methyl-2,4dioxohexahydrofuro[2,3-c]pyrrole-6-carboxylic acid methyl ester (490)

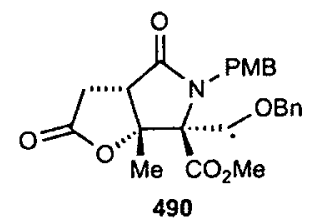

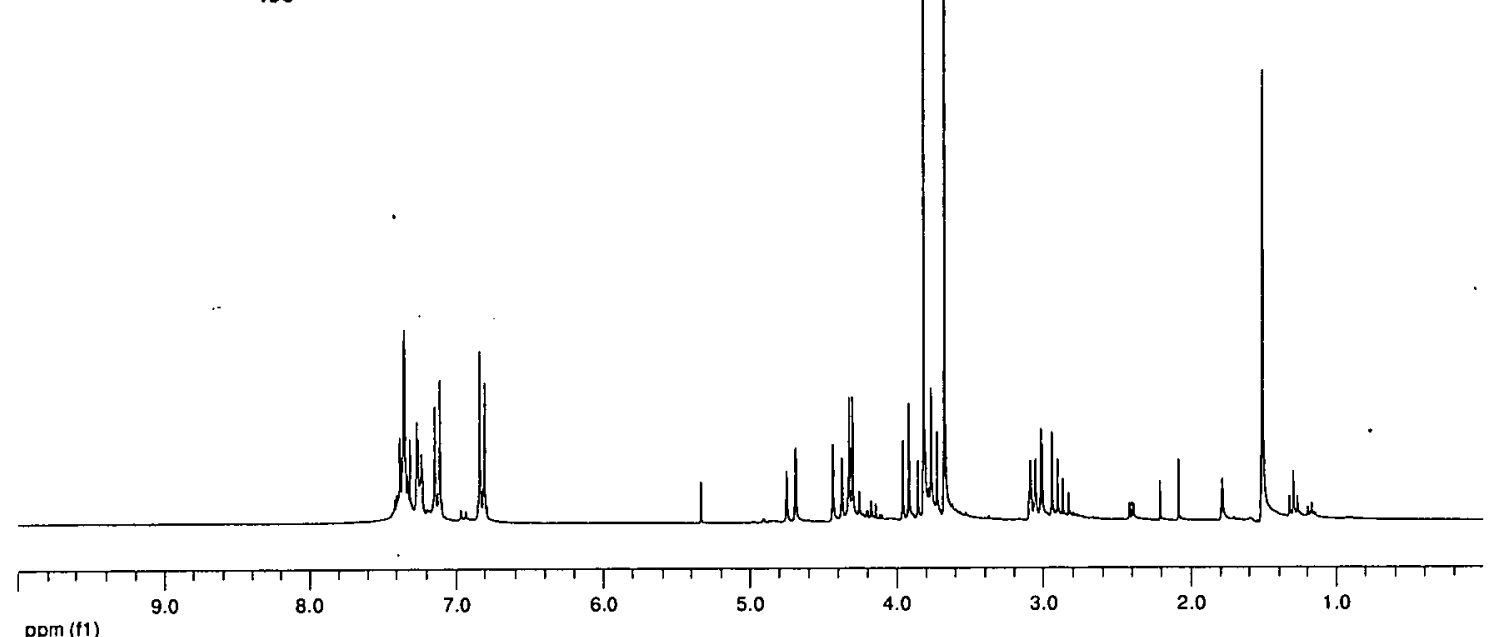

ppm (f1)
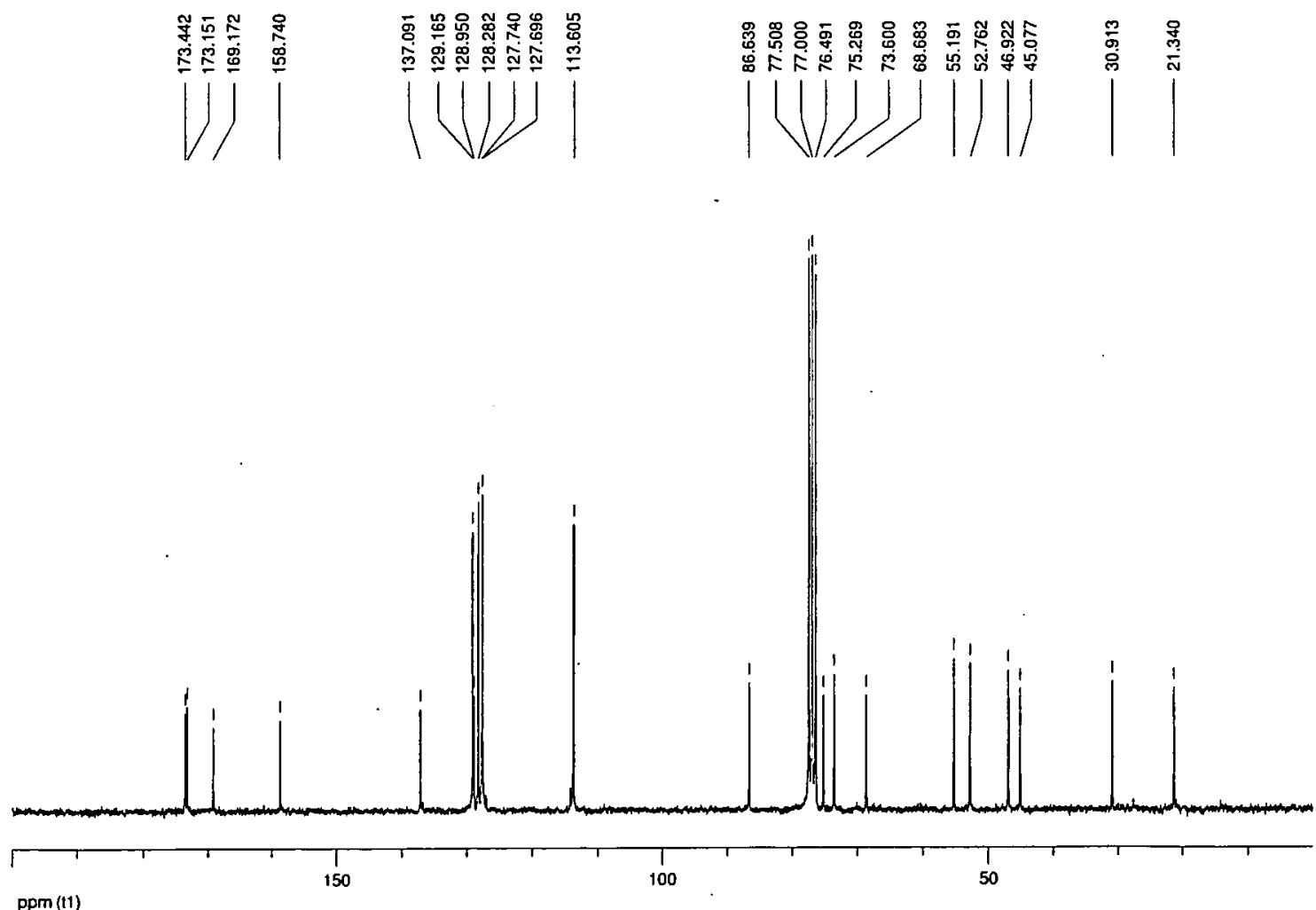
(3a $R, 6 R, 6 a S)-6-H y d r o x y m e t h y l-5-(4-m e t h o x y b e n z y l)-6 a-m e t h y l-2,4-$ dioxohexahydrofuro[2,3-c]pyrrole-6-carboxylic acid methyl ester (493)

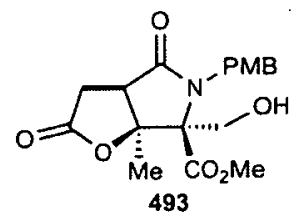

493

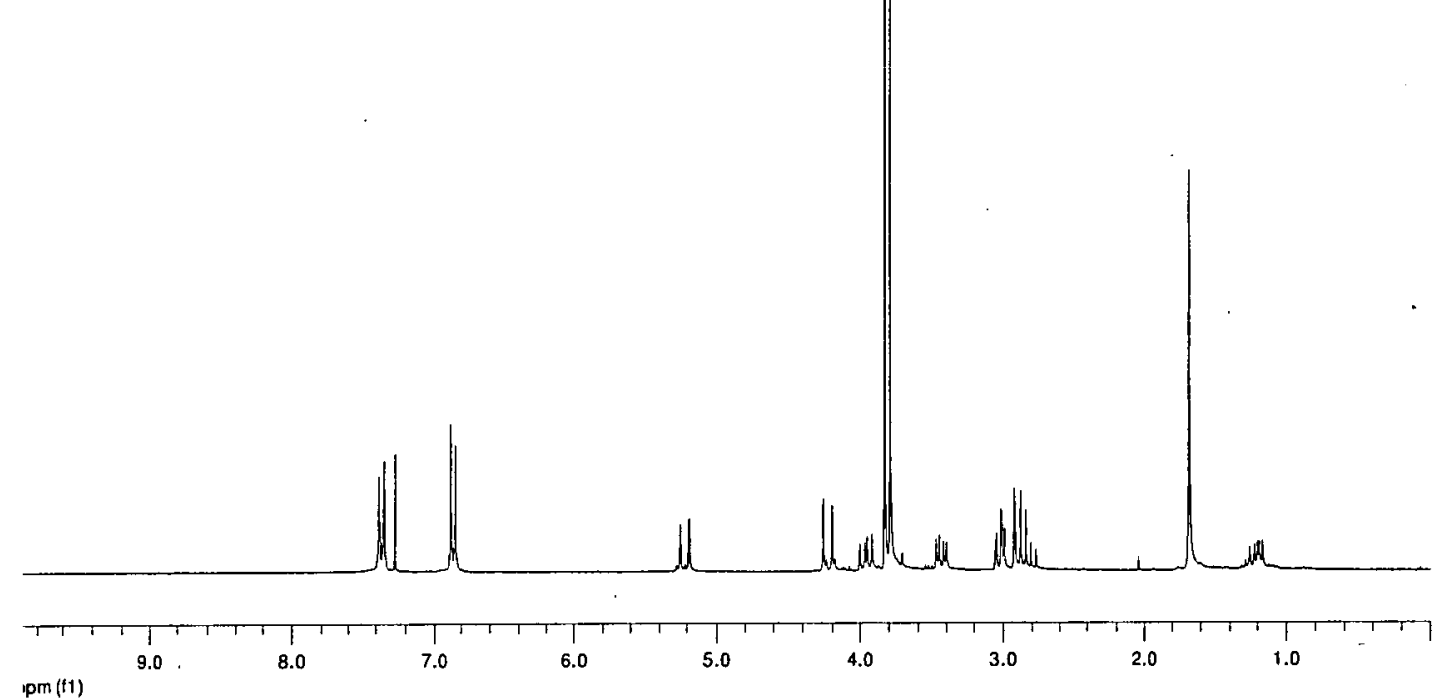

ipm (t1)
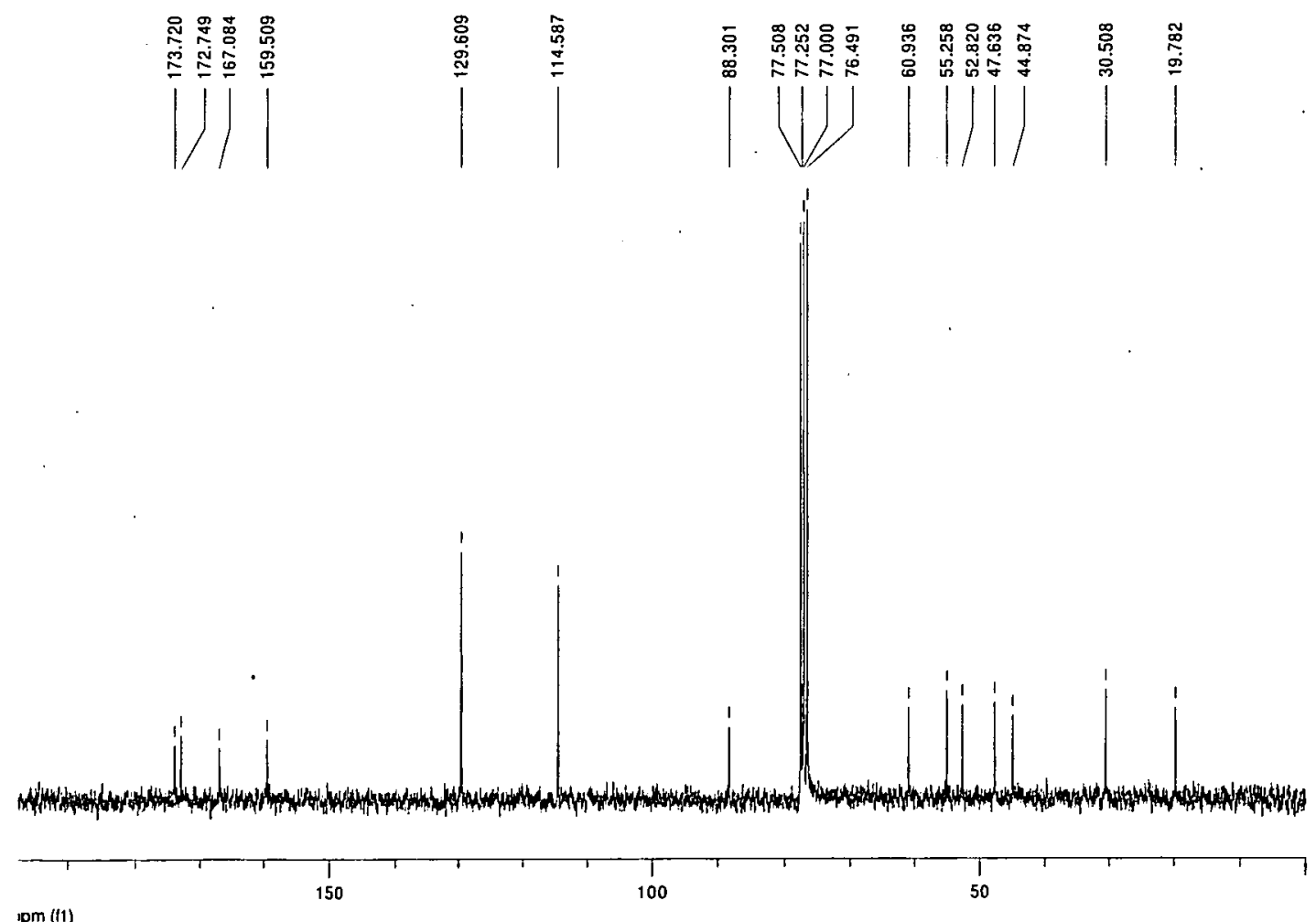
$(3 a R, 6 R, 6 a S)-6-F o r m y l-5-(4-m e t h o x y b e n z y l)-6 a-m e t h y l-2,4-$ dioxohexahydrofuro[2,3-c]pyrrole-6-carboxylic acid methyl ester (494)<smiles>CC(=O)N1C(=O)C2CC(=O)O[C@@]2(N(C(C)=O)C(=O)O)C1(C(=O)O)C(=O)O</smiles>

494

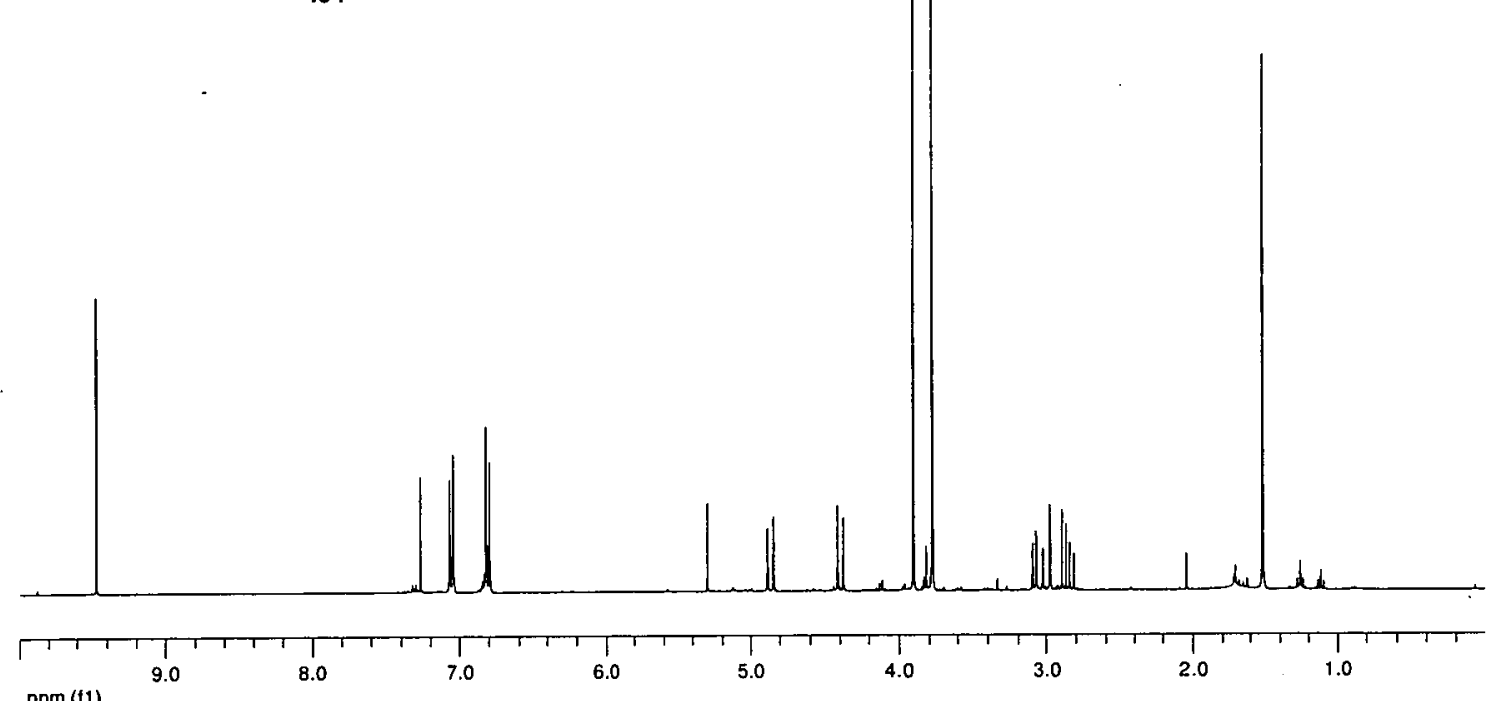
ppm (f1)
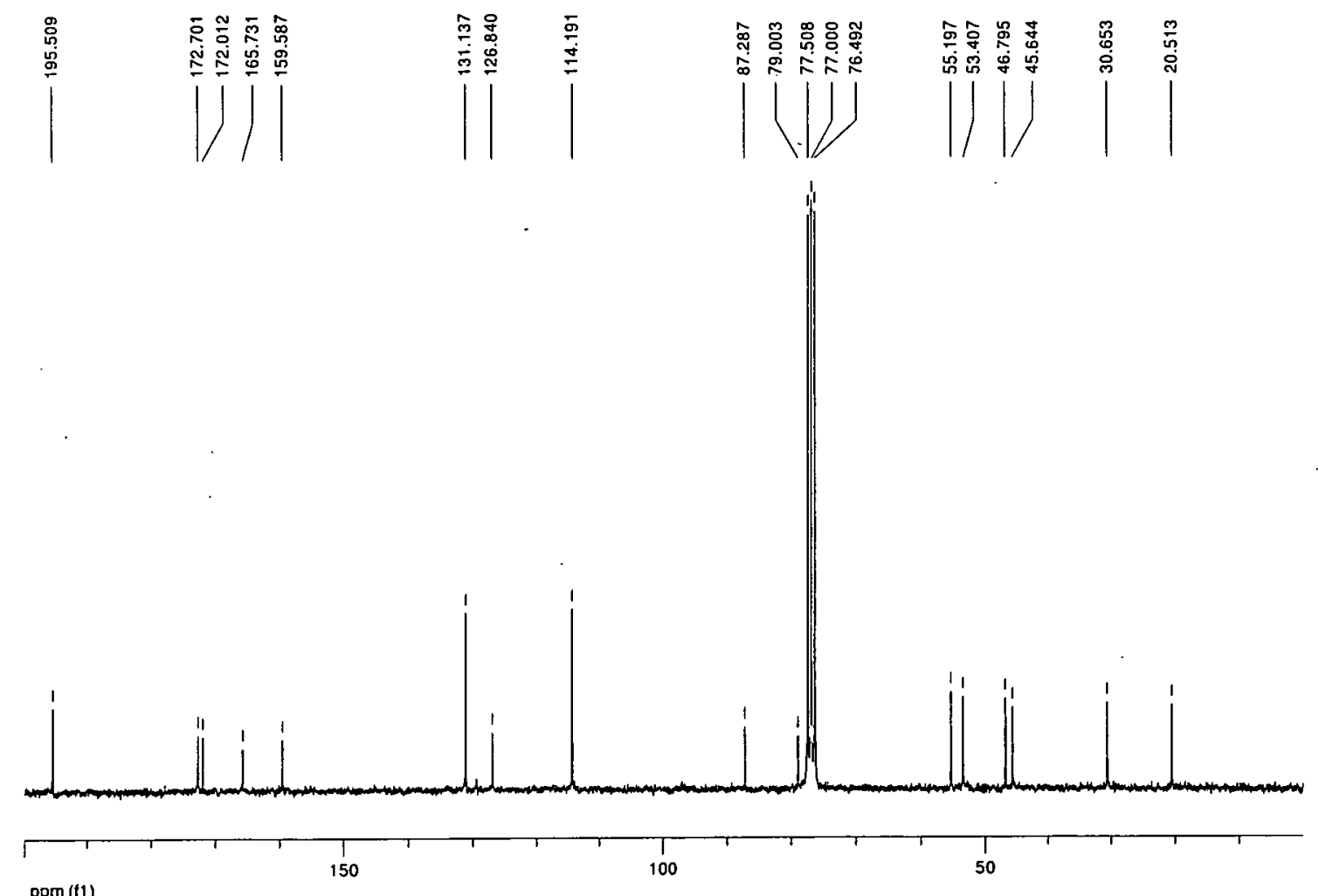

ppm (11) 
$(3 a R, 6 R, 6 a S)-6-[(S)-(S)-C y c l o h e x-2-e n y l h y d r o x y m e t h y l]-5-(4-m e t h o x y b e n z y l)-$ 6a-methyl-2,4-dioxohexahydrofuro[2,3-c]pyrrole-6-carboxylic acid methyl ester (495)

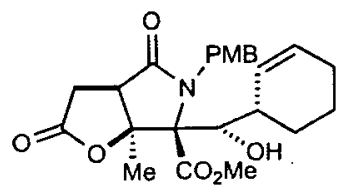

495

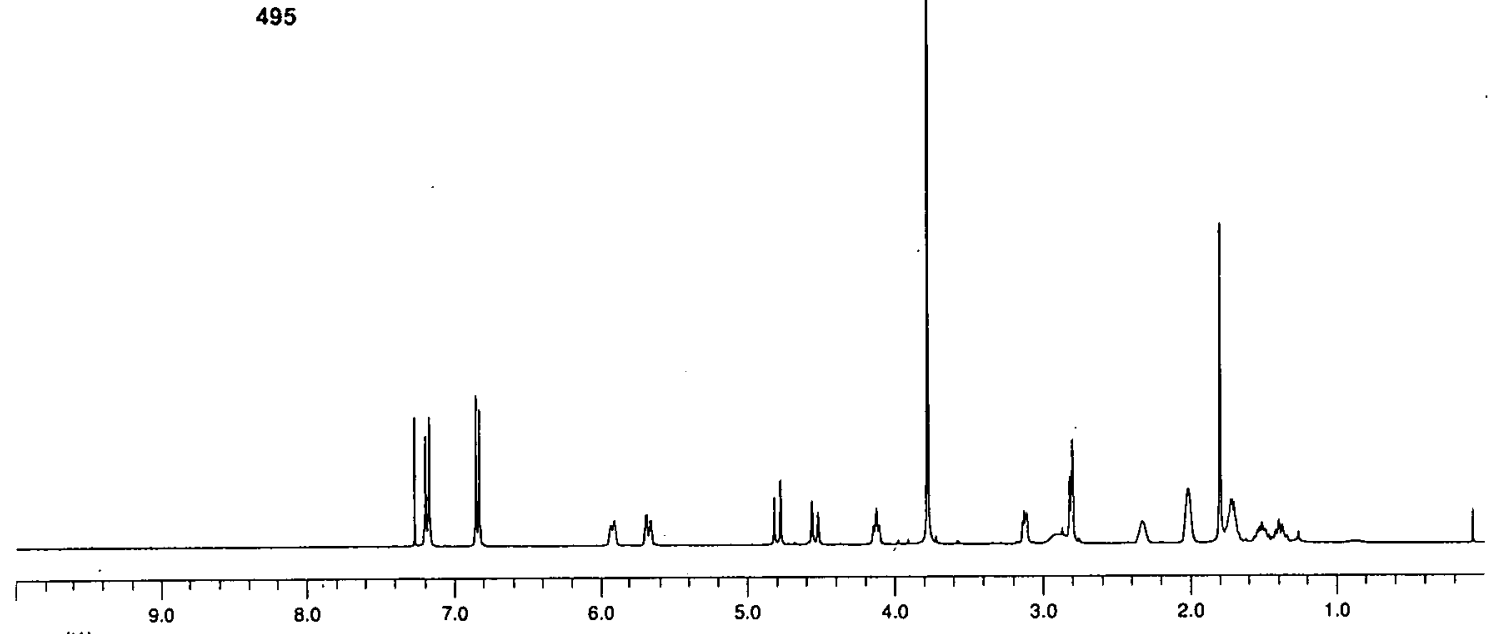

$\operatorname{ppm}(t 1)$
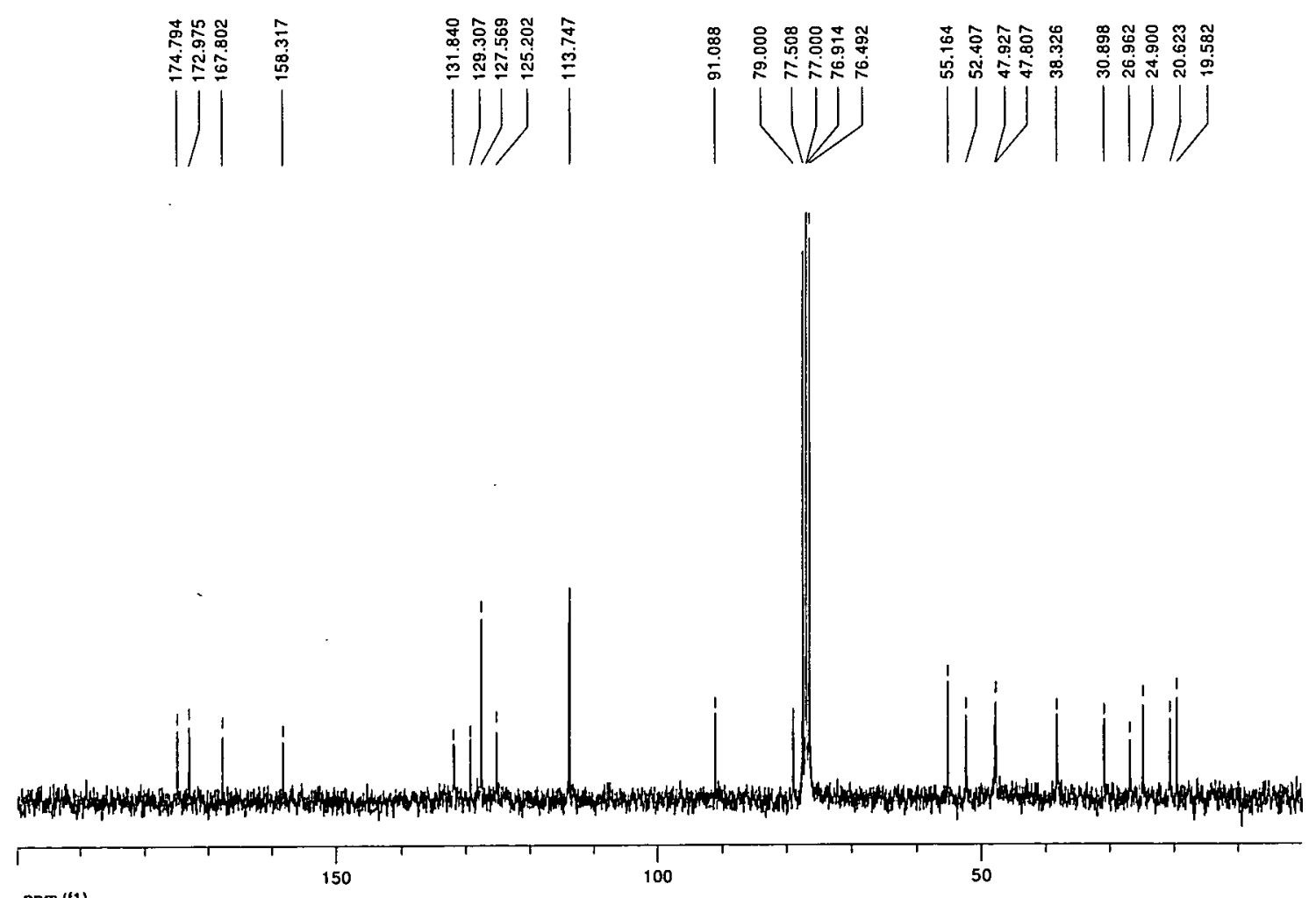

ppm (11) 
$(2 R, 3 S, 4 R)-2-[(S)-(S)-C y c l o h e x-2-e n y l h y d r o x y m e t h y l]-3-h y d r o x y-4-(2-$

hydroxyethyl)-1-(4-methoxybenzyl)-3-methyl-5-oxopyrrolidine-2-carboxylic acid methyl ester (26)

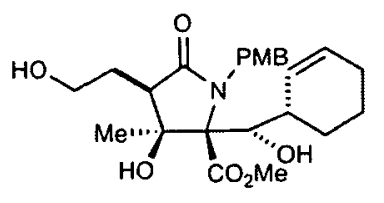

26

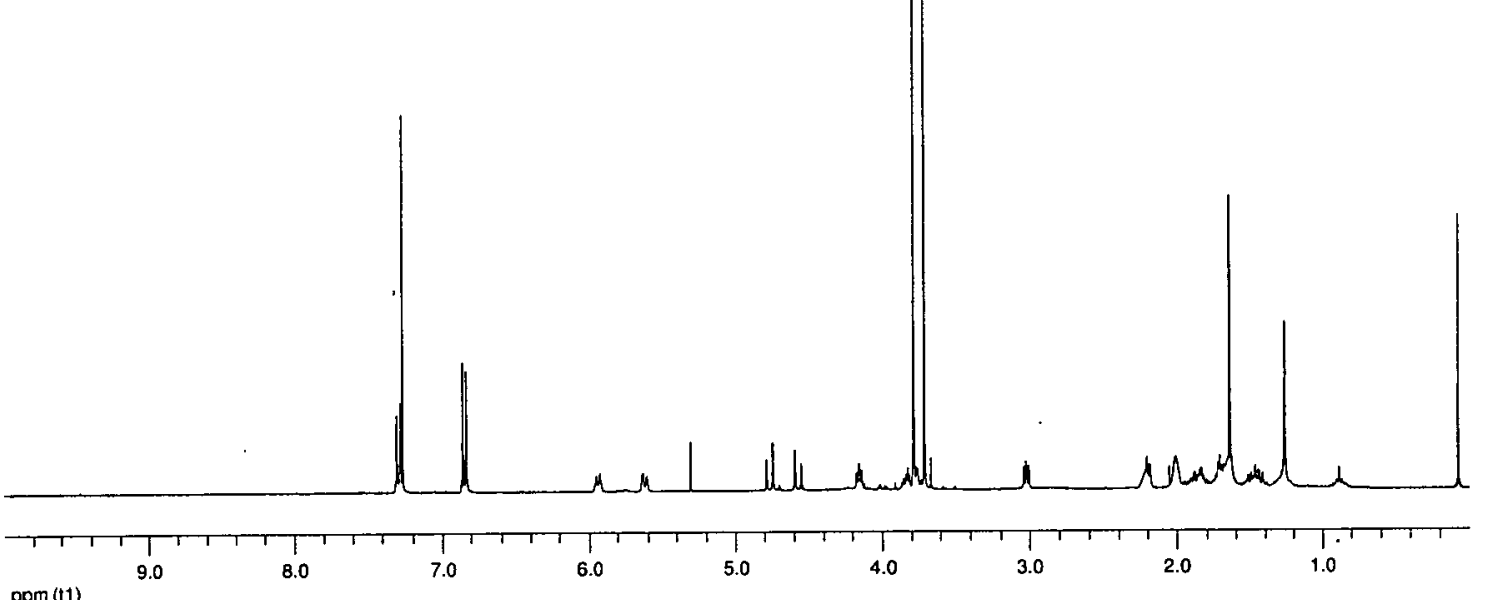

ppm (t1)
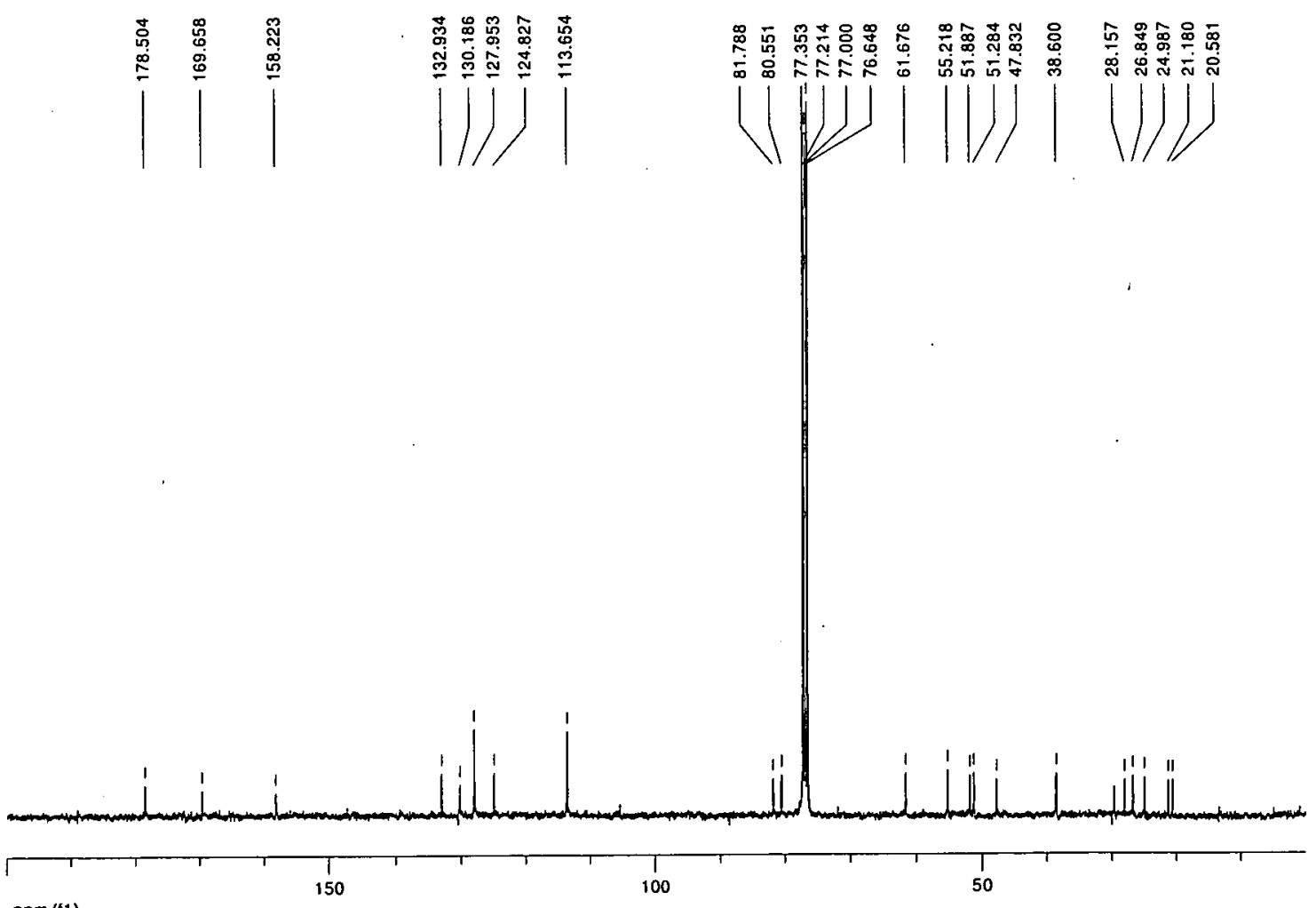

ppm (f1) 


\section{${ }^{13}$ C NMR Spectrum of triol 26 obtained by the Corey group}

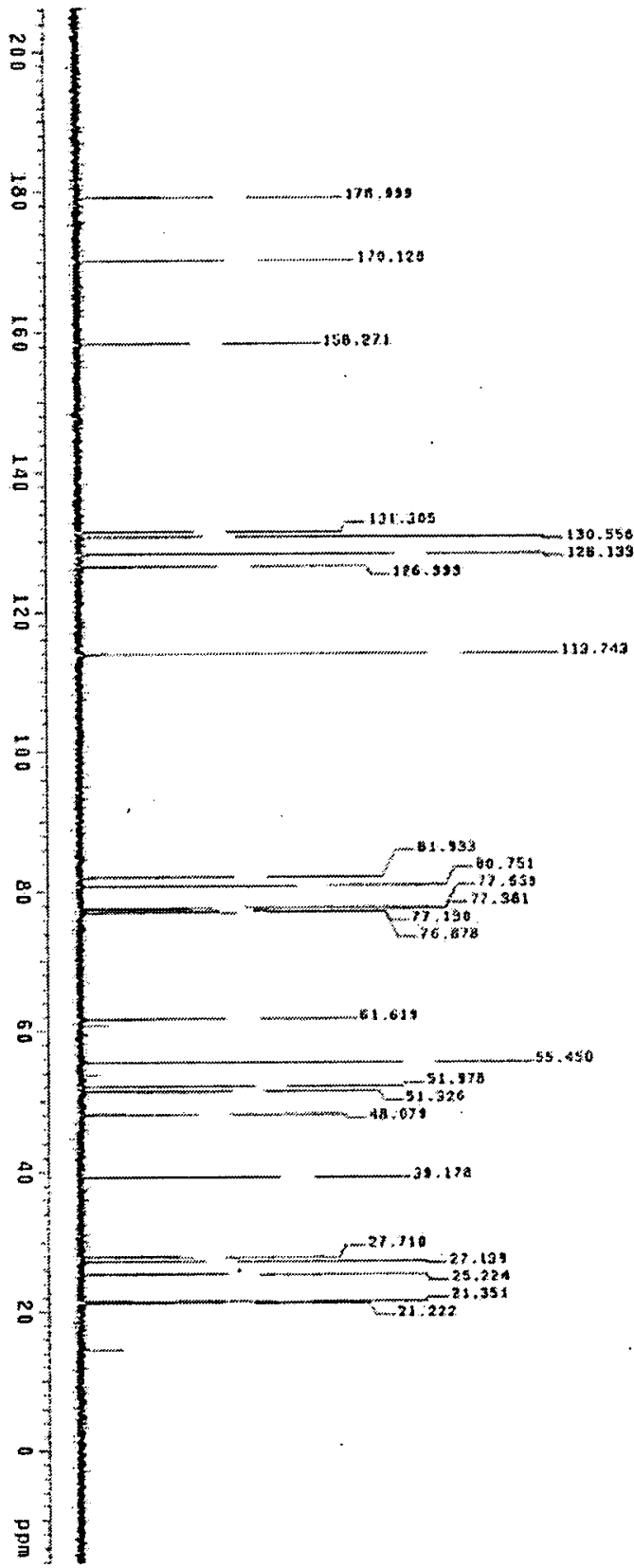

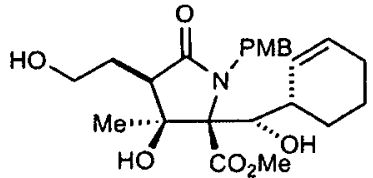

26 


\section{${ }^{13} \mathrm{C}$ NMR Spectrum of triol 26 obtained by the Pattenden group}
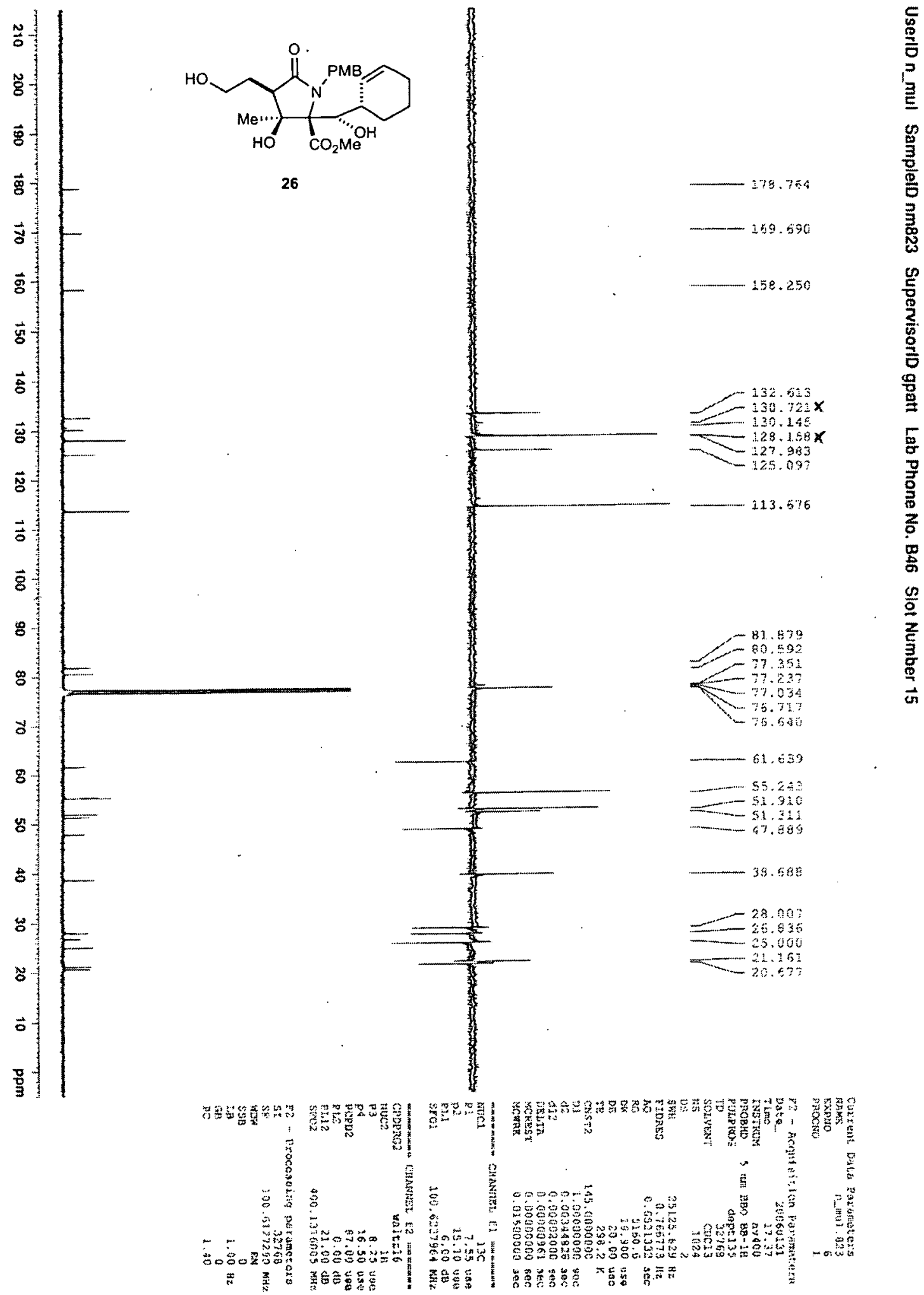


\section{References}

1. Feling, R. H.; Buchanan, G. O.; Mincer, T. J.; Kauffman, C. A.; Jensen, P. R.; Fenical, W. Angew. Chem., Int. Ed. 2003, 42, 355-357.

2. (a) Omura, S.; Fujimoto, T.; Otoguro, K.; Matsuzaki, K.; Moriguchi, R.; Tanaka, H.; Sasaki, Y. J. Antibiot. 1991, 44, 113-116.

(b) Omura, S.; Matsuzaki, K.; Fujimoto, T.; Kosuge, K.; Furuya, T.; Fujita, S.; Nakagawa, A. J. Antibiot. 1991, 44, 117-118.

3. Goldberg, A. L. Biochem. Soc. Trans. 2007, 35, 12-17.

4. Chauhan, D.; Catley, L.; Li, G.; Podar, K.; Hideshima, T.; Velankar, M.; Mitsiades, C.; Mitsiades, N.; Yasui, H.; Letai, A.; Ovaa, H.; Berkers, C.; Nicholson, B.; Chao, T.-H.; Neuteboom Saskia, T. C.; Richardson, P.; Palladino Michael, A.; Anderson Kenneth, C. Cancer Cell 2005, 8, 407-419.

5. Lee, D. H.; Goldberg, A. L. Trends Cell Biol 1998, 8, 397-403.

6. (a) Wilkinson, K. D. Proc. Natl. Acad. Sci. U. S. A. 2005, 102, 15280-15282.

(b) Ciechanover, A. Angew. Chem., Int. Ed. 2005, 44, 5944-5967.

(c) Rose, I. A. Proc. Natl. Acad. Sci. U. S. A. 2005, 102, 11575-11577.

(d) Hershko, A. Cell Death Differ. 2005, 12, 1191-1197.

7. Biel, M.; Wascholowski, V.; Giannis, A. Angew. Chem., Int. Ed. 2004, 43, 6414-6416.

8. Kisselev, A. F.; Goldberg, A. L. Chem. Biol. 2001, 8, 739-758.

9. Palombella, V. J.; Rando, O. J.; Goldberg, A. L.; Maniatis, T. Cell 1994, 78, 773-785.

10. Drexler, H. C. A.; Risau, W.; Konerding, M. A. FASEB J. 2000, 14, 65-77.

11. Oikawa, T.; Sasaki, T.; Nakamura, M.; Shimamura, M.; Tanahashi, N.; Omura, S.; Tanaka, K. Biochem. Biophys. Res. Commun. 1998, 246, 243-248.

12. Borissenko, L.; Groll, M. Chem. Rev. 2007, 107, 687-717.

13. (a) Fenteany, G.; Standaert, R. F.; Reichard, G. A.; Corey, E. J.; Schreiber, S. L. Proc. Natl. Acad. Sci. U. S. A. 1994, 91, 3358-3362.

(b) Fenteany, G.; Standaert, R. F.; Lane, W. S.; Choi, S.; Corey, E. J.; Schreiber, S. L. Science 1995, 268, 726-731. 
14. Groll, M.; Ditzel, L.; Loewe, J.; Stock, D.; Bochtler, M.; Bartunik, H. D.; Huber, R. Nature 1997, 386, 463-471.

15. Dick, L. R.; Cruikshank, A. A.; Grenier, L.; Melandri, F. D.; Nunes, S. L.; Stein, R. L. J. Biol. Chem. 1996, 271, 7273-7276.

16. Masse, C. E.; Morgan, A. J.; Adams, J.; Panek, J. S. Eur. J. Org. Chem. 2000, 2513-2528.

17. Corey, E. J.; Reichard, G. A. J. Am. Chem. Soc. 1992, 114, 10677-10678.

18. Corey, E. J.; Li, W.-D. Z.; Nagamitsu, T.; Fenteany, G. Tetrahedron 1999, 55, 3305-3316.

19. Corey, E. J.; Choi, S. Tetrahedron Lett. 1993, 34, 6969-6972.

20. Groll, M.; Huber, R.; Potts, B. C. M. J. Am. Chem. Soc. 2006, 128, 5136-5141.

21. Macherla, V. R.; Mitchell, S. S.; Manam, R. R.; Reed, K. A.; Chao, T.-H.; Nicholson, B.; Deyanat-Yazdi, G.; Mai, B.; Jensen, P. R.; Fenical, W. F.; Neuteboom, S. T. C.; Lam, K. S.; Palladino, M. A.; Potts, B. C. M. J. Med. Chem. 2005, 48, 3684-3687.

22. Reddy, L. R.; Fournier, J.-F.; Reddy, B. V. S.; Corey, E. J. Org. Lett. 2005, 7, 2699-2701.

23. Reddy, L. R.; Fournier, J.-F.; Reddy, B. V. S.; Corey, E. J. J. Am. Chem. Soc. 2005, 127, 8974-8976.

24. Hogan, P. C.; Corey, E. J. J. Am. Chem. Soc. 2005, 127, 15386-15387.

25. Williams, P. G.; Buchanan, G. O.; Feling, R. H.; Kauffman, C. A.; Jensen, P. R.; Fenical, W. J. Org. Chem. 2005, 70, 6196-6203.

26. Reddy, L. R.; Saravanan, P.; Corey, E. J. J. Am. Chem. Soc. 2004, 126, 6230-6231.

27. Endo, A.; Danishefsky, S. J. J. Am. Chem. Soc. 2005, 127, 8298-8299.

28. Ling, T.; Macherla, V. R.; Manam, R. R.; McArthur, K. A.; Potts, B. C. M. Org. Lett. 2007, 9, 2289-2292.

29. Mulholland, N. P.; Pattenden, G.; Walters, I. A. S. Org. Biomol. Chem. 2006, 4, 2845-2846.

30. Ma, G.; Nguyen, H.; Romo, D. Org. Lett. 2007, 9, 2143-2146.

31. Caubert, V.; Masse, J.; Retailleau, P.; Langlois, N. Tetrahedron Lett. 2006, 48, 381-384. 
32. Caubert, V.; Langlois, N. Tetrahedron Lett. 2006, 47, 4473-4475.

33. Corey, E. J.; Li, W.; Nagamitsu, T. Angew. Chem., Int. Ed. 1998, 37, 1676-1679.

34. (a) Crane, S. N.; Corey, E. J. Org. Lett. 2001, 3, 1395-1397.

(b) Saravanan, P.; Corey, E. J. J. Org. Chem. 2003, 68, 2760-2764.

35. (a) Dess, D. B.; Martin, J. C. J. Org. Chem. 1983, 48, 4155-4156.

(b) Dess, D. B.; Martin, J. C. J. Am. Chem. Soc. 1991, 113, 7277-7287.

36. Miyake, H.; Yamamura, K. Chem. Lett. 1992, 507-508.

37. (a) Fleming, I. Chemtracts: Org. Chem. 1996, 9, 1-64.

(b) Jones, G. R.; Landais, Y. Tetrahedron 1996, 52, 7599-7662.

(c) Fleming, I.; Barbero, A.; Walter, D. Chem. Rev. 1997, 97, 2063-2192.

38. (a) Sato, F.; Okamoto, S. Adv. Synth. Catal. 2001, 343, 759-784.

(b) Kulinkovich, O. G. Chem. Rev. 2003, 103, 2597-2632.

39. Reddy, L. R.; Saravanan, P.; Fournier, J.-F.; Reddy, B. V. S.; Corey, E. J. Org. Lett. 2005, 7, 2703-2705.

40. (a) Kablaoui, N. M.; Buchwald, S. L. J. Am. Chem. Soc. 1996, 118, 3182-3191.

(b) Mandal, S. K.; Amin, S. R.; Crowe, W. E. J. Am. Chem. Soc. 2001, 123, 6457-6458.

(c) Crowe, W. E.; Vu, A. T. J. Am. Chem. Soc. 1996, 118, 1557-1558.

(d) Quan, L. G.; Cha, J. K. Tetrahedron Lett. 2001, 42, 8567-8569.

41. Lee, G. H.; Choi, E. B.; Lee, E.; Pak, C. S. J. Org. Chem. 1994, 59, 1428-1443.

42. Reddy, B. V. S.; Reddy, L. R.; Corey, E. J. Tetrahedron Lett. 2005, 46, 4589-4593.

43. (a) Thottathil, J. K.; Moniot, J. L.; Mueller, R. H.; Wong, M. K. Y.; Kissick, T. P. J. Org. Chem. 1986, 51, 3140-3143.

(b) Hamada, Y.; Kawai, A.; Kohno, Y.; Hara, O.; Shioiri, T. J. Am. Chem. Soc. 1989, $111,1524-1525$.

(c) Hamada, Y.; Hara, O.; Kawai, A.; Kohno, Y.; Shioiri, T. Tetrahedron 1991, $47,8635-8652$.

44. Lee, M.; Kim, D. H. Bioorg. Med. Chem. 2002, 10, 913-922.

45. (a) Langlois, N.; Nguyen, B. K. L. J. Org. Chem. 2004, 69, 7558-7564.

(b) Mota, A. J.; Langlois, N. Tetrahedron Lett. 2003, 44, 1141-1143. 
46. Evans, D. A.; Ennis, M. D.; Le, T.; Mandel, N.; Mandel, G. J. Am. Chem. Soc. 1984, 106, 1154-1156.

47. Pfitzner, K. E.; Moffatt, J. G. J. Am. Chem. Soc. 1965, 87, 5661-5670.

48. Dieter Seebach, A. R. S. M. H. Angew. Chem., Int. Ed. Engl. 1996, 35, 2708-2748.

49. (a) Griffith, W. P.; Ley, S. V. Aldrichimica Acta 1990, 23, 13-19.

(b) Ley, S. V.; Norman, J.; Griffith, W. P.; Marsden, S. P. Synthesis 1994, 639-666.

50. Srebnik, M.; Ramachandran, P. V. Aldrichimica Acta 1987, 20, 9-24.

51. Simig, G.; Doleschall, G.; Hornyak, G.; Fetter, J.; Lempert, K.; Nyitrai, J.; Huszthy, P.; Gizur, T.; Kajtar-Peredy, M. Tetrahedron 1985, 41, 479-484.

52. Ponsford, R. J.; Southgate, R. J. Chem. Soc., Chem. Commun. 1979, 846-847.

53. Clemens, R. J. Chem. Rev. 1986, 86, 241-318.

54. Liu, J.; Wong, C.-H. Tetrahedron Lett. 2002, 43, 4037-4039.

55. (a) Carroll, M. F.; Bader, A. R. J. Am. Chem. Soc. 1952, 74, 6305-6305:

(b) Carroll, M. F.; Bader, A. R. J. Am. Chem. Soc. 1953, 75, 5400-5402.

56. Clemens, R. J.; Hyatt, J. A. J. Org. Chem. 1985, 50, 2431-2435.

57. (a) Davis, C. R.; Swenson, D. C.; Burton, D. J. J. Org. Chem. 1993, 58, 6843-6850.

(b) Lynch, C. L.; Hale, J. J.; Budhu, R. J.; Gentry, A. L.; Finke, P. E.; Caldwell, C. G.; Mills, S. G.; MacCoss, M.; Shen, D. M.; Chapman, K. T.; Malkowitz, L.; Springer, M. S.; Gould, S. L.; DeMartino, J. A.; Siciliano, S. J.; Cascieri, M. A.; Carella, A.; Carver, G.; Holmes, K.; Schleif, W. A.; Danzeisen, R.; Hazuda, D.; Kessler, J.; Lineberger, J.; Miller, M.; Emini, E. Org. Lett. 2003, 5, 2473-2475.

58. Smith, N. D.; Mancuso, J.; Lautens, M. Chem. Rev. 2000, 100, 3257-3282.

59. Evans, D. A.; Allison, B. D.; Yang, M. G.; Masse, C. E. J. Am. Chem. Soc. 2001, 123, 10840-10852.

60. Yamazaki, S.; Sakamoto, M.; Suzuri, M.; Doi, M.; Nakazawa, T.; Kobayashi, Y. J. Chem. Soc., Perkin Trans. 1 2001, 1870-1875.

61. Omura, K.; Swern, D. Tetrahedron 1978, 34, 1651-1660.

62. Donohoe, T. J.; Sintim, H. O.; Sisangia, L.; Harling, J. D. Angew. Chem., Int. Ed. 2004, 43, 2293-2296. 
63. (a) Reich, H. J.; Kulicke, K. J. J. Am. Chem. Soc. 1996, 118, 273-274.

(b) Sikorski, W. H.; Reich, H. J. J. Am. Chem. Soc. 2001, 123, 6527-6535.

64. Seebach, D. Angew. Chem. 1988, 100, 1685-1715.

65. Lam, H. W.; Murray, G. J.; Firth, J. D. Org. Lett. 2005, 7, 5743-5746.

66. Lam, H. W.; Joensuu, P. M. Org. Lett. 2005, 7, 4225-4228.

67. (a) Lam, H. W.; Joensuu, P. M.; Murray, G. J.; Fordyce, E. A. F.; Prieto, O.; Luebbers, T. Org. Lett. 2006, 8, 3729-3732.

(b) Joensuu, P. M.; Murray, G. J.; Fordyce, E. A. F.; Luebbers, T.; Lam, H. W. J. Am. Chem. Soc. 2008, 130, in press.

68. (a) Palomo, C.; Oiarbide, M.; Garcia, J. M. Chem. Eur. J. 2002, 8, 36-44.

(b) Casiraghi, G.; Zanardi, F.; Appendino, G.; Rassu, G. Chem. Rev. 2000, 100, 1929-1972.

(c) Machajewski, T. D.; Wong, C.-H.; Lerner, R. A. Angew. Chem., Int. Ed. 2000, 39, 1352-1374.

(d) Carreira, E. M. Comprehensive Asymmetric Catalysis I-III 1999, 3, 997-1065.

(e) Mahrwald, R. Chem. Rev. 1999, 99, 1095-1120.

(f) Groger, H.; Vogl, E. M.; Shibasaki, M. Chem. Eur. J. 1998, 4, 1137-1141.

69. Schinzer, D. Mod. Aldol React. 2004, 1, 311-328.

70. Prabhat; Qin, H. Tetrahedron 2000, 56, 917-947.

71. (a) Crimmins, M. T.; King, B. W.; Tabet, E. A. J. Am. Chem. Soc. 1997, 119, 7883-7884.

(b) Crimmins, M. T.; Chaudhary, K. Org. Lett. 2000, 2, 775-777.

(c) Crimmins, M. T.; King, B. W.; Tabet, E. A.; Chaudhary, K. J. Org. Chem. 2001, 66, 894-902.

72. (a) Evans, D. A.; Kozlowski, M. C.; Murry, J. A.; Burgey, C. S.; Campos, K. R.; Connell, B. T.; Staples, R. J. J. Am. Chem. Soc. 1999, 121, 669-685.

(b) Evans, D. A.; Tedrow, J. S.; Shaw, J. T.; Downey, C. W. J. Am. Chem. Soc. 2002, 124, 392-393.

73. Oppolzer, W.; Blagg, J.; Rodriguez, I.; Walther, E. J. Am. Chem. Soc. 1990, $112,2767-2772$. 
74. Abiko, A.; Liu, J.-F.; Buske, D. C.; Moriyama, S.; Masamune, S. J. Am. Chem. Soc. 1999, 121, 7168-7169.

75. Paterson, I.; Norcross, R. D.; Ward, R. A.; Romea, P.; Lister, M. A. J. Am. Chem. Soc. 1994, 116, 11287-11314.

76. (a) Denmark, S. E.; Stavenger, R. A. J. Am. Chem. Soc. 2000, 122, 8837-8847.

(b) Denmark, S. E.; Stavenger, R. A. Acc. Chem. Res. 2000, 33, 432-440.

(c) Denmark, S. E.; Pham, S. M. Org. Lett. 2001, 3, 2201-2204.

77. Mukaiyama, T.; Narasaka, K.; Banno, K. Chem. Lett. 1973, 1011-1014.

78. Nelson, S. G. Tetrahedron: Asymmetry 1998, 9, 357-389.

79. (a) Nakagawa, M.; Nakao, H.; Watanabe, K. Chem. Lett. 1985, 391-394.

(b) Yamada, Y. M. A.; Yoshikawa, N.; Sasai, H.; Shibasaki, M. Angew. Chem., Int. Ed. Engl. 1997, 36, 1871-1873.

(c) Yoshikawa, N.; Yamada, Y. M. A.; Das, J.; Sasai, H.; Shibasaki, M. J. Am. Chem. Soc. 1999, 121, 4168-4178.

(d) List, B.; Lerner, R. A.; Barbas, C. F., III J. Am. Chem. Soc. 2000, 122, 2395-2396.

(e) Trost, B. M.; Ito, H. J. Am. Chem. Soc. 2000, 122, 12003-12004.

(f) Yoshikawa, N.; Kumagai, N.; Matsunaga, S.; Moll, G.; Ohshima, T.; Suzuki, T.; Shibasaki, M. J. Am. Chem. Soc. 2001, 123, 2466-2467.

(g) Alcaide, B.; Almendros, P. Eur. J. Org. Chem. 2002, 1595-1601.

80. (a) Stork, G.; Rosen, P.; Goldman, N. L. J. Am. Chem. Soc. 1961, 83, 2965-2966.

(b) Stork, G.; Rosen, P.; Goldman, N.; Coombs, R. V.; Tsuji, J. J. Am. Chem. Soc. 1965, 87, 275-286.

81. Huddleston, R. R.; Krische, M. J. Synlett 2003, 12-21.

82. Revis, A.; Hilty, T. K. Tetrahedron Lett. 1987, 28, 4809-4812.

83. Chiu, P. Synthesis 2004, 2210-2215.

84. Nishiyama, H.; Shiomi, T. Top. Curr. Chem. 2007, 279, 105-137.

85. Matsuda, I.; Takahashi, K.; Sato, S. Tetrahedron Lett. 1990, 31, 5331-5334.

86. Taylor, S. J.; Morken, J. P. J. Am. Chem. Soc. 1999, 121, 12202-12203.

87. Taylor, S. J.; Duffey, M. O.; Morken, J. P. J. Am. Chem. Soc. 2000, 122, 4528-4529. 
88. Fuller, N. O.; Morken, J. P. Org. Lett. 2005, 7, 4867-4869.

89. Zhao, C.-X.; Duffey, M. O.; Taylor, S. J.; Morken, J. P. Org. Lett. 2001, 3, 1829-1831.

90. Duffey, M. O.; LeTiran, A.; Morken, J. P. J. Am. Chem. Soc. 2003, 125, 12666.

91. (a) Emiabata-Smith, D.; McKillop, A.; Mills, C.; Motherwell, W. B.; Whitehead, A: J. Synlett 2001, 1302-1304.

(b) Freiria, M.; Whitehead, A. J.; Tocher, D. A.; Motherwell, W. B. Tetrahedron 2004, 60, 2673-2692.

92. Freiria, M.; Whitehead, A. J.; Motherwell, W. B. Synthesis 2005, 3079-3084.

93. Isayama, S.; Mukaiyama, T. Chem. Lett. 1989, 2005-2008.

94. Baik, T.-G.; Luis, A. L.; Wang, L.-C.; Krische, M. J. J. Am. Chem. Soc. 2001, $123,5112-5113$.

95. Wang, L.-C.; Jang, H.-Y.; Roh, Y.; Lynch, V.; Schultz, A. J.; Wang, X.; Krische, M. J. J. Am. Chem. Soc. 2002, 124, 9448-9453.

96. Jang, H.-Y.; Huddleston, R. R.; Krische, M. J. J. Am. Chem. Soc. 2002, 124, 15156-15157.

97. (a) Jang, H.-Y.; Krische, M. J. Eur. J. Org. Chem. 2004, 3953-3958.

(b) Jang, H.-Y.; Krische, M. J. Acc. Chem. Res. 2004, 37, 653-661.

98. Huddleston, R. R.; Krische, M. J. Org. Lett. 2003, 5, 1143-1146.

99. (a) Koech, P. K.; Krische, M. J. Org. Lett. 2004, 6, 691-694.

(b) Marriner, G. A.; Garner, S. A.; Jang, H.-Y.; Krische, M. J. J. Org. Chem. 2004, 69, 1380-1382.

(c) Jung, C.-K.; Krische, M. J. J. Am. Chem. Soc. 2006, 128, 17051-17056.

(d) Han, S. B.; Krische, M. J. Org. Lett. 2006, 8, 5657-5660.

(e) Jung, C.-K.; Garner, S. A.; Krische, M. J. Org. Lett. 2006, 8, 519-522.

100. Huddleston, R. R.; Cauble, D. F.; Krische, M. J. J. Org. Chem. 2003, 68, 11-14. 101. (a) Miyai, T.; Inoue, K.; Yasuda, M.; Shibata, I.; Baba, A. Tetrahedron Lett. 1998, 39, 1929-1932.

(b) Inoue, K.; Ishida, T.; Shibata, I.; Baba, A. Adv. Synth. Catal. 2002, 344, 283-287.

(c) Shibata, I.; Kato, H.; Ishida, T.; Yasuda, M.; Baba, A. Angew. Chem., Int. Ed. 2004, 43, 711-714. 
102. Miura, K.; Yamada, Y.; Tomita, M.; Hosomi, A. Synlett 2004, 1985-1989.

103. Mahoney, W. S.; Brestensky, D. M.; Stryker, J. M. J. Am. Chem. Soc. 1988, 110, 291-293.

104. Chiu, P.; Chen, B.; Cheng, K. F. Tetrahedron Lett. 1998, 39, 9229-9232.

105. Chiu, P.; Szeto, C. P.; Geng, Z.; Cheng, K. F. Org Lett 2001, 3, 1901-1903.

106. Chiu, P.; Szeto, C. P.; Geng, Z.; Cheng, K. F. Tetrahedron Lett. 2001, 42, 4091-4093.

107. (a) Mori, A.; Fujita, A. Chem. Commun. 1997, $2159-2160$.

(b) Ito, H.; Ishizuka, T.; Arimoto, K.; Miura, K.; Hosomi, A. Tetrahedron Lett. 1997, 38, 8887-8890.

108. (a) Appella, D. H.; Moritani, Y.; Shintani, R.; Ferreira, E. M.; Buchwald, S. L. J. Am. Chem. Soc. 1999, 121, 9473-9474.

(b) Hughes, G.; Kimura, M.; Buchwald, S. L. J. Am. Chem. Soc. 2003, 125, 11253-11258.

(c) Rainka, M. P.; Aye, Y.; Buchwald, S. L. Proc. Natl. Acad. Sci. U. S. A. 2004, 101, 5821-5823.

109. (a) Lipshutz, B. H.; Servesko, J. M. Angew. Chem., Int. Ed. 2003, 42, 4789-4792.

(b) Lipshutz, B. H.; Servesko, J. M.; Petersen, T. B.; Papa, P. P.; Lover, A. A. Org. Lett. 2004, 6, 1273-1275.

(c) Lipshutz, B. H.; Servesko, J. M.; Taft, B. R. J. Am. Chem. Soc. 2004, 126, 8352-8353.

110. Lipshutz, B. H.; Chrisman, W.; Noson, K.; Papa, P.; Sclafani, J. A.; Vivian, R. W.; Keith, J. M. Tetrahedron 2000, 56, 2779-2788.

111. Chiu, P.; Leung, S. K. Chem. Commun. 2004, 2308-2309.

112. (a) Kimura, M.; Miyachi, A.; Kojima, K.; Tanaka, S.; Tamaru, Y. J. Am. Chem. Soc. 2004, 126, 14360-14361.

(b) Kimura, M.; Ezoe, A.; Shibata, K.; Tamaru, Y. J. Am. Chem. Soc. 1998, 120, 4033-4034.

(c) Molinaro, C.; Jamison, T. F. J. Am. Chem. Soc. 2003, 125, 8076-8077.

(d) Miller, K. M.; Huang, W.-S.; Jamison, T. F. J. Am. Chem. Soc. 2003, 125, 3442-3443. 
(e) Molinaro, C.; Jamison Timothy, F. Angew Chem Int Ed Engl 2004, 44, 129-132.

113. Chrovian, C. C.; Montgomery, J. Org. Lett. 2007, 9, 537-540.

114. Montgomery, J. Angew. Chem., Int. Ed. 2004, 43, 3890-3908.

115. Hratchian, H. P.; Chowdhury, S. K.; Gutierrez-Garcia, V. M.; Amarasinghe, K. K. D.; Heeg, M. J.; Schlegel, H. B.; Montgomery, J. Organometallics 2004, 23, 4636-4646.

116. Fournet, G.; Balme, G.; Gore, J. Tetrahedron 1991, 47, 6293-6304.

117. Marigo, M.; Juhl, K.; Jorgensen, K. A. Angew. Chem., Int. Ed. 2003, 42, 1367-1369.

118. (a) Maria Lumbierres, C. M. M. M.-M. Rosa M. S. A. V. E. L. E. M. Eur. J. Org. Chem. 2001, 2001, 2321-2328.

(b) Gimbert, C.; Lumbierres, M.; Marchi, C.; Moreno-Manas, M.; Sebastian, R. M.; Vallribera, A. Tetrahedron 2005, 61, 8598-8605.

119. (a) Luche, J. L. J. Am. Chem. Soc. 1978, 100, 2226-2227.

(b) Hart, D. J.; Li, J.; Wu, W.-L.; Kozikowski, A. P. J. Org. Chem. 1997, 62, 5023-5033.

120. Ichikawa, Y.; Egawa, H.; Ito, T.; Isobe, M.; Nakano, K.; Kotsuki, H. Org. Lett. 2006, 8, 5737-5740.

121. Reddy, L. R.; Corey, E. J. Org. Lett. 2006, 8, 1717-1719.

122. Fontana, A. J. Org. Chem. 2001, 66, 2506-2508.

123. Ito, M.; Clark, C. W.; Mortimore, M.; Goh, J. B.; Martin, S. F. J. Am. Chem. Soc. 2001, 123, 8003-8010.

124. Davies, S. G.; Haggitt, J. R.; Ichihara, O.; Kelly, R. J.; Leech, M. A.; Price Mortimer, A. J.; Roberts, P. M.; Smith, A. D. Org. Biomol. Chem. 2004, 2, 2630-2649.

125. Masuda, M.; Jonkheijm, P.; Sijbesma, R. P.; Meijer, E. W. J. Am. Chem. Soc. 2003, 125, 15935-15940.

126. Sergius, R. Ber. 1887, 20, 1210-1211.

127. Fuerstner, A. Synthesis 1989, 571-590.

128. Ocampo, R.; Dolbier, W. R., Jr. Tetrahedron 2004, 60, 9325-9374.

129. Orsini, F.; Sello, G. Curr. Org. Synth. 2004, 1, 111-135. 
130. Rieke, R. D. Acc. Chem. Res. 1977, 10, 301-306.

131. Maruoka, K.; Hashimoto, S.; Kitagawa, Y.; Yamamoto, H.; Nozaki, H. J. Am. Chem. Soc. 1977, 99, 7705-7707.

132. Stokker, G. E.; Hoffman, W. F.; Alberts, A. W.; Cragoe, E. J., Jr.; Deana, A. A.; Gilfillan, J. L.; Huff, J. W.; Novello, F. C.; Prugh, J. D.; et al. J. Med. Chem. 1985, 28, 347-358.

133. Sanchez, M.; Bermejo, F. Tetrahedron Lett. 1997, 38, 5057-5060.

134. Ruggeri, R. B.; Heathcock, C. H. J. Org. Chem. 1987, 52, 5745-5746.

135. Kanai, K.; Wakabayashi, H.; Honda, T. Org. Lett. 2000, 2, 2549-2551.

136. (a) Conan, A.; Sibille, S.; Perichon, J. J. Org. Chem. 1991, 56, 2018-2024.

(b) McHarek, S.; Sibille, S.; Nedelec, J. Y.; Perichon, J. J. Organomet. Chem. 1991, 401, 211-215.

137. Ding, Y.; Zhao, G. J. Chem. Soc., Chem. Commun. 1992, 941-942.

138. Kigoshi, H.; Kita, M.; Ogawa, S.; Itoh, M.; Uemura, D. Org. Lett. 2003, 5, 957-960.

139. Namy, J. L.; Girard, P.; Kagan, H. B. Nouv. J. Chim. 1977, 1, 5-7.

140. Girard, P.; Namy, J. L.; Kagan, H. B. J. Am. Chem. Soc. 1980, 102, 2693-2698.

141. Kagan, H. B.; Namy, J. L.; Girard, P. Tetrahedron 1981, 37, 175-180.

142. (a) Molander, G. A. Chem. Rev. 1992, 92, 29-68.

(b) Molander, G. A.; Harris, C. R. Chem. Rev. 1996, 96, 307-338.

(c) Krief, A.; Laval, A.-M. Chem. Rev. 1999, 99, 745-777.

(d) Steel, P. G. J. Chem. Soc., Perkin Trans. 1 2001, 2727-2751.

(e) Kagan, H. B. Tetrahedron 2003, 59, 10351-10372.

(f) Edmonds, D. J.; Johnston, D.; Procter, D. J. Chem. Rev. 2004, 104, 3371-3403.

143. (a) Imamoto, T.; Ono, M. Chem. Lett. 1987, 501-502.

(b) Molander, G. A.; Kenny, C. J. Org. Chem. 1991, 56, 1439-1445.

144. (a) Curran, D. P.; Fevig, T. L.; Jasperse, C. P.; Totleben, M. J. Synlett 1992, 943-961.

(b) Curran, D. P.; Totleben, M. J. J. Am. Chem. Soc. 1992, 114, 6050-6058.

145. (a) Hamann, B.; Namy, J.-L.; Kagan, H. B. Tetrahedron 1996, 52, $14225-14234$. 
(b) Hamann-Gaudinet, B.; Namy, J.-L.; Kagan, H. B. Tetrahedron Lett. 1997, 38, 6585-6588.

146. Inanaga, J.; Ishikawa, M.; Yamaguchi, M. Chem. Lett. 1987, 1485-1486.

147. Shabangi, M.; Flowers, R. A., II Tetrahedron Lett. 1997, 38, 1137-1140.

148. Hou, Z.; Zhang, Y.; Wakatsuki, Y. Bull. Chem. Soc. Jpn. 1997, 70, 149-153.

149. Rasmus J. Enemærke, T. H. T. S. K. D. Chem.--Eur. J. 2000, 6, 3747-3754.

150. Shabangi, M.; Sealy, J. M.; Fuchs, J. R.; Flowers, R. A., II Tetrahedron Lett. 1998, 39, 4429-4432.

151. Aoyagi, Y.; Yoshimura, M.; Tsuda, M.; Tsuchibuchi, T.; Kawamata, S.; Tateno, H.; Asano, K.; Nakamura, H.; Obokata, M.; et al. J. Chem. Soc., Perkin Trans. 1 1995, 689-692.

152. Fuchs, J. R.; Mitchell, M. L.; Shabangi, M.; Flowers, R. A., II Tetrahedron Lett. 1997, 38, 8157-8158.

153. Miller, R. S.; Sealy, J. M.; Shabangi, M.; Kuhlman, M. L.; Fuchs, J. R.; Flowers, R. A. J. Am. Chem. Soc. 2000, 122, 7718-7722.

154. Machrouhi, F.; Hamann, B.; Namy, J. L.; Kagan, H. B. Synlett 1996, 633-634.

155. Molander, G. A.; Alonso-Alija, C. J. Org: Chem. 1998, 63, 4366-4373.

156. Inanaga, J.; Yokoyama, Y.; Handa, Y.; Yamaguchi, M. Tetrahedron Lett. 1991, 32, 6371-6374.

157. (a) Molander, G. A.; Etter, J. B. J. Am. Chem. Soc. 1987, 109, 6556-6558.

(b) Molander, G. A.; Etter, J. B.; Harring, L. S.; Thorel, P. J. J. Am. Chem. Soc. 1991, 113, 8036-8045.

158. Qiao, L.; Wang, S.; George, C.; Lewin, N. E.; Blumberg, P. M.; Kozikowski, A. P. J. Am. Chem. Soc. 1998, 120, 6629-6630.

159. Benfatti, F.; Cardillo, G.; Fabböroni, S.; Gentilucci, L.; Perciaccante, R.; Piccinelli, F.; Tolomelli, A. Synthesis 2005, 61-70.

160. Shimada, T.; Yoshioka, M.; Konno, T.; Ishihara, T. Org. Lett. 2006, 8, 1129-1131.

161. Adrian, J. C., Jr.; Snapper, M. L. J. Org. Chem. 2003, 68, 2143-2150.

162. (a) Jin, H.; Uenishi, J.; Christ, W. J.; Kishi, Y. J. Am. Chem. Soc. 1986, 108, 5644-5646. 
(b) Takai, K.; Tagashira, M.; Kuroda, T.; Oshima, K.; Utimoto, K.; Nozaki, H. J. Am. Chem. Soc. 1986, 108, 6048-6050.

163. (a) Fuerstner, A.; Shi, N. J. Am. Chem. Soc. 1996, 118, 2533-2534.

(b) Fuerstner, A.; Shi, N. J. Am. Chem. Soc. 1996, 118, 12349-12357.

164. Furstner, A. Chem. Rev. 1999, 99, 991-1046.

165. Dubois, J.-E.; Axiotis, G.; Bertounesque, E. Tetrahedron Lett. 1985, 26, 4371-4372.

166. Wessjohann, L.; Wild, H. Synlett 1997, 731-733.

167. Wessjohann, L.; Wild, H. Synthesis 1997, 512-514.

168. Wessjohann, L.; Gabriel, T. J. Org. Chem. 1997, 62, 3772-3774.

169. Ichikawa, S.; Shuto, S.; Minakawa, N.; Matsuda, A. J. Org. Chem. 1997, 62, 1368-1375.

170. (a) Corey, E. J.; Zheng, G. Z. Tetrahedron Lett. 1997, 38, 2045-2048.

(b) Orsini, F.; Lucci, E. M. Tetrahedron Lett. 2005, 46, 1909-1911.

171. Halland, N.; Braunton, A.; Bachmann, S.; Marigo, M.; Jorgensen, K. A. J. Am. Chem. Soc. 2004, 126, 4790-4791.

172. Abiko, A.; Roberts, J. C.; Takemasa, T.; Masamune, S. Tetrahedron Lett. 1986, $27,4537-4540$.

173. Chang, A. H. C.; Horton, D.; Kovac, P. Tetrahedron: Asymmetry 2000, 11, 595--606.

174. Hirai, K.; Ooi, H.; Esumi, T.; Iwabuchi, Y.; Hatakeyama, S. Org. Lett. 2003, 5, 857-859.

175. Beccalli, E. M.; Marchesini, A.; Pilati, T. Tetrahedron 1992, 48, 5359-5374.

176. Sharma, A.; Chattopadhyay, S. J. Org. Chem. 1999, 64, 8059-8062.

177. Harpp, D. N.; Bao, L. Q.; Black, C. J.; Gleason, J. G.; Smith, R. A. J. Org. Chem. 1975, 40, 3420-3427.

178. Wang, P.; Tang, Y.; Tirrell, D. A. J. Am. Chem. Soc. 2003, 125, 6900-6906.

179. Jacobi, P. A.; Murphree, S.; Rupprecht, F.; Zheng, W. J. Org. Chem. 1996, 61, 2413-2427.

180. Minami, I.; Yuhara, M.; Watanabe, H.; Tsuji, J. J. Organomet. Chem. 1987, $334,225-242$.

181. Clarke, P. A. Tetrahedron Lett. 2002, 43, 4761-4763. 
182. Zimmerman, H. E.; Traxler, M. D. J. Am. Chem. Soc. 1957, 79, 1920-1923.

183. Kimura, M.; Ezoe, A.; Mori, M.; Iwata, K.; Tamaru, Y. J. Am. Chem. Soc. 2006, 128, 8559-8568.

184. Still, W. C.; Kahn, M.; Mitra, A. J. Org. Chem. 1978, 43, 2923-2925.

185. Wallner, O. A.; Szabo, K. J. Org. Lett. 2004, 6, 1829-1831.

186. Kende, A. S.; Fludzinski, P. Org. Synth. 1986, 64, 104-107.

187. Chih-Yuan Chuang, V. C. V. Z. M. R. G. I. O. Chirality, 2002, 14, 151-162.

188. Hyunsoo Han, C.-W. C. K. D. J. Chem.--Eur. J. 1999, 5, 1565-1569.

189. Lall, M. S.; Ramtohul, Y. K.; James, M. N. G.; Vederas, J. C. J. Org. Chem. 2002, 67, 1536-1547.

190. Kolb, H. C.; Kanamarlapudi, R. C.; Richardson, P. F.; Khan, G. U.S. Pat. Appl. Publ. 2002-263234, 2003153728, 20021002., 2003.

191. Barluenga, J.; Martinez-Gallo, J. M.; Najera, C.; Yus, M. Synthesis 1986, 678-680.

192. Zhu, G.; Negishi, E. i. Org. Lett. 2007, 9, 2771-2774.

193. Zhu, P. C.; Lin, J.; Pittman, C. U. J. Org. Chem. 1995, 60, 5729-5731.

194. Mattes, H.; Benezra, C. J. Org. Chem. 1988, 53, 2732-2737.

195. Villo, L.; Metsala, A.; Parve, O.; Pehk, T. Tetrahedron Lett. 2002, 43, 3203-3207. 